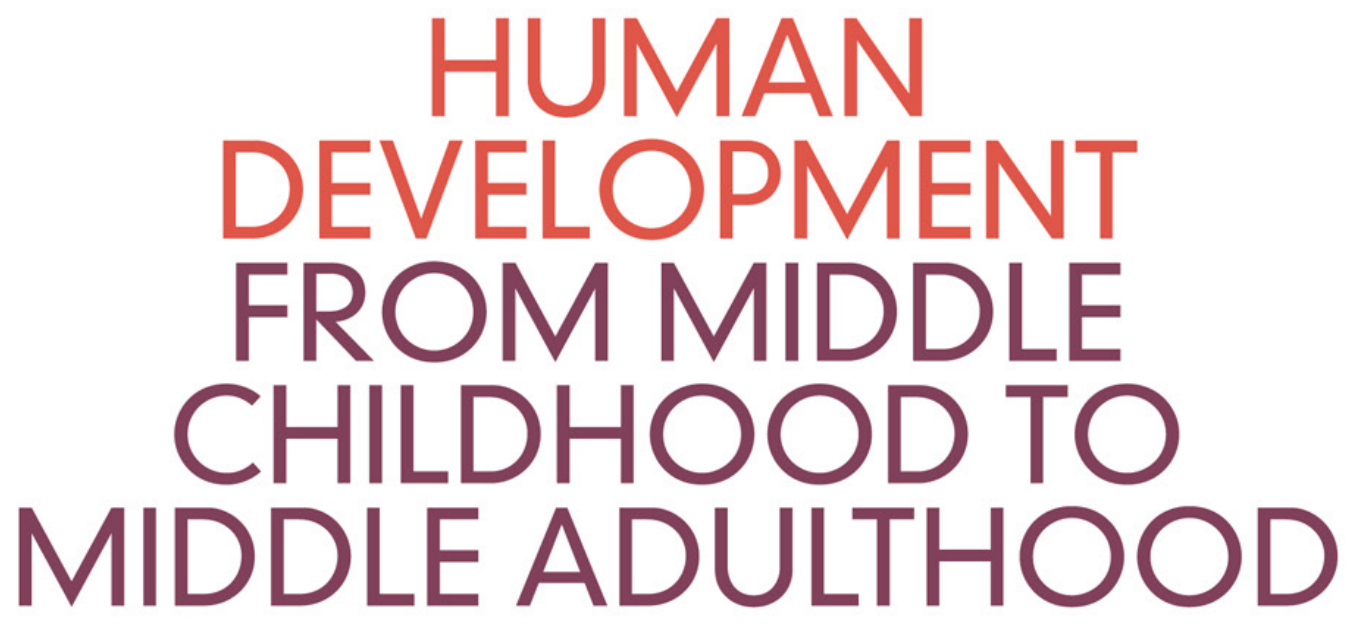

Growing Up to be Middle-Aged 


\section{HUMAN DEVELOPMENT FROM MIDDLE CHILDHOOD TO MIDDLE ADULTHOOD}

This seminal work focuses on human development from middle childhood to middle adulthood, through analysis of the research findings of the groundbreaking Jyväskylä Longitudinal Study of Personality and Social Development (JYLS). The JYLS project, which began in 1968, has generated extensive publications over many years but this is the first comprehensive summary that presents the conceptual framework, the research design and methodology, and the findings. The study looks at the development over time of issues related to personality, identity, health, anti-social behavior, and well-being and is unparalleled in its duration, intensity, comprehensiveness, and psychological richness.

The thorough synthesis of this study illustrates that there are different paths to adulthood and that human development cannot be described in average terms. The 42-year perspective that the JYLS provides shows the developmental consequences of children's differences in socioemotional behavior over time, and the great significance of children's positive socioemotional behavior for their further development until middle age.

Not only will the book be an invaluable tool for those considering research methods and analysis on large datasets, it is ideal reading for students on lifespan courses and researchers methodologically interested in longitudinal research.

Lea Pulkkinen, $\mathbf{P h D}$, is Professor of Psychology Emerita at the University of Jyväskylä, Finland. For 40 years, she has conducted a longitudinal study on personality and social development. Her interest has focused on the continuity of positive and problem behaviors over time, and transformation of findings into policy for improving the quality of life in childhood and adulthood. 
$\Longrightarrow$ Taylor \& Francis Taylor \& Francis Group

http://taylorandfrancis.com 


\section{HUMAN DEVELOPMENT FROM MIDDLE CHILDHOOD TO MIDDLE ADULTHOOD}

Growing Up to be Middle-Aged

Lea Pulkkinen

IN COLLABORATION WITH KATJA KOKKO

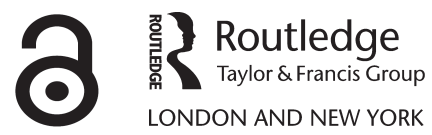


First published 2017

by Routledge

2 Park Square, Milton Park, Abingdon, Oxon OX14 4RN

and by Routledge

711 Third Avenue, New York, NY 10017

Routledge is an imprint of the Taylor E Francis Group, an informa business

(C) 2017 Lea Pulkkinen

The right of Lea Pulkkinen to be identified as the author of this work has been asserted by her in accordance with sections 77 and 78 of the Copyright, Designs and Patents Act 1988.

The Open Access version of this book, available at www.taylorfrancis.com, has been made available under a Creative Commons Attribution-Non Commercial-No Derivatives 4.0 license.

Trademark notice: Product or corporate names may be trademarks or registered trademarks, and are used only for identification and explanation without intent to infringe.

British Library Cataloguing-in-Publication Data

A catalogue record for this book is available from the British Library

Library of Congress Cataloging in Publication Data

A catalog record for this book has been requested

ISBN: 978-1-138-84014-0 (hbk)

ISBN: 978-1-138-84015-7 (pbk)

ISBN: 978-1-315-73294-7 (ebk)

DOI: $10.4324 / 9781315732947$

Typeset in Bembo

by Deanta Global Publishing Services, Chennai, India 
To the participants of the longitudinal study 
$\Longrightarrow$ Taylor \& Francis Taylor \& Francis Group

http://taylorandfrancis.com 


\section{CONTENTS}

List of figures

xiii

List of tables

List of boxes

xvii

Preface

xviii

PART I

Introduction to the Jyväskylä Longitudinal Study of Personality and Social Development

1 How the longitudinal study began 3

1.1 A historical context for the study of aggression 3

1.2 Positive behavior in an impulse control model and the goals of the study

1.2.1 The search for alternatives to aggression 6

$\begin{array}{ll}\text { 1.2.2 The impulse control model } & 7\end{array}$

1.2.3 The goals of the longitudinal study 9

1.3 Research conditions in Finland and in the longitudinal study 10

2 The execution of the longitudinal study 16

2.1 The research sample and the major waves of data collection 16

2.2 Attrition and the representativeness of the sample 19

2.3 Additional data collections $\quad 21$

2.4 Assessment methods $\quad 22$

2.4.1 Peer nomination and teacher rating 22

2.4.2 Personal interview $\quad 27$

2.4.3 Self-reports and other methods $\quad 30$

2.5 Data analysis 36 
3 Reflections on the research process 39

3.1 The evaluation of choices 39

3.2 Recommendations for launching a longitudinal study $\quad 40$

3.3 What is development? 43

PART II

The development of personality and psychological functioning

4 Theoretical frameworks for the study of socioemotional development 47

4.1 From cognitive control of impulses to self-control 47

4.2 Updated framework 49

4.2.1 The model for the Unfolding of

Socioemotional Behavior $\quad 49$

4.2.2 Self-regulation and executive functions $\quad 54$

4.2.3 Toward cumulative theoretical reasoning 58

4.3 Three layers of personality for organizing research findings $\quad 62$

5 The person as social actor 64

5.1 Questions to be answered 64

5.2 Socioemotional behavior in childhood and adolescence $\quad 65$

5.2.1 Agreement between informants $\quad 65$

5.2.2 Continuity in socioemotional behavior 67

5.2.3 The person-situation controversy 68

5.3 Children grow and adult styles of life emerge 71

5.3.1 The two-dimensional framework across generations $\quad 71$

5.3.2 The unfolding of the styles of life from age 8 to $27 \quad 74$

5.3.3 The developmental background of the styles of life $\quad 81$

5.3.4 A person-oriented approach to personal styles at age $27 \quad 85$

5.3.5 Personality profiles at ages 33 to $50 \quad 87$

5.4 Maturation, adjustment, or growth in adult personality 91

5.5 Childhood socioemotional behaviors as predictors of adult personality characteristics 96

5.5.1 Roots of the personality profiles and the clusters of personal styles 96

5.5.2 The roots of adult agreeableness and continuity in aggression 97

5.5.3 Paths from childhood socioemotional behavior to adult personality 
6 The person as motivated agent

6.1.1 Age-stratified or individualistic transitions?

6.1.2 Life changes in early adulthood 106

6.1.3 Patterns and timing of adult transitions 108

6.2 The length and timing of one's education 112

6.2.1 The structure of education $\quad 112$

6.2.2 Predictors of the choice of education 113

6.2.3 "Off-time" education and other post-comprehensive educational trajectories 116

6.3 Goals and values 117

6.3.1 Common elements in the life structure of adults $\quad 117$

6.3.2 Values in middle age 120

6.3.3 Individual life structures and unifying life themes 122

7 The person as autobiographical author and an

"experiencer" of life

125

7.1 A holistic approach to personality 125

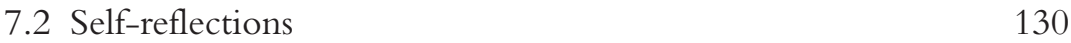

7.2.1 Identity formation 130

7.2.2 Personal control over one's own life 134

7.2.3 Optimism and self-esteem 135

7.3 Turning points 137

7.3.1 A study of turning points 137

7.3.2 Individual differences in the experience of turning points 138

7.3.3 A qualitative analysis of turning points $\quad 140$

8 Personality and psychological functioning 142

8.1 Personality and well-being 142

8.1.1 The concept of mental well-being 142

8.1.2 Continuity in well-being 144

8.1.3 The personality traits associated with mental well-being 145

8.2 The model for personality and psychological functioning $\quad 146$

8.3 Explanations for the associations between personality traits and psychological functioning 


\section{PART III}

\section{The development of social functioning}

9 Social development and social functioning 155

9.1 Conceptual approaches to social functioning 155

9.2 The spheres of life $\quad 157$

9.3 Processes in social functioning 158

10 Problems in social functioning 165

10.1 Antisocial development $\quad 165$

10.1.1 Antisocial behavior 165

10.1.2 From aggression to antisocial behavior 167

10.1.3 Proactive and reactive aggression as predictors of criminal offending 169

10.2 Accumulation of problems 171

10.2.1 Multi-problem predictors of problem behaviors 171

10.2.2 Dynamic processes in the accumulation of problems 175

10.2.3 Continuity from risk factors to social functioning problems 177

10.3 Offender groups compared 180

10.3.1 Offender groups 180

10.3.2 Differences in personality 182

10.3.3 Social background 184

10.3.4 Life success of the offender groups in adulthood 185

10.3.5 Conclusions and suggestions for prevention 187

11 Health behavior 189

11.1 Substance use 189

11.1.1 The start and continuity of the use of alcohol 189

11.1.2 Problem drinking in middle age 192

11.1.3 Socioemotional characteristics associated with drinking 194

11.1.4 Social backgrounds of the use of alcohol 195

11.1.5 Smoking cigarettes 196

11.2 Health risks 198

11.2.1 Health in early adulthood 198

11.2.2 The predictors of accidents and impairment 199

11.2.3 Subjective health and metabolic syndrome
in middle age

11.3 Temperance in adulthood 203 
12 The family and its care functions 207

12.1 A partnership in the family 207

12.1.1 The structure of the family 207

12.1.2 The relationship between the partners 209

12.1.3 Adult attachment 211

12.2 Parenting 213

12.2.1 Delights and stresses in parenting 213

12.2.2 Parental identity 214

12.2.3 Child-rearing practices 216

12.3 Relationships in the chain of generations 218

13 Work as a sphere of life 223

13.1 Occupational status and career line in women and men 223

13.2 Success in career path 228

13.2.1 Constructive behavior in childhood as the antecedent of success in the work domain 228

13.2.2 Satisfaction with work 231

13.2.3 Occupational well-being and personality 235

13.3 Job insecurity 236

13.3.1 Unemployment and psychological distress: selection or causation? 236

13.3.2 Health consequences of the unstable career line $\quad 240$

13.3.3 The consequences of economic strain for the family and parenting 241

13.4 Work-family balance 243

13.4.1 A stress theory approach to work-family interface 243

13.4.2 A role enhancement theory approach to workfamily interface 245

13.4.3 Successful reconciliation 247

13.5 Recovery from work and the content of free time 248

\section{PART IV}

Epilogue

14 Navigating through the spheres of life 255

14.1 Middle age: a prime time 255

14.2 Midlife transition 258

14.3 The person functioning in the different spheres of life $\quad 259$ 
xii Contents

15 The integrated model for individual differences in social and psychological functioning

16 A credo

References 273

Author index 302

Subject index 


\section{FIGURES}

1.1 A descriptive model of aggression 5

1.2 A framework for the analysis of aggressive and nonaggressive behaviors 8

4.1 The model for the Unfolding of Socioemotional Behavior 50

4.2 Executive functions and behavioral and emotional problems 57

4.3 Executive functions and adjustment 58

5.1 Developmental lines from childhood socioemotional behavior to adult styles of life $\quad 75$

5.2 Three clusters of personal styles for men and women at age $27 \quad 86$

5.3 Personality profiles characterized by personality traits at ages 33, 42, and 50

5.4 Personality profile groups located on a plane on the basis of their means in extraversion and conscientiousness (across ages 33, 42, and 50)

5.5 Continuity in behavioral activity from childhood through adolescence to adulthood

5.6 Continuity in well-controlled behavior from childhood through adolescence to adulthood

8.1 A model for personality and psychological functioning

9.1 A human being steering his or her functioning through different spheres of life

9.2 Childhood antecedents of adult social functioning: males

9.3 Paths from self-control and behavioral activity in childhood to middle-age psychological well-being: females and males 
xiv Figures

9.4 LISREL model of the cycle of maladaptation between aggression in childhood and long-term unemployment in adulthood

10.1 Interconnections among childhood risk factors and among adult social functioning problems. Two paths from childhood risk factors to adult social functioning problems for males

11.1 Means with 95 percent confidence intervals of the indicators of adult use of alcohol by the age of onset of drinking standardized across the whole sample

11.2 The continuity of habits to drink into intoxication from age 27 to 50; males and females

11.3 Trajectories of body mass index (BMI) in adulthood

13.1 Constructive behavior as an antecedent of success in work domain and psychological functioning: women

13.2 The participants' estimation of their household's financial situation at age 50 in relation to their monthly income in euros

13.3 The experience of work-family conflict and enrichment in relation to personality traits at age 42

15.1 The integrated model for individual differences in social and psychological functioning 


\section{TABLES}

2.1 The size of the sample in each of the major data collection waves: the sample was drawn for second-grade pupils at age 8

5.1 Concurrent validity and relative stability of socioemotional characteristics in peer nominations (PN) and teacher ratings (TR)

5.2 Means of parents and children with regard to the same socioemotional characteristics at the same ages; teacher ratings in 1968 and 1998

5.3 Correlations between fathers and sons in teacher ratings of socioemotional behaviors at ages 8 to 10

5.4 Significant connections between the styles of life at different ages and home backgrounds in adolescence

5.5 Items loaded significantly on a factor for child-centered parenting versus parent-centered parenting; based on interviews with the participants at the age of 14 and the interviews of one of each child's parents

5.6 Means (M) and standard deviations (SD) of the NEO-Five Factor Inventory (NEO-FFI) personality scales at ages 33, 42, and $50 ; n=95$ for women and 70 for men

5.7 Correlations between age 8 peer-nominated general childhood aggression and adult self-reported physical aggression by study and stratified by childhood parent occupational status and sex for the JYLS, Columbia County Longitudinal Study, USA (CCLS), and Child Development Project, USA (CDP)

6.1 Timing of adult transitions: cumulative percentages of women $(\mathrm{n}=132)$ and men $(\mathrm{n}=150)$ 
6.2 Comparison of the patterns of adult transitions in social and psychological functioning

6.3 How much pleasure do family, work, friends and leisure pursuits give people in relation to each other at different ages: rank orders from 1 to 4

6.4 Rank-order of the personal value domains at age 50

8.1 Probabilities of following low and high trajectories of psychological well-being

8.2 Means (M) and standard deviations (SD) of personality profiles averaged across ages 33, 42, and 50 in indicators of adult psychological functioning

10.1 Clusters of adjustment and their predictive value for educational career and criminal arrests: males $(\mathrm{M})$ and females $(\mathrm{F})$

10.2 Differences between the offender groups in adult social and psychological functioning: logistic regression analysis (p-values for significant odds ratios)

12.1 Official marital status in the age cohort group born in 1959 in Finland (data drawn in 2008) and in the JYLS sample at age 50 (in 2009)

13.1 Occupational status among the JYLS participants employed at ages 42 and 50, and in the age cohort group born in 1959 (data drawn in 2006) 


\section{BOXES}

1.1 The major stages and substages of the longitudinal study (JYLS) 14

2.1 Items for socioemotional behavior at ages 8 and 14 (peer nomination and teacher rating) and at ages 20,27,36, 42 , and 50 (self-rating)

5.1 Four developmental paths from childhood (C) through early adolescence (EA) and late adolescence (LA) to young adulthood (YA): research findings (Part II) summarized

5.2 Changes in the Big Five personality traits in men and women, and in the personality profile groups from age 33 to 50

6.1 Clusters for goal orientation with typical personal goals and life situation

10.1 The offender groups compared with the nonoffenders in socioemotional behavior at ages 8 and 14 , and in adult personality

15.1 Social functioning in different domains of life in relation to the participants' characteristics in childhood (C) and adulthood (A): research findings (Part III) summarized 


\section{PREFACE}

The purpose of this book is to present a synthesis of the results with regards to human development from the study entitled "The Jyväskylä Longitudinal Study of Personality and Social Development" (henceforth referred to as the JYLS). The study consists of several follow-up studies of the same individuals over the course of 42 years. The word Jyväskylä (Finnish pronunciation: "jyvaes kylae") in the title of the longitudinal study refers to the name of a town in Central Finland where the University of Jyväskylä, the home base of the study, is located. The rest of the title describes the major content areas of the research project.

The results of the study have been written up in numerous papers published over the past 50 years, beginning in the 1960s. The aim of this book is to give a broader picture of human development than can be found in the information included in the various articles. Metaphorically expressed, the process of writing the synthesis has been like assembling a huge jigsaw puzzle without seeing the completed picture in advance. The picture has been cut up into all sorts of odd shapes, and the challenge has been to figure out what picture all these pieces will make in the end. During the process of finding the pieces that fit together, the picture has slowly come into view. Gradually, the contours of the picture and the dominant figures have emerged, with the addition of small interesting details.

The work has been challenging for various reasons. First, dominant psychological theories and methods have changed and psychological knowledge has increased immensely over the past 50 years. Many concepts and research methods which are now commonly used were not available when this study began and were not available when many of its first follow-up studies took place. Second, the development of those who participated in the study has progressed from childhood (age 8) through to adolescence (age 14), late adolescence (age 20), early adulthood (age 27), young adulthood (age 36), and early middle adulthood (age 42), to the midst of middle adulthood (age 50). Third, human development has been followed 
in the multitude of the domains of life such as personality, education, work, family, health behavior, and social integration. Fourth, a great number of researchers with their personal interests have analyzed the data. Fifth, publishing practice in scientific journals guides researchers to analyze data with a limited focus. This then has increased the number of publications focusing on specific topics.

Writing a synthesis of the results of the longitudinal study has, consequently, demanded updating the terminology in the theoretical models, combining the results across different age ranges, integrating the findings across the domains of life, finding a good fit between the different interests of the researchers who have worked on the study, and coming to a view of how the view of development is augmented by each piece of information. In this book, references are made to around 180 publications based on the JYLS data. Some readers might expect that a book about human development will include biographies of individuals or that it will describe developmental stages. This book does not, however, present biographies. It was agreed with the participants of the longitudinal study that their individual life stories would not be published. Neither is this book a description of developmental stages. It has not been the goal of the study to research and describe developmental stages.

What is the picture that this book paints? The book illustrates that there are different paths to adulthood and that human development cannot be described in average terms. The 42-year perspective that the JYLS provides, shows the developmental consequences of children's differences in socioemotional behavior over time. The main contribution to the literature is to show the great significance of children's positive socioemotional behavior, also called "noncognitive skills," for their further development until middle age. Love given to the child provides him or her with a firm basis for the future. Life paths are not, however, predestined by childhood experiences; positive development may also start at a later age.

Fairy tales typically end with a phrase "and they lived happily ever after." This implies that after a happy wedding, nothing particularly special will happen in people's lives, and that the important developmental processes and events all take place before this. This kind of idea also used to be dominant in developmental psychology, which largely neglected the study of development during adulthood. At present, researchers are more aware of the entire human life-course and life-span development, and have a deeper and more profound knowledge of human development. This includes insights into how to make interventions which are benign and ethical for the development of individuals and the societies in which they live. Hopefully, this long-term longitudinal study contributes to an increased awareness of people's different paths in life toward a greater humanity, and inspires interventions to promote positive development.

This book is dedicated to the study participants who have made the longitudinal study possible by giving up their time for data collection, and by sharing their thoughts and experiences with researchers. The study started with 369 children, born in 1959, and the participation rate has remained high for the whole course of the study. A few of the participants have already passed away. 
The baseline study of this longitudinal project was a study of one age group, that is, a cross-sectional study, for my doctoral dissertation when the participants were 8 years of age, but it expanded into a follow-up study, that is, a longitudinal study, with the help of assistant, later Professor, Helena Hurme and a group of psychology students who were preparing their Master's theses (Pekka Aalto, Heleena Hurskainen, Anneli Kangas, Rauni Kanto, Arja Mönkkönen, and Anja Seila) from 1973 to 1974 under my supervision. The second follow-up study was also conducted with the help of the students (Ulla Kinnunen, Kari Rajaniemi, Sirpa Salo, and Anne Vedenoja) from 1979 to 1980. In the later stages of the study, tens of Master's degree students undertook data collection as interviewers. Ulla Kinnunen, Professor at the University of Tampere, Finland, became a collaborator in the area of work and family and she was the Co-Principal Investigator of the project from 2000 to 2002. Taru Feldt, Adjunct Professor at the University of Jyväskylä, has also been a long-term collaborator in the study on the area of work and personality.

The longitudinal data have been used in one or more internationally published articles included in a doctoral dissertation by: Anu Mustonen (media violence, 1997), Juha Perttula (experienced life, 1998), Anna Rönkä (accumulation of problems, 1999), Kaisa Aunola (parenting, 2001), Katja Kokko (unemployment, 2001), Marja Kokkonen (emotion regulation, 2001) , Kaisa Männikkö (attachment, 2001), Riitta-Leena Metsäpelto (parenting, 2003), Petri Juujärvi (aggression, 2003), Marja-Liisa Kinnunen (health, 2005), Anne Mäkikangas (distress, 2007), Tuuli Pitkänen (alcohol drinking, 2006), Päivi Fadjukoff (identity development, 2007), Johanna Rantanen (work-home interference, 2008), Eija Räikkönen (transition to adulthood, 2012), Jutta Viinikainen (personality and income, 2012), and Merja Hietalahti (values, 2016). These dissertations are electronically available at the University of Jyväskylä. They have had an invaluable significance for the analysis of the longitudinal data and publishing results.

Anna-Liisa Lyyra (statistician and data manager) has been responsible for maintaining and organizing the longitudinal data since the early 2000s. She and Asko Tolvanen have also participated in data analysis and have advised researchers about this aspect of the work. Their advice has been much needed in the complex processing and analysis of longitudinal data with rapidly developing software and computer capacities. During the 1980s and 1990s, Ari Mäkiaho was of great help to me with these tasks. Anna-Liisa Lyyra has also given me invaluable help with the preparation of figures and tables and additional data analyses for this book. I am also very grateful to Belinda Heys (editor) for her close reading of the manuscript and her English language editing.

In 1995, Katja Kokko joined the team for her Master's thesis, and continued her studies to doctoral degree level as part of this project. She participated in the design and execution of data collection in 2001, and has been committed to this study through her career. She was the Co-Principal Investigator of the project from 2009 to 2012, and has been the Principal Investigator since 2013. The responsibility for the longitudinal study has been transferred to the younger generation. Katja Kokko, Research Director at the Gerontology Research Centre of the University 
of Jyväskylä, has cooperated with national and international researchers who have been interested in the use of this longitudinal data in research, supervised students' theses based on this data, and has taken on the management of the project as a whole, including saving the data in the Finnish Social Science Data Archive. She has contributed to this book by providing additional data analyses, drafting the section on data analysis with Anna-Liisa Lyyra, and by making valuable comments and additions throughout the text, particularly in the area of mental well-being and work. Marja-Liisa Kinnunen, MD, PhD, was responsible for medical examinations when the participants were 42 and 50 years old.

I have been the Principal Investigator of the JYLS since its inception in the 1960s until 2012 and responsible for the text and the development of theoretical models. In this synthesis of the study, I have tried to consider the publications of my colleagues, as I am deeply aware that without the large team of researchers, it would not have been possible to conduct this longitudinal study. In some contexts, I use the first pronoun "I" because I have been responsible for a choice or the design of the study in question. When the team expanded, I have used collective pronouns to indicate this. This variation demonstrates the complexity of a longitudinal study in many respects.

The University of Jyväskylä, Finland has offered an excellent basis for research. The Academy of Finland has supported the study by awarding grants since the early 1970s (the latest grants - No. 127125 to Lea Pulkkinen and Nos. 118316 and 135347 to Katja Kokko). The Finnish Cultural Foundation awarded to me a special "eminentia grant" in 2015 for the purpose of writing up a synthesis from this lifetime's work, and I am very grateful for it.

Finally, I wish to thank those closest to me for their continued support and encouragement in this work.

Lea Pulkkinen 
$\Longrightarrow$ Taylor \& Francis Taylor \& Francis Group

http://taylorandfrancis.com 
PART I

Introduction to the Jyväskylä Longitudinal Study of Personality and Social Development 
$\Longrightarrow$ Taylor \& Francis Taylor \& Francis Group

http://taylorandfrancis.com 


\section{1}

\section{HOW THE LONGITUDINAL STUDY BEGAN}

\subsection{A historical context for the study of aggression}

Longitudinal studies were rare in the mid-1960s, but there were pioneering studies that had been initiated in the 1920s in California such as the Gifted Children Study by Terman (Terman \& Oden, 1959) and two Oakland studies, the Guidance Study by Macfarlane (1938) and the Oakland Growth Study by Jones (1938). Early longitudinal studies were often motivated by educational goals; that is, the possibility of advancing or steering child development. I had been interested in human development since childhood, when I had amused myself by trying to imagine what kind of adults the children whom I knew were going to become and, conversely, what kind of children the adults I knew had been when they were young. Through reading the longitudinal study conducted at the Fels Research Institute by Kagan and Moss (1962), I became interested in personality development. Children had been followed from birth up to adolescence and on into early adulthood. The study resulted in new insights into the stability of behavior. A child's behavior during the period from 6 to 10 years of age predicted at least moderately some aspects of that person's behavior during early adulthood (age 24).

Although I was familiar with the longitudinal study by Kagan and Moss, it did not occur to me to start a longitudinal study when I was planning my doctoral dissertation during the second half of the 1960s. My dissertation was a so-called cross-sectional study, because I collected data only at one time point. For the study of individual development, a longitudinal study is needed. The definition of a longitudinal study is that data are collected about the same set of individuals at least twice after an interval of time. In fact, I (my name was Lea Pitkänen until 1975) wrote in the Discussion section of my dissertation: "A longitudinal study would make it possible to examine the stability of the individual patterns of behaviour" 
(Pitkänen, 1969, p. 190). At that time, I could not have anticipated staying on the same track for over 40 years.

What were these individual patterns of behavior which I found so compelling that I felt the need to study their stability over time? To answer this question fully, I need to first describe the historical setting in which I was planning the dissertation. What were the theoretical thoughts and research instruments that were available to me as a student during my studies from 1958 onwards? The small Department of Psychology at the University of Jyväskylä offered only a few courses to students. Studying mostly consisted of reading textbooks and writing examinations based on the information they contained.

Professor Martti Takala, the only professor at the Department of Psychology, was interested in both experimental personality psychology and differential psychology; the latter refers to the study of individual differences. The textbooks he chose for students reflected his interests. I enjoyed reading the Swedish textbook Differentiell psykologi by Ekman (1952) so much that after the examination, my professor kindly said to me that it was not necessary to learn the content of the textbooks by heart. My first English textbook was Child Development by Olson (1949), which I read slowly with the help of a dictionary because I had only taken a short course in English at school. The book greatly affected my orientation to the subject of childhood. The most interesting textbook was, however, Theories of Personality by Hall and Lindzey (1957). It included in-depth descriptions of the grand personality theories by Freud, Lewin, Allport, Rogers, and many others, and the first factor theories of personality by Eysenck and Cattell. Regarding the latter, I was impressed by Eysenck's theory with his bipolar contrast between introversion and extraversion which describes differences in temperament between individuals.

In the field of learning theory, the prevailing theory at that time was a behavioristic stimulus response orientation that Dollard and Miller (1950) applied to personality. A basic tenet of this theory was the formation of behavioral habits as a result of reinforcement. The theory introduced me to the construct of a drive as a strong stimulus which impels the individual to behave or respond in a certain manner until the stimulus is reduced or eliminated. According to this theory, learning consists primarily of developing efficient means of reducing drive stimuli. The stimulus response theory was mainly based on animal experiments. The emergence of social learning theory as a result of the work by Bandura and Walters (1963) was a groundbreaking experience for me. It opened a new perspective on social learning. Later, the theories of motivation (e.g., Atkinson, 1964) and clinical child psychology also stimulated my thinking when I had to learn about them as part of my teaching responsibilities.

As a research assistant at the Department of Psychology, I collected data from children concerning their achievement-, affiliation-, and aggression-oriented behaviors and participated in experimental research on personality (Pitkänen, 1963b; Takala \& Pitkänen, 1963). I was puzzled and dissatisfied by the inconsistent findings that different measures resulted from a certain category of behavior. I became skeptical about interpreting aggression or achievement as a need or a 
personality trait. I found that they were not unified constructs. I became interested in the multidimensionality of personality constructs and the dependence of behavior on situational factors. As aggression was more visible and measurable than achievement, I chose aggression for a closer examination in my doctoral dissertation. I began my independent research work for my dissertation in 1964, inspired by the book on aggression by Arnold Buss (1961). At that time, I was a mother to two daughters born in 1961 and 1964, which also motivated my study on children.

I conducted a Kindergarten Study with 216 six-year-old boys whose behavior kindergarten teachers (26) observed and rated using 32 items on aggression (Pitkänen, 1966, 1969, p. 29). For sampling these variables, I devised a "cone-shaped biscuit" model to describe different components of aggression (Figure 1.1). One dimension consisted of the intensity of aggression (the height of the cone). Two other dimensions were the motivational sequence of an aggressive act, and the direction of aggression (diameters of the cone). Different modes of aggression (physical, verbal, facial) were also depicted in the descriptive model of aggression as vectors.

The motivational sequence referred to the initiation of an aggressive act (offensive meaning proactive) or a response to an aggressive act (defensive meaning reactive). The direction of aggression referred to whether aggression was targeted at another person directly or indirectly via mediating events, for instance, by gossiping about

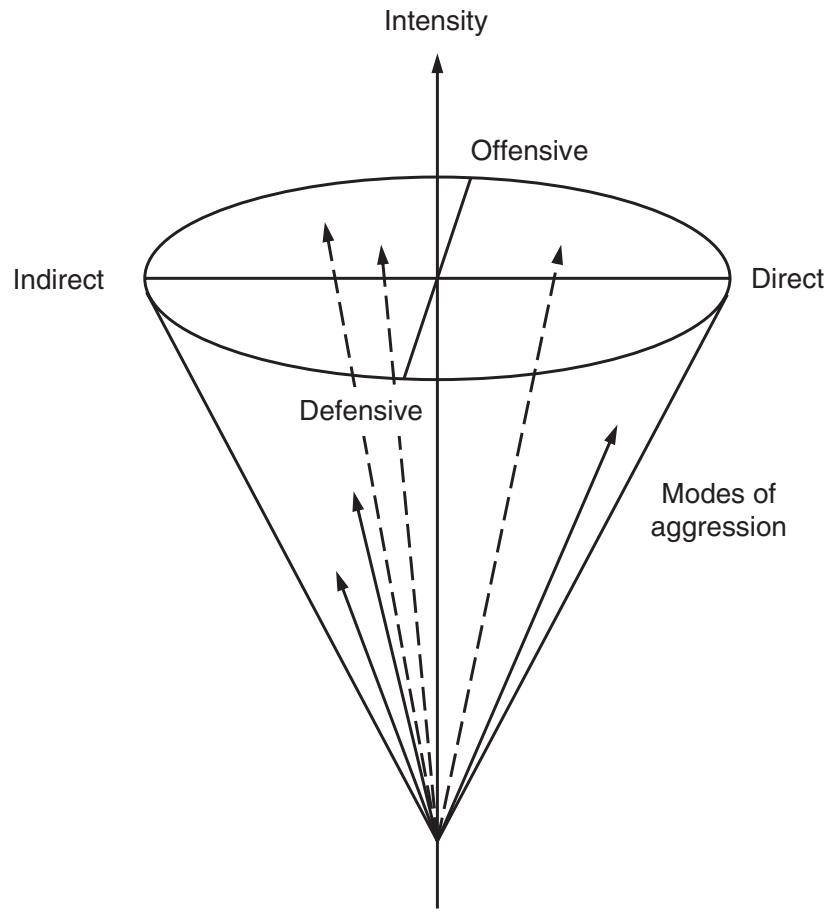

FIGURE 1.1 A descriptive model of aggression.

Source: Pitkänen (1969: Figure 1, p. 29). Reproduced with permission. 
the target. These components can be used for the analysis of aggressive acts. For instance, in the act, "He hurts somebody without any reason, e.g., by tripping, pulling hair, pinching, or striking in passing," the purpose of the act is offensive (proactive), the direction is direct, the mode is physical, and the intensity can be observed.

The results confirmed the relevance of the dimensions for individual differences. They also indicated that the most differentiating quality in boys' aggressive behavior was the purpose of the aggression. The children who were perceived as being most aggressive were proactively aggressive, and they generally also defended themselves if attacked. Aggression for the purpose of self-defense was to some extent acceptable but it was critical that the intensity of the defense matched with the intensity of the offense which triggered it. Tremblay (2000) acknowledged that the differentiation between offensive and defensive aggression was a pioneering piece of research that other researchers have taken further using the terminology proactive and reactive aggression.

\subsection{Positive behavior in an impulse control model and the goals of the study}

\subsubsection{The search for alternatives to aggression}

The Kindergarten Study previously described revealed that only a few children were frequently aggressive. This observation stimulated my interest in alternative, nonaggressive behaviors which are used to cope with conflict situations, and led me to develop a new study that formed the second part of my dissertation (Pitkänen, 1969). The JYLS was built on the basis of this second cross-sectional study.

First, I needed to learn more about individual differences in social behavior. In the literature, I discovered that researchers were more interested in problem behaviors than in positive behaviors. The concepts used to describe positive behaviors in children were vague, such as describing someone as "a nice child," but what does one actually mean by this? The boom in studying prosocial behavior did not begin until the late 1970s (e.g., Mussen \& Eisenberg-Berg, 1977; Staub, 1979). The orientation toward positive psychology only began at the beginning of this millennium (Seligman \& Csikszentmihalyi, 2000). In 1967, there was no model which conceptualized positive alternatives to aggression and, therefore, I had to develop it.

From animal studies, a nonaggressive option to "a fight" was "a flight," as described in Miller's (1959) theory of approach-avoidance conflict presented within the conceptual framework of the stimulus response behavior theory. In a conflict situation, there are two tendencies, either to approach the situation or to avoid a negative stimulus. The approach tendency is sustained by a drive stimulus that has its origin in the internal physiological condition of an organism. The avoidance tendency is motivated by fear and is an acquired drive. In human psychology from the point of view of psychodynamic theories, aggression was seen as an expression of aggressive energy and its display functioned in a "cathartic" way while reducing the 
energy of the aggression (Feshbach, 1964). Nonaggression was generally referred to as an "inhibition of aggression," and it was not viewed in a positive light due to the assumption that inhibition might result in a personality pathology. Aggressive energy could be channeled by a great variety of acts, even by nonaggressive acts. Thus, it became difficult to assess which behavior was an expression of channeled aggressive energy and which was motivated by nonaggressive urges or reasons.

Researchers had tried to classify nonaggressive responses to frustrations using constructs, such as submissiveness and sympathy (Wittenborn, 1956); withdrawal (instigation alteration), limitation (frustration depreciation), and substitution (goal response alteration) (McClelland \& Apicella, 1945); and the approval motive that makes the behavior of approval-dependent individuals conforming (Crowne \& Marlowe, 1964). Feshbach (1964) put forward the idea that the reduction of anger and the aggressive drive might be accomplished by displaced aggression, changing the stimulus condition or its meaning, and mediating responses that are incompatible with anger. Lazarus' (1966) analysis of coping process was along similar lines and this made an important contribution to my thinking. He distinguished three major coping reaction patterns: direct actions, defensive reappraisals, and anxiety-reaction patterns. Direct actions contained actions aimed at strengthening the individual's resources against being harmed, attack patterns, and avoidance patterns. Defensive reappraisal involved thought processes of many kinds and anxiety-reaction patterns appeared as a threat reaction when no clear action tendency was generated.

In 1967, after an intensive period of thinking, I gained an insight into a way of describing differences in children's behavior covering both aggressive and nonaggressive behaviors. I reasoned that the human brain allows for more variation in social behavior than just the "fight or flight" response and inhibition of anger. I speculated that a characteristic of human beings is that they can self-reflect their own behavior, intentions, and emotions and exercise control over how these are expressed. I concluded by considering the increased recognition of the interface between emotion and cognition (Schachter \& Singer, 1962), that it is a human being's capacity for cognitive control over his or her emotional behavior which makes him or her able to appraise a stimulus situation, become motivated in a new way, and decide freely between alternative behaviors. My thinking anticipated the current understanding of the executive control of forebrain, which was not known at that time.

\subsubsection{The impulse control model}

I devised a model based on two orthogonal dimensions: overt activity and control of behavior (Pitkänen, 1969, p. 102; “a compass model”). I assumed that there were three layers of constructs in the control of an impulse to act aggressively: psychological processes, theoretical constructs, and behavioral indicators (Figure 1.2; Pitkänen, 1969, p. 112; Pulkkinen, 1982). The hypothetical psychological processes were (1) suppression of the extrinsic or behavioral aspect of an impulse and (2) neutralization of the intrinsic or emotional aspect of an impulse. The activation of one or both of these processes defines the theoretical constructs for selecting 


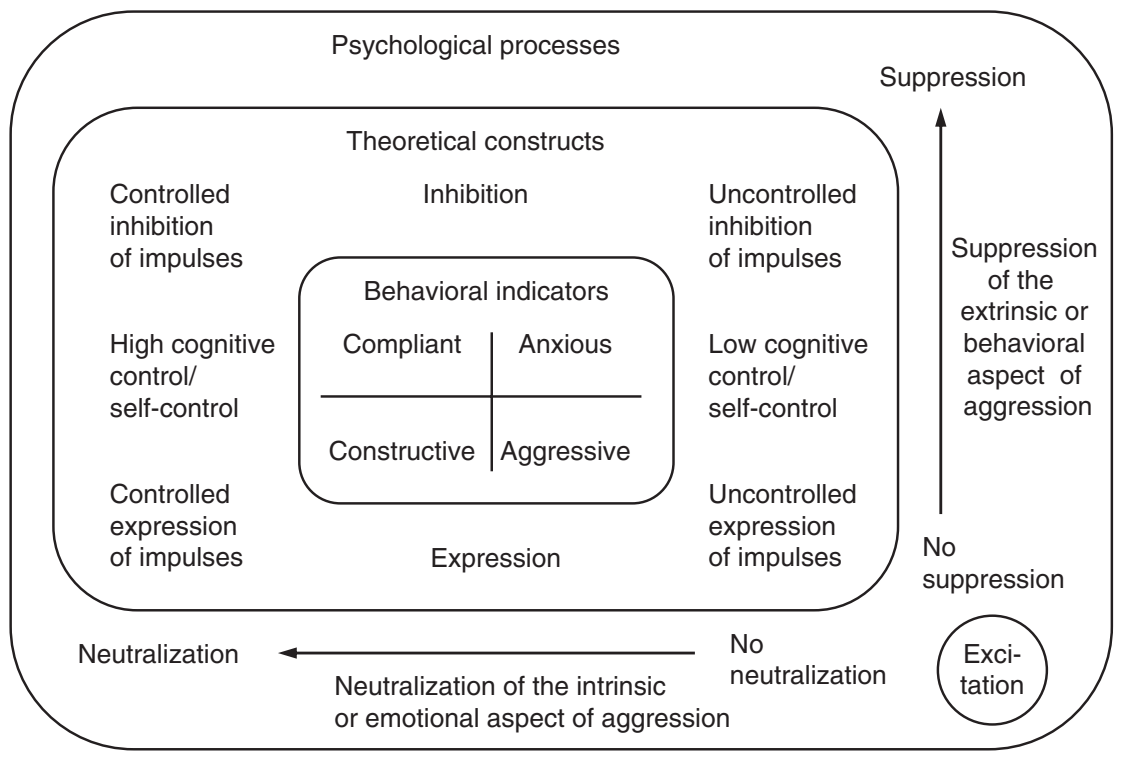

FIGURE 1.2 A framework for the analysis of aggressive and nonaggressive behaviors.

Source: Pulkkinen, L. (1982). Self-control and continuity from childhood to adolescence. In B. P. Baltes \& O. G. Brim, Jr. (Eds.), Life-span development and behavior (Vol 4, pp. 63-105). (Figure 1, p. 68). New York, NY: Academic Press. (C) 1982, by Academic Press, Inc. Reproduced with permission.

alternative behaviors, and the behavioral indicators which are depicted in the core of the model.

I postulated, first, that the primary effect of a threatening stimulus on an organism is to activate an impulse to act and the emotions associated with it. The emotional state may consist of different combinations of anger and anxiety, depending on the relative strength of the approach and avoidance tendencies. Theoretically, there were two main behavioral options for a low level of control of behavior:

1. Uncontrolled expression of impulses where the goal of the action in a threatening situation is to eliminate a noxious stimulus immediately. It corresponds with the approach tendency defined in animal studies. Action may be manifested in many aggressive forms.

2. Uncontrolled inhibition of impulses where individual's responses are characterized by avoidance behavior referring to the avoidance tendency. Activation aroused by the stimulus is bound to emotions, such as anxiety indicated by fearfulness and helpfulness such as crying. Responses enabling an individual to eliminate the threat are suppressed.

The second hypothesis was that human behavior may be steered by cognitive processes. An individual may be able to appraise the stimulus situations he encounters, including the perspectives of other parties and the factors that have caused the 
annoying situation, and to decide on how to act by weighing up various behavioral alternatives. In other words, the emotional state can be neutralized by cognitive control and the way of reacting to the situation can be chosen consciously, guided by cognitive control. I assumed that cognitive control is motivated by an attempt to behave in a socially acceptable manner. Consequently, I postulated two behavioral alternatives for a high level of control of behavior:

3. Controlled expression of impulses where an emotional state is neutralized by cognitive control, but the behavioral aspect to eliminate the aggression stimuli is active. An individual searches for nonaggressive, constructive ways to solve the conflict by using coping behaviors, including positive thinking, active confrontation, and consideration of others with empathy.

4. Controlled inhibition of impulses where an individual suppresses his or her negative emotions and behavior stimulated by the situation, but neutralizes the emotional arousal by, for instance, rationalizing his motives or trying to find compensatory activities. Consequently, the individual's behavior is characterized by compliance, which includes not showing negative emotions, avoidance of situations that might arouse negative affect by doing something else, and balanced mood.

My approach was situation specific and I thought children learn from experience different ways of coping with impulses instigated by threats and conflicts. I did not connect the concept of impulse to the personality trait "impulsivity" (Murray, 1938), a "cognitive style" (Kagan, 1965), or an "impulse" in psychoanalytic theory in which an impulse means a discharge of psychic energy, the source of which is the Id. If arousal (or excitation) raises the level of tension, the Id tries to return the organism to a constant level of energy through the discharge of energy. The Id functions according to the pleasure principle. The ego, the part of the Id which has been modified by influences from the external world, seeks to reduce the level of tension within the Id through the reality principle, that is, by considering demands in the real world (see a review in Pulkkinen, 1986).

\subsubsection{The goals of the longitudinal study}

I thought that an individual's typical way of reacting to an excitation was associated with his or her temperament characteristic known as extraversion/activity or introversion/passivity which can be modified by cognitive control. Thus, a behaviorally passive individual is likely to react to a threatening situation with inhibition, by becoming anxious or complying, whereas a behaviorally active individual is likely to react to the situation with expression, that is, by behaving in an aggressive or a constructive way. I also hypothesized that the level of cognitive control that an individual can bring to bear is associated with maturation, current situational factors, external control, and internal control based on learning experiences in previous situations and internalized guidance. 
I studied the existence of these patterns of behavior in 8-year-old children's behavior (Pitkänen, 1969). The results confirmed the model, and led to the idea of following up the development of the participants in the study. A cross-sectional study transitioned into becoming a long-term longitudinal study, namely the JYLS. The details of the goals of the study have evolved over the years, but at that time the main goals were to study the following:

- the differences between children in their ways of interacting with other people in emotion-triggering situations;

- the stability of individual patterns of behavior over years, and the life conditions which supported these patterns of behavior; and

- the significance of differences in children's behavior in childhood for the development of their psychological and social functioning as adults, including a specific focus on positive development as a unique contribution to the literature.

The terminology used in the impulse control model was updated during the course of the study, but the basic assumptions about individual differences have remained the same. The core of the model is the control of the expression of emotion and behavior (Pulkkinen, 2014), which at different stages has been called cognitive control, self-control and self-regulation. How the model has been updated is described in Chapter 4.

\subsection{Research conditions in Finland and in the longitudinal study}

In the peer review process of a manuscript, Finnish researchers sometimes encounter the problem that a reviewer is skeptical about the research conditions that exist in Finland. Therefore, it is relevant at this point to explain that Finnish research findings are comparable to those obtained in other Western countries. This has been shown in a number of comparative studies.

"A living laboratory." Someone who lives abroad but is well-acquainted with Finland may well have a clear view of this country. I discovered such a description in an article by Richard Rose, Professor Emeritus of the University of Indiana, Bloomington, USA (Rose, 2006a). He has worked with Finnish researchers on twin studies for more than 30 years. I have had the pleasure to be one of his collaborators in the longitudinal study of twin children (FinnTwin 12) since the early 1990s. In his analysis entitled "Finland as a research setting," he mentions that "Finland is an extraordinary living laboratory for behavioral and genetic research. With its Nordic neighbors, it offers unusual advantages for longitudinal studies" (Rose, 2006a, p. 21).

Inspired by Rose's article, I will first provide some general information about Finland, and then describe the research setting of the present longitudinal study. Finland is located in Northern Europe with the Swedes as neighbors on the west side, the Russians to the east, the Norwegians to the north, and the Estonians to 
the south. Finland is a sparsely populated country with 5.5 million inhabitants. The average density of population is 17 persons per sq. $\mathrm{km}$. The population is concentrated in southern Finland where the capital, Helsinki, is located. The demographic pyramid is similar to many Western countries in that the proportion of aging people is rapidly growing in comparison to the proportion of children. In 2014, the proportion of people who were 75 years or older was 8.7 percent. Life expectancy is high, particularly in women: it is 83.8 years for girls born at the present time. For boys, it is 77.8 years, lower than in other Nordic countries. Heart disease, suicide, heavy drinking of alcohol, and accidents shorten male life expectancy.

About 90 percent of people living in Finland speak Finnish. Until recently, there has not been much immigration. Other languages due to immigration are spoken by 5 percent. The Finnish language shares its roots with Hungarian and Estonian. For historical reasons, the country is officially bilingual: the two languages are Finnish and Swedish. Swedish is spoken by 5 percent of the population. English is widely spoken in the country.

Finland became an independent republic in 1917. It was part of the Kingdom of Sweden for centuries but, as a consequence of a war between Sweden and Russia, it became an autonomous Grand Duchy with the Russian Empire for about a hundred years (1809-1917). In the Second World War, Finland successfully defended its independence against occupation. It has never belonged to the Eastern Bloc, the name used for the former communist states of Central and Eastern Europe. Finland does not belong to NATO, but cooperates with it on peace and security operations. Finland has been a member of the European Union since 1995, and its currency has used the euro since 2002. Finland has a parliamentary form of government and its president is elected for a 6-year term.

The country is technologically advanced. Finland scores very highly in innovation. It used to be an agrarian society, dependent on agriculture and forestry. Forests cover nearly 70 percent of the entire country, and of the total area of Finland, 10 percent, including 188,000 lakes, is water. Finnish people have a close relationship with nature. Finland's transition to having a high-tech industry was rapid. Its global competitiveness is remarkable considering its small population. The country underwent severe economic pressure in the early 1990s following the collapse of the former Soviet Union. At the same time, trade with the Soviet Union all but collapsed. However, it recovered from this challenge through the success of high-tech companies such as Nokia, which became a world leader in the design and production of cellular phones. With its small population, the country is, however, vulnerable to global economic markets and pressures, as became visible when Nokia lost its position as the market leader.

Finland has invested in its children's education and citizen's welfare and has offered free education for everybody right up to the highest university degrees. Finland's compulsory public education system is known for its superior achievements in the Programme for International Student Assessment (PISA) drawn from the member countries of the Organization for Economic Cooperation and Development (OECD). Finland has a large network of universities (14) and polytechnic 
colleges/universities (24) spread all over the country. In the 2013 Ranking of National Higher Education Systems, the top four countries in Europe were found to be Sweden, Switzerland, Denmark and Finland (Universitas 21, 2013). Education and the teaching profession are both highly valued in Finland. Finnish culture is rich in music, visual arts, design, and literature. Most people (about 75 percent) are baptized at birth in the Lutheran Church, but few Finns are regular churchgoers.

In terms of equality, Finland is among the best countries in the world (Unicef, 2016; Wilkinson, 2011). Finland's constitution guarantees basic economic, social, and educational rights with free education available to all people resident in the country. Along the same lines as the Nordic welfare model, the social protection system with its services and income security is funded mainly by taxation. Of total gross domestic product, 7 percent is allocated for health services. Free maternity and children's health clinics were established in the 1940s to support the birth and development of the new generation after the turmoil of the Second World War. Children have also received free daily lunch at their school since the 1940s.

Most women (nearly 80 percent) are engaged in full-time employment; parttime work for mothers is rare. Parental leave consists of 158 weekdays, of which 105 days are for the mother and the rest are negotiable between the mother and the father. Childcare is organized in municipal daycare centers and in private daycare homes. One academic year of kindergarten education is arranged for 6-year-olds before they start public schooling at age 7 . Corporal punishment of children at school was outlawed by legislation in the 1920s and in the home in the 1980s.

It is fair to say that Finland is one of the Western countries with a higher standard of living and education. Finnish people tend to respond positively when asked to participate in research. One of the advantages for longitudinal research in Finland is the Population Register Centre (PRC) that contains data on all Finnish citizens. Since 1969, each newborn individual has been given a unique personal identifying number. Important to a twin researcher is that this number incorporates the date of a baby's birth and an alphanumeric linkage to their biological mother, as described by Rose (2006a). Furthermore, this register contains a current residential address for all Finnish citizens as well as information on family structure and changes in it. Another advantage for longitudinal research is a good statistical system within Statistics Finland, the public authority established for statistics, which has made it possible to collect age cohort data to estimate the representativeness of the research sample at different adult ages.

Research conditions in the longitudinal study. The research setting of the JYLS has improved over the years. For instance, the PRC had not yet been established when the study began in the 1960s, which meant that personal identifying numbers were not available for the first follow-up study. The participants had to be traced using other means. For later follow-ups, the PRC has been of great help. Also, funding conditions have improved. Funding improved significantly only in the mid-1980s and beyond.

The history of the longitudinal study coincides with the development of digitalization. In the 1960s, data processing was done manually. Scores were marked on 
lists and data were analyzed with electronic calculating machines. The possibility of moving scores from the lists to punch cards, and the use of punch card machines, were steps forward and helped in sorting out the data. In the 1960s, the University of Jyväskylä signed a contract with a local state-owned metal industry (VALMET) that enabled researchers to use a computer that VALMET had purchased. Conditions improved in the 1970s. Personal computers were not available for researchers at the university in the early 1980s, but I bought my first computer privately in 1984. I had visited professor Urie Bronfenbrenner at Cornell University and saw how enthusiastic he was about his computer. I thought that if he had learned to use it, I would also be able to do so. My computer was an Apple IIc and it was soon my most important domestic appliance. It could only be used for text processing; the memory was not sufficient for calculations.

Technical advancements took place at a fast pace over the course of the longitudinal study, which caused concerns about how to maintain the longitudinal data and take care of its transfer to new systems. We managed to do this successfully. The archive level data, which does not contain any personal identification information about the participants, are now backed up and saved in the Finnish Social Science Data Archive where specialists maintain and take care of the data. It is available to researchers upon request to the Principal Investigator of the study. In general, all data have been used and saved without identification information. Artificial code numbers are used to connect the data from the same person.

In the 1960s and 1970s, financial resources for research were still very limited in Finland. The country was slowly recovering from the Second World War and the war reparations which Finland had agreed to pay to the Soviet Union in the Moscow Armistice in 1944. The last train full of goods for paying for the war reparations left Finland in 1952. "Approximately 340,000 railroad carloads were needed to deliver all reparations" (Wikipedia, 2016). In the 1960s and 70s, the higher education system was expanding through the establishment of new universities, and the research funding system was being developed.

I received a young researchers' grant for the period from 1968 to 1969 during which I collected and analyzed the data (Stages $1 \mathrm{a}-\mathrm{b}$, see Box 1.1) for my doctoral dissertation. While being employed as an associate professor at the university, I was awarded small research grants by the Academy of Finland for the collection of follow-up data at ages 14 and 20 (Stages $2 \mathrm{a}-\mathrm{c}$ ). The grants covered the costs of some of the materials, but was not sufficient to cover the travel expenses that interviewing all participants would have incurred. Therefore, a subsample was selected for the interview. In addition, due to the lack of funding, the number of tapes that could be purchased for recording the interviews was limited. Data collection was conducted with the help of psychology students who prepared their Master's theses on the data. Political discussions were animated in the field of social sciences in the 1970s. The prevailing atmosphere was to find explanations for human behavior in social structures and inequalities in line with socialist ideology. It was not fashionable to study individual differences and internal processes, which might have reduced my chances of securing funding for the study. 


\section{BOX 1.1 THE MAJOR STAGES AND SUBSTAGES OF THE LONGITUDINAL STUDY (JYLS)}

1. 1a. The collection of baseline data at age 8 (in 1968) with the initial sample $(\mathrm{N}=369)$

1b. A sub-sample of boys ( $n=60$; extreme groups in their aggressive and nonaggressive behaviors) was selected for an intensive study at age 8 (1968)

1c. Follow-up of the sub-sample of boys at age 9 (1969)

2. 2a. Follow-up of the initial sample at age 14 (in 1973-74)

2 b. Interview study with a selected sub-sample of boys and girls at age14 (1974) and one of the parents

2c. Follow-up of the sub-sample of the boys and girls at age 20 (in 1979-80)

3. Follow-up of the initial sample at age 27 (in 1986)

4. The initial sample participated at age 33 (in 1992) in a study for the standardization (analyzing the usability) of the NEO Personality Inventory in non-Indo-European languages

5. Follow-up of the initial sample at age 36 (in 1995)

6. An offspring study with the participants (and their spouses) at age 38-40 (in 1997-1999) and their school-aged children

7. Follow-up of the initial sample at age 42 (in 2001)

8. Follow-up of the initial sample at age 50 (in 2009)

Rumors about the ongoing longitudinal study spread to the National Board of Health, and one day in 1981, I received a telephone call in which I was offered a grant to analyze the data from the point of view of substance use. The offer brought me great joy, and meant that health behaviors became one of the focus areas in the study.

The atmosphere for research became more favorable in the 1980s and, in general, funding opportunities improved. In 1986, I was awarded two grants for data collection (from the Academy of Finland and the Finnish Cultural Foundation), which made it possible to trace the whole initial sample at age 27 and conduct personal interviews with all participants who agreed to be interviewed (Stage 3, Box 1.1). The Academy of Finland also supported data collections in the follow-up studies in 1995 and 2001 (Stages 5 and 7) and appointed me as Academy Professor from 1996 to 2001. This appointment included a grant that was used for the Offspring study (Stage 6). A Program for National Centers of Excellence was created in the 1990s and our group was successful in applying for this status, which came with a good amount of funding from the Academy of Finland and the University of Jyväskylä. I was Director of the center called "Human Development and Its Risk Factors" from 1997 to 2005, until I was forced to retire due to the prevailing retirement legislation at the time. 
After 1995, research funding was awarded in many forms including funded positions for doctoral students and postdoctoral researchers. Funding made it possible to build a research team. I also continued as part of the research team while living off my pension. The Academy of Finland awarded a new grant for Stage 8 conducted from 2009 to 2012, for which I worked as Principal Investigator and Katja Kokko as Co-Principal Investigator. She was an Academy Research Fellow funded by the Academy of Finland. Her funding was partly used for the 2009 data collection of the JYLS.

International collaboration has involved comparative studies with Canadian (Richard Tremblay), Swedish (Britt af Klinteberg, Lars Bergman), US (e.g., Eric Dubow, Rowell Huesmann, Janet Landsford), and UK (Ingrid Schoon) data sets; they have confirmed the comparability of the study's findings across different countries. From 2003 to 2013, Katja Kokko and I were members of the Center for Analysis of Pathways from Childhood to Adulthood (CAPCA) coordinated by Michigan University, USA, which focused on the comparative analyses of longitudinal data sets. 


\section{2}

\section{THE EXECUTION OF THE LONGITUDINAL STUDY}

\subsection{The research sample and the major waves of data collection}

In choosing the age of the initial research sample, I set as the first criterion that the children whose behavior I was going to study would be able to respond to a peer nomination. Peer nomination is a technique where children are asked to choose from a list of their classmates the children who fit the definition given by the researcher, for instance, "Which of your classmates always tries to be friendly to others?" I was interested in observing children's behavior, but I understood that because I was working on my own on my dissertation, I would only have been able to observe a small group of children. This meant that the sample would have remained too small for statistical analyses and the generalization of results. My methodological choices were to collect data from the class teacher and classmates, both focusing on the same behaviors. I thought that together these two approaches would result in valid information about individual differences. In Finland, the school starting age is in the fall of the year in which the child turns 7. Most children learn to read during the first semester and thereafter the development of their reading and writing skills progresses quickly. However, the first grade was considered to be too early for data collection. The same evaluation applied to my second criterion, the establishment of a social structure in a class in a way that would reveal children's typical behaviors. My third criterion was that the teacher would know the pupils well. Taking these criteria into account, I estimated that the second grade (age 8) would be a good time for data collection.

The school authorities of the town where I planned to collect the data accepted my study plan and its methods. They agreed that I could conduct the study in the second-grade classes drawn randomly from the schools of the town, and that the teachers of these classes and classmates could be asked to assess the typical behaviors of the children in these classes. The parents' consent was not needed in 1968 when the study began. 
For the sample size, I set as the first criterion that both boys and girls would be included in the sample. It was quite common that aggressive behavior tended only to be studied in boys. The second criterion was that the sample would include pupils from the town center and different suburban areas. The third criterion was that the number of boys and girls would be sufficient for statistical analyses, but that the number would not be too high for a doctoral student who would be doing all the coding and the data processing on her own. Technical equipment was limited at that time and, therefore, the majority of the work had to be done manually.

Age 8 (in 1968). The average size of a second-grade school class was 30 students. I estimated that a sufficient sample size could be reached by including 12 school classes in the study. I drew the classes randomly from the town's schools. Half of the classes were located in the town center and half in the suburban areas. There were 369 students in these classes of whom 93.5 percent were born in 1959, 3.8 percent in 1958 , and 2.7 percent in 1960 . I intended to carry out the study focusing on them. At the time of data collection, their average age was 8.3 years. All the participants were native Finns; Finland was ethnically very homogeneous in the 1960s. The whole population of second-grade pupils in Finland was about 75,000 at the time.

The participation rate was 100 percent, because data were collected about all the students in the selected classes. Thus, the initial sample size was 196 boys and 173 girls, the same as the size of the intended sample. This participation rate provided a good basis for achieving a representative sample of second-graders. Often, a significant attrition takes place at this initial phase of a study which then biases the initial sample. Table 2.1 presents the sample sizes at the point of each major data collection wave, using a terminology that considers the intended, initial, eligible, and effective (or actual) sample sizes (Pulkkinen \& Kokko, 2012).

The socioeconomic background of the participants was assessed based on the father's occupation (and the mother's if the mother was a sole provider). The town in Central Finland from where the sample was randomly drawn is a university and industrial town. The number of inhabitants was about 60,000 when the study began, and was growing due to migration from the rural areas to the urban areas. The sample represented the social structure of Finnish population in the 1960s as described by the nine-point classification system by Rauhala (1966) based on valuations of the different occupations. One-fourth of the participants had parents in more highly valued occupations, 58 percent in the middle level occupations, and 17 percent in less highly valued occupations (Pulkkinen, 1989a). Compared with the present time, fewer parents had obtained formal vocational education or had gone to university, occupational skills were often learned at work, and white-collar occupations were less frequent. This situation was common in Finland and other countries at that time (see Kokko et al., 2014). Therefore, professional (whitecollar) occupations were relatively rare (about 30 percent) among the parents, while the majority of the parents were in nonprofessional (or working-class) occupations.

At age 14 (in 1974), the eligible sample was equal to the initial sample. When the first data were collected at age 8 , the unique personal identification numbers 
TABLE 2.1 The size of the sample in each of the major data collection waves: the sample was drawn for second-grade pupils at age 8

\begin{tabular}{|c|c|c|c|c|c|c|c|c|c|c|c|c|}
\hline \multirow{2}{*}{$\frac{\text { Age }}{\text { Gender }}$} & \multicolumn{2}{|l|}{8} & \multicolumn{2}{|l|}{14} & \multicolumn{2}{|l|}{27} & \multicolumn{2}{|l|}{36} & \multicolumn{2}{|l|}{42} & \multicolumn{2}{|l|}{50} \\
\hline & $M$ & $F$ & $M$ & $F$ & $M$ & $F$ & $M$ & $F$ & $M$ & $F$ & $M$ & $F$ \\
\hline Intended sample & 196 & 173 & & & & & & & & & & \\
\hline Participation rate \% & 100 & 100 & & & & & & & & & & \\
\hline Initial sample & 196 & 173 & 196 & 173 & 196 & 173 & 196 & 173 & 196 & 173 & 196 & 173 \\
\hline Eligible sample & 196 & 173 & 196 & 173 & 196 & 173 & 194 & 173 & 186 & 157 & 174 & 149 \\
\hline Participation rate \% & 100 & 100 & 96 & 97 & 85 & 90 & 83 & 88 & 81 & 85 & 83 & 85 \\
\hline Effective sample & 196 & 173 & 189 & 167 & 166 & 155 & 161 & 152 & 151 & 134 & 144 & 127 \\
\hline Retention rate \% & & & 96 & 97 & 85 & 90 & 82 & 88 & 77 & 77 & 73 & 73 \\
\hline
\end{tabular}

Note:The number of participants given in the table presents the number of those participants from whom some data were obtained. In adulthood, the participation fluctuated to some extent depending on the assessment method. More details about the number of participants are given in specific articles.

were not available. The Population Register Centre (PRC) was established a year later. Therefore, the tracing of the sample was made by using school records and the local school authority, local records of the parents' addresses, and church and civil records regarding the house moves of families. The participants were found to be located in 78 school classes in several municipalities. The consent to collect data in these schools and classes using peer nomination and teacher rating was received for 96 percent of boys and girls of the sample. The effective sample, that is, the number of those participants for whom information was gathered for the follow-up study consisted of 189 boys and 167 girls. The participation rate of the eligible sample and the retention rate of the initial sample were close to 100 percent.

At age 27 (in 1986), the eligible sample was still the same as the initial sample. The participants' addresses were obtained through the PRC; the unique personal identifying numbers were collected in the context of the data collection at age 14 . For those who had not updated their addresses at the PRC, their parents' addresses were obtained, telephone directories were searched, and local records of house moves were used. A letter was sent to the participants whose addresses were identified. The written consent was received from 166 men and 155 women. The participation rate was 85 percent for men and 90 percent for women. More than half (57 percent) were living in the center of the province from where the initial sample was drawn, and one-tenth in other parts of the same province; 3 percent north of the province, 26 percent south of the province, and 3 percent abroad (mainly in Sweden) (Pulkkinen, 1989a).

At age 36 (in 1995), the eligible sample size was smaller for the men than in the initial sample, because two of the men had died. The sample of participants was approached again with a request to continue their participation in the study. The consent was received from 161 men and 152 women. The participation rate was 
83 percent for men and 88 percent for women. The retention rates of the initial sample were 82 and 88 percent, respectively.

At age 42 (in 2001), the eligible sample was 7 percent smaller than the initial sample (186 men and 157 women), because 5 men and 1 woman had died, and 5 men and 15 women had withdrawn from the study. The sample was approached with a request to participate in the continuation of the study. The consent was received from 151 men and 134 women. The participation rate of the eligible sample was 81 percent for men and 85 percent for women, and the retention rate of the initial sample was 77 percent for both genders.

At age 50 (in 2009), the eligible sample was 174 men and 146 women; 8 men and 4 women had died ( 3.3 percent of the initial sample), and 14 men and 20 women had withdrawn from the study (10 percent of the initial sample). The sample was approached with a request to participate in the continuation of the study. Consent was received from 144 men and 127 women who participated in the study. The participation rate was 83 percent for men and 85 percent for women, and the retention rate was 73 percent for both genders. Until the end of the year 2015 (age 56), 12 men (6.1 percent of 196) and 5 women (2.9 percent of 173) had died (4.6 percent of 369$)$.

Many participants had gone to study and work outside Central Finland, but many of them returned to Central Finland at a later age if work conditions allowed it. In middle age, two-thirds of the participants lived in Central Finland and 17 percent in the Helsinki capital region. About 3 percent lived abroad. The rest were spread around the country.

\subsection{Attrition and the representativeness of the sample}

In a long-standing longitudinal study, it is natural that all data cannot be collected from all members of the initial sample. Some participants die, some cannot attend data collection in a certain wave due to illness or other reasons, and some decide not to participate. Therefore, it is important to analyze whether the attrition, that is, the reduction in the number of participants, had been selective and had therefore affected the representativeness of the sample.

The first question that we wanted to clarify was: for how many of the participants of the initial sample whom we had studied in childhood had data also been collected during their adulthood? We were pleased to find out that this percentage was 94.6 (Metsäpelto et al., 2010). With regard to these participants, data were collected both in childhood (at age 8 and 14) and at some point during their adulthood.

The second question to analyze was the latest age when the data were collected from each participant. Participants might have skipped a wave and appeared again in the following wave. We found out that in adulthood, the latest data were collected at age 50 for 73.3 percent of the initial sample; at age 42 for 8.7 percent, at age 36 for 9.3 percent, and at age 27 for 3.3 percent. Thus, for 82 percent of the initial sample data were collected in mid-adulthood, at the earliest at age 42 . 
The third question was: what proportion of the initial sample had participated in all major data collection waves? We found out that almost two-thirds (60.2 percent) of the initial sample had participated in all six major data collection waves (at age 8, 14, 27, 36, 42, and 50); 17.8 percent had participated in five waves; 10.8 percent in four waves; and 2.2 percent in three waves. There was one participant (0.3 percent) who was studied only once, at age 8 , and 19 participants (5.1 percent) who were studied twice, at age 8 and 14 .

The fourth question was whether the participants who took part in the data collection at age $50(n=271)$ differed from those who did not take part in it $(n=98)$ in terms of the characteristics of their social behavior which had been assessed at 8 years of age. No significant differences were found. Neither were there differences in school success at age 8 , nor in the socioeconomic status of the family of origin. Thus, at age 50, the studied sample was strongly representative of the initial random sample, in terms of the relevant variables of this study. The results were similar to those obtained when the comparisons were made at age 42 (Pulkkinen et al., 2003). The only reason for attrition was found between the ages of 42 and 50: the participants who at age 42 were heavy users of alcohol were less likely to participate in data collection at age 50 than other participants due to having died or for other reasons, such as being unable to participate in the study due to heavy drinking.

The fifth question was how well the JYLS sample studied at ages 42 and 50 represented the whole age cohort born in Finland in 1959 ( $\mathrm{N}$ 75,000). Comparisons were made using demographic variables drawn from Statistics Finland (Pulkkinen et al., 2003; Metsäpelto et al., 2010). The comparisons showed that the distribution of demographic variables in the sample studied in middle age corresponded very well to the distributions in the age cohort group born in the same year.

We found that the unemployment rates were almost equal in these groups at age 50 in 2009: 8.9 percent of the JYLS participants, and 8.2 percent of the age cohort group were unemployed. The same was true with the average number of children that had been born. It was 1.85 to the JYLS participants and 1.87 to the age cohort group. When we looked at marital status, we found that 60 percent of the JYLS participants and 58 percent of the age cohort group were married. We found that somewhat fewer (68 percent) of the JYLS participants belonged to the Lutheran Church than the age cohort group (76 percent). There are geographical and urban and rural differences in religious activity. In looking at the differences in education and occupational status between the JYLS participants and the age cohort group, we did not find differences for men, but for women there was a slight difference. The JYLS women had attended a vocational college more often than the women in the age cohort group. No difference existed when we looked at higher education. The sample was drawn from an urban area, and this difference reflects better educational opportunities for women in the urban area than on average in Finland in the 1970s.

The analysis of the attrition and the representativeness of the sample showed that the sample studied in middle age was a good representation of the initial random sample, and the distributions of demographic variables corresponded with those in the age cohort group born in 1959 in Finland. It is also notable that the whole 
intended random sample participated in the study, and thus, there was no initial attrition. This kind of opportunity for a longitudinal study is hardly possible anymore. The consent of school authorities was sufficient in 1968 for collecting data at school. Parental consent was required in 1974 when a subsample of the participants and one of the parents of each child were mostly interviewed in their homes. At later ages, participants were asked for written informed consent in each data collection wave. The Ethical Committee of the Central Finland Health District has approved the JYLS data collection (most recently in 2009).

\subsection{Additional data collections}

At age 8, the question of whether children with peer- and teacher-rated aggressive and nonaggressive patterns of behavior would differ in their test responses was posed. I formed a subsample of 60 boys whose behavior most typically represented the following patterns: 10 aggressive, 10 anxious, 10 constructive, 10 compliant boys, 10 boys who were both aggressive and anxious, and 10 boys who were both constructive and compliant (i.e., six “extreme groups," Pitkänen, 1969; cf. Figure 1.2). I asked each of the members of the extreme groups to complete three types of questionnaire that I had devised to study their reactive and proactive aggression directly and more indirectly, as described in Section 5.2.3.

Age 9. The same intensively studied sample of boys was studied again to investigate the stability of their patterns of behavior as assessed by their teachers and to study situational effects on their nonverbal reactive aggression. I devised an "Aggression Machine" with lamps for stimulus presentation and buttons for responses (Pitkänen, 1973a; see Section 5.2.3).

Ages 14 and 20. I had theoretical assumptions about the reasons for the emergence of individual differences in aggressive and nonaggressive behavior and, therefore, I wanted to collect background data about their family and upbringing. At age 14 (in 1974), a subsample of 43 percent of the participants (77 boys, 77 girls) and one parent of each of the teenagers were separately studied more intensively through the process of interviewing them. For financial reasons, the interviews were limited to the participants who had belonged to any of the extreme groups at age 8 or 14 on the basis of peer nominations and teacher ratings (Pulkkinen, 1982). It was the most economical way to study the development of behavior among the participants in the selected categories. Altogether, 201 students were selected. The parents of this sample were asked to agree to interviews with the child and either of the parents. The agreement was obtained from 154 parents (response rate, 76.6 percent). Boys and girls were evenly distributed across the extreme groups: aggressive (10 percent); anxious (8 percent); both aggressive and anxious (7 percent); constructive (12 percent); compliant (10 percent); and both constructive and compliant (11 percent). Nearly half of the sample (42 percent) did not belong to any extreme group. Thus, the selected sample was enriched by the participants who belonged to the six extreme groups at age 14. At age 20, 135 participants (68 men, 67 women) were interviewed again. 
The standardization of a personality test. At age 33 (in 1992), the sample was invited to take part in an international study concerning the standardization of the NEO Personality Inventory (Costa \& McCrae, 1985) in non-Indo-European languages (Pulver, Allik, Pulkkinen, \& Hämäläinen, 1995). The participants were sent the questionnaire by post, and it was returned by 123 men (63 percent of the men) and 126 women (73 percent of the women).

Offspring study. From 1997 to 1999, the participants who had school-aged children were invited to visit the laboratory for the study of their children. The number of participating families was 109; 55 men and 54 women from the initial sample (Pulkkinen et al., 2000). The children who were studied were around of the age when one of their parents had participated in the study. The focus of interest was on the neuropsychological basis and generalizability of children's self-regulation across different domains of functioning.

\subsection{Assessment methods}

In the JYLS, a great number of assessment methods have been used up until the participants turned 50. Baseline data concerning children's aggressive and nonaggressive behavior at age 8 was collected using peer nominations and teacher ratings. Follow-up studies have focused on five major domains: personality and well-being, education and work, family, health behavior and health, and problems in social functioning. Methods used include personal interviews, the life situation questionnaire, inventories, medical examination, and register data. Internationally available research methods have been used in data collection whenever possible. In their translation into Finnish, a back-translation method was used. It means, for example, that an English test was translated into Finnish, and the Finnish version was then translated into English, after which the original and translated English versions were compared for obtaining possible problems in the translation.

The methods used for obtaining the results presented in this book, and those who developed them, are introduced in this chapter. References to the methods are not repeated in later chapters. More information on methods can be found in the original publications and in the Finnish Social Science Data Archive, which has a list (Pulkkinen \& Kokko, 2017) of methods used at different ages.

\subsubsection{Peer nomination and teacher rating}

Peer nomination and teacher rating were the methods that I chose for collecting data at age 8 to test the hypotheses concerning individual differences in aggressive and nonaggressive behavior. Cronbach (1960) argued that in many cases peer ratings furnish more useful information than teacher ratings. According to Walker (1967), the correlations of peer ratings with teacher ratings are fairly high, and the scale scores for peer ratings are even more stable than those for teacher ratings after a one 1-year interval (Walker, 1967). In an educational system where each primary school class is taught by one class teacher, it was not possible to gather ratings from 
two teachers about the same children for validating the teacher ratings, but peer ratings of the child's classmates could be used for this purpose.

Age 8 peer nomination. I called the peer rating method "peer nomination." I modified it from the "Guess who technique," originally presented by Hartshorne, May, and Maller (1929). The ratings (altogether 33) concerned aggressive and nonaggressive behavior. They took the form of: Which of your classmates always tries to be friendly to others? Which of your classmates may attack somebody without any reason? For data collection, I prepared a pad for each participant consisting of 35 successively numbered pages. An answer was given to each question on a separate page by underlining the names that the pupil chose from the list (the two first tasks were exercises, for instance: "Who is absent from the school today?"; the pupils underlined the names of those who were absent). Self-selection was not allowed. I personally collected the peer nomination data in all 12 classrooms by reading aloud the question and observing that all pupils had understood the task and responded to it before turning the next page and reading a new question. The questions were not printed on the pages, only the number of the question that I mentioned before reading it out.

The girls' pad contained the first name of each girl in the class, written in capital letters, and the boys' pads contained the boys' names, also written in capital letters. There were about 15 same-sex pupils in each class. I thought that children would make sharper discriminations if they were only allowed to choose their same-sex peers, and that it would eliminate a stereotypical nomination of boys or girls to certain behaviors. The number of peers to be chosen for each question was left relatively undefined, although an emphasis was placed on the importance of choosing at least three names for each answer. According to Bjerstedt (1963), lower-grade pupils are usually capable of making at least three choices. A fixed number of choices were not expected, because some forms of aggression might be rare. Unlimited peer nominations were also recently recommended by Gommans and Cillessen (2015).

I performed the sampling of the aggression variables in the framework of the model of the components of aggression (Figure 1.1) by utilizing the Kindergarten Study preceding this study, so that different types of aggression were represented. I also formulated items to match with those used in previous studies, if possible. Items in common with the study by Walder, Abelson, Eron, Banta, and Laulicht (1961) made it possible to carry out a comparative study on aggression 40 years later when the participants of both studies had reached middle age (Kokko, Pulkkinen, Huesmann, Dubow, \& Boxer, 2009).

In the sampling of the nonaggression variables, I employed the framework for the analysis of aggressive and nonaggressive behaviors (Figure 1.2) and the definitions given to both controlled expression of impulses and controlled and uncontrolled inhibition of impulses in Section 1.2. In addition, I chose variables for reference variables: emotional lability and stability, and behavioral activity and passivity. Box 2.1 presents most items for socioemotional behavior included in the Peer Nomination Inventory (PNI) and the Teacher Rating Form of the PNI 


\section{BOX 2.1 ITEMS FOR SOCIOEMOTIONAL BEHAVIOR AT AGES 8 AND 14 (PEER NOMINATION AND TEACHER RATING) AND AT AGES 20, 27, 36, 42, AND 50 (SELF-RATING)}

Constructive behavior

Age 8: Active coping with a problem:

Tries to act reasonably even in an annoying situation

Positive thinking and active confrontation:

Thinks that if one negotiates, everything will be better

Consideration of others with helpfulness and empathy:

Sides with smaller and weaker peers

Age 14: Tries to solve annoying situations reasonably, negotiates, conciliates, strives for justice

Adult: I solve difficult situations sensibly; I negotiate, and take others into account in what I do

Compliant behavior

Age 8: Suppression of negative emotions:

Never quarrels with others

Regulation of emotions with compensatory activity:

Dislikes squabbling company and leaves it for something else

Prudence:

Is peaceable and patient

Readily apologizes even when one has not done anything very wrong

Age 14: Is peaceable, patient, adjustable

Adult: I give in easily, avoid quarrels, and usually behave according to expectations

Aggressive behavior

Age 8: Direct reactive physical and verbal aggression:

May hurt another child when angry, for example, by hitting or kicking Direct proactive aggression:

May attack somebody without any reason

Says naughty things to other children even when they have done nothing wrong

Indirect and displaced aggression:

Kicks pieces of furniture or other objects when angry at something

Teases smaller and weaker peers

Age 14: Attacks others without a reason, teases others, says naughty things (Defends oneself if teased, but does not attack without a reason) ${ }^{1}$

Adult: I often get angry and easily land in disputes or fights 
Anxious behavior

Age 8: Social helpfulness:

Is afraid of other children

Overwhelmed by unwanted emotions:

Easily starts crying if others treat them nastily

Who do you think easily cries, say at the dentist

Avoidance behavior:

Is too withdrawn and timid (teacher rating only)

Age 14: Is fearful, helpless in others' company, target of teasing, unable to defend

Adult: I am shy, feel stressed in the company of others and avoid new situations

Self-regulation, reference variables for emotional lability

Age 8: Is sometimes very touchy and sometimes a nice chum

Disobeys the teacher

Is unsteady and lacks concentration in work and attentiveness (teacher rating only)

Age 14: Is impulsive, lacks concentration, changes moods

Adult: I find it difficult to concentrate on the topic, my moods fluctuate, and I am easily upset

Reference variables for emotional stability

Age 8: Is considered a reliable classmate

Always tries to be friendly to others

Age 14: Is reliable, keeps promise, does not get excited

Adult: I am reliable and balanced. I can stay calm in unexpected situations

Excitation/inhibition, reference variables for activity

Age 8: Is always busy and plays eagerly with other children

Age 14: Keeps moving, always on the go, has a lot of energy, plays with others

Adult: I am energetic, active, and spend a lot of time with other people and hobbies

Reference variables for passivity

Age 8: Is always silent and does not care to be busy

Age 14: Does not move much, stands alone, silent

Adult: I am quiet, withdrawn, and spend a lot of time on my own

Social skills. An opening question of the peer nomination and teacher rating: Let us imagine that the teacher tells you to name the classmate who would be a good leader of an excursion that the class is going to make: 
Age 8: Who do you think would be a good leader? Whom would you never choose as a leader of the excursion?

${ }^{1}$ Defensive or reactive aggression defined in this way which excludes proactive aggression correlates with constructive behavior rather than with aggressive behavior, and indicates assertiveness rather than aggressiveness (see Section 10.1.3)

at ages 8 and 14, and corresponding items for the Self-Rating Form of the PNI in adulthood. Box 2.1 shows what aspects of constructive, compliant, aggressive, and anxious behavior were assessed at age 8. All items are listed in Pitkänen (1969: Appendix, Part 2).

Age 8 teacher rating. The variables used in the peer nomination were included in the Teacher Rating Form of the Peer Nomination Inventory. Teachers were also asked to rate children on eight additional variables concerning antisocial behavior, withdrawal, impulsiveness, and school achievement by ranking boys and girls separately in the order of school success. They were also asked to provide the father's (and/or mother's) profession for each child based on the information contained in the school records.

The response pats were the same as in the peer rating. The procedure was, however, different. The variables were presented in the form of a statement, for instance: "Always tries to be friendly to others"; "Attacks somebody without a reason." Girls and boys were rated separately by writing a number from the scale of 3 to 0 after the name of each pupil. Number 3 was to be given to those pupils for whom the characteristic in question was very prominent, and 0 to those pupils in whom the teacher had never observed the characteristic in question. The behavior of the girls/boys was to be compared with that of girls/boys of the same age in general. The time allowed for the rating was two weeks, as for many variables rating required accurate observations of the pupils' behavior. The teachers (12) were encouraged to make observations of their pupils during the breaks.

School success. A teacher was asked to rank order boys and girls separately on the basis of their school success. The rank orders were transformed into five-point normal distributions.

Age 14 peer nomination. I reduced the number of items from 33 to 9 on the basis of the correlative and factor analytical results on the interrelationships between the items at age 8 (Pitkänen, 1969, pp. 182-3). At age 8, separate items for each act were used, for example, "attacks," "teases," and "says naughty things," but a combined formulation "Attacks without reason, teases others, says naughty things" was used at age 14 (see Box 2.1). By reducing the number of items, I tried to reduce the workload of pupils and teachers and also facilitate the execution of data collection in the 78 school classes to which the participants had spread during the interval of 6 years. Peer nomination was completed for the whole class if there was even one study participant in the class by including the names of all the pupils in the class on the list grouped into boys and girls. In the peer nomination, pupils were asked 
to nominate three same-sex classmates who were best described by each item. Self-selection was not allowed. From these responses, nominations concerning the study participants were calculated and included in the study. At the time, it was not possible to handle the massive amount of data collected by peer nominations in 78 classes concerning all students in all the classes, numbering around 2,300 students in total.

Age 14 teacher rating. The same nine items worded as statements were used in the teacher rating. Teachers were asked to assess only the study participants, not the whole class. They were asked to think of 100 same-sex pupils and a scale where the number 1 referred to the pupil whose behavior the item described very little and number 100 to the pupil whose behavior the item described very well. The study participant had to be compared with them and a score from 1 to 100 had to be given to him or her. The framework of 100 students was expected to make the ratings comparable, because in many cases, a teacher rated only one or two participants. Due to a move from the class-teacher system in the primary school to the subject-teacher system in the lower secondary school, there were several teachers who taught the class. An effort was made to find the teacher who knew the study participant best and to ask him or her to make the ratings. The total number of teachers who assigned the ratings at age 14 was around 130 .

Teacher questionnaire. The teachers were also given a teacher questionnaire in which he or she was asked to respond to questions concerning the pupils' popularity in the class; his/her interest in school work; specific difficulties in the pupil's learning or life conditions; his/her reactions to failure; punishments at school; truancy; observations about the pupil's activities in their free time, substance use, and contact with the police; the teacher's contacts with the pupil's parents; and changes in the pupil's behavior and school achievements.

School success. Data on school reports at the end of the comprehensive (obligatory) school at around age 15 were collected by researchers from the school archives (Pulkkinen, 1989a).

\subsubsection{Personal interview}

Ages 14, 20, and 27. The interviews were conducted by discussing chosen themes with a participant without strictly formulating the questions, and by following the order in which the participant wanted to speak about the topics. I had been at Sussex University, UK for postdoctoral studies during the academic year 1972-3 under the supervision of Professor Marie Jahoda. During my postdoctoral year, we conducted a study with Asian people who had been expelled from Uganda by Idi Amin and had immigrated to England. There I learned how to conduct a nonintrusive interview, known as a thematic interview, which I later brought back to my own university. The thematic, semi-structured interview was expected to give more insight into the life and experiences of the participants than the structured questionand-response options designed at the desk of a researcher. Little was known about the background of different behavior patterns and the transition into adulthood, 
because these questions had hardly been studied in psychology by the middle of the 1970s and the thematic interview gave us greater insight into these processes. My doctoral students applied this interview method to their doctoral dissertations and wrote a widely read textbook about the method (Hirsjärvi \& Hurme, 1980).

In the JYLS, there were about 12 interviewers in each data collection wave who I trained to use the thematic interview technique. Most interviewers were students doing their Master's degree in psychology. Interviewers mostly participated in data collection only in one of the waves, which meant that interviews were technically independent. The interviews were tape-recorded and they were coded into categories formed on the basis of the response variation found in the interviews. These variables were used in quantitative data analyses, but data were also analyzed qualitatively in the students' theses and dissertations.

At age 14, the themes discussed individually with the participant and one of the child's parents (parent interview) were selected to shed light on the formation of individual differences in socioemotional behavior. They concerned both the child's and the parent's perspective on the interaction between the parents and the child (the parents' trust in the child, consideration of the child's opinion, shared activities with the child), the parents' interest in and control over the child's school attendance and leisure activities, the type of guidance provided, the punishments given, and the family's living conditions. In addition, various aspects of the child's behavior were covered, such as his/her interest in school, activities during his/her free time, friends, substance use, and the child's relationship with the parents.

At age 20, the themes of the interview were selected to increase the researchers' understanding of the formation of the young people's lifestyles and growing independence from their parents. The interview themes covered the participant's relationships with parents and friends, romantic relationships, independence from the parents, economic dependency, life attitudes, education, leisure activities, use of alcohol and cigarettes, and plans and expectations for the future. Some themes focused on the participant's childhood environment and upbringing to replicate the findings obtained earlier.

At age 27, the transition into adulthood was studied more thoroughly. The themes discussed concerned living conditions and moving home, marital status, children and child-rearing, education and work, relationships with the parents and childhood memories, experiences of being an adult, personal coping styles, smoking and use of alcohol, and life goals.

Ages 36, 42, and 50. The interviews were more structured at ages 36, 42, and 50 than at age 27 , because the coding of the thematic interviews turned out to be too time-consuming and the comparability of the thematic interviews between participants was more difficult to achieve than it was by using a more structured interview. The coding of the age 27 interview helped to formulate relevant response categories for a more structured interview. We built the interviews to include a set of inventories that the participants filled in during the interview; some inventories were given to them to be completed at home and then brought in to the interview, 
or sent back by post afterwards. With the use of inventories, we aimed to increase the comparability of the findings with international studies.

The topical areas of the interview and the inventories were kept as similar as possible at ages 36,42 , and 50 , but some changes were made due to the age-specific topics or other reasons such as the issues prevalent at the time. The latter refers, for instance, to questions concerning the effects of the economic recession and the use of modern information technology, and to removing the topic of health that was included in the interview at age 36 , because the participants underwent a separate medical examination at ages 42 and 50. An example of one of the agespecific changes was the topic of moving into middle age, which was added to the interview at age 42, including questions about, for example, a possible experience of a mid-life crisis. In general, the interview included the Identity Status Interview, the Life History Calendar, and discussion, for instance, about important domains and turning points in people's lives, marital relationships, employment, and working conditions. During the interview sessions, the participants were presented with several self-administered inventories that often were shortened versions of the original ones. They concerned, for instance, emotion regulation, personality, health, substance use, free time activities, and child-rearing, as described in more detail in this chapter.

The interviewers were mostly psychology students doing their Master's degree in psychology. There were 12 to 15 interviewers in each wave. The interviews were also conducted by senior researchers. All the interviewers were trained to conduct the interviews. The participants were interviewed in their homes, in a university laboratory, or in other places of the participants' choosing. The participants' travel costs to the University of Jyväskylä were reimbursed. If necessary, the interviewers travelled to interview a participant, including going abroad. The length of the interview varied from 2 to 5 hours depending on the participant's need and willingness to speak about his or her life, and the time that was taken to fill in the inventories. The interviews were tape-recorded. The interviewer coded his or her interviews according to set guidelines. Transcriptions were written from answers to questions which did not include response options.

Identity interview. The interviews at ages 27, 36, 42, and 50 included the Identity Status Interview (Marcia, 1966) focusing on five domains at ages 27, 36, 42, and 50: religious beliefs, political ideology, occupational career, intimate relationship, and lifestyle, with the addition of parenting at ages 36, 42, and 50 (Fadjukoff, Pulkkinen, \& Kokko, 2016). The Identity Status Interview was conducted in the context of the discussion on the domain in question. For instance, occupational identity was explored during the discussion about work. Each participant's identity status was assessed for each domain using two criteria: the firmness of commitment and the presence or absence of a period of exploration, and was then coded into identity status categories (identity diffusion, foreclosure, moratorium, and identity achievement).

Life Events and the Life History Calendar. A Life Event Inventory was included in the Life Situation Questionnaire at ages 27 and 36. In addition, turning or branching points in life were studied at age 36 (Birren \& Hedlund, 1987) by asking the 
participants to describe significant life events that had affected their choices and decisions. At ages 42 and 50, the personal interview was begun by introducing the Life History Calendar (Caspi et al., 1996). It was intended to produce information about the participants' residence, family (marital relationships and children), educational and work history, and other life events, logging what had occurred each year from ages 15 to 50. At age 42, the participant observed the interviewer using a life history table as a basis of discussion about what had happened to the participant since he or she had turned 15. On the vertical dimension of the table, there were the topics listed that they were going to discuss and on the horizontal dimension, the years from 15 to 42. The topics were: living arrangements, co-habitation/marriage, children, education, work, and unexpected life events.

For each topic, there was a life line on which the interviewer marked the age when the event started and when it finished. For instance: "Where did you live when you were 15 ?" The place was written down. "How long did you go on living there?" The line was drawn from 15 until the age the participant mentioned, for instance, until 20 years, which was marked by an X. "Where did you live after that? For how long?" until age 42. The following questions tried to clarify when the participant moved away from the parental home and formed an intimate relationship: "When did you move away from your parents? With whom did you live? Did you live with the same partner? Did you get married? When? When did you have your first child, your second child?"

At age 50, the time after age 42 was screened using The Life History Calendar, and for those participants who did not attend the interview at age 42, the Life History Calendar was used to map the timing of major life events from age 15 to 50 . The calendar data were used, for instance, in the study of adult transitions.

\subsubsection{Self-reports and other methods}

Life Situation Questionnaire. In adulthood, the participants were first sent a Life Situation Questionnaire (LSQ) by post (Pulkkinen, 2006). It was developed for data collection at age 27 (LSQ1), and it was used again at ages 36 (LSQ2), 42 (LSQ3), and 50 (LSQ4). These versions were kept as similar as possible across the adult years to ensure the comparability of the data collected at different ages, but also allowed for some adaptations to the participant's age and the current events at the time. Even slight changes in response options make the comparison difficult. The LSQ included about 200 items concerning, for instance, education and work, living conditions, family relationships, leisure activities, use of alcohol and smoking, and several self-rating scales. The scales concerned, for instance, satisfaction with life, socioemotional behavior, alcoholism, health, life events, personal control over one's development, and future orientation. These topics were deepened in the personal interview. The LSQ provided comparable background information on all the participants.

Personality traits. Two personality inventories were presented to the pupils during their school lessons at age 8 . One of them was the Personality Inventory for 
the Lower Forms of the Primary School based on the work by Cattell and Coan (1957). It had been standardized for Finland (Ylinentalo, 1965). The other inventory was the Junior Eysenck Personality Inventory (Junior NESI; Eysenck, 1965). It was being standardized for Finland with a modified scale for extraversion divided into impulsive and social extraversion. The remaining scales in this inventory were the neuroticism and lie scales.

At age 27, the Eysenck Personality Questionnaire (EPQ; Eysenck \& Eysenck, 1975) standardized for Finland (Haapasalo, 1990), was administered. It included the scales for extraversion, neuroticism, psychoticism referring to impulsive and callous behavior, and a lie scale.

At ages 33, 42, and 50, personality traits were assessed using the Five Factor Model Framework. Our Estonian colleagues had initiated the standardization of the Neuroticism, Extraversion, Openness Personality Inventory (NEO-PI; Costa \& McCrae, 1985) for non-Indo-European languages, Estonian and Finnish, and invited the JYLS to participate in it. The version presented with the participants comprised 315 items including additional items to increase cultural relevance among Estonian and Finnish people (Hämäläinen, Pulkkinen, Allik, \& Pulver, 1994; Pulver et al., 1995). On the basis of item analyses in both language groups, a version with 181 items was formed. It is an authorized adaptation of the NEO-PI in which about onequarter of the items were substitutes for the original American items. A shortened version was formed from it to correspond to the shortened 60-item NEO-Five Factor Inventory (Costa \& McCrae, 1989). In the Finnish NEO-FFI, only three items were substitutes for original items. It was administered to the participants at ages 42 and 50. For age 33, responses to the items of the NEO-PI which were included in the shortened version of this test, NEO-FFI, were considered.

Vulnerability traits. The concept of vulnerability traits refers to personality correlates of some psychiatric disorders such as depression. A vulnerability trait, for instance aggression or anxiety, may cause risks for social and personal adjustment. Vulnerability traits were measured using the Karolinska Scales of Personality (KSP; Schalling, 1986) that were administered at ages 36, 42, and 50. The KSP consists of 15 scales that can be grouped into four broader classes: (1) introversion-extraversion-related scales: impulsiveness, monotony avoidance, and detachment; (2) conformity-nonconformity scales: social desirability and socialization; (3) anxiety-related scales: psychic anxiety, somatic anxiety, muscular tension, inhibition of aggression, and psychasthenia; and (4) aggression-related scales: verbal aggression, indirect aggression, irritability, suspicion, and guilt. Aggression was also assessed at ages 36, 42, and 50 using the Aggression Questionnaire by Buss and Perry (1992), complemented by a few items for the study of physical and verbal aggression and the self-control of emotions (Kokko \& Pulkkinen, 2005).

Emotion regulation. I devised for data collection, at age 20, a Self-Control Inventory by bringing together items from several existing measures of anxiety, aggression, and social desirability, and a 64-item Self-Control Check List (Pulkkinen, 1982), because inventories for the study of emotion regulation were not available. At age 27, the Sensation Seeking Scale (SSS; Zuckerman, 1979) standardized for 
Finland (Haapasalo, 1990), was administered. It included the scales for experience seeking, thrill and adventure seeking, and disinhibition.

At ages 36 and 42, emotion regulation was studied using new measures that were available for the study of the regulation of emotions. The participants completed the following state measures during the semi-structured interview: the Brief Mood Introspection Scale (BMIS); Mayer \& Gaschke, 1988) and a combined metascale including the Meta-Evaluation Scale (MES) and the Meta-Regulation Scale (MRS) (Mayer \& Stevens, 1994). The BMIS consisted of 15 adjectives that measured the participant's current mood at the beginning of the interview. The MES assessed the person's conscious evaluation of his/her current mood on the scales of clarity, typicality, influence, and acceptance. The MRS measured conscious emotion regulation with subscales for repair, dampening, and maintenance. Emotional ambivalence was studied with the Ambivalence Over Expressiveness Questionnaire (AEQ; King \& Emmons, 1990). Self-assessments of socioemotional behavior were asked at ages 27, 36, 42, and 50 in the items for adults presented in Box 2.1.

The Self-Control Schedule (SCS; Rosenbaum, 1980) was administered only at age 36. At age 42, the scales of Rumination, Emotional Inhibition and Benign Control of the Emotion Control Questionnaire by Roger and Nesshoever (1987) were administered, as well as the Self-Report Measure of Emotional Intelligence by Schütte et al. (1998). The latter was based on the analysis of emotional intelligence by Mayer and Salovey (1997) covering emotional perception and expression and emotional facilitation and management.

Temperament. Temperament characteristics were studied at age 42. The Adult Temperament Questionnaire (ATQ; Rothbart, Ahadi, \& Evans, 2000) was handed to the participants in the context of a personal interview. The ATQ consists of several scales that can be grouped as follows: surgency, including the subscales for sociability and positive affect; effortful control, including the subscales for activation control, attentional control, and inhibitory control; and negative affectivity, including the subscales for fear, sadness, and discomfort. In the 1960s, when the participants were children, temperament measures were not available.

Self and life structure. At ages 27, 36, 42, and 50, a questionnaire called the Personal Control Over One's Development was included in the Life Situation Questionnaire. I devised it based on Brandtstädter's model (1984). The items were divided into five factors: self-confidence, social support, low self-worth, blaming others for one's own failure, and contentment with one's present achievements (Pulkkinen \& Rönkä, 1994). Self-esteem was studied using the Self-Esteem Scale (Rosenberg, 1965) at ages 36, 42, and 50. At ages 36 and 42, the Strategic Attribution Questionnaire (Nurmi, Salmela-Aro, \& Haavisto, 1995) including scales for mastery beliefs, failure expectations, and task-irrelevant behavior, was also administered.

For the study of the participants' life structure, that is, the importance of various things in their lives, I developed a Life Structure Inventory which was administered in the context of the Life Situation Questionnaire at ages 27, 36, 42, and 50. It includes several parts. One part, the Personal Value Task, concerns the assessment of the personal value of arts, politics, science, technology, religion, sports, 
economics, nurturing/education, and social work. The second part is the Pleasure Ranking Task, in which family, work, friends, and leisure activities are asked to be ranked according to the amount of pleasure they gave to the respondent. The third part of the Life Structure Inventory consists of the Future Orientation Questionnaire. It includes questions about (1) to what extent the participants considered that their future depended on, for instance, themselves, their past, their home and parents, the world situation, and God; (2) how worried they were about the future in respect of, for instance, financial problems, relationship problems, diseases, and war; and (3) how important they considered issues such as economic success, their health, cultural life, and world peace for the future.

A Goal Pattern Schedule, later entitled a Personal Life Investment Schedule (Staudinger, 1996; Staudinger \& Fleeson, 1996), was presented to the participants at age 36. It included ten content areas: health, cognitive fitness, hobbies or interests, relations with friends, sexuality, well-being of family, professional activity, independence, life, and death. The participants were asked to rate on a five-point scale how much they thought or did something about each of the topics. The measure was extended in the JYLS to include financial matters, religious matters, physical fitness, fulfillment of goals and capacities, and wisdom (understanding of life) at ages 42 and 50.

At age 50, values were examined with the 57-item Schwartz Value Survey (Schwartz, 1992) translated and adapted for Finland by Puohiniemi (1995). Selfreflection was studied by asking the participants how meaningful they found their current life (Read \& Suutama, 2008). Also, the Spiritual Meaning Scale, including questions about the purpose of life (Mascaro, Rosen, \& Morey, 2004) and the scale for Regrets (Jokisaari, 2007) were presented. For the latter, the participant was asked to look back on his or her life and think of the things he or she would have done differently and why they regretted not having done these things.

Mental well-being and ill-being. Well-being has different components, such as emotional well-being that refers to positive affect and satisfaction, and psychological wellbeing, which is defined as an individual's realization of his or her "daimon" (true nature), and it describes an individual's positive functioning (Ryan \& Deci, 2001). Emotional well-being was assessed with a Life-Satisfaction Scale included in the Life Situation Questionnaire at ages 27, 36, 42, and 50. These items concerned satisfaction with seven life domains: housing, financial situation, choice of occupation, present employment, present intimate relationship or lack of it, content of leisure time, and present state of friendship. In the interview at ages 36, 42, and 50 an overall level of satisfaction and happiness at the time was also described on the Mood Line (Perho \& Korhonen, 1993). At age 50, the Satisfaction with Life Scale (Diener, Emmons, Larsen, \& Griffin, 1985), and the International Positive and Negative Affect Schedule-Short Form (I-PANAS-SF; Thompson, 2007) were also presented.

Psychological well-being was measured at ages 36, 42, and 50 using the Scales of Psychological Well-Being (Ryff, 1989) with three items for each of the six components of psychological well-being (self-acceptance, positive relationships with others, mastery of one's environment autonomy, purpose in life, and personal growth). 
In addition, social well-being was assessed at ages 42 and 50 using the Scales of Social Well-Being (Keyes, 1988), and low mental well-being was assessed at ages 36, 42, and 50 with a Depression Scale included in the General Behavior Inventory (GBI) developed by Depue (1987). This measure of depressive symptoms can be used with clinical and nonclinical populations. The Generativity and Integrity scales by Ryff and Heincke (1983) was presented at ages 42 and 50 for the study of caring for the next generation. Positive subjective experiences or the sense of coherence was studied using the Orientation to Life Questionnaire (Antonovsky, 1987) at age 42.

Family. Child-rearing was studied at ages 36 and 42 with a 28-item Child-Rearing Practices Questionnaire that I composed for the study of child-centered versus adult-centered parenting drawing on several sources. Items measuring encouragement of independence, rational guidance, supervision of the child, expression of affection, authoritarian control, and control through the use of anxiety were drawn from the Child Rearing Practices Report (Roberts, Block, \& Block, 1984) used by Chao \& Kohlberg (1994) and Kochanska (1990). Parental depression, punishment and conformity, and affection and attachment were assessed by means of items drawn from the inventory by Gerris et al. (1993). The latter inventory included four items for parenting stress developed by Abidin (Lloyd \& Abidin, 1985). The items and factor structure of the Child Rearing Practices Questionnaire have been published in the study by Aunola, Nurmi, Onatsu-Arvilommi, and Pulkkinen (1999). The 4-item Parenting Stress Scale, with a few child-centered parenting items, was also presented at age 50 .

Intimate relationships were studied using the Dyadic Adjustment Scale developed by Spanier (1976) and revised by Busby, Christensen, Crane, and Larson (1995). It included the scales of consensus, satisfaction, cohesion, and happiness. Marital hostility was defined on the basis of the Conflict Tactics Scales invented by Straus (1992) at age 36 and its revision (Straus \& Hamby, 1996) at ages 42 and 50. It concerns marital disagreement and ways of settling differences between the partners, from negotiation to verbal and physical aggression. Jealousy was studied at age 42 through the use of a questionnaire by Kontula and Koskela (1991). Adult attachment styles were studied using a four-category model of adult attachment using the Relationship Questionnaire by Bartholomew and Horowitz (1991).

Work. The significance of work using a typology of Kahn and Wiener (1967) was explored during the interview at ages 36, 42, and 50. Inventories concerning control at work, used in a national study of living conditions conducted in 1994, and job exhaustion (Maslach \& Jackson, 1986) were presented to the participants at ages 36, 42, and 50. Furthermore, a series of questions were presented at ages 42 and 50 about time demands at work using a Job Stress Survey adapted from Karasek (1979) and Elo, Leppänen, Lindström, and Ropponen (1990), and about skill discretion referring to the degree of creativity, learning, and highly developed skills required in a job using a Job Content Questionnaire by Karasek (1985). An inventory about work-home interaction (Frone, Russell, \& Cooper, 1992) was presented at ages 36 and 42, and one on work and mental health was presented at age 36 (Warr, 1990). 
At age 50, inventories on the themes of the reconciliation of work and family life (Geurts, 2000; Van Steenbergen, Ellemers, \& Mooijart, 2007) and the experiences with recovery from work (Sonnentag \& Fritz, 2007) were presented. In addition, work engagement was assessed using a short version of the Utrecht Work Engagement Scale (UWES; Schaufeli, Bakker, \& Salanova, 2006), and workaholism was explored through the use of the ten-item version of the Dutch Work Addiction Scale (DUWAS; Schaufeli, Taris, \& Bakker, 2008). The use of modern technology was also studied via the Life Situation Questionnaire at ages 42 and 50.

Health behavior. For health behavior, two alcoholism screening tests were presented. One of them was the CAGE questionnaire developed by Ewing and Rouse (Ewing, 1984). It consists of four questions: (a) "Have you ever felt the need to Cut down on your drinking?"; (b) "Have you felt Annoyed by criticism of your drinking?"; (c) "Do you feel Guilty about your drinking?"; and (d) "Have you ever had a drink in the morning to get rid of a hangover (an Eye opener)?" The second test (Malmö modification of the brief MAST; mm-MAST) had been adapted by Kristenson and Trell (1982) from the Michigan Alcoholism Screening Test (MAST; Selzer, 1971) for use in Scandinavia. The participants were also asked to fill in a quantityfrequency table where the horizontal quantity options consisted of different portion estimates per occasion (e.g., a bottle of beer) and seven possible vertical frequency options for each quantity option (e.g., several times a day, once a day) (Pitkänen et al., 2008). The annual consumption of alcohol can be estimated from these responses.

Leisure activities were explored through the Life Situation Questionnaire at ages $27,36,42$, and 50 and were also studied by means of a questionnaire containing various ways of spending leisure time at ages 20 and 50. Choices of television programs (Van den Bulck, 1995) were studied in more detail at age 36, as were the participants' viewing motives, via a method adopted from Rubin (1983). The Self-Report Delinquency Scale by Junger-Tas, Terlouw, and Klein (1994) was administered at age 36 .

Medical examinations. A medical examination was conducted by a physician and a nurse at ages 42 and 50. It contained an interview about medication, diseases, injuries, hospitalization, physical symptoms, family history, and self-rated health. In addition, blood pressure, heart rate, height, weight, and waist and hip circumference were measured. For the study of the personal experience of health, subjective health assessments were made at ages 27, 36, 42, and 50, and a Symptom Check List (Aro, 1988) was administered at ages 36, 42, and 50.

The participants were also invited to take laboratory tests at ages 42 and 50 . These tests yielded information about total cholesterol, high-density lipoprotein (HDL), low-density lipoprotein (LDL), triglycerides, blood sugar, an adrenal androgen dehydroepiandrosterone-sulfate (DHEAs), and 12-hour urine norepinephrine. Most of the blood samples were analyzed in the clinical laboratory of the Hospital of Central Finland and the rest were sent to the clinical laboratories of other hospitals.

Register data. With the consent of the Ministry of Justice, criminal records (both from the government register and local police records) of the initial sample were 
looked into at age 20. The age at which individuals can be accused of crimes (criminal responsibility age) is 15 years of age in Finland. Those below this age limit come under the jurisdiction of child welfare authorities. The government register was searched every 5 years up to age 46 . Only certain offenses remain permanently on the register. The Tax Authority registers were searched at age 43 and from age 45 to 50 .

\subsection{Data analysis}

A thorough understanding of data, based on looking at distributions, means, standard deviations, and correlations of variables, and on explorative factor analyses, lays a foundation for testing hypotheses with more advanced methods. From the beginning, mainly quantitative methods of data analysis have been used in the JYLS. At the time when the JYLS was started, the development of the technique of factor analysis inspired personality researchers to examine dimensions of variables (e.g., extraversion) in which differences between individuals can be described. This approach is called variable-oriented. An alternative approach, called person-oriented, focuses on a person as the unit of analysis and identifies basic categories of people to classify people instead of variables into latent groups (Bergman, Magnusson, \& El Khouri, 2003). Both approaches were employed in the JYLS. The availability of particular statistical tools has favored the variable-oriented approach. Also in the JYLS, a wide variety of variable-oriented methods have been used; of these, the main methods are listed in this section.

In order to reduce the large number of variables available for the studied constructs, explorative and confirmatory factor analyses have been used. The obtained factor scores have been used, or the loadings have guided the formation of new sum scores. The focus might also have been on simultaneous relations between different areas of functioning, or in predictive associations such as whether developmental background or childhood characteristics predict adult functioning. In these cases, linear or logistic regression analysis, path analysis, or a structural equation modeling (SEM) have been applied. These methods have also been used for the analysis of the relative stability of a characteristic across time, such as personality traits and mental well-being. The main statistical software used in the JYLS have been SPSS, SAS, Stata, LISREL, and Mplus (see, e.g., Muthén \& Muthén, 1998-2005). Details of statistical analyses and references to methods can be found in the original publications presented in this book.

In the study of group differences, mean levels of characteristics have been compared using a t-test, and univariate and multivariate analysis of variance (ANOVA and MANOVA). In an investigation of the stability and change over time in the mean level of a characteristic, the repeated measures analysis of variance has been employed. More recently, we have applied a latent growth curve analysis for the study of mean level changes. It allows for obtaining both the level of the latent characteristic (e.g., a personality trait) and the shape of its change. There is an aspect of a longitudinal study which is worth noticing, and this concerns repeated 
measurements. The comparison of absolute stability and changes can only be made if the measures are exactly the same at each point of measurement. Nevertheless, the use of the same measures or the same response scales is not always possible in psychology, because the indicators of a characteristic often change over time. Therefore, the estimation of changes is often relative, based on the comparison of changes in other individuals. A construct analysis is needed for assessing whether measurements at different time points are dealing with the same phenomenon or theoretically related phenomena.

Following the development of statistical software, LISREL models combining factor and regression analyses were used in the JYLS from the 1980s to analyze relations between variables. For example, in the study by Kokko and Pulkkinen (2000), the latent factors for aggression at age 8 and school maladjustment at age 14 were formed, and their relationships to adult long-term unemployment were examined by considering the measurement errors. Using SEM, a path analysis has been applied to the study of mediating and moderating factors, and to the study of cross-lagged associations between different characteristics across time. During this millennium, advanced statistical software such as Mplus, combining SEM and categorical data analyses have been available.

The use of variable-oriented approach has also been criticized of the loss of a holistic view of an individual. In the JYLS, the person-oriented approach has also been applied in data analysis, particularly for finding different personality types and profiles. Methods such as a hierarchical cluster analysis, a latent profile analysis, and configural frequency analysis have been employed. Furthermore, a trajectory analysis (Nagin, 1999) has been applied to the study of subgroups in developmental trajectories in, for example, neuroticism to find out individuals who are high, moderate, or low in that characteristic at all ages.

There are many participants who have taken part in the majority, but not all, of the data collection phases or whose records contained missing information for a certain data collection phase. The missing and incomplete data have been treated in various ways. The possibility of replacing missing data with corresponding information from an earlier phase or a parallel method at the same age has been considered. Replacement is possible for some types of information, such as the highest degree of education obtained until adulthood, never using alcohol until a certain age, or the duration of unemployment. In cases where data were lacking, a traditional way was to exclude incomplete data list- or pairwise, which means that only the participants were included in the analysis for whom the requested data were available. Recently, more advanced methods for treating missing data have been applied; they include simple and multiple imputation methods, and the full-information maximum likelihood estimation method.

The methods of handling study data, and computing capacities, have hugely developed during the almost half a century that the JYLS has been taking place; from manual computing to punch cards and the mainframe computer system, through personal computers and floppy disks, to very effective laptops. In the 1960s, even factor analyses were done manually. At the beginning, statistical 
analysis methods mainly facilitated testing the relation of two variables. Later, software for multivariate analysis became possible and easier to use. In this millennium, very complicated modeling with discrete and continuous, and observed and latent variables simultaneously has become possible, of which one could only have dreamed in the early days. 


\section{3 \\ REFLECTIONS ON THE RESEARCH PROCESS}

\subsection{The evaluation of choices}

Almost 50 years have passed since the beginning of this longitudinal study. It is a time to reflect on (1) whether the longitudinal study was worth undertaking, (2) what should have been done differently, and (3) what are the lessons that have been learned? Regarding the first question, responsible researchers are not the right people to assess the value of the study; it is the task of the scientific community both of the present and, particularly, of the future. The value of a study depends on the contribution that the study makes to science through the sustainability of its results and theoretical ideas.

The second question, what should have been done differently, is also difficult to address, because the choices were made in certain contexts that often were beyond the control of the researchers. The choices mostly concerned methods, and five examples (A to E) of these choices are presented in this chapter. Example A concerns the choice of research instruments. From the present perspective, one might recommend the use of certain measures, such as temperament tests in childhood or the NEO Personality Inventory in adolescence, but the present knowledge base for their assessment was not available in the 1960s and 1970s. One cannot utilize today the knowledge and methods that will be achieved and published years into the future. The time perspective easily disappears when the choices that were made in a longitudinal study are discussed.

Example B concerns the scope of the study. It would have been interesting to include cognitive tests into the set of measures in the study, but the focus of the JYLS was on the development of socioemotional behavior, and there were no human or material resources for expanding the study to include cognitive development; some cognitive tests were presented at ages 42 and 50. Increasing our understanding of the holistic process of development could possibly be achieved by 
combining research findings across studies. Example C concerns new theoretical and methodological approaches to human development that have emerged during the course of the study, such as molecular genetics, which has grown enormously in importance, including in the field of psychology. Until now, it has not been possible to do genetic analyses in the JYLS, but saliva samples have been collected from those JYLS participants who have given their consent for this, and they have been frozen and saved for possible use in the future. The sample size is not sufficient for an epidemiological study, but for a study of specific hypotheses the sample might make a contribution.

Example D concerns the research sample. It would have been valuable to conduct the intensive follow-up study with the whole original sample also in adolescence, but it had to be limited to a selected sample due to insufficient funding in the 1970s. It was, however, important to receive some funding, because it encouraged the continuation of the study, although it was not sufficient for the execution of the full study at that time. Example E concerns the timing of the data collection. One might ask why data collection was not timed with regular intervals but was conducted at ages $8,14,20,27,36,42$, and 50 and how this choice affects the results. The starting age was chosen on the child developmental basis as explained in Section 1.2. Thereafter, the aim has been to collect data in developmentally interesting phases. Data collections cover childhood, two stages of adolescence, two stages of young adulthood, and two stages of middle age. Small irregularities in the intervals, due to external conditions, were not expected to affect results, because the aim of this study was not to study certain developmental stages but rather to study developmental processes. One might remark that there is a gap in data collection during early childhood, and it is true that important development takes place in children before the age of 8 . For understanding development holistically, research findings from different studies must be integrated, which in this case means, particularly, understanding the formation of individual differences in socioemotional behavior before the age of 8 .

The third question, what are the lessons that have been learned can be presented in the form: what can be recommended for someone who would like to launch a longitudinal study? Experienced longitudinal researchers such as Block (1971), Magnusson and Bergman (1988), and Rose (2006a) have discussed this matter. There are also useful handbooks about research methods in developmental studies such as that by Laursen, Little, and Card (2012).

\subsection{Recommendations for launching a longitudinal study}

Five recommendations are presented based on our experiences by utilizing the article by Pulkkinen and Kokko (2012). After each recommendation, the solutions found in the JYLS are described and reflected upon.

The first recommendation is to carefully consider whether one has a research problem for which a longitudinal study is really needed. Many age group comparisons can be made with cross-sectional studies. A longitudinal study is needed 
if one is interested in consistency and change in individuals, and in developmental consequences of individual characteristics over time, such as the accumulation of success or problems. In the JYLS, the significance of the characteristics of individuals for their future development was the target of the study. It was a question that could only be studied longitudinally.

The second recommendation is to consider whether research problems concern phenomena that are sustainable, independent of the theoretical constructs used for their interpretation. Theoretical constructs created in a specific intellectual climate are vulnerable to becoming outdated, but they can be updated with advancing knowledge and theory, if the problem is relevant. In the JYLS, core problems concerning individual differences in socioemotional behavior and the developmental processes associated with them have been sustainable. The theoretical constructs to explain these differences and processes have been slightly changed to adjust to the intellectual climate of the time and a new knowledge base.

The third recommendation concerns the careful choice of a sample by weighing up different viewpoints. A longitudinal study may be a nationwide cohort study or it may be limited to a smaller sample. The size of the sample is directly related to the costs and often to the intensity of the study. A large cohort study demands substantial funding over several years, as well as an expert team drawn from different disciplines to conduct it. Individual researchers may, however, conduct useful longitudinal studies in psychology because the understanding of psychological processes may be increased through an intensive study of a smaller sample, if its size remains sufficient after the unavoidable attrition that occurs in longitudinal studies. A biased attrition limits the generalizability of results even when the sample is originally large. Tracing the participants for a follow-up study and ensuring that they are willing to attend the study again and again requires a lot of time and attention. With this sample size, it has been possible to conduct an intensive study and keep the participants involved in it. In the JYLS, the sample was randomly drawn from a certain area of the country. Finland was a homogeneous country in the 1960s in terms of the ethnic background and the socioeconomic status of the people. This explains the similarity of the demographic data even at age 50 in the JYLS sample and in the whole age cohort born in Finland in the same year, 1959.

The fourth recommendation concerns the choice of measures. The accumulation of scientific knowledge presupposes that research findings in different studies are comparable. A way to increase comparability is to use internationally acknowledged measures that are carefully developed. Another way is to harmonize measures between different studies so that they have common elements that warrant comparisons of the results or joint analyses. The quality of data achieved is the ultimate criterion for the value of a longitudinal study. If the baseline data have not been collected with reliable and valid measures, there is no basis for the follow-up study. The use of self-made inventories without careful development work risks the reliability and the validity of the measures and compromises the comparison of research findings. 
In the JYLS, several internationally known inventories were used in adulthood. Key personality measures were adapted to, or standardized for, Finland, and for the rest, careful translations and back-translations were used for securing their usability in another language culture. The harmonization of measures was carried out for peer nomination by partially using the same items as in a US longitudinal study. A choice was made to collect the baseline data by using teacher ratings and peer nominations, although there was pressure in the 1960s to use psychological tests for data collection. There were projective techniques such as the Rosenzweig Picture Frustration Study, Children's Apperception Test, and sentence completion tests (Pitkänen, 1963a, 1968), but relationships between children's responses to projective tests and their overt behavior had turned out to be complicated (e.g., Olweus, 1969). There were also personality inventories, but it was generally assumed that a social desirability set affects an individual's responses and lowers the validity of responses in respect to overt behavior (Crowne \& Marlowe, 1960; Edwards, 1957). Special scales for response sets and lying were added to personality inventories. As both aggressive and nonaggressive behaviors were studied in the JYLS, it was not relevant to assume that children's presentation of themselves in positive ways was always a result of lying or that it was motivated by a tendency to respond in socially desirable way (i.e., social desirability set). More fundamental motivations and differences between individuals were expected to exist. Furthermore, responding to personality inventories is demanding because it presupposes that an individual can make relevant self-assessments. For a child, this may be difficult for a variety of reasons. Two personality inventories were, however, presented to the participants at age 8 , but they turned out to have low validity when peer and teacher ratings were used as criteria, particularly in emotional behavior or emotion regulation (Pitkänen, 1969, p. 123). Their use would not have formed a firm basis for the continuation of the study of socioemotional behavior.

The fifth recommendation is to use advanced statistical methods for data analysis. It was one of the major goals of the JYLS. Prospective data collected in a longitudinal study provide more opportunities for the interpretation of causalities between phenomena than data collected at the same time point, but even in a longitudinal study, the interpretation of a causal relationship between phenomena is complex; there may be confounding factors that affect both antecedents and consequences. Advanced statistical methods help to control factors that might affect the relationships and estimate the amount and direction of the contribution of one factor to another factor. Advanced statistical methods also help to compensate for missing data, which is caused when an individual's data are lacking on some measures or measurement points. The recommendation to use advanced statistical methods concerns quantitative analyses, but there are also advanced methods for the use of qualitative data. A qualitative analysis is useful for deepening one's understanding of developmental processes and the unique experiences of individuals. Quantitative and qualitative analyses should be used in a complementary way more often than is customary at present. 


\subsection{What is development?}

As a final point, some reflections are presented here on the study of development based on a thorough discussion of this matter in the 1980s (reviewed by Pulkkinen, 1985). Studies of human development often result in stability coefficients, either for the absolute stability of a characteristic over time or the relative stability between individuals. A statistically significant but low stability correlation (e.g., 0.30) shows, however, that only a small proportion of the variation ( 9 percent) is explained by the stability of the phenomenon, while 91 percent of the variation is unexplained due to the unreliability of measures and true changes. Still, the latter finding is often neglected. The study of stability stems partly from the dispositional or trait orientation to personality with expectations about the stability of traits. Theoretical assumptions about the development of personality characteristics are rarely presented. The study of stability is also related to the statistical methods available. As Baltes (1983, p. 101) remarks, "many methods contained in the methodological arsenal of psychology are ill-suited to the study of developmental change." Instability should be at least as interesting as stability. Or, as Thomae (1979) spells out, "the essential component of a concept of development refers to changes of different degrees." New types of software are available for the study of these changes, but more development work along these lines is needed. Also, an analysis of instability in single cases may enrich our understanding of the developmental process in question and provide inspiration for the development of new theories.

Baltes (1983), the major proponent of the life-span developmental approach, has argued that the conception of developmental change has been influenced by a biological notion of developmental growth and, therefore, development has been traditionally defined in terms of sequentiality, unidirectionality, an end state, irreversibility, and so on. He posited that the maturational conception is too restrictive from the point of view of life-span development. Rapid biological maturation in childhood allows for the interpretation of almost all changes as development. But, in adulthood, when maturation becomes slower or turns into a biological decline, a distinction between biological maturation and psychological development becomes necessary. The sources of changes involve both the innerbiological and outer-ecological levels of the context within which the individual is embedded (Lerner, 1983). Humans have a capacity for change across the entire life-span.

Both normal and pathological functioning can be studied from a developmental perspective if all sequences of change are interpreted as development, but it has been questioned whether it is correct to speak about, for instance, antisocial development. Kaplan (1983) argued that it was necessary to have the purpose or telos of "development" in mind. Development, as distinct from change, ought to be comprehended as "movement toward perfection" (1983, p. 204). It refers to a movement toward freedom, autonomy, and individuation. Kagan (1983), in turn, pointed out problems in Kaplan's metaphysical ideals toward which people should grow. Ideals depend on the culture and its view of the human being, and a 
44 Introduction to the JYLS

developing individual may also have personal ideals toward which he or she would like to develop.

Individuals are unique and each individual has his or her own strengths. Average descriptions of development easily hide this fact. A goal of the JYLS has been to find conceptual constructs that are relevant for the description and explanation of individual differences in development toward different directions. In general, individual differences in personality and social behavior bring richness to the human community. 
PART II

The development of personality and psychological functioning 
$\Longrightarrow$ Taylor \& Francis

Taylor \& Francis Group

http://taylorandfrancis.com 


\section{4}

\section{THEORETICAL FRAMEWORKS FOR THE STUDY OF SOCIOEMOTIONAL DEVELOPMENT}

\subsection{From cognitive control of impulses to self-control}

In 50 years, psychology has advanced as a science and new theoretical approaches to human development have been presented. In this chapter, the following question is reviewed: what has happened during these years to the conceptual constructs of the two-dimensional model (described in Chapter 1) as the framework for the study of children's socioemotional behavior? It is followed by a question: how to describe developmental processes within the two-dimensional model?

Amendments that have been made to the model have concerned theoretical constructs, but not the basic hypotheses on individual differences. First, I changed the concept of the cognitive control of behavior to the concept of self-control, which I understood to include a strong cognitive component (Pulkkinen, 1982). The theoretical roots of the construct of self-control came from the study of cognitive processes in children's control over motor behavior and speech (Vygotsky, 1962). According to Luria (1961), self-control consists of a process of choosing a course of action and an internal evaluation of the success of the choice made. The concept of self-control was also employed in cognitive-behavioral therapy (Kanfer \& Karoly, 1972), where self-control refers to a person's attempt to find a controlling response in a specific situation. Furthermore, Bandura (1973) used the term selfcontrol in his theory of observational learning, where mediational responses convey information to the observer about the characteristics of the appropriate behavior. I used the concept of self-control to mean the same as cognitive control, covering both the control of emotions and the control of behavior, which Eisenberg and Fabes (1992) considered to be an important distinction.

Second, I relabeled the model as a model of emotional and behavioral regulation (Pulkkinen, 1995). The study of emotional regulation had grown rapidly during the 1990s. Emotional regulation helps to maintain internal arousal within 
a manageable, optimal performance range, whereas behavior regulation helps one to adjust one's reactions to external circumstances. In this context, I expanded the analysis of an individual's situation-specific behavior to include a description of individuals' characteristic behaviors. The reason for this change was that when the study continued to be longitudinal, findings showed that children's reaction styles tended to be consistent over time.

Third, the model was drawn in a circumplex form (Pulkkinen, 1995) inspired by the presentation of Eysenck and Rachman (1965). The vertical axis depicted expression versus inhibition of behavior (extraversion versus introversion) and the horizontal axis depicted low versus high self-control (neuroticism versus emotional stability). I maintained the original hypotheses on children's different ways of coping with conflict (aggressive, anxious, constructive, and compliant), but included more behavioral options such as impulsive and thoughtful behavior in the model. In this way, the situation-specific control of impulses could be connected with other characteristic behaviors of individuals. The circumplex shape helped to illustrate that the two-dimensional framework does not present a typology of behavior, but rather it shows that behavior may involve different magnitudes of activity and self-control. It also helped to demonstrate, for instance, relationships between externalizing and internalizing problem behaviors (Achenbach \& Edelbrock, 1983; Cicchetti \& Toth, 1991), which share variance of low self-control, but differ in overt activity. A very distressed child may be both aggressive and anxious (displaying externalizing and internalizing problem behaviors). It was seen that recurrent pain in 11-year-old twin boys and girls was most strongly associated with the co-morbidity (simultaneous existence) of internalizing and externalizing problems (Vaalamo, Pulkkinen, Kinnunen, Kaprio, \& Rose, 2002). Correspondingly, a child may display both more active and less active well-controlled behavior, which is seen in high adjustment (both constructive and compliant behavior; Pulkkinen, Kaprio, \& Rose, 1999).

Interestingly, in their recent article, Duckworth and Steinberg (2015) distinguished two processes contributing to self-controlled behavior: volitional processes and impulsive (or, in their terminology, impulsigenic) processes, bearing similarities to self-control and behavioral activity, respectively, from the two-dimensional model. According to Duckworth and Steinberg, volitional processes include executive functions and metacognitive strategies such as planning. Executive functions help one to cope with temptations in a self-controlled way by suppressing impulses and keeping long-standing goals alive. Impulsigenic processes weaken self-control and are focused on short-term rewards and sensation seeking. Impulsigenic forces can be domain general (e.g., anxiety) or domain specific (a certain act) and they are involuntary, that is, activated without any conscious desire to do so. Both reactive tendencies to under-control and reactive tendencies to over-control can be found in children at an early age. "In either case, volitional processes are required to override impulsigenic tendencies that are essentially reactive rather than reflective" (Duckworth \& Steinberg, 2015, p. 34). My ideas almost 50 years earlier were along these lines, but the means of studying these processes were less advanced at that time. 


\subsection{Updated framework}

\subsubsection{The model for the Unfolding of Socioemotional Behavior}

In the context of writing this synthesis of the longitudinal study, I found it necessary to amend the two-dimensional framework model by adding a developmental perspective to it. Originally, the two-dimensional model depicted situation-specific behavior, but the longitudinal study had revealed developmental continuities that needed a theoretical integration of the model. Although individuals may vary their behavior from situation to situation, there is a tendency toward continuity in human behavior both across situations and across time. Continuity refers to the maintenance of psychological processes or functions (Kagan, 1980), which may manifest in phenotypically similar behavior (meaning that behavior looks similar, such as aggression at age 8 and 20) or in phenotypically different behaviors (e.g., aggression at age 8 and drunken driving at age 20). In the latter case, when the predictor (aggression) and the outcomes (drunken driving) are phenotypically different, they should be theoretically connected in order to interpret that there is continuity between them. Both types of continuity (homotypic in case of phenotypically similar and heterotypic in case of phenotypically different behaviors) can be understood within the revised two-dimensional model.

The revised model is labeled as the model for the Unfolding of Socioemotional Behavior (USB). The concept of socioemotional behavior refers to the expression and regulation of emotions and behavior in human relationships and the concept of socioemotional development refers to the unfolding of a person's typical ways of interacting with others. What is new in the revised model is that the USB model presents, besides individual differences in socioemotional behavior, different developmental directions from childhood socioemotional behaviors to adult styles of life (Figure 4.1). A style of life means an organized whole of the individual's socioemotional behavior, life attitudes, and everyday activities. They represent, respectively, affective, cognitive, and behavioral components of human action which constitute a triangular model of lifestyle (Pulkkinen, 1992a).

Compared with the impulse control model (Figure 1.2), impulsive behavior replaces aggressive behavior in the USB model, because results have shown that the expression of aggression is not limited to the uncontrolled expression of impulses (Pulkkinen, 2017). Furthermore, the concept of self-regulation replaces the concept of self-control. The term self-regulation was used in the literature, for instance, in the context of mood management (Morris \& Reilly, 1987) and emotion regulation (Saarni, 1989), and Kopp (1982) had argued that self-regulation is a more mature form of control than self-control. I had, however, continued to use the concept of self-control in order to maintain consistency in the presentation of the results of the longitudinal study and argued on the basis of the results of the JYLS that self-control is at the heart of successful development (Pulkkinen, 2014b). The construct of self-control was introduced to the psychological literature earlier than self-regulation, and these constructs have had different histories, as reviewed by 


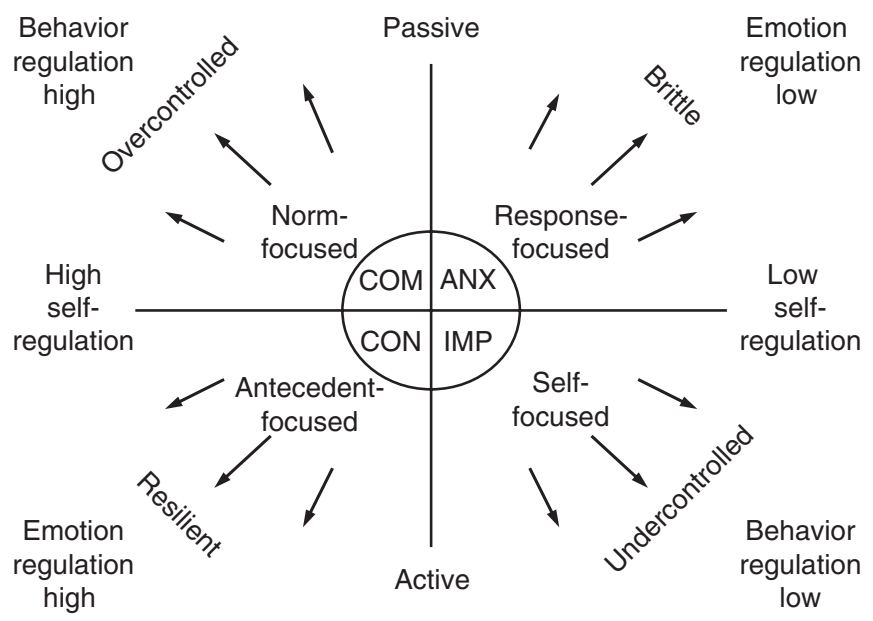

FIGURE 4.1 The model for the Unfolding of Socioemotional Behavior.

Note: $\mathrm{COM}=$ Compliant; $\mathrm{ANX}=$ Anxious; $\mathrm{CON}=$ Constructive; $\mathrm{IMP}=$ Impulsive .

Pulkkinen and Pitkänen (2010). As noted by Vohs and Baumeister (2004), the concepts of self-control and self-regulation are often used interchangeably.

A reason for the adoption of the construct of self-regulation at this point is that there is a need to advance communication between researchers through terminological adjustments. Research into self-regulation has increased and has deepened our understanding of processes beyond individual differences. Selfregulation was used in cybernetic theory to refer to a mechanism's (such as a thermometer's) capacity to regulate processes by making adjustments according to set standards. Self-regulation in human behavior refers to an individual's capacity to maintain harmony with his/her social and physical environment through the conscious and nonconscious regulation of thoughts, attention, emotions, and behaviors (Vohs \& Baumeister, 2004). Peterson and Seligman (2004, p. 500) state accordingly that "self-regulation refers to how a person exerts control over his or her responses [thoughts, emotions, impulses, performances] so as to pursue goals and live up to certain standards [ideals, norms, performance targets, expectations of other people]."

The self-regulation of socioemotional behavior is analyzed in the USB model in two respects which depict different ways of making an effort to maintain control over oneself and to adapt to one's environment: (1) behavior regulation, that is, how much an individual's behavior is affected by his/her own impulses (is self-focused) or how much an individual tries to adapt his/her needs to other individuals' needs and expectations (is norm focused); and (2) emotion regulation, that is, whether an individual handles emotions in an anticipatory way to enable him or her to function adaptively (is antecedent focused) instead of being overwhelmed (response focused) by unwanted emotions. A harmony presupposes that an individual maintains control over his/her behavior and emotions and adjusts the expressions of personal 
needs and affects to the context in which the behavior is taking place. Children's general low self-control, as assessed with the Self-Control Rating Scale by Kendall and Wilcox (1979), correlated positively with low behavior regulation (externalizing problem behaviors including aggressiveness, hyperactivity-impulsivity, inattention) and with low emotion regulation (internalizing problem behaviors including anxiety and depressive symptoms). High self-regulation, in turn, correlated positively with adaptive behaviors (including constructive and compliant behavior and popularity). This study was conducted with the offspring of the JYLS participants (Kokkonen \& Kinnunen, 2006).

Behavior regulation. A study of children's capacity to inhibit impulsive behavior and delay gratification indicated that impulsive children act before thinking, and that children who can delay gratification can wait for a preferred reward for a certain time (Mischel, 1974). The child's capacity to delay gratification develops with age and is associated with, for instance, cognitive performance (Mischel \& Ayduk, 2004). A small child typically behaves in a self-focused way and follows his/her immediate needs and experiences. In the socialization process of socioemotional behavior, consequences of behavior are highlighted and the child is expected to adjust his or her behavior to the rules and norms. Norms vary in different cultures, stages of life, and historical periods of time and define what kind of behavior is expected in society and what is considered to be outside the norm.

Self-focused, impulsive behavior in childhood may result in direct aggressive behavior in a conflict situation. Continuity in self-focused behavior and ignoring social rules increases the risk of escalating conflicts with people at home, at school, and in society. It tends to lead to the style of life which is termed Undercontrolled in the USB model. In research, much attention has been paid to low behavior regulation, because externalizing problem behaviors are visible, harmful, and costly to society.

Norm-focused behavior means that an individual complies with social expectations and accepts them cognitively, as indicated by compliance in childhood in the USB model. There are differences between individuals in how strictly they follow norms. Consistent and strict norm-focused behavior may lead to the style of life termed Overcontrolled in the USB model.

The likelihood of low behavior regulation is increased by neuro-developmental risk factors, such as hyperactivity (Moffitt, Caspi, Rutter, \& Silva, 2001), which are more common in males than in females. Tests for the assessment of behavioral adjustment, hyperactivity-impulsivity, and other indicators of externalizing problem behaviors have been devised, such as Rutter Child Scale (Rutter, 1967), the Child Behavior Checklist (Achenbach \& Edelbrock, 1983), the Strengths and Difficulties Questionnaire (Goodman, 1997), and Multidimensional Peer Nomination Inventory (Pulkkinen et al., 1999). The last-mentioned measure includes items for both low and high behavior regulation and low and high emotion regulation.

Emotion regulation. It used to be common to interpret emotions as states of subjective emotional experience resulting from physiological changes, as evoked by external and internal events (Frijda, 1986). At the end of the 1980s, a functional approach to the study of emotions and mood regulation emerged (Morris \& Reilly, 1987) and 
emotions were seen as processes which establish, maintain, or disrupt relationships between people (Campos, Campos, \& Barrett, 1989). Cicchetti, Ganiban, and Barnett (1991, p. 15) defined emotion regulation as a process "by which emotional arousal is redirected, controlled, modulated, and modified to enable an individual to function adaptively in emotionally arousing situations." (The term "emotion regulation" is used in the literature in the meaning of the regulation of emotions instead of the term "emotional regulation.") Emotion dysregulation, in turn, may exist as an over- and under-regulation of emotions. It is seen as having difficulty in modulating the experience and expression of emotion in response to contextual demands, and in controlling the effects of emotional arousal on the organization and quality of thoughts, actions, and interactions (Cole, Michel, \& Teti, 1994).

It has been found that the timing of emotion regulation so that it is antecedent focused (aiming at the reinterpretation of emotional stimuli) rather than response focused (e.g., suppression of emotion aroused by the situation) is important to a person's adaptive behavior (Larsen \& Prizmic, 2004). Emotion regulation involves emotion information processing, which together with socioemotional learning, "serves as a continuous source of emotion-regulatory strategies" (Izard, Stark, Trentacosta, \& Schultz, 2008, p. 160). They also argue that emotion utilization indicated by constructive thought and action may stem from modulated emotion experiences.

In the USB model (Figure 4.1), emotion regulation is described as antecedentfocused behavior which refers to the appraisal of the situation and the reinterpretation of emotional stimuli. The aim of processing emotions is to control emotional arousal in an anticipatory way in order to enable an individual to function adaptively. Anticipatory processing results in becoming aware and accepting of one's own and others' emotions, making adequate social observations about the situational context, and taking time before reacting to the situation by means of internal control and, one could also say, through the use of one's free will. Effective processing of emotions involves flexibility in behavior according to situational cues and demands and it is seen in constructive behavior, which may lead to the style of life called Resilient. A resilient person is able to confront new situations and handle emotions in a way that advances social interaction and harmony with the people involved.

Low emotion regulation is called response-oriented behavior in the USB model. It refers to reactions to emotions aroused by the situation. An individual may try to suppress or escape unwanted emotions and feels anxiety due to helplessness in handling the situation and himself/herself. Being overwhelmed by negative emotions may be associated with making inadequate social observations and assumptions about the intentions of others. As articulated by Izard et al. (2008, p. 160), "maladaptive emotion information processing distorts emotion knowledge and degrades emotion regulation and utilization." Lacking skills of handling emotions may lead to the style of life known as Brittle.

Studies of children's emotion regulation show that children who are better regulated tend to be more resilient, that is, in terms of the ability to deal with stress adaptively (Eisenberg, 2014). People use different techniques to regulate their moods. Salovey, Mayer, and Caruso (2005) list effective techniques such as physical 
exercise, listening to music, social interaction, and pleasant distractions (e.g., hobbies, shopping, reading); and less effective techniques such as passive mood management (e.g., television viewing, food, sleep), avoiding the unpleasant thing or person which caused the bad mood, and substance use (alcohol, drugs). In her socioemotional selectivity theory, Carstensen (1993) proposed that the selection of social relationships may be an effective way of regulating emotions, because it limits the escalation of negative affect in social interaction. It has been found to be an increasingly effective and common strategy throughout life (Carstensen, Pasupathi, Mayr, \& Nesselroade, 2000).

One of the techniques for emotion regulation may be cognitive mood management. Mayer and Stevens (1994) developed a seven-item meta regulation scale that includes three active, conscious, and cognitive strategies: (1) repair, which refers to an individual's active attempt to turn a negative emotion in a more positive direction by planning, recalling, and imagining something desirable; (2) dampening, which refers to the dampening down of positive emotions, for instance, by reminding oneself of reality; and (3) maintenance, which is used to preserve an ongoing emotion without changing it. This scale was used with the JYLS sample in adulthood. As analyses for the present book showed, the three strategies functioned differently so that maintenance was an adaptive strategy, whereas repair and dampening were not. The maintenance of the ongoing emotion correlated positively with a calm and positive mood, the acceptance and clarity of one's mood, one's mood having a minimal influence on one's thinking and behavior, and cognitive control (e.g., "When I am faced with a difficult problem, I try to approach it in a systematic way"). On the contrary, one's efforts to escape the ongoing emotion by repair and dampening had negative correlations with the calm and positive mood and the other mood variables but positive correlations with neuroticism and anxiety, as well as with emotional ambivalence and unsuccessful control (e.g., "When I am faced with a difficult decision, I prefer to postpone it even if I have all the facts"). Maintenance also correlated positively with good subjective health, whereas repair correlated with poor subjective health (M-L; Kinnunen, Kokkonen, Kaprio, \& Pulkkinen, 2005).

An interesting question is why the strategy of repair, turning a negative emotion into a more positive direction, is not adaptive, whereas maintenance is adaptive. The difference in the effectiveness of the strategies may be related to their differences in the antecedent-focused and response-focused characteristics (Larsen \& Prizmic, 2004). The maintenance indicates a successful strategy in which emotional stimuli are reinterpreted and a resulting emotion is accepted, whereas the repair strategy indicates a less successful strategy in which the person tries to manipulate and deny an emotion.

The concept of the antecedent-focused regulation through appraising the stimulus situation has been included in the two-dimensional model of impulse control from its inception.

An individual may be able to appraise the stimulus situation he encounters and to decide between alternatives; i.e., the emotional aspect can be 
neutralized by cognitive control ... It is assumed that this kind of behaviour is motivated by an attempt to behave in a socially acceptable manner.

(Pitkänen, 1969, p. 103)

Children can be advised to create antecedent-focused perceptions of a situation that help them in coping with the situation. This was demonstrated by a program conducted with a group of three psychology students who were doing their Master's theses under my supervision. Together, we devised a program for improving children's self-control or, in today's terminology, their emotion regulation (Pulkkinen, Heikkinen, Markkanen, \& Ranta, 1977). The program was tested in daycare centers with 4- to 6-year-old children. It consisted of 36 teaching units (each lasting around 20 minutes). Fairy tales and pictures were used as materials for holding discussions with the children. The sessions covered three major areas:

- Self-understanding (understands one's individuality, one's own feelings, one's own reactions)

- Understanding others (understands others' differences, others' feelings, others' reactions)

- Understanding of social skills (understands cooperation, sharing and taking turns, thinking of others)

The comparison of pre- and post-test observations of children in the experimental and control groups revealed that the experimental group children improved in empathy, helping behavior, taking turns, obeying rules, and controlling anger (Pitkänen-Pulkkinen, 1977). The program was widely used for years in Finnish daycare centers. This program, developed by using a controlled study, was perhaps one of the first programs for improving emotion regulation in children. Salovey, Mayer, and Caruso (2005) list a number of educational intervention programs for improving emotional intelligence and mention that the oldest social and emotional learning programs date back to the 1990s.

Salovey et al. (2005) describe the Four-Branch Model of Emotional Intelligence, which includes the categories of emotional perception and expression, emotional facilitation of thought, emotional understanding, and emotional management. My ideas were similar for constructive behavior, but I did not call it emotional intelligence. As Salovey et al. (2005) state, emotional intelligence should be tested as an ability, which is not an easy task. Perhaps intellectual processes beyond emotional regulation are not abilities, but executive functions.

\subsubsection{Self-regulation and executive functions}

Differences between children in the self-regulation of socioemotional behavior raised a question whether self-regulation is a general capacity in a child that is also seen in cognitive performances. We found with the Offspring sample that in boys' help-seeking behavior in cognitive tasks, a shorter reflection time before asking 
questions was associated with higher impulsivity, that is, with lower self-regulation (Puustinen, Kokkonen, Tolvanen, \& Pulkkinen, 2004). Executive functions in brain control performances. We were interested in whether executive functions also control socioemotional behavior. In order to study this assumption, we had first to study possible dimensions of executive functions in children (Lehto, Juujärvi, Kooistra, \& Pulkkinen, 2003).

The study was based on (1) the view that the term "executive functioning" refers to a multidimensional behavioral concept covering higher order cortical functions such as attentional control and (2) the conception that "a small set of executive functions (EF) utilizes the neural capacity of the prefrontal and adjacent areas for protecting the temporal order of subsequent behaviours" (Lehto et al., 2003, p. 296), that is, that executive functions control the display of behavior. Our hypotheses on the dimensions of executive functioning were based on a review of the literature by Miyake et al. (2000) concerning research with adults. The review proposed that the taxonomy of executive functioning consists of at least three basic functions: shifting, updating, and inhibition. Shifting refers to an ability to change from one mental set or task to another. Updating consists of the updating and monitoring of the working memory, which, in turn, refers to the storage of information, and inhibition means the ability to inhibit dominant responses when necessary.

We studied the dimensions of executive function with 108 children (56 percent boys) whose mean age was 10.5 years. They were offspring of the JYLS participants: one of each of the children's parents was a participant in the JYLS. The methods were (for details of methods and their references see Lehto et al., 2003): Trail Making Test, Auditory Attention and Response Set, Word Fluency task, Matching Familiar Figures, Mazes, and CANTAB Working Memory and Planning battery including Psychomotor Screening Task, Spatial Span task, Spatial Working Memory task, and Tower of London. Intellectual capacity (IQ) was controlled by a short-form Wechsler Intelligence Scale for Children-Revised (WISC-R), including Vocabulary and Block Design.

By using confirmatory factor analyses, we extracted three executive components identified as working memory, inhibition, and shifting; their eigenvalues were, respectively, 2.8, 1.1, and 0.99 (Lehto et al., 2003). We concluded that the findings were in good agreement with the view that executive functions are simultaneously uniform and diverse. Diversity found in that performance in executive tests was accounted for by three factors, and unity in the correlations between the factors.

At the next step, we studied connections between executive functions and socioemotional behavior. The following results are based on a manuscript by Lehto, Pulkkinen, and Juujärvi (2002). It was submitted for publication, but peer-reviewers criticized it for the lack of a theory on connections between socioemotional behavior and executive functions and the manuscript remained unpublished. At that time, there was not enough understanding of the possible role of executive functions in the explanation of self-regulation in socioemotional behavior.

Teacher ratings of children's behavior were made using a 35-item Teacher Rating Form of the Multidimensional Peer Nomination Inventory (TR-MPNI; 
Pulkkinen, et al., 1999) in all the school classes where the 108 children were based. All children were in different classes. The teacher rating instrument was developed for the purpose of the study of Finnish twins as an extension of the Peer Nomination Inventory used in the baseline data collection of the JYLS. A total of eight scales were used in this study: hyperactivity-impulsivity, inattention, aggression, depressive symptoms, social anxiety, compliance, constructiveness, and social activity (e.g., active with other children, popular). A factor analysis yielded the same three factors as with the large twin sample (Pulkkinen et al., 1999): behavioral problems, emotional problems, and adjustment. The latter covered both aspects of adaptive behavior, as well as compliant and constructive behavior.

The teachers were also asked to rate the children's self-control on a 20-item version of the Self-Control Rating Scale (SCRS) developed by Kendall and Wilcox (1979) (e.g., "When the child promises to do something, can you count on him or her to do it?"). Its correlations with the MPNI scales validated the role of selfcontrol in socioemotional behavior. The SCRS correlated highly negatively with behavioral problems ( -0.83 for hyperactivity-impulsivity, -0.62 with aggression; and -0.54 with inattention) and positively with adaptive behavior $(0.67$ with compliance, 0.66 with constructiveness, and 0.41 with social activity [including popularity]; Lehto et al., 2002).

The executive functions included in the study by Lehto et al. (2002) were updating and inhibition, the eigenvalues of which were $>2.0$. Updating contained working memory and shifting (Lehto et al., 2003) and was positively loaded by Spatial Span, Mazes, Word Fluency, and Auditory Attention and Response Set and negatively by errors in Spatial Working Memory and the Trail Making Test. Inhibition was loaded positively by the Matching Familiar Figure Test and Tower of London.

There were no previous studies that could have provided us with guidelines as to how to model executive functions and socioemotional behavior. We adopted the rather intuitive assumption that cognitive factors (i.e., executive functions) would form the grounds of observable behavior. Structural equation modeling was carried out separately for behavioral and emotional problems and adjustment, because there was a high negative correlation between these problems and adjustment, suggesting that they were at opposite ends of the self-regulation dimension (as depicted in Figure 4.1).

The results on the relationships between executive functions and externalizing (behavioral) and internalizing (emotional) problem behaviors indicated (Figure 4.2) that low inhibition explained behavioral problems via hyperactivity-impulsivity and low updating explained emotional problems via social anxiety. Inattention was explained by both low updating and inhibition (the latter indirectly via hyperactivity-impulsivity). The figure also shows that aggression was associated both with externalizing problems (hyperactivity-impulsivity and inattention) and internalizing problems via depressive symptoms. The fit indices RMSEA (the root mean square error of approximation) and CFI (the comparative fit index) confirmed the goodness-of-fit of the model to the data. The values of good fit to the model are .95 or greater for CFI and .06 or lower for RMSEA (Hu \& Bentler, 1999). 


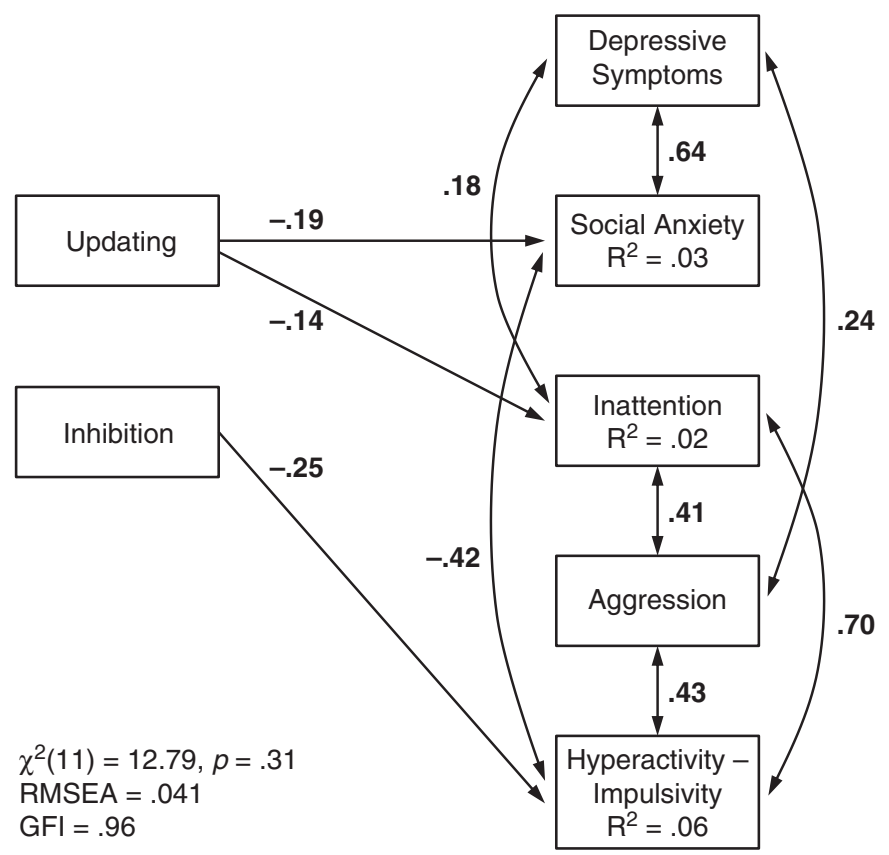

FIGURE 4.2 Executive functions and behavioral and emotional problems.

The results on executive functions and adjustment showed with high goodness-of-fit indices (Figure 4.3) that both updating and inhibition explained constructive behavior, whereas updating explained social activity (popularity) and inhibition explained compliance. As there were significantly different correlations between inhibition and social activity in girls and boys, we tested the possibility that gender would be a moderating factor in this relationship and added the interaction term into the model (Figure 4.3). The results showed that a high capacity to inhibit responses in cognitive tasks (inhibition) was positively related to social activity only in girls (Lehto et al., 2002). Different cognitive functions beyond social activity of girls and boys may explain some differences in the future outcomes in the development of socially active girls and boys, such as the stronger association of social activity with career orientation in females and with risk behavior in males (Pulkkinen, 2009). Extraverted children may have strong impulses that they must learn to regulate, as Shiner (2006) notes, and the risks for unsuccessful regulation may be higher in males.

Conclusions. The results supported the assumptions presented in the model for the USB.The executive function called inhibition underlay, particularly, self-focused versus norm-focused behavior (hyperactivity-impulsivity versus compliance), whereas the executive function called updating underlay, particularly, antecedent-oriented versus response-oriented behavior (constructiveness versus anxiety). Updating involved working memory and shifting that might advance making adequate social observations and increase flexibility in emotional behavior. The association of 


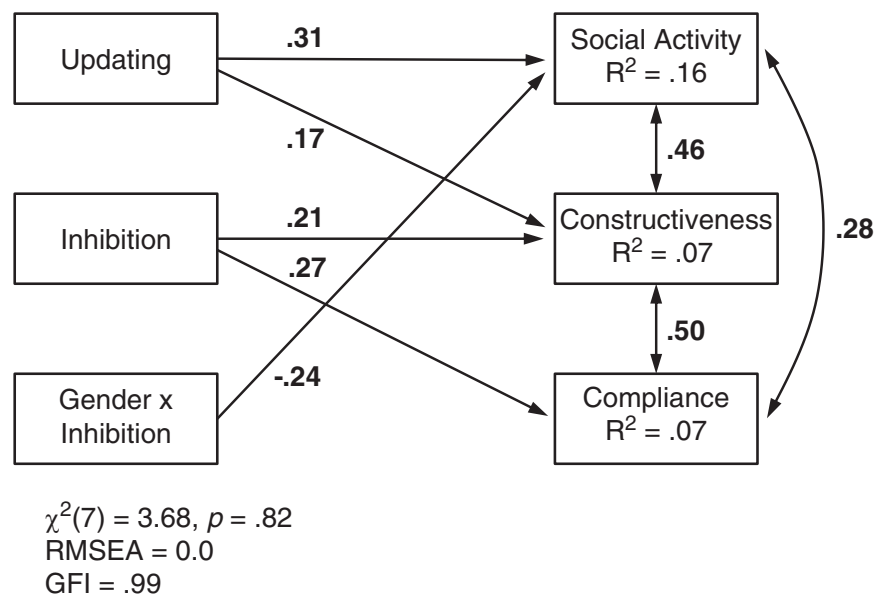

FIGURE 4.3 Executive functions and adjustment.

self-regulation including executive functions and social and emotional competence is increasingly acknowledged as the foundation for adjustment to school (Blair \& Raven, 2015).

\subsubsection{Toward cumulative theoretical reasoning}

The third question concerned the comparability of the theoretical constructs in the USB model to the theoretical reasoning of other researchers and the adjustment of terminology with the purpose of increasing the communication between researchers. Two theoretical approaches are particularly relevant in this context. One of them concerns the relationship between socioemotional behavior and temperament, and the other, the assumptions concerning the developmental directions toward the styles of life in the USB model.

Temperament. I made the assumption that the basis of individual differences in socioemotional behavior lies in the individual's temperament, but that constitutional factors alone are not sufficient to explain the development of behavioral patterns (Pulkkinen, 1982, p. 68); socialization experiences shape behaviors. A temperament theory that I find most interesting from the point of view of socioemotional behavior was presented by Rothbart and her colleagues (see Rothbart, 2011). Rothbart and Derryberry (1981) defined temperament as constitutional differences in reactivity and self-regulation. The term "constitutional" covers heredity, but also maturation and experience. Reactivity means the functional state of internal central nervous systems as reflected in various response parameters such as threshold and intensity and self-regulation means higher level processes that modulate by enhancing or inhibiting the reactive state of the central nervous systems. Individual differences exist in emotional, motor, and attentional reactivity and self-regulation. Rothbart and Bates (1998) 
remark that temperament processes are open systems. It means that experiences influence the development of temperament and, therefore, the border between temperament and socioemotional behavior may become somewhat difficult to discern.

The two dimensions of the framework model from the JYLS, the degree of social activity and self-regulation, bear some similarity to the definition of individual differences in temperament. When I began the longitudinal study, Rothbart's temperament theory and the associated measures developed for assessing individual differences in temperament were not available. Therefore, a parallelism between temperament and socioemotional behavior exists on a general conceptual level. In my understanding, temperament affects the individual's readiness to react to his/her social environment, but the quality of the socioemotional behavior depends on the interaction of biological self-regulatory processes, maturation, social observations of a situational context, and socialization experiences. Socioemotional behavior is more situation specific and modifiable by environmental factors than temperament.

Developmental directions. The USB model in Figure 4.1 depicts that individual differences in socioemotional behavior tend to lead to different styles of life. The terms used for styles of life refer to Jack Block's (2002) theory of personality as an affect-processing system. The theoretical constructs were originally presented by Jeanne and Jack Block (1980). They were interested in the development of the ability to control impulses, effective coping, and varieties of adaptive behavior, as I was. The Blocks used the construct of ego-control to describe the degree of impulse control and modulation. Their ego structures framework consisted of a combination of a psychoanalytic view of the structure of personality containing the Id, which generates impulses and the ego, which restrains the expression of these impulses; and Kurt Lewin's theory on the properties of boundaries: permeability and elasticity. Excessive boundary permeability (under-control) is associated with insufficient modulation of impulses, whereas boundary impermeability (over-control) is associated with the inhibition of action and affect. Elasticity (ego-resiliency) refers to the capacity of a boundary to change its characteristic level of permeabilityimpermeability, whereas the opposite end (ego-brittleness) implies little adaptive flexibility. The Blocks used the concept of the ego and not the self in reference to the agent of regulation; the concept of self "should be reserved for the meaning of self as object of appraisal, as implying the presence of self-awareness" (Block, 2002, p. 28). This would mean a suggestion to use the term "ego-regulation" rather than self-regulation.

The Blocks originally included the expression and inhibition of both action and affect in the same dimension (under-control versus over-control). Ego-resiliency versus ego-brittleness was, in turn, defined by the Blocks as elasticity (i.e., a capacity to change the expression of action and affect). However, development in psychological science toward the study of emotions affected Jack Block's thinking when he formulated his theory on personality as an affect-processing system (Block, 2002). In this theory, he analyzes the relations between action and affect in a way 
which is close to the USB model. Block believes that cognitive mechanisms are important in the adaptations, but writes the following:

Rather than awarding preeminence to cognition [...] I believe that cognition [...] should be viewed as only one way of responding to affective imbalances. Affect is not in the service of cognition; instead I look at cognition [...] as a way $[\ldots]$ of reacting and responding to affect.

(Block, 2002, p. xiii)

He gives a long list of terminologies that researchers have used for reflection or absence of reflection before carrying out the behavior, such as delay of gratification, underregulation, emotional reactivity, self-control, inhibitory control, willpower, and behavioral disinhibition. "Their common denominator is that they all relate to the way impulse is monitored, by degree of restraint or expression" (Block, 2002, p. 8).

Block prefers the terms undercontrol and overcontrol for behavior regulation. He describes the undercontroller as having, for instance, the following characteristics (Block, 2002, p. 91): tends toward immediate gratification or insufficient modulation of desires, is overly expansive and spontaneous, immediate and direct in expression of motivations, disorderly, emotional fluctuations, social customs disregarded. The overcontroller is characterized by being excessively constrained, delaying gratifications, interpersonal relations are distant, minimal expression of emotions, highly organized, planful, categorical in thinking, single-minded, and overconforming (Block, 2002, pp. 91-2).

Behavior regulation alone is not sufficient for adaptable behavior. "An adaptable individual will regulate behaviors so as to be controlled or to reduce control as a function of the evocative quality or contextual cues afforded by the existing situation" (Block, 2002, p. 12). As worded by Carver (2005) in his review of the Blocks' theory, good adaptation has two aspects: "learning to restrain impulses when such impulses would create problems" and "knowing when to restrain yourself and when to behave instead more freely and spontaneously" (2005, p. 313). Researchers have used different terminologies to describe human adaptability such as emotional regulation, social intelligence, executive functions, self-regulation, self-efficacy, competence, coping, and effortful control, as listed by Block (2002, p. 10). He notes that "the broad labels self-regulation or emotional regulation seem to have come into use in psychology to characterize such adaptation" (2002, p. 11), but remarks that these constructs often have an unclear meaning. It is not clear whether they refer to "dynamic and resourceful adaptability" or "behavioral control," or "some confound of adaptation with impulse control." Block prefers the construct resilience in reference to what is called emotion regulation or self-regulation by others (Block, 2002, p. 95).

Resilience is a latent factor for indicators of resourceful adaptability. "Resilient individuals are dynamically resourceful when confronted by the strain set by new and yet unmastered situations [...] unresilient or brittle individuals have little adaptive flexibility" (Block, 2002, p. 95). Brittle individuals are, in turn, 
"disquieted," they "perseverate," become "behaviorally diffuse" or "anxious when facing competing demands," and they have "difficulty in recouping from traumatic experiences." The likelihood of brittleness increases in an environment that creates anxiety and discomfort and where the development of attentional focus and the ability to resist attentional interference is disturbed (Block, 2002, pp. 136-8).

Block (2002) remarks that in psychiatry, the term resilient has been used to mean "invulnerable" or "stress-resistant" that involves an unexpected observation that in spite of several risk factors in their lives, children appear to be healthy psychologically. This use of the concept of resilience is also found in many empirical studies such as Schoon (2006) and Werner and Smith (1992). Thus, the term "resilient" has two meanings. The psychological definition given by the Blocks when they introduced this construct did not connect resilience to risk factors: "resilient implies the resourcefulness, adaptability, and engagement with his or her world" (Block, 2002, p. 26).

In the USB model, the concept of resilience has the same wide meaning of resourceful behavior as the Blocks have defined it. There might be a need for a construct that describes resilience in a more specific case when risk factors are identified. Conversely, the same construct can be used with the specifications of the risk factors, because it is likely that resilient individuals are most resilient in the case when risk factors are threatening their development.

Inconsistency in the use of the constructs. In the study of personality types or clusters, three labels which are often used are: Resilient, Overcontrolled, and Undercontrolled (Donellan \& Robins, 2010). The Resilient profile has been associated with positive outcomes such as good social relations, whereas the Overcontrolled profile has been associated with unfavorable outcomes such as internalizing problems. The Undercontrolled profile has been associated with externalizing tendencies. These labels come from the Blocks' theory, but the construct of "Ego-brittleness" has been ignored. The construct of Overcontrolled has been used to cover a set of characteristics that are typical of the Brittles (anxious, neurotic, vulnerable). A reason for the ignorance of Brittles in the choice of labels may be in the terminology that Jack Block (1971) used when he gave labels to his personality types. He did not use the construct of "brittle" at that time. The construct "Ego-brittleness" was used in a later theoretical analysis with Jeanne Block (Block \& Block, 1980).

In the USB model, the construct of the Brittle profile is used to refer to an individual's tendency to experience internalizing problems, whereas the Overcontrolled profile refers to behavior that is excessively constrained, highly organized, planful, and conforming, as described by Block (2002).

Conclusions. Jack Block speaks about "jangle fallacy," which was already identified in psychological literature in the 1920s, and states that:

An enduring problem in personality psychology is that equivalent or highly related recognitions or findings have not been recognized as such. [...In the] compartmentalized scientific field of psychology, bodies of relevant 
past or contemporaneous research have not been encountered by serious investigators.

(Block, 2002, p. 12)

He quotes Freud, who noted "the narcissism of minor differences" that leads to unique terminology. An effort was made here to bring together theoretical reasoning and knowledge across researchers in this field. Psychological phenomena are universal, but it takes time before the terminology for the description of them stabilizes and becomes more uniform.

\subsection{Three layers of personality for organizing research findings}

Divergent developmental processes from childhood to adulthood cover many functions and one of them is unfolding an individual's personality. Personality psychology and developmental psychology have historically had little to do with each other, as McAdams and Adler (2006) remark. For personality psychologists, the personality exists as if it were enduring, whereas developmental psychologists are interested in meaningful changes. Many definitions have been given to the concept of personality. A well-known and comprehensive definition of personality was presented by Allport (1937, p. 48): personality is "the dynamic organization within the individual of those psychophysical systems that determine his unique adjustments to his environment."

McAdams and Pals (2006) highlight individual uniqueness in personality and call personality “psychological individuality." McAdams (2015, p. 253) applies the system of three layers (McAdams \& Pals, 2006) to the analysis of personality development along three separate but interacting lines running from early childhood through to adulthood. He states that "Over time, a person becomes a social actor, a motivated agent, and an autobiographical author" (2015, p. 253). In each of these lines, developmental processes exist, and the lines combine in an individual in a unique way that is recognized as personality. To become a unique person means becoming "a certain kind of actor, a certain kind of agent, and a certain kind of author of the self" (2015, p. 253).

- The person as social actor refers to the person's broad dispositional traits that account for consistencies of behavior, thought, and feelings across situations and over time. In the development of a social actor, temperament features in early childhood provide socioemotional material for the formation of dispositional personality traits for adulthood. At a later age, social roles become increasingly important for the development of personality traits of social actors.

- The person as motivated agent refers to motivational perspectives and characteristic adaptations such as motives, goals, plans, values, will, and virtues. The development of a motivated agent also starts in childhood. During the first three years of life, a child acquires the theory of mind, which means a "notion that human beings have particular desires and beliefs in their minds and, as 
a result, human beings act upon those desires and beliefs in a goal-directed fashion" (McAdams, 2015, p. 256). Cognitive and social development in middle childhood advances the development of autonomy, competence, and relatedness. They are fundamental needs in individuals, according to the selfdetermination theory by Ryan and Deci (2006). A motivated agent is free to plan, choose goals, and strive for them, but socializing agents are needed to help structure the goals. Conflicts between goals and their achievement may emerge, but their minimization is facilitated by the selection, optimization, and compensation of the goals, as Baltes (1997) describes in his research into the behavior of midlife adults.

- The person as autobiographical author refers to integrative life narratives, a reconstruction of past, present, and anticipated future. The development of an autobiographical author starts from episodic memory in the third or fourth year of life and progresses to the development of a life story and narrative identity. "Narrative identity is the story that a person composes about how he or she came to be the person he or she is becoming" (McAdams, 2015, p. 259).

Erikson (1950) described personality development in terms of developmental stages. The view of McAdams and his colleagues (McAdams \& Adler, 2006; McAdams \& Olson, 2010) is different. According to them, there is no single course of development or universal stage sequence in the development of personality; development on each line from childhood to old age takes place individually. They distinguished four developmental milestones in the life course in which the developmental lines are combined in unique ways. During the first years of life, the child moves from attachment to the emergence of an agentic, autobiographical self, when the child starts to become a goal-directed striver and the narrator of his or her experiences. The second milestone is the transition from late childhood to adolescence when goals and motives become clearer and children become able to evaluate the value and progress of their goal attainment. The third milestone is the period of emerging adulthood that marks the exploration of, and commitment to, new goals and a new understanding of one's life story. The fourth milestone is midlife when personal agency may be distributed across many goals and responsibilities and life stories express either energizing themes of agency and growth or disappointment and frustration. In each milestone, features related to traits, adaptations, and life stories appear, change or interact with each other in important ways.

The three layers of personality (social actor, motivated agent, and autobiographical author) can be applied to the organization of the accumulated knowledge about personality concerning, for instance, continuity and change in personality (McAdams \& Olson, 2010). In the following chapters, research results of the JYLS with regard to personality are presented within the three-layer framework. The broad scope of the JYLS has enabled data analysis that has resulted in findings on developmental processes in each of the three lines and their interactions. 


\section{THE PERSON AS SOCIAL ACTOR}

\subsection{Questions to be answered}

This chapter presents the development of personality focusing on the layer of "person as social actor" as introduced in Chapter 4. McAdams (2015) describes this layer in the personality by posing questions such as "How do I act? What do I feel at present?" He argues that development through maturational growth aims toward greater self-regulation and efficacy in social and emotional performance. This argument is a good fit with the model for the Unfolding of Socioemotional Behavior (USB) (Figure 4.1), the validity of which is discussed in this chapter in the light of the JYLS findings.

In Chapter 3, on methodological reflection, it was mentioned that the quality of data is the ultimate criterion for the value of a longitudinal study. The choice of peer nomination and teacher rating as methods for collecting baseline data in the JYLS and their contents were explained in Section 2.4.1. Their qualities as measures are first described below.

When I collected the baseline data for the JYLS, I had 12 school classes of second grade pupils who were sitting at their desks trying to answer my 33 questions of, for instance, "Who tries to be always friendly to others?" and "Who attacks other children without a reason?" I asked them to choose at least three same-sex classmates as a response to each question. The teacher was asked to rate how typical the kind of behavior described in the item was for each child. In the data analysis, it was interesting to see whether the peers' choices (a kind of vote) pointed to the same classmates in each question, and whether the average number of choices corresponded to teacher ratings of the typicality of behavior of each child. This question and the many other questions listed are discussed in this chapter. In addition, the issue of whether boys and girls, and men and women, are similar in personality is explored in some depth. 
1. Do the children's peer nominations correspond to the teacher ratings?

2. Do the assessments made in childhood predict these pupils' behavior in adolescence?

3. Is the child's behavior with regard to a certain characteristic, such as aggression, general across different types of situations, or does it depend on with whom he or she is interacting?

4. Is the model for the USB valid for describing children's differences in socioemotional behavior across generations?

5. Does socioemotional development progress in the directions depicted in the USB model and result in the expected styles of life?

6. Do environmental factors such as upbringing affect the development of the styles of life?

7. Does a variable-oriented approach to the participants' styles of life in young adulthood produce similar results to a person-oriented approach to their personal styles? The former refers to grouping descriptive characteristics, whereas the latter refers to grouping the individuals themselves.

8. How should one describe the personality of the study participants when they have reached middle age?

9. Does maturational growth aim toward greater self-regulation and efficacy in social and emotional performance?

10. Does socioemotional behavior in childhood predict adult personality?

These questions are answered based on the publications of the JYLS research team. As this book is the synthesis of results, findings across publications have been combined and generalized. The terminology has been updated to match the USB terminology, as indicated in the text. Research methods were described and relevant references were given in Chapter 2. More detailed information on methods and on the literature concerning each question can be found in the original publications. For some questions, answers were found by conducting additional data analyses for this book.

\subsection{Socioemotional behavior in childhood and adolescence}

\subsubsection{Agreement between informants}

The main criteria for the quality of data are that the measurement techniques used are reliable and that those measures assess the phenomena that were intended to be studied. The latter refers to the validity of a measure that is often estimated in relation to an external criterion. As regards the reliability of the peer nomination technique, which was studied for each item by dividing boys and girls separately into two groups and comparing the nominations given by them (Pitkänen, 1969, p. 118), high correspondence was found. It showed the internal reliability of the measure. The validity of peer nominations was studied using simultaneously conducted teacher ratings as external criteria, which is termed concurrent validity. 
TABLE 5.1 Concurrent validity and relative stability of socioemotional characteristics in peer nominations $(\mathrm{PN})$ and teacher ratings (TR)

\begin{tabular}{|c|c|c|c|c|c|c|c|c|}
\hline & \multicolumn{4}{|c|}{ Concurrent validity } & \multicolumn{4}{|c|}{ Relative stability } \\
\hline & \multicolumn{2}{|c|}{ PN/TR (Age 8) } & \multicolumn{2}{|c|}{ PN/TR (Age 14) } & \multicolumn{2}{|c|}{$P N(8-14)$} & \multicolumn{2}{|c|}{$\operatorname{TR}(8-14)$} \\
\hline & Boys & Girls & Boys & Girls & Boys & Girls & Boys & Girls \\
\hline Aggression & $0.73^{\star \star \star}$ & $0.39^{\star \star \star}$ & $0.48^{\star \star \star}$ & $0.41^{\star \star \star}$ & $0.37^{\star \star \star}$ & $0.37^{\star \star \star}$ & $0.37^{\star \star \star}$ & 0.13 \\
\hline Compliance & $0.62^{\star \star \star}$ & $0.41^{\star \star \star}$ & $0.36^{\star \star \star}$ & $0.30^{\star \star \star}$ & $0.29^{\star \star \star}$ & $0.22^{\star}$ & $0.34^{\star \star \star}$ & 0.18 \\
\hline Constructiveness & $0.60^{\star \star \star}$ & $0.50^{\star \star \star}$ & $0.31^{\star \star \star}$ & $0.40^{\star \star \star}$ & $0.29^{\star \star \star}$ & 0.11 & $0.23^{\star \star \star}$ & $0.19^{\star}$ \\
\hline Anxiety & $0.41^{\star \star \star}$ & $0.35^{\star \star \star}$ & $0.22^{\star}$ & 0.17 & $0.48^{\star \star \star}$ & $0.42^{\star \star \star}$ & 0.11 & $0.25^{\star \star \star}$ \\
\hline
\end{tabular}

Adapted from Pitkänen-Pulkkinen (1981b).

Note: ${ }^{\star \star \star} \mathrm{p}<0.001 ;{ }^{\star} \mathrm{p}<0.05$.

The agreement between peer nominations and teacher ratings (concurrent validity) was high for boys' aggressive behavior at age $8(r=0.73$; Table 5.1), which indicated that peers and teachers had made very similar observations of boys' differences in aggressive behavior. Validity was also good for boys' compliant and constructive behavior, but lower, although still significant, for anxious behavior (Pitkänen-Pulkkinen, 1981b). For girls, the agreement between peers and teachers in these variables was lower but significant. A reason for the gender difference in the agreement between peers and teachers may be in more visible differences in boys' when compared with girls' behaviors; girls may comply to rules and control and inhibit the expression of their emotions more than boys.

At age 14, the concurrent validity correlations were lower for boys, but significant. The difference in the size of the validity correlations between ages 8 and 14 is understandable, because the teachers at age 8 were class teachers, but at age 14 , the behavior was being rated by subject teachers, who had fewer opportunities to observe the children. When there are limited opportunities to make observations, ratings may be confounded by other factors such as school success (Pulkkinen \& Pitkänen, 1993).

The correspondence of peer nominations and teacher ratings concurred with the size of agreement (0.44) between peer nominations and teacher ratings reported by Achenbach, McConaughy, and Howell (1987) in their meta-analysis on crossinformant agreement; for the agreement between teacher and parent assessments they reported a lower correlation (0.27). Peers and teachers see children in the same context whereas teachers and parents see them in different contexts, and parents have fewer opportunities for making observations of many children.

Conclusions regarding question 1 . The correspondence between peer nominations and teacher ratings by class teachers at age 8 was highly significant. The choice of peer nominations and teacher ratings as measures can be considered as providing a good basis for the present longitudinal study on socioemotional development. 


\subsubsection{Continuity in socioemotional behavior}

When the children grew older, the question arose about whether their typical characteristics remained similar over time. This question concerns the so called differential continuity (Caspi \& Shiner, 2006), which is also known as "relative stability." It is indicated by a correlation between two measurements at different points in time and it shows the degree to which the order of individuals is maintained in the amount of the studied characteristic over time. The concepts of continuity and stability have been used interchangeably in the literature. Kagan (1980) has made a distinction between them by defining stability to be a structural concept, meaning that an individual retains the same relative position on a dimension over time. Continuity, in turn, is a process construct that refers to the maintenance of psychological processes or functions.

Relative stability was analyzed separately for peer nominations and teacher ratings (Pitkänen-Pulkkinen, 1981b). Peer nominations at age 8 predicted aggressive and anxious behaviors at age 14 well among boys and girls (Table 5.1), whereas teacher ratings predicted aggressive behavior better in boys than in girls, but anxious behavior better in girls than in boys. Significant relative stability of socioemotional behaviors across 6 years is notable, because the participants had spread from 12 school classes to 78 classes when moving from the second grade to the eighth grade. The ways in which a person behaves tended to continue in the context of a new class. A person-level analysis at age 8 revealed that 55 percent of very aggressive boys (and 30 percent of very aggressive girls) had maintained their very aggressive behavior at age 14 . None of them had become very compliant (the polar opposite to aggression in the two-dimensional model) and only a few had become anxious. In most cases, the changes that had occurred were toward average behavior (Pulkkinen, 1977). Cairns and Cairns (1994) have also demonstrated stability in highly aggressive boys' and girls' behavior during middle childhood and preadolescence.

A low coefficient for relative stability may result from real changes in a characteristic or from problems with the measurement of it. For constructive behavior in girls (Table 5.1), low stability most likely indicates a real change, because the agreement between peer nominations and teacher ratings with regard to girls' constructive behavior was highly significant at ages 8 and 14, but relative stability was low both in peer nomination and teacher rating.

The meta-analysis by Olweus (1979) concerning aggression in males indicated that the average size of the stability coefficient depends on the length of the interval. As the interval increases, the stability coefficient tends to decrease. It was possible to test this argument with the JYLS data. The items were formulated differently at ages 8 and 14, as explained in Chapter 2 (see Box 2.1). At age 8, several items were presented for each category of socioemotional behavior (e.g., "attacks," "teases," and "says naughty things" for aggression) but at age 14, single items were combined on the basis of the results at age 8 (e.g., "Attacks without reason, teases others, says naughty things"). The combined items had already been used at age 9 in a followup study of boys (Pitkänen, 1973c). Therefore, it was possible to analyze whether 
the relative stability of behavior depended on the formulation of the items or on the length of the interval between the measurements.

For boys, the correlations showed that the relative stability of socioemotional behaviors did not depend on the formulation of the items (separated items summed up or combined into one item), but it did depend on the interval between the measurements. When the interval was only 1 year (from age 8 to 9 and the formulations were different), the correlations (e.g., 0.60 for aggression) were higher than the correlations (e.g., 0.37 for aggression) when the interval was 5 years (from age 9 to 14 and the formulations were similar). As a class teacher system (at ages 8 and 9) had changed to a subject teacher system (at age 14) so that teachers had different opportunities to make observations of the pupils, the interval does not only explain lower correlations.

When focusing on the question of relative stability one may also consider the absolute stability of a characteristic (e.g., aggression) over time. In the JYLS, the means of peer nominations and teacher ratings at ages 8 and 14 were not comparable, because the variables used in the teacher ratings and peer nominations were different at ages 8 and 14. Therefore, knowledge of the stability of the amount of a certain type of socioemotional behavior during school age was not available through this study.

Conclusions regarding question 2. Relative stability in socioemotional behavior from childhood to adolescence was generally significant. The exception was girls' constructive behavior in which real changes most likely occurred during their school years.

\subsubsection{The person-situation controversy}

In the 1960s, trait theories were dominant in the area of personality. At the same time, learning theories and other cognitive approaches were developed, as a consequence of which, an emphasis was laid on situational factors. An attack on trait approaches was made by Mischel (1968), who became known for his criticism of traits as stable and enduring characteristics that imply that people's behavior remains fairly unchanged over time and across situations. He argued that changes in environmental or external conditions modify how people behave, and that individuals' behavior is relatively situation-specific. He began a discussion that became known as the person-situation controversy.

In the early 1960s, I had also become critical of the trait approach to aggression, and studied aggression as a situation-specific behavior as the following sequence of three lines of study demonstrates. In the Kindergarten Study with boys (Pitkänen, 1966, 1969: Part I) teachers were asked to assess for each of the 32 aggression items: who had been the boy's target of aggression and in what context the aggression was displayed. The options for the target of aggression were: a boy of the same size, a taller boy, a smaller boy, a girl, or a teacher; and the options for the contexts were: free play outdoors, free play indoors, and periods of directed activity such as formal group work, meals, or music sessions. A boy of the same size was the most typical 
target of aggression, and aggression toward him was most typically displayed during periods of free play outdoors and indoors (see Pulkkinen, 2017).

Later, in the context of collecting the baseline data for the JYLS, I continued the study into the situational effects on aggressive behavior using experimental studies with a subsample of 60 boys (Pitkänen, 1969, pp. 141-76). I devised three series of questions and personally presented them to the boys. The series of questions that focused on reactive aggression included direct questions about different types of aggression (physical, verbal, facial, direct, indirect), for instance: "You certainly know what it is like when somebody hurts you, say, by hitting, pushing or throwing something. What would you do if one of the boys in your class, who is of the same size as you, hurt you?" The target was varied in the same way as in the Kindergarten Study (i.e., taller boy, smaller boy, girl, mother, teacher). For proactive aggression, the questions took the following form: "Do you attack a boy of your size or try to hurt him in any way, even if he had done you no harm, just to tease him?" The third question series included stories for which each boy was asked to provide a solution to a conflict. This was a more indirect measure.

Sensitivity to the targets of aggression came out in the responses of the 8-yearold boys. Both reactive and proactive physical aggression were most expressed toward a boy of the same size, and toward a smaller boy rather than to a taller boy. Differences between the levels of aggression targeted at the victims were smaller when it came to verbal and indirect aggression. Around 10 years later, these test responses were compared with the participants' self-ratings of aggressiveness (Pitkänen-Pulkkinen, 1981a). At age 8, proactive aggression of any type and reactive aggression displayed in a direct physical way most highly predicted self-ratings of aggression at age 20. Verbal defense and reactions to frustrating stories had no predictive validity for aggression at age 20 .

Inspired by the findings on the situational effects on aggression, I designed a so-called "Aggression Machine" (PAM) (Pitkänen, 1973a). In the PAM, the inclining side of the apparatus has two parallel rows: one consisting of lamps for stimulus presentation, the other consisting of buttons for the participants to press. The lights were programed by means of a built-in program disc. Each stimulus was recorded on the recording tape along with the pressing of the button. The physically aggressive attacks/responses were illustrated by pictures and words on a scale of the intensity of aggression (do nothing, pushes a little, pinching, slapping, knocking down, pulling hair, hitting with a stick, and punching). The attacker (a boy of the same size, taller boy, smaller boy, girl, father, female teacher) was also depicted by a picture. The participant was asked to press a button as a response to an attack. Data were collected from the extreme groups at age 9 .

A large part of the variance of the intensity of aggression in the PAM was explained by the situational variations. When the attacker was a boy of the same size, the intensity of aggression correlated most highly with teacher-rated aggressiveness. Boys who were rated as aggressive by teachers also made sharper discriminations between situations than did nonaggressive boys (Pitkänen, 1973b). The PAM responses at age 9 were compared with self-ratings on 
aggression and self-control 10 years later (Pitkänen-Pulkkinen, 1980b). It was found that the intensity of the PAM aggression toward a boy of the same size had predictive validity with self-rated aggression at age 20 , and that the length of time before the reaction took place, as measured by the PAM, was associated with later self-control. This means that taking a longer time to react before choosing an aggressive response indicated more consistent self-control. Interestingly, PAM aggression toward a boy of the same size was associated with school maladjustment at age 14 and substance use at age 20, whereas PAM aggression toward a girl correlated with experiencing conflicts with the mother at age 14 and an early move away from home. Aggression expressed toward an authority figure (teacher, father), in turn, correlated with social anxiety at age 14 and a lack of plans for the future at age 19/20. Thus, the target of aggression may reflect different dynamics of aggression.

The PAM was computerized in the late 1990s and used in a doctoral dissertation by Juujärvi (Juujärvi, 2003; Juujärvi, Kooistra, Kaartinen, \& Pulkkinen, 2001). The data collection took place as part of the Offspring Study of the JYLS. The sample included 109 children (56 percent boys; average age 10.5 years) from the JYLS participants. There were three general findings that were common for boys and girls.

First, in accordance with previous findings, a status difference between the attacker and the defender affected the intensity of an aggressive response; in addition, this study showed that a status difference affected the intensity of aggressive responses similarly in boys and girls. Same-sized peers (of the same sex and opposite sex) and taller peers evoked less situational control in aggressive reactions than a smaller peer or the parent. Attacks by a same-sized peer of the same sex were countered with the highest intensity. This shows the effects of situational control factors on the intensity of aggression.

Second, the comparison of aggressive and nonaggressive children (categorized on the basis of teacher ratings) showed that aggressive children reacted more intensively than nonaggressive children toward three opponents: a same-sized peer of the same sex, a smaller child, and a same-sized peer of the opposite sex.

Third, in the situation of a minor provocation differences between aggressive and nonaggressive children appeared more clearly than in the situation of a major provocation. Aggressive children reacted to mild attacks (from 0 to 3; on a scale from 0 to 7) more intensively than nonaggressive children. It suggests that particularly in situations of minor provocation, aggressiveness with low behavior regulation determines response intensity. The same trend was seen in regard to any opponent, although it was most significant when the opponent was a same-sized peer of the same sex.

Conclusions regarding question 3 . The highest continuity of aggression over time was found (1) when aggression was measured in childhood through direct questions about aggressive behavior, or by using a nonverbal test, and (2) when the target of aggression was a same-sized peer of the same sex. These findings supported Mischel's (1968, p. 36) statement that "behaviors sampled in closely similar situations yield the best correlations." 


\subsection{Children grow and adult styles of life emerge}

\subsubsection{The two-dimensional framework across generations}

Structural validity. The validity of the two-dimensional structure in the model for the USB was studied at age 8 by calculating the intercorrelations of teacher ratings and peer nominations, and by a factor analysis (Pitkänen, 1969). The results confirmed that the first two factors depicting bipolar dimensions for activity versus passivity and high versus low self-regulation explained the majority of individual differences. (When the number of factors was increased, specific components appeared, depending on the similarity of variables.) Activity explained the variance of both aggressive and constructive behavior, and at the other end of the axis, passivity explained the variance of both anxious and compliant behaviors. Correspondingly, low self-regulation explained the variance of aggressive and anxious behavior, and high self-regulation explained the variance of constructive and compliant behavior. Each variable was differentially weighted by activity and self-regulation, and the relationships of the variables were interpreted as following the circular law of order.

Factor analytical results confirmed that the different patterns of behavior were not categorical concepts (types) per se, but rather they signified the four corners of the two-dimensional map, as presented in the middle of Figure 4.1. A personoriented analysis (a cluster analysis) showed, however that there were participants whose behavior was mainly characterized by aggressive, constructive, compliant, or anxious behavior (Pulkkinen 1995).

There was structural stability in socioemotional behavior from age 8 to 14: the corresponding structure was found in teacher ratings at age 14. Intercorrelations of teacher ratings indicated that ratings on activity correlated positively with ratings on aggressive and constructive behavior (Pulkkinen, 1995). The dual nature of extraversion was also noticed by Eysenck and Eysenck (1963) when they made a distinction between social and impulsive extraversion. Correspondingly, ratings on passivity correlated positively with ratings on anxious and compliant behavior. Emotional lability (low control), in turn, correlated positively with anxiety and aggression but negatively with compliance and constructiveness, whereas these correlations were the opposite for emotional stability. The reference axes for activity and self-regulation were largely independent of each other.

Socioemotional behavior across generations. The Offspring Study conducted as part of the JYLS in 1998 allowed for a comparison between parents' socioemotional behavior in 1968 with their children's behavior in 1998 by using the same items and the same technique (teacher rating). Table 5.2 presents the means of the teacher ratings for the children and the ratings of one of each child's parents at around the same age (from 8 to 10 years old). It was found that in both generations, boys were rated as being more aggressive and disobedient to the teacher than girls, whereas girls were rated as more compliant, concurring with the findings by Hyde (2014). Over the generations, the same gender differences were found in behavior regulation: boys were lower in behavior regulation than girls, although teachers were encouraged to rate a pupil's behavior in comparison with 
TABLE 5.2 Means of parents and children with regard to the same socioemotional characteristics at the same ages; teacher ratings in 1968 and 1998

\begin{tabular}{|c|c|c|c|c|c|c|c|c|}
\hline \multirow{3}{*}{$\begin{array}{l}\text { Socioemotional } \\
\text { behavior }^{1}\end{array}$} & \multicolumn{2}{|l|}{1968} & \multicolumn{2}{|c|}{1998} & \multicolumn{4}{|l|}{ t-values } \\
\hline & \multirow{2}{*}{$\frac{\text { Father }}{(49)}$} & \multirow{2}{*}{$\frac{\text { Mother }}{(51)}$} & \multirow{2}{*}{$\frac{\text { Son }}{(55)}$} & \multirow{2}{*}{$\frac{\text { Daughter }}{(47)}$} & \multirow{2}{*}{$\begin{array}{l}\text { Father/ } \\
\text { mother }\end{array}$} & \multirow{2}{*}{$\begin{array}{l}\text { Son/ } \\
\text { daughter }\end{array}$} & \multicolumn{2}{|c|}{$1968 / 1998$} \\
\hline & & & & & & & Males & Females \\
\hline Aggressive & 0.59 & 0.22 & 0.62 & 0.33 & $3.66^{\star \star \star}$ & $2.20^{\star}$ & n.s. & n.s \\
\hline Anxious $^{2}$ & 0.57 & 0.57 & 1.09 & 1.16 & n.s. & n.s. & \multicolumn{2}{|c|}{ (Not comparable) } \\
\hline Constructive & 1.33 & 1.49 & 1.72 & 1.95 & n.s. & $-1.73^{\dagger}$ & $-2.86^{\star \star}$ & $-2.93^{\star \star}$ \\
\hline Compliant & 1.44 & 1.73 & 1.41 & 1.75 & $-1.79^{\dagger}$ & $-2.73^{\star \star}$ & n.s. & n.s. \\
\hline Stable & 1.48 & 1.59 & 2.13 & 1.90 & n.s. & n.s. & $-2.61^{\star \star}$ & $-3.95^{\star \star \star}$ \\
\hline Labile & 0.73 & 0.41 & 0.37 & 0.38 & $2.31^{\star}$ & n.s. & n.s. & n.s. \\
\hline Disobedient & 0.47 & 0.10 & 0.58 & 0.23 & $3.43^{\star \star \star}$ & $2.71^{\star \star}$ & n.s. & n.s. \\
\hline Active & 1.53 & 1.80 & 2.49 & 2.43 & n.s. & n.s. & $-5.51^{\star \star \star}$ & $-6.94^{\star \star \star}$ \\
\hline Passive & 0.45 & 0.55 & 0.56 & 0.74 & n.s. & n.s. & n.s. & n.s. \\
\hline
\end{tabular}

Note: ${ }^{\star \star \star} \mathrm{p}<0.001 ;{ }^{\star \star} \mathrm{p}<0.01 ;{ }^{\star} \mathrm{p}<0.05 ;{ }^{\dagger} \mathrm{p}<0.10$.

${ }^{1}$ Rated on a scale from $0=$ "never observed in the pupil in question" to $3=$ "very characteristic of the pupil"; averaged across items.

${ }^{2}$ Items for anxious behavior were different in 1968 and 1998.

the behavior of pupils of the same sex. Consistent gender differences across generations did not emerge in social activity nor in emotion regulation (constructive and anxious behavior).

In addition, the comparisons of the levels of socioemotional behaviors across generations revealed an interesting result. Both boys and girls in 1998 were significantly more active (always together with others versus shy) emotionally balanced (friendly and reliable), and constructive (acts reasonably, negotiates, defends weaker peers; see Box 2.1), that is, higher in emotion regulation, than their parents in 1968. Unfortunately, comparisons cannot be made with regard to anxiety, for which the items differed in 1968 and 1998. Differences did not exist in behavior regulation (aggressive and compliant behaviors). The positive changes in activity and emotion regulation were most likely due to changes in parenting, that is, due to increasing child-centeredness and more guidance provided by Finnish parents. In 1983, legislation was passed that forbade corporal punishment in the home and positive parenting was highlighted. The connections between child-centered parenting and constructive behavior are described in Section 5.3.3. The wording of the Act of Child Custody in 1983 was highly consistent with the results of the JYLS published in a Finnish book by Pulkkinen (1977) about upbringing and connections between child-centered parenting and constructive behavior.

(1) The purpose of child custody is to ensure the welfare and balanced development of a child in accordance with the child's individual needs and wishes. 
The purpose is also to secure a close and affectionate relationship in particular between the child and his or her parents.

(2) A child must be ensured good care and upbringing as well as supervision and protection appropriate for his or her age and stage of development. A child should be brought up in a secure and stimulating environment and receive an education that corresponds with his or her inclinations and wishes.

(3) A child must be brought up with understanding, security and affection. A child must not be subdued, corporally punished or treated offensively in any other way. The growth of a child towards independence, responsibility and adulthood must be supported and encouraged.

Act on Child Custody and Right of Access (361/1983).

(C) Ministry of Justice, Finland. Available online at www.finlex.fi/fi/laki/kaannokset/ 1983/en19830361.pdf (accessed 8 June 2016)

Correlations between fathers and sons. The Offspring Study also made it possible to compare the similarity between parents and their children in socioemotional behavior at the same age. Analyses showed that the father's socioemotional behavior in childhood correlated with their son's behavior at the corresponding age (30 years later), but not with their daughters' behavior. The mother's socioemotional behavior did not correlate with their son's or daughter's behavior. The correlations between the fathers and sons (31 father-son pairs) concerned low selfregulation (Table 5.3). The father's disobedience to the teacher at age 8 correlated positively with the son's disobedience to the teacher, lability of moods, and aggressive behavior, and negatively with emotional stability and constructive behavior. In general, the sons' low self-regulation (disobedience and emotional lability) was

TABLE 5.3 Correlations between fathers and sons in teacher ratings of socioemotional behaviors at ages 8 to 10

\begin{tabular}{lcrrrrrrrrr}
\hline \multicolumn{10}{c}{ Sons (1998) } \\
\hline Fathers (1968) & 1 & 2 & 3 & 4 & 5 & 6 & 7 & 8 & 9 \\
\hline 1 Aggressive & 0.26 & 0.23 & $-0.60^{\star \star \star}$ & -0.22 & $-0.43^{\star}$ & $0.40^{\star}$ & $0.54^{\star \star}$ & $-0.32^{\dagger}$ & 0.28 \\
2 & Anxious & 0.13 & -0.03 & -0.10 & 0.14 & 0.16 & 0.24 & 0.15 & -0.04 & -0.02 \\
3 & Constructive & -0.05 & -0.06 & 0.27 & 0.28 & $0.31^{\dagger}$ & $-0.42^{\star}$ & $-0.42^{\star}$ & 0.14 & -0.12 \\
4 & Compliant & -0.17 & $-0.40^{\star}$ & $0.33^{\dagger}$ & 0.27 & $0.34^{\dagger}$ & $-0.40^{\star}$ & $-0.39^{\star}$ & 0.10 & -0.16 \\
5 & Stable & 0.12 & -0.08 & 0.22 & 0.21 & 0.16 & -0.16 & -0.14 & 0.04 & -0.01 \\
6 & Labile & 0.21 & 0.12 & $-0.33^{\dagger}$ & -0.26 & $-0.35^{\dagger}$ & $0.46^{\star}$ & $0.48^{\star \star}$ & -0.12 & 0.14 \\
7 & Disobedient & $0.37^{\star}$ & 0.07 & $-0.44^{\star}$ & -0.23 & $-0.51^{\star \star}$ & $0.44^{\star}$ & $0.53^{\star \star}$ & -0.17 & 0.04 \\
8 & Active & 0.14 & $0.40^{\star}$ & -0.29 & 0.04 & -0.27 & 0.04 & 0.21 & -0.13 & 0.19 \\
9 & Passive & 0.21 & -0.11 & 0.09 & 0.02 & 0.16 & 0.12 & 0.01 & 0.02 & -0.07 \\
\hline
\end{tabular}

Items for anxious behavior were different in 1968 and 1998.

Note: ${ }^{\star \star \star} \mathrm{p}<0.001 ;{ }^{\star \star} \mathrm{p}<0.01 ;{ }^{\star} \mathrm{p}<0.05 ;{ }^{\dagger} \mathrm{p}<0.10$. 
associated with the fathers' low self-regulation, and the sons' constructive behavior was less likely if the father had been aggressive. These correlations suggest that the fathers' low self-regulation did not promote the development of emotion regulation in their sons. The similarity between the father and son did not concern specific behaviors such as aggression or activity.

It is to be noted that only one of the sons' parents belonged to the JYLS participants and therefore, it was not known what the sons' mothers had been like as children when the similarity between the fathers and sons were studied. Children may be affected by maternal psychopathology by genetic factors, neuroendocrine processes, maladaptive cognitions, and parenting, as reviewed by Hautmann et al. (2015), who also showed that parenting of mothers is more strongly associated with child antisocial behavior than parenting of fathers. Besides genetic and socialization factors between the father and son, an assortative mating through the choice of the wife might have strengthened the connection in low self-regulation between the fathers and sons. It was found in the study by Rönkä, Kinnunen, and Pulkkinen (2001) that low self-regulation as children in men was associated with their problem behaviors as adults, and that men who had a higher level of problems of social functioning typically had a problematic partner. A problematic partner had drinking problems, was unemployed, or expressed aggression toward the partner. The parents' problem behavior causes the risk of parent-centered parenting, which is, in turn, associated with low self-regulation in children. The similarity of sons to fathers, often in terms of negative behaviors, is known in many proverbs where it is expressed in a hidden way such as: The apple does not drop far from the tree.

Conclusions regarding question 4 . Empirical data has confirmed the validity of the two-dimensional structure with social activity versus passivity and low versus high self-regulation as reference axes. Over generations, boys were rated to be lower in behavior regulation (more aggressive and disobedient) than girls who were rated as being more compliant. Changes across generations were found toward increasing emotion regulation and social activity in boys and girls, possibly due to legislation that guided parenting toward greater child-centeredness. The father's low self-regulation (e.g., disobedience) in childhood correlated with his son's low selfregulation 30 years later, suggesting the accumulation of problems in the father's life reflected in the son's development.

\subsubsection{The unfolding of the styles of life from age 8 to 27}

Methodological remarks. Answers to the fifth question: whether socioemotional development progresses in the directions depicted in the model for USB (Figure 4.1) were found in two studies. First, the development of children's socioemotional behavior toward adult styles of life was studied by forming large data sets at ages 8,14 , and 20. They concerned personality characteristics, life attitudes, and everyday activities. The first two bipolar factors to depict the major dimensions of individual differences were extracted at each age (Pulkkinen, 1982). Second, for the age 27 data, new software made it possible to use a confirmatory LISREL factor analysis, meaning that 
the structure of the model could be tested with statistical criteria (Pulkkinen, 1990a). At ages 8 and 27, data were available for the whole original sample, and at ages 14 and 20 for the subsample, which was interviewed at these ages.

The relationships between the factors obtained at each age were studied by calculating correlation coefficients between the factor scores across ages. Significant correlations between the factors obtained at each age level confirmed that it was possible to distinguish four expected developmental lines from age 8 to 27 . They are schematically presented in Figure 5.1. The developmental lines depicted by arrows are based on highly significant correlations $(p<0.001)$ between successive ages obtained in studies by Pulkkinen (1982, 1990a). In spite of these general lines of continuity, some participants had turned in other directions. This could be seen in small or trend-like correlations $(\mathrm{p}<0.05)$ across paths, which are marked by dotted lines in Figure 5.1.

Developmental paths. The unfolding of the styles of life progressed from differences in children's socioemotional behavior to their diverging orientations to life in early adolescence. These orientations paved a way to emerging styles of life

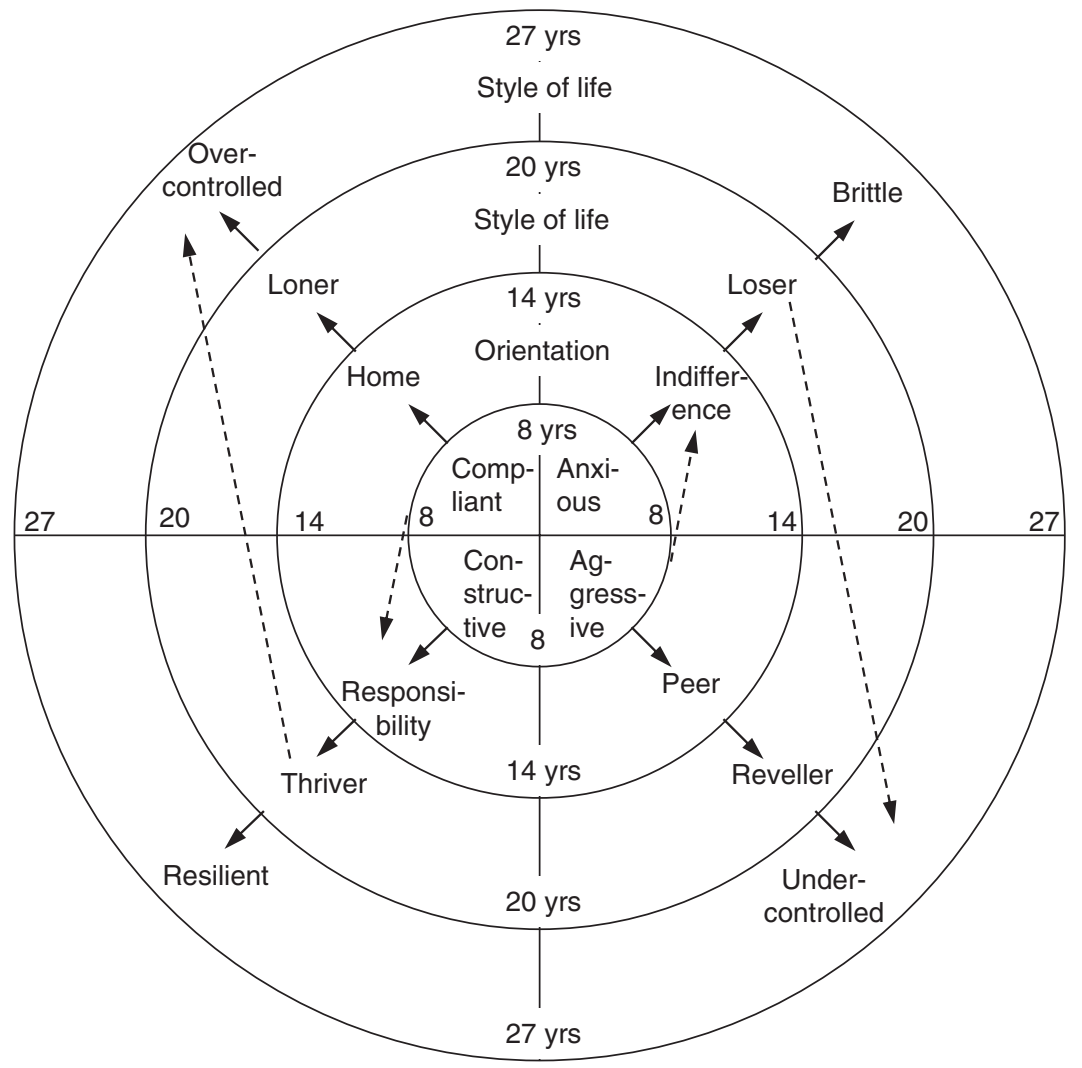

FIGURE 5.1 Developmental lines from childhood socioemotional behavior to adult styles of life. 
in late adolescence, which further resulted in the lifestyles of young adults. The adult lifestyles were closely connected to the participants' personality characteristics assessed with the Eysenck Personality Questionnaire (EPQ) and Zukerman's Sensation Seeking Scales (SSS). They are relabeled here as the Undercontrolled versus Overcontrolled styles of life and the Resilient versus Brittle styles of life, respectively, to fit with the terminology adopted in this book.

Childhood. Individual differences in socioemotional behavior at age 8 were largely explained by two bipolar factors, which were labeled as Constructive, socially skilled behavior versus Anxious, socially helpless behavior, and Aggressive, shortsighted behavior versus Compliant, prudent behavior (Pulkkinen, 1982). Constructive, socially skilled behavior comprised of peer nominations and teacher ratings of various aspects of constructive behavior, social activity and fitting for leadership, and school success. In contrast, Anxious and socially helpless behavior comprised of peer nominations and teacher ratings of being unfit for leadership, afraid of others, and withdrawn; crying easily at the dentist and if one was treated nastily by others; and by a lack of concentration. Aggressive, short-sighted behavior comprised of peer nominations and teacher ratings of different types of aggressive behavior, disobeying the teacher, changing moods, and poor concentration. Antisocial symptoms as observed by the teacher and restlessness and impulsive extraversion assessed with personality inventories also belonged to this pattern of behavior. In contrast, Compliant and prudent behavior comprised of peer nominations and teacher ratings of quarreling rarely and leaving a squabbling group, and being peaceable, quiet, withdrawn, friendly, reliable, and someone who apologized easily. Higher scores in dependency, femininity, submissiveness, and altruism in personality inventories also belonged to this pattern.

The pair of the factors obtained at age 8 confirmed the structure of the twodimensional model by demonstrating that the combination of self-regulation and social activity might occur in different ways. Constructive behavior depicted high emotion regulation associated with social activity, whereas anxious behavior depicted low emotion regulation associated with social passivity. Correspondingly, aggressive behavior depicted low behavior regulation associated with social activity, whereas compliant behavior depicted high behavior regulation associated with social passivity. These differences continued up to young adulthood, as summarized in Box 5.1.

Early adolescence. Different orientations emerged in the participants' lives when they themselves and one of their parents were interviewed at age 14. Peer nominations and teacher ratings of socioemotional behavior were also considered. Again, two bipolar factors largely explained individual differences. They were labeled as Orientation toward responsibility versus Indifferent attitudes toward one's obligations, and Orientation toward peers versus Orientation to home (Pulkkinen, 1982).

Orientation toward responsibility was shown by constructive behavior, good school reports, interest in school attendance, and doing one's homework, being fit for leadership, having many friends and taking part in organized activities. Chatting about free-time activities with their parents and having a close relationship with the father also belonged to this pattern. In contrast, Indifferent attitudes toward one's 


\section{BOX 5.1 FOUR DEVELOPMENTAL PATHS FROM CHILDHOOD (C) THROUGH EARLY ADOLESCENCE (EA) AND LATE ADOLESCENCE (LA) TO YOUNG ADULTHOOD (YA): RESEARCH FINDINGS (PART II) SUMMARIZED}

(1) From constructive behavior to the Resilient style of life

C: Constructive behavior involving acting reasonably, negotiating, siding with smaller and weaker peers, and being socially active, reliable, and fit for leadership; having good school results.

EA: An orientation to taking responsibility for one's school work and other activities.

LA: The style of life of Thriver: the effective use of social and cognitive resources, school success, optimism, and continuing with one's studies.

YA: The Resilient style of life: extraversion, a sense of control over one's development, long education, high self-confidence, identity achievement in ideological matters, and valuing the arts and sciences.

(2) From anxious behavior to the Brittle style of life

C: Socially anxious behavior involving being afraid of others, being withdrawn, crying easily, and lacking concentration.

EA: Indifferent attitude towards one's obligations, particularly school; lacking hobbies; lacking concentration, changeable moods, being fearful and unable to defend oneself but displaying some aggressive behavior; conflicts with parents.

LA: The style of life of Loser: being afraid of the future and disappointed in oneself, lacking plans for the future and having no sense of responsibility toward society.

YA: The Brittle style of life: high neuroticism, a sense of failure, blaming others for one's failures, identity diffusion, unclear plans for the future, pessimism, dissatisfaction with one's present life, and having the feeling that other people do not support one.

(3) From aggressive behavior to the Undercontrolled style of life

A: Aggressive, short-sighted behavior involving different types of aggressive behavior; changing moods, poor concentration, and disobeying the teacher.

EA: Orientation to peers more than to the home including hanging around with peers, an early onset of smoking, drinking and dating, having a distant relationship with the parents, getting involved in fights, and school maladjustment.

LA: The style of life of the Reveller: smoking, use of alcohol, being often drunk; socially active and making friends easily, but also getting angry easily; conflicts with one's parents. 
YA: The Undercontrolled style of life: extraversion, experience seeking, disinhibition, the excessive use of alcohol, regular smoking, committing offenses, limited education and low occupational status, unemployment, and dissatisfaction with life.

(4) From compliant behavior to the Overcontrolled style of life

C: Compliant, prudent behavior involving quarreling rarely, avoiding children who were squabbling, being peaceable, quiet, friendly, reliable, and apologizing easily.

EA: Orientation to home more than to one's peers indicated by spending free time at home, having a close relationship with the mother, being often alone, refraining from smoking and drinking, respecting the norms at school, and being peaceful.

LA: The style of life of Loner: low levels of social activity, a strong adherence to social norms, refraining from the use of alcohol and smoking, having normative conceptions about sexual behavior, being tense and withdrawn, and dependent upon one's parents.

YA: The Overcontrolled style of life: introversion and low levels of experience seeking, spending leisure time engaged in home-oriented hobbies, and refraining from substance use, entertainment seeking, and the breaking of rules.

obligations were indicated by low interest in school attendance; being lazy about one's homework, truanting weekly; having poor school reports, and not having hobbies. Lacking concentration; having changeable moods; being fearful and unable to defend oneself but also displaying some aggressive behavior belonged to this pattern. The participants had friends whom the parents disliked, and they did not discuss their activities in their free time with their parents. The parents perceived the child as being difficult.

Orientation toward peers was shown by the adolescents hanging out with their peers, having many friends whom the parents disliked, spending free time on the streets and in discos, starting to smoke, drink, and go out with a boy/girlfriend at an early age, and experiencing conflict and a distant relationship with the mother. The pattern also included aggressive attacks on others and participating in fights, but the adolescent was also suited to being a leader of a group. School maladjustment was indicated by receiving punishments at school, and spending pocket money on entertainment demonstrated a short-sighted style of life. In contrast, orientation to home at age 14 was shown by spending one's free time at home; being often on one's own, having few friends, and having no friends whom the parents disliked; refraining from smoking, using alcohol, and dating; respecting the norms at school; being peace-loving and never fighting with others; being unfit for leadership; and having a close relationship with the mother. 
Playmates and peers are important to children, and "the enemy is not our children's peers but peer orientation," as Neufeld and Maté (2004) spell out. They base their argument on their clinical experience and findings in research. A relatively higher orientation to peers than to the parents at an early age involves emotional risks in terms of attachment relations and behavioral risks in terms of models and pressures to which children become vulnerable. A balance between parent- and peer-orientation is needed for children's development as shown by the participants' lifestyles (Reveller and Loner) at age 20.

Late adolescence. At age 20, two pairs of lifestyles explained the large part of variation in the participants' behavior. One pair of lifestyles was labeled as the Thriver versus Loser, and the other pair as the Reveller versus Loner (Pulkkinen, 1982). The style of life of Thriver was characterized by optimism about the future; good school success; continuation of studies or being in regular employment; clear plans for the future; positive and realistic life attitudes; and satisfaction with choices. Further characteristics were nonsmoking; feeling responsible toward society; having opinions about politics; and making friends easily. Personality characteristics included high self-control, constructiveness, a balanced mood, sociability, and realism with respect to one's own character. In contrast, the style of life of Loser was characterized by being afraid of the future; disappointed with oneself; dissatisfied with one's choices; and having low self-confidence. School success had been poor and having no occupational education after the obligatory school was common. Plans for the future were lacking, living was day by day along with a risk of unemployment. It was typical of a Loser to watch TV for many hours a day, to have difficulty in making friends, and to be passive in respect to society. The Losers had problems in psychological adjustment (self-concept and social adaptation) as assessed by the interviewer.

The Reveller style of life was characterized by similar features to those at age 14 in peer orientation: spending one's free time in discos and pubs, excessive smoking, the use of alcohol many times a week, and being frequently drunk (Pulkkinen, 1983b). The Revellers had conflicts with their parents and they did not want to be dependent upon them. They started dating and sexual activity at an early age, and accepted living with a partner without being married; in 1980, this was against the prevailing norms. In terms of personality characteristics, the pattern included social activity and making friends easily, but also getting angry easily. The Reveller style of life was also associated with the number and types of offenses that the male participants had committed up to age 20 (Pulkkinen, 1983a). These associations were lower in females. In contrast, the Loner style of life was indicated by not using alcohol and not smoking cigarettes; normative conceptions on sexual behavior; no romantic relationship as yet; being tense, quiet, and withdrawn, and having difficulty in making friends; being dependent upon one's parents and having no conflicts with them; and perceiving that parents had a strong hold over their children.

Young adulthood. When the participants of the JYLS became 27 years old, a question arose whether their styles of life in late adolescence predicted their adult styles of life. The confirmatory analysis showed that two factors (Resilient versus Brittle, 
and Undercontrolled versus Overcontrolled) explained the variance in the styles of life satisfactorily. Their correlations with the age 20 styles of life were calculated and they showed significant continuity as depicted in Figure 5.1.

The Thriver style of life predicted the Resilient style of life, in which extraversion and experience seeking were associated with a sense of control over one's development; gaining a high level of education; high self-confidence; identity achievement in relation to religion and politics; clear plans for the future; an orientation toward studying; and appreciating the arts and sciences. The Loser style of life predicted, in turn, the Brittle style of life that was characterized by low levels of experience seeking and low extraversion (high introversion); high neuroticism, a sense of failure, and blaming others for one's failures. This style of life was also characterized by low self-confidence, identity diffusion, a lack of plans for the future, and the sense of not being supported by others; as well as being pessimistic and dissatisfied with one's present life. Social problems such as having a limited level of education, low occupational status, an early entry into the workforce, and having experienced periods of unemployment, were associated with the Brittle style of life.

The Reveller style of life at age 20 predicted the Undercontrolled style of life at age 27. This included extraverted behavior and experience seeking associated with the excessive use of alcohol, seeking entertainment, regular smoking, committing offenses, disinhibition (SSS), and psychoticism (EPQ) in terms of to low adherence to social norms and high impulsivity. Social and personal problems connected to the Undercontrolled behavior were revealed by, for instance, having a limited education and a low occupational status, periods of unemployment, fear of personal difficulties in the future, and dissatisfaction with one's present life. The Loner style of life predicted, in turn, the Overcontrolled style of life, characterized by introversion and low levels of experience seeking; spending leisure time engaged in homeoriented hobbies; and refraining from substance use, entertainment seeking, and breaking the rules.

Some male and female Losers developed toward the Undercontrolled style of life, and some Thrivers toward the Overcontrolled style of life (dotted lines in Figure 5.1). In addition, gender-specific changes after adolescence, which are not depicted in the figure, were found to go "clockwise" in women and "anti-clockwise" in men (cf. Figure 5.1). In women, an orientation toward responsibility at age 14 also preceded the Overcontrolled style of life, and an orientation to feeling indifferent toward one's responsibilities preceded the Undercontrolled style of life. In men, home-orientation at age 14 also preceded the Resilient styles of life, and peer-orientation preceded the Brittle style of life. These gender-specific correlations were significant at the $\mathrm{p}<0.01$ level.

Conclusions regarding question 5. Socioemotional development progressed in the directions depicted in the USB model (Figure 4.1). Constructive behavior in childhood was the beginning of a developmental path to the Resilient style of life in young adulthood, whereas social anxiety in childhood led to the Brittle style of life. Aggressive behavior, in turn, began a path toward the Undercontrolled style of life, whereas compliant behavior led to the Overcontrolled style of life. 


\subsubsection{The developmental background of the styles of life}

The roots of the different styles of life were explored in terms of the connections between the developmental lines and the children's home backgrounds, including their upbringing and living conditions when they were 14 years old (Pulkkinen, 1982). Factor analyses were made separately for upbringing and living conditions and two factors were extracted for both: Child-centered versus parent-centered parenting and Indifference versus concerned parenting for upbringing; and socioeconomic status and steadiness of conditions for living conditions. Table 5.4 shows the significant associations between the different components of the participants' home backgrounds and the developmental lines until the age of 20 , which were obtained by calculating the correlations between the factor scores.

Upbringing. Parent-centered versus child-centered parenting was consistently connected with all the developmental lines, as can be seen in Table 5.4. The content of child-centered versus parent-centered parenting is presented in Table 5.5. The label "child-centered parenting" refers to the parents' love and support for, and care of, the child, understanding of the child's needs and setting consistent and justified boundaries for the child's behavior. Child-centered parenting has the same qualities as authoritative child-rearing in Baumrind's (1971) terminology. The label "parent-centered parenting," in turn, refers to having little time for and little

TABLE 5.4 Significant connections between the styles of life at different ages and home backgrounds in adolescence

\begin{tabular}{|c|c|c|c|c|c|c|c|c|c|}
\hline & \multirow[b]{3}{*}{ Age } & \multicolumn{8}{|c|}{ Home background at age 14} \\
\hline & & \multicolumn{4}{|c|}{ Upbringing } & \multicolumn{4}{|c|}{ Living conditions } \\
\hline & & $\begin{array}{l}\text { Parent } \\
\text { centered }\end{array}$ & $\begin{array}{l}\text { Child- } \\
\text { centered }\end{array}$ & Unconcerned & Concerned & $\begin{array}{l}\text { Low } \\
\text { SES }\end{array}$ & $\begin{array}{l}\text { High } \\
\text { SES }\end{array}$ & $\begin{array}{l}\text { Un- } \\
\text { steady }\end{array}$ & Steady \\
\hline Aggression & 8 & $\star \star \star$ & & & & & & & \\
\hline Peer-orientation & 14 & $\star \star \star$ & & & & & & & \\
\hline Reveller & 20 & $\star \star \star$ & & & & & & $\star \star \star$ & \\
\hline Compliance & 8 & & $\star \star \star$ & & & & & & \\
\hline $\begin{array}{l}\text { Home- } \\
\text { orientation }\end{array}$ & 14 & & $\star \star \star$ & & & & & & \\
\hline Loner & 20 & & $\star \star \star$ & & & & & & $\star \star \star$ \\
\hline Constructiveness & 8 & & $\star \star \star$ & & $\star \star$ & & $\star \star \star$ & & $\star \star$ \\
\hline Responsibility & 14 & & $\star \star \star$ & & & & $\star \star$ & & $\star \star$ \\
\hline Thriver & 20 & & $\star \star \star$ & & & & $\star \star$ & & \\
\hline Anxiety & 8 & $\star \star \star$ & & $\star \star$ & & $\star \star \star$ & & $\star \star \star$ & \\
\hline Indifference & 14 & $\star \star \star$ & & & & $\star \star$ & & $\star \star \star$ & \\
\hline Loser & 20 & $\star \star \star$ & & & & $\star \star$ & & & \\
\hline
\end{tabular}

Adapted from Pulkkinen (1982).

$\star \star \star$ Correlation coefficient was significant at the $\mathrm{p}<.001$ level (between 0.29 and 0.53 ).

$\star \star$ Correlation coefficient was significant at the $\mathrm{p}<.01$ level (between 0.22 and 0.25 ). 
TABLE 5.5 Items loaded significantly on a factor for child-centered parenting versus parentcentered parenting; based on interviews with the participants at the age of 14 and the interviews of one of each child's parents

\begin{tabular}{|c|c|c|c|}
\hline Loading & Child-centered parenting & Informant & Parent-centered parenting \\
\hline 0.64 & Parents trust the child & Child & $\begin{array}{l}\text { Parents are sometimes or often } \\
\text { distrustful }\end{array}$ \\
\hline 0.64 & $\begin{array}{l}\text { Parents know the child's free-time } \\
\text { company }\end{array}$ & Child & $\begin{array}{l}\text { Parents do not know or know } \\
\text { sometimes }\end{array}$ \\
\hline 0.60 & $\begin{array}{l}\text { Parents know where the child } \\
\text { spends his/her leisure time }\end{array}$ & Child & $\begin{array}{l}\text { Parents do not know or know } \\
\text { sometimes }\end{array}$ \\
\hline 0.60 & No conflicts about child's leisure & Child & Conflicts about child's leisure \\
\hline 0.57 & $\begin{array}{l}\text { Parents interested in the child's } \\
\text { school }\end{array}$ & Child & $\begin{array}{l}\text { Parents indifferent to the child's } \\
\text { school }\end{array}$ \\
\hline 0.56 & No spanking & $\begin{array}{l}\text { Child, } \\
\text { Parent }\end{array}$ & Spanking still in teenage years \\
\hline 0.53 & $\begin{array}{l}\text { Parents sympathetic in child's } \\
\text { failure }\end{array}$ & Child & Parents punitive in child's failure \\
\hline 0.49 & Parents consider child's opinion & Child & $\begin{array}{l}\text { Parents do not consider or consider } \\
\text { sometimes }\end{array}$ \\
\hline 0.47 & $\begin{array}{l}\text { Conversation between parents } \\
\text { and child daily }\end{array}$ & Child & $\begin{array}{l}\text { Rare conversation between parents } \\
\text { and child }\end{array}$ \\
\hline 0.46 & Parents consistent in child rearing & Child & Parents inconsistent in child rearing \\
\hline 0.43 & Just restrictions and sanctions & Child & Unjust restrictions and sanctions \\
\hline 0.36 & $\begin{array}{l}\text { In case of misbehavior, advice } \\
\text { given }\end{array}$ & Parent & $\begin{array}{l}\text { In case of misbehavior, punishments } \\
\text { given }\end{array}$ \\
\hline 0.32 & Parents praise if child is successful & Parent & No praise or material rewards \\
\hline
\end{tabular}

Adapted from Pulkkinen (1982).

Note: For each item, both the child's and the parent's responses drawn from the interviews were included in the analysis. As shown by the Informant, the factor for child-centered versus parent-centered parenting was mostly loaded by the child's perspective. Factors for living conditions were more highly loaded by the parent's perspective.

interest in the child, showing little consideration of the child's needs, and being inconsistent and harsh in disciplining the child. Parent-centered parenting covers authoritarian behavior and the neglect of children's needs.

Child-centered parenting was associated with the developmental line from constructive behavior to the Thriver style of life and with the line from compliant behavior to the Loner style of life (Pulkkinen, 1982). Both lines represent high self-regulation in the USB model (Figure 4.1), the former representing emotion regulation and the latter behavior regulation. The parents' trust in the child, their awareness of his or her activities, their interest in, and support of, the child's school attendance, their consideration of the child's opinions, and many other aspects of positive parenting encouraged the development of high self-regulation in children. In Table 5.5, it can also be seen that in most cases, the informant was the child. The parents were asked the same questions, but it was most important to understand 
how the child experienced their parents' behavior. When he/she experienced it to be loving and caring, this had positive effects on their behavior.

Parent-centered parenting was associated with the developmental line from aggressive behavior to the Reveller style of life and with that from anxious behavior to the Loser style of life. Both lines represent low self-regulation in the USB model (Figure 4.1), the former representing behavior regulation and the latter emotion regulation. The parents' lack of emotional support for the child, their low level of interest in the child's activities, and the harsh and inconsistent discipline did not promote the development of self-regulation in children.

The other component of parenting, unconcerned versus concerned parenting, was only associated with children's behavior at age 8 . This parenting component was mostly formed by the interviewers' interpretations of the family atmosphere (Pulkkinen, 1982), being a monitoring aspect of parent- versus child-centered parenting. For unconcerned parenting, which was linked to the child's anxious behavior, the interviewer assessed the atmosphere at home as laissez-faire. The interviewer's assessment was associated with the parents' little support to the child and opinion that it was up to the child to choose his/her friends, and with children's perceptions about disagreements between their parents about child-rearing and the inconsistencies in this area. In concerned parenting, which was associated with children's constructive behavior, the parents encouraged their children and helped them with their homework. The mother controlled the child's choice of friends and set the time at which the child had to be home, and attended cultural events with the child.

Living conditions. Two components of significant living conditions emerged in a factor analysis (Pulkkinen, 1982): socioeconomic status of the parents and steadiness of living conditions. Higher socioeconomic status was associated with the developmental line from constructive behavior to the Thriver style of life (Table 5.4). Higher status was indicated by the father's and mother's higher status occupations and academic training, and the father having a permanent job. The quality of the dwelling was higher. The parents regularly took part in cultural and political activities, and family members shared leisure activities. They financially supported the child's hobbies. There were fewer children in the family. The parents' lower socioeconomic status was associated with the developmental line from social anxiety to the Loser style of life. The parents were less educated and their occupational statuses were lower. The father had experienced one or more periods of unemployment. Density was high in the home due to its small size and the many children. The parents were culturally and politically less active. The family members shared activities more rarely: everybody tended to have their own leisure activities. Parents did not financially support the child's hobbies.

Correlations of the steadiness of living conditions with the developmental lines showed that steady living conditions were associated with children's constructive behavior and an orientation toward taking responsibility, whereas unsteady living conditions were associated with children's anxious behavior and indifference (signs of anticipatory marginalization). In steadier life conditions, the mother worked less frequently outside home; she was at home when the child returned from school 
and she had sufficient time for the child. The child had lived in the same dwelling with his/her biological family without experiencing house moves. The parents were older and they smoked and drank alcohol less frequently than the parents with unsteady conditions. Unsteady conditions were characterized by the following features of family life. The mother had been employed during the child's lifetime, and she had not had sufficient time for child (from the child's point of view). In early childhood, the participant had several daycare arrangements and caretakers. While the child was of school age, he/she had to spend several hours alone at home each day after school because of the parents' working hours and the time they spent commuting. Pets at home were there to comfort the child. The family had lived in several homes. In unsteady conditions, the participant was more likely to live in a divorced or remarried family or with a sole provider. The parents were younger and they smoked, and the father drank alcohol more excessively than did the parents with steady living conditions.

Unsteady conditions at age 14 correlated with the Reveller style of life in late adolescence, and not with the Loser style of life. This implied along with the other connections shown in Table 5.4, that social marginalization indicated by the Loser style of life was not associated with external living conditions but rather with parent-centered parenting and lower socioeconomic status. The Reveller style of life, in turn, was associated with parent-centered parenting and unsteady external conditions created by a younger parent (or parents), which when combined did not promote norm-oriented behavior.

Likewise, steady conditions at age 14 correlated with the Loner style of life in late adolescence and not with the Thriver style of life. The Thriver style of life was promoted by child-centered parenting and higher socioeconomic status, whereas the Loner style of life was associated with child-centered parenting and steady conditions created by older parents. Possibly very steady conditions are not optimal for the development of the resilience of the Thrivers but that some changes in life are good for the child's development as Bronfenbrenner (1979, pp. 103-4) has argued. A discriminant analysis with highly aggressive, anxious, constructive, and compliant children at age 14 (Hurme 1976; Pitkänen-Pulkkinen, 1980a) confirmed that parents had encouraged free-time activities by constructive children more than the parents of the compliant children.

Case studies. As the parenting and living conditions were studied only at age 14, it was not possible to analyze the stability of the developmental context across years. Some information about the effects of changes on children was received from the six individuals in whom the lifestyle was the opposite of the style expected (Pulkkinen, 1982). In the case of a very aggressive 8 -year-old boy, who had become a Loner by the age of 20, the parents' increased awareness of the need to supervise the child's behavior broke the developmental line toward the Reveller style of life. The participant developed poor health that strengthened his dependence upon his parents. In another case in which a very compliant 8-year-old girl became a Reveller by the age of 20 (instead of a Loner), the relationship between the mother and daughter became worse due to the mother's new and demanding role as an employer. 
The mother was preoccupied with her work and hobbies, while the daughter spent her time with her peers and started smoking and drinking.

In the cases of two girls whose development progressed from constructive behavior to the Loser style of life (instead of the Thrivers), the families encountered a traumatic experience (i.e., the father dying or having a bad accident) that affected the whole family financially and emotionally. The daughters did not attend a vocational college after their compulsory schooling, and were vulnerable to unemployment. In two other cases in which anxious behavior progressed toward the Thriver style of life, the families with many children lived in very cramped homes when the children were young. In a small apartment where a parent needed to sleep during the day after working a night shift, the children's life was very limited and the atmosphere was strained. The conditions improved later when the parent had to stop working shifts due to ill-health and the family atmosphere improved.

Conclusions regarding question 6. The children's upbringing and living conditions were associated with the unfolding of the styles of life. Child-centered parenting with the parents' higher socioeconomic status promoted high self-regulation toward the Thriver style of life, and with steady living conditions toward the Loner style of life. On the contrary, parent-centered parenting with low socioeconomic status promoted low self-regulation toward the Loser style of life, and with unsteady conditions toward the Reveller style of life.

\subsubsection{A person-oriented approach to personal styles at age 27}

During the course of the JYLS, I have tried to describe adult personality holistically in different ways instead of using single personality traits. The analysis of the styles of life previously described represents a so-called variable-oriented approach. Correlation coefficients, factor analyses, and factor scores were used to find connections between the variables. Another approach, called person-oriented, was used to categorize individuals into groups based on their similarity with regard to selected variables. A person-oriented approach was introduced by Block (1971) in his analysis of personality types, and Magnusson (1998) has strongly advocated the use of this approach.

A hierarchical clustering technique applied to the JYLS data collected at age 27 (Pulkkinen, 1996a) limits the number of variables that can be included in a cluster analysis. Therefore, the rich data collected had to be reduced to a manageable number of variables. Three components of human action: affective, cognitive, and behavioral, were considered. Within each component, the number of variables was reduced using a factor analysis: four composites for personality characteristics; three for life orientations; and five for behavioral activities. The first two clusters divided men and women to those who had difficulties in adapting to life (conflicted adaptation: 25 percent of the women and 22 percent of the men) and to those who were positively adapted. It further divided into two clusters when the hierarchical cluster analysis was continued (Figure 5.2). 


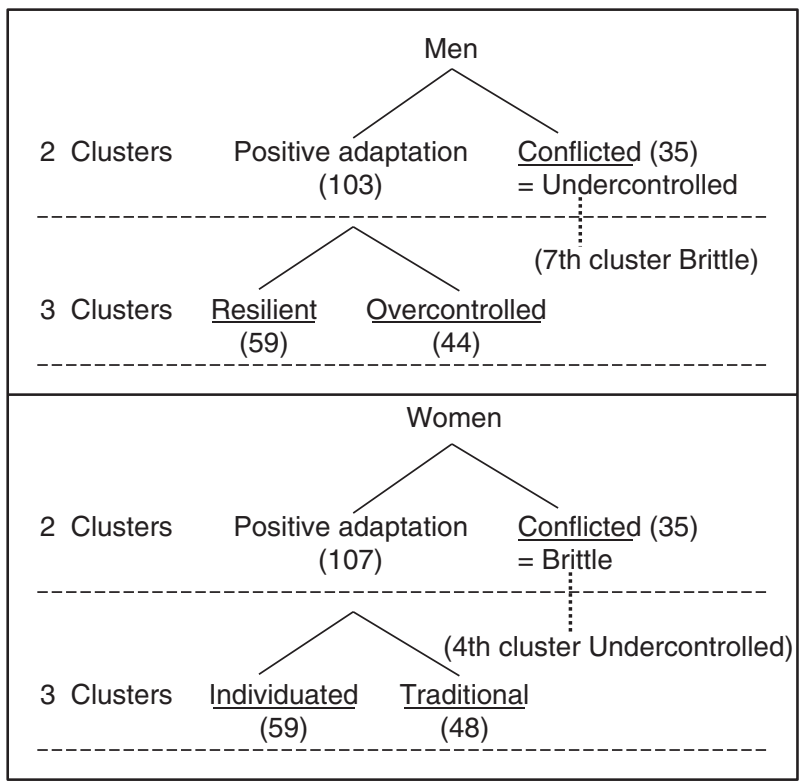

FIGURE 5.2 Three clusters of personal styles for men and women at age 27.

Source: Pulkkinen, L. (1996a). Female and male personality styles: A typological and developmental analysis. Journal of Personality and Social Psychology, 70, 1288-306. (C) American Psychological Association. Adapted with permission.

Note: The label 'Overcontrolled' for men was entitled 'Introvert' in Pulkkinen (1996a). The label 'Brittle' for men was entitled 'Neurotics' in Pulkkinen (1996a). The label 'Traditional' for women was entitled 'Feminine' in Pulkkinen (1996a).

Clusters for men. The male Conflicted cluster differed from the other male clusters particularly in the following features: higher sensation seeking, impulsive and risk taking behavior; higher neuroticism; lower social integration including an unstable work history and criminal arrests; and more negative life attitudes; heavier drinking and sedentary leisure time; and identity exploration. The conflicted cluster was specified for males who were labeled as Undercontrolled.

The two clusters that showed positive adaptation in men differed in extraversion. The extraverted men were low in neuroticism, had positive life attitudes, and were family-oriented and well-integrated into work life and society in general. This cluster was labeled as Resilient. The introverted men were low in sensation seeking and impulsivity but high in agreeableness and social integration. This cluster was labeled as Overcontrolled.

Clusters for women. In women, the cluster for conflicted adaptation was very high in neuroticism and low in extraversion. Other characteristics of this cluster when compared with the other female clusters were more negative life attitudes, heavier drinking and sedentary leisure time, lower levels of reflectiveness, lower social integration, and fewer intellectual pursuits. The conflicted cluster was specified for females by the label Brittle. 
The two clusters for Positive adaptation obtained in women were lower in neuroticism and heavy drinking, and higher in extraversion than the Brittles. They did not differ from each other in extraversion as did the corresponding male clusters, but they did differ in intellectual interests, family life, nonconscientiousness, contentment, and family life. One of these clusters was characterized by high intellectual interests and low family orientation. Compared with the other women, the cluster also differed in higher sensation seeking and impulsivity and exploration of identity. The cluster was labeled as Individuated.

Women in the other cluster for positive adaptation were characterized by being occupied with family life and were content with their present achievements and did not have developmental goals. They were very low in impulsivity, risk taking and sensation seeking, and had few intellectual interests. The cluster was labeled as Traditional. The terms "Individuated" and "Traditional" women were used by York and John (1992), who also used the label "Conflicted."

When the clustering was continued until seven clusters had formed, more specific clusters emerged (Pulkkinen, 1996a). The cluster for Conflicted adaptation divided into two clusters for women, when the Undercontrolled type separated from the Brittle (at the level of four clusters). In the men, the Brittle differentiated from the Undercontrolled at the level of seven clusters. There was correspondence between the seven clusters extracted in the JYLS and the types extracted by Block (1971) with the US sample born in the 1920s, as compared by Pulkkinen (1996a).

Conclusions regarding question 7 . A person-oriented approach conducted with a clustering technique differentiated participants along the dimension of self-regulation, adapted, and conflicted (cf. Figure 5.1), and resulted in categories that shared characteristics of personality styles presented in Section 5.3.2, particularly for males. Low self-regulation characterized the clusters for conflicted adaptation interpreted as Undercontrolled for men and Brittle for women. High self-regulation characterized the clusters for positive adaptation to life: the Resilient and Overcontrolled men, and the Individuated and Traditional women. Positive adaptation in women was associated with female roles (career- or home-orientation). The role-based adaptation did not come out in the variable-oriented analysis in which both men and women were included.

\subsubsection{Personality profiles at ages 33 to 50}

It is common to describe an individual's personality by using trait names (e.g., a person is extraverted), but in reality, individuals have simultaneously several personality traits that possibly function together, rather than separately. Less is known about their common functions, although a person-oriented approach (clustering technique) has also been applied to personality traits (e.g., Donellan \& Robins, 2010). In the JYLS, a further question was explored, which was whether individuals have the same personality profile across adulthood (Kinnunen et al., 2012). The analysis of personality profiles was based on the big 5 personality traits assessed at ages 33, 42, and 50 using the 60-item NEO-Five Factor Inventory 
(NEO-FFI; see Chapter 2). The five factors and their subtraits are as follows (McCrae \& Costa, 2003):

- Neuroticism (anxiety, hostility, depression, impulsivity, vulnerability)

- Extraversion (warmth, excitement-seeking, positive emotions)

- Openness to experience (fantasy, aesthetics, feelings, ideas, values)

- Conscientiousness (competence, order, dutifulness, achievement striving, selfdiscipline, deliberation)

- Agreeableness (trust, straight-forwardness, compliance, modesty, tendermindedness).

Personality profiles were analyzed by using a latent profile analysis (LPA) (Kinnunen et al., 2012). This technique provides new and more exact opportunities for defining personality profiles and comparing them across ages than a cluster analysis. The aim of the latent profile analysis was to examine whether there were homogeneous subgroups of individuals with distinctive profiles in the Big Five personality traits, and to compare the continuity of these profiles across the ages of 33,42 , and 50 . The measurements of the personality traits at each age were included in the LPA models. The estimation was performed step by step starting from one class solution and continuing to estimate the parameters for $\mathrm{k}$ class solution until statistical criteria were met for the sufficient number of classes. Based on statistical criteria, the most parsimonious solution with five classes, that is, five personality profiles were chosen.

The personality profiles were different (see Figure 5.3), and the shapes of the profiles remained very similar across the ages of 33, 42, and 50 (Kinnunen et al., 2012). The first profile class was low in neuroticism and high, in comparison to the other profiles, in extraversion and conscientiousness. It was interpreted as the Resilient profile. The second profile class was opposite to the first profile. It was high in neuroticism and low in extraversion, and lower than average in openness, conscientiousness, and agreeableness. It is relabeled here as the Brittle profile. The third profile class was also low in extraversion and openness, but it differed from the second profile in being lower in neuroticism and higher in conscientiousness and agreeableness. The profile depicts nonneurotic constrained behavior. It was relabeled here as the Overcontolled profile. The fourth profile class was opposite to the third profile; it was high in openness and extraversion and low in conscientiousness. It was interpreted as the Undercontrolled profile. The fifth profile class was characterized by a zero profile in all personality traits, that is, the mean scores in all personality traits. It is interpreted as the Ordinary profile. The reason for relabeling the Brittle and Overcontrolled profiles was related (as explained in Section 4.2.3) to the observation of the hazardous use of the label of Overcontrolled in the literature (and also in our article) and the lack of the use of the Brittle label.

The Ordinary profile was the largest profile; this profile group consisted of 44 percent of the participants. The second largest profile group was the Resilient profile (21 percent). The Undercontrolled and Brittle profile groups were of the same 


4.5 Resilient $\quad$ Brittle Overcontrolled Undercontrolled $\quad$ Ordinary

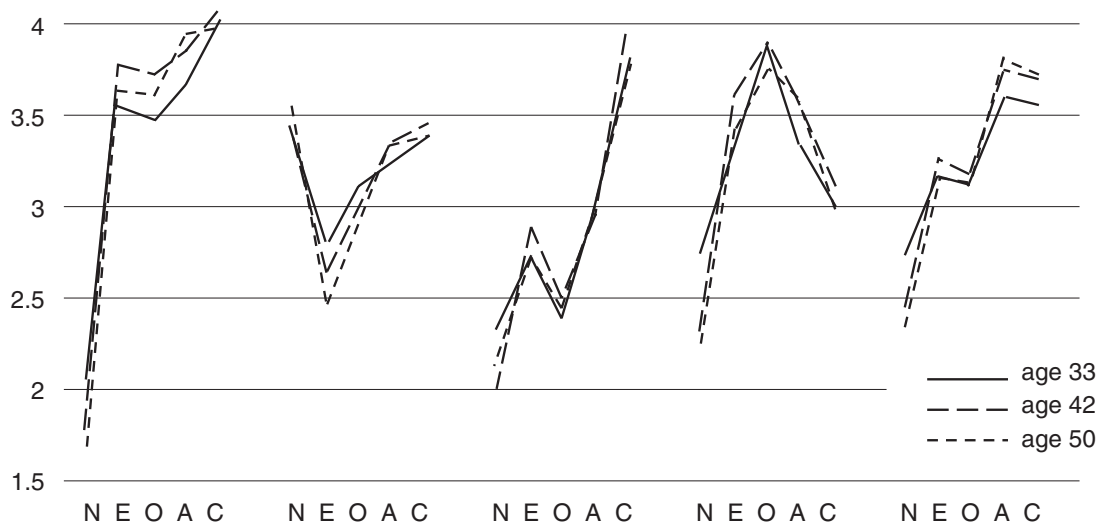

FIGURE 5.3 Personality profiles characterized by personality traits at ages 33,42 , and 50 . $\mathrm{N}=$ neuroticism, $\mathrm{E}=$ extraversion, $\mathrm{O}=$ openness, $\mathrm{A}=$ agreeableness, $\mathrm{C}=$ conscientiousness

Source: Adapted from Kinnunen, M.-L., Metsäpelto, R., Feldt, T., Kokko, K., Tolvanen, A., Kinnunen, U., Leppänen, E., \& Pulkkinen, L. (2012). Personality profiles and health: Longitudinal evidence among Finnish adults. Scandinavian Journal of Psychology, 53, 512-22. Figure 1 (p. 517). (C) 2012 The Authors. Publisher: Blackwell Publishing Ltd. Adapted with permission.

Note: The label 'Brittle' was entitled 'Overcontrolled' in Kinnunen et al. (2012). The label 'Overcontrolled' was entitled as 'Reserved' in Kinnunen et al. (2012).

size (14 percent and 13 percent, respectively); and the smallest profile group was the Overcontrolled profile (8 percent). Male and female participants were distributed equally across the Ordinary, Resilient, and Brittle profiles. In the Undercontrolled profile, women were overrepresented, and in the Overcontrolled profile, men were overrepresented.

A framework for the differences between the profiles. In order to invent a framework in which the relationships between the personality profiles could be described, I analyzed differences and similarities between the profile groups in terms of their personality traits. This work led to the insight that the trait dimensions that differentiated the five personality profiles across ages 33, 42, and 50 in the most unambiguous way were extraversion and conscientiousness (Figure 5.4). When the means of the profiles (averaged across the three ages) were compared with the means of the whole sample in extraversion and conscientiousness, the Resilient profile was found to be high (i.e., above the means of the sample) in both extraversion and conscientiousness, and the Brittle profile was low (i.e., below the means) in both of them. The Undercontrolled profile was found to be higher than the sample mean in extraversion but lower in conscientiousness, whereas the Overcontrolled profile was lower in extraversion but higher in conscientiousness. For the other personality traits, the Brittle profile was highest in neuroticism, and the Undercontrolled profile was highest in openness. Furthermore, both the Resilient and Overcontrolled profiles were higher in agreeableness than the Brittle and Undercontrolled profiles. 


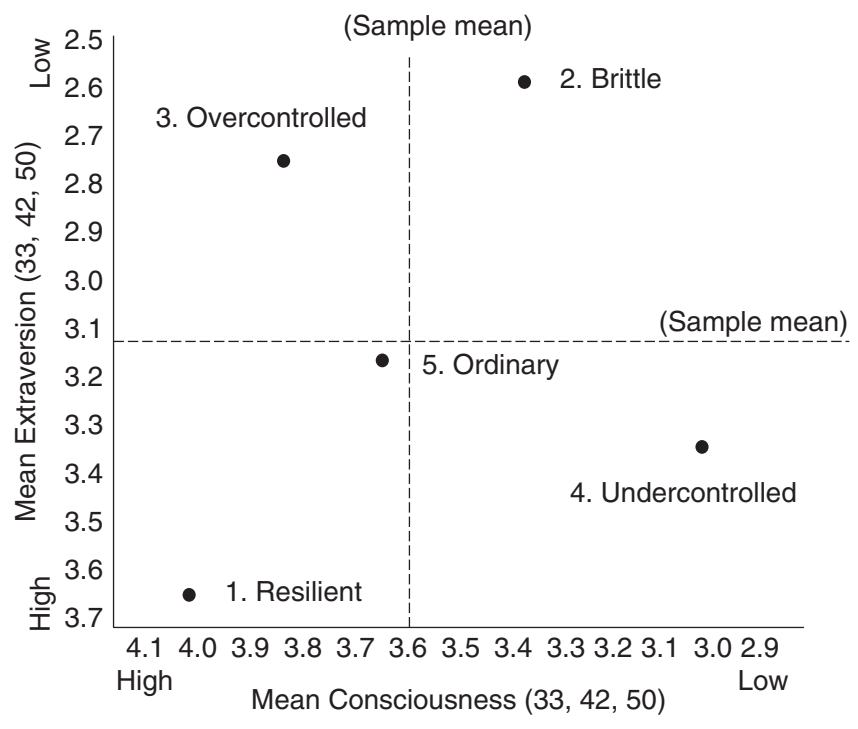

FIGURE 5.4 Personality profile groups located on a plane on the basis of their means in extraversion and conscientiousness (across ages 33, 42, and 50).

The validity of the framework presented in Figure 5.4 was demonstrated by comparing the clusters for personal styles obtained at age 27 (Section 5.3.4) in the Big Five personality traits at ages 33 and 42 (Pulkkinen, Feldt, \& Kokko, 2005). Compared with the other men, the men with the Resilient personal style were higher in extraversion and conscientiousness; the men with the Undercontrolled personal style were higher in extraversion but lower in conscientiousness; and the men with the Overcontrolled personal style were lower in extraversion but higher in conscientiousness. Thus, the male clusters for personal styles located on the plane (Figure 5.4) in the same way as the personality profiles although personal styles were extracted from very different data sets, not including the Big Five personality traits (Pulkkinen, 1982). For females, personal styles could not be described so clearly in the framework of Figure 5.4. Women's personal styles were associated with role behaviors more highly than with personality traits.

An idiographic approach to personality. The person-centered approach to personality was further complemented by an approach known as idiographic in contrast to nomothetic (Allport, 1937). The latter refers to the usual measure of personality traits on certain scales, whereas "idiographic" refers to individuals' descriptions of themselves in their own terms. Allport argued that individuals are unique, although they have traits with common aspects, and that typically there are five to ten central traits that people use when they are asked to describe themselves. To explore how the participants would describe themselves we asked them the following questions in the interview at age 36: What are your typical characteristics? The number of responses varied from 0 to 10 with the mean number being 4.9 for women and 
4.4 for men (Pulkkinen, Männikkö, \& Nurmi, 2000). In the content analysis of responses, they were categorized into the Big Five personality traits.

The most frequently mentioned descriptors concerned characteristics related to agreeableness, such as being honest and reliable, and they were the same for women and men. Gender specific positive and negative characteristics were, for instance, compliant and being mean for women, and generous and being domineering for men. Only one-fifth of responses that were categorized as agreeableness were negative. The same ratio applied to the other factors except for emotional stability/neuroticism for which negative descriptors exceeded positive descriptors. The use of positive descriptors correlated with high self-esteem in both genders. Self-descriptors correlated more highly with the test scores of personality traits (NEO-FFI) in men than in women, and correspondingly, the personality profiles extracted from self-descriptors compared with those extracted from the NEO-FFI scores were more similar in men than in women (Pulkkinen et al, 2000).

Conclusions regarding question 8 . The profile analysis indicated that personality traits are combined in five stable ways across the ages, and these were labeled as Resilient, Overcontrolled, Undercontrolled, Brittle, and Ordinary. Differences between the profiles can be described on the basis of the amount of extraversion and conscientiousness involved in each profile. The framework also applies to the description of the differences between male personal styles. Self-descriptions of participants' personality mostly consisted of positive descriptors concerning agreeableness.

\subsection{Maturation, adjustment, or growth in adult personality}

The average changes in personality traits in adulthood are often presented by stating that people become more conscientious, agreeable, and emotionally stable (less neurotic) with age. These changes have been interpreted as following a maturity principle (Caspi, Roberts, \& Shiner, 2005). McAdams (2015) describes maturational growth toward greater self-regulation and efficacy in social and emotional performance. Staudinger and Kunzman (2005) use the concept of adjustment (instead of maturation) to refer to positive development in an individual's adaptation to societal circumstances such as rules, norms, and societal expectations. They separate adjustment from growth, which refers to positive development toward ideal endpoints such as integrity and wisdom. Adjustment and growth are not independent of each other; a certain level of adjustment is necessary for growth, and adjustment is expected to contribute to the smooth running of everyday life, maintaining wellbeing, increasing life success, and even longevity.

Methodological remarks. Data on continuity in personality characteristics (personality traits, NEO-FFI at ages 33, 42, 50); vulnerability traits (Karolinska Scales of Personality [KSP] at ages 36, 42, 50); and socioemotional behaviors (selfratings at ages 36, 42, and 50) were analyzed (list-wise) by Kokko for this book with the participants who had responded to this measure three times, that is, at each age, to see whether continuity or discontinuity in personality occurs in 
the same individuals. Although the participation rate was high in the JYLS, the participants in different data collections were not always exactly the same group of people. The means of the scales of the NEO-FFI presented here, and in some previously published works, are slightly different, because the formation of age groups has been made on a different basis, such as by using the maximum number of participants at each age (e.g., Kokko, Tolvanen, \& Pulkkinen, 2013). The participation in all measurements was associated with higher agreeableness but not with other traits.

In the comparison of the means of traits between the ages, a repeated measures ANOVA method was used separately for men, women, and all of the participants. In the analysis of relative stability, Product moment correlations were calculated. They are lower estimations of relative stability than coefficients, based on the Structural Equation Modeling with latent factors and item parcels for each trait, as presented in the articles by Kokko, Rantanen, and Pulkkinen (2015), Rantanen, Metsäpelto, Feldt, Pulkkinen, and Kokko (2007), and Rantanen, Tillemann, Metsäpelto, Kokko, and Pulkkinen (2014).

Personality traits: absolute stability. The means of the personality traits are presented in Table 5.6. In the comparison of the means, the criterion for a significant change was kept high $(\mathrm{p}<0.01)$ to avoid the over-interpretation of possibly random differences. Comparisons at each age showed that women and men did not differ in neuroticism and extraversion, but women were higher than men in agreeableness at all ages, in openness at ages 33 and 42, and in conscientiousness at age 42 .

The stability of the means of the traits over time was studied for both genders and all participants, and in addition for each personality profile group. The latter analyses were carried out for an unpublished conference paper (Pulkkinen, Räikkönen, Kinnunen, \& Kokko, 2013). The results of the comparisons of the means are summarized in Box 5.2.

Changes in the means of the traits indicated that, on average, self-regulation increased in adulthood: neuroticism decreased and agreeableness increased. However, differences in self-regulation became more accentuated between the adults because the increase of self-regulation did not take place in the profile groups that were lowest in self-regulation. Increasing age did not seem to compensate for a characteristic that had a low profile in an individual. More specifically:

1. Neuroticism decreased in the men and women from age 33 to 42 and remained thereafter on that level. The decrease was found in all profile groups except in the Brittle who were most neurotic.

2. Agreeableness increased in the men from age 33 to 42 , and in the women from age 33 to 50 . The increase was found in all profile groups except the Brittle and Undercontrolled who were least agreeable.

3. Individual differences increased in extraversion that increased in the Undercontrolled profile (which was already high in extraversion) and decreased in the Brittle profile (which was already low in extraversion). 


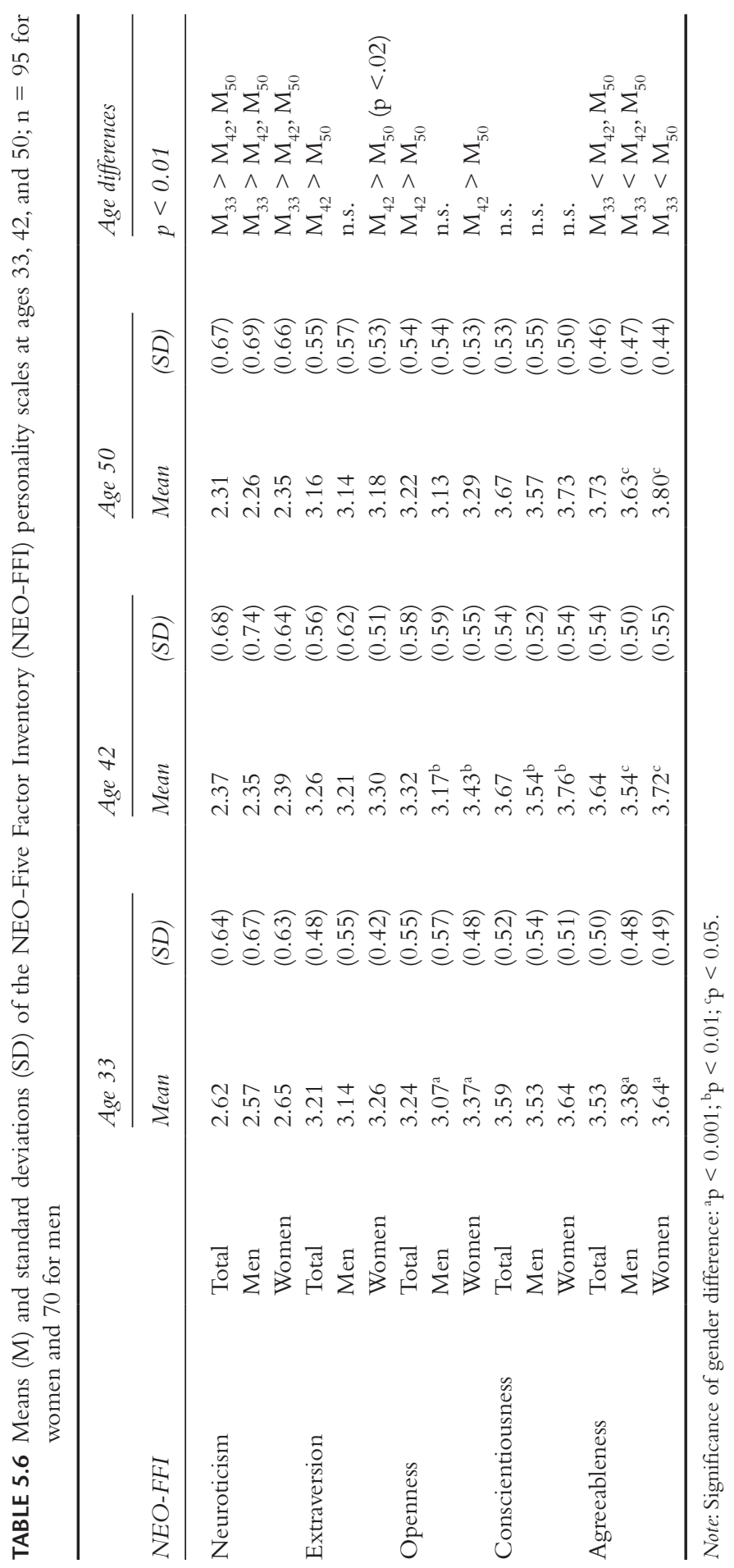




\section{BOX 5.2 CHANGES IN THE BIG FIVE PERSONALITY TRAITS IN MEN AND WOMEN, AND IN THE PERSONALITY PROFILE GROUPS FROM AGE 33 TO 50}

\section{- Neuroticism}

Men and women: neuroticism decreased from age 33 to 42

Profile groups:

Resilient, Overcontrolled, Ordinary, and Undercontrolled: neuroticism decreased from age 33 to 42

Brittle (the most neurotic): neuroticism did not decrease

\section{- Agreeableness}

Men: agreeableness increased from age 33 to 42 ; women: from age 33 to 50

Profile groups:

Resilient: agreeableness increased from age 33 to 50

Overcontrolled: agreeableness increased from age 42 to 50

Ordinary: agreeableness increased from age 33 to 42

Brittle and Undercontrolled (the least agreeable): agreeableness did not increase

\section{- Extraversion}

Women: extraversion decreased from age 42 to 50

Profile groups:

Undercontrolled: extraversion increased from age 33 to 42

Brittle: extraversion decreased from age 33 to 50

Other profiles: no change

- Openness to experience

Women: openness to experience decreased from age 42 to 50

Profile groups:

Resilient: openness increased from age 33 to 42 and decreased from age 42 to 50

Other profiles: no change

\section{- Conscientiousness}

Men and women: conscientiousness did not change

Profile groups:

Ordinary: conscientiousness increased from age 33 to 42

Other profiles: no change 
An increasing conscientiousness is one of the assumed components of the maturity principle together with an increasing agreeableness and a decreasing neuroticism (Caspi et al., 2005), but the JYLS results did not show an average age-related increase in conscientiousness. Conscientiousness did increase in the large Ordinary category, but not in the other profile groups. Another difference to what is generally believed in terms of developmental trends was that openness to experience did not increase with age. Rather, average openness decreased in women in middle age. An increase in openness was found in the Resilient profile group from age 33 to 42 , but thereafter, the change declined. In the other profile groups, significant changes did not occur. These findings concur with those by Roberts, Walton, and Viechtbauer (2006): in middle age, openness remains at the same level or decreases. Openness to experience has theoretically been connected with personality growth (Staudinger \& Kunzmann, 2005). In the JYLS data, Kokko and Shehadeh (in press) found that individuals who were high in openness to experience from age 42 to 50 were higher in emotional and social well-being, wisdom, and spirituality. They were also higher in occupational status than individuals who were lower in openness. The authors concluded that personal growth may concern a small group of individuals, and possibly become more visible at an older age.

Personality traits: relative stability. High relative stability in personality traits was found from age 42 to 50, which means, for example, that extraverted individuals as measured at one point in time were also extraverted at another point in time when compared with other individuals. This finding concerned all personality traits among men and women. The correlations varied between 0.70 and 0.80 . The high relative stability from age 42 to 50 was in line with the statement by Caspi et al. (2005), but high stability was reached later in women than in men in neuroticism and extraversion. At an earlier age, relative stability was therefore higher among men than among women in these traits. The results concerning the higher stability of neuroticism and extraversion among men than among women from age 33 to 42 (Rantanen et al., 2007), and the levelling off at this difference after age 42 (Kokko et al., 2015) were also obtained by using structural equation modeling of the JYLS data.

Vulnerability traits. Vulnerability traits were assessed by using the KSP at ages 36, 42, and 50. The KSP includes 15 scales that are designed to highlight certain vulnerability factors, which might help to identify individuals at risk and aid our understanding of the interactions between biological dispositions and situational factors. The scales can be grouped into four broader classes: (1) extraversion-related scales: impulsiveness, monotony avoidance, and detachment; (2) anxiety-related scales: psychic anxiety, somatic anxiety, muscular tension, inhibition of aggression, and psychasthenia; (3) aggression-related scales: verbal aggression, indirect aggression, irritability, suspicion and guilt; and (4) conformity-nonconformity scales: social desirability and socialization (af Klinteberg, 1996).

Comparisons of the means of the scales at different ages showed high absolute stability for men and women. Age related-changes in the means of the scores were found only in one scale (out of 15) for men and in four scales for women. In men 
and women, monotony avoidance decreased; gradually in women from age 36 to 50 and from age 42 to 50 in men. It means that the participants felt less of a need for external stimulation and change when they grew older. The other changes for women were an increase in sociability (less detachment) from age 36 to 50 and a decrease in indirect aggression and irritability from age 42 to 50.

The average scores of personality characteristics were very similar in men and women in middle age. The only gender difference at age 50 (among 28 comparisons) was that women scored higher than men in sociability (lower detachment). More gender differences existed in earlier adulthood, but they all levelled off apart from sociability. The relative stability of the KSP scales from age 36 to 42 and from age 42 to 50 was high for males: $\sim 0.85$ for extraversion and anxiety, and $\sim 0.78$ for aggression and conformity. More variety was found in the stability coefficients for women; the average level was $\sim 0.73$.

Socioemotional behavior. A third measure consisted of 8 self-ratings of socioemotional behavior (aggression, anxiety, constructiveness, compliance, emotional stability, emotional lability, activity, and passivity; the items are listed in Box 2.1) at ages 36,42 , and 50 . It was found that the stability of means was also high in selfratings from age 36 to 50 . For the whole group, there were only two significant differences. First, anxiety decreased from age 36 to 42, which concurs with the decrease of neuroticism, and second, emotional stability increased from age 36 to 50. The latter was due to the changes experienced by women; among men there was no change.

Conclusions regarding question 9. The absolute and relative stability of personality characteristics was high in adulthood. Significant changes indicated the average increase of self-regulation (increasing agreeableness and decreasing neuroticism and anxiety), as proposed by McAdams (2015). These changes improved emotional and social performance and were in accordance with results in other studies (e.g., Denissen, van Aken, Penke, \& Wood (2013). The contribution of the JYLS to the literature was to show the increasing individual differences in self-regulation, because self-regulation did not increase in the personality profile groups that were lowest in emotion regulation (the Brittle) and behavior regulation (the Undercontrolled). The constructs of maturity and growth in personality involve broad processes that cannot solely be described by personality traits.

\subsection{Childhood socioemotional behaviors as predictors of adult personality characteristics}

\subsubsection{Roots of the personality profiles and the clusters of personal styles}

Participants in different personality profile groups in adulthood had differed in socioemotional behaviors in their youth in the ways that fitted the characteristics of the profiles. This was found in the comparison of the personality profiles in socioemotional behavior rated by teachers at age 14 (Pulkkinen et al., 2013; cf. Figure 5.4): 
- The most extraverted profile groups, Resilient and Undercontrolled, and the Ordinary profile group had been higher in social activity than the Brittle profile group.

- The most neurotic Brittle profile group had been higher in social anxiety than the Resilient and Ordinary profile groups.

- The most conscientious profile groups, Resilient and Overcontrolled, and the Ordinary profile group had been higher in emotional stability than the Undercontrolled profile group.

- The least conscientious profile groups, the Undercontrolled and Brittle individuals, had been emotionally the most labile.

Thus, adult personality profiles had their roots in adolescent behaviors. It is to be noted that teacher ratings at age 8 did not predict individuals' personality profiles, but only the teacher ratings completed at age 14 differentiated them. It suggests that the structure of personality develops during the school years and becomes more predictive of adult personality profiles.

For personal styles, it was found that the participant who had been high in self-regulation in childhood were likely to have a personal style at age 27 , which indicated positive adaptation, whereas low self-regulation in childhood predicted conflicted adaptation (Pulkkinen, 1998). However, two-thirds of the boys and girls who had belonged to the low self-regulation clusters in childhood were positively adapted in adulthood; only one-third of them displayed conflicted adaptation. It indicates general positive development. Only the boys who had been scored in the highest quartiles for aggressiveness and the girls who had been scored in the highest quartiles for anxiety were at high risk of experiencing troubled adaptation in adulthood.

\subsubsection{The roots of adult agreeableness and continuity in aggression}

Connections between childhood socioemotional behavior and adult characteristics were studied for agreeableness and aggression. The roots of adult agreeableness were searched for in childhood data, whereas for aggression, continuity from childhood to adulthood was investigated.

Roots of agreeableness. Agreeableness is a personality trait that includes trust, straight-forwardness, altruism, compliance, modesty, tender-mindedness, and low hostility (McCrae \& Costa, 2003). The roots of adult agreeableness were searched for using configural frequency analysis and their background with discriminant analysis (Laursen, Pulkkinen, \& Adams, 2002). Socioemotional behaviors at age 8 (activity, aggression, anxiety, compliance, constructiveness, and emotional stability assessed through teacher ratings and peer nominations) and adulthood personality variables at age 36 (impulsivity, inhibition of aggression, and socialization assessed by the KSP) were included in this analysis.

Two major types of adult participants in respect to agreeableness were found: (1) highly agreeable adults who were well-socialized into society; and (2) less 
agreeable adults who were more impulsive and less socialized. Continuity from childhood to adulthood in these behavior patterns was significant. They had different developmental backgrounds. Highly agreeable adults had been emotionally more stable and compliant as children and were more rarely aggressive than the adults who were less agreeable. Highly agreeable individuals functioned more positively both in childhood and adulthood.

Less agreeable adults had been less stable and compliant and more aggressive in childhood. They had been more disobedient toward their teacher, and they had had more concentration problems than highly agreeable adults. Less agreeable men had poorer school success and displayed more problem behaviors than highly agreeable men. In adulthood, less agreeable individuals experienced more problem drinking, more criminal arrests, a more unstable career path, and more depressive symptoms than the highly agreeable adults.

The results indicated that children who are compliant and restrained become involved in positive interactions, whereas problems in behavior regulation increase the risk of problem behaviors. Single characteristics did not make an individual's personality; rather, personality consists of combinations of several characteristics, and when these patterns are considered, the predictability of behavior increases over time (Laursen et al., 2002).

Continuity in aggression. It has been generally believed that there is continuity in aggression from childhood to adulthood. A review by Olweus (1979) is often quoted in this context. He found stability in aggressive behavior while reviewing 16 studies conducted with males, and drew a conclusion that there was considerable longitudinal consistency in male aggressive behavior from childhood to early adulthood, comparable to the stability of intelligence. Long-term longitudinal results on continuity in aggression were not available in the 1970s. In the 2000s, the JYLS and the Columbia County Longitudinal Study (CCLS; USA) offered an exceptional opportunity for the parallel testing of continuity in aggression within long-term longitudinal studies. Due to the harmonization of the measures, there were several identical items on aggression included in the peer nomination used for collecting baseline data in these two studies (in 1961 for the CCLS, and in 1968 for the JYLS), as explained in Section 2.2.

In the first comparative study, a general peer-nominated aggression score covering different types of aggression at age 8 was computed in both samples (Kokko, Pulkkinen, Huesmann, Dubow, \& Pulkkinen, 2009). In adolescence, peer-nominated aggression at age 14 was used in the JYLS, and peer-nominated aggression at ages 18 to 19 in the CCLS. At ages 36 and 42, the items of the aggression inventory were divided into three subscales: physical aggression (e.g., "Given enough provocation, I may hit another person"), verbal aggression (e.g., "When people annoy me, I may tell them what I think of them"), and low selfcontrol of anger (e.g., "I have trouble controlling my temper"). In the CCLS, aggression scores were derived at ages 30 and 48 from self-ratings of serious physical aggression and verbal aggression, and in addition at age 48, from selfratings of lack of self-control. 
In both samples, aggression in childhood significantly predicted aggression in adolescence for males and females, and aggression in adolescence significantly predicted physical aggression in adulthood. These connections were higher in the US sample than in the Finnish sample. In neither sample did aggression at age 8 predict adult aggression directly. Adult aggression was predicted only through the level of aggression in adolescence. This means that childhood aggression only predicted aggression in early and late adolescence (Kokko et al., 2009). The explained variance of adult aggression was higher in the US sample (49 percent for males and 24 percent for females) than in the Finnish sample (12 percent for males and 8 percent for females).

The results indicated significant country differences. In the second study (Kokko et al., 2014), the country differences were re-examined by adding another US sample, Child Development Project (CDP; Dodge, Bates, \& Pettit, 1990) to the comparison. Corresponding items of aggression at age 8 were selected carefully for the predictors, and early adult aggression (at ages 21 to 30) was assessed by variables that described actual physical aggression committed by the participant. Adolescent aggression was not included in the model.

Correlations showed that there was continuity for both genders from general aggression at age 8 to physical aggression in early adulthood in both US samples but not in the Finnish sample (Table 5.7). The significance of continuity in aggression was also tested with SEM analyses (Kokko et al., 2014). The results indicated, first, that there was equal and significant continuity of aggression in the two US samples for participants who were from nonprofessional family backgrounds, but not in the corresponding Finnish sample. Second, there was no significant continuity of aggression in participants from either country who came from a professional family background.

It can be concluded that the continuity in aggression was only evident in the US children whose family occupational status was nonprofessional, including blue-collar unskilled and skilled workers. Socioeconomic status did not moderate the continuity of aggression from childhood to middle age in the Finnish sample, where continuity from general aggression in childhood to actual physical aggression in early adulthood (at age 27) did not exist in children from a family where a parent had a nonprofessional or a professional occupational status.

The similarity of the findings between the two American samples was remarkable because the participants represented different age cohorts, one born in the 1950s (CCLS) and the other in the 1980s (CDP), they were located in different parts of the country, and they had been assessed by different study teams with different methods. A possible explanation for the differences between the USA and Finland is that the school environment functions as a moderator (Kokko et al., 2014). In Finland, schools are less different with regard to the socioeconomic backgrounds of the children's families than in the USA. The Finnish housing policy encourages equality between residential areas, and the residential area in which the family is based is the major factor determining the child's school in Finland. Furthermore, different subcultures of behavioral norms are almost 
TABLE 5.7 Correlations between age 8 peer-nominated general childhood aggression and adult self-reported physical aggression by study and stratified by childhood parent occupational status and sex for the JYLS, Columbia County Longitudinal Study, USA (CCLS), and Child Development Project, USA (CDP)

\begin{tabular}{|c|c|c|c|c|c|c|c|}
\hline & & \multicolumn{2}{|l|}{$J Y L S$} & \multicolumn{2}{|l|}{ CCLS } & \multicolumn{2}{|l|}{$C D P$} \\
\hline & & $r$ & $N$ & $r$ & $N$ & $r$ & $N$ \\
\hline \multicolumn{2}{|l|}{ Full sample } & 0.02 & 292 & $0.20^{\star \star \star}$ & 391 & $0.22^{\star \star \star}$ & 385 \\
\hline \multirow[t]{2}{*}{ Correlations by sex } & Males & 0.07 & 150 & $0.24^{\star \star \star}$ & 192 & $0.21^{\star \star}$ & 193 \\
\hline & Females & -0.05 & 142 & $0.12^{\dagger}$ & 199 & $0.17^{\star}$ & 192 \\
\hline \multirow{2}{*}{$\begin{array}{c}\text { Correlations by parent } \\
\text { occupational status }\end{array}$} & Nonprofessionals & -0.02 & 203 & $0.23^{\star \star \star}$ & 242 & $0.28^{\star \star \star}$ & 275 \\
\hline & Professionals & 0.09 & 89 & 0.16 & 98 & -0.04 & 119 \\
\hline
\end{tabular}

From Kokko et al. (2014). Country, sex, and parent occupational status: Moderators of the continuity of aggression from childhood to adulthood. Aggressive Behavior, 40, 553-67. (Table III, p. 561) (C) 2014 Wiley Periodicals, Inc. Reproduced by permission.

Note: Both aggression measures were natural log-transformed for this analysis.

${ }^{\star \star *} \mathrm{p}<0.001 ;{ }^{\star \star} \mathrm{p}<0.01 ;{ }^{\star} \mathrm{p}<0.05 ;{ }^{\dagger} \mathrm{p}<0.10$.

nonexistent in Finland, whereas in countries with high inequality of income, their existence is common (Wilkinson \& Pickett, 2010). This may cause differences between schools. Educational possibilities across socioeconomic status groups are more equal in Finland than in the USA, because college and university education is free and supported by the state in Finland. Income inequality is associated with social problems, and the association is higher in the USA than in Finland (Wilkinson, 2011).

Differences in the continuity of aggression between the two studies (Kokko et al., 2009, 2014) are explained by the inclusion of adolescent aggression in the model in the first study and by the exclusion of it from the second study. Additional analyses have shown that continuity of childhood aggression to adulthood only appeared in males who were highly aggressive (above 75th percentile) both at ages 8 and 14; continuity did not exist in females. Being above the 75th percentile in aggression at age 8 but below it at age 14 did not predict adult aggression (Pulkkinen, 2017). Collecting adolescent data at a later age in the CCLS (at ages 18 to 19) than in the JYLS (age 14), and differences in the adult measures and age points, might also have contributed to a higher continuity of aggression in the US sample than in the Finnish sample.

\subsubsection{Paths from childhood socioemotional behavior to adult personality}

The analysis of paths to adult personality (Pulkkinen, Kokko, \& Rantanen, 2012) assumed that an individual's temperament forms a basis for his/her childhood socioemotional behavior and adult personality characteristics. Socioemotional behavior 
is not identical to temperament, but the temperament characteristics of reactivity and self-regulation affect socioemotional behavior as discussed in Section 4.2.3.

Models for analysis. In the study of paths from childhood socioemotional behavior to adult personality, hypotheses were based on the review of literature by Caspi et al. (2005). They proposed that (1) a surgency factor (referring to high activity and positive emotionality; Rothbart, 2011) in the child's temperament may lead to adult traits in the extraversion and positive emotionality domain; (2) the temperament dimension of anxious, fearful, and irritable distress may lead to neuroticism or negative emotionality; and (3) childhood capacities for effortful control, focused attention, and behavioral inhibition may underlie the development of conscientiousness and agreeableness.

In the JYLS, three variables for socioemotional behavior in childhood were formed according to the categorization by Caspi et al. (2005): (1) behavioral activity (e.g., "is energetic, always on the go, often has contact with others"); (2) negative emotionality (subscales for aggressiveness, anxiety, and emotional lability); and (3) well-controlled behavior (subscales for constructiveness, compliance and emotional stability). They were based on teacher ratings at ages 8 and 14 .

Information about the adult personality was composed of five personality traits (NEO-FFI), four vulnerability traits formed from the KSP: aggressiveness, anxiety, sociability, and conformity; and three temperament traits formed from the subscales of the Adult Temperament Questionnaire (Rothbart, Ahadi, \& Evans, 2000): surgency (sociability and positive affect), effortful control, and negative affectivity (fear, sadness, and discomfort). The intercorrelations of the adult personality characteristics at age 42, calculated separately for males and females, revealed three groups of variables that were confirmed with a principal factor analysis: (1) Extraversion and surgency correlated positively with each other and both of them correlated with openness to experience and sociability. (2) Neuroticism and negative affectivity correlated positively with each other and both of them correlated with anxiety and aggression. All these indicators of negative affect correlated negatively with effortful control and conscientiousness. (3) Conscientiousness and effortful control correlated positively with each other and both correlated with conformity. In addition, in men, agreeableness correlated with effortful control. Agreeableness correlated with conformity and sociability in men and women.

The paths from childhood socioemotional behavior to adult personality characteristics were studied using three sets of variables. Three models were tested using structural equation modeling (Pulkkinen et al., 2012):

- from behavioral activity to extraversion and positive emotionality in adulthood;

- from negative emotionality to neuroticism and negative affectivity in adulthood; and

- from well-controlled behavior to conscientiousness and effortful control in adulthood. 
Developmental paths. For behavioral activity in males, the results showed that there was significant continuity from age 8 to adulthood as indicated by links to temperament (surgency), personality traits (extraversion and openness to experience), and sociability (Figure 5.5). These connections were not significant for females, but the results went in the same direction as for the men. Female activity was associated with sociability in adulthood, but not directly from age 8 ; the age 8 behavioral activity predicted activity at age 14 , which was linked to adult sociability.

For negative emotionality, the expected connections were found in males between the ages of 8 and 14. Furthermore, negative emotionality at age 14 was linked to aggressiveness in adulthood. In females, the expected links did not exist. Neuroticism in adulthood was not linked to childhood behaviors.

Well-controlled behavior in males at the age of 8 was significantly linked to conformity in adulthood (Figure 5.6). In women, only well-controlled behavior at age 14 was significantly linked with conscientiousness in adulthood. Although the significant links had to do with different variables in males and females, they went in the same directions for both genders.

The results were in accordance with expectations, although the connections were higher in males than in females. Gender differences in significant developmental paths and correlation coefficients suggest that temperament-based differences in activity were more observable by teachers in 8-year-old boys' than in girls' behavior. Possibly, the gender differences were due to socialization experiences, as proposed by Kagan and Moss (1962). Girls may feel pressure to control their behavior more than boys do. The significant paths in females, which started at age 14,

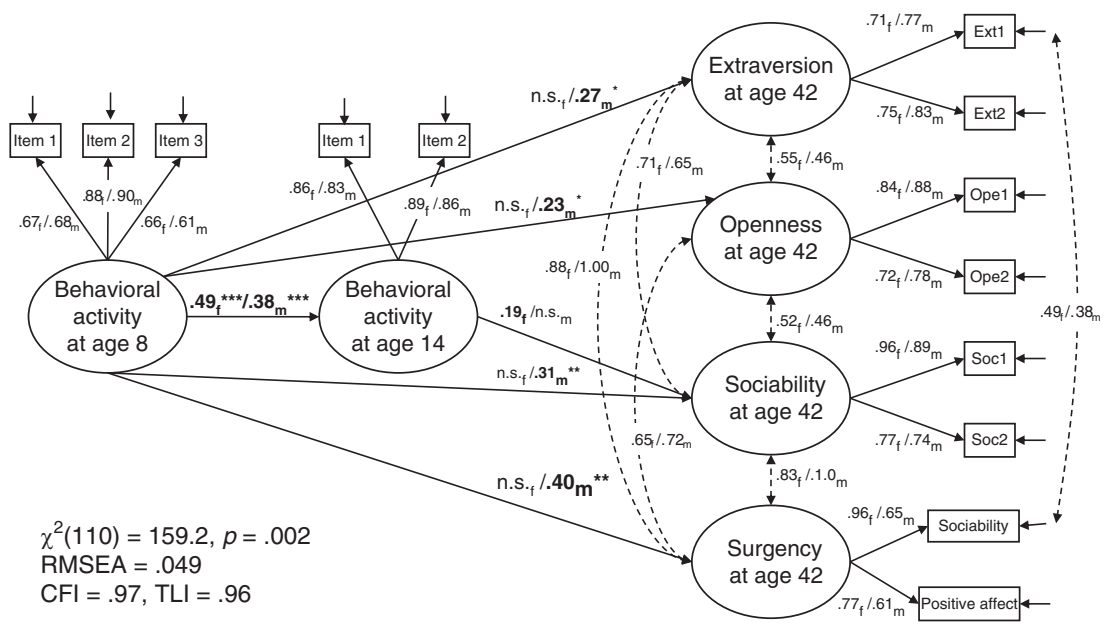

FIGURE 5.5 Continuity in behavioral activity from childhood through adolescence to adulthood.

Source: From Pulkkinen, L., Kokko, K., \& Rantanen, J. (2012). Paths from socioemotional behavior in middle childhood to personality in middle adulthood. Developmental Psychology, 48, 1283-91. (Figure 1, p. 1287). (C) 2012 American Psychological Association. Reprinted with permission. 


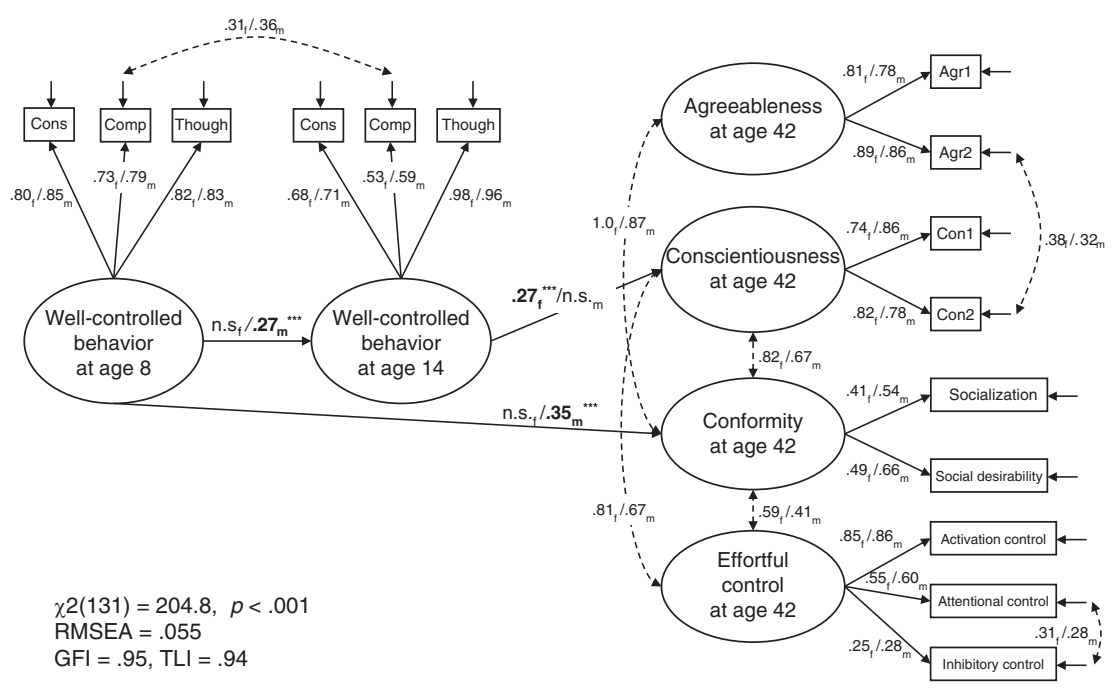

FIGURE 5.6 Continuity in well-controlled behavior from childhood through adolescence to adulthood.

Source: From Pulkkinen, L., Kokko, K., \& Rantanen, J. (2012). Paths from socioemotional behavior in middle childhood to personality in middle adulthood. Developmental Psychology, 48, 1283-91. (Figure 2, p. 1288). (C) 2012 American Psychological Association. Reprinted with permission.

show that girls' behavior develops (or girls start to display their unique personality more clearly) during their school years, so that differences in sociability and conscientiousness predict their adult personality. Changes in girls' constructive behavior between the ages 8 and 14 were found in Section 5.2.2.

Correlations. The results confirmed our hypothesis that there is continuity from childhood behavior to adult personality characteristics, particularly in behavioral activity and well-controlled behavior. There was a similarity with the findings of Hampson and Goldberg (2006) who found the highest stability coefficients from childhood to middle age (across 40 years) in extraversion (0.30) and conscientiousness $(0.26)$ and zero coefficient in neuroticism. In the JYLS, the correlation for extraversion was of the same size for males $(0.31, \mathrm{p}<0.001$; between behavioral activity and surgency from age 8 to 42 ), and the correlation between well-controlled behavior and conformity was 0.35 ( $\mathrm{p}<0.001)$. For negative emotionality, the correlation was zero. In females, no significant correlations were found between socioemotional behaviors at age 8 and adult characteristics, but well-controlled behavior at age 14 correlated positively with conscientiousness at age $42(\mathrm{r}=0.21$, $\mathrm{p}<0.05)$, which was in line with the thought by Eisenberg, Duckworth, Spinrad, \& Valentine (2014) that self-control underlies the emergence of conscientiousness. There were gender differences in continuity in the JYLS in the sense that childhood behavior predicted adult characteristics more robustly and from an earlier age in males than in females. This finding was contrary to the statement by Caspi et al. (2005) on gender similarity in the continuity of personality characteristics. 


\section{Personality and psychological functioning}

It has been common to speak about a child's temperament and an adult's personality as separate entities. Studies of temperament and personality were kept theoretically and empirically separate for a long time. In recent years, the understanding of the relationships between temperament and personality has, however, increased as a result of the intensive work of researchers aiming at achieving a synthesis between these lines of research, as McAdams and Olson (2010) note. The study about the paths from childhood socioemotional behavior to adult personality traits shows continuity, but also discontinuity; the analysis of discontinuity is in need of further research.

Conclusions regarding question 10 . The answer to the question of whether socioemotional behavior in childhood predicts adult personality is positive with certain limitations. For the patterns of adult characteristics indicating differences in self-regulation, roots in socioemotional behaviors were found in childhood, but for the patterns of personality traits, roots were found in early adolescence. Also, continuity in single characteristics from childhood to adulthood went via adolescence. It suggests that personality characteristics were formed during school age. An exception was male extraversion, which was highly continuous from age 8 to middle age. Continuity in aggression was more limited than generally believed (see also Pulkkinen, 2017). 


\section{6}

\section{THE PERSON AS MOTIVATED AGENT}

\subsection{The transition to adulthood}

\subsubsection{Age-stratified or individualistic transitions?}

The second line of personality development, according to McAdams (2015, p. 260-261), is related to questions of "What do I want? What do I value?" with the present and the future as the temporal focus. People are not only social actors with personality characteristics and emotions, but they have "a motivational agenda," that is, desires, goals and values to pursue. Motivational perspectives in personality psychology focus on characteristic adaptations rather than on dispositional traits (McAdams \& Pals, 2006). In this chapter answers to the following questions are presented based on the JYLS data: (1) what kind of characteristic adaptations could be found in the participants during their transitions to adulthood; (2) how did the participants build their future by means of education; and (3) what kind of personal goals and values were included in the participants' life.

The first question to be discussed in this chapter concerns the transitions into adulthood. An analysis of them was started within the JYLS in the 1980s, when age stratification as an explanation for the transition into adulthood was widely discussed in sociological literature. Neugarten, Moore, and Lowe (1965) had presented a view that age norms define the range of years in an individual's life during which it is regarded as appropriate to undertake various types of roles and behaviors, such as getting married. Social pressures for entering into and exiting from roles when people grow older, stem from prevailing norms enforced with legislation regulating, for instance, compulsory school attendance, the minimum age for employment and marriage, and full legal age. They produce similarities in the timing of life events (age-regularities) in people' lives. Historical time may change the norms concerning the appropriate time of particular transitions or the order of the transitions in our lives, as can be seen in relation to attitudes toward living together and having a child before marriage. 
In each society, there are expectations about the age at which certain roles are taken up and when others are avoided, but there are also individual factors, such as maturation that affect role behaviors. Havighurst (1953) developed the idea of tasks to be achieved by a certain age and introduced the construct of a "developmental task" for each stage of development from early childhood to late adulthood. In early adulthood (age 21 to 27), the major developmental tasks are achieving autonomy from one's parents, career choice, marriage, childbearing, and work.

The aspirations and plans of young people themselves may also have a significant impact on their transition into adulthood. An individual may consciously delay or complete a transition at a certain stage. Therefore, the transition into adulthood is better described as a developmental process rather than as an event (Hogan \& Astone, 1968). Marini (1984) concurred with these ideas while arguing that adult role transitions, such as finishing school and joining the workforce, marriage or parenthood, may occur in a different order depending on an individual's socioeconomic origins, gender, educational attainments, birth cohorts, and culture. The occurrence, timing, and sequence of social roles define the unique course of an individual's life.

Levinson (1986) described individual transitions in terms of a "life structure," which refers to an individual's most significant relationships with various others during a certain period. The other may be a person, an institution, or an object. Every relationship shows both stability and change. The life structure is created during a transitional period to involve those significant others that are most significant to an individual during that particular period. This is then followed by a more static period for some years. It comes to an end as a new transitional period begins, which then creates the possibility for a change in the life structure, and for a new period to begin. According to Levinson, the entry life structure for early adulthood is the time during the twenties when an individual sets up an initial mode of adult living.

More recently, Arnett (2000) asked his study participants to evaluate the importance of various criteria for the transition into adulthood, which included role transitions (e.g., marriage), family capacities (e.g., caring for children), norm compliance (e.g., avoiding drinking), biological transitions, and individualistic transitions (e.g., accepting responsibility for one's actions, deciding on one's values, becoming financially independent, and establishing an equal relationship with one's parents). The participants, who represented different age groups from adolescence to middle age, regarded individualistic transitions as the most likely markers of the transition into adulthood. In all age groups, role transitions were ranked the lowest in importance for the transition to adulthood. The period from dependent adolescence to independent adulthood was mostly experienced positively (Arnett, 2004).

\subsubsection{Life changes in early adulthood}

Self-perceived adulthood. In the JYLS, participants were asked an open-ended question at age 27: what were the distinctive characteristics of adulthood and youth (Pulkkinen 1989b). The responses showed that individualistic transitions were most important, in line with Arnett's (2000) findings: 
- $\quad$ an increased responsibility (61 percent) for finances, work, one's spouse and children, and other people;

- maturation of thinking (31 percent);

- $\quad$ independent decision making (21 percent);

- increased self-confidence and consolidating self-image (20 percent);

- a feeling of freedom (18 percent); and

- calming down (17 percent).

At age 27, women (91 percent) generally felt themselves to be independent of their parents in terms of their decision making; 79 percent of the men felt this way. Nevertheless, not everyone regarded him/herself as being an adult (this applied to 9 percent of women, 16 percent of men). One's orientation toward the future develops through the influence of cognitive maturation, social experiences in one's family, school, or work, and the complex interaction between situational and person variables (Trommsdorff, 1986). The JYLS participants' optimistic expectations about the future at age 20 were associated with the number of years of education, a sense of responsibilities toward society, positive experiences of child-centered parenting at home and with other aspects of the Thriver style of life, whereas pessimistic expectations were associated with the Loser style of life (Pulkkinen, 1990b). A pessimistic future orientation can be found in youth whose environment does not offer them a sufficient number of opportunities in which to test their abilities and to experience success and acceptance.

Adult transitions. In the literature on transitions to adulthood, the transitions that are typically expected to signify adult status are called adult transitions. This term commonly refers to five transitions: moving from the parental home to living independently, completing one's education, starting to work full-time, establishing an intimate relationship, and becoming a parent. Among the JYLS participants, almost everybody (96 percent of the women and 88 percent of the men) had moved out of the parental home by the age of 27 , although one-sixth of the participants reported that they had moved out and then back to their parental home again due to their studies, workplace, and other life situations (Pulkkinen, 1989b). In Finland, young people tend to move out of the parental home at a fairly young age; women at 20 years and men at 21 years (Nikander, 1998). An important reason for this is that the Finnish social welfare system provides a housing allowance for students and for individuals who are on a low income (and are not students) to live in rented accommodation.

The median age of starting a full-time job was 20 to 21 years for men and 2 years later for women due to their longer education. The length of time spent in education also affected the entry into parenthood. Women with less education were more often ( 80 percent) mothers by the age of 27 than women who had studied at a university ( 25 percent). The median age at which the first child was born for women with a minimum level of compulsory education was 20 to 21 years and for those with shorter or longer vocational education it was 24 to 25 years. The youngest mother was 15 years old when she had her first child. At age 27 , half of the women and 40 percent of the men were married, a quarter 
TABLE 6.1 Timing of adult transitions: cumulative percentages of women $(\mathrm{n}=132)$ and men $(\mathrm{n}=150)$

\begin{tabular}{lllllll}
\hline & & \multicolumn{2}{c}{ Timing of undergoing adult transitions (\%) } & Not undergone \\
\cline { 3 - 4 } & & By age 27 & By age 36 & By age 42 & At age 42 \\
\hline \multirow{2}{*}{ All four adult transitions } & Female & 31.8 & 65.9 & 70.5 & 29.5 \\
& Male & 20.0 & 54.7 & 57.3 & 42.7 \\
Educational degree & Female & 68.9 & 81.1 & 83.3 & 16.7 \\
& Male & 65.3 & 71.3 & 71.3 & 28.7 \\
\multirow{2}{*}{ Intimate relationship job } & Female & 97.7 & 98.5 & 98.5 & 1.5 \\
\multirow{2}{*}{ Child } & Male & 96.0 & 98.7 & 98.7 & 1.3 \\
& Female & 87.1 & 97.7 & 97.7 & 2.3 \\
& Male & 77.3 & 91.3 & 92.7 & 7.3 \\
& Female & 55.3 & 84.1 & 85.6 & 14.4 \\
\hline
\end{tabular}

From Räikkönen, E., Kokko, K., \& Rantanen, J. (2011). Timing of adult transitions: Antecedents and implications for psychological functioning. European Psychologist, 16,314-23. (Table 1, p. 318.) Reprinted by permission from European Psychologist (C) 2011 Hogrefe Publishing.

were cohabiting, 7 percent had divorced, and one-fifth lived on their own. Every possible combination in the order of the three transitions (entering the workforce, marriage, and parenthood) existed (Pulkkinen, 1989b). However, the vast majority of the participants with a lower level of education had gone through them in the conventional order: entering the workforce, marriage, and parenthood. The completion of higher levels of education had delayed the transitions and had brought about changes in the conventional order. The place of entry into the workforce in the chain of marriage and parenthood varied in women more than in men.

The years from 27 to 36 are busy with adult transitions. As seen in Table 6.1, 66 percent of women and 55 percent of men had navigated all four transitions (being awarded an educational degree, entering full-time work, committing to an intimate relationship, and having one's first child) by the age of 36; one-third of the women and one-fifth of the men by age 27 (Räikkönen, Kokko, \& Rantanen, 2011). However, at the age of 42 , close to half (43 percent) of men and 30 percent of women had not undergone all adult transitions. The first child was lacking in 19 percent of the men and 14 percent of the women, but most often, an educational degree was what was lacking; in 29 percent of the men and 17 percent of the women.

\subsubsection{Patterns and timing of adult transitions}

Certain transitions tend to co-occur in people's lives. Possible patterns of transitions were studied by considering five transitions up to the age of 27: moving out of the parents' home, completing one's education, entering full-time work, beginning an intimate relationship, and the birth of the first child (Räikkönen, Kokko, 
Chen, \& Pulkkinen, 2012). Three "patterns of adult transitions" (latent classes) were obtained: Almost half (46 percent) of the participants were members of the class entitled Work-orientation with delayed parenthood. The other half divided into two classes: Traditional work and family (35 percent) and Academic orientation with no children (19 percent). Almost all the participants in the three classes were living independently (95.9 percent) and thus moving from the parental home did not differentiate between the classes.

The typical characteristics of the three patterns were as follows:

1. Work-orientation with delayed parenthood. Members of this class were working full-time. They had either completed a vocational qualification or had no post-comprehensive education. No one was married; they were all single or cohabiting. Mostly, they did not have children.

2. Traditional work and family. Members of this class had typically undergone all five transitions. They lived independently, had entered the workforce, and had at least one child. None of them were single and almost everyone was married. Most of them had completed vocational training.

3. Academic orientation with no children. Most members of this class had completed a college or university degree. Some of them were still full-time students. None of them had children. Being single was more typical than being married or cohabiting.

When studying the consequences of the different patterns of adult transitions for later life, the work-orientation pattern differed most significantly from the other transition patterns (Table 6.2). Comparisons were made at ages 27, 36, and 50 in psychological functioning (depression and life-satisfaction) and social

TABLE 6.2 Comparison of the patterns of adult transitions in social and psychological functioning

\begin{tabular}{lll}
\hline & Age & Significant differences \\
\hline Depression & 27 & Work-oriented $>$ Traditional \\
& 42 & No differences \\
Life satisfaction & 50 & Work-oriented $>$ Traditional \\
& 27 & Traditional, Academic $>$ Work-oriented \\
Career stability & 42 & Traditional $>$ Work-oriented \\
& 50 & Traditional $>$ Work-oriented \\
& 27 & Traditional $>$ Work-oriented \\
Heavy drinking & 42 & No differences \\
& 50 & Wo differences \\
& 27 & Work-oriented $>$ Traditional \\
& 42 & Work-oriented $>$ Academic-oriented \\
& 50 & Academic-oriented \\
\hline
\end{tabular}

Note: Comparisons at age 27 are based on Räikkönen et al. (2012). Comparisons at ages 42 and 50 were made for the present purpose. 
functioning (career stability and heavy drinking) (for age 27, see Räikkönen et al., 2012; for ages 36 and 50, the data were analyzed for the present purpose). It was found that:

- Participants belonging to the work-orientation pattern were, relative to the traditional pattern, less satisfied with their lives throughout adulthood, were more depressive at ages 27 and 50, and were heavier drinkers in young adulthood. They had a more unstable career path, but this was only in young adulthood.

- Participants belonging to the traditional work and family pattern were doing the best psychologically. Having a child and family life at an early age seemed to be positive from the point of view of their psychological functioning.

- Participants belonging to the academic orientation pattern did not differ from those in the other patterns in middle age except from those in the workorientation pattern in the more controlled use of alcohol. This difference did not exist at age 27 when the academic-orientation and work-orientation participants were typically single and childless, which may explain the similarity in their drinking behaviors.

The participants who had completed all transitions (traditional pattern) were doing best psychologically at age 27. It was consistent with the findings by Salmela-Aro, Kiuru, Nurmi, \& Eerola (2014): postponing transitions was associated with more depressive symptoms. People, however also complete adult transitions after age 27. In the JYLS, it was possible to study whether individuals who complete adult transitions at a later age reach the same level of psychological functioning. In the study by Räikkönen et al. (2011), three "timing of transition groups" were formed on the basis of the timing of transitions up to the age of 42: (1) on-time transitions (all transitions undergone by age 27); (2) late transitions (at least one transition occurred after age 27); and (3) partial transition (not all transitions had been completed by age 42 ).

The comparison of the different timing of transition groups at age 42 in terms of their psychological functioning (psychological well-being, life satisfaction, depressive symptoms, self-esteem, and identity achievement) (Räikkönen et al., 2011) confirmed that the participants who had undergone all transitions by the age of 27 were high in psychological functioning. However, those participants who had undergone all adult transitions later in life, by the age of 42 , had a similar profile and the means did not differ significantly from those of the on-time transition group.

The participants, who had only partially completed the adult transitions, were significantly lower than the on-time and late transition groups in psychological well-being, life satisfaction, and self-esteem. Those in the partial transitions group also tended to have more depressive symptoms than those in the late transitions group. Thus, both the comparisons of the adult transition patterns and the timing of transition groups suggested that psychological functioning is better among individuals who undergo adult transitions than among those who do not. The timing of the transitions is not decisive. 
Studies of the background factors of the transition patterns up until the age of 27 and the timing of transitions up until the age of 42 revealed the following connections (Räikkönen et al., 2011, 2012):

1. Family background. The three transition patterns did not differ in terms of the socioeconomic status of the family of origin or the family structure (living with one parent or with both parents), but the timing of transition groups did differ. Participants with late transitions had more often a high socioeconomic status (SES) background than the other participants. They had spent a long time in academic education, which then delayed the adult transitions. In contrast, partial transitions were associated with a blue-collar family SES; an educational degree was most commonly lacking in the case of partial transitions.

2. School success. Participants who were part of the pattern of academic orientation with no children had achieved better school success at the end of comprehensive school and had higher educational aspirations than those from the other patterns, independent of the socioeconomic status of the parents.

3. Sex. Women were more likely to be in the traditional work and family pattern than in the academic- and work-orientation pattern and they were overrepresented in the group who had had on-time transitions.

4. Personality. Participants who had undergone all transitions either on-time or late by the age of 42 had been more active socially at age 8 than participants with partial transitions.

Adult transitions shape individuals' ways of life through changing life routines as was found in the study by Pulkkinen (1989b). The concept of the way of life refers to a system of activities characteristic of people who live under certain material and nonmaterial conditions (Takala, 1979). Transitions may be associated with a person's functioning in many spheres and affect satisfaction with life which explains the differences of transition patterns in psychological functioning. Three types of orientations in the JYLS participants' ways of life were distinguished at age 27.

Concentration on one's family was associated with the participant's early entry into family roles. Marriage and children changed the basic source of satisfaction from friends to home and family and oriented the use of leisure time to household chores instead of to social gatherings. For men, an early entry into family roles meant a stable working life and an awareness of increasing responsibility. This orientation included, for instance, the early purchase of a home. An early entry into family roles was related to an early move away from the parents' home, which gave male participants a sense of personal control over their development. Interestingly, in women, a sense of personal control over their development was related to having a high level of education rather than to an early entry into family roles. Activities were oriented to household chores more often in women than in men who spent more time on their hobbies. Women had hobbies that they focused on at home, such as handicrafts, or they participated in cultural activities, whereas men spent more time out of doors or watched television. Other than for chores related to 
the home, spouses hardly shared their free-time activities with one another. The participants admitted that the wife did all or at least more of the household chores than did the husband. (The spouses' roles reflected the situation during the first half of the 1980s; since then, roles have become more equal.)

Career and cultural orientation was associated with higher education and a late entry into the workforce. For women, this life path also meant a late entry into family roles. Women had a sense of personal control over their development and they found a source of satisfaction in their work, whereas men found more satisfaction in their leisure time. For both men and women, a stable working career was related to the notion that the future depends on oneself, and to an increased awareness of one's responsibility for universal matters.

The accumulation of problems was found among those who entered the workforce at an early age. Indifference toward school attendance in early adolescence had led the participants to finish schooling at the end of comprehensive school. A lack of secondary education resulted in an early entry into the workforce and an increased risk of social and personal problems. Problems included unemployment, dissatisfaction with life, sense of failure, identity diffusion, pessimism, and fears of one's own lack of strength, health problems, and financial matters. A high number of nonnormative life events was further associated with risky behaviors such as drinking and committing criminal offenses.

Conclusions. Adult transitions were more individualistic than age-stratified in respect to the order and the timing of the transitions. There were, however, transition patterns that differed in people who were oriented more strongly toward work, family, or education. Transitions shape individuals' ways of life. Participants who had undergone all the adult transitions were higher in psychological functioning than those who had undergone only some of the transitions. The timing of the transitions was not decisive. Spending longer in education postpones the transitions.

\subsection{The length and timing of one's education}

\subsubsection{The structure of education}

The second question concerned education as a building block of life. It was shown that the length of education affected the content and timing of adult transitions and that a lack of an educational degree was the most frequent reason for a partial transition into adulthood up until the age of 42. A partial transition was associated with lower psychological and social functioning. Education in Finland is free, but all participants did not continue studies after the compulsory education. Factors which predicted success or failure in the attainment of an educational degree are analyzed in this chapter.

In the Finnish education system, the school starting age is 7, and the compulsory education (comprehensive school), including primary and lower secondary education, ends at age 16. Thereafter, the student may enter a general upper secondary school or a vocational upper secondary school, so called 
post-comprehensive education. Around 10 percent of the relevant age group does not start upper secondary studies immediately after the compulsory education (Ministry of Education, 2016; Sahlberg, 2011). After completing one's schooling at the general upper secondary school, a student may apply to study at a university or at a vocational college, which is now known as a polytechnic or applied university. To improve an individual's eligibility in the labor market, training via short courses is available for those who have no vocational training or are unemployed.

In the JYLS, the majority of the participants had completed post-comprehensive education. A shorter educational path into the labor market via vocational upper secondary school education was more common (48 percent) among men than among women (28 percent), whereas a longer path via general upper secondary school and vocational college was more common among women (32 percent) than among men ( 8 percent). This difference in terms of women's longer vocational training corresponded to differences in the age cohort group born in Finland in 1959, according to Statistics Finland. Girls attended the general upper secondary school more frequently than did the boys and thus postponed their choice of vocational training. Gender differences did not exist in terms of going to university after the general upper secondary school. Men and women had also equally (32 percent of men and 27 percent of women) only attended comprehensive school. They entered the work market without a vocational degree. A lack of vocational education was more common in the 1970s than today due to the improvement of opportunities in vocational education.

University studies are generally completed at an older age in Finland than in some other countries for several reasons. First, men must complete compulsory military service (which is now also voluntary for women). Second, the minimum academic qualification for employment in higher white-collar occupations is an MA degree (not a BA). Third, entrance exams for tertiary education are arranged for specified fields (not for university studies in general) in a way that may force a student who fails an exam to have to wait for a year to sit the exam again. Fourth, free studies, student and housing allowances, and other benefits related to medical care and transport costs help students to study for longer. Fifth, many students work during their university studies in order to contribute to their living costs.

\subsubsection{Predictors of the choice of education}

Home background. The study of the predictors of the length of education completed indicated, as could be expected, that school success at comprehensive school at age 8 and at the end of the school predicted the time spent in education for men and women (Pulkkinen, 1989a,b). The socioeconomic status of the parents affects children's choices of educational paths less in Finland than in many other countries as shown by a cross-cultural comparison (Duncan et al., 2012). The length of the parents' and their children's schooling were only weakly correlated in the JYLS compared with US, 
British, and Swedish samples. Finland had promoted equality of opportunity more than the other countries (i.e., the United States, the United Kingdom, and Sweden). Results from the JYLS show consistently that the socioeconomic background of the family of origin accounts only a little for the participants' social functioning except that the higher socioeconomic background is associated with the developmental path from constructive behavior to the Thriver style of life (cf. Section 5.3.3).

The selection of an educational path after comprehensive school was mainly associated with the school success, but the parents' socioeconomic status indirectly affected children's choices (Pulkkinen, 1989a). First, most of the men and women who had decided not to continue their formal education after comprehensive school came from the group with the lowest socioeconomic status, particularly, when their grade point average (GPA) was low. Second, at the end of comprehensive school, the mean level of the GPA was higher in girls than in boys and girls tended to postpone their choice concerning their future occupation by continuing their studies in the general upper secondary school. However, after this, girls chose vocational studies rather than university studies if their mother's education was of a low level. They made this decision independently of their school success. Third, a boy's decision to attend a vocational upper secondary school rather than a general upper secondary school tended to be more likely if the father's socioeconomic status was low, but if a boy's GPA was high, he tended to choose the general upper secondary school and enter university. Boys' choices of university studies depended more on their success at school than on their socioeconomic background.

Education of the parents of the participants. The parents of the JYLS participants attended school during an earlier school system, which included two streams, one academic and one nonacademic. The nonacademic stream consisted of seven grades in primary school. It was locally accessible and free. After this, the student could enter a vocational school or the workforce. In order to enter the academic stream, the child had to pass an entrance exam at lower secondary school at about 11 years old. Secondary schools were mainly situated in towns and the parents had to pay tuition and other costs. The parents of the participants mostly (70 percent) had completed their schooling in the 7-year primary school. In contrast, only 3 percent of their daughters and 12 percent of their sons had spent less than 9 years at school. Furthermore, the rate of graduation from the general upper secondary school was only 8 percent among the parents, but was 47 percent among their daughters and 22 percent among their sons. In one generation, the level of academic education had risen rapidly. As a result of the school reform that moved from the parallel school system to the comprehensive school system, social mobility increased in Finland. The participants of the JYLS attended school during this major reform toward free comprehensive schooling and tuition free vocational education at all levels.

Individual characteristics. The study of possible individual characteristics that might be associated with educational attainment revealed that socioemotional behavior at age 8 predicted the length of a person's schooling. The most socially anxious boys and girls were over-represented among the participants who finished their schooling at the end of comprehensive school, whereas the most socially constructive 
children were likely to go on to attend general upper secondary school (Pulkkinen, 1989a). Likewise, at age 14, an orientation toward responsibility, particularly in girls, and the style of life of the Thriver at age 20 were positively associated with success at school. At age 27, the Resilient style of life was associated with a long education in males and females (see Section 5.3.2) and, consequently, with entering late into full-time work, which was characteristic of the adult transitions on the academic track. The individual characteristics that were positively associated with educational attainment included high emotion regulation, in the model for the Unfolding of Socioemotional Behavior.

The behavioral regulation dimension was associated with the length of education among boys but not among girls. Boys who were above average in aggressiveness at age 8 were overrepresented among the participants who finished their schooling after comprehensive school (Pulkkinen, 1989a). A relatively higher orientation to one's peer group than to one's home at age 14 and the Reveller style of life at age 20 were associated with shorter education among boys. The Undercontrolled style of life at age 27 was associated with social and personal problems, including a limited education, early entry into the workforce, and experiences with unemployment, which were characteristics of the work-orientation transition track.

Teachers' estimations of pupils' positive development on the item: "The teacher feels that this pupil will certainly find his/her way later on in life" correlated positively with the length of education until age 27 ( $r=0.26$ for girls and 0.34 for boys, $\mathrm{p}<0.001$, for each) (Pulkkinen, 1989a). For some boys who at age 8 had received the prediction of a high likelihood of positive development, school success had, however, weakened during their years at comprehensive school. At age 27, they were using alcohol in excess. Teachers equally predicted boys' and girls' unstable working careers, but they were less able to predict girls', as opposed to boys', stable working careers. Among males, their work career was more directly associated with their school success than among females whose career depended more on their role as a mother in young adulthood.

The teacher's prediction of the pupils' development later on in life did not correlate with the father's occupational status (Pulkkinen, 1989a), but the teacher's positive predictions did correlate with the pupils' school success $(\mathrm{r} \sim 0.60)$, as could be expected. In addition, the teacher's positive prediction correlated highly $(\mathrm{r} \sim 0.70)$ with constructive behavior versus anxious behavior (a bipolar variable), but nonsignificantly with aggressive versus compliant behavior. These results mean that teachers had observed pupils' constructive behavior and school success, which correlated highly $(0.72)$ with each other at age 8 and estimated them to be resources for the pupils' finding their way in the future, both in terms of their education and their career path, independent of their socioeconomic background. In contrast, pupils' socially anxious behavior and poorer school success made teachers more uncertain about their resources for finding their way in the future. The results on the connection of academic success with constructive behavior were in line with the study by Caprara, Barbaranelli, Pastorelli, Bandura, and Zimbardo (2000). Their study confirmed the central role of early prosocialness (as they called it) in 
academic achievement and, in addition, that there was no connection between early academic achievement and later academic achievement if early prosocialness was controlled for, that is, if it was not considered.

\subsection{3 "Off-time" education and other post-comprehensive educational trajectories}

A question of whether completing one's education later in life could compensate for the consequences of the educational choices made at a younger age can be analyzed based on the study by Kokko, Pulkkinen, Mesiäinen, and Lyyra (2008). Four trajectories of post-comprehensive education between the ages of 15 and 42 were obtained in this study by using semiparametric mixture modeling (Nagin, 2005): (1) no or early post-comprehensive education (until age 20; 37 percent of the participants); (2) on-time education (before the late twenties; 33 percent); (3) off-time education (peak time in mid-thirties; 21 percent); and (4) continuing education (9 percent). Men were overrepresented in the no or early post-comprehensive education trajectory.

The off-time education participants (Group 3) had a higher occupational status at age 42 than the participants with no or early education (Group 1). Off-time education increased optimism among women: at age 27 , their optimism was at the lowest level, but at the ages of 36 and 42, it was at the highest level of all the women. The comparisons revealed the many positive consequences of off-time education for women and suggested that opportunities to start studying for a degree should be available for individuals after the usual period in one's youth. These results concur with the findings by Shulman et al. (2015), which show that changes that people make in their career may be "an embarkation on a new path toward a new goal" (p. 17). Paternal support helps young people find their way within the occupational domain.

In men, an increase in optimism was not found in Group 3. Men who had engaged in continuing education (Group 4) were most optimistic after age 36. Young men tend to pursue their plans more consistently than women whose career is more fluctuating (Shulman et al., 2015). The continuing education participants remained in education longer than the on-time education participants. Both groups had more favorable developmental backgrounds and better school success than the two other groups, and they were in higher occupational status positions at ages 27, 36, and 42. Activity and self-regulation in childhood had been highest in the continuing education participants. Thus, both cognitive and noncognitive capacities in childhood were high in the continuing education participants.

Conclusions. Choices of education were mainly affected by school success, although compliance with the parents' socioeconomic status was seen in the lack of post-comprehensive education and in the choice of vocational studies. Individual characteristics associated with emotion regulation correlated with educational attainments. Teachers' predictions about the success in life of their pupils were based on their school success and constructive behavior independent of their socioeconomic background. 


\subsection{Goals and values}

\subsubsection{Common elements in the life structure of adults}

A third question concerned the personal goals and values included in the participants' life structures. A life structure is formed by the people, institutions, and objects that are significant to an individual (Levinson, 1986). It was possible to assess what common elements were included in the life structures of middle-aged people using several measures administered to the JYLS participants.

What gives you the most pleasure? At ages 27, 36, 42, and 50, the participants were administered the pleasure ranking task, in which they were asked to rank family, work, friends, and leisure pursuits according to the amount of pleasure they gave them. The highest ranking was given by women and men to family at all ages (Table 6.3; Pulkkinen \& Polet, 2010b). Changes in the rankings can be seen as indicators of average changes in life structures. The rankings showed that the importance of life areas became somewhat different between men and women with age, except for family, which was the most important source of satisfaction at all ages. Profession or work as the source of satisfaction lost its importance after the mid-thirties, at age 36 in men and at age 42 in women, and at the same time, leisure pursuits among men, and friends among women, increased in importance.

What is most important to you? The significance of the family also came out when the participants were asked via an open question in the personal interview at age 36 , what was the most important thing to them at the moment; and what if any, were the second and third most important? The most important thing was family (Pulkkinen, Nurmi, \& Kokko, 2002). The second was profession, the third was health, and, thereafter, leisure time and friends appeared with equal means. Health was not included in the pleasure ranking task (Table 6.3), but an open question produced it. Among the hopes for the future, health was mentioned most often followed by family, child-rearing, and earnings. Health exceeded all other domains in the participants' fears with regard to the future. Global problems were next, far behind health.

How much do you think about? Another measure was the Personal Life Investment Schedule, in which the participants were asked to assess how much they thought or

TABLE 6.3 How much pleasure do family, work, friends and leisure pursuits give people in relation to each other at different ages: rank orders from 1 to 4

\begin{tabular}{|c|c|c|c|c|c|c|c|c|}
\hline \multirow[t]{2}{*}{ Rank } & \multicolumn{4}{|l|}{ Men } & \multicolumn{4}{|l|}{ Women } \\
\hline & 27 years & 36 years & 42 years & 50 years & 27 years & 36 years & 42 years & 50 years \\
\hline 1. & Family & Family & Family & Family & Family & Family & Family & Family \\
\hline 2. & Work & Work & Leisure & Leisure & Work & Work & Friends & Friends \\
\hline 3. & Friends & Leisure & Friends & Friends & Friends & Friends & Work & Leisure \\
\hline 4. & Leisure & Friends & Work & Work & Leisure & Leisure & Leisure & Work \\
\hline
\end{tabular}

Adapted from Pulkkinen \& Polet (2010b). 
did something about each of the topics, that is, how much energy and effort they invested in the ten life domains. The domains and their means at age 50 on the five-point scale were: welfare of the family (4.23), health (3.83), professional tasks (3.81), friends (3.46), life reflection (3.43), leisure (3.42), cognitive fitness (3.34), sexuality (3.22), independence (3.25), and death (2.06) (Hietalahti, Tolvanen, \& Kokko, 2015). Correspondingly, at age 36, the participants reported to invest most energy in family (4.42). The next was profession (4.02) and third was health (3.67) (Pulkkinen et al., 2002). Thus, family was the leading domain in terms of the participants' personal investments of thought and effort. In accordance with the increasing focus on health was the result, found in the analysis of the items added to the Life Investment Scale, that thinking about one's physical fitness increased from age 42 to 50; 46 percent of the participants thought a lot or quite a lot about their physical fitness at age 42 , and 55 percent at age 50. For comparison, this figure was 88 percent for the welfare of the family at both ages.

How much do you value? The participants were asked in the Personal value task to estimate the personal value of the following domains at ages 27, 36, 42, and 50: politics, art, science, technology, religion, economy, child-rearing, social work, physical fitness, and (hedonistic) pleasure in life. Physical fitness and pleasure were the domains that were most valued at age 50 for men and women (Table 6.4; Pulkkinen $\&$ Polet, 2010b). From the third place onwards, gender differences appeared in a stereotypical manner. Among men, technology, sports, and economy were equally valued. Among women, child-rearing and arts were next in importance. The least important domain for women was politics; for men, it was religion.

TABLE 6.4 Rank-order of the personal value domains at age 50

\begin{tabular}{|c|c|c|c|c|c|c|c|}
\hline \multicolumn{4}{|c|}{ Females } & \multicolumn{4}{|l|}{ Males } \\
\hline Rank & Domain & $M$ & $\begin{array}{l}\text { Significant } \\
\text { differences }\end{array}$ & Rank & Domain & $M$ & $\begin{array}{l}\text { Significant } \\
\text { differences }\end{array}$ \\
\hline 1. & Physical fitness & 3.64 & $1>2>11$ & 1. & Physical fitness & 3.41 & $1>2-11$ \\
\hline 2. & Pleasure seeking & 3.36 & $2>3-11$ & 2. & Pleasure seeking & 3.30 & $2>3-11$ \\
\hline 3. & $\begin{array}{l}\text { Care and child } \\
\text { rearing }\end{array}$ & 3.13 & $3>4-11$ & 3. & Technology & 2.98 & $3>6-11$ \\
\hline 4. & Arts & 2.75 & $4>8-11$ & 4. & Sports & 2.95 & $4>8-11$ \\
\hline 5. & Economy & 2.63 & $5>10-11$ & 5. & Economy & 2.87 & $5>8-11$ \\
\hline 6. & Social work & 2.62 & $6>10>11$ & 6. & $\begin{array}{l}\text { Care and child } \\
\text { rearing }\end{array}$ & 2.79 & $6>8-11$ \\
\hline 7. & Sports & 2.53 & $7>11$ & 7. & Science & 2.73 & $7>8-11$ \\
\hline 8. & Technology & 2.48 & $8>11$ & 8. & Politics & 2.29 & $8>11$ \\
\hline 9. & Science & 2.48 & $9>11$ & 9. & Social work & 2.27 & $9>11$ \\
\hline 10. & Religion & 2.40 & $10>11$ & 10. & Arts & 2.23 & $10>11$ \\
\hline 11. & Politics & 2.12 & & 11. & Religion & 1.98 & \\
\hline
\end{tabular}

Adapted from Pulkkinen \& Polet (2010b). 
The personal value of the studied domains was stable from age 27 to age 50 (Pulkkinen \& Polet, 2010b). If there were changes in the value of a life domain, these changes mainly occurred between the ages of 36 and 42 , and not after age 42 : the value increased from age 36 to 42 for the arts among both genders, for science among women, and for religion among men (the latter between ages 27 and 42). The value of politics was low and stable in women, but in men, there was a fluctuation: the value of politics increased from 27 to 36 , then decreased from age 36 to 42 , and then increased again from age 42 to 50 . The fluctuation was most likely associated with the economic recession that was serious in Finland when the participants were 36 and 50 years old (in the mid-1990s and late-2000s, respectively). The recession activated the men's political interests.

What does your future depend on? The participants were asked in the Future Orientation Questionnaire to assess how much their future depended on their home background, fate, and some other factors. Their assessment at age 50 was that their future depended more highly on them than on any other source of strength. Thereafter, equally influential were the world situation, external conditions, luck, and other people and the third group was formed by one's past life, home background, fate, and God (Pulkkinen \& Polet, 2010b). Men found God to have less of an influence on their future than any other listed source of strength.

The idea that "the future depends on me" was stable from age 27 to 42 in both men and women, but thereafter, men's trust in themselves significantly decreased (Pulkkinen \& Polet, 2010b). At the same time, their picture of the significance of the situation in the world for their life strengthened. The changes in men indicated that although they, at age 50, still felt sure that their future depended on them, they gradually allowed more space for the idea that the future also depended on the world situation and on other people. The conceptions were more stable in women than in men.

Findings across different measures showed that family was the most significant element in the participants' life structure through adult years: it was in first place as a source of satisfaction; it was emotionally the most important thing to them; it was the leading domain in the personal investment in thoughts and actions; and hopes for the future were related to the family. Also, in the study by Staudinger (1996), family was the domain in which the age group of 35 to 54 years was most invested and profession was the second. Profession was also significant in the JYLS, although it lost its meaning as a source of satisfaction toward mid-life. Health was high in importance and fears for the future were related to health. Concerns about health and the high value placed on physical fitness concurred with the finding that the participants considered their future to be dependent, in the first place, on themselves.

Religion was lowest in importance, particularly among men who considered that God had the least influence upon their future. Death was not a topic that was thought about. The low importance of religion, also among women, was indicated by the low number (around 10 percent) of men and women who considered that religion was very important to them from the age of 27 to 50. The Protestant church (Lutheran) is the state church in Finland and around 70 percent of the 
participants and in the age cohort group (more women than men) belonged to it (Metsäpelto et al., 2010). Secularization in Finland has increased and going to church is not a common activity. However, about a quarter of the participants admitted having spiritual experiences regarding, for instance, a higher power that guides their actions and, even more frequently, they had a sense that they had been given a special task or purpose in the world. Most participants (90 percent) found their life meaningful at age 50 .

\subsubsection{Values in middle age}

Values are guiding principles for actions, they assist people in making choices-and people's actions reflect their values. Values were studied in the JYLS using the model by Schwartz $(1992,2012)$. The model is theoretically arranged in the shape of a circle with two pairs of opposite value sectors comprising ten motivationally different value domains, which, in turn, consisted of 57 values.

1. Self-enhancement sector comprises of two value domains: power (social status and prestige, control, and dominance over people and resources) and achievement (personal success, competence according to social standards) versus selftranscendence sector comprising two value domains: universalism (understanding, tolerance, and protecting the welfare of people and nature) and benevolence (preservation and enhancement of the welfare of people in an individual's in-group).

2. Conservation sector is comprised of three value domains: security (safety, harmony, stability of society and relationships, and stability of self), conformity (restraint of actions and impulses that might upset or harm others and violate social norms), and tradition (respect and acceptance of the customs and ideas of traditional culture and religion) versus openness to change sector comprising three value domains: self-direction (independent thought and action, creating, exploring), stimulation (excitement, novelty), and hedonism (pleasure for oneself).

In the JYLS, values were examined at age 50 by using the 57 -item Schwartz Value Survey. The description of the results on the level of the value domains is based on the report by Pulkkinen and Polet (2010b) and on the value level in the article by Hietalahti et al. (2015). The values were very similar among men and women. The existence of the four value sectors in the Finnish sample was confirmed by Hietalahti et al. (2015) with a higher-order factor analysis, in spite of differences in the structure of the domains in which universalism divided into societal concern, tolerance, and protecting nature, and benevolence divided into caring and dependability.

Self-transcendence was the most highly valued sector among the four value sectors and, within it, benevolence received the highest score among all value domains. The highest values within benevolence were: "being honest," "being 
responsible," and "true friendship." The dominance of self-transcendence was also seen in the high value placed on the domain of universalism. The highest values within universalism were "inner harmony" and "social justice." These values fit into the ideology of a welfare state that is dominant and trusted in Finland; in addition, Finland is one of the countries in the world that has the least corruption.

Self-enhancement, the opposite sector to self-transcendence, was the least valued sector among the four sectors and the domain of power within it was the least valued domain. Values representing power include, for instance, "social power" and "authority." "Power" received the lowest score of importance among 57 values. Achievement, the other value domain within self-enhancement, also received a low mean score, particularly for the value "being influential." Thus, the participants did not value people's efforts to be influential and to exercise power over other people.

The pair of value sectors, conservation versus openness to change, received scores falling between self-transcendence and self-enhancement. The second most important value sector (after self-transcendence) was conservation, which received high scores in the domain of security, particularly, for the values "family security" and "healthy"; and, in the domain of conformity, particularly, in "honoring parents and elders." The third domain, tradition, was not highly valued. The value "devout in spiritual life," which was included in tradition, received the second lowest value score among 57 items, which concurred with the assessments of the low significance of religion as presented previously.

Openness to change was in third place among the value sectors in importance. It comprises the domains of self-direction (e.g., "self-respect" and "freedom") and hedonism (e.g., "privacy" and "enjoying life"), which were more valued than the third domain, stimulation (e.g., "daring" and "exciting life"). The participants were 50 years old, which may explain the lower value of stimulation. At a younger age, it might have been higher in value. According to Schwartz (2012), adolescents are less concerned than adults with promoting the welfare of others (benevolence) and society (universalism), but give higher priority to achievement and power (selfenhancing values), and excitement and pleasure.

The results indicated that self-transcendence was typical of middle-aged Finnish participants, including the acceptance of other people and concern for their wellbeing. Also, conservation, including "protecting traditional practices" and "stability," was more valued than openness to change (independence of thought, action and emotion), and self-enhancement (aiming at success and dominance). The single values that were clearly above the other values in importance were: "family security," "healthy," "inner harmony," "responsible," and "true friendship." Family and health were also the most significant elements in the life structure of middleaged participants as shown previously.

Connections between values and personality traits studied by Hietalahti, Tolvanen, and Kokko (2016) suggested different personal ways of relating to values: (1) the personality trait agreeableness was associated with values in the sectors of self-transcendence (concerning benevolence indicated by caring) and 
conservation (concerning conformity); (2) extraversion was associated with values in self-engagement (concerning achievement and power) and openness to change (stimulation); and (3) openness to experience was associated with values in selftranscendence (universalism concerning tolerance and protecting nature) and with openness to change (self-direction in thoughts and action-the latter in women). The pattern describes people well who are activists in, for instance, promoting sustainability. Neuroticism was not associated with values and the associations of conscientiousness with values were negligible.

\subsubsection{Individual life structures and unifying life themes}

People steer their development by setting both specific and more general personal goals. Individuals' engagements and disengagements have been studied in terms of such constructs as "personal projects" (Little, 1983) and "personal strivings" (Emmons, 1986). The concept of a "personal goal” was used in the JYLS. By definition, personal goals refer to a goal construction that involves both individual motives and social opportunity structures for their actualization (Nurmi, 1989). We expected that differences between individuals existed in how they structured their personal goals with regard to their life situations and studied this issue based on the data collected at age 36 (Pulkkinen et al., 2002).

Personal goals. Several measures were used for the study of personal goals, such as open questions about the most important things in one's life, hopes and fears, and sources of happiness and the Personal Life Investment Schedule. A clustering technique was applied to obtain goal clusters. The five clusters that were identified were compared in terms of the participants' current life situation and personality (see Box 6.1).

The goal clusters describe differences in the participants' life structures at age 36 . Goals were associated with the participant's life situation, and, in some cases, with personality. One differentiating aspect in the life situation was the extent to which the participants had accomplished typical developmental tasks concerning education, marital status, childbirth, and employment. In the cluster for transpersonal or love-orientation, the individuals had completed these developmental tasks. Difficulties in the completion of developmental tasks were found in the basic needs-orientation (see Box 6.1) with regard to education and in the self-orientation with regard to intimate relationships. In both cases, related problems occurred. As regards the participants' personality, the transpersonal orientation was associated with social activity in women, the basic needs orientation with problem behaviors and the self-orientation with an ambiguous set of neuroticism in both genders. Participants who had materialistic goals were more often men than women.

Unifying life themes. In the long personal interviews with the participants at age 36, which lasted from 2 to 4 hours, the interviewers (14 in total) were asked to write down what, if any, they perceived as a unifying life theme of an interviewee. The unifying life theme refers to the personal goals around which an individual's behavior is organized (Bühler, 1933; Murray, 1938). It describes personal goals on 


\section{BOX 6.1 CLUSTERS FOR GOAL ORIENTATION WITH TYPI- CAL PERSONAL GOALS AND LIFE SITUATION}

- $\quad$ Transpersonal-orientation (21\%)

Personal goals: family, occupation, child-rearing; fears: death of a significant other

Life situation: lengthy education, married, children, employed, cultural activities, political interests

Personality: socially active behavior at age 14 and extraversion for women but neither of them for men

- $\quad$ Basic needs-orientation (30\%)

Personal goals: concerns about health, livelihood, and getting older

Life situation: difficulties in dealing with the developmental tasks of education, low education, financial problems, divorce, early pensioner

Personality: aggression in childhood, poor school success in adolescence, problem drinking in early adulthood

- $\quad$ Self-orientation (9\%)

Personal goals: self-related concerns such as human growth, human relationships, lifestyle

Life situation: difficulties in dealing with the developmental tasks of forming intimate relationships, unmarried, being a single parent, unstable career, depressed and in need of mental health services, frequent drunkenness, interested in the arts

Personality: openness to experience, agreeableness, neuroticism

- Global-orientation (16\%)

Personal goals: concerned with global issues such as war, emphasis on leisure time activities

Life situation: distanced themselves from striving for conventional goal of achieving career success, unemployed, self-employed, continuing education, maternity leave

- $\quad$ Others (24\%)

Personal goals: materialistic, such as buying a residence, low means in other goals, particularly regarding human relationships and human growth

Life situation: living in a cohabitated relationship, children

Source: Adapted from Pulkkinen, Nurmi, \& Kokko (2002). 
a general level. Unifying themes were found in about 80 percent of the interviews (Pulkkinen et al., 2002). It meant that the interviewee repeatedly raised a topic that was seemingly important to him or her, for instance, unemployment, familywork balance, and children. The clusters for personal styles obtained at age 27 (cf. Section 5.3.4) were compared in the participants' unifying life themes 9 years later, at age 36 .

The Resilient men presented life themes that focused on their profession, leisure activities (or lack of free time), and family. Because of the economic recession in Finland in the 1990s, many Resilient men had experienced unexpected financial problems (e.g., bankruptcy), which shocked them and forced them to reappraise their values. For the Overcontrolled men, the most characteristic life theme concerned their lifestyle; that is, a quiet life without demanding long-term goals, children and their own parents, and health. The life themes that came out in the interviews with the Undercontrolled men more often than with the other men, concerned spousal conflicts and divorce, unemployment, alcohol use, and suicidal thoughts.

The Traditional women had accepted the traditional female role oriented toward the home and were focused on family-centered life, and experienced a strain between work and family. They mentioned "seeking balance between work and family life" as a life theme more often than the Brittle women. The Individuated women presented their profession as a life theme by emphasizing independence and efficiency more often than the other women. However, many Individuated women had experienced life changes that had typically shifted their values toward family-centeredness and away from their profession after age 27 , and they also mentioned seeking balance between work and family as a life theme more often than the Brittle women. The life themes, which came out in the interviews with the Brittle women, concerned unemployment, depression, and divorce more often than the other women.

In general, the unifying life themes focused on the conflicts and constraints that limited the attainment of age-graded developmental tasks. Individuals who were on the paths of positive adaptation had the capacity to cope with the developmental tasks of work and family life despite temporary problems. In contrast, individuals who were on the path of conflicting adaptation were struggling with problems to do with their profession, social relationships, and lifestyles.

Conclusions. The participants' life structures included several common elements in their adult years. The highest importance was given to family, which concurs with high self-transcendent values such as concerns for the well-being of others. Health and physical fitness were increasing in importance, which is understandable considering that the participants believed that their future mainly depended on themselves. Religion was low in importance and did not provide the participants with much strength for the future. Goal-orientation and unifying life themes reflected the participants' personal strengths and constraints, which limited the attainment of developmental tasks. 


\section{THE PERSON AS AUTOBIOGRAPHICAL AUTHOR AND AN "EXPERIENCER" OF LIFE}

\subsection{A holistic approach to personality}

In the framework of McAdams (2015), the third line of personality development is the person as autobiographical author. It refers to integrative narratives by reconstructing the past, present, and the anticipated future. McAdams describes the development of an autobiographical author starting from episodic memory and then moving to a life story and narrative identity. In this chapter, also self-reflections of some aspects of life and turning points of life are described as the indicators of an individual's reconstruction of their life.

In the early part of the twentieth century, a holistic view of personality was strongly advocated by Allport, Binet, Stern, Lewin, Jung, and other distinguished psychologists, but in the middle of the century this went out of fashion, writes Magnusson (2000, p. 33). He mentions Nevitt Stanford as a person who used the term holism in 1965, and Rae Carlsson, who asked in 1971: Where is the person in personality research? Thereafter, there have been an increasing number of researchers who have supported a more holistic view. One of them was Jack Block (1971), who studied personality types from a holistic perspective.

Since the mid-1990s, Magnusson has highlighted the need for approaching a human being as a whole, both theoretically and empirically. He uses the term "holistic interactionism" for the person-environment system that functions as an integrated totality. "At each level, the totality derives its characteristic features and properties from the interaction among the elements involved, not from the effect of each isolated part on the totality" (Magnusson, 2000, p. 42). A holistic model offers a theoretical foundation for the planning and interpretation of empirical studies. The JYLS with its broad vertical and horizontal scope has aimed at a holistic view of a human being, although empirical results have been published in pieces. The overall purpose of this book is to give a holistic picture of human development, but in this chapter, results are presented based on qualitative analyses of semi-structured 
interviews conducted at age 27. The analyses were made for a sample of men (Perttula, 1998), and for a sample of women (Rokka and Sundelin, 1993).

Men. Perttula made a phenomenological psychological analysis of 26 men's interviews, and developed a framework for it which he called a multi-triangular model. The model is rooted in the hermeneutic tradition of Husserl, and is based on the holistic conception of man (Rauhala, 1983). The model includes three principles: the principle of nestedness, the principle of holism, and the principle of pluralism. The principle of nestedness means that there are ontological presuppositions in psychological research, and a researcher cannot avoid making ontological commitments. The principle of holism means that a human being always operates as a whole; and the principle of pluralism means that while a human being is a whole, structurally, there are various ways to be a human being. He modified the phenomenological psychological method, originally presented by Giorgi (1988), to include two broad parts, which can be conceptualized as 14 specified methodological steps. The first part was named individual-specific, and the second part was termed general. In the former, each research participant was an autonomous research unit, whereas in the latter, the researcher aimed to describe the common core (or the various types) of the empirical phenomenon.

Perttula introduced the construct of "experienced life-fabric," which resembles the construct of "life-structure" by Levinson (1986). "Experienced" refers to the experiential nature of the empirical phenomenon of the study and "life-fabric" to the extensiveness of the situation. In principle, the situation of the experienced life-fabric is the whole life-course of each of the research participants. His research questions were: (1) What are the unique ways that each research participant experientially organizes his life-course? (2) Could it be possible to formulate one or more descriptions about the common ways in which the research participants organized their life-courses experientially?

Perttula's analysis did not result in only one common core of the phenomenon, but delineated five types of male experiences and a few subtypes. The main types can be briefly summarized as follows:

Type I. The experienced life-fabric is organized around an active personal control over one's life, which is organized further by independence and freedom of self, and self-directness of life. A sense of personal control over life originates in a particular event, transition or turning point. There is a decisive discontinuity between the family of origin and the present life. The individual is generally satisfied with his present life. (3 men)

Type II. The family of origin provides no supportive sense of meaning when constructing life in young adulthood. Although the life course is organized around the positive development of oneself, the experience of life as a whole is negative. The desire to have personal control over one's life is strong, but the opportunities to do so are limited. In young adulthood life is limited to areas of life which one can control. The areas which are beyond one's personal control, be they impossible to control or not of interest are excluded from one's life. Focusing on everyday life is preceded by a life course in which personal difficulties require a lot of attention. (5 men) 
Type III. The experienced life-fabric is organized around the present impossibility to integrate work and family into a satisfactory whole. Work is the prerequisite of prosperity, which is, in turn, the prerequisite of family welfare. At present, more is being invested in work than in the family. The relation to the parents is close, which forms the essential basis for the present life. ( 2 men)

Type $I V$. The experienced life-fabric is organized around the stability and continuity of the life course together with the importance of ordinary things and everyday life. Work has a crucial meaning in life. The family of origin, above all, directs the way life is constructed. The relation between the self and external reality is characterized by control over life. A change toward active personal control over one's life is found difficult. In any case, the way of experiencing life remains positive. ( 8 men)

Type $V$. The experienced life-fabric is organized around continuous conflicts in oneself with conflicted relationships with the external world. At one extreme of the type is a strong personal desire to be independent in relation to other people. This is associated with a subtle personal balance. At the opposite extreme is total despair about one's own life together with a totally passive adaptation to life. At the latter extreme, there are no alternatives for living. Adulthood is characterized by excessive uses of alcohol. The life course forms a continuous totality, even though life in the family of origin did not support the construction of the person's future life. The relationship to the father was remote and filled with conflict. In adolescence, education in general was undervalued and avoided, where possible. Regardless of the varied importance given to work in adulthood, prosperity is always an important goal of life. (4 men)

(Perttula, 1998, pp. 126-7)

These types have some associations with the styles of life and personal styles extracted at age 27 (cf. Section 5.3.2): the Resilient (Type I) and the Overcontrolled (Type IV) for positive adaptation, and the Undercontrolled (Type V) for conflicted adaptation. I had selected the 26 male participants (out of 166) whose interviews Perttula analyzed - without informing him of this in advance-so that the three personal styles were overrepresented in the sample.

Perttula (1998, p. 128) distinguished six nonquantifiable continua with two opposite meanings at the extremes that differentiate between the types. Every type appears as a unique combination of these six features. They are:

1. Active versus passive personal control over one's life.

2. Continuity versus discontinuity between life in the family of origin and one's present life.

3. Constancy versus transitions in one's life course.

4. Current balance versus conflicts with one's self.

5. Consistency versus developmental change in one's self.

6. Satisfaction versus dissatisfaction with one's life.

He concludes that in young adulthood, the reflective mode of experiential living presupposes three developmental processes: an experiential discontinuity between 
life in the family of origin and one's present life (number 2 in the list); transitions in one's life-course (3); and a developmental change in oneself (5). On the contrary, continuity between life in the family of origin and one's present life, a lack of transitions, and a lack of changes in oneself leads to a nonreflective mode of experiential living in young adulthood. Thus, discontinuity and change are needed for the reflective mode of experiential living that provides better possibilities than that provided by the nonreflective mode to change one's life in young adulthood.

With thankfulness for his work, I add to this description that Juha Perttula was an independent thinker who conducted the phenomenological psychological analysis as his doctoral dissertation. He became a professor of psychology at the University of North Finland at a young age. He passed away of a serious illness in 2015 at the age of 50 years, during the time when I wrote this chapter for the book.

Women. The analysis of the interviews with women was also based on the holistic conception of the human being as formulated by Rauhala (1983). Rauhala's conception of the human being includes the idea that as individual beings, human existence is realized through the existential modes of consciousness, materiality, and situatedness. Consciousness refers to existence, materiality to organic processes, and situatedness to living in relation to the world. The method of analysis was based on the Grounded Theory and procedure by Strauss (1990) including three stages of categorization. The material is analyzed into units, coded and reorganized, and during this process, the research progresses from description to understanding, and to detecting the core categories.

Rokka and Sundelin (1993) applied this method to 20 women's interviews when they were aged 27 . They were selected (without informing the researchers in advance) so that conflicted adaptation was overrepresented. The researchers argued that in order to understand how an individual's life is evolving, the meaning of his or her own experiences must be understood.

The analyses revealed that women's lives were organized around their daily roles. The foundations of life were formed by experiences with motherhood, child rearing, single parenting, being a spouse, living in an intimate relationship, divorce, and being single. Women's own experiences of being a child and a daughter were reflected in their present roles. Each woman's uniqueness was overshadowed by their roles, and they only vaguely considered their own wishes for the future. Their own uniqueness was, however, evolving, although they lived their present life in their close circles without having clear plans for the future or making efforts to change their lives. They felt as though they were being driven in their present life situation. They lived day by day without considering things in advance.

Rokka and Sundelin (1993) distinguished three lines of development among the women with conflicted adaptation: married women, divorced women, and single women. The organization of life into the categories of married, divorced, and single women was also found among adaptive personal styles, which described family-oriented traditional women and career-oriented individuated women in rather typical ways. In this context, it is only described how the different marital statuses were associated with the conflicted personal style (Brittle). 
For married women, motherhood was a source of satisfaction. Women felt that the child belonged to the mother, and that other people were not able to affect this relationship very much. Forming an intimate relationship - often after a short acquaintance-had been a way of changing one's life and getting away from one's family of origin. They wished to be more independent from their husband. For women who were employed, work provided them with the necessary independence and gave them a rest from family life. Their conceptions of female and male roles were conventional, but they felt themselves tired by all their responsibilities. Their relationship with their own mother had improved, but their relationship with their father was distant if he abused alcohol.

Divorced women expressed their relief about their present situation: they found life to be easier without their former husband. The relationship had been difficult due to, for instance, heavy drinking. The relationship had been established too early. Despite the divorce, the former husband was having an impact on their life, which they found dissatisfactory. Other adult contacts were rare. Although the former husband took part in child care, the women felt the burden of responsibility for everything. They felt exhausted. The women had ambivalent feelings toward the child; the child both limited their life and was the fixed point of their life. The woman's relationship with her parents was distant. The father had been an alcohol abuser, and the mother had not taken their children's opinions into consideration. The women felt bitter toward society that had not provided them with help and services when they needed them. They argued that services were provided in an unequal way.

Single women had chosen their life situation. They believed and wished to establish a romantic relationship and family sometime later in life. At the time of the interview, they did not feel themselves to be mature enough to do these things; they felt that they still needed to develop themselves and to experience something before they settled down. To them, having a family meant settling down, which was a strange idea to them. The women felt that marriage was there to support the upbringing of a new generation. They wished that as wives they would be regarded as their own unique selves. Their relationship with their mother had been good and they were dependent upon their mother, who also tried to take an active part in the daughter's life. The mother had been a sole provider. The daughter felt that the mother had been too permissive and had not set the necessary boundaries, which had caused the daughter to feel insecure. They wanted to set stricter boundaries for their own children. They did not yet want to commit themselves to child-rearing, and they did not want to bring their children up alone.

Conclusions. The phenomenological psychological analysis of men's interviews revealed five types of male experiences and three of them had similarities to the personal styles of Resilient, Overcontrolled, and Undercontrolled. Possibilities for changing one's life in young adulthood were associated with the reflective mode of experiential living based on the discontinuity and transitions of external conditions and a developmental change in oneself. The analysis of women's interviews by 
employing the Grounded Theory, revealed that the foundations of their lives were formed by experiences with daily roles such as motherhood or being a spouse or single, which overshadowed young women's uniqueness. Three lines of development were distinguished among the women with conflicted adaptation and positive adaptation: married, divorced, and single women.

\subsection{Self-reflections}

The collection of autobiographical data and their qualitative analysis limit the number of participants who can be included in a study. In psychology, various measures have been developed to capture individuals' self-reflections on the past, present, and the anticipated future concerning, for instance, identity, personal control over life, optimism, and self-esteem, to be included in quantitative analyses. Although the JYLS participants were not autobiographical authors in a literal sense, when they were interviewed and when they filled in the inventories, their selfreflections were based on the reconstructions of their lives. In this chapter, answers to the following questions are outlined: (1) Does an individual's identity develop in adulthood? (2) What constitutes an individual's sense of personal control over life? (3) How stable is optimism and self-esteem in adulthood?

\subsubsection{Identity formation}

A sense of identity is an integrative experience that is based upon past and present experiences, and the anticipation of the future. In the framework of personality by McAdams and Pals (2006), identity is seen as rooted in the stable levels of dispositional traits (person as social actor) and characteristic adaptations (person as motivated agent), and constructed in interaction with the environment. In the JYLS, the analysis of identity formation was conducted following Marcia's (1966) identity status approach that was based on Erikson's (1950) conceptualization of identity.

According to Marcia, an individual's characteristic adaptation to life appears in his or her relationships to various life domains. These relationships are part of an individual's identity organized by his/her abilities, beliefs, and individual history (Marcia, 1980). Erikson (1950) considered occupational and ideological domains essential to identity, but Marcia (1966) further divided the ideological domain into political and religious subdomains. Interpersonal domains were later added to the concept of identity. There is no general agreement about what would constitute a specific set of domains that would comprehensively encompass the concept of identity.

In the JYLS, five domains, referring to religious beliefs, political ideology, occupational career, intimate relationships, and life style were included in all of the identity interviews at ages 27, 36, 42, and 50 (Fadjukoff, Pulkkinen, \& Kokko, 2016). At ages 42 and 50, parenting was added to them (Fadjukoff, Pulkkinen, Lyyra, \& Kokko, 2016). The identity interview was conducted as part of a personal interview. The opening question in the identity interview was of the following 
type: "Do you have a personal relationship to religion?" "Do you have a political viewpoint?" In addition, the participants were asked about how they had acquired their views; whether they had explored different alternatives or whether they had adopted their ideas, for example, from significant others (Fadjukoff, 2007).

Using two criteria, the presence or absence of a period of exploration and the firmness of personal commitment, four identity statuses were defined for each domain: (1) diffuse: when there was no exploration and no commitment; (2) foreclosure: when there was no exploration, but the person had a commitment that he or she had acquired from other people or from a culture; (3) moratorium: when the person was exploring alternatives but had not made a commitment; and (4) identity achievement: when the person had explored alternatives, made a choice, and had committed to one of them. Besides domain-specific status classifications, the overall identity of an individual was also determined.

Development of identity. Longitudinal patterns of identity formation were analyzed from age 27 to 50 in the five domains. The overall identity for each person was assessed with successive steps. If the person had the same status classification in three domains out of five, that status was regarded as dominant. In other cases, the overall status classification was decided upon by analyzing the salience of the domains, considering information about the individual's life situation. The dominant status was found in 80 percent of the participants at age 50, but only 60 percent of the participants at age 27 had the same status classification in three domains.

The results indicated that the identity achievement status increased with age, although more slowly among the men than among the women (Fadjukoff, Pulkkinen, \& Kokko, 2016). At age 50, achievement was the most frequent status in every domain for women and in the occupational, political, and intimate relationship domains for men. Identity diffusion was at its highest at age 27, and was found in around 25 percent of the participants. Diffusion was frequent in both genders in political and religious identity, and it was more persistent in men than in women. It remained at the level of about 20 percent at ages 42 and 50 among the men, but decreased among women. Moratorium was also at its highest at age 27, but thereafter it decreased and almost disappeared by the age of 42 . In early adulthood, moratorium was mostly related to occupational identity, but, in general, the exploration of identity is not typical in adulthood. Identity foreclosure, which means commitment without exploration, was also more common in men than in women in young adulthood.

As a whole, the study by Fadjukoff, Pulkkinen, Lyyra, and Kokko (2016) showed that gender differences diminished by the age of 50 . Women developed a mature identity at an earlier age than men, while men's identity formation continued in middle age and became similar to that of women. Only diffusion was more common in men than in women at the age of 50. The results confirmed the findings in other studies, as reviewed by Kroger (2000) that identity development does not end in adolescence but continues into adulthood. Gender differences in identity formation among the JYLS participants raised the question about the reasons for these. As speculated upon by Fadjukoff, Pulkkinen, and Kokko (2016), it may be possible 
that the domains were less salient for men than for women or that in these domains, women found more choices and were able to exert more control than men.

Typical development proceeds from diffusion to foreclosure, to moratorium, and to achievement as suggested by Marcia (1966) and confirmed theoretically and empirically (e.g., by Kroger, Martinussen, \& Marcia, 2010). Also in the JYLS, development in the expected sequence from diffusion to achievement was the most common; regression was predominant only in political identity (Fadjukoff, Pulkkinen, \& Kokko, 2005). There may also be variability in a mature identity between foreclosure and achievement (FAFA-cycles) as found in adolescence (Stephen, Fraser, \& Marcia, 1992) or between moratorium and achievement (MAMAcycles) as found in adulthood (Pulkkinen \& Kokko, 2000). A cycle means that individuals who have reached the status of identity achievement, tend to return (regress) to the previous status (either moratorium or foreclosure) and to re-explore a domain before making a new commitment.

In general, regression in identity formation was not found to be typical of any domain. The status over time was either stable, as was found for religious identity, or there was progression, as was found for the lifestyle identity. In some domains, gender differences existed, as in an intimate relationship where commitment was reached early by women and the status remained stable, whereas progression was found in men. There was also fluctuation in the status, which indicated sensitivity to historical time (Fadjukoff, Kokko, and Pulkkinen, 2010): occupational identity regressed at age 50 in both genders during the economic recession in the late 2000s, when the employment situation became more uncertain. Political identity, in turn, regressed in men in the early 2000s when there was an economic boom and they became less interested in politics, whereas the importance of politics peaked during the recession (cf. Section 6.3.1).

Background and context. Antecedents of adult identity development were searched for by considering the individual's family background and his/her school success and life context. The parents' higher occupational status contributed to the participants' identity achievement at ages 27 , whereas the contribution of their own school success to their identity achievement started at 36 when their own occupational statuses had been established (Fadjukoff \& Pulkkinen, 2006). Spending a prolonged period in education and then entering full-time work yielded positive effects on identity achievement, which is most likely associated with the level of education attained by the individuals (Fadjukoff, Kokko, \& Pulkkinen, 2007).

The timing of entering adulthood was associated with identity achievement. Taking on adult roles at an earlier age in the domain of family formation was associated with higher identity achievement at age 27 for both genders, which further preceded higher identity achievement at ages 36 and 42 (Fadjukoff, Kokko, \& Pulkkinen, 2007). Furthermore, self-perceived adulthood at age 27 was associated in women with identity achievement, although self-perceived adulthood was not related to the external markers of transitions into adulthood. It was consistent with Arnett's (2004) finding regarding the importance of internal criteria such as inde- 
pendence in decision making and responsibility in the transition to adulthood. It means that, on one hand, identity formation in women was associated with family roles, but on the other hand, it was associated with internal criteria.

Personal styles and identity. The stability of identity was also studied by comparing the clusters for personal styles obtained at age 27 (cf. Section 5.3.4) in identity status at ages 27, 36, and 42 (Fadjukoff, Pulkkinen, \& Feldt, 2007). An individual's identity status across the domains was determined based on the number of domains (out of five) in which the individual fell into a particular status.

Men. The Resilient men were characterized at age 27 by high identity achievement and low moratorium, diffusion, and foreclosure. They exceeded the Overcontrolled men in identity achievement at ages 36 and 42 and were lower in diffusion. The Overcontrolled men were higher than other men in identity diffusion at age 27, and diffusion was typical of them until age 42. Another status that characterized them at age 42 was foreclosure.

Interestingly, the development of identity in the Undercontrolled men revealed positive trends. They were high in moratorium and low in achievement at age 27, but moratorium declined sharply until the age of 36 , and at the same time, identity achievement started to increase and reached the level of the Resilients at age 42. Behavior regulation had been low in the Undercontrolled men since childhood and heavy drinking continued in adulthood, but toward middle age, their career stability, self-esteem, and mastery beliefs increased (Pulkkinen, Feldt, \& Kokko, 2005), and identity achievement also increased.

Women. The Individuated women were characterized by the highest identity achievement at all ages. They were low in foreclosure and diffusion. The Traditional women were characterized by identity foreclosure at ages 27 and 36, but thereafter, foreclosure declined and identity achievement increased. The Brittle women were lower in identity achievement and higher in diffusion than the Individuated women at all ages.

The Brittle women and the Overcontrolled men were less extraverted (more introverted) as adults, and more anxious as children than their counterparts (Fadjukoff et al., 2007). They did not demonstrate the progressive identity development toward achievement to the same extent as the other gender groups, but were higher in diffusion than them. This finding concurs with Marcia's (2002) suggestion that diffusion promotes a sense of personal and interpersonal stagnation when one feels isolated from others, while identity achievement leads to generativity in middle age.

Conclusions. Individuals' identity developed in adulthood, and faster in women than in men, but toward middle age, gender differences diminished. Typical identity development across domains proceeded from diffusion to foreclosure, to moratorium and to achievement. School success, the level of education, and entering adulthood at an early age were associated with identity achievement. Introverted individuals (Overcontrolled men and Brittle women) did not show the same kind of advancement in identity development as their more extraverted counterparts. 


\subsubsection{Personal control over one's own life}

Control over one's own life was one of the central features in young adults' life experiences according to Perttula's (1998) analysis of male interviews. Personal control over one's life was studied in two ways in the JYLS. One method was the Personal Control over One's Development Questionnaire, which I developed by utilizing Brandtstädter's (1984) model (Pulkkinen \& Rönkä, 1994). The model shows the structure and interrelationship of the cognitive, emotional, and behavioral aspects in an individual's sense of control over subjectively important areas of his/her personal development. It was administered at ages $27,36,42$, and 50 . The other measure was a 13-item version of the Orientation to Life Questionnaire, which was used to assess the person's sense of coherence. The background to this questionnaire stems from health psychology where positive subjective experiences have gained increasing attention. It was administered at age 42 .

A sense of control over one's development. The following subscales were designed for the Personal Control Questionnaire to cover the different types of sense of control that one can exert over one's development: self-confidence due to success (positive internal control); low self-worth (depressive mood) due to experienced incompetence (negative internal control); thankfulness for social support (positive external control): blaming others for one's failure (negative external control); and contentment with present achievements without setting further developmental goals.

The mean scores of the subscales for self-confidence, social support, and blaming others did not change between ages 27, 36, and 42 (Fadjukoff \& Pulkkinen, 2006). The only aspect of a sense of control in which changes took place was negative internal control; that is, low self-worth or depressive mood. It reduced significantly between ages 36 and 42 , which fits the decrease of neuroticism during this period. Contentment with present achievements without setting additional developmental goals increased from age 27 to 36 and from age 36 to 42 . A composite Personal Control over Development measure was constructed by averaging the scores of the subscales (reversed scoring for the subscales of low self-worth, blaming others, and contentment). The path coefficients were significant from age 27 to $36(0.39)$ and from age 36 to $42(0.45)$, indicating continuity in the participants' sense of control over their development.

School success and growing up in a child-centered home contributed to the participant's sense of control over their development at age 27 (Fadjukoff \& Pulkkinen, 2006). A child-centered home included good parental relationships when the participant was 14 years of age, the participant's good relationship with the father, maternal support and supervision, and a lack of physical punishment (Kokko \& Pulkkinen, 2000).

A sense of coherence. Another method that we used for the study of personal control over one's own life was based on the concept of a sense of coherence. It refers to a feeling of confidence that things are predictable, that resources are available to meet demands, and that the demands are worthy of investment (Antonovsky, 1987). 
These three components are termed: a sense of comprehensibility, manageability, and meaningfulness.

Our studies have shown that a sense of coherence is one of the indicators of positive psychological functioning in adulthood (Pulkkinen, Feldt, Kokko, 2006). The study of the developmental background of a sense of coherence in adulthood, indicated that an accepting and emotionally warm parental attitude toward the child, combined with guidance and supervision (i.e., child-centered parenting at age 14), contributed to the attainment of a meaningful, comprehensible, and manageable life orientation in adulthood. This contribution took place via a chain of school success, a higher level of education, and a higher stability of the person's career path, the development of which the child-centered parenting supported (Feldt, Kokko, Kinnunen, \& Pulkkinen, 2005).

The sense of coherence is highly (path coefficient $=0.85$ ) associated with low neuroticism (i.e., emotional stability), and moderately associated with extraversion and agreeableness (0.40) (Feldt, Metsäpelto, Kinnunen, \& Pulkkinen, 2007). The association between a sense of coherence and emotional stability (low neuroticism) is so high that it might be argued that these measures are measuring the same characteristic. These constructs and their measures are, however, based on conceptually and theoretically distinct layers of personality. Items in the inventories are also different. The association between the indicators of these constructs show that personality at the dispositional and experiential levels function in a highly-synchronized way. Individuals who are very neurotic do not experience their life to be as meaningful, comprehensible, and manageable when compared with emotionally stable individuals.

Conclusions. Personal control over one's life was studied in respect to the sense of control over one's development and to the sense of manageability, comprehensibility, and meaningfulness in life. Both the sense of control over one's development and the sense of coherence had their roots in child-centered parenting and good school success. The repeated measures of the sense of control over one's development indicated that this sense is rather stable from age 27 to middle age.

\subsubsection{Optimism and self-esteem}

The third question was whether optimism and self-esteem are stable in adulthood. It was found that the sense of coherence was associated with optimism (Feldt, Mäkikangas, \& Aunola, 2006). People with optimistic expectancies believe that things will work out, and they make a real effort to reach their goals, whereas those with pessimistic expectancies feel that things will end up with a negative outcome, and they are at greater risk of giving up (Scheier \& Carver, 1985).

Optimism. Optimism was assessed at ages 27, 36, and 42 using a five-item scale that included statements such as "I believe things will turn out fine," "I am able to make my goals come true," and "Generally speaking are your expectations of the future 1 = very pessimistic to 4 = very optimistic?" It was found that relative stability in optimism was high from age 27 to 42 (Feldt et al., 2006). Optimism had its 
roots in school success at age 14 and in a child-centered home, including adequate supervision and warm relationships between family members. Furthermore, childcentered parenting was associated with the increase of optimism from age 27 to 36 . Also, feeling satisfied with important areas of one's life (one's choice of occupation, work, leisure, finances, housing) contributed to optimism across the life-span (Feldt et al., 2006).

There was no change in the average level of optimism, but the development of optimism was different in individuals with different personal styles obtained at age 27 (cf. Section 5.3.4). In women who had the Individuated personal style, optimism decreased from age 27 to 42, whereas it in increased in the women with the Traditional personal style (Pulkkinen et al., 2006). The unexpected decrease of optimism among the career-oriented Individuated women may reflect difficulties experienced by them in achieving their career goals or an imbalance between the different spheres of life. Optimism was lowest among the women with the Brittle personal style. Optimism was higher in the men with the Resilient personal style than in the other men.

Self-esteem. Self-esteem was assessed using the Self-Esteem Scale by Rosenberg (1965) at ages 36, 42, and 50. Self-esteem refers to positive or negative evaluation of oneself, and indicates the degree to which one experiences oneself as worthy and capable. We found that high self-esteem was an indicator of good psychological functioning with, for instance, a sense of coherence (Pulkkinen et al., 2006). High self-esteem was preceded by success at work. Thus, self-esteem reacts to experiences. We found the same when we studied the relationships between self-esteem and psychosomatic symptoms (Kinnunen, Feldt, Kinnunen, \& Pulkkinen, 2008). Higher numbers of psychosomatic symptoms at age 36 predicted lower self-esteem at age 42; higher self-esteem at age 36 did not predict lower numbers of psychosomatic symptoms at age 42 .

As was found for optimism, self-esteem was connected to personal styles at ages 36 and 42. Low self-esteem was associated with the Brittle personal style in women, and high self-esteem was associated with the Resilient style in men (Pulkkinen et al., 2005). Relative stability of self-esteem from age 36 to 42 and to 50 was high for both genders, and there were no gender differences in the level of self-esteem at any age. Self-esteem increased from age 36 to 42 in both genders, but decreased in both genders from age 42 to 50 to the same level at which it was at age 36 .

The decrease of self-esteem in both genders between ages 42 and 50 is an interesting phenomenon because self-esteem peaked 10 years later, at age 60 , in a representative US sample (Orth, Trzesniewski, \& Robin, 2010), and a decline occurred only after the age of 60 . In that study, a three-item version of the Self-Esteem Scale by Rosenberg was used with the reliability coefficient of 0.58 . In the JYLS, the ten-item scale was used with the reliability coefficient of 0.84 . The reasons for the early decline in self-esteem should be further investigated (after checking that the findings remain similar to the three-item version used in the JYLS). Ageing is not positively valued in the Finnish labor market where attitudes toward older workers (reaching age 50) include undervaluing their capacities. This can be seen, 
for instance, in the limited opportunities for re-employment for older workers. This general atmosphere may be reflected in people's self-esteem. One's level of achievement at work explains one's level of self-esteem (Pulkkinen et al., 2006).

Conclusions. Optimism was very stable during the adult years. It was rooted in school success and child-centered parenting. Self-esteem increased until the age of 42 but declined after this, possibly due to attitudes toward older people in the labor market. There were consistent differences between individuals with different personal styles. The Resilient style in men was associated with higher optimism and self-esteem, and the Brittle style in women was associated with lower optimism and self-esteem.

\subsection{Turning points}

In the development of a narrative identity, the emergence of autobiographical reasoning is a key factor according to McAdams (2015). He says, referring to Habermas and Bluck, that this reasoning means "a wide set of interpretative operations through which people derive personal meaning from their own autobiographical memories" (p. 259). For example, a person may interpret a specific period from the past to be a turning point, after which he or she was not the same. In this chapter, an answer is sought for the question of whether there were turning points in the participants' lives that affected their future.

\subsubsection{A study of turning points}

Important life events, such as getting married, may open up new opportunities or give rise to a new path in development. These events are known as positive turning points. The concept of turning points refer to important life events, which cause a lasting alteration in the developmental trajectory or some reorientation in how the person prioritizes their activities (Clausen, 1995). Rutter (1996) has remarked that turning points include both experiences over which an individual has no control and those that he or she can consciously choose.

In the JYLS, we were interested in looking into what kind of subjective conception of change in the lives of individuals might, in their 30s, push them off their previous pathways (Rönkä, Oravala, \& Pulkkinen, 2003). Information on turning points was collected at age 36 in the personal interview that covered many life topics. The interviewer introduced the concept of turning points and how they function, describing them as something that brings structure to one's life in the domains of education, work, family, and so on. The participants were told that turning points are like coming to a crossroads in one's life where one makes decisions and choices that will direct one's life and make changes. The participants were asked "What kind of significant life events can you identify in your own life, and the decisions and choices you made with regard to these?" The interviewee was encouraged to mention at least three turning points, and for each turning point, the participant was asked to specify: how much personal choice he/she had had when 
it happened; how old he/she was when it happened, how he/she had evaluated it when it happened (immediate reactions), and how he/she evaluated the consequences of the turning point in hindsight.

The responses were coded into the following main categories: education, work, family, health, interpersonal relationships, quest for identity, loss, standard of living, social transitions, world (e.g., wars), social transitions (e.g., move to another community, military service), and lifestyle (e.g., new hobbies, committing a crime). In addition, the amount of personal choice that the individual had over each turning point was assessed, and each turning point was evaluated on a five-point scale from very positive to very negative in regard to how the person had experienced the event at the time when it took place and what were its long-term consequences.

All participants except for four men mentioned turning points (with nine being the maximum number of turning points that were mentioned). The average number of turning points was 3.8. The mean age at which the turning points had occurred was 24; 12 years before the interview was conducted.

For both men and women, most of the turning points related to family life; 83 percent of the participants mentioned marriage/common-law marriage, separation/divorce, building a family, or entering a new intimate relationship. Educationrelated turning points, including the choice of occupation, were mentioned by 50 percent, work-related turning points by 41 percent, and social transitions by 39 percent. Work-related transitions included positive and negative experiences such as job seeking and job loss, as well as entrepreneurial activity and bankruptcy. The frequencies at which the other categories were mentioned were lower, at 17 percent or less.

Gender differences were found in the perceptions of important turning points. Women more often than men mentioned changes related to building a family, such as the birth of a child, social transitions such as traveling and living temporarily in another country, and changes in the health of people who were close to them. Men, in turn, mentioned turning points in the work sphere more often, particularly related to the reevaluation of their own goals, military service, and lifestyle. Most of the turning points were the normative role transitions of early adulthood: leaving home, ending school, starting a new job, starting or ending a relationship, becoming a mother or father. Nonnormative life events, such as the death of a person close to one, was seldom mentioned as a turning point. Gender differences reflected differences in gender roles.

\subsubsection{Individual differences in the experience of turning points}

In general, the participants experienced that they had made personal choices about the turning points they mentioned. No personal choice was included in the illnesses and deaths of others close to them and their parents' divorce. The experience of having a higher level of personal choice was associated with the more positive evaluation of the turning point (Rönkä et al., 2003). In the cases in which the participant experienced that he or she had plenty of personal choice with 
regard to the turning point, both the immediate reactions to, and the evaluation of, the consequences of the turning point were positive. In those cases in which the participant had no choice regarding the turning point, the immediate reactions were more negative, but the lasting impressions were almost as positive as in the cases when the participant had a high degree of choice. This shows that people have the capacities to cope with unexpected life changes, and are able to see the positive outcomes in these cases. This capacity might help to maintain psychological well-being and satisfaction with life. However, a high number of nonnormative life events in adulthood correlated with depressive symptoms, but only in men (Immonen \& Kokko, 2008).

A lack of important turning points may also affect individuals' lives. This assumption can be made based on the analysis of the regrets that the participants expressed at age 50 (Lahtela, Rajala, Kokko, Räikkönen, \& Feldt, 2014). Half of the participants reported that they regretted something in their lives. Regrets were classified into six categories: studies and education, work and career, family and parenting, relationships and marriage, lifestyle and personal characteristics, and living and finances. The participants who had no regrets reported more ego integrity and life satisfaction than the participants who had regrets. In particular, those regrets regarding studies and education were associated with low ego integrity (measured using the scale by Ryff \& Heincke, 1983) and low satisfaction with life.

The role of turning points in the participants' lives was studied in the light of different developmental trajectories that were formed by cross-tabulating three categories for the number of risk factors ( 0 to 1,2 to 3 , and 4 or more risk factors) and two categories for the number of problems in social functioning ( 0 to 1 and 2 or more problems) resulting in $3 \times 2$ trajectories (Rönkä, Oravala, \& Pulkkinen, 2002). The risk factors comprised aggressiveness, anxiety, problems in adjustment to school, poor school success, low school motivation, low socioeconomic status of the family of origin, and parental drinking problems. Social functioning problems comprised poor financial standing, poor social relationships, poor intimate relationships, unemployment, drinking problems, and criminality.

The analysis revealed that one of the six trajectories could be identified as "very resilient" because the participants on this trajectory had no social functioning problems despite having many risk factors, whereas one trajectory was identified as "vulnerable," because these participants who had social functioning problems also had many risk factors.

The six trajectories did not differ with regard to the total number of turning points mentioned nor in the mean age at which the turning points happened. The trajectories that depicted good social functioning at age 36 were, however, typified by more positive evaluations of both the immediate reactions and the long-term consequences of the turning points than the people on the trajectories of poorer social functioning, particularly the vulnerable subgroup.

The participants who were coping well with their lives, despite several risk factors in childhood, reported positive experiences and plenty of choice in relation to their turning points. They tended to mention turning points relating to their 
standard of living and interpersonal relationships. On the contrary, participants in the vulnerable subgroup perceived turning points more negatively and reported few opportunities for personal choice. They often mentioned nonnormative life events, such as losses and failures, as turning points in their lives. For instance, 40 percent of the vulnerable subgroup and 7 percent of the very resilient subgroup mentioned the death of someone close to them as a turning point in their lives.

\subsubsection{A qualitative analysis of turning points}

A qualitative analysis of the responses of the participants revealed that turning points were often described as a chain of events (Rönkä et al., 2002). The responses included a description of an environmental change (the outer domain), a change in lifestyle (the behavioral domain), and a personal experience (the inner domain). The turning points of the participants on the resilient and vulnerable trajectories were analyzed more closely, as described in the section that follows.

The very resilient individuals underlined an environmental change (the outer domain) as a means of bringing about a positive change. Environmental change included, for instance, finding a partner, giving birth to a child, moving to another place, divorcing from a violent partner, stepping out of a peer network, and finding a person, father-in-law, employer, or somebody else "who takes care of one." The new environment offered more supportive social relationships, positive models, and new roles. They motivated a change in lifestyle (the behavioral domain), as expressed by the participants who said that they had "matured," "grown up mentally," or "settled down."

The turning-point experiences (the inner domain) were described in three ways: in terms of a growth in self-esteem, in the increased ability to cope with difficulties, and in the increase of personal control over one's life. An experience of the growth in self-esteem might have related, for example, to being good at sport, which then opened an opportunity to join a special group for athletics in the army. An experience of the development of coping skills might have related to difficulties she or he had overcome (such as parental drinking problems, living with a violent partner) that had made him or her strong. Furthermore, an experience of the increase of personal control over life was related to one's own active role (planning, choice) in changing one's lifestyle: as a participant said "it was a time of change in my life."

The vulnerable individuals, who had several risk factors in childhood and adolescence and experienced problems in social functioning in adulthood, reported turning-point experiences in the environment (the outer domain) that were negative: exclusion from education or work (e.g., dropping out of school), negative role models (e.g., marriage to an alcoholic partner), little social support (e.g., living alone with a baby), teenage pregnancy, bankruptcy, the placement of one's child in a foster family, or the death of people close to one. The person's lifestyle (the behavioral domain) included drinking, drug, and health problems. A few of the participants asked God for help. In the inner domain, it was typical that the participants had a sense of having little control over life events and a lack of motivation 
to face the future. Sometimes, the individual had experienced a series of life events that he or she had no control over. Positive events in these individuals' lives also occurred, but the general impression of their life course was more negative than that of the very resilient.

Conclusions. The analysis of turning points in the participants' lives demonstrated the important role of planning and control in the lives of individuals with risk factors. This is in line with the principles of the life-course approach to human development that sees individuals as active contributors to their own development (Elder, 1988). Sources of this ability and motivation seem to be in the experience of some form of success, accomplishment, or the ability to experience pleasure in certain activities. They enhance personal confidence and the sense of competence and help one to deal with the dilemmas of life (Quinton and Rutter, 1988; Rutter, 1996). A sense of competence can be fostered by steps that make it more likely that people feel in control of their lives (Rönkä et al., 2002). 


\section{8}

\section{PERSONALITY AND PSYCHOLOGICAL FUNCTIONING}

\subsection{Personality and well-being}

\subsubsection{The concept of mental well-being}

In previous chapters the development of personality was described on three levels: the person as actor, motivated agent, and autobiographical author. The next question is how individuals experience their current life situation: are they satisfied with it and do they feel that their life fulfills the wishes they have for their lives? These experiences have been described in different ways, for example as happiness and well-being. They form part of psychological functioning, which refers to the internal criteria for overall adaptation to life. Other indicators of psychological functioning include emotional and cognitive performance, spiritual experiences, and other existential aspects of life. In this chapter, psychological functioning in the adult years is discussed in relation to individuals' personality characteristics.

The concept of well-being refers to a good or satisfactory condition of existence; a state characterized by health, happiness, and prosperity (from dictionary.com). There are several theories of well-being. In psychology, a tripartite model of mental well-being proposed by Keyes (2002) has received support across many cultures. It covers hedonic, psychological and social well-being. As reviewed by Kokko, Korkalainen, Lyyra, and Feldt (2013), the hedonic view of well-being has been called subjective or emotional well-being, referring to positive feeling states. It is indicated by satisfaction with life overall or satisfaction with different aspects of life, as well as by personal happiness, which is a kind of summary of the presence of positive mood and the absence of negative mood (Ryan \& Deci, 2001).

The psychological view refers to an individual's wishes to realize his/her inner voice for striving toward actualizing his/her potential and growth in an ethically respected way. Carol Ryff (1989) separated out six components in psychological 
well-being (and the respective measures) to describe challenges that an individual encounters in his or her efforts to function positively. The components are selfacceptance, positive relations with others, environmental mastery, autonomy, purpose in life, and personal growth. Three components of these, self-acceptance, environmental mastery, and positive relations, are positively associated with happiness and life satisfaction (Ryff \& Keyes, 1995), which shows that there is a significant overlap between emotional well-being and psychological well-being, but that there is also a difference between the two. Emotional well-being was not considered sufficient for covering existential well-being.

Keyes (1998) argued that in addition to emotional well-being and psychological well-being, which represent a private and personal phenomenon, social wellbeing should be considered in the conceptualization and assessment of well-being. Social well-being focuses on social challenges that an individual may encounter in his or her environment and includes the way these challenges are resolved. Keyes proposed five components of social well-being that resemble the inner components of psychological well-being: social acceptance, social coherence, social integration, social contribution, and social actualization. They describe the more public and societal criteria of positive functioning and mental well-being than the components of emotional and psychological well-being. Social well-being correlates, but not strongly, with life satisfaction and happiness, as well as with psychological well-being (Kokko, Korkalainen et al., 2013). Structurally, it is distinct from them.

In the tripartite model, emotional well-being indicates positive feeling states and psychological well-being and social well-being indicate positive functioning. All of them, if there is an absence of mental illness, characterize an individual's mental health, as reviewed by Kokko et al. (2013). In the cases when the construct of mental well-being is used in reference to mental health, indicators of mental health problems, such as depression or anxiety, should be controlled for. The construct of mental well-being is used here to cover emotional, psychological, and social wellbeing and the absence of mental health problems. Several measures have been used in the JYLS for assessing mental well-being (cf. Section 2.2). Emotional well-being was indicated by mood, life satisfaction, and happiness during the last years. Life satisfaction was an average score of satisfaction with seven life domains. Psychological well-being was assessed by using the Scales of Psychological Well-Being, and social well-being by using the Scales of Social Well-Being. The absence of mental illness was indicated by lack of depressive symptoms assessed by using the Depression scale of General Behavior Inventory.

The findings by Kokko et al. (2013) confirmed that well-being at ages 36 and 42 had three components: emotional well-being, psychological well-being, and a lack of depression. Emotional well-being covered happiness, life satisfaction, positive mood, and negative mood (reversed scoring). When social well-being was added to the analysis at ages 42 and 50, social well-being also contributed significantly to mental well-being (Kokko, Rantanen, \& Pulkkinen, 2015). 


\subsubsection{Continuity in well-being}

People have a high tendency to experience well-being in a similar way across time. Some people experience more well-being than others over time, as shown by the high path coefficient (0.84) both from age 36 to 42 (Kokko, Korkalainen et al., 2013) and from age 42 to 50 (Kokko et al., 2015). The high path coefficient means that over 70 percent of the variance of mental well-being at a later age was explained by mental well-being 6 years earlier in each case.

Further analyses by Kokko concerning the comparison of the means of the scales at different ages indicated that there were no significant differences in the means of psychological well-being and depression between ages 36 and 50 among women or men but there was a trend that psychological well-being was lower at age 42 than at age 36 (Kokko, 2010b). Neither were there changes among women in emotional well-being for positive and negative mood and happiness, but, among men, happiness increased significantly $(\mathrm{p}<0.01)$ from age 42 to 50 . Happiness was at the lower level among men when compared with women at age 42 but reached the happiness level of women at age 50. This change in emotional well-being among men was confirmed by changes in their mood $(p<0.05)$ : an increase in positive mood and a decrease in negative mood when ages 36 and 50 were compared. Due to changes, mainly in men, significant $(\mathrm{p}<0.01)$ improvement in positive mood and happiness occurred from age 42 to 50 in the total group, also including the women. At the same time, social well-being increased significantly $(p=0.000)$ in men and women. As social well-being was not assessed at age 36, it is not known whether the increase of social well-being was progressive throughout adulthood.

Satisfaction with life was studied at ages 36,42 , and 50 by asking the participants to assess their satisfaction with career, job, relationship, habitation, leisure time, and friendships on a four-point scale. It allowed for the comparison of satisfaction with different spheres of life. The ratings showed that less than 5 percent of men and women were very dissatisfied and that most commonly participants were quite satisfied in these areas. Satisfaction was higher with regard to one's current habitation situation, intimate relationship, choice of occupation, and current job than with regard to one's current financial situation and the content of one's leisure time. Gender differences in satisfaction were marginal.

The average life satisfaction across different domains increased from age 36 to 42 and remained on that level until age 50 in the women and the men. There were, however, some changes within single domains (Pulkkinen \& Polet, 2010b). A progressive increase was found in satisfaction with habitation, which shows that participants had been able to move in to more spacious and pleasant flats or houses during the course of their life. Satisfaction with income improved from age 36 to 42 and stayed on that level until age 50 . In terms of satisfaction with the content of leisure time, an increase was found in women from age 36 to 42 , but there was a decrease in men.

Average scores may hide differences between subgroups. Observations on differences in well-being at age 42 between the clusters for personal styles extracted at age 27 indicated that psychological well-being and life satisfaction were higher 
in the Resilient men than in the other men; and that psychological well-being was lower and depression was higher in the Brittle women than in the other women (Pulkkinen et al., 2005). Adult individuals did not consist of a homogeneous group in respect to their well-being.

\subsubsection{The personality traits associated with mental well-being}

It is a common observation that extraverted people look happier than neurotic people. Accordingly, higher emotional and psychological well-being was found to be associated with higher extraversion in the study by Kokko, Tolvanen, and Pulkkinen (2013), whereas higher neuroticism was associated with lower emotional and psychological well-being. Also, conscientiousness was positively, but not highly, associated with emotional and psychological well-being, and openness to experience with psychological well-being. The associations of agreeableness with well-being were the lowest in the study. The study of longitudinal associations between personality traits and well-being were based on the personality traits assessed at ages 33, 42, and 50, and psychological well-being and emotional wellbeing (life satisfaction) assessed at ages 36, 42, and 50. Associations were analyzed with correlation coefficients and bivariate latent growth curve analysis.

These results were in accordance with meta-analytic findings based on 347 samples (Steel, Schmidt, \& Shultz, 2008), which showed that neuroticism is most consistently, but negatively, associated with various components of emotional wellbeing such as life satisfaction, affectivity, and happiness, whereas extraversion is associated with them positively. Openness, conscientiousness, and agreeableness are less strongly associated with emotional well-being, or the connections are more specific. Most studies included in the meta-analysis have been cross-sectional, which means that data on personality traits and well-being have been collected at the same time point. The Big Five personality traits explained 40 to 60 percent of the variance of emotional well-being.

The study by Kokko et al. (2013) also revealed that the initial level of personality traits (at age 33) contributed to psychological well-being at later ages (ages 36, 42, and 50). This means that the higher the initial level of extraversion, the higher the level of well-being was at a later age and, correspondingly, the higher the initial level of neuroticism, the lower the level of psychological well-being was at a later age. Personality traits affect well-being and not vice versa. Openness, however, increased from age 33 to 50 in individuals whose initial level of psychological wellbeing was high. Well-being may give one strength to be open to experiences.

The aforementioned results are based on associations between variables. To analyze whether different groups of individuals in both well-being and personality traits exist, and whether these groups have mutual links, a person-centered method, namely, semi-parametric mixture modeling, was used (Kokko et al., 2015). In these analyses, different developmental trajectories of personality traits and well-being, differing in both the level and developmental course of the characteristics in question, were examined. Three trajectories were obtained for each personality trait as 
TABLE 8.1 Probabilities of following low and high trajectories of psychological well-being

\begin{tabular}{lllr}
\hline Personality traits groups & & \multicolumn{2}{c}{ Psychological well-being groups } \\
\cline { 3 - 4 } & & Low & High \\
\hline \multirow{2}{*}{ Neuroticism } & Low & 0.00 & 0.88 \\
\multirow{2}{*}{ Extraversion } & High & 0.77 & 0.00 \\
& Low & 0.96 & 0.00 \\
Conscientiousness & High & 0.00 & 0.84 \\
\multirow{2}{*}{ Openness } & Low & 0.44 & 0.17 \\
\multirow{2}{*}{ Agreeableness } & High & 0.20 & 0.66 \\
& Low & 0.41 & 0.18 \\
& High & 0.05 & 0.50 \\
& Low & 0.48 & 0.37 \\
\hline
\end{tabular}

Adapted from Kokko, Rantanen, \& Pulkkinen (2015).

Note: Only low and high groups are presented here. For Moderate groups, see Kokko et al. (2015).

well as for psychological well-being: low, moderate, and high. In the low trajectory group, participants had received low scores at each age $(33 / 36,42,50)$ in the variable in question and in the high trajectory group, the participants had received high scores at each age. Table 8.1 shows the probabilities of following the low and high trajectories of psychological well-being for each personality trait in the low and high trajectories of the personality traits.

The probabilities presented in Table 8.1 show that participants who were on the high trajectory of extraversion were most probably ( 84 percent) on the high wellbeing trajectory. Also, the participants who were on the low trajectory of neuroticism were most probably (88 percent) on the high trajectory of psychological well-being. For agreeableness, conscientiousness, and openness, the trajectories of personality and well-being were less strongly linked. These findings were in line with previous results (Kokko et al., 2013; Steel et al., 2008): the associations of personality traits with wellbeing were highest with extraversion (positively) and neuroticism (negatively).

\subsection{The model for personality and psychological functioning}

Differences between the personality profiles extracted from the Big Five personality traits were described in Figure 5.4 (Section 5.3.5) in terms of extraversion and conscientiousness. The comparison of the personality profiles revealed that they differed in the subjective assessments of health (Kinnunen et al., 2012). Low subjective health was associated with the Brittle profile and high subjective health with the Resilient profile. The profiles did not differ in the objective indicators of health such as blood pressure, cholesterol, and body mass index. As self-assessment of health may indicate psychic well-being, the personality profiles were also compared by using several other indicators of psychological functioning in order to see 
whether there was consistency in the differences between the personality profiles (Pulkkinen, Räikkönen, Kinnunen, \& Kokko, 2013).

Table 8.2 presents the means of the profile groups in several indicators of psychological functioning. Consistent differences were found between the Resilient and Brittle profiles. Concurring with the differences in self-assessed health, the Resilient profile individuals were highest in psychological functioning and the Brittle profile individuals were lowest.

- The Resilient profile participants were highest in self-assessed health, psychological well-being, optimism, personal control over their development, and life satisfaction, but lowest in anxiety and depressive symptoms.

- The Brittle profile participants were lowest in self-assessed health and the indicators of positive psychological well-being, but highest in anxiety and depressive symptoms.

- The Undercontrolled and Overcontrolled individuals fell between the Resilient and Brittle individuals in these indicators of positive psychological functioning and in the level of the anxiety and depressive symptoms they experienced.

At each age $(36,42$, and 50), an analysis was carried out to see how much of the variation between the personality profiles was explained by psychological functioning using the multivariate analysis of covariance (MANCOVA) with gender as a covariate (Pulkkinen et al., 2013). The following aspects of psychological functioning were included in the analysis: psychological well-being, optimism, personal control over one's development, depressive symptoms, and anxiety. Psychological functioning related to mental well-being as indicated by these variables, explained the variation between the personality profiles significantly at each age with an increasing effect size with age. For age 36: $F(20,691)=6.82$, $\mathrm{p}<0.001$, effect size $=$ 0.14 ; for age $42: F(20,681)=8.52, \mathrm{p}<0.001$, effect size $=0.17$; and for age 50 : $F(20,651)=11.13, \mathrm{p}<0.001$, effect size $=0.22$.

The differences in psychological functioning between the profile groups are depicted in Figure 8.1 within the framework of extraversion and conscientiousness (Figure 5.4). The Resilient profile was high in all aspects of psychological functioning, whereas the Brittle profile was low in them. The indicators of psychological functioning related to mental well-being did not differentiate the personality profiles in the dimension from the Undercontrolled profile group to the Overcontrolled group. Therefore, their differences were researched using behavioral indicators (Pulkkinen et al., 2013). It was found that the Undercontrolled individuals were higher than the other profiles (particularly the Overcontrolled) in monotony avoidance at ages 36, 42, and 50. Likewise, in impulsivity, the Undercontrolled profile was highest. The Overcontrolled profile was lowest in impulsivity at age 50. Monotony avoidance and impulsivity explained the variation between the personality profiles significantly with the highest effect size being at age 42. For age 36: $F(8,464)=4.18, \mathrm{p}<0.001$, effect size $=0.07$; for age 42: $F(8,440)=8.07, \mathrm{p}<0.001$, effect size $=0.13$; and for age $50, F(8,414)=4.82$, $\mathrm{p}<0.001$, effect size $=0.09$. 


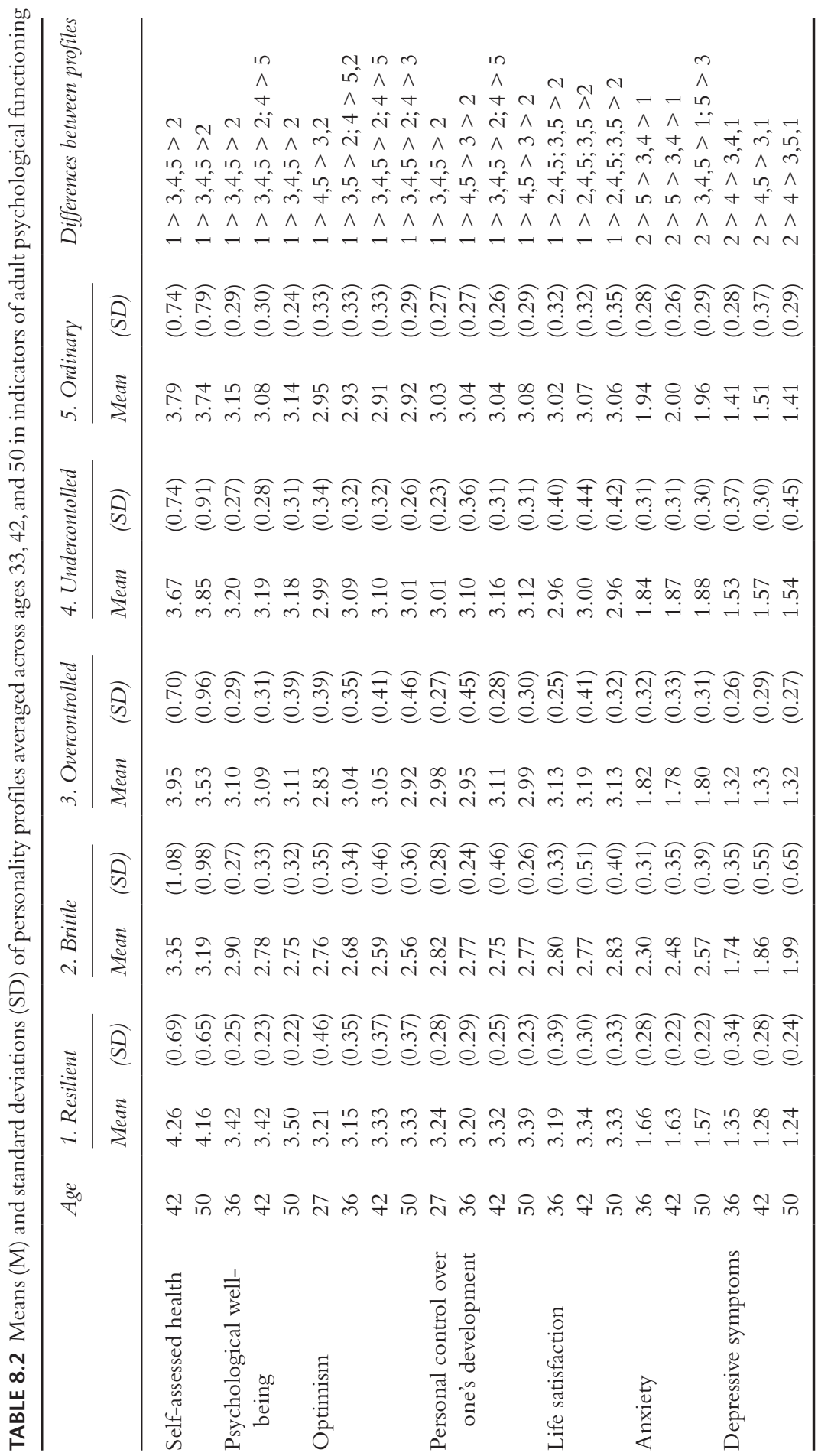




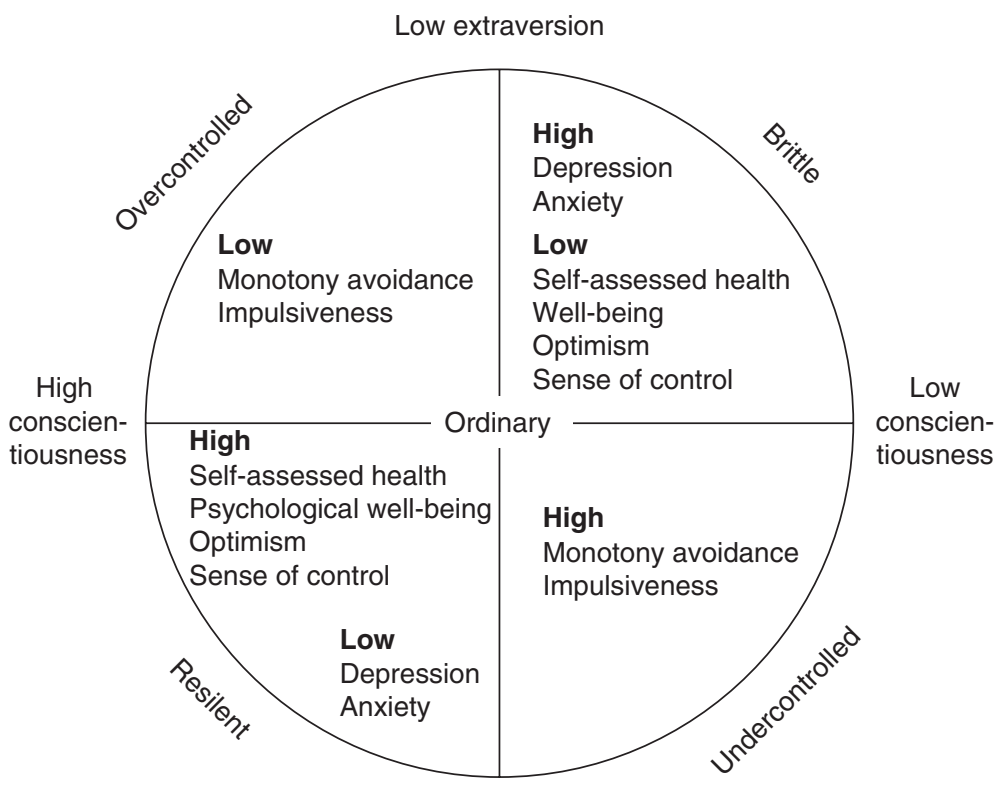

High extraversion

FIGURE 8.1 A model for personality and psychological functioning.

On the basis of the Model for Personality and Psychological Functioning, a number of hypotheses can be generated on individual differences in other indicators of psychological functioning, as well as in social functioning. Undercontrolled and Overcontrolled profile groups can be expected to differ in social functioning. These connections are presented in Part III. The integrated model, which includes both psychological and social functioning, is presented in Part IV.

\subsection{Explanations for the associations between personality traits and psychological functioning}

Explanations for the associations between personality traits and psychological functioning can be looked for at different levels of human functioning and with different conceptual perspectives, such as dopamine circuits in the brain (DeYoung, 2014), the use of antecedent-focused emotion regulation strategy for the reinterpretation of emotional stimuli (Larsen \& Prizmic, 2004), and temperament (Rothbart, 2011). A close link between two dimensions of the Big Five (extraversion and neuroticism) and the two-dimensional model of emotions with positive affect and negative affect (Watson \& Tellegen, 1985) has been found in the literature, as reviewed by De Raad and Kokkonen (2000). They demonstrated the commonality between those systems by making use of circumplex representations resembling the model of Personality and Psychological Functioning, but in the latter model, 
the two-dimensional framework is formed by extraversion and conscientiousness. In this context, three conceptual perspectives on the explanation of associations between personality traits and psychological functioning are presented for discussion: extraversion, conscientiousness, and emotion regulation.

Extraversion. High extraversion, when it is combined with high conscientiousness, is associated with positive psychological functioning. The connection between extraversion and well-being starts from childhood. As shown by Kokko et al. (2015), behavioral activity at age 8 accounted for behavioral activity at age 14, which was further linked to mental well-being at age 42. Mental well-being was formed by different indicators of emotional well-being (positive and negative mood, life satisfaction, and happiness), psychological well-being, social well-being, and low depressive symptoms.

The connection of mental well-being with active, extraverted behavior might mean that there is a common biological basis for this. Behavioral activity in children has a strong component of temperament. Watson and Clark (1997) reviewed evidence that both positive affectivity and other component traits of extraversion are reflections of a single integrated system related to approach and avoidance behaviors. They refer to Tellegen, who has argued that extraversion and positive affectivity levels may "represent individual differences in sensitivity to pleasurable stimuli" (Watson \& Clark, 1997, p. 787).

Explanations for the associations of extraversion and neuroticism with mental well-being, presented by Steel et al. (2008) are on the same line, but also offer other views. It is possible that personality and well-being share a biological mechanism or a neural substrate such as Gray's behavioral inhibition and activation systems, and the neurotransmitters of dopamine and serotonin. Dopamine is connected to extraversion and positive experience, and serotonin to neuroticism and depression. Another explanation might be in genetic information that personality traits and well-being share via common genes.

Indirect associations may be caused by the consequences of extraverted or neurotic behavior that may predispose individuals to situations and life events that bring psychological or emotional well-being or ill-being with them. A recent study by Hietalahti, Rantanen, and Kokko (2016) using the JYLS data provides an example. It was found that extraversion and mental well-being correlated and that both of them were associated with goals: leisure-related goals in women and performance-related goals in men. Furthermore, it was found that goals mediated the connection between personality traits and well-being. Extraversion was associated with the pursuit of, and investment in, goals, which, in turn, contributed to psychological well-being.

Conscientiousness. The trait that has been found to play a significant role in health is conscientiousness, as reviewed by Friedman and Kern (2014). They write "Perhaps the most exciting recent discovery to emerge in the area of personality, well-being, and health is the lifelong importance of conscientiousness" (p. 731). Conscientious individuals are dependable, well organized and persistent and they stay healthier and live longer. According to Friedman and Kern, explanations 
for the connection between conscientiousness and health have been sought from healthier behaviors concerning smoking, eating, and wearing seat belts; situation selection by choosing healthier environments and healthier human relationships; more meaningful careers, better education, and higher income; better emotion regulation ability; and gene-by-environment interactions. Serotonin level affects personality and conscientiousness and regulates bodily functions such as sleep.

In the literature, explanations for the connections between personality traits and well-being have been focused on individual traits, extraversion, conscientiousness, and neuroticism. The combination of extraversion and conscientiousness has received little attention. Inconsistent findings concerning the connection between extraversion and health (as reviewed by Kinnunen et al., 2012) become understandable when the trait profiles are considered: high extraversion combined with high conscientiousness (in the Resilients) is associated with high subjective health, but extraversion combined with low conscientiousness (in the Undercontrolled) is associated with lower subjective health. Low conscientiousness with high extraversion and openness to experience may make the Undercontrolled vulnerable to a lifestyle that triggers poorer health.

Emotion regulation. The model for Personality and Psychological Functioning (Figure 8.1) concurs with the model for the Unfolding of Socioemotional Behavior (USB) (Figure 4.1). The USB model suggests that socioemotional development toward adult styles of life could be described in the framework of behavioral activity and self-regulation. In adulthood, extraversion and conscientiousness can be seen as approximating these dimensions. The explanatory concept for the development of the Resilient and Brittle styles of life is emotion regulation in the USB model: antecedent-oriented emotion regulation for the Resilient and responseoriented regulation for the Brittle. Emotion regulation involves anticipatory cognitive processes that affect the adaptability of emotions to the situational context. Indicators of high psychological functioning are most likely associated with effective emotion regulation, whereas indicators of low psychological functioning are most likely associated with negative emotions, which an individual finds difficult to work through.

Neuroticism is the personality trait that is most highly associated with low psychological and emotional well-being (Kokko et al., 2013), and high neuroticism is most likely to be found in the Brittle individuals who are low in extraversion and conscientiousness (cf. Section 5.3.5). Neuroticism reflects low emotion regulation and neurotic individuals are vulnerable to being overwhelmed by unwanted emotions. Neuroticism is low in the Resilient individuals but it is also low in all other personality profiles when compared with the Brittle. The reason for this most likely is that the Neuroticism scale is not bipolar: it does not include a question for antecedent-focused emotion regulation. A zero score in the Neuroticism scale does not yet indicate an active emotion processing in an anticipatory way. Different types of items would be needed for assessing positive thinking, active and constructive confrontation, and empathy toward other people. For the differentiation of the whole population, the power of neuroticism alone is limited. 
152 Personality and psychological functioning

Behavior regulation, the other component of self-regulation in the USB model, did not differentiate the profile groups in those aspects of psychological functioning that concerned positive or negative emotions and well-being. Differences were in the control of behavioral impulses and in the need for external stimuli. The function of behavior regulation in human development is analyzed in Part III in the context of the development of social functioning. 


\section{PART III}

\section{The development of social functioning}


$\Longrightarrow$ Taylor \& Francis

Taylor \& Francis Group

http://taylorandfrancis.com 


\section{9}

\section{SOCIAL DEVELOPMENT AND SOCIAL FUNCTIONING}

\subsection{Conceptual approaches to social functioning}

Interest in social development has gradually expanded in psychological literature from the maturation of children's social competence to the conceptualization of an individual as an active agent in his or her environment. Interest has also expanded from the socialization of children to children's capacities for balancing internal needs and external demands for optimal functioning; in other words, from "other" regulation to self-regulation (Collins, 2003). Extensively studied areas researched in social development, for instance, comprise of family-related questions such as attachment; peer relations; social and moral reasoning; cooperation, competition, and bullying; and attitudes and prejudices. Thin (2016) has analyzed social behavior on four levels: individual, interpersonal, organizational, and societal or global level. For instance, at the individual level, social capability involves prosocial attitudes, empathy, attachment, active engagement, and social confidence, whereas at the organizational or communal level, social capability involves fair commitment and engagement in goal setting. Thus, the concept of social behavior covers a wide range of behaviors. In the JYLS, the focus has been on the development of social functioning.

There is no consensus about the taxonomy of social functioning capacities. Social functioning may be seen in playing various roles, handling money, work and other daily tasks, and in participation in social life and leisure activities, coping with substance use, and feeling a sense of belonging. The concept of social functioning is more commonly used in social work than in psychology. Kananoja (1983), who introduced the concept of social functioning to Finnish social work, analyzed it using two parts of the concept. "Social," which refers to living in relationships with other people and assuming responsibility for other people; and "functioning," which refers to the awareness, resources, and personality 
of an individual. Awareness means understanding oneself as a person who has goals and understanding the surrounding world. Resources may be social skills, cultural, economic, and power related. Personality and its development include both the capacity to function in relation to other people and the capacity for self-regulation. She defined self-regulation as one's capacity to regulate psychic activity in relation to one's needs and emotions. The background of the concept of social functioning was in the International Classification of Impairments, Disabilities and Handicaps by WHO (1980), as a consequence of which, attention was turned to circumstances that may reduce or increase disabilities and to the clarification of the definition of goals of rehabilitation as a process that aims at enhancing social functioning to cope with social situations and participate in social relationships. An individual both has his/her social relationships and is an active social actor in society, and they together define his/her social functioning (Heikkinen, 2013).

Positive social functioning is an implicit goal in several theoretical approaches to human development: growth models, life-span models, and life-course models (Pulkkinen \& Caspi, 2002). Life-span specifies the temporal order of life stages, such as childhood, adolescence, and adulthood, whereas life-course specifies the demands of social roles at different stages. In order to understand social development, one should simultaneously consider physical maturation, psychological development, social-role demands, and life context. Life-span developmental trajectories for biological, cognitive, emotional, and social functioning are intertwined with each other and with life-course trajectories concerning, for instance, family, education, and work (Pulkkinen, 2000).

Common to different models is the analysis of the ways in which individuals try to handle age-graded tasks (e.g., developmental tasks at different ages), although they differ in the extent to which the goals of successful development have been specified. Key constructs include, for instance, successful resolution of psychosocial crises (Erikson, 1950); accomplishment of developmental tasks (Havighurst, 1953); realization of one's potential (Maslow, 1954); social trajectories (Elder, 1998); and the maximization of desirable goals or outcomes and the avoidance of undesirable outcomes (Baltes, Lindenberger, \& Staudinger, 1998). A comprehensive description of the developmental stages from the child's birth to old age and successful psychosocial outcomes was presented by Erikson (1950). His assumption was that a successful passing into the next phase presupposes leaving behind or resolving the conflicts of the previous stage. The entire life-span is required for all the functions of psychological growth to appear and to become integrated.

One way to make a distinction between personality development and social development is to define their differences in the function of development. Personality development concerns the individuation of a person in becoming a unique personality, whereas social development concerns the adaptation of an individual into society to enable him or her to function successfully with other individuals (Pulkkinen, 2002). As noted by Masten and Coatsworth (1995), "[h]uman individuals as living organisms, must maintain coherence and organization as a unit, while they interact with the environment, including other individuals, and also 
function as part of a larger system” (1995, p. 715). Correspondingly, psychological functioning has internal criteria, whereas social functioning has external criteria for functioning in life. Internal criteria comprise subjective experiences such as mental well-being and self-reflections on past, present, and the future. External criteria in turn consist of actions in human relationships and society through its institutions such as family and work, acceptance of social norms, and agency, that is, by being an active actor and agent in one's personal life and society.

\subsection{The spheres of life}

Social functioning takes place in different spheres of life. The complex context of social functioning was described by Pulkkinen (2010a) with a metaphorical picture of a person who is steering his or her life through different domains (Figure 9.1). The energy of the engine comes from the person's characteristics of acting, feeling and thinking, their goals and values, existential experiences of the past, present, and future, mental well-being, and, in general, from his or her psychological and social functioning capacities in the actual context.

The main domains of adult life are summarized as propellers for family, work, leisure, and health. The domains of life are not separate entities but are related to each other in many ways, such as work-family balance, recovery from work through one's activities on one's free time, health-promoting or healthcompromising behavior during one's free time, and participating in the care functions of the family. Even though there is only one engine depicted in the picture,

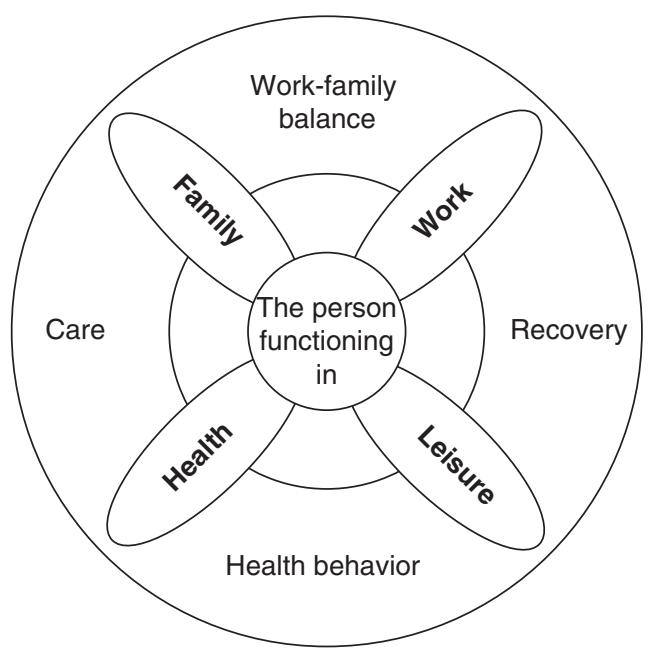

FIGURE 9.1 A human being steering his or her functioning through different spheres of life.

Source: Pulkkinen (2010a). Miltä keski-ikä näyttää? [What does middle-age look like?] In L. Pulkkinen \& K. Kokko (Eds.), Keski-ikä elämänvaiheena [Middle age as a stage of life.] (Reports from the Department of Psychology, No 352, pp. 97-106). University of Jyväskylä, Finland. Adapted with permission. 
each individual acts in society in interaction with other individuals, and lives in a certain culture or subculture. These domains create frameworks of action for individuals through opportunity structures, rules and legislation, and various institutions and services.

The domain of life that gives most the pleasure to adults is family, when the participants were asked to prioritize family, work, friends, and leisure pursuits at ages 27, 36, 42, and 50 (Section 6.3.1). The care activities in people's lives are focused on family. Depending on the definition of the family and the social services available, the care functions may extend from the partner relationship and children, to grandchildren and parents. New family constellations extend the concept of family and care functions from biological parenting to social parenting.

Work took second place as the source of pleasure at ages 27 and 36, but later it dropped to fourth place. The average decline of work as a source of satisfaction is an interesting question. Work and family may be in conflict with each other in terms of the use of time and energy, but on the other hand, work was in second place as a source of satisfaction when the participants' children were young. Lower satisfaction with work in middle age may be associated with the content of work, the challenges of which do not meet the needs of middle aged people. Recovery from work at an older age may also become more difficult and, therefore, leisure pursuits become more important sources of satisfaction later on in life.

The domain that increases in significance with age is health. It was not included in the comparison of family, work, friends, and leisure, but the importance of health came out in other comparisons. People generally see that their future depends on themselves and they are worried about their health.

In the following chapters, core findings of the JYLS on social functioning in these spheres in middle age are presented. The next chapters are, however, devoted to processes and problems in social functioning across different domains. Individuals have resources for positive social functioning, but they also meet with various risk factors that tend to reduce the likelihood of positive functioning. The hypothesis is that risk factors and their negative consequences tend to accumulate in individuals whose self-regulation, particularly behavior regulation, is low.

\subsection{Processes in social functioning}

Life experiences and personal characteristics may support positive social functioning, which has gained more attention as a result of the emergence of positive psychology at the beginning of this millennium. The concept "positive psychology" focuses on the study of positive subjective experiences, positive individual traits, and institutions such as education that enable positive experiences and traits (Seligman \& Csikzentmihalyi, 2000). Several framework models have been developed within the positive psychology movement to understand the optimal functioning of individuals, as reviewed by Rusk and Waters (2014). In the JYLS, the study of positive social functioning began in the 1970s and was connected to the study of socioemotional development covering both positive and problem behaviors. 
In empirical psychological research, various problems in functioning often receive more attention than positive functioning. Problems may concern hyperactivity, aggression, trouble with the law, and school or job failure (so-called externalizing problem behaviors), or depression, anxiety, and negative self-perceptions (so-called internalizing problem behaviors). One can regard the former as social functioning problems and the latter as psychological functioning problems. They are typically treated in society in different ways: social functioning problems receive punishment and less therapeutic treatment than psychological functioning problems. Within the model for Unfolding of Socioemotional Behavior (Figure 4.1) and empirical research on developmental paths (Figure 5.1) with their backgrounds (Table 5.3), it can be understood that externalizing problems (associated with the Reveller style of life) and internalizing problems (associated with the Loser style of life) may have a common origin in unsupportive and stressful life circumstances, but individuals' temperamental differences affect variations in the quality of their reactions to adversity either outwards or inwards.

Antecedents of an individual's positive development may be found in so-called resource factors, which include both individual factors such as cognitive capacities and personality characteristics, and external factors such as education and parenting (Masten \& Coastworth, 1995). Risk factors, which increase the likelihood of problem behaviors, can, likewise, be found both in individual and external factors (Pulkkinen, 2002). Furthermore, there may be protective factors, which may balance, compete with, compensate for, or reduce the impact of risk factors (Garmezy, 1985; Schoon, 2006). They prevent negative chain reactions and open up new opportunities (Werner \& Smith, 1992). Protective factors operate to buffer a child or ameliorate the effects of adversity.

We have studied resource factors for social functioning in different ways as illustrated by three studies. The first study (Pulkkinen, Nygren, \& Kokko, 2002) concerned antecedents of social functioning on a general level and the second study (Pulkkinen, Lyyra, \& Kokko, 2011) on a specific level. The third study (Kokko \& Pulkkinen, 2000) was focused on risk and protective factors of social functioning.

Study 1. In the study by Pulkkinen et al. (2002), positive development was defined on the basis of (1) social functioning using external criteria such as adaptation to society through work, acceptance of social norms, and responsible behavior; and (2) psychological functioning using internal criteria such as subjective measures of mental well-being and self-reflections. We searched indicators of positive social functioning from the participants' work, socialization to society, and the use of alcohol when they were 36 years old and chose the following indicators for them: stability of career path, responses to an inventory that assesses the respect of social norms (Karolinska Scales of Personality, Socialization) and controlled use of alcohol. The indicators of positive psychological functioning were satisfaction with life, self-esteem, and psychological well-being, which were assessed with inventories. We tried to find factors in these participants' childhood that might explain positive development in adulthood. We expected that the participants' 
self-regulation in childhood would be one of the resource factors and school success another one.

Furthermore, we thought that good family circumstances would promote positive social and psychological development. We chose the parents' socioeconomic status, controlled use of alcohol, and child-centered home atmosphere to indicate good circumstances. The variable for child-centered home was based on the participants' recollections of parenting practices and home atmosphere at age 14, when they were 27 years old (Kokko \& Pulkkinen, 2000). A composite score of the following variables was used: good parental relationship, good relationship with the father, maternal support, maternal supervision, and no physical punishment used in one's upbringing. A comparison of memories about parenting and the home atmosphere at age 27 with the prospective data on them collected at age 14 with a subsample of 154 participants (42 percent of the original sample) revealed significant correlations on the level of individual variables (Pulkkinen, 1990b) and on the level of a scale for child-centered versus parent-centered parenting (Männikkö \& Pulkkinen, 2001). We chose to use retrospective data in order to keep the sample size as large as possible.

The results (LISREL model for men in Figure 9.2) showed that latent factors for social functioning and psychological functioning were formed by assumed criteria. Psychological functioning correlated with social functioning, but was not directly explained by the developmental antecedents in childhood. Only the level of social functioning was highly explained by a broad latent childhood factor, called favorable developmental backgrounds. These consisted of indicators of individual development such as high self-regulation in childhood and high school success in adoles-

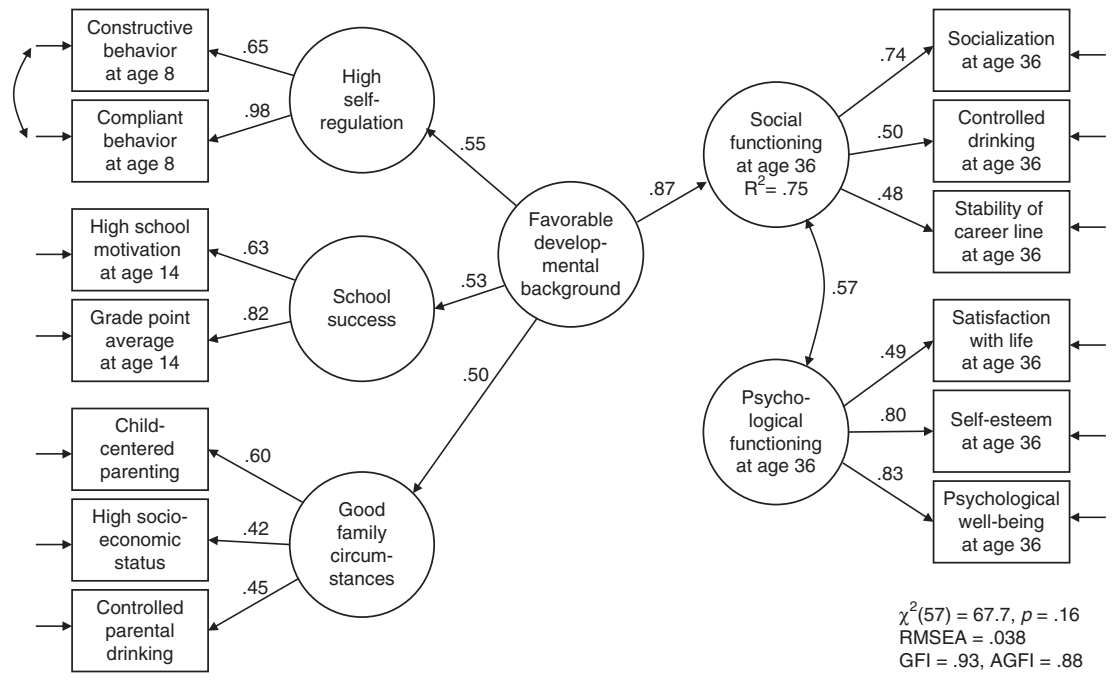

FIGURE 9.2 Childhood antecedents of adult social functioning: males.

Source: From Pulkkinen, L., Nygren, H., \& Kokko, K. (2002). Successful development: Childhood antecedents of adaptive psychosocial functioning in adulthood. Journal of Adult Development, 9, 251-65. (Figure 2, p. 260). (C) 2002 Plenum Publishing Corporation. Reprinted with permission. 
cence; and of good family circumstances covering child-centered home, controlled parental drinking, and high socioeconomic status. The connection between favorable developmental backgrounds at age 14 and social functioning at age 36 was so high that there were hardly any participants whose social functioning would have been poor if their developmental backgrounds were good (Pulkkinen, 2004).

In the model for women, a general latent factor for favorable developmental antecedents did not exist. Instead, there was a more specific latent childhood factor formed by constructive behavior and school success that directly explained the stability of the individual's career path. Another specific latent childhood factor, formed by child-centered home and controlled parental drinking, explained women's socialization and controlled drinking. The socioeconomic status of the family of origin did not explain women's social functioning. The differences in the latent childhood factors between the male and female models showed that the antecedents of social functioning accumulated more in males than in females.

Study 2. In the study by Pulkkinen et al. (2011), antecedents of the quality of adult social relationships were searched from childhood and their connections with later psychological functioning were examined. The results confirmed that self-regulation, covering both compliance and constructiveness at age 8 explained the breadth of the social network at age 27 in men and women (Figure 9.3).
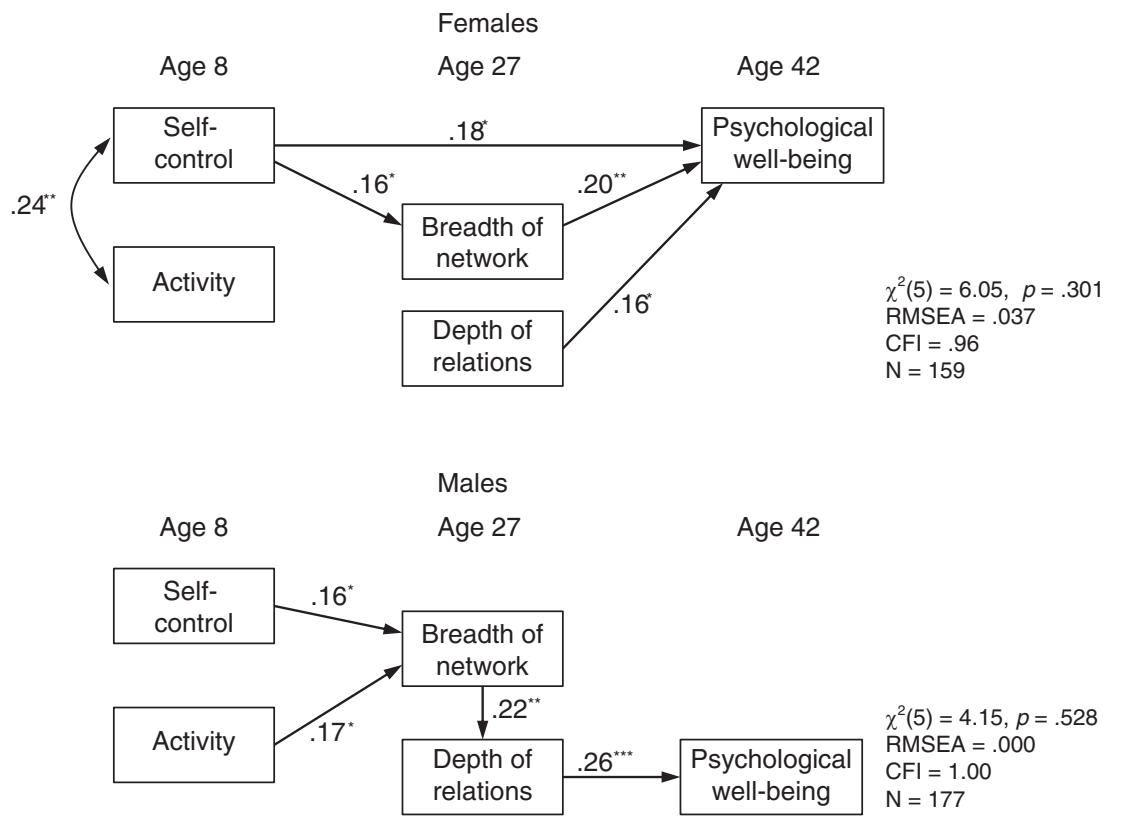

FIGURE 9.3 Paths from self-control and behavioral activity in childhood to middle-age psychological well-being: females and males.

Source: Pulkkinen, L., Lyyra, A.-L., \& Kokko, K. (2011). Is social capital a mediator between self-control and psychological and social functioning across 34 years? International Journal of Behavioral Development, 35(6), 475-81. (Figure 1, p. 479 and Figure 3, p. 480.) Publisher: SAGE. (C) 2011 The Authors. 
The breadth of network referred to one's membership in voluntary organizations, the diversity of visitors at home, the frequency of visiting others, greeting and chatting with neighbors, frequent contacts with relatives, support from the authorities, and voting in elections. The other aspect of social relationships, the depth of relations, was not directly explained by childhood self-regulation. The depth of relations included variables concerning the number of one's own close relatives and one's spouse's relatives; the number of close friends one had and people to confide in; meeting workmates, friends, or neighbors at home; and receiving help and support from friends, workmates, or neighbors.

Positive functioning in human relationships was associated with psychological well-being with the gender difference that in women, the breadth of network explained psychological well-being, but in men, it was the depth of human relationships that explained psychological well-being. Furthermore, with self-regulation at age 8 , both aspects of social relationships made independent contributions to psychological well-being in women. It was also found out that the breadth of one's network partially mediated self-regulation's connection with well-being. This means that when self-regulation was high, the network tended to be larger and it contributed to the person's well-being.

Gender differences in the connections of social relationships with problems in social interaction were similar to those for psychological well-being. Having a broad network reduced the risk of aggression in women. In men, it was the depth of relations that reduced the risk of aggression. Also, high self-regulation in childhood reduced the risk of men's aggression in middle age.

Study 3. In the study by Kokko and Pulkkinen (2000), risk factors for social functioning and the ways in which protective factors might operate were studied in relation to long-term unemployment (lasting for more than 24 months). It was found that aggressive behavior at age 8 explained long-term unemployment via school maladjustment at age 14 and via problem drinking at age 27 (Figure 9.4). School maladjustment was indicated by poor school success and low interest in schoolwork, sanctions at school, and truancy. It predicted long-term unemployment both directly and indirectly, via problem drinking and the lack of occupational alternatives. Thus, childhood aggressive behavior did not directly explain problems in adult social functioning, but there was a cumulative process of problem behaviors by adolescence that was directly and indirectly associated with adult behaviors. The sample included males and females.

Protective factors were sought out from possible moderators, which would provide aggressive children with a buffer against becoming unemployed. Both environmental and personal protective factors were considered. It was assumed that individuals, who live in a supportive parenting environment and are capable of prosocial behavior in spite of their aggressive tendencies, may be able to cope with critical life situations more positively than individuals who are less capable of prosocial behavior.

Personal protective factors were sought in self-regulation (called prosociality by Kokko \& Pulkkinen, 2000). These covered constructive behavior (high emotion 


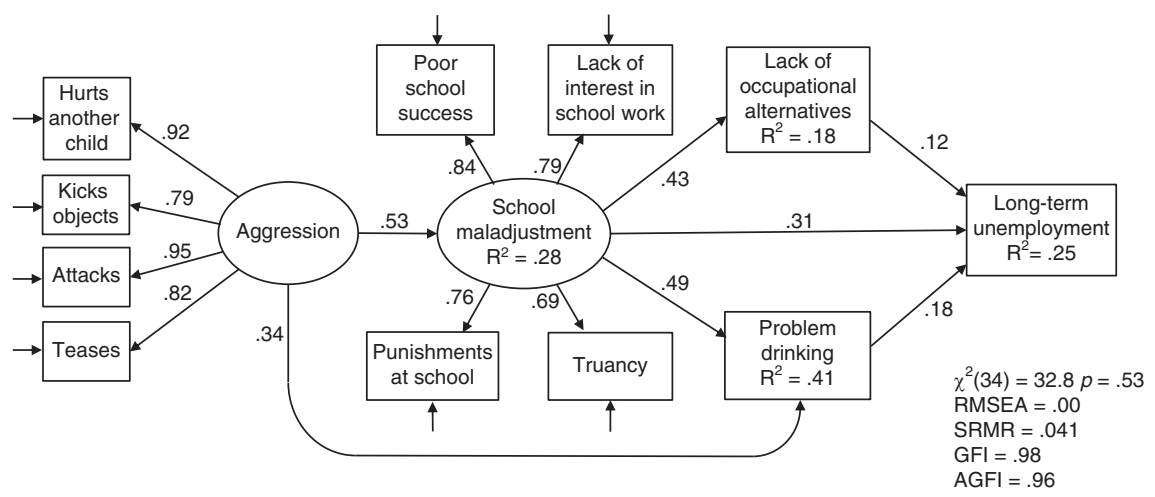

FIGURE 9.4 LISREL model of the cycle of maladaptation between aggression in childhood and long-term unemployment in adulthood.

Source: Kokko, K., \& Pulkkinen, L. (2000). Aggression in childhood and long-term unemployment in adulthood: A cycle of maladaptation and some protective factors. Developmental Psychology, 3, 463-72. (Figure 1, p. 467). (C) 2000 American Psychological Association. Reprinted with permission.

regulation with items for positive coping with conflict, active confrontation, and consideration of others with empathy and helpfulness), mood stability, being a good leader, and lack of disobedience toward the teacher. Parenting and relationships between family members were chosen to represent environmental factors, because child-centered parenting is related to positive development (Pulkkinen, 1982). The scale for the participants' recollections of parenting practices and home atmosphere at age 14 when they were 27 years was developed by Kokko and Pulkkinen (see Study 1).

The results showed that for individuals who belonged to the top quarter of the aggression score distribution as children, both child-centered parenting and the child's high self-regulation protected the participants from becoming longterm unemployed up until the age of 36. The probability of becoming long-term unemployed was high (i.e., 45 percent), among the aggressive children whose self-regulation was very low (more than one standard deviation [SD] below the mean of aggressive children) and the parents' child-centeredness was also very low (1 SD below the mean of the aggressive children). The probability of becoming long-term unemployed was only 1 percent among the aggressive children whose self-regulation was higher (1 SD above the mean of the aggressive children) and the parents' child-centeredness was also high (1 SD above the mean). Thus, the aggressive children who had received more child-centered parenting and who, in general, had more self-regulation than aggressive children, were less likely to become long-term unemployed.

The effects of child-centered parenting and the child's self-regulation operated both directly and in conjunction with the risk factor, here aggression. This double function of protective factors has also been found by other researchers (Freitas \& 


\section{The development of social functioning}

Downey, 1998; Rutter, 1994; Werner \& Smith, 1992). It means that the same factors may be general resource factors for development and function as specific protective factors when the child is exposed to a risk situation. The interaction between self-regulation and aggressive tendencies as the predictors of long-term unemployment is possible, because these tendencies are not mutually exclusive. Individuals may vary their behavior in respect to situational factors and those who show relatively more flexibility are in a better position. The interaction between parenting and aggression shows, in turn, that parents may support children who are aggressive in childhood to avoid the formation of the cycle of maladjustment and pathways that lead to long-term unemployment. 


\section{0}

\section{PROBLEMS IN SOCIAL FUNCTIONING}

\subsection{Antisocial development}

\subsubsection{Antisocial behavior}

The first question to be analyzed in this chapter is whether aggressive behavior in childhood predicts antisocial development. Problems in self-regulation and social functioning do not generally appear in isolation; therefore, the second question is how aggressive behavior is patterned with other problem behaviors and how the patterns predict crime and other problems in social functioning. The third question concerns the future of criminal offenders, that is, in what respect the individuals who had committed offenses at different times in their life course differed from nonoffenders in personality characteristics and social and psychological functioning in middle age.

There is no unanimous definition of the concept of antisocial behavior; each discipline has its own conceptual systems and ways of collecting data. The age of the person whose behavior is being observed also affects the selection of the markers of antisocial behavior. Antisocial behavior is a large umbrella concept that encompasses not only delinquency and crime, but also disruptive behavior of children below the age of criminal responsibility (Pulkkinen, 2001; Rutter, Giller, \& Hagel, 1998).

Developmental psychologists generally use the concept of problem behavior for antisocial behavior and include in it various forms of externalizing problem behaviors such as aggression and disobedience, and norm-breaking behaviors such as dishonesty, stealing, and truancy. The younger the children are, the more their "antisocial" behavior extends beyond acts that break the law. Antisocial behavior is assessed on a quantitative dimension; an individual's position on it depends on how typical certain behaviors are of him or her. Informants are often teachers or parents. Developmental approaches to antisocial behavior are also focused on the developmental antecedents and accumulation of problem behaviors. 
Clinical assessment of antisocial behavior in children is based on specific categorical constructs and their criteria, such as oppositional defiant disorder in smaller children, including temper tantrums and irritable behavior, and conduct disorder in older children referring to a persistent pattern of behavior that violates the rights of others or age-appropriate societal norms. Information about psychopathological syndromes is mostly received from parents. A third diagnosis, antisocial personality disorder, can be applied to individuals who are at least 18 years of age.

Criminologists categorize antisocial behavior on the basis of offenses committed by a person who has passed the crime responsibility. This age varies from 7 to 18 years in different countries; it is 15 years in Finland, which means that crimes of children under that age are not registered. The most common crime among young people is theft. In a meta-analysis of factor analytic studies of antisocial behavior in young people, four major categories were found: (1) aggression, such as assault and cruelty; (2) property violations, such as stealing and vandalism; (3) oppositional behavior, such as angry and stubborn; and (4) status violations, such as substance use and truancy (Frick et al., 1993).

In the JYLS, antisocial behavior has been approached developmentally since the early 1980s. Criminal records were examined in two registers: the government register and the police register. The government register, which includes offenses where sentences have been imprisonment, was examined at age 20 and thereafter, once in 5 years. The local police register was examined at ages 20 and 26. It contains all arrests, including petty offenses for which the person was not prosecuted, although the police might have sought punishment. At age 20, 36 percent of all 196 men in the initial sample and 10 percent of all 173 women were found in the local police register; 19 men and 4 women were also in the government register (Pulkkinen, 1983a). By age 32, the number of registered men had increased to 48 percent of the men and the number of women to 16 percent of the women (Hämäläinen \& Pulkkinen, 1995).

Criminal offenses were highly concentrated. A small number of men accounted for half of all arrests found in the male participants of the JYLS until age 27: 4.1 percent of men (Pulkkinen, 1988). For comparison, this figure was 5.5 percent of males in England in the Cambridge Study in Delinquent Development at the corresponding age (Farrington, 1986). The convictions in the JYLS were even more concentrated. Only 3 men of the 32 convicted men until age 32 accounted for half of the convictions of these 32 men. It means 1.5 percent of the whole male sample. In the female sample, 6 women had been convicted, and 2 of them accounted for 82 percent of all their convictions (Hämäläinen \& Pulkkinen, 1995).

Men altogether had committed 171 crimes by age 32; violations against property (theft, fraud, forgery) was the largest category (52 percent of all crimes). Other offense categories were traffic offenses (27 percent of crimes), violent offenses (19 percent), and drug offenses ( 2 percent). Several men (44 percent of the convicted) had only one conviction (Hämäläinen \& Pulkkinen, 1995). Out of the six convicted women, two women had been convicted of several thefts, frauds, and assaults, and the rest had committed one type of offense, such as drunken driving or theft. 
The number of different individuals who appeared in criminal registers at least once in a year reached its peak at age 17 after which the prevalence remained high until age 20 with another peak at age 24 (Pulkkinen, 1988). The sudden peak at age 24 was caused by a group of the first-time offenders who were arrested for drunkenness, traffic offenses, and frauds. For females, there was no peak (Hämäläinen \& Pulkkinen, 1995). After early adulthood, the prevalence of criminal offending declined and offending was concentrated in a few individuals.

\subsubsection{From aggression to antisocial behavior}

Aggressive behavior is generally considered an antecedent of criminal behavior. Correlations between aggressive behavior in childhood and criminal offending in adulthood was studied at ages 20 and 27. At age 20, offenses were categorized into theft offenses, violent offenses, and alcohol offenses (Pulkkinen, 1983a). The category for alcohol offenses included arrests because of drunkenness, illegal disposal and sale of alcohol, and drunken driving. In the 1970s, drunken behavior in public places was not allowed and it caused arrests by police. It was the most frequent reason for an arrest. Scales were constructed for alcohol, theft, and violence offenses by weighting offenses based on their severity.

Different types of aggression at age 8, except facial aggression, significantly correlated in males with different types of offenses, as well as with the total number of offenses. The highest correlation was between physical aggression and the total number of offenses (0.37; Pulkkinen, 1987). Peer-nominations and teacher ratings on boys' aggressive behavior at age 8 correlated with the number of arrests until age 27 at the same level (Hämäläinen \& Pulkkinen, 1996; Pulkkinen, 1998). The latter study also showed that for females, the correlation between aggressive behavior in childhood and arrests was zero.

A correlation coefficient indicates the amount of shared variance between variables, but it does not indicate (1) how accurate the prediction is on the individual level; and (2) whether the relationship is linear. Both aspects were studied more closely. In addition, the predictability of one-type and multi-type offending was compared.

Accuracy. To study the accuracy of predictions, male participants were divided into two categories of offenses (yes/no) and aggression (high/low), using for the latter the mean of aggression as a cutting point (Pulkkinen \& Hurme, 1984). The participants were cross-tabulated $(2 \times 2)$ for each offense category based on their aggressiveness and offenses committed by age 20 . If the high aggression group had committed offenses, the prediction was true positive, if not, then the prediction was false positive. Correspondingly, if the low aggression group had committed offenses, the prediction was false negative, and if not, it was true negative.

The errors in the identification of offenders and nonoffenders (false positives plus false negatives) were about 30 percent for alcohol offenses, and 20 percent for both theft and violent offenses. The prediction did not essentially improve from age 8 to 14 . Correspondingly, true predictions (true positives plus true negatives) were about 70 percent and 80 percent. In other studies reviewed by Loeber and Dishion 
(1983), the errors in the identification of offenders with teacher ratings of aggressiveness as predictors have also varied from 20 percent to 30 percent.

Linearity. To study whether an increase of aggression progressively increases the likelihood of criminal offending, male participants were divided into four quartiles on the basis of the amount of peer-nominated and teacher-rated aggression at age 8 , and the quartiles were compared in the number of arrests at age 27 (Pulkkinen \& Pitkänen, 1993). The relationship was not linear. Only the highest quartile differed from the other quartiles in the number of arrests; the other clusters did not differ from each other. The highest quartile of aggressiveness affects the formation of the significant correlation coefficients between aggression and the number of arrests. When the most aggressive quartile of boys was excluded from the analysis, the correlation between the age 8 aggression and arrests until age 27 dropped to 0.03 (from 0.33 ) (Pulkkinen, 1998, pp. 175-6). It means that only very high amounts of aggression in childhood predicted criminal offenses. The same phenomenon concerned low self-regulation more generally. It can be concluded that the relationship between the amount of aggression and offenses was not linear: only accentuated problems in self-regulation in childhood predicted criminal offending in adulthood.

One-type and multi-type offending. Aggressive behavior at age 8 did not differentiate between the individuals who had committed only one type of offense from those who had not committed any offenses. This was found when the offender groups were divided into those who had committed one type, two types, or three types of offenses (categorized into alcohol, theft, and violent offenses) (Pulkkinen, 1983a). Only the children who later committed 2 to 3 types of offenses had been more aggressive at age 8 than nonoffenders. They had also been less compliant than the nonoffenders. In another study (Hämäläinen \& Pulkkinen, 1995), it was also found that those individuals who had committed offenses at the youngest age (15 to 16 years) registered in Finland had been the most aggressive at age 8. However, the age 14 aggression did differentiate between the one-type offenders from the nonoffenders. It suggests that children's differences in aggressive behavior became more indicative of antisocial behavior during school years.

Personal views. Prospective data on the future offenders was available from personal interviews conducted at age 14. The future male offenders stated that their moods changed easily, they became angry easily, and they often teased others and had fights with them (Pulkkinen, 1983a). Also, the parent interviewed at age 14 confirmed that the future male offenders differed from the nonoffenders in behavior regulation. The parents of the male offenders considered that their sons behaved more aggressively in case of failure, were less able to laugh at themselves, and had generally been more difficult compared with the ratings of the nonoffenders. The choice of friends of the future male offenders caused worries to the parents, and the male offenders had been conscious of this worry. The parents considered the influence of peers important. The interviews concerning leisure activities indicated that the future male offenders had spent their leisure in streets and discos more often and were at home more rarely than the nonoffenders. They smoked more and had been drunk more often, and their peers used more alcohol and smoked more than 
the peers of the nonoffenders. They had started heterosexual interaction at an early age. They admitted that they were illicitly absent from school and did not take care of their homework. Consequently, success at school was poor. Some differences were also found between the future female offenders and nonoffenders in interview variables. The future female offenders had the conception that their parents had not much knowledge of their whereabouts. They also had fewer hobbies than the female nonoffenders. The female offenders felt themselves as being more passive than the other girls.

Both the future female and male offenders watched TV for excitement more than the others. Data were collected in 1974, when personal computers, smart phones, and other modern communication appliances were not yet available. A study on the causal links between violence viewing in adulthood and behavioral characteristics of the viewers suggested, based on the JYLS data, that aggression was more likely an antecedent than a consequence of media violence viewing among adults (Mustonen, 1997). Aggressive individuals were more interested in violent viewing than less aggressive individuals, and their major motives to watch violence were finding excitement, relaxation, and escape. Viewing violence may cause anxiety, because violence viewing at age 33 was positively related to viewers' anxiety at age 36 .

\subsubsection{Proactive and reactive aggression as predictors of criminal offending}

In the descriptive model of aggression (see Chapter 1, Figure 1.1), three major dimensions of aggression were distinguished: intensity of aggression, purpose of aggression (proactive or reactive), and direction of aggression (direct or indirect). Kindergarten teachers observed that the boys who were proactively aggressive (attacked others without a seeming reason), also generally defended themselves if attacked. There were also boys who defended themselves when attacked, but did not attack others. In the latter case, aggressive behavior was reactive and limited to self-defense. A question that is discussed in this chapter is whether the different purposes of aggression (proactive and reactive) predicted antisocial behavior in different magnitudes.

At age 14, a differentiation between proactive and reactive aggression was aimed at by formulating aggression items as follows. An item for proactive aggression was: "Who attacks without reason, teases others, says naughty things?" and an item for reactive aggression without proactive aggression (henceforth: reactive aggression) was: "Who defends oneself if teased, but does not attack without reason?" These two variables correlated by -0.29 for boys and by -0.24 for girls, $\mathrm{p}<0.01$ for each (Pulkkinen, 1987). The correlations of proactive and reactive aggression with criminal offending in men were different. Proactive aggression correlated with the sum score of offenses and the number of different types of criminal offenses until age 20 by 0.33 , but all correlations between reactive aggression and offenses were zero.

At age 20, proactive aggression assessed at age 14 was significantly connected with the total number of criminal offenses and the number of different types of 
offenses that male participants had committed by age 20 (Pulkkinen, 1983a, 1987). This finding for men was confirmed at age 27, when participants were grouped into proactively aggressive, reactively aggressive, and nonaggressive participants based on the age 14 assessments, and the groups were compared in the number of arrests by age 27, excluding alcohol-related offenses (Pulkkinen, 1996). The proactively aggressive men had more arrests than reactively aggressive and nonaggressive men; the latter groups did not differ from each other. Female groups did not differ from each other.

Differences between proactive and reactive aggression are illustrated by their correlations with other socioemotional characteristics (Pulkkinen, 1987). Concurrent correlations at age 14 showed that both teacher-rated and peer-nominated proactive aggression

- correlated positively with low self-regulation ("is impulsive, lacks concentration, changes moods"), but negatively with compliance, constructiveness, and high self-regulation ("is reliable, keeps promises, does not get excited"); and

- was positively associated with maladjustment to school (punishment at school, truancy), smoking, low frustration tolerance, and giving up after failure, and negatively with school success and attentiveness and carefulness.

The correlations were reversed for reactive aggression without proactive aggression rated by peers and teachers. It was positively associated with

- constructive and compliant behavior and other indicators of high selfregulation, but negatively with the indicators of low self-regulation; and

- interest in school attendance, school success, and attentiveness and carefulness, but negatively with punishment at school.

Various forms of aggressive behavior at age 8 predicted proactive aggression at age 14 but correlated negatively with reactive aggression (Pulkkinen, 1987). The study conducted at age 27 strengthened the view that males and females who were nonaggressive or displayed only reactive aggression in adolescence had been characterized by higher behavior regulation at ages 8, 14, and 27 than those who displayed proactive aggression (Pulkkinen, 1996). It means that reactive aggression for self-defense without proactive aggression was accepted as part of social interaction.

In the literature, reactive aggression has been presented as more pathological than proactive aggression. Proactive aggression has been said to have instrumental goals, whereas reactive aggression has been connected to the display of anger (Dodge, 1991). Dodge assumed that a proactively aggressive child would have a better prognosis than a reactively aggressive child. The results of the JYLS data do not support this assumption. Proactive aggression (which mostly emerges with reactive aggression) had a poorer prognosis than reactive aggression without proactive aggression. The prognosis of proactive aggression was comparable to school bullying (Ttofi, Farrington, \& Lösel, 2012). They examined 18 studies in which 
children who bullied at school were identified and compared with nonbullies in respect to their criminal behavior. School bullying was a strong risk factor for later criminal behavior. A person-oriented approach is needed for the study of individual patterns of aggression in regard to proactive and reactive aggression. In further studies, special attention should also be paid on the threshold of reactive aggression. Intensive reactive aggression to mild stimuli may have different dynamism (such as a biased social cognition) compared with the situation when the intensity of reactive aggression is adjusted to the intensity of an attack.

Conclusions regarding question 1. Childhood aggression predicted multiple offending, but aggression was a significant predictor of crime only when it was very intensive, and only proactive aggression predicted crime. Reactive aggression without proactive aggression did not predict crime. For females, aggression was not associated with the number of offenses. Insights about aggression as the predictor of further aggression and criminal behavior have been summarized in Pulkkinen (2017).

\subsection{Accumulation of problems}

\subsubsection{Multi-problem predictors of problem behaviors}

The second question concerns the patterning of aggressive behavior with other problem behaviors and the significance of different patterns as predictors of crime and other problems in social functioning. The accumulation of problems in self-regulation was analyzed in a person-oriented way by grouping individuals with a clustering technique in regard to the types of problems in their behavior. These analyses were made across time and culture. One international comparison was made with the JYLS and two Canadian male samples that varied in age (6, 8, and 10 years), culture (Finnish and Canadian), and decade (1960s, 1980s) (Pulkkinen \& Tremblay, 1992). Another international comparison in respect to patterns of problem behaviors was made with Swedish findings by Magnusson and Bergman (1988).

Patterns for males. In the comparison of the Finnish and Canadian samples, the first aim was to identify significant patterns of boys' social behavior using five dimensions: aggression, anxiety, hyperactivity, inattentiveness, and prosociality. The second aim was to verify the replicability of the patterns across ages, cultures, and decades. The third aim was to determine the stability of the patterns from one age to another, and the fourth aim was to analyze the predictive value of the behavior patterns in antisocial behavior. Nine patterns were extracted using a cluster analytic procedure. High comparability was found in eight of them across ages and cultures. Aggression divided into three clusters: Bully, Multi-problem, and Uncontrolled (Pulkkinen \& Tremblay, 1992). Other clusters were called Anxious, Passive, Inattentive, Nervous, and Normal. Stability of the patterns over 4 years was analyzed with the Montreal sample. It was found that the participants tended to remain in the same cluster from age 6 to 10 years, or at least, in one of the aggressive or nonaggressive clusters. 
The outcome variables at age 11 in the Canadian samples, and at ages 14, 20, and 27 years in the JYLS, included disruptive behavior, delinquency, school success, stability of work line, and problem drinking. The patterns had predictive validity. For instance, the Normal boys who were scored low on all deviant behaviors were also scored low in the outcome criteria for deviant behavior. On the contrary, the Multi-problem boys were scored highest on disruptive behavior, their school success was lowest, their working career was most unstable, and they had more often committed theft offenses than the participants in the other patterns. Other clusters were between these two in the amount of deviant behavior. The patterns showed continuity in deviant/nondeviant behaviors.

These results concurred with the findings by Stattin and Magnusson (1989) that aggressive boys often are socially maladjusted at an early age. They are restless and have concentration difficulties, show low school motivation, and have poor relations. Magnusson and Bergman (1988) demonstrated that aggression in its extreme forms usually occurs together with other conduct problems, and that there is a longitudinal relationship between the multi-problem pattern and later criminal offending.

The patterns extracted in Sweden by Magnusson and Bergman were replicated with the JYLS data (Pulkkinen, 1992a,b). As in the Swedish study, aggressiveness in males appeared as a problem in four patterns out of seven (Table 10.1). Aggression appeared with restlessness (Cluster 3); with poor concentration (Cluster 5); with restlessness, poor concentration, poor school motivation, and poor school success (Cluster 6); and with all these problems plus poor peer relations (low popularity and leadership) (Cluster 7). In Cluster 7, problems were multi-dimensional and, additionally, poor peer relations and a lack of concentration were prominent.

The highest proportion of males (45 percent of 20 males; see note in Table 10.1) were arrested in Cluster 7, called Multi-problem. About 30 percent had been arrested both in Cluster 6 and Cluster 5, and 24 percent in Cluster 3. In Cluster 4 , which was characterized by severe problems in school, the arrest rate was 33 percent, independent of aggression. It shows that aggressiveness was not a necessary element in the problem pattern that predicted offending. Aggressive behavior had a different predictive value depending on the pattern of behavior. When aggressiveness was only combined with restlessness in childhood, it was less predictive of antisocial behavior than when it was combined with several problem behaviors in adolescence. It seems critical in whether aggressive behavior becomes patterned with other problem behaviors during school years.

A lack of concentration was a common element in the male clusters where the arrest rates were highest. It indicates problems in self-regulation. The risk of arrests was higher when self-regulation was lower, independent of whether aggression belonged to the pattern. These findings concurred with the general theory of crime by Gottfredson and Hirschi (1990). They argue that individuals possessing high self-control would be "substantially less likely at all periods of life [to] engage in criminal acts" (p. 89). Individuals who lack self-control tend to be impulsive, insensitive, risk-taking, and short-sighted, "and they will tend therefore to engage 


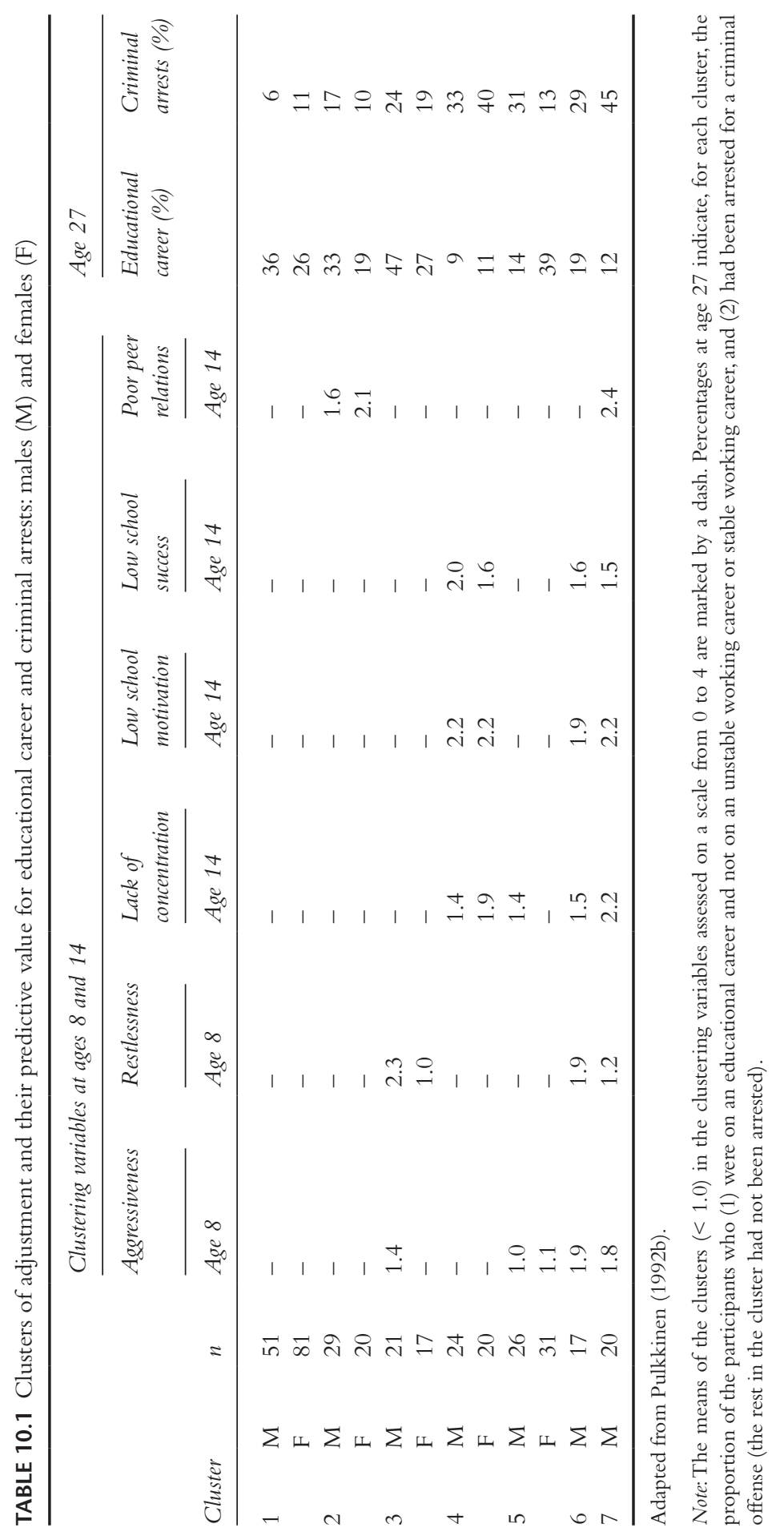


in criminal and analogous acts" (p. 90). They assumed that low self-control can be identified prior to the age of responsibility for crime, and it tends to persist through life. In the JYLS, low self-control was identified prior to the age of responsibility for crime.

Patterns for females. A cluster analysis was also conducted with females based on data collected at ages 8 and 14, and five clusters were extracted (Pulkkinen, 1992b). The female clusters were less multi-dimensional than the male clusters. The coverage of the cluster was widest in Cluster 4 (Table 10.1). The arrest rate was highest (40 percent) in this cluster, including a lack of concentration, low school motivation, and poor school success. Aggressive behavior did not appear in it. Aggression only appeared in Cluster 5, where other problems did not exist, and for which the arrest rate was 13 percent. Thus, a lack of concentration as an indicator of low self- regulation was also included in the female pattern that predicted criminal arrests.

It is noteworthy that the rate of women who had an educational career at age 27 was highest (39 percent) in Cluster 5 formed only by aggressiveness. Correspondingly, the rate of educational career was highest in males in Cluster 3 that was formed by aggressiveness and restlessness at age 8. Educational career at age 27 meant that at least half of the last 7-year period was spent for education.

The results were contrary to expectations: female aggression did not contribute to criminal offending but to educational career. Therefore, the connection between aggressiveness and educational career was more closely investigated for females (Pulkkinen, 1992b). It was found that high-aggressive girls might have either educational or unstable careers. These careers were associated with the type of aggression that the girls displayed. Physically aggressive girls had been less motivated toward school attendance, and the youngest mothers were found among them. Early motherhood had interrupted their education, and many of them had an unstable career. There were, however, women among them who began their occupational training at a later age, and therefore, they were on an educational career at age 27. Most women who had an educational career had a better school success than the average. Among them, there were girls who had been rated as verbally or facially more aggressive than on average at age 8 , and who had been rated by their teachers as socially more active and energetic at age 14 than the girls who were less career oriented.

Some girls (and boys) might have been perceived to be aggressive as children possibly due to their activeness, or their aggressiveness was a temporary reaction to life pressures. They did not exist in criminal registers if there was no problem behavior in adolescence. Criminal offenses were more likely found if aggressive behavior was patterned with problems in concentration and in school motivation and success.

Nonprosociality. The concept of multi-problem behavior is commonly understood to refer to the presence of several problem behaviors. It has been, however, found in the JYLS that multi-problem may also involve a lack of a characteristic that protects from further problems. In the comparative study of the Canadian 
and Finnish male samples, nonprosociality (low constructive behavior) belonged with aggressiveness, inattention, and hyperactivity to the pattern that was predictive of deviant behavior, including criminal offending (Pulkkinen \& Tremblay, 1992). The role of a lack of prosocial behavior in the development of antisocial behavior was also seen in the study by Pulkkinen and Hämäläinen (1995) where the number of arrests until age 27 correlated negatively with the indicators of high self-regulation: compliance, stable mood, and constructive behavior in males, and with constructive behavior in females.

Escalating adjustment problems including norm-breaking behavior, poor school success and either aggressiveness or low prosociality (operationalized by constructive behavior at age 8) predicted the highest number of arrests by age 27 (Hämäläinen \& Pulkkinen, 1996). A further logit analysis of the independent and interactive effects of behavioral problems revealed two independent predictors of arrests. First, normbreaking behavior (indicated by disobedience at age 8 ; and truancy, punishments at school, and substance use at age 14) had a strong main effect on arrests. Second, low constructive behavior alone had a significant main effect on arrests. On the contrary, aggressive behavior had no independent effect on arrests; it functioned in the interaction with poor school success. Aggressiveness at age 8 did not predict arrests without the comorbidity of other problems.

The significant main effect of low constructive behavior on arrests is a remarkable finding. It is in accordance with the findings that risks of antisocial behavior are higher in the Loser style of life than in the Thriver style of life (Pulkkinen, 1983a; cf. Figure 5.1). This dimension of styles of life has roots in childhood anxious versus constructive behavior, interpreted to indicate low versus high emotion regulation. Risks of antisocial behavior are not limited to low behavior regulation (the Reveller style of life versus the Loner style of life). Low self-regulation in both components (behavior and emotion regulation) simultaneously, or in one of them, is a risk of antisocial behavior.

In the study by Hämäläinen and Pulkkinen (1996), lack of concentration was not, unfortunately, controlled for. The study by Kooistra, Tolvanen, Mäkiaho, and Pulkkinen (2001) showed the significance of low concentration as a predictor of offending at age 36. The analysis was conducted with latent growth curve modeling. The results indicated that poor concentration and poor (and worsening) school success explained 53 percent of the variance of criminal convictions in men and 44 percent in women. Low self-control defined by attentional control was also associated with the emergency and persistence of unemployment in British cohort studies (Daly, Delaney, Egan, \& Baumeister, 2015). Altogether, these studies show that there are different paths to criminal offending and that low self-regulation in one or another form is a core in these paths.

\subsubsection{Dynamic processes in the accumulation of problems}

Problems in social functioning do not generally appear in isolation. In the study of maladaptive social functioning, the co-occurrence of various forms of problem 
behavior and unfavorable background conditions has been documented by several researchers. They have introduced theoretical constructs to describe the way in which problems tend to accumulate. Rönkä (1999) described and reviewed the constructs in the $2 \times 2$ framework. One dimension consisted of a researcher's interest, either in an individual or in population, whereas the other dimension consisted of the temporal perspective, either at a certain point of time or over time. If the focus of interest is on an individual at a certain point of time, the co-occurrence (or co-morbidity) of problems may be found, whereas if the focus of interest is on an individual over time, the formation of the chain of adversities (or amplification of problems) may be found. In case the focus of interest is in a population at a certain point of time, the concentration of problems in a subgroup may be found, whereas if the population is studied over time, the process of polarization may be found.

Jessor and Jessor (1977) were among the first researchers to formulate a problem behavior theory. Their main idea was that various types of problem behaviors in adolescence and young adulthood, such as delinquency and drug use, tend to co-occur and form the so-called problem behavior syndrome. In line with the problem behavior theory, Bergman and Magnusson (1997) underlined that the balance between risk factors and protective factors affect a cumulative process. They introduced a problem gravitation hypothesis of adjustment problems, which means that adjustment problems tend to concentrate within a small group of people. Those who suffer from one kind of adjustment problem tend to have other problems. They also argued that problems tend to be polarized so that both well-adjusted pattern and the pattern with multiple adjustment problems become more frequent over time in relation to other patterns. With age, people either outgrow mild problems or the problems get worse and accumulate.

Another view of the accumulation of problems is to distinguish several pathways and cyclical chain effects where a risk factor results in some negative outcomes, which, in turn, causes difficulties (Caspi, Bem, \& Elder, 1989). A cumulative process may involve contemporary continuity, which refers to a case where a characteristic, such as impulsivity, makes an individual prone to risk-taking in different settings. Thus, there is the continuity of a characteristic that causes difficulties across different contexts. The process may also involve interactive continuity when an individual's style evokes a reciprocal response, for example, when aggressive behavior in children evokes aggressive reaction from other people; or it may involve cumulative continuity, when consequences of behavior, such as poor adjustment to school, limit opportunities for further education. This process may be caused by spreading of difficulties, that is, by a developmental cascade of adverse consequences (Masten et al., 2005). "Cascading effects" refers to the situation where problems or changes in one domain may bring about problems or changes in other related domains. For instance, children whose low behavior regulation causes difficulties in the adaptation to school work and poor school success, face difficulties in further education and the labor market. The cumulative process may also exist in external circumstances, whereby circumstances affect a person's behavior, which further triggers certain circumstances. For instance, early adverse circumstances may affect the way a person sees himself or herself, and low 
self-worth may, in turn, result in school failure, which may cause difficulties in his or her educational career, which affects the subsequent working career and employment.

Maughan and Champion (1990) distinguished two basic strands through which childhood risks are transferred into adulthood: the outer strand of risk factors referring to environmental continuity, and the inner strand of risk factors referring to the increase of inner vulnerability as a consequence of the exposure to risk factors. The outer strand of risk factors means that continuity in, and linking of, poor environments may predispose individuals to the experience of poor social circumstances and lack of support. Poverty and antisocial parents are examples of conditions that may function in this way. The conditions narrow options for managing life in a socially acceptable way, and they may limit the quality and extent of interpersonal contacts. Thus, environmental continuity explains individuals' behavior.

The inner strand of risk factors refers to inner vulnerability, such as low selfesteem and low self-efficacy, as a consequence of the exposure to risk factors. Caprara and Zimbardo (1996) have paid attention to the child's causal attributions, expectations, and perceived opportunities as a result of the reactions of other people to what a child does. This kind of cognitive-emotional information processing view is also included in the theory of self-efficacy by Bandura (1982), who argues that an individual's conception of his or her performance mastery in personally valued areas increases the likelihood of success or failure.

\subsubsection{Continuity from risk factors to social functioning problems}

Social functioning at age 27 was conceptualized in the study by Rönkä and Pulkkinen (1995), following the definition by Rutter, Quinton, and Hill (1990), as the way an individual copes with developmental tasks linked to (1) work (unstable career line); (2) social relations (lack of friends, confidants, and social support); (3) financial standing (poor); and (4) social norms (alcohol abuse and criminal offenses excluding alcohol-related offenses). Information about social functioning problems was obtained through interviews and inventories.

It was found that an unstable career line played a key role in men's social functioning problems with heavy drinking and criminal arrests (Figure 10.1; Rönkä \& Pulkkinen, 1995). In women, an unstable career line did not play as strongly a key role in social functioning problems as in men. The studied problems in social functioning were generally less strongly interrelated in women than in men. However, criminal arrests and drinking problems were also interrelated in women.

Continuity from risk factors to social functioning problems was studied by Rönkä and Pulkkinen (1995) by choosing, based on previous studies, the following childhood risk factors as possible predictors of the problems of adult social functioning:

- Socioemotional behavior at age 8: aggressiveness and anxiety.

- Negative school experiences at age 14: poor school success; poor peer relations; and problems in adjustment to school (sanctions at school, truancy, contact with police, teacher estimation of adjustment problems). 


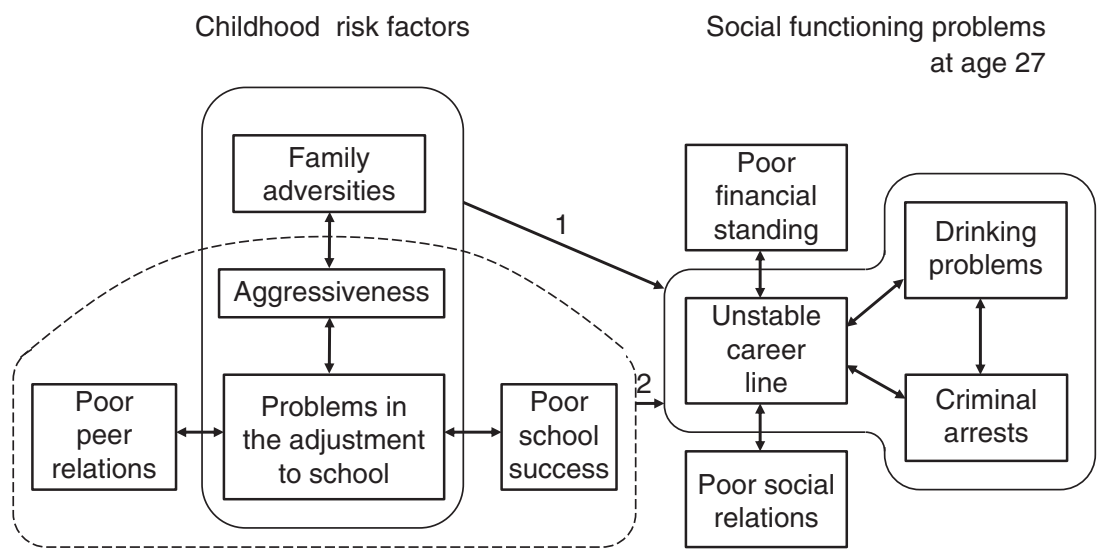

FIGURE 10.1 Interconnections among childhood risk factors and among adult social functioning problems. Two paths from childhood risk factors to adult social functioning problems for males.

Source: Adapted from Rönkä \& Pulkkinen (1995).

- Family background: low socioeconomic status of the family of origin and problems in the family at age 14 (at least one of the following adversities: parental divorce or death, parental use of alcohol, teacher estimation of disadvantaged home conditions).

The childhood risk factors were interconnected. The core of the risk factors consisted of problems in adjustment to school (see Figure 10.1). For male participants, adjustment problems were reciprocally linked to poor school success, poor peer relations, and aggressiveness, and aggressiveness was further linked to family adversities, such as parental abuse of alcohol and divorce. In females, problems in adjustment to school were similarly linked to poor school success and peer relations, but in their case, problems in school adjustment were more closely connected to family adversities, which were further linked to aggressiveness.

There were two pathways from childhood risk factors to the pattern of adult social functioning problems (unstable career, drinking problems, and criminal arrests) in men as seen in Figure 10.1. (Pattern analyses could not be conducted for women due to the small number of women with this pattern of adult social functioning problems.) Pathway 1 included poor external circumstances (family adversities, aggressiveness at age 8, and problems in adjustment to school at age 14), whereas pathway 2 consisted of behavioral vulnerability (aggressiveness at age 8 , problems in adjustment to school at age 14, poor peer relations at age 14, and school success at age 14).

Risk factors as predictors of poor social functioning were always combined; no single risk factor predicted cumulative problems in adulthood, neither did social functioning problems exist in isolation of each other in men-they tended to interact and co-occur with further problems. The two pathways demonstrate 
cascading effects in two ways. In the first path, interactional continuity exists in the connection between family adversities and the child's aggressiveness, which evokes reciprocal responses at school causing problems in adjustment to school, and furthermore, spreads into difficulties with social norms and the career line. The second pathway demonstrates the cascade effects of low self-regulation, which interferes with learning in a classroom and relationships with peers, and further decreases opportunities in the labor market and social adaptation. Common to these pathways was the link between the adjustment to school and unstable career line, which may be interpreted as the outer strand of risk factors through poor social circumstances and lack of support (Maughan and Champion, 1990). It means that negative outcomes of risk factors caused difficulties and problems in men's social functioning, which, in turn, caused further problems (Rönkä \& Pulkkinen, 1995).

The outer strand was also found in women (via unstable career line), but only in adulthood (Rönkä, Kinnunen, \& Pulkkinen, 2001). Career instability functioned as a mediator that explained the increase of the accumulation of problems in men and women. The process can be described as follows: social functioning problems at age 27 increased the risk of career instability experienced after age 27, which further increased the risk of other social functioning problems. Career instability may be interpreted as an outer strand or poor circumstances in the continuity of problems.

Another factor that explained the increase of social functioning problems in women was partner quality assessed at age 36 (Rönkä et al., 2001). Having a nonproblematic partner at age 36 protected women against problems of social functioning, while having either a problematic partner or no partner was associated with an increase of problems from age 27 to 36 . A problematic partner was unemployed or had drinking problems, or expressed physical aggression toward the partner. The moderator effect was not found in men, but men who had a problematic partner or no partner at all had, at both ages (27 and 36), a higher level of problems of social functioning.

Childhood risk factors predicted early timing of parenthood in both genders, but only in women did early parenthood predict career instability and the increase of problems of social functioning at age 36 (Rönkä, Kinnunen, \& Pulkkinen, 2000). Early motherhood was a mediating factor of later social functioning problems. It functioned in the same way as a sense of failure indicating the inner strand of risk factors in the accumulation of problems (Rönkä, 1999). In men, becoming a father at an early age did not explain continuity in social functioning problems. The inner strand in continuity of problems was only found in women. It means that inner vulnerability indicated by a sense of failure was associated with problems of social functioning at age 27 and further explained the accumulation of social functioning problems at age 36 (Rönkä et al., 2000).

Social functioning problems (poor financial standing, unemployment, poor social relationships, poor intimate relationships, drinking problems, and criminality) at ages 27 and 36 were more common in men than in women, but gender differences narrowed with age, because problems increased in women over time (Rönkä et al., 2001). The proportion of women who had no problems, decreased in 9 years from 67 percent to 41 percent; three times more than in men (from 39 percent to 
30 percent). In most cases, the increase concerned the emergence of one problem. Thus, having no problems at age 27 did not protect women from having problems at age 36 , whereas men with no problems at age 27 tended not to have problems at age 36 . Also, the state of having accumulated problems at age 27 continued to age 36 in men. If problems were connected to each other, then they tended to persist or even get worse.

The polarization of problems, which means that "no problems" and "several problems" would have become more frequent over time in relation to others, was not found. Instead of it, the concentration of serious problems within a small group of people with time was obtained. Social functioning problems may accumulate during adulthood to the extent that an individual is permanently disengaged from the economic mainstream. Of the initial JYLS sample of 369 participants, 17 percent lived below and 71 percent above the poverty line at age 43 based on the government tax register (information was lacking for 12 percent). Poverty was defined by having annual income below 60 percent of the median. For 80 percent of the participants for whom information about income was available at age 50, the income level remained the same from age 43 to 50 .

In a study by Savolainen, Mason, Lyyra, Pulkkinen, and Kokko (2017), socioeconomic exclusion was operationalized in terms of (1) poverty, as defined at ages 43 and 50 on the basis of a government tax register of annual income, and (2) labor market exclusion indicated by disability pension or premature death. The major criterion for social exclusion was living below the poverty line at one or both time points. Disability pension was commonly associated with it; 6 percent had a disability pension and 3 percent had died. Antecedents of socioeconomic exclusion were low educational and occupational attainment on the one hand, and low self-regulation on the other hand, covering high aggressive and rule-breaking behavior in adolescence and adult crime. These pathways were independent of each other except for the cascade effect of poor academic performance to criminal behavior in adulthood.

Conclusions regarding question 2. The multi-problem patterns of behavior indicating low self-regulation, not necessarily including aggressive behavior, were most predictive of further problems of social functioning. Empirical findings supported theoretical assumptions about an interactive continuity in the accumulation of problems in social behavior where an individual's characteristics evoke a reciprocal response, and cumulative continuity, which includes cascading (spreading) effects of adverse consequences. The outer strand of risk factors, which means that negative outcomes of risk factors cause problems in social functioning, was found in males from childhood to adulthood and in females in adulthood via an unstable career. For women, the inner strand was also found, which refers to a sense of failure.

\subsection{Offender groups compared}

\subsubsection{Offender groups}

The third question of this chapter concerned the future development of individuals who commit offenses. This question was studied by forming offender groups in 
middle age and comparing them in prospective data about their development from childhood on, and in their adult outcomes. More specifically, the questions were as follows (Pulkkinen, Lyyra, \& Kokko, 2009):

1. What offender categories could be found on the basis of the onset and duration of offending when all data is utilized on criminal offending collected by middle age?

2. Did offenders in these categories differ in their personality characteristics?

3. Did offender categories differ in the consequences of antisocial behavior for life success in middle age?

Moffitt (1993) has made a well-known distinction between life-course persistent and adolescence-limited antisocial behavior and described their different developmental histories. The categories were based on the age of onset of offending. Moffitt assumed that adolescence-onset offenders become involved in antisocial behavior within adolescent peer group dynamism without having a history of antisocial behavior in childhood. Adolescence-onset offenders desist from antisocial behavior as a function of maturing into adulthood and adopting a conventional lifestyle. On the contrary, life-course persistent offenders have a history of antisocial behavior in childhood that has developed when high-risk young children have lived in a high-risk social environment. The pattern of problem behaviors includes a lack of control of behavior, difficult temperament features, hyperactivity, emotional lability, and cognitive and motor deficits (Moffitt, Caspi, Dickson, Silva, \& Stanton, 1996). Results on the predictors of the hypothesized paths have supported theoretical assumptions on them (Moffitt, Caspi, Harrington, \& Milne, 2002).

It was expected that the paths for life-course persistent and adolescence-limited offenders could also have been found in the JYLS participants. Offender categories were formed using several sources of information on offenses committed by the participants: the police and government registers, personal interviews with the Life-History Calendar at age 42, and Self-Report Delinquency Scale, administered at age 36 .

Four offender groups were formed based on the onset and duration of offending (Pulkkinen et al., 2009). In addition to adolescence-limited and persistent offenders, a category for adult-onset offenders was found:

1. Adolescence-limited offenders. Offenses only in youth, at age 15 to 20: males 53 (27 percent of 196), females 27 (16 percent of 173)

2. Persistent offenders. Offenses in youth and adulthood, at age 15 to 42: males 57 (29 percent), females 11 (6 percent)

3. Adult-onset offenders. Offenses only in adulthood, at age 21 to 42 : males 32 (16 percent), females 25 (14 percent)

4. Nonoffenders. Males 54 (28 percent), females 110 (64 percent).

Age 21 was selected as a cut-off point, because 15 to 20-year-old offenders are considered youth criminals in Finnish legislation with special provisions. Criminal 
responsibility age is 15 and the offenses of the younger ones are not registered. Speeding and arrests for drunkenness were the most common self-reported offenses in all offender groups. They were not used as criteria for the categorization if they had appeared only once.

Other frequently reported offenses were vandalism, fighting, and shoplifting. Theft and assault appeared among the persistent offenders, and disturbing general order among the adolescence-limited offenders. The adult-onset offenders exhibited a greater diversity of specialized offenses, such as document forgery and sexual offenses.

\subsubsection{Differences in personality}

The offender groups were compared in socioemotional characteristics at ages 8 and 14 and in personality characteristics assessed by the Eysenck Personality Questionnaire and the Zuckerman Sensation Seeking Scale at age 27, the NEO-Fife Factor Inventory at ages 33 and 42, the Karolinska Scales of Personality at ages 36 and 42, and some other scales (see Pulkkinen et al., 2009). Box 10.1 presents the variables for socioemotional behavior and personality in which the offender groups differed from the nonoffenders.

All offender groups differed from the nonoffenders in higher social activity at age 14, in higher neuroticism (Eysenck Personality Questionnaire) at age 27, and in higher verbal aggression at age 42 (Pulkkinen et al., 2009). In addition, the persistent offenders differed from the nonoffenders in a broad spectrum of low self-regulation indicated by a lack of concentration, low emotional stability, and high aggressive, norm-breaking, disinhibited, and risk-taking behavior from childhood to adulthood, combined with poor school success, low anxiety, and early initiation of the use of alcohol. The adult-onset offenders were also more aggressive and disinhibited than the nonoffenders. The adolescence-limited offenders differed from the nonoffenders in lower constructive behavior and poorer school success in childhood, lower anxiety in adolescence, and in early initiation of the use of alcohol.

In comparison with the persistent offenders, the adolescence-limited offenders displayed less disinhibited and risk-taking behavior, were less aggressive in childhood and adolescence, and more passive and compliant at age 8 but no more in adolescence. Early onset of drinking was characteristic of both offender groups, as well as lower social anxiety in adolescence. Among the persistent offenders, drinking behavior was associated with other norm-breaking behavior, whereas in the adolescence-limited offenders, drinking more likely developed as part of a social activity with the peer group. Both the adolescence-limited offenders and the persistent offenders had poorer school success than the nonoffenders at age 8 , but only the persistent offenders had poorer school success at age 14 , which suggests cognitive maturation in the adolescence-limited offenders.

The adult-onset offenders had behaved in childhood and adolescence in a more socially acceptable way than the persistent offenders. They were more anxious, 


\section{BOX 10.1 THE OFFENDER GROUPS COMPARED WITH THE NONOFFENDERS IN SOCIOEMOTIONAL BEHAVIOR AT AGES 8 AND 14, AND IN ADULT PERSONALITY}

The persistent offenders differed from the nonoffenders by being

- $\quad$ higher in aggressiveness, particularly proactive aggression, at ages 8 and 14;

- $\quad$ lower in compliance at ages 8 and 14;

- lower in school success at ages 8 and 14;

- $\quad$ lower in self-regulation (concentration, emotional stability) at age 14;

- $\quad$ higher in social activity at age 14;

- lower in anxiety at age 14;

- $\quad$ higher in norm-breaking behavior at age 14;

- younger at the onset of drinking;

- $\quad$ higher in sensation seeking (experience seeking, disinhibition, boredom susceptibility) and impulsivity (risk-taking, psychoticism);

- lower in agreeableness, conscientiousness, socialization, social desirability;

- $\quad$ higher in somatic anxiety, muscular tension, and suspicion; and

- $\quad$ higher in neuroticism.

The adult-onset offenders differed from the nonoffenders by being

- $\quad$ higher in proactive aggression (teases, attacks others) at ages 8 and 14;

- $\quad$ higher in social activity at age 14;

- $\quad$ higher in various forms of aggressiveness and disinhibition in adulthood;

- $\quad$ higher in extraversion and risk-taking; and

- $\quad$ higher in neuroticism.

The adolescence-limited offenders differed from the nonoffenders by being

- lower in constructive behavior at age 8;

- lower in school success at age 8;

- $\quad$ higher in social activity at age 14;

- lower in anxiety at age 14;

- younger at the onset of drinking;

- $\quad$ higher in (verbal) aggressiveness in adulthood;

- lower in agreeableness;

- $\quad$ higher in neuroticism.

compliant, and careful with their school work, and in adulthood, they were higher in social desirability and lower in irritability. In comparison with the adolescencelimited offenders, the adult-onset offenders were, however, more bullying, more disobedient to the teacher, more labile emotionally, and socially more active; in 
adulthood, differences between their personality characteristics did not exist, except for higher orienting sensitivity in the adult-onset offenders.

Differences between the offender groups can be summarized as follows. In the dimension of low behavior regulation, the persistent offenders represented the lower end of behavior regulation, and the nonoffenders represented the higher end of the dimension. The profile of the adolescent-limited offenders was closest to that of the nonoffenders, whereas the profile of the adult-onset offenders was closer to the persistent offenders. The differences between the persistent offenders and the adolescence-limited offenders were in line with the assumption by Moffitt (1993).

\subsubsection{Social background}

The offender groups did not differ in their parents' occupational status or family structure (intact family/parents divorced when the participant was 14 years) (Pulkkinen et al., 2009). Having parents in blue-collar occupations and intact family structure were equally distributed across the offender groups. In a British study, persistent offenders came from a lower socioeconomic background than nonoffenders, and family income was lower in the homes of persistent offenders during their adolescence than in the homes of adolescence-limited offenders (Farrington, Ttofi, \& Coid, 2009). Finland is a country where social benefits for sole providers are available and economic equality is high (Wilkinson, 2011). Parental socioeconomic status explains less of the variance of children's behavior, and school achievement, than in most other countries, as revealed by the PISA study (Schleicher, 2011).

There were, however, differences between the offender groups in family functioning. Pulkkinen (2012a) described these differences in terms of social capital. High social capital was indexed by child-centered parenting and home atmosphere (Kokko \& Pulkkinen, 2000); this scale was a combination of 5 variables assessed at age 27 based on the participants' recollections of, for instance, relations between the parents, memories of the father, supervision from the mother, and physical punishment. In the homes of persistent offenders, atmosphere was less child-centered than in the homes of adolescence-limited offenders (Pulkkinen et al., 2009). These retrospective findings corresponded to the prospective findings of the Cambridge Study in Delinquent Development, where harsh discipline in childhood, parental conflict, and the father not being involved with his son in adolescence were higher in persistent offenders than in adolescence-limited offenders.

In the JYLS, the parents of persistent offenders had been heavier drinkers than those of nonoffenders when the participants lived at home. Thus, the persistent offenders had both heavy-drinking parents and low social capital at home. Heavy drinking increases conflicts between family members and reduces social capital of a family.

On the basis of the comprehensive review of literature on family factors and crime, Farrington (2010) groups family factors into six categories: (1) criminal and antisocial parents and siblings; (2) large family size; (3) child-rearing methods; (4) abuse and neglect; (5) parental conflict and disruption; and (6) other parental 
factors such as young age and substance use. He shows that these categories involve risks of offending and other problems in children. The strongest predictor of antisocial behavior is antisocial parents. Other rather strong predictors are large family size, poor parental supervision, parental conflict, and disrupted families. The findings in the JYLS fit to the outcome of the review except for antisocial parents. This information was not available in the JYLS.

A transmission of low self-regulation from the father to the son was found in the JYLS offspring study: the father's disobedience and aggressive behavior at age 8 correlated with his son's disobedience at the corresponding age (cf. Section 12.2.2). The impact of the father's low self-regulation on the son may accumulate through assortative mating. As found by Rönkä et al. (2001), men with a higher level of problems of social functioning at ages 27 to 36 , were likely to have a problematic partner (e.g., a heavy user of alcohol). As reviewed by Pulkkinen (2012a), antisocial adults are more likely to have children with conduct problems, and having two antisocial parents, witnessing severe partner conflicts, and experiencing maltreatment further increases the risk of conduct problems in children. Nevertheless, children's conduct problems alone do not predict conduct problems in the children of a subsequent generation. The maintenance of an antisocial lifestyle, including deviant peer and partner relationships, is an important mechanism of intergenerational continuity of antisocial behavior. Continuity across three generations depends on whether conduct problems in childhood lead to antisocial behavior in early adulthood.

\subsubsection{Life success of the offender groups in adulthood}

Comparisons of the offender groups in adult functioning (Pulkkinen et al., 2009) indicated that the persistent offenders differed from the nonoffenders in lower socioeconomic status, lower education, higher unemployment, lower financial status, heavier drinking, and lower consensus in partner relationship (Table 10.2). In comparison with the nonoffenders, the adult-onset offenders had a lower financial status and more use of alcohol, whereas the only difference between the nonoffenders and adolescence-limited offenders was that the latter had more children.

The adolescence-limited offenders were doing better financially than the persistent offenders; they used less alcohol, and they had a better partner relationship with marriage. Financial situation of the adolescence-limited offenders was also better than that of the adult-onset offenders; other differences did not exist. There were fewer differences between the adult-onset offenders and the persistent offenders; the occupational status was higher in the adult-onset offenders and the consensus between the partners was better.

The offender groups were also compared in psychological functioning (Pulkkinen et al., 2009). All offender groups had more psychosomatic symptoms than the nonoffenders. The persistent offenders had lower psychological well-being and selfesteem than the nonoffenders. Both the persistent offenders and the adult-onset offenders had a sense of lower self-worth and contentment with their achievements than the adolescence-limited offenders. The profile of the adult-onset offenders 
186 The development of social functioning

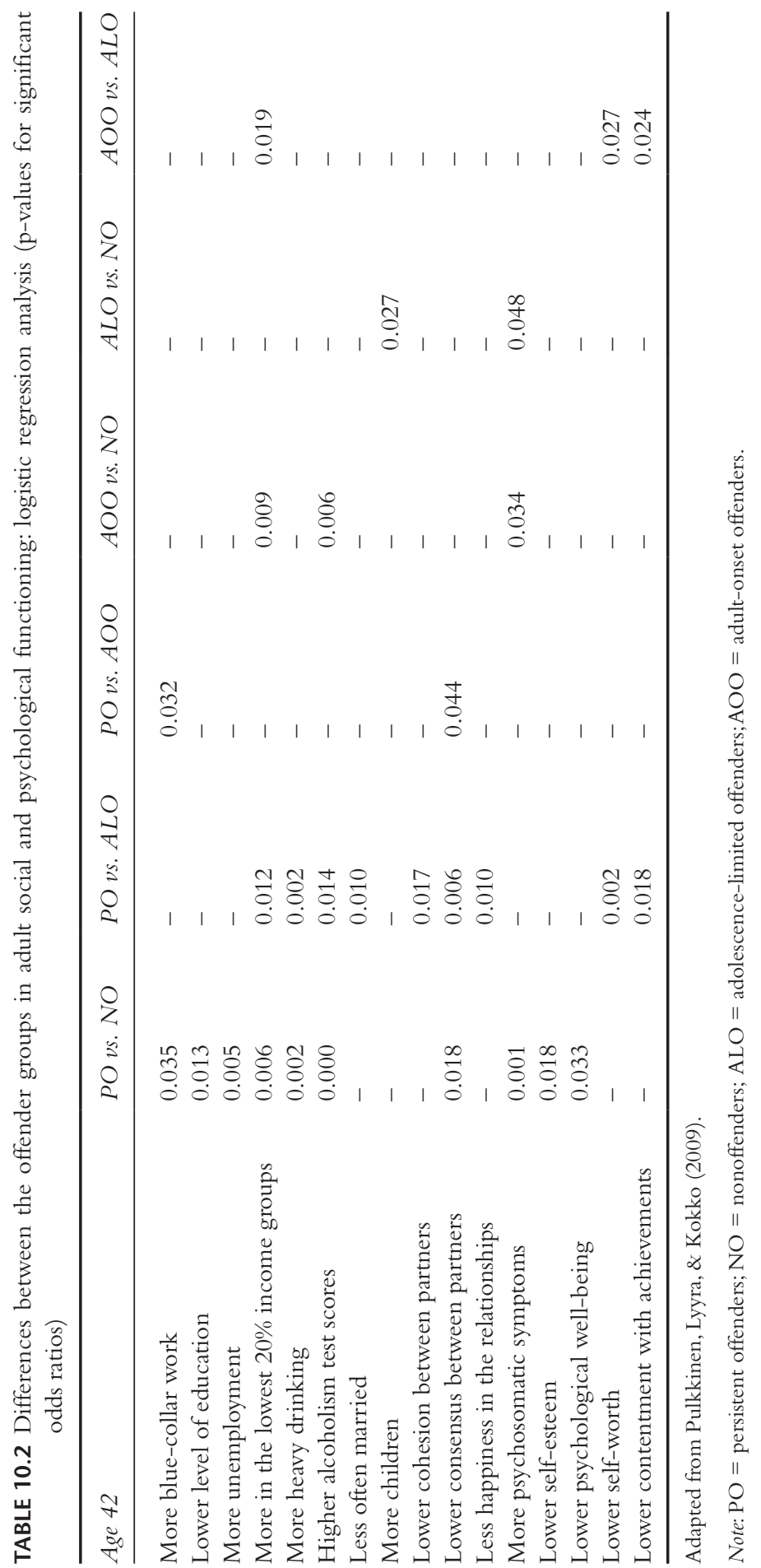


was less clear than the profiles of the persistent and adolescence-limited offenders. The adult-onset offenders were socially inhibited in adolescence, as also found by Beckeley et al. (2016), which might have protected them from offenses. In adulthood, they were dependent on alcohol in both studies. Beckeley et al. (2016) also found mental health problems.

In general, the results suggested that committing offenses only in adolescence did not have long-term negative consequences for life success, whereas persistent offending was associated with many kinds of failures in social and psychological functioning in adulthood. Persistent offending was registered in this study only starting from age 15, but the persistent offenders had displayed antisocial behavior in early school age as indicated by the bullying-type of proactive aggression and norm-breaking behavior. Information about their early childhood behavior was not available. In the Dunedin Longitudinal Study, New Zealand, childhood problem behavior at age 5 predicted both boys' and girls' antisocial behavior at age 15 (Moffitt, Caspi, Rutter, \& Silva, 2001). Neurocognitive risk factors that predicted antisocial behavior were the same in boys and in girls, but there were more risk factors in boys. Conduct disorder at age 11 predicted antisocial behavior at age 21 in males; in females, this diagnosis was rare. Problem behaviors patterned more infrequently in girls than in boys in the JYLS.

\subsubsection{Conclusions and suggestions for prevention}

The different profiles of the offender groups resulted in the following conclusions regarding question three, and suggestions for the prevention of juvenile delinquency (Pulkkinen, 2015).

First. Half of the juvenile delinquents, that is, the adolescence-limited offenders, are quite normal adolescents. They do not differ in personality and social functioning from the nonoffenders. A difference might be in delayed cognitive and emotional maturity as shown by poor school success and low constructive behavior in childhood. Glueck and Glueck (1950) presented a debated maturation hypothesis to explain desistance or ceasing from crime based on their study of 500 juvenile delinquents. Their idea was that as the individual matured, he or she began to make more responsible decisions and understand that "crime does not lead to satisfaction." They found roots of delinquency in children's problem behaviors, but they believed that children mature out of these problems. They did not distinguish different subgroups among juvenile delinquents, that is, the life-course persistent and adolescence-limited offenders that may be a reason for a criticism of the maturation hypothesis.

Persistent offenders start antisocial behavior in childhood indicating low selfregulation, whereas adolescence-limited offenders engage in antisocial behavior in adolescence without the similar profile of low self-regulation as found in the persistent offenders. The maturation hypothesis by Glueck and Glueck might be applicable to the adolescence-limited offenders, whereas the self-control hypothesis presented by Gottfredson and Hirschi (1990) in their general theory of crime 
would be more applicable to the persistent offenders. Gottfredson and Hirschi suggested that people who lack self-control tend to be risk-taking and shortsighted and, therefore, they will tend to engage in criminal and analogous acts. The self-control theory on crime as an explanation of life-course persistent offending has also received support from findings in other longitudinal studies such as the Dunedin Study (Piquero, Moffitt, \& Wright, 2007), and the Pittsburgh Youth Study (Loeber, Slot, \& Southamer-Loeber, 2006).

Second. Advancing the maturation out of offending can be aimed at with pedagogical means at school. They may concern provisions of special support for overcoming learning difficulties, such as dyslexia and concentration problems, and educative means for supervising group activities such as bullying. Also, extracurricular activities may be influential (Pulkkinen, 2016). In the United States, it has been shown for a risk group of offending that a participation in extracurricular activities at school reduced dropout from the school and antisocial behavior, and that extracurricular activities at school had longstanding positive effects on students' behavior via reducing truancy and the use of alcohol and other drugs, as well as increasing interest in the continuation of studies (Mahoney, Larson, \& Eccles, 2005).

Third. The primary context for the development of children is the home. Parents can advance children's maturation by their upbringing. There are many critical aspects in upbringing for the development of emotion and behavior regulation in children. Good and regular care and child-centered parenting promote emotion regulation by giving the child the basic security feeling, secure attachment to the mother and other primary care takers, and advice and support for coping with emotion-provoking situations. For the development of behavior regulation, the child needs adequate supervision when he or she starts to move and become a member of the social system.

Fourth. A child may have serious behavior problems that can be detected at an early age. They may concern neuropsychological problems in cognitive processes and behavior regulation, learning difficulties, motor clumsiness, attention deficits and hyperactivity (ADHD), aggressive behavior, and related symptoms. Although all childhood problems do not lead to later antisocial behavior, they commonly exist in persistent offenders (Moffitt, 1993). Therefore, early detection and proper treatment are needed.

Fifth. A high risk of antisocial behavior among adolescents is associated with the use of alcohol and other drugs. Both types of juvenile offenders started the use of alcohol at an earlier age than the nonoffenders. The adolescence-limited offenders later reduced their alcohol use, whereas the persistent offenders continued heavy drinking and developed alcohol dependency. The adult-onset offenders initiated drinking at the same age as adolescents normally do, but their drinking often developed to a heavy consumption of alcohol. At least in Finland, most offenses are committed while being intoxicated by alcohol. More conscious attitudes toward under-age drinking, parental supervision, educational opportunities for young people, and the adolescents' own awareness of their responsibility for their behavior and their future are means of improving the quality of adolescence and prevention of antisocial development. 


\section{1}

\section{HEALTH BEHAVIOR}

\subsection{Substance use}

\subsubsection{The start and continuity of the use of alcohol}

Health is one of the main domains of life (Chapter 9) and it increases in significance with age (Chapter 6). This chapter starts with the analysis of substance use, focusing on the use of alcohol and its risks for health. Longitudinal associations of socioemotional behavior and styles of life with health behavior and health are presented. The chapter ends with a discussion of temperance as a virtue.

Young people's experimentation with alcohol and tobacco has been seen as part of transition-marking behaviors in adolescent development (Jessor \& Jessor, 1977), as well as imitation of adult behavior, an attempt to achieve peer acceptance, and an effort to master age-typical difficulties in psychological growth (Jessor, Donovan, \& Costa, 1991; Silbereisen \& Noack, 1988). It has been assumed that adolescents "mature out" of abusive drinking patterns as the responsibilities of later adulthood supervene. The results of the JYLS show whether this pattern also existed in Finland.

The use of alcohol was assessed in the JYLS at ages 14, 20, 27, 36, 42, and 50. At all ages, the use of alcohol was discussed in the personal interview; at age 14, teachers' perceptions on the participants' use of alcohol was also asked. In addition, questions of drinking were included in the Life Situation Questionnaire (the Frequency $\times$ Quantity table), which was filled in before the adult interviews, and two alcoholism screening tests were administered: CAGE and the Malmö Modification of the Michigan Alcoholism Screening Test (mm-MAST). Data on alcohol-related arrests were collected in police registers at ages 20 and 26, and convictions in government register up to middle age.

The age of onset of drinking ranged from 10 to 30 years with the mean age of 15.3 years for boys and 15.6 years for girls (Pitkänen, Lyyra, \& Pulkkinen, 2005). The legal age of the use of alcohol in Finland is 18 years for beer and wine and 
20 years for spirits. The early start of drinking correlated with adult alcohol use. For outlining what was the critical age of onset of drinking as a predictor of adult alcohol use, the participants were categorized into four groups on the basis of the age of onset of drinking: 13 years or younger (17 percent); 14 to 15 years (44 percent); 16 to 17 years ( 24 percent); and 18 years or older (14 percent). Men and women were equally distributed in these groups. The groups were compared in the frequency of drinking, drinking into intoxication, and two alcoholism screening tests at age 42 .

The results showed that the earlier the use of alcohol had been started, the more alcohol these individuals used in adulthood (Figure 11.1). A critical age was16 years: if drinking had been started at age 16 or later, the risk of heavy adult drinking was smaller than if drinking had been started at a younger age. Despite the gender differences in the level of drinking, the same pattern existed in both genders.

The early start of drinking predicted the frequency of drinking to intoxication in adulthood (Pitkänen, 2010). For the participants who were drunk several times a week at age 27, the average age of onset was 14.2 years; for those who were drunk weekly, 15 years; for those who were drunk once a year at most, 15.8 years; and for those who used alcohol only a little at age 27, 17.1 years. The early start of drinking was studied more closely by constructing a variable for the use of alcohol at age 14 using self-reports, teacher interviews, and retrospective data (Pitkänen, 2006; Pitkänen, Kokko, Lyyra, \& Pulkkinen, 2008). The criterion for drinking was that the participant had been drunk or admitted using alcohol once in a while; mere tasting alcohol was not considered to indicate the use of alcohol. The use of alcohol at age 14 was coded: no (63 percent) and yes (37 percent). Drinking at age 14 predicted drinking at age 20 and problem drinking at ages 27 and 42 (T. Pitkänen
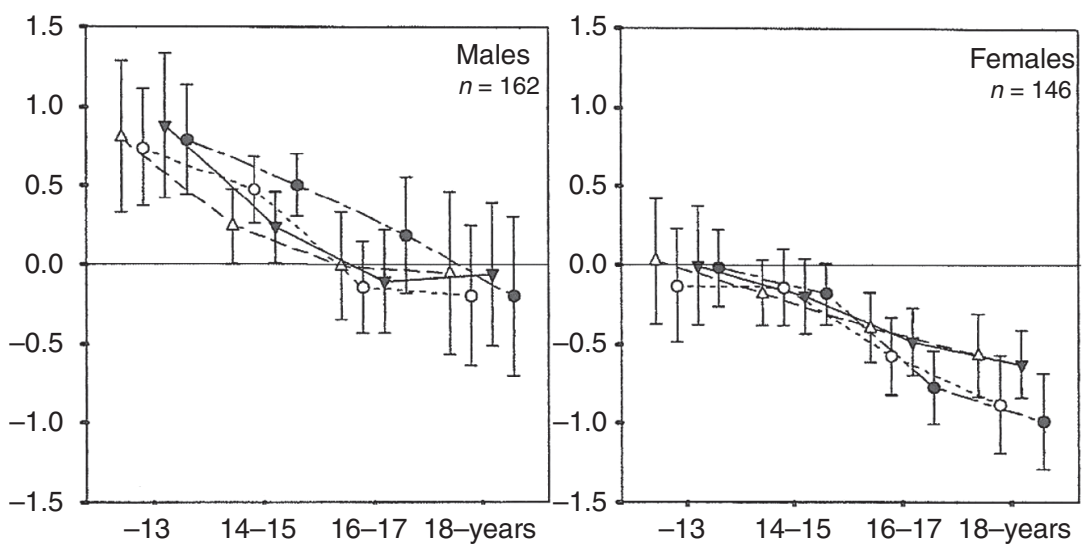

FIGURE 11.1 Means with 95 percent confidence intervals of the indicators of adult use of alcohol by the age of onset of drinking standardized across the whole sample.

$\Delta=$ Frequency of drinking, $\bigcirc=$ binge drinking, $\nabla=$ CAGE, $\bigcirc=$ mm-Mast.

Source: From Pitkänen, T., Lyyra, A.-L., \& Pulkkinen, L. (2005). Age of onset of drinking and the use of alcohol in adulthood: a follow-up study from age 8 to 42 for females and males. Addiction, 10, 652-61. (Figure 1, p. 658) (C) 2005 Society for the Study of Addiction. Publisher: John Wiley and Sons. Reprinted with permission. 
et al., 2008). Problem drinking was defined on the basis of problems that drinking had caused to the participants such as accidents and absence from work.

It is generally believed that the use of alcohol diminishes with age and becomes more controlled. The comparison of the consumption of alcohol at age 36 and 50 revealed, however, that the magnitude of consumed alcohol among the JYLS participants had increased significantly (Pitkänen, 2010). The increasing trend was also found in the frequency of drinking between the ages 42 and 50. At age 42, men reported using alcohol, on average, on 132 days a year; and at age 50, on 149 days a year. The same figures for women were on 80 days a year and on 90 days a year.

At age 50,69 percent of the participants used alcohol at least once a week, and one-third of the men and one-tenth of the women had been drunk at least once a week during the last 12 months; 20 percent of the men and 37 percent of the women had not been intoxicated by drinking (Pitkänen, 2010). Men used alcohol more often than women and in larger magnitude, but the heaviest drinking men (10 percent of the men) and women (4 percent of the women) consumed, on average, 37 liters of pure alcohol a year. Among the rest, average consumption of alcohol was 4.5 liters a year for men and 2 liters a year for women. Thus, the consumption of alcohol was highly concentrated on a small fraction of participants: 10 percent of the men consumed alcohol eight times more than the rest. For women, 4 percent consumed 18 times more than the other women. Men used higher quantities of alcohol at a time than women. One portion includes 12 grams of pure alcohol and is equivalent to a bottle $(33 \mathrm{cl})$ of beer or a glass $(12 \mathrm{cl})$ of wine. One-fourth of the men reported that they drank 20 portions of alcohol at a time at least a few times a year.

In order to understand these results, the Finnish drinking culture is shortly described. The use of alcohol, particularly spirits, has been part of accepted male behavior. Drinking among women was rare until the 1970s. The increase in drinking among women and in the whole population co-occurred with the change of legislation in 1969, which eased the restrictions of sale and consumption of alcohol by introducing "medium strength beer" (4.5 percent alcohol). Unlike other alcoholic beverages, it could be obtained through outlets other than the State Alcohol Monopoly and licensed restaurants. As a result, consumption of alcohol increased and differences in the number of abstainers between the genders diminished during the 1970s (Ylälahti, 1981). In 1960, 3.7 liters of 100 percent alcohol per capita were consumed in Finland, 9 liters in 1980, and 12.7 liters in 2005. Thereafter, there has been a declining trend. The annual consumption was 10.8 liters per person (15 years or older) in 2015 (Statista: The Statistic Portal, 2017).

The change can also be seen in so-called epidemiological risk limit set for health reasons. The limits were high until recently: 280 grams of pure alcohol a week for men and 190 grams a week for women, which makes 15 liters a year for men and 10 liters a year for women (Sillanaukee, Kiianmaa Roine, \& Seppä, 1992). The recommendations in the Current Care Guidelines (Duodecim 4.11.2015) are half of that for men and almost one-third for women: 140 grams of pure alcohol (or 12 portions) a week for men and 70 grams (or 6 portions) for women. 
The trends of drinking on the population level can also be seen in adolescent use of alcohol. Drinking began to increase in the 1970s. The European School Survey Project on Alcohol and Other Drugs (Hibell et al., 1997) showed that the heavy use of alcohol had become common also among girls: 31 percent of 16-year-old Finnish girls had been drunk at least 10 times during last 12 months, which was a leading figure together with Danish girls (30 percent). Among boys, the figures were of the same magnitude. In 2004, 22 percent of Finnish boys and girls had been drunk at least once a month, but thereafter, drinking has gradually reduced so that in 2015, only 10 percent had been drunk at least once a month (National Institute for Health and Welfare, 2017). Information on the risks of the early age of onset of drinking for adult heavy use of alcohol has been widely spread. The results of the JYLS study have contributed to increasing awareness of the risk of heavy drinking in adulthood if one begins drinking at an early age.

The participants of the JYLS were born in 1959 and they were in their adolescence when the use of alcohol began to increase in Finland. They have used more alcohol in their youth than young people at present. The early age of onset of drinking increases heavy drinking in adulthood, which may explain heavy drinking in the JYLS when the participants were in middle age.

\subsubsection{Problem drinking in middle age}

At age 50, all participants admitted that they had used alcohol at some point of their lives (Pitkänen, 2010). Participants were considered nondrinkers if they had been abstinent for more than 1 year, which often happened for health reasons. Almost half of the men and one-fourth of the women admitted at age 50 that the use of alcohol had caused one or more concrete problems to them, most often problems in an intimate relationship (32 percent of the men; 13 percent of the women admitted that they had experienced this problem). Other problems included other conflicts (men 27 percent; women 12 percent); absence from work (men 9 percent; women 1 percent); risks with work contracts (men 5 percent; women 1 percent); fights (men 18 percent; women 3 percent); and excessive (binge) drinking for several days (men 12 percent; women 3 percent).

The threshold of experiencing, recognizing, or admitting problems is individual. A high consumption of alcohol at age 50, and drinking into intoxication in particular, was associated with subjectively experienced problems caused by drinking. Problems caused by drinking and high consumption of alcohol were found in 18 percent of the men and 6 percent of the women (Pitkänen, 2010). These figures show the rate of serious drinking problems, assessed with an external and subjective criterion.

The average amount of problems due to drinking had decreased from age 42, but this finding underestimated problems caused by drinking by age 50 as can be argued on the basis of attrition. The men who did not participate in the study at age 50, but who did participate in it at age 42, included several heavy drinkers who exceeded the epidemiological risk limit in the quantity of drinking at age 42 
(Metsäpelto et al., 2010). The annual consumption of alcohol had been higher at age 42 in the nonparticipating men than in the participating men. The nonparticipating men had also been drunk more often at age 42 than the participating men. For women, some indicators of heavy drinking among those who did not participate were also found. Some heavy drinkers declined the request to be interviewed and some were difficult to reach although they had accepted the request, or they were not in the condition to be interviewed at the appointment. Several heavy users of alcohol had also died by age 50 . The use of alcohol was most likely related to several deaths. By 2016, 7.6 percent of the men (initially 196) had died and 2.9 percent of the women (173).

For the participating men and women, there was continuity in drinking into intoxication from age 27 to 50 (Pitkänen, 2010). The participants were categorized into six groups for males and five groups for females at age 27 on the basis of the frequency of being drunk. The scale ranged from $0=$ never, $1=$ once a year or less, to $5=$ several times a week. Figure 11.2 shows the means of the frequency of intoxication at ages $27,36,42$, and 50 for each group. Although differences between the groups diminished, there was continuity in the frequency of intoxication. The assumption that the frequency of intoxication decreases when people enter adulthood, was not supported by these findings. In some individuals, drinking decreased and in some individuals, it increased.

Correlations between drinking into intoxication and negative consequences were 0.59 for males and 0.40 for females, and between the frequency of drinking and negative consequences were 0.37 and 0.08 , respectively. Thus, the frequency of the use of alcohol was less strongly connected with negative consequences among the women. The frequency of drinking, particularly in women, indicated different culture in the use of alcohol compared with drinking a high amount of alcohol in one sitting. Possibly for this reason, the frequency of drinking in adulthood was less predictable on the basis of the early onset of drinking than problem drinking. Frequency of drinking and problem drinking indicate different aspects of drinking behavior. T. Pitkänen
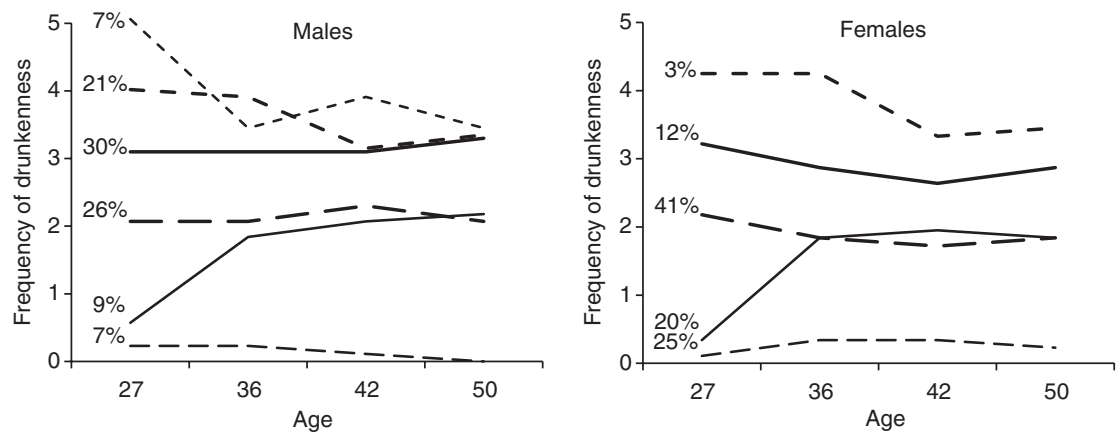

FIGURE 11.2 The continuity of habits to drink into intoxication from age 27 to 50 ; males and females.

Source: Pulkkinen (2010a). Milta keski-ika nayttaa? [What does middle-age look like?] In L. Pulkkinen \& K. Kokko (Eds.), Keski-ikä elämänvaiheena [Middle age as a stage of life.] (Reports from the Department of Psychology, No 352, pp. 97-106). University of Jyvaskyla, Finland. Adapted with permission. 
et al. (2008) suggest that for the evaluation of problem drinking, it is important to assess whether an individual has a control over the quantity of the consumed alcohol and whether there are hazardous consequences of drinking. They also suggest that despite significant correlation between the two alcoholism screening tests used in the JYLS (0.70 for men and 0.53 for women at age 42; Pitkänen, Lyyra, \& Pulkkinen, 2005), several other methods should also be used for assessing problematic alcohol use. They include the frequency of being drunk, the magnitude of alcohol consumed in one setting, and problems caused by drinking.

\subsubsection{Socioemotional characteristics associated with drinking}

Low self-regulation (indicated by low constructive and compliant behavior, and high emotional lability and aggressive behavior) at age 14 predicted problem drinking (defined by the number of problems caused by drinking) at ages 27 and 42 in both men and women (T. Pitkänen et al., 2008). Studies of the associations of drinking with the styles of life indicated that at age 20, heavy drinking was part of the Reveller style of life in both genders and at age 14, part of peer orientation in the men (Pulkkinen, 1983b). In adulthood, heavy drinking was part of the Undercontrolled and Brittle styles of life; the latter particularly in women (Pulkkinen, 1990b). Both styles of life involve several indicators of low self-regulation; the Undercontrolled style of life low behavior regulation and the Brittle style of life low emotion regulation (cf. Section 5.3.2).

High anxiety in childhood predicted female problem drinking at age 27 , whereas it was low anxiety and conduct problems in adolescence that predicted male problem drinking at age 27 (Pulkkinen \& Pitkänen, 1994). This difference suggests different dynamics of problem drinking in men and women: drinking related to the Undercontrolled lifestyle in men and to the Brittle lifestyle in women. No socioemotional precursors were found for social drinking characterized by the use of alcohol at dinner and social gatherings without experienced difficulties caused by drinking.

In men, drinking problems were a part of a larger pattern of conduct problems with underlying low behavior regulation. Similar results were received in a study by Pulkkinen, Virtanen, af Klinteberg, and Magnusson (2000) in which three groups were compared: (1) noncriminal male participants; (2) criminal offenders without an alcohol record; and (3) offenders with an alcohol record. The results showed that, both in the JYLS and a Swedish sample, the offenders with an alcohol record received (compared with the two other groups) higher scores on psychopathy-related personality characteristics, as indicated by higher impulsivity, muscular tension, and lower socialization. They also displayed higher aggressiveness in childhood than the noncriminal group. The interpretation was that there are underlying psychobiological mechanisms and possibly a common genetic basis in externalizing problems and alcohol problems. A Finnish twin study (FinnTwin 12) confirmed that the overlap between childhood conduct problems and later alcohol problems is due largely to shared genetic factors (Dick et al., 2009). 
Male drinking in early adolescence was explained by environmental factors, particularly by unshared environmental factors (Rose, Dick, Viken, Pulkkinen, \& Kaprio, 2001). Experiences that are not shared with a twin pair may include, for instance, effects of personal friends. Once drinking was initiated (from age 16 onwards), genetic factors also explained drinking behavior in men. In early adulthood (from ages 23 to 27) alcohol-related problems were largely influenced by genetic factors. Among females, genetic factors explained the use of alcohol at age 14 more highly than among males.

\subsubsection{Social backgrounds of the use of alcohol}

The connections of children's use of alcohol at age 27 to their parents' use of alcohol were investigated in two studies, the results of which are described separately for each gender. In the first study, the parents were categorized into four groups: low consumption (57 percent of the participants), mild (16 percent), moderate (15 percent), and high (13 percent) severity of alcohol problems (Pulkkinen \& Pitkänen, 1994). The category for high severity included parents whose children at age 27 described one or both parents as an "alcohol addict," "more often drunk than sober," and "drank to death." High severity of alcohol problems was more typical of fathers than of mothers. No distinction was made as to whether alcohol abuse existed in the father or mother or a stepparent (if the stepparent had lived at home when the participant still lived at home). In the second study, a stepwise regression analysis was conducted in which parental substance use was dichotomized (low use of alcohol/problematic use of alcohol; based on data collected at ages 14, 20, 27, 36, and 42) and the mother's smoking cigarettes was added to the predictors of the participant's problem drinking at age 42 (T. Pitkänen et al., 2008).

Males. It was found that parents' drinking was the risk factor of their sons' problems with drinking at age 27 covering the number of difficulties caused by drinking, the number of times being drunk, alcoholism screening test, and high quantity of drinking. The sons of the parents whose alcohol consumption was low had significantly less problems with drinking than the sons of the parents who had mild, moderate, or high severity of alcohol problems (Pulkkinen \& Pitkänen, 1994). The effects of the parents' alcohol problems on the sons' problems with drinking were similar at all levels of the severity of the parents' alcohol problems, which means that the effects did not depend on the severity of the parents' alcohol problems. Parents' drinking may function as a drinking model to the son, but it may also affect the son's life through other mechanisms, such as by increasing the risk of a poor relationship between the son and his father, partly because of the higher risk of the parents' divorce. The average rate of divorce in the sample was 22 percent by the time the participant was 27 years old, but the rate was 54 percent if the severity of the parents' alcohol problems was high and 13 percent if the parents' alcohol consumption was low.

The second study confirmed that the effects of the parents' heavy drinking on the sons' drinking continued beyond age 27; parental drinking explained the 
sons' higher scores in the alcoholism screening test, CAGE, at ages 27 and 42 (T. Pitkänen et al., 2008). This study also showed that the parents' heavy drinking was associated with parent-centered parenting (cf. Section 5.3.3), which does not promote the development of self-regulation in children. The parents' socioeconomic status did not explain their sons' drinking problems. However, their sons' own lower education was associated with higher problem drinking in adulthood, as additional analyses by T. Pitkänen indicated. Heavy use of alcohol from an early age is the risk of one's lower interest in studies and the accumulation of problems.

Females. The family backgrounds also explained significantly the amount of problems caused by drinking and higher scores in the alcoholism screening test, CAGE, in daughters (T. Pitkänen et al., 2008). The significant aspects of the backgrounds were low child-centeredness and the mother's smoking. No associations were found between the parents' use of alcohol and the daughter's problem drinking when the effect of the mother's smoking was controlled for (T. Pitkänen et al. 2008). It is possible that smoking mothers used alcohol more heavily than nonsmoking mothers. The variable for parental drinking concerned the parents in general without separating the father's and mother's drinking and in most cases the father used alcohol more heavily than the mother. The mother's model may have a specific effect on her daughter.

The parents' lower socioeconomic status was associated with the earlier age of the onset of the use of alcohol in daughters (T. Pitkänen et al., 2005) and low childcentered parenting was associated with the daughters' heavy drinking at age 20, and with higher number of problems caused by drinking and higher scores in the alcoholism screening test, CAGE, at age 42 (T. Pitkänen et al., 2008). These findings were in line with the results of the FinnTwin12 study in which low parental monitoring of children's behavior was found to be a risk factor for substance use at age 14 and in which girls turned out to be more vulnerable to the consequences of reduced parental monitoring than boys (Rose et al., 2001). This study also showed that when monitoring was lower, the influence of genetic factors was higher.

\subsubsection{Smoking cigarettes}

Smoking cigarettes was common in Finland when the participants were in their adolescence (in the 1970s), but when they were 50 years old (in 2009), smoking was restricted in public buildings, buses, trains, and many workplaces and the price of cigarettes had risen. Awareness of health risks concerning smoking cigarettes had increased during the previous 40 years.

Frequency. Age variation in smoking behavior of the JYLS participants was associated with historical time and smoking in the whole country. In 1973, 20 percent of 14-year-old girls and boys smoked cigarettes in the whole country of Finland (Rimpelä, 1980). In the JYLS data, the figures were of the same size in 1974 when the participants were 14 years old. At ages 18 to 20, the proportion of regular smokers was 30 percent both in the JYLS sample and in the whole country (Pulkkinen, 
1983b). The proportion of participants who had never smoked by age 27 was the same for men and women, 15 percent (Pulkkinen \& Kallio, 1988).

At age 27, regular smoking was more common among men (41 percent) than among women (26 percent) in the JYLS, but the gender difference diminished by age 50, when 33 percent of men and 22 percent of women were regular smokers. Thus, the cessation of smoking while approaching middle age was more common among men than among women. The median age of cessation to smoking was 28 years in women and 35 years in men. Lower education was associated with heavier smoking in men and women (Pitkänen, 2010). It suggests that more educated participants were more conscious of information on health risks associated with smoking.

Dependency. Dependency on smoking was high according to two criteria (Pitkänen, 2010). One criterion was to smoke ten or more cigarettes a day. Almost all men who had smoked cigarettes met this criterion, as did 80 percent of the smoking women. Regularly smoking men smoked from 2 to 50 cigarettes a day, women from 3 to 20 cigarettes; the median numbers were 15 and 10 cigarettes a day, respectively. The median age when the participant had smoked 10 cigarettes for the first time was 16 years.

Another criterion of dependency on smoking is how soon after waking in the morning a person wants to smoke a cigarette. Half of the men and 38 percent of the women who smoked cigarettes at age 50 said that they smoked the first cigarette during the first half an hour after waking; 78 percent of the men and 58 percent of the women within 1 hour. The participants who had a strong dependency on smoking represented 24 percent of all male participants and 12 percent of the female participants.

The use of other substances was rare among the participants. Ten percent of the participants admitted at age 50 that they had used one type of drug or medicine for intoxication, and 2 percent mentioned the use of several substances. Experimenting with or using drugs was mainly found in those participants who were daily smokers and consumed alcohol a lot. Generally, substance use had been limited to youth years. Only a few had used cannabis or other substances in the 2000s (while being 40 years or more).

The median age when experimenting with smoking began was 14 years. If the participant had not experimented with smoking by age 18, it was rare to do it later (Pulkkinen \& Kallio, 1988). Experimenting with smoking had taken place around 1.5 years before experimenting with alcohol. Regular smoking and the use of alcohol correlated positively, but the correlation was higher at age 14 (0.64) than at age 20 (0.37). In adulthood, heavy drinkers who drank to intoxication generally smoked and abstainers did not smoke. Still at age 50, smoking was associated with problem drinking, but not with the frequency of drinking (Pitkänen, 2010). Occasional smoking was said to be related to the situations in which alcohol was consumed.

Socioemotional behavior and smoking. The male and female participants who had not even tried smoking by age 20 had been more compliant at age 8 and more 
home-oriented at age 14 than smokers. At age 20, refraining from substance use was a characteristic of the Loner and Thriver styles of life in both men and women. Smoking cigarettes developed with the Reveller style of life, particularly in males, and with the Loser style of life, particularly in females (Pulkkinen, 1983b). The male participants who smoked regularly at age 20 had been more aggressive at age 8 and more peer-oriented at age 14 than the nonsmokers, whereas home conditions (low child-centred parenting) were associated with regular smoking in female participants. High emotional lability and aggressive behavior and low compliant and constructive behavior predicted the early start of smoking in males but not in females (Pulkkinen \& Kallio, 1988).

Smoking and drinking were connected to each other and associated with other problem behaviors in the Undercontrolled style of life at age 27. They correlated with disinhibition, experience seeking, and boredom susceptibility in men and women (Pulkkinen, 1990a). Smoking was also more common in women characterized by the Brittle style of life than in women characterized by the Resilient styles of life. Participants mentioned in the interview that main reasons for smoking were the feeling of relaxation, smoking habit, and smoking company.

\subsection{Health risks}

\subsubsection{Health in early adulthood}

Health of the JYLS participants was investigated at age 27 in the context of a personal interview. They were asked to describe their present state of health and the following: what problems they had with health; whether they used any medicine regularly; whether they had been in any accident and, if so, in what kind of accident and when; whether they had undergone surgery and why; whether they had headaches, sleeping disorders, or other feelings of being unwell; and whether they suffered from stress and, if so, for what reasons.

Early adulthood is generally a period of physical fitness and health. In the JYLS sample, the health of the participants at age 27 was generally good, but women estimated their health to be better than men; 86 percent of the women assessed it as good, 12 percent as moderate, and 1 percent as weak; of the men, respectively, 73, 23, and 4 percent (Pulkkinen, 1990c). Regular medicine users were around one-tenth of the participants. One-fifth of the participants had suffered from respiratory diseases or diseases of the digestive system and a few percent from heart diseases or diseases of the circulatory system. One-fifth had experienced diseases such as migraine, allergic rhinitis, and asthma and two-thirds (more often women than men) psychosomatic symptoms such as headaches, stomachaches, and sleeping problems. A feeling of stress had been experienced by two-thirds of men and women. Men were stressed by pressure at work and financial problems, whereas women were stressed by household chores, children's day-care arrangements, monotony of life, studies, and work. A few participants mentioned three or four causes for stress. 
Men's health was most often compromised by diseases or injuries of the locomotor system; 44 percent of the men (14 percent of the women) had been in an accident. The accidents had mostly taken place at age 18 to 20 . Only nine men mentioned that accidents had occurred under age 15, three of them before school age. All types of accidents had been more frequent in men than women: 20 percent of the men ( 8 percent of the women) had been in a traffic accident and 30 percent of the men (5 percent of the women) in other accidents (sport, work, play, violence). One-fifth of the men (5 percent of the women) mentioned that accidents had affected their health rather severely or severely.

Eight variables were constructed to describe the participants' health at age 27: subjective health, use of medicine, number of operations, unwell for psychosomatic symptoms, illnesses, the number of accidents, injuries as consequences of accidents, and stress. The men and women were separately grouped into clusters with a clustering technique. The same clusters could be identified for both genders except for one (Pulkkinen, 1990c): Healthy, Stressed, Medicators, Worried, and Injured. The sixth cluster was Risk-takers for men and Surgeries for women.

The comparison of the clusters showed, for instance, that female Medicators (regular users of a medicine) had started smoking earlier than other women and they smoked more than other women through adolescence. Also, heavy drinking in adolescence predicted the regular use of medicine. Female Medicators mentioned that they used alcohol for regulating their well-being. The male Medicators and Injureds (had been injured by accidents) had been more often drunk than the Healthys. Heavy drinking of male adolescents was related to problems with health because heavy drinking increased the risk of accidents.

\subsubsection{The predictors of accidents and impairment}

The predictability of risks of accidents on the basis of individuals' socioemotional characteristics was analyzed more closely by considering all peer nominations and teacher ratings at ages 8 and 14 (Pulkkinen, 1995). The results indicated that the most generalized predictor of the number of different types of accidents was noncompliance, that is, disobedience toward a teacher at age 8. Other characteristics in childhood and adolescence that contributed to the prediction of accidents were low anxiety and poor school success for males, but high anxiety for females. The gender difference in anxiety as a predictor of accidents was in accordance with other findings in the JYLS. Problem behaviors, such as heavy drinking in men, were associated with low anxiety involved in the Undercontrolled style of life, whereas problem behaviors in women tended to be associated with high anxiety in the Brittle style of life.

Negative consequences of heavy drinking (called problem drinking) increased the risk of different types of accidents by age 27 . In women, problem drinking alone explained the number of different types of accidents, but in men, disobedience at age 8 also was a significant predictor of accidents. Noncompliance in childhood was also the most generalized predictor of impairment in an accident 
among the women. Physical impairment was classified on the basis of the ratings of the interviewees into five categories (from $0=$ no accident or injuries to $4=$ very severe impairment, for example, loss of vision, several surgeries needed, impaired working capacity). Among the men, peer-nominated and teacher-rated aggression at age 8 and conduct problems at age 14 (including teacher ratings of sanctions at school, truancy, smoking, drinking, and contacts with police) were together significant predictors of impairment. The unique variance of aggression as a predictor of male impairment but not of the number of accidents, suggests that severe impairment involves a specific affective dynamics, not only low behavior regulation. Correspondingly, Romanov et al. (1994) has found that hostility was related only to violent deaths, not to traffic accidents. Early deaths by middle age among the men, but not among the women, were significantly associated with low self-regulation in childhood, which increases risks of accidents and unhealthy lifestyles.

Associations of health risks with personality profiles fitted the model for the Unfolding of Socioemotional Behavior (Section 4.2.1). The participants who had the style of life of Reveller tended to experience different types of accidents, possibly associated with sensation seeking typical of the Undercontrolled style of life (Section 5.3.2). Good health was associated with the style of life of Thriver (cf. Section 5.3.2), which precedes the Resilient style of life. The resilient participants exceeded other participants, particularly the brittle ones in different indicators of psychological functioning in adulthood (cf. Section 8.2). The resilient individuals felt well despite stresses, which they coped with using active behavior, whereas the brittle individuals tended to medicate themselves.

\subsubsection{Subjective health and metabolic syndrome in middle age}

Subjective health. Self-assessments of health (called subjective health) of the participants remained at around the same level from age 27 to 42 , but declined in middle age; good health was found in 81 percent of the women (72 percent of the men) at age 42 but in 70 percent of the women ( 62 percent of the men) at age 50 . Poor health increased from 2 percent to 10 percent in the men, but remained on the same level (4 percent) in the women. Subjective health became more moderate in the women (M-L. Kinnunen, 2010; Pulkkinen et al., 2003; Sinkkonen \& Pulkkinen, 1996).

At age 42, health estimation was also made by a doctor in the context of health examination. When the doctor's estimations were compared with self-rated health, it was found that the health estimations of the doctor were better (Pulkkinen et al., 2003). The doctor regarded the participants' health to be very good in 44 percent (18 percent in self-assessed health) and moderate or poor in 9 percent (22 percent in self-assessed health). The doctor said that she estimated the health of the participants in respect to the population of this age. The determinants of self-rated health at age 36 were studied by examining paths from age 14 socioemotional behavior to self-rated health by considering heavy drinking (frequency of being drunk), regular smoking of cigarettes, disabilities that affect functional capacity, physical 
(psychosomatic) symptoms, physical exercises, chronic diseases, use of medicine, and accidents (Kokkonen, Kinnunen, \& Pulkkinen, 2002).

The results showed that poor self-assessed health at age 36 was linked to heavy drinking among the men and to regular smoking among the women, which were associated with low self-regulation at age 14. Furthermore, psychosomatic symptoms such as headache, stomach-ache, loss of appetite, trembling hands, muscular pain, and irregular heartbeat correlated with poorer self-assessed health. The psychosomatic symptoms were also preceded by the use of alcohol (in men) and smoking (in women). Thus, the interpretation was that the individuals, whose behavior regulation was low, tended to resort to substance use, and the substance use explained the experience of physical symptoms and poorer assessments of health. Chronic diseases, use of medicine, and other variables did not explain selfassessed health and physical exercise did not explain or mediate better self-reported health outcomes.

Metabolic syndrome. Heavy drinking increases a risk of metabolic syndrome, which is a well-known risk factor for cardiovascular disease and diabetes. Indicators of the metabolic syndrome risk comprise insulin resistance, abdominal obesity, hypertension, dyslipidemia, and microalbuminuria (M-L. Kinnunen, Kokkonen, Kaprio, \& Pulkkinen, 2005). Waist circumference is a simple but strong indicator of metabolic syndrome. Other indicators are found in blood samples for the low level of high-density lipoprotein (HDL) cholesterol, and high triglycerides and plasma glucose, as well as in high systolic and diastolic blood pressures. Metabolic syndrome risk is estimated to exist when several of these criteria are met (M-L., 2010). The metabolic syndrome was more common among the JYLS men than among the women. It more than doubled in both sexes from age 42 to age 50 (from 20 percent to 48 percent for men, and from 12 percent to 26 percent for women).

The study of the connections between the metabolic syndrome and subjective health at age 42 revealed a high association between them (M-L. Kinnunen et al., 2005). This connection was understandable through substance use, which affected both the metabolic syndrome and subjective health as the third factor. A metabolic syndrome is not sensed as a pain or otherwise, it is defined based on laboratory results.

Why are heavy drinking, regular smoking, and the metabolic syndrome associated with self-ratings of poorer health? It was speculated that the self-assessment of good health simply characterizes a physically and psychologically well-functioning person who has a high resistance to stress and risk-taking behavior (M-L. Kinnunen et al., 2005). As presented in Section 8.2, which introduced the model for Personality and Psychological Functioning, self-rated health was highest in the Resilient personality profile and lowest in the Brittle personality profile. Self-rated health belonged to a pattern of several well-being indicators that were highest in the Resilient individuals and lowest in the Brittle individuals. An interpretation offered here is that emotion regulation underlying this dimension explains differences in subjective health and part of differences in substance use. This interpretation was confirmed by the findings that the individuals who are able to speak about 
their problems with others, who feel that they have control over their emotions, and who accept their mood and emotions, feel better, assess their health to be better, and have less psychosomatic symptoms than the individuals who have difficulties in emotion regulation (M-L. Kinnunen et al., 2005). These results suggest that personality factors should be added to the study of the explanations of subjective health.

Obesity. Overweight is indicated by a body mass index (BMI) that is calculated as the rate of a person's weight $(\mathrm{kg})$ and height (m, squared). If $\mathrm{kg} / \mathrm{m}^{2}$ is 25 or more, the BMI shows overweight and if it is less than 25, weight is normal. At age 50, half of the women (49 percent) but only a quarter (25 percent) of the men had a normal weight. Most men (41 percent) were overweight and one-third (34 percent) were obese $\left(\mathrm{BMI}>30 \mathrm{~kg} / \mathrm{m}^{2}\right)$. Among the women, the corresponding figures were 26 percent and 25 percent (M-L. Kinnunen, 2010). The higher BMI among the men than among the women concurred the gender difference in metabolic syndrome.

Individuals tended to remain in the same BMI-category throughout adulthood. Three trajectories, labeled Normal Weight (47 percent), Overweight (43 percent), and Obese (10 percent) were obtained (M-L. Kinnunen, Pietiläinen, \& Rissanen, 2006). All three trajectories were linearly ascending, which means that BMI increased in all categories, but the Normal Weight trajectory ascended least and the Obese trajectory ascended most (Figure 11.3). Participants who already were overweight in young adulthood gained the most.

Information on weight was available for the JYLS participants at ages 7 and 14 . Those who weighed more than the others at age 7 , fell more often into the trajectories of Overweight and Obese from age 27 to 42 compared with the trajectory of Normal Weight (M.-L. Kinnunen et al., 2006). Additionally, 14-year-olds who weighed most were more likely to follow the trajectory of Obese than the trajec-

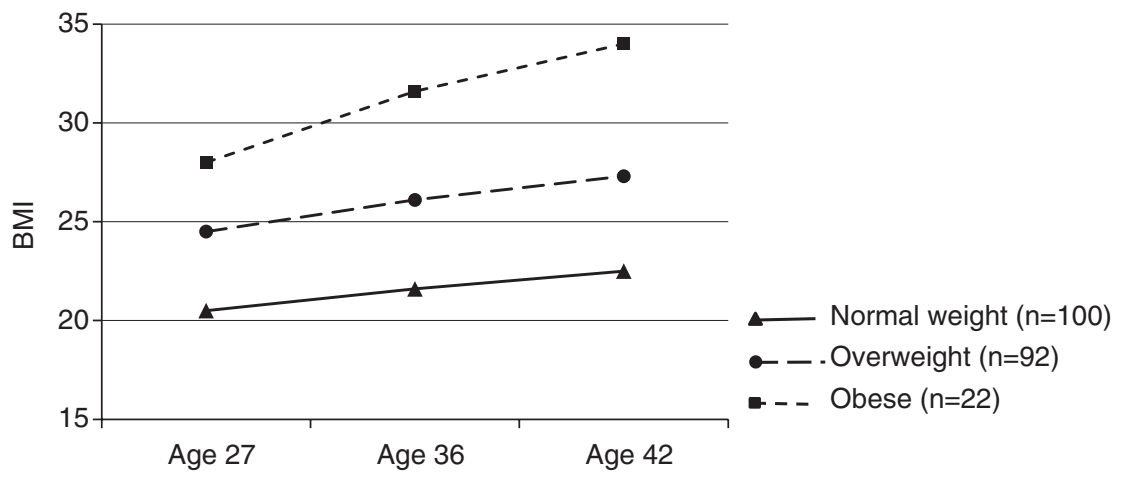

FIGURE 11.3 Trajectories of body mass index (BMI) in adulthood.

Source: From Kinnunen, M-L., Pietiläinen, K., \& Rissanen, A. (2006). Body size and overweight from birth to adulthood. In L. Pulkkinen, J. Kaprio \& R. J. Rose (Eds.), Socioemotional development and health from adolescence to adulthood (pp. 95-107). (Figure 4.2, p. 102.) New York: Cambridge University Press. (C) 2006 Cambridge University Press. Reprinted with permission. 
tory of Normal weight. BMIs calculated at different ages correlated significantly with each other, although the relative stability of BMI increased with age.

A study of associations of socioeconomic status with two indicators of metabolic syndrome risks, BMI, and blood pressure, indicted that the participant's lower education explained a higher BMI at age 42, and that the higher BMI explained a higher systolic blood pressure at the same age (Kivimäki et al., 2004). The socioeconomic status of the home of origin did not explain the level of the participant's BMI, but it did explain the level of his or her education. Thus, the home background was indirectly associated with BMI through the person's education, but the person's own education through his or her occupational status was the direct predictor of BMI.

The study of Kivimäki et al. (2004) also showed that systolic blood pressure in adolescence was the stronger predictor of systolic blood pressure at age 42 than the level of education. Genetic factors affect blood pressure, as well as the experience on one's health. Silventoinen, Lahelma, and Kaprio (2006), who analyzed the precursors of self-rated health with twin samples, concluded that socioeconomic differences are associated with self-rated health in Finland, but the "background of these differences is, however, not in family background but rather in genetic factors. An important question for further studies is whether genetic factors affect self-rated health independently or in interplay with home environment or other environmental factors" (p. 171).

\subsection{Temperance in adulthood}

Self-regulation is consistently associated with the characteristics of personality and lifestyles. A high self-regulation identified in childhood predicts positive social and psychological functioning, whereas a low self-regulation predicts problems in them. As a researcher of self-regulation for tens of years, I was very impressed by the finding that self-regulation had been included in the list of universal virtues (Peterson \& Seligman, 2004). Self-regulation was identified there as a character strength of temperance as one of the virtues. The concept of virtue entered the terminology of psychology with the emergence of positive psychology in the early 2000s. One of the goals of positive psychology is to provide a common vocabulary for communication about the good life, positive subjective experiences, and positive traits.

World philosophies and religions have conceptualized virtues for individuals' and society's benefit as described by Peterson and Seligman (2004) in their thorough analysis of character strengths and virtues. Some 2,400 years ago, Plato proposed that four core virtues of the ideal Hellenic city were anchored in individuals' souls. The virtues were wisdom, courage, self-restraint, and justice. Plato's student, Aristotle, included the original Platonic four (courage, justice, temperance, wisdom) in his list of virtues, but, to these, he added a number of others. In Christian theology, described in Thomas Acquina's doctrinal work (Summa Theologiae) in the thirteenth century, seven holy virtues include the cardinal virtues of 
wisdom, courage, temperance, and justice, and three theological virtues: charity or love, faith, and hope. Also, Muslim and Buddhist doctrines and Hinduism include temperance (or moderation or restraint) as a virtue when personal improvement is highlighted.

Peterson and Seligman (2004) collected character strengths that had to meet ten criteria, such as fulfilling an individual, being morally valued, and being trait-like in the sense of having a degree of generality across situations and stability across time. They classified 24 character strengths under six broad virtues that consistently emerge across history and culture:

1. Wisdom, referring to cognitive strengths; the respective character strengths are creativity, curiosity, open-mindedness, love of learning, and perspective as the product of knowledge and experience.

2. Courage, referring to emotional strengths; the respective character strengths are bravery, persistence, integrity, and vitality.

3. Humanity, referring to interpersonal strengths: love, kindness, and social intelligence are the respective character strengths.

4. Justice, referring to civic strengths; the respective character strengths are citizenship, fairness, and leadership.

5. Temperance, referring to strengths that protect against excess; the respective character strengths are forgiveness and mercy, humility and modesty, prudence and self-regulation.

6. Transcendence, referring to strengths that forge connections to the large universe and provide meaning; the respective character strengths are the appreciation of beauty and excellence, gratitude, hope (optimism), humor (playfulness), and spirituality (religiousness, sense of purpose).

The character strength, self-regulation, which has been the focus of the JYLS since 1968 is subsumed under the fifth virtue, called temperance. The character strengths of temperance are defined in terms of what a person refrains from doing against hate, arrogance, short-term pleasure, and destabilizing emotional extremes. Temperance balances or moderates activities and emotions between two extremes rather than suppressing them. According to a dictionary "A person who shows temperance has a strong mind and does not eat too much, drink too much, or do too much of anything." "If a person's behaviour is temperate, it is calm and reasonable, so that they do not get angry or lose their temper easily" (Collins Cobuild: English Dictionary for Advanced Learners, 2001, p. 1606).

The nature of temperance was studied by Pulkkinen and Pitkänen (2010) with three groups of the male and female participants of the JYLS, formed by using four criteria in adulthood: problems in the consumption of alcohol, smoking cigarettes, adult crime, and BMI. The purpose was to investigate whether the participants who displayed excessive behavior in several ways differed in the indicators of selfregulation from the participants who behaved in more conventional and controlled ways. The groups were: 
1. Temperate. The most controlled group (20.4 percent of 348; 42 women and 29 men). The following four criteria had to be met: intoxication (drunkenness) less than once a month; no smoking; no registered crime after age 20; and BMI $<25 \mathrm{~kg} / \mathrm{m}^{2}$ for women and BMI $<30 \mathrm{~kg} / \mathrm{m}^{2}$ for men.

2. Middle group (58.9 percent; 87 women and 118 men) between the most controlled and least controlled groups.

3. Intemperate. The least controlled group (20.6 percent; 31 women and 41 men). In this group, women met two to four criteria and men three to four criteria out of the following; intoxicated at least once a week, regular smoking, registered for crime after age 20, and BMI $>30$.

The groups were compared in the teacher ratings of socioemotional behavior at ages 8 and 14; Eysenck Personality Questionnaire (EPQ) and Sensation Seeking Scale by Zuckerman (SSS) at age 27; Karolinska Scales of Personality (KSP) and NEO-FFI at age 42; indicators of psychological and social functioning in adulthood; and family backgrounds. The following comparison concerns the temperate and intemperate groups; the middle group fell between these two groups in all variables. The middle group differed significantly from both groups only in three variables: school success and conduct problems at age 14, and self-rated health at age 42 (Pulkkinen \& Pitkänen, 2010). The following differences were found when the intemperates were compared with the temperates:

- Socioemotional behavior. The intemperates had been higher than the temperates in teacher-rated aggressiveness (men at age 8 and women at age 14), and lower in constructive and compliant behavior at age 14 (men already at age 8).

- Adult Personality. The intemperates had been higher than the temperates in disinhibition (SSS) and psychoticism (EPQ) at age 27; and in aggression and impulsiveness (KSP) at age 42, but lower in conformity (KSP) and conscientiousness (NEO-FFI).

- Self-control. The intemperates had been higher than the temperates in inhibitory control (KSP) and benign control at age 42; the latter refers to deliberate behavior (Emotion Control Questionnaire).

- Psychological functioning. The intemperates assessed their health to be poorer than the temperates at age 42 , and they had more eating-related psychosomatic problems. The intemperate men assessed their life satisfaction, psychological well-being, and self-esteem to be poorer at age 42 than the temperate men.

- Social functioning. The intemperates had, compared with the temperates, poorer school success at age 14 (women already at age 8) and their education was shorter, their career line was less stable, and their income level was lower at age 43 . They had more conduct problems at age 14 and they had started the use of alcohol at an earlier age.

- Family backgrounds. The intemperate women came from a lower socioeconomic background than women in the temperate group; this difference did not exist between intemperate and temperate men. The parents of the 
intemperates used alcohol and smoked cigarettes more than the parents of the temperates. The parents of the intemperates were younger than those of the temperates. The intemperates had experienced less parents' interest in school work (males) and child-centered parenting (women).

Conclusions. Low self-regulation manifested itself in these comparisons in numerous ways. The intemperates (one-fifth of the participants) displayed lower behavior and emotion regulation at school age, disinhibited personality in adulthood, and problems in social functioning. On the contrary, the temperates (one-fifth of the participants) were consistently on a track of high self-regulation and adaptation to society. Self-regulation develops in human relationships where the role of the parents as a model and supervisor is important.

Basic virtues are universal and they have a long history. In recent times, it has not been fashionable to speak about virtues in general and temperance in particular, at least in the Western world. Strengths of temperance are seldom praised. On the contrary, excessive behaviors and experiences have been popular, for instance, in mass media. Some change is, however, taking place in respect to increasing responsibility for sustainability regarding nature and human life (Caradonna, 2014). Self-regulation is also acknowledged, more often than in previous times, to be a core capacity of a child.

Individuals may have social and ethical ideals. It is typical of ideals that they direct one's attitudes and regulate his or her behavior internally without external control. The internalization of ethical ideals is part of an individual's personal growth. People are called virtuous if they have internalized ethical ideas. Temperance could be an ethical ideal. It has positive consequences for an individual's life. 


\section{2}

\section{THE FAMILY AND ITS CARE FUNCTIONS}

\subsection{A partnership in the family}

\subsubsection{The structure of the family}

Family is the domain of life that gives most pleasure to people throughout adulthood (Chapter 6). In this chapter, reasons for it can be found in one's relationships with the partner and children, and in intergenerational relationships. Associations of present relationships and parenting with socioemotional development, personality characteristics, and psychological functioning are analyzed. The aim is to find the emotional wholeness of intimate relationships.

Identification information enquired in studies often includes a person's marital status and the number of children. One could think that it is simple to collect data on them, but, in reality, it is complex. Traditional categories may not be sufficient for the description of the different ways people are living.

Marital status. The official categorization of marital status in Finland is based on the legislation concerning marriage: single, married, divorced, widowed, and registered relationship; the latter has been possible for same sex relationships since 2002. Marital status concerns the current situation without covering the marriage history (such as a remarriage).

Table 12.1 shows the distribution of the marital status at age 50 in the JYLS sample and in the whole age cohort born in the same year, 1959 (information for the latter were drawn from Statistics Finland in 2008) (Metsäpelto et al., 2010). The distributions were similar: close to 60 percent were married, around a fifth were divorced, and close to a fourth were single; the rates of the rest were marginal. Many single and divorced participants (22 percent of the men and 14 percent of the women) lived in a common-law marriage at the time when they were 50 years of age. When the common-law marriage was considered, the rate of the participants who lived as single was 10 percent of both men and women. Around 30 percent had divorced by age 50 , but some of them had remarried. Of the participants who 
TABLE 12.1 Official marital status in the age cohort group born in 1959 in Finland (data drawn in 2008) and in the JYLS sample at age 50 (in 2009)

\begin{tabular}{|c|c|c|c|c|c|c|}
\hline \multirow[t]{2}{*}{ Marital status (\%) } & \multicolumn{3}{|c|}{ Age cohort groups } & \multicolumn{3}{|c|}{ JYLS sample } \\
\hline & Men & Women & All & Men & Women & All \\
\hline Single & 27 & 19 & 23 & 28 & 19 & 24 \\
\hline Married & 56 & 59 & 57 & 54 & 58 & 56 \\
\hline Divorced & 17 & 20 & 19 & 18 & 22 & 20 \\
\hline Widowed & 1 & 2 & 1 & 0 & 0.8 & 0.4 \\
\hline $\mathrm{n}$ & 37740 & 37209 & 74949 & 142 & 124 & 266 \\
\hline
\end{tabular}

Adapted from Metsäpelto et al. (2010).

Note: The categories for single and divorced include participants who lived in a common-law marriage. The table does not include a registered relationship due to its rare frequency.

were married, 16 percent were remarried after the divorce and the rest were in their first marriage (Pulkkinen, 2010b).

The number of children and the structure of the household. The question: "How many children do you have?" may be difficult to answer, if it is not specified what children are meant. One may understand it as a question of how many biological children he or she has independent of their age and where they live, or how many children are living in the household. In the latter case, one has to decide whether to count the following: only one's own biological children or also spouse's children; children who live in the household full-time; or the children who live in the household part-time due to a joint custody that may concern one's own or the spouse's children. Some parents also have adopted children and some are foster parents of children taken into custody.

Most JYLS participants (88 percent of the women, 83 percent of the men) had biological children at age 50 (Pulkkinen, 2010b). The average number of children per person was 1.90 for the women and 1.84 for the men; 17 percent had one child and 43 percent had two children. A few percent had four children or more. The most fertile time of the participants was at ages 30 to 32 when almost half of all children of the participants were born. Women had their first child at a significantly younger age (on average at age 25) than men (at age 28). The proportion of young parents who were under 20 years when the first child was born was 6 percent; 75 percent of them were women. The age to become a mother varied remarkably, from the age of 15 to 47.

At age 50, almost a half of the participants had three or four people in the household, and 10 percent had a larger family of up to eight members. The members of the household mostly consisted of the spouse and their common children, but a fifth also had children from the participant's or the souse's previous relationship, and 2 percent had "part-time" children. Older children had already moved to live on their own; 87 percent of the participants had children who had reached the age of majority, which is 18 years in Finland. In a few cases, the respondent's mother (3.5 percent) or father (1.7 percent) lived in the same household. 


\subsubsection{The relationship between the partners}

The average age of the first marriage was 26 years for men and 25 years for women, but the range was from 15 to 42 years (Pulkkinen, 2010b). About 75 percent of the participants had lived in a common-law marriage at some point of their life. Many common-law couples get married when children are born to secure legislative security for the family and children.

Satisfaction with the relationship. The participants were asked at age 50 how satisfied they were with their present partner relationships or lack of it (Pulkkinen, 2010 b). Men and women were either very satisfied (41 percent) or quite satisfied (47 percent). Only 12 percent were dissatisfied. The level of satisfaction remained the same from age 36 to 50. Single individuals and those who lived as divorced or separated were least satisfied and remarried people were most satisfied. The number of children was not associated with the satisfaction.

A question: How happy are you in your relationship? was presented at age 50 with the participants who had a permanent relationship. The participants were happy (31 percent), very happy (30 percent), or extremely happy (29 percent); some of the participants ( 2 percent) found the relationship even perfect. Only 8 percent were unhappy. The happiness question was included in the Dyadic Adjustment Scale, which also included subscales for Consensus and Cohesion. Responses to these scales supported the conception of a satisfactory and happy relationship. The Consensus Scale concerns the agreement between the partners in everyday matters. The highest agreement between the spouses was when they were making major decisions, and it was also high in child-rearing and dealing with in-laws; around 70 percent agreed mostly or always. The disagreement that was most frequent was household tasks (18 percent). Also, the use of alcohol caused disagreement (16 percent). These figures were very similar at age 42 . The cohesion subscale, which was presented with the participants at age 42, comprised questions of shared activities. Almost all couples shared a calm conversation with each other either every day or almost every day, and more than half of them enthusiastically exchanged thoughts almost every day. Many couples (40 percent) shared work, but less often hobbies. Consensus and cohesion correlated with each other by 0.57 . They also correlated with partner satisfaction.

Jealousy is a feeling that may disturb an intimate relationship. It was studied at age 42. The questions concerned the consequences of the respondent's own jealousy for the relationship. Women and men had equally (27 percent) experienced jealousy to the extent that it had caused at least one of the problems of the following types: frequent quarrels, physical violence, depression, termination of the relationship, increasing use of alcohol, and need for psychiatric help (Suomalainen, Kokkonen, \& Pulkkinen, 2004). Jealousy did not correlate with the partner satisfaction in the men, but in the women, there was a small negative but significant correlation $(-0.20)$. Partner satisfaction correlated negatively with neuroticism, but jealousy did not correlate with neuroticism.

Divorce. At age 50, the respondents were asked: "How often have you considered divorce, separation, or terminating your relationship?" "Do you ever regret 
that you are married (or living together)?" "How often do you and your partner quarrel?" and "How often do you and your mate get on each other's nerves?" Only about 7 percent of the men and women responded to these questions "often" or "very often." Half of the participants (56 percent) had never regretted the present relationship and 39 percent had never considered its termination. Quarreling and getting on each other's nerves were more common; only less than one out of ten had never experienced them. Thus, there may be quarrels in a happy relationship. These figures were very similar at age 42 .

Among the participants who were interviewed at age 50, a third of the women (31 percent) and a fourth of the men (26 percent) had divorced at some point of time. The proportion of the participants who had separated from their commonlaw marriage was about the same: 32 percent of men and women (Pulkkinen, $2010 \mathrm{~b}$ ). The same individuals might have separated from a common-law marriage and, therefore, the rates cannot be summed up to show how many had ever separated or divorced.

The age of the participants at the time of the divorce varied from 21 to 50 years and there was no clear peak age of divorce. Half of the divorces had taken place until the age of 36 in the women and until the age of 39 in the men. A few individuals had divorced twice. Half of the separations from the common-law marriage took place by age 27 in the women and by age 29 in the men.

Self-regulation and the partner relationship. A question of whether the quality of romantic relationship can be predicted on the basis of individuals' socioemotional characteristics in childhood was studied when the participants were 36 years old (U. Kinnunen \& Pulkkinen, 2003). The divorced participants differed, on average, from those living in the intact marriage in childhood socioemotional behavior. Among the men and women, the divorced group had been assessed to be more aggressive by their teachers at age 8 , and men also at age 14 , and they had rated themselves to be less agreeable at age 27. A logistic regression analysis indicated that being assessed as very aggressive (above 75 th percentile) at age 8 multiplied the divorce risk by 4.8 for women and by 2.7 for men compared with the assessment as being not aggressive. Aggression at age 14 and low agreeableness at age 27 did not improve the explanation of divorce.

Compliance at age 14 helped men to remain in the relationship: an increase of 1 standard deviation in the scale of compliance decreased the odds of subsequent divorce by 58 percent. Compliance was an even more powerful predictor of nondivorce in men than aggression was the predictor of divorce. Thus, high behavior regulation was a resource factor for a romantic relationship in the men.

Among the women, a 1-year increase in the age at the time of marriage decreased the odds of subsequent divorce among the women by 28 percent when aggression at age 8 was controlled for. It suggests that 1 year increased women's maturity for the relationship (and possibly the choice of a partner).

The quality of partnership may also vary in intact marriages. It was studied by defining marital quality on the basis of the participant's satisfaction with the relationship and the amount of hostility in the relationship (U. Kinnunen \& Pulkkinen, 
2003). It was found that the women who were living in a good marriage (close relationship, no conflicts) compared with those who lived in a poor marriage (not close, a lot of conflicts) at age 36, had been rated by their teachers at age 8 as more constructive and active and socially less anxious and passive. Constructive behavior (positive confrontation, active coping, and consideration of others) involves high emotion regulation. The good quality of women's marriage was connected to high emotion regulation. Among the men, the men who were stably married and satisfied with the relationships had been rated as less aggressive, anxious, and labile at age 8 than those who were stably married but dissatisfied. The good quality of men's marriage was associated with a lack of the indicators of both components of low self-regulation (emotion and behavior regulation).

The men and women in the JYLS were not couples with each other and, therefore, the characteristics of their spouses were not known. The results show, however, that the amount and quality of self-regulation, even in one of the spouses, contributed to the quality of the perceived relationship.

\subsubsection{Adult attachment}

Attachment theories were developed to describe a child-parent relationship, but they have been extended to also describe an adult-adult relationship. Attachment refers to an enduring and intensive affective bond between particular individuals (Bartholomew, 1990). It includes four observable components: (1) proximity seeking, which means approaching, staying near, and making contact; (2) separation protest, which means resisting separations and being distressed when they occur; (3) safe haven, which means a possibility to turn to the other for comfort and support in time of distress; (4) and secure base, which means a possibility to trust in the availability of the other and, hence, to engage in nonattachment and explorative behavior (Ainsworth, Blehar, Waters, \& Walls, 1978).

Different models of measuring attachment have been developed. Children's attachment has mainly been studied in terms of anxious/avoidant, secure, and anxious/ambivalent attachment patterns (Ainsworth et al., 1978). A fourth class was suggested by Main and Weston (1981) to cover disorganized and highly anxious attachment behavior that cannot fit the three patterns of Ainsworth et al. (1978). A four-category model of adult attachment (Bartholomew, 1990) was applied to the JYLS data when the participants were 36 and 42 years of age. In Bartholomew's model, the four adult attachment styles are defined as the combinations of positive or negative working models of both self and others. A positive model of self means that a person perceives himself or herself as worthy of love, whereas a negative model means that the perception of oneself is unworthy of love. Correspondingly, a positive model of others means that others are seen as caring and available, whereas a negative model means that others are seen as uncaring and distant. A working model guides interpersonal behavior and information processing.

The four attachment styles (with two opposing pairs in terms of the positivity of the self and other models) were: (1) the secure attachment style, which is based 
on the positive model of both self and others; it means that the person is comfortable with intimacy and autonomy; (2) the fearful attachment style, in which both models are negative, the person has fear of attachment and is socially avoidant; (3) the preoccupied attachment style, which is based on having a negative model of self but a positive model of others; it means that the person is overly preoccupied in the attachment behavior and dependent on others; and (4) the dismissing attachment style, in which the model of self is positive but the model of others is negative; it means that the person denies attachment and dependence.

The definitions of the four attachment styles were modified from the Relationship Questionnaire by Bartholomew and Horowitz (1991) and presented with the JYLS participants in the context of the personal interview at ages 36 and 42 . The participants were asked to indicate how well the definitions described them in relation to those who are of (1) their same sex and (2) their opposite sex. Secure attachment was defined as being emotionally close; fearful attachment as being uncomfortable getting close to others; preoccupied attachment as being completely emotionally intimate with others; and dismissing attachment as being comfortable without close emotional relationship (Männikkö, 2001).

The results obtained when the participants were 36 years old showed that the attachment styles were rather similar in relation to the same and opposite sex (Männikkö, 2001). The attachment styles were also similar across time. The similarity across genders and time indicates that the attachment style represents a more general way of relating to others than just to the current romantic partner. The secure attachment style was most frequent; two-thirds of the participants assessed that it described them well or very well. The preoccupied and dismissing attachment styles were least frequent, altogether around 10 percent of the participants had assessed that they described them well. The distributions were very similar for men and women.

Associations of the attachment styles at age 36 with personality traits and psychological functioning were studied by comparing four adult attachment clusters extracted by a clustering technique (Männikkö, 2001, p. 88). The comparison of the attachment clusters in the Big Five personality traits showed that secure attachment was associated with high extraversion and low neuroticism, whereas fearful attachment was associated with introversion and high neuroticism. The attachment styles did not differ in conscientiousness. The participants who had either fearful or preoccupied attachment style (both of them are based on a negative model of self) were more neurotic than those who had either secure or dismissing attachment styles (a positive model of self in both of them). The results were in agreement with earlier studies as reviewed by Männikkö (2001).

The secure and fearful attachment styles also differed from each other in psychological functioning. The participants who had the secure attachment style were characterized by higher self-esteem and psychological well-being than the participants who had the fearful attachment style. The fearful attachment style was also associated with depressive symptoms, alexithymia (i.e., problems in emotional communication), anxiety, irritability, guilt feelings, low socialization, health problems, 
and heavy drinking (Männikkö, 2001, p. 94-101). These differences concurred with differences in psychological functioning (Figure 8.1) between the Resilient and Brittle personality profiles. Secure attachment can be seen as involving more emotion regulation than fearful attachment. Fewer differences were found between the preoccupied and dismissed attachment styles.

Bartholomew (1990) presented hypotheses concerning the origin and development of the different adult attachment patterns, for instance, that warm and responsive parenting would result in the secure adult attachment style, whereas the parents' negativity to the child would lead to the fearful adult attachment style. In the light of other results in the JYLS, this hypothesis was confirmed. The Resilient individuals have experienced more child-centered parenting than the Brittle individuals (cf. Section 5.3.3). Furthermore, studies on attachment between parents and children (e.g., Benoit \& Parker, 1994; Steele, Steele, \& Fonagy, 1996) have shown that parents with the secure attachment style generally have securely attached children. They are sensitive to their children's needs, which is a prerequisite of the development of attachment. Positive or negative experiences are stored in memory affecting conceptions and expectations on other people (Van Ijzendoorn, 1995). They guide emotional reactions and behavior in later relationships.

Conclusions. Partner relationship in middle age was, on average, very good. The quality of partnership had roots in self-regulation in childhood; high behavior regulation was associated in men with staying in marriage, and high emotion regulation in women was associated with better marital quality. Secure attachment was associated with good psychological functioning indicating high emotion regulation.

\subsection{Parenting}

\subsubsection{Delights and stresses in parenting}

Becoming a parent is one of the major adult transitions. Participants who had undergone five adult transitions, moved out from the parents' home, completed vocational education, entered work and marriage, and got a child were doing well psychologically (Section 6.1.3). Parenting brings about delights and responsibilities and, perhaps, also stress.

At age 42, the participants who had children were asked to describe the delights and worries that children brought for them (Pulkkinen et al., 2003). Almost everybody mentioned some kind of delight and worry. Most commonly mentioned delights were the positive development of the child and his or her positive characteristics. Children enrich the parents' lives. Worries were also often mentioned. They were mostly related to the children's well-being and their coping with life in the future. Parents were also worried about children's future, free-time activities, and their own parenthood. The latter included, for instance, knowledge about being a good parent and sufficiency as a parent.

Parenting stress is a construct that refers to parents' feelings of powerlessness and insufficiency in the face of parenting (Loyd \& Abidin, 1985). In the study 
by Rantanen, Tillemann, Metsäpelto, Kokko, and Pulkkinen (2015), parenting stress was conceptualized in terms of the parents' perception of their distress and incompetence as parents. It was studied when the respondents were 36, 42, and 50 years old with four items such as "I have a lot more problems raising my child than I expected." This study showed that the experience of parenting stress was stable from age 36 to 50; the correlations across ages varied from 0.57 to 0.62 , indicating that the participants who experienced parenting stress more than others at one age, tended to experience it at another age. More neurotic individuals tended to perceive more parenting stress, and more extraverted individuals tended to experience less parenting stress at each age.

Women and men did not differ in parenting stress (Fadjukoff, Pulkkinen, Lyyra, \& Kokko, 2016). The perception of high parenting stress at age 36 was found to be associated with low self-esteem and low mastery orientation (Aunola, Nurmi, Onatsu-Arvilommi, \& Pulkkinen, 1999). The mastery orientation refers to the extent to which a respondent believes that he or she has personal control over the situation (Nurmi, Salmela-Aro, \& Haavisto, 1995). Parenting stress seemed to belong to the pattern of the Brittle personality profile (cf. Figure 8.1).

There was reciprocal influence between parenting stress and personality showing that they influenced each other. High neuroticism at age 33 predicted high parenting stress at age 42, and high parenting stress at age 36 predicted high neuroticism at age 42 (Rantanen et al., 2015). Likewise, high extraversion predicted low parenting stress, and low parenting stress predicted high extraversion at the respective ages. The reciprocal cycles were centered on early middle age (from age 33/36 to 42 ), that is, on a period when most participants had children at home and were likely to be more committed to child-caring than later in midlife (age 42 to 50).

\subsubsection{Parental identity}

An adult's characteristic adaptation to life appears, among others, in his or her relations to various life domains. These relations have been described in terms of identity status (Marcia, 1966; cf. Section 7.2.1). In previous literature, parenting has not been among the domains in which identity development has been studied, but we included it in these domains (together with religious beliefs, political ideology, occupational career, intimate relationship, and life style) at ages 36, 42, and 50. A hypothesis was that the development of parental identity would coincide with the development of the identity status in respect to other domains, and indicate the person's maturation into the role of the parent.

Parental identity interview was part of the personal interview with an opening question: "Do you have an opinion of how to rear children?" Thereafter, the participants were asked the following: how they had acquired their view; whether they had ever thought of childrearing; whether they had had conflicting ideas about the issue; whether they had had influential people around or other sources for opinions; and how they had ended up with their present views. The participant's identity status was assessed by using two criteria: the firmness of personal commitment 
(no/yes) to an opinion and the absence or presence of a period of the exploration of opinion. In the identity diffusion status, commitment and exploration were lacking; in the foreclosure status, a person has an opinion learned at home or somewhere else without ever personally exploring alternative opinions; in the moratorium status, a person is exploring opinions without yet making a commitment to anything; and in the identity achievement status, the person has made the commitment to an opinion after the exploration.

The results showed that the most intensive progression in parental identity took place between ages 36 and 42 (Fadjukoff et al., 2016). At age 36, the foreclosure and achievement statuses appeared almost equally, but by age 42 , the identity achievement status became more common. This progression took place in the women and men, but more clearly in the women who outnumbered men in the identity achievement status at ages 42 and 50. Identity diffusion and moratorium statuses were rare among the participants.

The levels of the participant's education and occupation were not associated with parental identity status at any age level. But parental identity status was associated with being a parent. Commitment, which means having an opinion either after the exploration (achievement status) or without exploration (foreclosure status), was associated with having more children. In contrast, parental identity diffusion status was common if the participant had no children, particularly among the men. These findings supported the assumption that parental identity status would be associated with maturation as a parent.

The development of the parental identity status is a process that could be seen in a reciprocal relationship between nurturance and identity status (Fadjukoff et al., 2016). Nurturance was assessed at ages 36 and 42 using the Child Rearing Practices Questionnaire, and it was indicated by responses to questions concerning the parents' expression of appreciation, encouragement of independence, showing affection, and respecting the child's opinion. The participants who were high in nurturance at age 36 were likely to have parental achievement status at ages 42 and 50. Likewise, a person's identity commitment (seen either in the foreclosure or achievement status) at age 36 predicted higher nurturance at age 42. Parental identity diffusion was associated with low nurturance. The parents' awareness of the child's friends, whereabouts, and daily schedule (at ages 36 and 42) was also associated with identity achievement status at age 50 . Nurturance and awareness of the child's activities are two aspects of child-centered parenting (cf. Section 5.3.3). The women were higher in child-centered parenting (nurturance and awareness) than men, and their level of identity achievement was higher than in the men.

Parental identity was also studied in relation to the person's generativity, which refers to the adult's concern for, and commitment to, guiding and caring for the next generation (Fadjukoff et al., 2016). It was assessed by using the Generativity scale (e.g., "I am concerned about providing guidance and direction to younger people"). The results showed that generativity at ages 42 and 50 was highest in the men and women whose parental identity status was achieved at age 42 . Women and men did not differ in the level of generativity. In regard to parenting 
stress and psychological well-being, significant connections were found in the men. Men's identity commitment (foreclosure or achievement status) was associated with low parenting stress; the identity achievement status was associated with psychological and social well-being; and parental diffusion was associated with high parenting stress.

\subsubsection{Child-rearing practices}

In the interview of the participants at age 14 and one of their parents, a childrearing dimension entitled child-centered parenting versus adult-centered parenting was obtained (cf. Section 5.3.3; Pulkkinen, 1982). It was mainly based on children's (who were participants of the JYLS at age 14) perspective on parenting not excluding the parents' view. Child-centered parenting has been found in the JYLS to be longitudinally connected with high self-regulation in children, whereas its opposite, parent-centered parenting, has been found to be the risk factor of low self-regulation. Metsäpelto and Juujärvi (2006, p. 211) defined child-centered parenting in a comprehensive way:

Child-centered parents were consistently more capable of organizing their behavior from the perspective of the child by providing a context in which the child could feel the parents' warmth and acceptance, sustained involvement and interest in the child's activities, consideration of the child's opinions, and responsiveness to the child's needs for comfort and guidance.

The concept of child-centered parenting has also been used in the JYLS to describe more generally child-centered home atmosphere in the study by Kokko and Pulkkinen (2000). It was defined retrospectively at age 27 based on the memories of the participants of the time when they were around 14 years old (cf. Section 9.3). These memories concerned parenting practices, parents, and their relationships and they correlated with prospectively collected data on child-centered parenting (Männikkö \& Pulkkinen, 2001).

A third approach to child-rearing was made through the Child-Rearing Practices Questionnaire (LSQ), which was composed for the JYLS (cf. Section 2.4.3) and administered to the participants when they were adults. A factor analysis conducted in the study by Aunola et al. (1999) yielded three factors: parenting stress and two factors for a parenting style. They were named as authoritative and authoritarian parenting styles following Baumrind's (1971) terminology (see Maccoby \& Martin, 1983). The authoritative style was indicated by affirmative answers to questions of nurturance and guidance. The authoritarian style emphasis the parent's power over the child (e.g., "It is important that children obey their parents"). Authoritative parenting style is called child-centered parenting in the JYLS. Parentcentered parenting includes authoritarian parenting but also indifference to the child; in both cases, parents' own needs are highlighted and children's needs are 
ignored or seen in the parents' eyes. In the study by Aunola et al. (1999), men were more authoritarian than women, and the parents' lower education was associated with the authoritarian style. Women were more authoritative than men, and the authoritative style was associated with the parent's higher self-esteem and mastery orientation (the latter defined by Nurmi et al., 1995).

Parents were found to differ in the degree of the aspect of child-centered parenting called nurturance and they tended to maintain their position in it across situations relative to other parents (Metsäpelto, Pulkkinen, \& Poikkeus, 2001). These results were received in the Offspring Study of the JYLS, in which the participants with their school-aged child were observed in three interactive situations. The parents maintained their parenting style across situations. The parents' personality traits were associated with their parenting style. The parents' extraversion, together with their high openness to experience and low neuroticism, was associated with their selfrated higher nurturance (Metsäpelto \& Pulkkinen, 2003). There were, however, two other perspectives on the association between parenting style and extraversion. The observers of the parents' nurturance in a family interaction situation connected the mother's nurturance to extraversion, but the father's nurturance to introversion (Metsäpelto \& Pulkkinen, 2005). Children's estimations of family atmosphere and parenting were more positive when the parent was introverted and nurturing than when she or he was extraverted and nurturing. The parents' extraversion may involve power assertion that reduces the child's experience of positive parenting.

Another aspect of child-centered parenting is the parents' knowledge of children's whereabouts. A lack of that knowledge is a well-known predictor of children's problem behavior. However, it has been found in Sweden (by Stattin \& Kerr, 2000) that parental knowledge is based on the child's willingness to disclose to parents. To shed more light on the role of parental knowledge in the child's socioemotional development, information about parental knowledge was obtained from both the parents and children. It was found in the Offspring Study of the JYLS that a factor that explained the accuracy of the parents' knowledge of their children's whereabouts was family atmosphere (Metsäpelto \& Juujärvi, 2006). The more positively children experienced family atmosphere, the more knowledgeable they reported their parents to be. The interpretation was that a warm and supportive relationship motivates the parent to stay informed and creates a context in which the child can freely disclose to the parent. In contrast, the parents had less knowledge of their children's whereabouts in the families in which children experienced a lack of warmth, support, trust, and openness. Although the parents considered themselves to be knowledgeable, children reported that their parents were unaware of their activities. Possibly, children in these families create an illusion of disclosure by employing partial disclosure or lying.

The results by Metsäpelto and Juujärvi (2006) also indicated that when the parents reported to be knowledgeable of their children's daily schedule, activities, and interests and the children perceived their home atmosphere as positive, the children's behavior was characterized by high adaptive behavior including constructive, compliant, and socially active behavior. Child-reported negative atmosphere 
at home was, in turn, associated with externalizing problem behaviors in children. Parental knowledge of their children's activities supported the development of self-regulation in children.

Conclusions. A person's conception of being good enough as a parent (low parenting stress) was associated with extraversion and positive psychological functioning, whereas high parenting stress was associated with neuroticism. Parental identity status develops toward identity achievement with the experience of being a parent. The achieved identity status was associated with generativity and two aspects of child-centered parenting: nurturance and awareness of children's whereabouts. Children's perceptions of positive home atmosphere affect the disclosure to parents, as well as adaptive behavior.

\subsection{Relationships in the chain of generations}

Societal changes, such as urbanization and industrialization, have changed the lives of families in many respects and increased life expectancy. The time that generations share has lengthened and, therefore, there are several generations living simultaneously. There were altogether five generations $(G)$ to be considered in the description of relationships across generations in the JYLS. Information about the relationships between generations was received from G3.

- $\quad \mathrm{G} 1=$ the JYLS participants' grandparents

- $\quad \mathrm{G} 2=$ the JYLS participants' parents

- $\mathrm{G} 3=$ the JYLS participants

- $\mathrm{G} 4=$ the JYLS participants' children

- $\quad \mathrm{G} 5=$ the JYLS participants' grandchildren.

Relationships between generations can be analyzed in terms of cohesion. Silverstein and Bengtson (1997) classified intergenerational cohesion into functional exchange, opportunity structure, and affinity. Functional exchange consists of instrumental assistance and help. The help may be given in the form of child care, help with household tasks, shopping, renovation, giving money or property, mental or emotional support, or advice. Opportunity structure refers to geographical distance and contact between generations, and affinity means emotional closeness and agreement between generations.

In some countries, grandparents have an important role in childminding, but in Finland, rapid urbanization and geographical mobility after the Second World War reduced its significance in daycare arrangements. In the 1950s and 1960s, when Finnish women worked full-time to help the country to recover from the Second World War, it was common to hire a girl from the countryside to help in the household and childminding. The state-supported daycare system was created in the 1970s. When the JYLS participants were under school age, present daycare arrangements were not yet available and, therefore, their caretaking arrangements were varied. Children form the social security base of the parents in many cultures. 
In Finland and other Nordic countries, pensions and social welfare services have been developed to allow for aging people to live independently. Middle age has been called a "sandwich generation" when people have a dual role to take care of their children and parents while doing full-time work. The JYLS data allowed for the investigation into what extent Finnish 50-year-old people were burdened by this dual role in their lives.

Grandparents (G1-G3). The grandparents of the JYLS participants had passed away by the time when the participants were 50 years. In fact, almost all grandfathers (98 percent) and grandmothers ( 87 percent) had already died by the time when they were 36 years. At that age, the respondents did not have frequent contacts with their grandparents; in most cases, only a few times a year. The participants were asked at age 36 how they would assess their grandparents' influence on their lives and development. Memories were mostly either very positive (30 percent) or positive (30 percent). One-third (32 percent) assessed that the grandparents had no influence on them and 3 percent had critical or negative memories. Some participants (8 percent) had no memories of their grandparents because they had died early.

The relationship had been closest with the maternal grandmother; she had been chosen to be the closest grandparent in 43 percent. After her, the order was as follows: the paternal grandmother, the maternal grandfather, and the paternal grandfather (12 percent). The grandparents' caretaking and support on quite a regular basis, warmth, consideration of the child, and shared activities, and visits to the grandparents or longer stays with them (during holidays) left positive memories. The estimations of the grandparents' little influence on the participants were associated with distant relationships, infrequent meeting, and the grandparents' illness. A negative relationship between a grandparent and the participant's parent left negative memories, for instance, when the mother's mother and her daughter did not get along with each other. In some cases, the grandfather was too authoritarian and intolerant to children.

The support function was very central in the most positive memories. In some cases, it could be seen as a protective factor against developmental risks caused by difficult home conditions. The protective function of the grandparents on the grandchildren was found by Werner and Smith (2001) in their longitudinal study on Kauai, one of the islands of Hawaii. They defined protective influence by positive development of children who lived in risky conditions and were otherwise at a risk for negative development. Grandparents may also be seen as a model: the respected head of the family, professional model, or the model of a man.

The functions of grandparents presented in the literature (e.g., Bengtson, 1985; Hurme, 2006; Mueller \& Elder, 2003) most likely also affected the relationships in the JYLS:

- Securing biological continuity. The grandparents secure the transmission of their genes when they help their offspring.

- Conveying positive emotions and experiences. Grandparents do not have the same obligations toward grandchildren as they had toward their own children 
at a corresponding age. Therefore, they more easily can convey positive emotions to them and positive experiences with grandchildren increases the well-being of the grandparents.

- Securing social continuity. The grandparents create historical continuity by building a bridge to the past for their grandchildren.

- Acting as support persons. Grandparents may take care of their grandchildren while the parents work or there are other obstacles of the parents to do it.

- Acting as models. The grandparents show with their own lifestyles how older people act and think, give advice and convey knowledge about how to live.

Parents (G2 and G3). Parents are providers of care and support to their children. The influence of the parents on the participants' lives was described in Section 5.3.3 in terms of upbringing and living conditions. Child-centered parenting and steady living conditions supported the development of self-regulation and through it positive social functioning. The socioeconomic status of the home of origin had less impact on the respondents' development than parenting and living conditions in Finland, where economic and educational equality is high.

The mothers of the 50-year-old respondents in the JYLS were mostly alive (71 percent), but less than a half of the fathers (48 percent) were alive. Both parents were alive in 39 percent and both parents had died in 20 percent. The parents who were still alive had generally good functioning capacities and they lived in their own homes. A few percent lived in service flats or nursing homes. The mothers were born on average in 1932, the fathers in 1929. As the data were collected in 2009, their average age was 77 and 80 years, respectively, at the time of the study.

The most common type of support from the parents to their 42-year-old children was mental support: 75 percent said that they received mental support, encouragement or advice; 55 percent received help with child care; 42 percent received financial help; and 40 percent said that they received help with household chores or renovation (Hurme, 2006). In addition, Werner and Smith (2001) reported that emotional support from the parents to their middle-aged children was common (57 percent received it). The support received from the parents was contingent upon the relationship to the mother but not to the father; the JYLS respondents who did not get along with their mother received less help and support from their parents.

Few middle-aged children ( 3 percent) gave weekly support to their parents in personal matters such as dressing and washing. The participants reported that 87 percent of the mothers and 92 percent of the fathers did not need this kind of help. Neither had they seen their parents' need for financial support. Gender differences did not exist in providing support to the parents, except for emotional support. The daughters saw their parents' need for emotional support more often than the sons, particularly, in the mothers. One-fourth of the adult daughters (13 percent of the sons) reported giving emotional support to their mothers weekly, and 16 percent of the daughters (4 percent of the sons) to their fathers. Information and counseling, for instance, in computer use was the second frequent form of support and equally given by sons and daughters to their mothers and fathers. 
Giving and receiving support tends to be reciprocal. In the JYLS, reciprocity was found in the help to the parents and the help from the parents when the respondents were 42 years of age (Hurme, 2006). Those children who give a lot of help also received a lot. Reciprocity was especially clear for mental and emotional support. Of all respondents, 48 percent had both given and received mental and social support from their parents, whereas 20 percent reported that they had neither received nor given it. In 18 percent of the cases, the parents had given mental or social support, but this had not been reciprocated by their adult children. Correspondingly, in 15 percent of the cases, the adult child had given support, but this had not been reciprocated by their parents.

To the question: "How do you get along with your mother at the moment?" 36 percent responded "extremely well" and 45 percent "very well." The figures for the father were similar. The relationship with a mother or father was broken in 1 percent. Thus, family relationships were, in general, described as very positive when the participants were 50 years.

Participants (G3 and G4). The older children of the participants had already moved to live on their own; 87 percent of the participants had children who had reached the age of majority, which is 18 years in Finland. These children lived mostly (74 percent) within 50 kilometers distance. Contacts between the parents and children were held daily (43 percent) or weekly (46 percent). The participants described their relationships with their own children generally to be very good at age 50. It was asked with a question for each child: "How do you get along with your child." More than 50 percent of the participants chose the option "extremely well" and 30 percent "very well." Either "not very well" or "not at all" was chosen by 2 percent.

Parenting styles of the participants were described elsewhere in this book. It was found, for example, that child-centered parenting in terms of being nurturing and interested in the child's activities promoted adaptive behavior in their children. Parenting is vulnerable to pressures such as economic difficulties in the parents' lives, which may be reflected in the quality of parenting if these pressures raise negative emotions in the parents.

The participants as grandparents (G3-G5). Middle age is the time to become grandparents; 22 percent of the women and 8 percent of the men had biological grandchildren at age 50 . Some participants ( 6 percent) were also "social" grandparents, that is, the grandparents of their spouse's children. Grandchildren were mainly born in the 2000 s when the participants were over 40 years, but one-third of the grandchildren were born before 2000. The earliest age to become a grandparent was 33 to 35 years.

The participants' relationships with their grandchildren were asked separately for each grandchild. In regard to the first grandchild, the women's relationships were generally very close (79 percent), but in 13 percent, the relationships were very or quite distant. Among the male respondents, the relationships were very close in 50 percent and distant in 10 percent. The gender difference may indicate the grandmothers' higher emotional involvement in their small grandchildren. Both women and men had taken part in the care of some of the grandchildren; one-fourth had not taken part. 
The participants' parents as grandparents (G2-G4). The role of the participants' parents as grandparents was studied when the participants were 42 years old. A geographical distance of the grandparents to their grandchildren (generation 4) was less than 50 kilometers in two-thirds and more than 200 kilometers in onefifth of families. Only 2 percent of the grandparents lived with the families of their 42-year-old children.

The participants were asked to describe their children's contacts with both maternal and paternal grandparents, not only with the participants' own parents but also with their spouse's parents. The responses showed that children met with their different grandparents rather similarly, but they met with their maternal grandmother most frequently (38 percent met at least weekly) and their paternal grandfather least frequently ( 20 percent met at least weekly). It was rare to meet with the grandparent daily and it was also rare to meet them hardly ever.

The participants were also asked about their observations of the closeness of the relationships between their children and grandparents. The distributions were quite similar for different grandparents, but the relationship was described to be very close most often with the maternal grandmother and least often with the paternal grandfather. It was controlled that relationships between the grandparents and grandchildren did not depend on the participants' relationships with their parents (Hurme, 2006). The differences in the closeness of the relationship between different grandparents corresponded to the participants' experiences with their own grandparents and findings in the literature as reviewed by Hurme (2006).

Conclusions. Relationships between the generations were generally good. Positive memories of the grandparents were based on concrete activities such as caretaking. Relationships with maternal and paternal grandparents were equally active, but the closest relationship was with the maternal grandmother. Emotional support was reciprocated between the participants and their parents. At age 50, a general pressure to take care of both one's own children and parents, known as a "sandwich generation," was not common in Finland, where public daycare is available for children and social welfare services allow for older people to live independently. In addition, a spouse is considered the caretaker of his or her spouse who is in need of help. 


\section{3}

\section{WORK AS A SPHERE OF LIFE}

\subsection{Occupational status and career line in women and men}

How the participants fared in the domain of work is described from five perspectives in this chapter: occupational status and the consequences of early childbearing for career development; personality characteristics associated with success in career path; the consequences of job insecurity for individuals; balancing demands on work and family; and recovery from work and the content of one's free time.

The occupational status of the participants was assessed with a question: "What is your latest occupational title?" The answers were coded into four categories: blue-collar occupations (e.g., cleaners and factory workers); lower white-collar occupations (e.g., nurses and technicians); higher white-collar occupations (e.g., managers and physicians); and entrepreneurs. The distributions of occupational statuses were very similar at ages 42 and 50: the men were significantly more often than the women in blue-collar occupations and the women were more often than the men in lower white-collar occupations (Table 13.1; Metsäpelto et al., 2010). Gender differences did not exist in the higher white-collar occupations. The similar distributions of occupational status were also found in the spouses' occupations studied at age 42 (Pulkkinen et al., 2003). The women's spouses were more often in blue-collar occupations (36 percent) than the men's spouses (16 percent), whereas the men's spouses were more often in lower white-collar occupations than the women's spouses (54 percent and 19 percent, respectively). In many marriages, the wife's occupational status was higher than the husband's occupational status. As seen in Table 13.1, the similar gender differences in the distributions also existed in the whole age cohort group born in 1959.

The gender differences in the occupational status concurred with gender differences in education until age 50: the JYLS women had a vocational college education (the total length of studies was 13 to 15 years) more often than the men who had a vocational training at a lower level (11 to 12 years). Gender differences did not exist 
TABLE 13.1 Occupational status among the JYLS participants employed at ages 42 and 50, and in the age cohort group born in 1959 (data drawn in 2006)

\begin{tabular}{|c|c|c|c|c|c|c|c|c|c|}
\hline \multirow{2}{*}{$\begin{array}{l}\text { Occupational } \\
\text { status (\%) }\end{array}$} & \multicolumn{3}{|c|}{ Age 42} & \multicolumn{3}{|c|}{ Age 50} & \multicolumn{3}{|c|}{ Age cohort, age 47} \\
\hline & Men & Women & Total & Men & Women & Total & Men & Women & Total \\
\hline $\mathrm{n}$ & 150 & 133 & 283 & 143 & 124 & 267 & 30,905 & 31,414 & 62,319 \\
\hline Blue-collar & 47 & 11 & 29 & 43 & 8 & 27 & 41 & 20 & 30 \\
\hline Lower white-collar & 16 & 59 & 38 & 22 & 60 & 39 & 20 & 50 & 35 \\
\hline Higher white-collar & 23 & 23 & 23 & 23 & 23 & 23 & 23 & 22 & 22 \\
\hline Entrepreneur & 13 & 7 & 10 & 12 & 9 & 11 & 16 & 9 & 13 \\
\hline
\end{tabular}

Adapted from Metsäpelto et al. (2010) and Pulkkinen et al. (2001).

in the rate of higher academic training (16 years or more) (Pulkkinen \& Polet, 2010a; for the Finnish education system, see Section 6.2.1). It is common to highlight the need for life-long learning to increase people's readiness to work in changing society and changing occupations. More than half of the women (57 percent) and one-third of the men (35 percent) had received some kind of education or training during the past 12 months when they were 50 years of age (Pulkkinen \& Polet, 2010a). It covers studies in the Open University and evening classes, and in-company training. The women had been more active than the men in further education.

The participants' satisfaction with their choices of occupation was generally good at age 50 (very good, 31 percent, and rather good, 56 percent). The satisfaction was not associated with the type of vocational education the person had received, and there were no gender differences in the distribution of satisfaction. The average level of satisfaction with the choice of one's occupation did not change from age 36 to 50 .

The stability of one's career path may vary on all occupational levels. We have categorized the stability of career paths considering the time used for education, the type of work, and experiences of unemployment following the categories distinguished by Sinisalo (1986). In the study by Rönkä, Kinnunen, and Pulkkinen (2000), the following categories were defined based on the participants' work histories from age 27 through age 36 . The same categories were applied to the periods from age 37 to 42 and from age 43 to 50 (Kokko, 2010c).

1. Stable career line: a participant had worked in one's own field without periods of unemployment, or a career had become stable during the follow-up period; mothers who returned to their jobs from maternity leave.

2. Changeable career line: a participant had shifted from work to occupational training during the follow-up period; or removed herself/himself from the labor market to care for children.

3. Unstable career line: a participant's jobs varied and for most part did not correspond to the fields for which he/she had been trained, and had been unemployed for more than half a year but less than 4 years. 
4. Unemployment (stagnated) career line: a participant had been unemployed for more than 4 years.

The stable career line was most common: 69 percent of the participants had a stable career line at age 27, 59 percent at age 36, 74 percent at age 42, and 76 percent at age 50 (Kokko, 2010c). Variability in these figures was caused by severe economic recession during the first half of the 1990s, when the stable career line was less frequent and the changeable career line more frequent (19 percent at age 36) than at ages 27,42 , and 50 ( 8 percent to 9 percent). The increase of the changeable career was explained by the participants' decisions to continue studies or stay at home and take care of their children. The unstable career line decreased from age 27 to 50 (from 20 percent to 11 percent), and the stagnated career line was least frequent at all ages (6 percent to 7 percent at ages 36 and 42; 3 percent to 4 percent at ages 27 and 50).

There was a general dependence between the length of the educational route and the career lines: choosing a general upper secondary school was linked to an educational career, which was distinguished at age 27 and which later developed to the stable career line. A lack of post-comprehensive education increased the risk of the stagnated career line. Other career lines, except the stable career, were at age 27 connected in women but not in men with the starting of a family life (Pulkkinen, 1989a).

Early parenthood. According to the literature, entering into parenthood affects social functioning more in women than in men through closing off opportunities for vocational education and career consolidation, or may even set a chain of cumulative disadvantages in motion. Early childbearing occurs often among adolescents from disadvantaged family backgrounds. This selectiveness increases the risk of later problems amongst young mothers (Baldwin, 1993; Furstenberg, Brooks-Gunn, \& Morgan, 1987; Werner \& Smith, 1992). Forming a family at a young age may also serve a need to escape an unstable and unsupportive family situation (Rutter, Quinton, \& Hill, 1990), or early motherhood may be a consequence of risk-taking behavior (Serbin, Peters, McAffer, \& Schwartzman, 1991). It has also been argued that early motherhood is a career "choice" when possibilities for creating a career are few (Merrick, 1995).

The role of the timing of parenthood amongst the JYLS participants was investigated in respect to educational opportunities and career development. We formed three groups of women at age 27 based on their age at the first childbirth: (1) 21 or younger (21 percent of the women); (2) 22 to 27 years (37 percent); and (3) no children by age 27 (41 percent) (Rönkä \& Pulkkinen, 1998). At age 27, there were no differences between the groups in psychological functioning indicated by satisfaction with life, self-confidence, and depression. However, the youngest mother group did differ from the two other groups at age 27 in

- lower education and more unstable career line;

- more social functioning problems (drinking behavior and criminal arrests); and

- poorer developmental backgrounds as indicated by a higher cumulative index for risk factors such as problems in adjustment to school, poor school success, 
and parental drinking, although the groups did not differ in the socioeconomic status of the family of origin.

Also in the long-term, by age 42, the women who had become mothers relatively early (before age 25) differed in work-related matters and social functioning from the women who were late mothers (the first childbirth after age 29) (Kokko, Pulkkinen, \& Mesiäinen, 2009). The early mothers had lower occupational status and more unstable career line, and they used more alcohol than the late mothers. Differences between the early and late mothers, however, levelled off with age in the stability of career line.

Early motherhood was associated with several risk factors in women's developmental background, but consequences of early motherhood on later social functioning depended on whether women succeeded in continuing with education and finding their places in the labor market (Rönkä \& Pulkkinen, 1998). According to the study by Kokko et al. (2009), problems with career development and the use of alcohol that seemed to be caused by early motherhood, were explained by other factors than the timing of the birth of the child. Low self-regulation in childhood was associated with the use of alcohol, and low school success was associated with problems in career development. Early child bearing might be associated with both low self-regulation and low school success, but it was not a reason for drinking or career problems; school success was more decisive. The early mothers with good school success reached the same level of education than the late mothers if there were opportunities for studies. Women who had a university degree had a higher grade point average (GPA) at the end of the comprehensive school than those who did not have a university degree, no matter when their first child was born. As also noted by Jaffe (2002), early child bearers often experience interpersonal and mental health problems, not only because they have a child at a young age, but there are other factors in these women's life histories that explain their problems.

In the analysis of the timing of education (cf. Section 6.2.3) it was found that women on the trajectories of "no or early education" and "off-time education" (peak time in mid-thirties) typically had the first child early, had started to use alcohol at an earlier age, and used it more heavily in adolescence than the women on the trajectories of "on-time" and "continuing" education (Kokko, 2006; Kokko, Pulkkinen, Mesiäinen, \& Lyyra, 2008). The "off-time education” women showed, however, an upward social mobility, from blue-collar to lower white-collar occupations, due to having an educational degree. It was associated with optimism. Post-comprehensive education contributed to women's career line, when time for completing post-comprehensive education was extended until age 42 .

According to these results, education at a later age strengthened women's positive development in spite of risk factors in their background. These findings suggest that negative consequences of early motherhood could be minimized by encouraging young mothers to continue their education. Most likely, education would also increase women's positive parenting with their own children. Parenting in the 
home of origin had been less child-centered in the early childbearing women than in the on-time education women.

Early parenthood also affected male functioning, but the effects were opposite to those for women. It was typical to be single at age 42 if a man had not received any vocational degree, whereas "no-degree women" typically had a child early (Kokko, 2006). The early timing of parenthood in the men was linked to career stability, not to career instability as in the women, and consequently, to a low number of social functioning problems (Rönkä et al., 2000). Men had become parents at a very early age more rarely than women, and thus, many of them had already finished their training as skilled workers. Men did not stay at home with their children, but tried to find a place in the labor market, whereas the majority of young mothers did stay at home with their children, most of them for several years. The "off-time education" did not result in an upward social mobility, from blue-collar to lower white-collar occupation in the men, and it was not associated with better psychological functioning such as increased optimism in the men, which was found in the women (Kokko et al., 2008).

Post-comprehensive education and risk behaviors. A lack of post-comprehensive education is the risk factor of one's lower occupational status and unstable career line. To study whether the consideration of other types of risk factors added to the risk of an individual's problems in social and psychological functioning, we made two sets of comparisons at age 50. We compared (1) the participants who had and who did not have post-comprehensive education until age 27, and (2) the participants who had and who did not have several risk factors (Pulkkinen \& Kokko, 2015) in occupational status. The risk factors included poor school success and poor school adjustment at age 14; onset of drinking at age 15 or younger; criminal offending; and a lack of post-comprehensive education. Around 5 percent of the men and women had all five risk behaviors, and close to 30 percent had no risk behaviors.

The comparisons of the groups indicated that post-comprehensive education alone was significantly associated with the higher future occupational status, and the consideration of the numbers of risk factors did not add to this association. The consideration of other risk factors had additional value to career stability and psychological functioning in the men, but not in the women; perhaps career stability depends on family-related choices more in women than in men. However, the higher numbers of risk factors were associated with the heavier use of alcohol in the men and women.

Conclusions. Women were more often in lower white-collar occupations than men who were more often in blue-collar occupations, which concurred with longer vocational education of the women compared with the men. School success and education are major factors affecting the occupational status. Early motherhood was associated with several risk factors in the women's developmental backgrounds, but the consequences of early motherhood for occupational status depended on whether women succeeded in continuing with education and finding their place in the labor market. 


\subsection{Success in career path}

\subsubsection{Constructive behavior in childhood as the antecedent of success in the work domain}

Studies with the JYLS sample have repeatedly shown that constructive behavior in childhood is a significant antecedent of his/her success in the work domain. Constructive behavior was assessed by using peer nomination and teacher rating considering three aspects: active coping with a problem; positive thinking and an active confrontation; and consideration of others with helpfulness and empathy (see Box 2.1). As presented in the model for the Unfolding of Socioemotional Behavior (Section 4.2.3) and confirmed by the results (Section 5.3.2), constructive behavior is an antecedent of the Resilient lifestyle. Constructive behavior is interpreted to indicate high emotion regulation.

Occupational attainment. The antecedents of occupational attainment by middle age were searched in a comparative study between the JYLS sample and a US sample (the Columbia County Longitudinal Study [CCLS]) (Dubow, Huesmann, Boxer, Pulkkinen, \& Kokko, 2006). Both samples were followed from age 8 to middle age. Occupational attainment at age 42 (JYLS) and at age 48 (CCLS) was defined in terms of occupational status. Educational status was assessed at ages 27 to 30 years, and the participants' socioemotional characteristics were assessed in childhood and adolescence. In addition, the family's occupational status in childhood was considered.

As could be expected, occupational status in middle age was most highly predicted (in both samples and both genders) by educational status in early adulthood, which, in turn, was predicted by cognitive-academic functioning at age 8 and the family's occupational status. It is notable, however, that the participant's personal characteristics related to self-regulation contributed to success in the work domain. In the JYLS, higher emotion regulation (indicated by constructive behavior) in adolescence predicted a higher occupational status in the women and a higher educational status in the men. A variable that approximated constructive behavior in the CCLS was the child's popularity. It predicted a higher educational status. The child's aggressive behavior (low behavior regulation), in turn, tended to explain a lower educational status independent of cognitive functioning in the US sample, but not among Finnish men. Another Finnish cohort study by Alatupa et al. (2013) shows, correspondingly, that low social adjustment predicts low educational attainment and occupational status at ages 30 to 36 .

The high significance of the participants' constructive behavior for their occupational attainment was also seen in the study by Pulkkinen, Feldt, and Kokko (2006). Success in the work domain at age 42 divided into two components: achievement level and employment. Achievement level was a latent variable for the length of education and occupational status, whereas employment was a latent variable for the stability of career line and the present employment situation (full-time, parttime, nonworking). The results showed the following connections: 
- In the women, constructive behavior alone contributed to their success in the work domain, but in regard to the level of achievement, not in regard to employment. The higher level of achievement explained women's positive psychological functioning (Figure 13.1).

- In the men, a broad latent factor for self-regulation covering constructive behavior, compliant behavior, and emotional stability from age 8 to 14 predicted both the level of achievement and employment at age 42. The level of achievement predicted positive psychological functioning.

Psychological functioning was indicated by psychological well-being and selfreflections (self-esteem, sense of coherence, and trust in life). Trust in life was assessed with questions concerning fears for the future, expectations on the future, and control over life events. The results on the association between achievement level and psychological functioning were in accordance with findings on the impact of education (which affects the occupational status) on emotional well-being, as reviewed by Keyes and Waterman (2003), and with findings on the association between the occupational status (through the quality of work) and well-being (Karasek \& Theorell, 1990).

Another study in which constructive behavior contributed to career orientation (defined with the same variables as occupational attainment; Pulkkinen, Ohranen, \& Tolvanen, 1999) showed that career orientation in women at age 36 was preceded by constructive behavior and social activity at ages 8 and 14, and extraversion at age 27. Men's career orientation, in turn, was preceded by high self-regulation more broadly covering both constructive and compliant behavior, and agreeableness at age 27. A typical extravert is social and active, and these characteristics may be needed in female career development. Activity in men may, in turn, involve a tendency to externalize problems, which compromise their career orientation (Pulkkinen, 2009; Pulkkinen et al., 1999). Therefore, agreeableness that involves

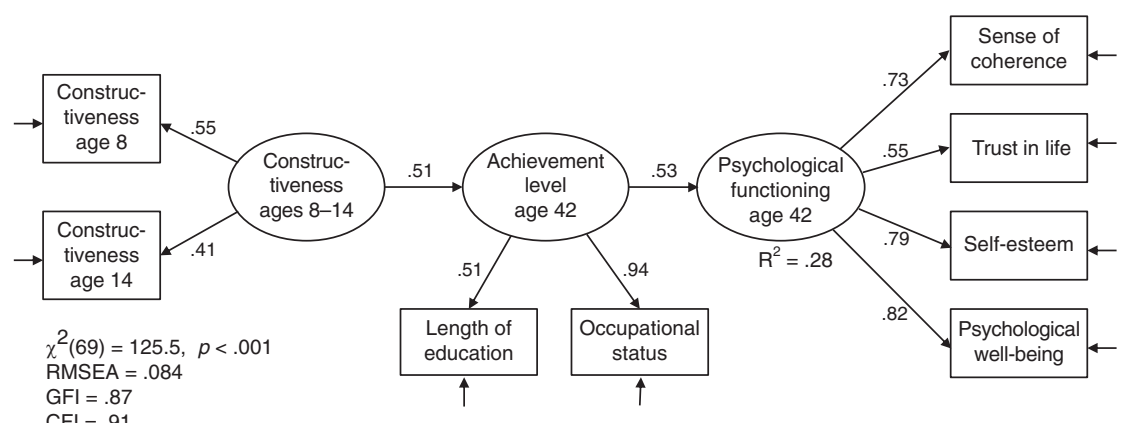

FIGURE 13.1 Constructive behavior as an antecedent of success in work domain and psychological functioning: women.

Source: From Pulkkinen, L., Feldt, T., \& Kokko, K. (2006). Adaptive behavior in childhood as an antecedent of psychological functioning in early middle age: Linkage via career orientation. Social Indicators Research, 77, 171-95. (Figure 3, p. 187.) (C) 2006 Springer. Reprinted with permission. 
compliance, modesty, and tender-mindedness may secure men's career orientation. In contrast, compliance in women may involve passivity, which does not promote success in their career line. Anxious and passive behavior in childhood and neuroticism in adulthood were associated with low occupational attainments in women, whereas emotional lability and poor school success contributed to low occupational attainments in men.

Income level. The role of one's constructive behavior in childhood in respect to his/her success in the labor market was also seen in the study of the antecedents of labor market income (Viinikainen, Kokko, Pulkkinen, \& Pehkonen, 2010). The results showed that extraversion in adulthood and constructive behavior in childhood were positively associated with income; an increase of 1 standard deviation in extraversion at age 42 was associated with about 9 percent increase in income at age 43; and an increase of 1 standard deviation in constructive behavior at age 8 was associated with a 10 percent increase in income at age 43 after controlling for gender, education, work experience, and unemployment history.

Other factors that affected the level of income were the level of education, occupational status, the stability of working career, low number of unemployment spells, and the male gender. When education, employment history, and childhood constructiveness were included in the model, adult extraversion did not improve the prediction of income and could be omitted from the model. In contrast, childhood constructive behavior in both genders contributed to higher income in middle age despite the interval of 36 years. It shows that constructiveness in childhood involved characteristics that were favorable for success in work and higher income. Other childhood characteristics included in the study indicating behavior regulation (inattentiveness, aggression) and social activity were not significant predictors of income. Neither had the other adult personality traits (neuroticism, openness, agreeableness, and conscientiousness) significant contributions to income when work history was controlled for.

Dropouts. Education and degrees have consequences for the labor market in terms of employment and earnings. The interruption of education is the risk of problems in the labor market as shown with the JYLS data (Viinikainen, Kokko, Pulkkinen, \& Pehkonen, 2014). A dropout was defined as a person who for some reason had decided to discontinue his or her studies at any level of education; he or she might have continued studies later, but was still considered a dropout; 16.5 percent of the participants were defined as dropouts. It was found that the dropouts and nondropouts did not differ in school achievement, annual earnings, and socioeconomic status of their parents, but they did differ in the length of education, which was longer in the nondropouts, and in self-regulation (constructiveness and emotional stability) at age 8 , which was higher in the nondropouts compared with the dropouts. A higher score in constructive behavior at age 8 was associated with a higher number of years of employment in adulthood and with fewer years of unemployment. The dropouts had experienced more unemployment, they had less work experience, and their working careers were less stable than in the nondropouts. 
Dropping out was associated with weaker labor market performance over a long period of time. There was, however, variation in this association between individuals, and this variation was explained by their personality characteristics. The dropouts who had been higher in constructive behavior at age 8 had better labor market success than the dropouts who were lacking constructiveness. For the latter, dropping out was associated with lower cumulative earnings and more years of unemployment (Viinikainen et al., 2014). Thus, constructive behavior (and emotion regulation involved in it) can be seen as a characteristic that increases resiliency in the labor market in spite of dropping out.

Along the same lines, emotional intelligence assessed at age 42 was associated with positive attitudes toward work at ages 36 and 42 (Granberg, 2003). A high score in the Self-Report Measure of Emotional Intelligence, and particularly perception of one's own and others' feelings, correlated with success expectations in men and women and slightly with occupational status. Having control over one's job and having a job that demands creativity and highly developed skills were also associated with emotional intelligence in men. In women, emotional intelligence was associated with general mastery beliefs and low task-irrelevant behavior in the Strategic Attribution Questionnaire. Women's scores in emotional intelligence were higher than men's scores.

\subsubsection{Satisfaction with work}

Significance of work. The meaning given to work was assessed in the JYLS at ages 36, 42, and 50 using the typology by Kahn and Wiener (1967). The participants were asked to assess the significance of work for them by selecting the description out of four that best corresponded to their own feelings about work. In parentheses, selections made at age 50 are indicated in percentage for males and females:

1. Work is a necessity: "I do it only to get paid and earn my living. The central meaning of my life is related to events outside of my job." (M 22 percent; F 10 percent)

2. Work is an occupation or profession: "At least to some extent, professional skill, occupational pride, self-respect, and orientation to develop are related to my work." (M 49 percent, F 72 percent)

3. Work is a career: "Work is a significant source of satisfaction in my life and the basis of planning my future. Work and free-time are not necessarily clearly separated. My efforts to achieve, develop, and succeed in my career are strong." (M 17 percent, F 12 percent)

4. Work is a calling or a vocation: "Work constitutes the essential content of my life and the basis of my self-expression. The difference between work and free-time is almost nonexistent or they are firmly linked to each other." (M 12 percent, F 6 percent)

Most participants - more women than men-regarded work as a profession, which means that personal needs for development were connected to work. Work as a career 
was equally selected by women and men, but more men than women regarded work as a necessity. Participants had most unfrequently felt that work was a calling or a vocation, implying that they were especially suited to do the job or to fulfill a particular role in life. There was only a small variation in the meaning of the work across ages.

The occupational status was associated with the significance of work: work was typically seen as a necessity by participants in blue-collar occupations; as a profession by those in lower white-collar occupations; as a career by those in higher white-collar occupations; and as a calling by entrepreneurs (Sainio, 2013). The higher the rating was, the more internal was the participant's work orientation. Compared with women, men tended to have more internal work orientation, but on the other hand, there were also more men for whom work was just a job.

Satisfaction with income. Work is an important means of earning one's livelihood. The financial situation of the participants was studied at age 50 (in 2009) by asking them to choose the category of monthly salary that best fitted their salaries before tax deductions. The median income level was higher (2,501 to 2,800 euros per month) in the men than in the women (2,201 to 2,500 euros; Pulkkinen \& Polet, 2010a). Also, among the highest earning participants (in this study more than 4,600 euros per month), the proportion of men was three times higher than that of women. Income differences between the men and women increased between the ages 42 and 50. Among the least earning participants, a gender difference did not exist: earnings less than 1,000 euros per month were in 8 percent of the men and women at age 50 .

A salary from the same work should normally be the same for men and women, but in typical female occupations, salary level is lower than in male occupations. There are also fewer women than men in leadership positions, where women also tend to earn less than men. Women earned, on average, 20 percent less than men (Viinikainen et al., 2010). Thus, despite the higher occupational status of women than men-in terms of more women in lower white-collar occupations and more men in blue-collar occupations-women earned less. Trade unions in Finland have not had gender-equal goals. Satisfaction with income was, however, generally (60 percent) quite good without a gender difference (Pulkkinen \& Polet, 2010a). The proportions of very dissatisfied and very satisfied were also the same in the women and men: less than 10 percent at ages $27,36,42$, and 50 .

Satisfaction with income (on the scale from very dissatisfied to very satisfied) was associated with the level of income, and most highly at both ends of the range of income. The largest jumps in satisfaction were in both ends of the range of income: when income increased from the level less than 1,000 euros a month to the level of 1,000 to 1,300 euros a month, and when the income increased from 4,000 to 4,600 euros a month to more than 7,000 euros. In the middle of the range, satisfaction increased only slightly when income increased. Nevertheless, the participants' estimations of the financial situation of their household (on a 4-point scale from very tight to very good) improved with the increase of monthly income only up until 4,000 to 4,600 euros a month; thereafter, the improvement levelled off (Figure 13.2). Possibly, satisfaction with income did not secure a good financial situation of the family if its needs exceeded available resources. 


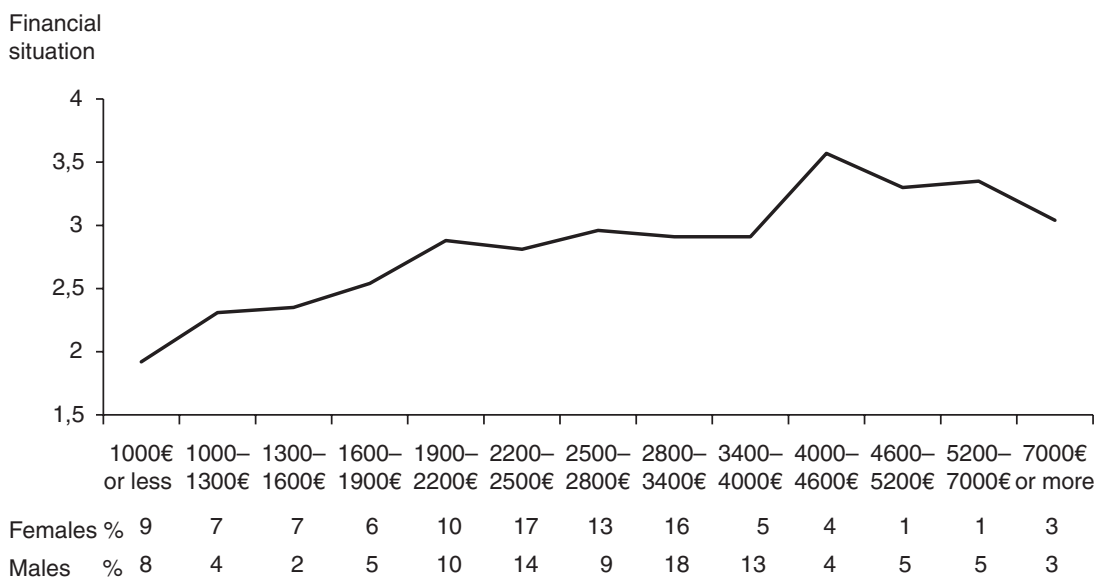

FIGURE 13.2 The participants' estimation of their household's financial situation at age 50 in relation to their monthly income in euros.

Source: Pulkkinen, L., \& Polet, J. (2010a). Koulutus, toimeentulo ja asuminen [Education, livelihood, and habitation]. In L. Pulkkinen \& K. Kokko (Eds.). Keski-ikä elämänvaiheena [Middle age as a stage of life]. (Reports from the Department of Psychology, No 352, pp. 20-25). University of Jyväskylä, Finland. Adapted with permission.

Job satisfaction. Working full-time as an employee was typical of both men and women at age 50; only 7.1 percent of the JYLS women and 1.4 percent of the men were working part-time (Kokko, 2010c). It corresponds to average figures in Finland; also, parents of small children mostly work full-time. A regular day-shift was in 75 percent of those employed. The other employees worked in two or three shifts (12 percent) and the rest had other arrangements of working hours. The number of hours the participants worked in a week was higher in the men (42.1 hours) than in the women (38.0 hours), but the women spent more time for household chores than the men (12.7 and 7.9 hours a week, respectively). The average number of working hours per week was 37.8 in Finland in 2011. Many JYLS participants worked overtime. Almost one-fifth of the male participants and 10 percent of the female participants worked 50 hours or more a week. One-fourth of the men and women had other activities on a voluntary basis in sports organizations, parish, politics, culture, and so on. Men spent around 11.6 hours a week for them, women 9.5 hours.

High job demands and low well-being at work were widely discussed topics in Finland at the time of the JYLS data collection in 2009, when the participants were 50 years. The discussion was activated by political goals to extend work lines and postpone the age of retirement. In the same context, the current work conditions and recovery from work became topics of debate. Well-being at work among the JYLS participants at age 50 was assessed from the perspectives of satisfaction with income, job satisfaction, job exhaustion, workaholism, and work engagement.

The participants were asked at age 50 how satisfied they were with their present work or employment situation (on the four-point scale). One-fourth (26 percent) 
were very satisfied and 60 percent quite satisfied (Rantanen \& Räikkönen, 2010). Four percent were very dissatisfied. The distribution of satisfaction was similar at ages 27,36 , and 42 .

Sources of satisfaction were studied in relation to job strain. According to the Job Demand-Control model (Karasek \& Theorell, 1990), job strain is expected to result from the combination of high demands and low decision control at work. Time demands were studied with five items (e.g., "I have to rush in order to complete my allotted tasks"), and control at work with seven items (e.g., "How much influence do you have on the tasks that constitute your job?"). Having influence over the work was associated with job satisfaction in men (Rantanen \& Räikkönen, 2010). Satisfaction was not associated with time pressures. About 55 percent of the men and women felt that they had quite or much influence over the content, order, pace, method, and division of the work, and around one-third of the participants had experienced time pressures in work at ages 36, 42, and 50. Having influence over the work was more common in the higher white-collar occupations than in the blue-collar occupations, but also time pressures were more common in higher white-collar occupations (45 percent) than in lower white-collar and blue-collar occupations (24 percent for both groups).

Job satisfaction was also studied in respect to having a job with high skill discretion. Job skill discretion refers to the degree of creativity, learning, and highly developed skills required in a job as well as the variety of skills needed and the possibility for personal development (Karasek \& Theorell, 1990). It was assessed with a Job Content Questionnaire (e.g., "My work requires advanced skills”). Job skill discretion, which is most typical of higher white-collar occupations, was positively related to job satisfaction at age 50 (particularly in the men; Rantanen \& Räikkönen, 2010), and to a good recovery experience and relaxation after work (Jalonen, Kinnunen, Pulkkinen, \& Kokko, 2015). Satisfaction with work was also associated with the amount of support and help that a participant received in his or her work (tasks) from a supervisor and co-workers: the correlation was 0.36 for the men $(\mathrm{p}<0.001)$ and 0.26 for the women $(\mathrm{p}<0.05)$ at age 50 . Receiving support was more common in higher white-collar occupations than in blue-collar occupations (Rantanen \& Räikkönen, 2010). Thus, having influence over work, the high quality of work (job skill discretion), and receiving support in work favored higher occupational groups and explained connections to job satisfaction.

The Demand-Control model postulates that the level of job demands and job control are the key factors impacting workers' health outcomes. Health consequences of long-term job strain were investigated in a study by M.-L. Kinnunen, Feldt, U. Kinnunen, Kaprio, and Pulkkinen (2006). The association between job strain and metabolic syndrome was not found in the men or the women. Rather, in higher white-collar occupations, metabolic syndrome risk was lower in the individuals who experienced higher job strain. An increase in metabolic syndrome may be associated with aspects of lifestyle more than with the quality of work.

Job exhaustion. Job exhaustion concerning feelings of fatigue that develop as one's emotional energies become exhausted (e.g., "I feel emotionally drained at my work"), was rare among the participants at age 50 : from 3 percent to 8 percent of the participants felt often or continuous mental fatigue because of work; being worn out 
at the end of working day; and/or being tired in the morning while facing another day at work (Rantanen \& Räikkönen, 2010). Job exhaustion had reduced with age; at ages 36 and 42, these feelings were expressed by 15 percent of the participants.

Workaholism. Excessive and compulsive working without feeling a pleasure about the work was called workaholism by Spence and Robbins (1992), which was studied using the ten-item version of the Dutch Work Addiction Scale (DUWAS). A comparative analysis between the JYLS sample and a Dutch sample (Rantanen et al., 2015) indicated that there were two major components in workaholism: working excessively and working compulsively. Working excessively included working frantically (e.g., "I stay busy and keep many irons in the fire") and working long hours (e.g., "I find myself continuing to work after my co-workers have called it quits"). Working compulsively included feeling uneasy if not working (e.g., "I feel guilty when I take time off') and obsessive work drive (e.g., "I feel obliged to work hard, even when it is not enjoyable"). Workaholism was rather rare amongst the participants at age 50: 19 percent experienced it often or continuously (Rantanen \& Räikkönen, 2010). The participants who were in higher white-collar occupations reported being more dependent on their work, that is, working longer and more compulsively, and recovering fully less often (56 percent), than the participants who were in lower white-collar occupations (72 percent); the participants in blue-collar occupations fell between them. Workaholism correlated with job exhaustion, but it did not correlate with job satisfaction and work engagement (Mäkikangas et al., 2015).

Work engagement. Positive experiences with work were more common among the JYLS participants at age 50 than negative experiences. Work engagement that is defined as a positive, fulfilling, and work-related state of mind correlated positively with job satisfaction and negatively with job exhaustion (Mäkikangas et al., 2015). It was assessed by using a short version of the Utrecht Work Engagement Scale (UWES), which comprises three subdimensions: vigor ("At my work, I feel bursting with energy"), dedication (e.g., "I am enthusiastic about my job”), and absorption (e.g., I get carried away when I'm working”). Nearly all participants (89 percent) had these feelings at age 50 at least once a week, one-third almost daily (Rantanen \& Räikkönen, 2010). However, the participants who regarded work as a necessity had fewer feelings of work engagement than those who regarded work as a profession, career, or calling (Sainio, 2013).

\subsubsection{Occupational well-being and personality}

Job satisfaction, work engagement, job exhaustion, and workaholism assessed at age 50 were included in a JYLS study in which the relationships between occupational well-being and the Big Five personality traits were analyzed (Mäkikangas et al., 2015). Four types of occupational well-being were distinguished using latent profile analysis: engaged (30 percent of the participants), who were high in work engagement and job satisfaction; burned-out (7 percent), who were high in job exhaustion and low in work engagement; "bored-out" (9 percent), who were low in job satisfaction and work engagement; and ordinary (54 percent), who had mean scores in all variables. Men and women were equally represented in these types, and 
the same concerned occupational status groups. Neither were there differences in the number of weekly working hours between the occupational well-being types.

The occupational well-being types were compared with the personality profiles extracted from the Big Five personality traits (see Section 5.3.5). Interdependencies between personality profiles and occupational well-being types showed dependencies as follows: the Resilient profile and the Engaged type (Adjusted residual [AR], 3.5); the Brittle profile and the Burned-out type (AR, 3.2); the Undercontrolled profile and the "Bored-out" type (AR, 2.9); and the Ordinary profile and the Ordinary type (AR, 2.6) (Mäkikangas et al., 2015).

High psychological well-being was found to be typical of the Resilient profile as depicted in the model for Personality and Psychological Functioning (Section 8.2), which fits well with the Engaged occupational well-being type. On the contrary, high anxiety, depressive symptoms and other indicators of low psychological functioning were found typical of the Brittle profile. It fits well with the Burned-out occupational well-being type. The Ordinary personality profile individuals had average scores in different personality traits fitting well with the Ordinary occupational well-being type. The Undercontrolled personality profile was high in openness to experience, monotony avoidance, and impulsiveness. It fits well with the "Bored-out" occupational well-being type.

Thus, occupational well-being could be described with the model for Psychological and Social Functioning (cf. Section 8.2) except for occupational well-being in the Overcontrolled profile characterized by high conscientiousness and tolerance of monotony, and by low extraversion and impulsivity. Only four occupational well-being types were extracted. Perhaps different measures would be needed for assessing the occupational well-being type of the Overcontrolled individuals such as satisfaction with a job where patience and persistence are needed. Possibly, there are also components in workaholism that could fit the Overcontrolled profile. Workaholism as operationalized in this study was largely independent of personality traits, and it did not differentiate between the occupational well-being types. The expected workaholic type did not emerge (Mäkikangas et al., 2015).

Conclusions. Constructive behavior in childhood was a significant antecedent of occupational attainment and income level. It also protected from dropout and its negative consequences. Having influence over work and job skill discretion, which refers to the degree of creativity and highly developed skills in work, are associated with job satisfaction. Occupational well-being could be described within the model for Psychological and Social Functioning.

\subsection{Job insecurity}

\subsubsection{Unemployment and psychological distress: selection or causation?}

The majority of participants (74 percent of men, 71 percent of women) were in the position of employees at age 50 (Kokko, 2010c). Less than 10 percent were 
entrepreneurs including farmers. The participants had mostly (70 percent or more) been on the stable career line from age 27 to 50. A somewhat lower stability (60 percent) was found at age 36, which was explained by economic recession during which the unemployment rate rose from 3.4 percent (in 1990) to 18.2 percent (in 1994) in Finland. It increased the changeable career line, particularly in women. The unstable career line was highest at age 27 (23 percent) and lowest at age 50 (15 percent).

Unemployment. The unemployment rate was 7.1 percent for men and 8.7 percent for women at age 50; 1.4 percent of the men were additionally on forced leave. Around 7 percent were pensioners for health reasons or they received support for rehabilitation, and around 4 percent were freelancers or they were temporarily out of the work market for various reasons, for example, to study. At age 42, the unemployment rate was 12 percent for men and 6 percent for women; and at age 36, 17 percent for men and 13 percent for women. One-third of the men and one-fourth of the women admitted at age 50 that they felt the threat of less work and income, unemployment, or layoffs (Kokko, 2010c). The most common reason for the latest period of unemployment was the end of the job contract (when asked at age 50). Other reasons included the employer's financial difficulties, such as bankruptcy, and the participant's difficulties with finding a job after finishing studies or child care at home. Only one-tenth of the participants had given their notice. Until recently, unemployment has hit blue-collar workers more than white-collar workers in Finland, as was also seen in the JYLS.

The period of ongoing unemployment had lasted, on average, for 10 months in the women, and for 63 months in the men (Kokko, 2010c). In some men, unemployment had even lasted 10 years. Men were long-term unemployed much more often than women. The majority of participants ( 67 percent of men, 55 percent of women) had experienced unemployment at some point of their lives; in one-quarter of them, an unemployment experience was before they were 25 years old. Many participants had been unemployed for several periods. There was also continuity in long-term unemployment, which means that a person who had been long-term unemployed was at a higher risk to be long-term unemployed later in life compared with those who had not been long-term unemployed. The length of an unemployment period tends to increase when participants become older, which indicates that it becomes more difficult to be re-employed at an older age (Kokko, 2006).

The participants' experiences of unemployment were asked at age 42 (2001). The experiences were generally negative, such as causing economic difficulties and increasing negative moods (anxiety, depression, irritability), but unemployment also had positive consequences, such as increasing time for studies and other people (Pulkkinen et al., 2003). Short-time unemployment could be taken as a kind of leave from work with the support of unemployment benefits, if the economic situation was not seriously deteriorated. When unemployment continues, risks for negative consequences increase as shown by research since the 1930s (e.g., Jahoda, Lazarsfeld, \& Zeisl, 1933/1972). 
Selection or causation. Causality between unemployment and distress has been studied with two alternative hypotheses, selection and causation, as proposed by Rutter (1994). The selection hypothesis assumes that prior distress predisposes an individual to unemployment, whereas the causation hypothesis assumes that unemployment causes distress. In order to test these hypotheses, longitudinal data are needed (Kokko, 2001, 2006). Both the selection and causation hypothesis have received some support from studies based on the JYLS data, but findings depend on the length of unemployment, the age of the unemployed people, and many other factors, as presented with a seven-step ladder.

First, short-time unemployment ( $<6$ months) was not preceded by child individual characteristics (Kokko, Pulkkinen, \& Puustinen, 2000); many external factors, such as fluctuations in the national economy, affect the employment situation.

Second, long-term (> 12 months) unemployment at a younger age (age 27) was explained by low education attained until age 27 (Kokko, Bergman, \& Pulkkinen, 2003). Participants' low self-regulation in childhood (such as aggression and emotional lability) did not directly explain unemployment but it did explain selection onto a lower educational track, which, in turn, explained unemployment. This result on the indirect link between low self-regulation and long-term unemployment through lower education was received with Finnish and Swedish samples in a comparative study of the JYLS and the IDA (Individual Development and Adjustment).

Third, very long-term (> 24 months) unemployment at age 36 was explained by the accumulation of problem behaviors (Kokko \& Pulkkinen, 2000). Participants' low behavior regulation in childhood (indicated by physical aggression at age 8) preceded maladjustment to school in early adolescence (indicated by poor school motivation and success, truancy, and punishments), which further predicted longterm unemployment at age 36. Maladjustment to school was also linked to problem drinking at age 27 and to the low number of occupational alternatives. Both of them also contributed to the risk of long-term unemployment at age 36. In another study by Kivimäki et al. (2003), childhood aggression did not predict unemployment at age 36; a difference between these studies was that in the study by Kokko and Pulkkinen (2000), childhood aggression was connected to the accumulation of problems in adolescence that was not considered in the study by Kivimäki et al. (2003). Neither study showed a direct link from childhood aggression to long-term unemployment.

Fourth, when low self-regulation in childhood was defined more broadly, indicated by both aggression and emotional lability, it was directly linked to very longterm (> 24 months) unemployment at age 36 (Kokko et al., 2000). In addition, in British cohort studies, childhood self-control defined without aggressive behavior or with including conduct problems in it, was associated with long-term unemployment across four decades (Daly, Delaney, Egan, Baumeister, 2015). Unemployment rate was high in Finland when the participants were 36 years old due to economic recession. There was a high competition for the few available jobs. It can be assumed that more unfavorable personal characteristics related to low selfregulation made the situation more detrimental by affecting the person's capacity to 
exit unemployment. For those who have better social capacities for re-employment, the period of unemployment may not last as long. Anxiety and passivity in childhood were also associated with long-term unemployment, but the link was indirect via poor school success and low educational level. Anxious children were at risk of poor school success that increased the risk of long-term unemployment.

Fifth, high self-regulation in childhood is a protective factor against long-term unemployment (Kokko \& Pulkkinen, 2000). High self-regulation at age 8 (indicated by constructive behavior, emotional stability, and the teacher's assessment of good coping capacities), and child-centered parenting buffered against a very aggressive child (above 75th percentile) becoming a long-term unemployed adult. The probability of an aggressive child becoming long-term unemployed was 1 percent if both self-regulation and child-centered parenting were one standard deviation above the values obtained for average aggressive children; whereas it was 45 percent if they were one standard deviation below those of average aggressive children. High self-regulation in childhood and child-centered parenting are individuals' resource factors for employment, but they can also be protective factors against long-term unemployment in case the child's aggressive behavior is a risk for the accumulation of problems.

Sixth, openness to experience is a risk of the higher number of unemployment spells (Viinikainen \& Kokko, 2012). Openness to experience at age 33 was associated with the higher number of unemployment spells between the ages of 33 and 50 , and therefore, also with the longer cumulative duration of unemployment until middle age. An increase of one standard deviation in openness was associated with around 4 to 5 months of increase in the cumulative employment. In contrast, a similar increase in extraversion was associated with a 3 to 5 month decrease in cumulative unemployment. Also, higher agreeableness was associated with a decrease in cumulative unemployment. The opposite connections of openness (covering fantasy, aesthetics, feelings, ideas) and extraversion (covering warmth, excitement-seeking, positive emotions) are notable, because these traits generally function in the same direction. Viinikainen and Kokko (2012) see the results as suggesting "that a higher level of openness might cause individuals to seek out new experiences," or "Individuals with a higher level of openness might also tend to choose occupations in which the risk of unemployment is higher" (p. 1214).

Seventh, the causation hypothesis was supported by findings that long-term unemployment causes psychological distress, but it was not the length of unemployment per se that was associated with psychological distress. Distress was higher if unemployment resulted in economic difficulties or lowered self-esteem (Kokko \& Pulkkinen, 1998). Both lower self-esteem and poorer economic situation increased depressive symptoms and psychological ill-health. The hypothesis was confirmed by controlling for the level of self-regulation in childhood (Kokko et al., 2000). The unemployed reported, at age 36, an increasing level of anxiety, depression, and psychological ill-health (assessed by the General Health Questionnaire).

Personality traits may increase vulnerability to unemployment as shown by Viinikainen and Kokko (2012). Neuroticism (covering anxiety, hostility, 
depression) at age 33 was associated with the duration of single unemployment spells between ages 36 and 50. It suggests that it is more difficult for a neurotic person to exit unemployment, but unemployment may also increase neuroticism. The connection may include different types of causalities.

Temporary jobs. Temporary job contracts have rapidly increased in Finland during the past decades, along with many changes in economic activity. In the JYLS, most of the participants (87 percent) who were employed at age 42 had a permanent contract; for the rest, the job was temporary. Possible predisposing factors for temporary work and whether the selection might explain mental health problems associated with it were searched at age 42 (Virtanen et al., 2005).

It was found that children with higher anxiety at age 8 were 2.8 times more likely than those with lower anxiety to be in temporary employment after controlling for gender, parental socioeconomic status, school success, and education. Aggressive behavior at age 8 did not predict temporary employment, but, together with low education, it did predict temporary employment. Anxiety indicating low emotion regulation is an antecedent of problems in psychological functioning, and thus, the selection into a temporary job may explain mental health problems associated with it. The results, which also applied to another sample included in the study by Virtanen et al. (2005), supported the health-related selection hypothesis. It was remarked, however, that "selection found in this study does not undermine the argument that labor market disadvantage may deteriorate health...In order to prevent accumulation of labor market disadvantage, the selection tendency should also be noticed in the employment strategies influencing temporary employment" (Virtanen et al., 2005, p. 2093).

External conditions increase instability of career lines, but there were also individual characteristics that were associated with career lines. It was found that the stability of career line tended to be associated with personality profiles fitting the model for self-regulation and psychological well-being. The Resilient individuals tended to have a more typically stable career line, and the Brittle individuals, an unstable or changeable career line.

\subsubsection{Health consequences of the unstable career line}

Unemployment causes psychological distress as previously stated. It may also cause physical health problems. It was found in a JYLS study (M.-L. Kinnunen, Kaprio, \& Pulkkinen, 2005) that an unstable career line between ages 27 and 36 was associated with increased health risks at age 42 . In this study, allostatic load was compared in individuals who had different career lines.

The concept of allostasis refers to an individual's capacity to maintain internal stability in his or her body through a change of states, but sometimes, conditions may become so stressful that their cumulative effects in the body causes an allostatic load (McEwen \& Stellar, 1993). Allostatic load was assessed at age 42 with eight parameters consisting of primary mediators and secondary outcomes. Primary mediators included an adrenal androgen dehydroepiandrosterone-sulfate (DHEA-S) as 
a neuroprotective agent and overnight 12-hour urinary norepinephrine, and an index of sympathetic nervous system activity. The secondary outcomes comprised, for instance, blood pressure, high-density lipoprotein cholesterol, and waist-to-hip ratio. A high-quartile method was employed for the definition of the allostatic load. It means that it was calculated in how many of the eight parameters a participant belonged to the highest quartile. Thus, the score varied from zero to eight, and a score of three or more indicated a high allostatic load. One-third of both men and women had an elevated allostatic load.

Participants with unstable careers indicated by unemployment, varied jobs, or jobs not corresponding to one's field, had an elevated risk for high allostatic load compared with the participants with a stable career history (M-L.Kinnunen et al., 2005). Men had higher allostatic load than women. The frequency of heavy drinking was associated with allostatic load, but occupational status and smoking were not. Participants who had high allostatic load also reported more psychosomatic symptoms than those with low allostatic load.

The unstable career line was found to have long-term effects on health in both genders. It is to be noted, however, that causality may not be simple. Although theoretically, allostatic load develops slowly from primary stress reactions via health risk factors to actual diseases, there may be some predisposing factors in an individual's personality to an elevated allostatic load, as there are selection factors for long-term unemployment.

\subsubsection{The consequences of economic strain for the family and parenting}

Economic stress caused by economic recession and unemployment has adverse effects on families (Elder, 1974). Economic stress is a multidimensional concept that comprises both objective and subjective aspects of employment and income. The objective aspects include employment instability (e.g., periods of unemployment), whereas the subjective aspects include employment uncertainty and economic strain (e.g., evaluation of one's financial situation; Voydanoff, 1990). It is not only unemployment that has consequences for families; job insecurity, if related to marital dysfunction, has consequences for the family, as reviewed by U. Kinnunen and Pulkkinen (1998).

Marital relations. Poor economic situation affects marital quality negatively if the partners perceive strain in the situation, which causes negative emotions, as proposed in the theoretical model by Conger, Ge, and Lorenz (1994). In the JYLS, two components were distinguished in the subjective evaluation of economic strain: evaluation of the current financial situation ("How good is your/your family's financial standing?") and expected economic strain in the future ("How afraid are you of financial difficulties in the future?"). Based on the spillover theory (e.g., Zedeck \& Mosier, 1990), it was expected that experiences in one area of life, such as unemployment, are carried to another area of life, such as marriage, and based on the stress theory (e.g., Lazarus \& Folkman, 1984), it was further hypothesized 
that it was the perceived strain rather than the objective situation that produces the adverse impact on the individuals such as depression and hostile marital interaction.

As in other studies, the proposed model was more pronounced for men than women (Conger et al, 1994). The JYLS results indicated, for men, that objective circumstances, as indicated by the participant's current employment situation, affected the perception of the current economic strain, which, in turn, affected the anticipation of the future strain (U. Kinnunen \& Pulkkinen, 1998). This strain fostered depressive mood and hostile marital interaction, and both of them had a direct impact on marital quality. Marital quality was assessed with questions concerning the closeness of the relationship, the amount of conflicts, and thinking of divorce. In women, financial strain had no impact on their perception of marital quality, but their long-standing unstable career line had direct effects on their depression, marital hostility, and marital quality. The stable career line forms a basis for women's financial independence, and a lack of it increases the risk of negative emotions and problems in the marital relationship. In another Finnish study conducted with couples (U. Kinnunen \& Feldt, 2004), economic stress was related to increased psychological distress and marital problems in men and women.

Children. During the economic recession in Finland in the 1990s, worries about its consequences for children gave rise to research that evaluated the influences of economic hardship on the mental health of children (Solantaus, Leinonen, \& Punamäki, 2004). Information was gathered from around 500 children aged 12 years and their mothers and fathers. The results showed that it was not the change of the economic situation that affected children, but the consequences of the economic strain for the parents. It decreased the parents' mental health and weakened marital interaction and the quality of parenting, and children's mental health was poorer. Thus, the parents' job-related emotions and marital and parenting quality mediate the effects of economic strain to children. A supportive partner relationship provides support for parenting and reduces the negative impacts of economic pressures on children. U. Kinnunen and Rantanen (2006), who reviewed these results, concluded that "parents' psychological reactions to job loss and unemployment are critical factors in determining whether job loss will indeed affect family relationships and child behavior" (p. 232).

The influence of job-related emotions on functioning as parents was investigated in the JYLS with 36-year-old participants who were employed and had children. Several aspects of job characteristics were considered: meaning of work, time demands, one's control over work, job insecurity, and work-family conflict. They were expected to cause negative job-related affects, which, in turn, would increase parenting stress and reduces child-centered parenting (U. Kinnunen \& Pulkkinen, 2001). The study confirmed two assumptions: (1) work experience is of the critical value in understanding the link between work and family, and (2) the effects of work experience on family functioning are indirect. Mediating processes concern emotional reactions to work experience and they tend to spillover to family roles. More specifically, high time demands and low control over work were linked to negative job-related affect (job exhaustion and negative mood at work), which 
were further related to higher parenting stress, and via it, to lower child-centered parenting and knowledge of children's friends, activities, and whereabouts.

The impact of job exhaustion on parenting was studied more closely with the offspring sample of the JYLS. Children (average age 12 years) of the participants were studied when the parents were 38 to 40 years old (U. Kinnunen \& Rantanen, 2006). Data allowed for testing the spillover model longitudinally from age 36 to 42. It was expected that the higher the level of job exhaustion, the higher the level of parenting stress. Furthermore, it was expected that both job exhaustion and parental stress would predict a lower level of child-centeredness in parenting behavior causing more problem behaviors in children. A significant continuity in the levels of job exhaustion and parenting stress from age 36 to age 42 was found. The more job exhaustion the parents reported, the more they reported simultaneous parenting stress. And the more they reported job exhaustion, the less they knew about their children's friends, activities, and whereabouts 4 years later, as reported by their children. It means that if the parents experience fatigue and parenting stress, they are less likely to be involved in their children's lives. It indicates the spillover of negative emotions from one life domain to another.

Higher parental knowledge was associated with children's higher self-regulation indicated by teacher-rated adaptive behavior (constructive, compliant, and socially active behavior). Furthermore, it was found that children may increase their parents' burden: externalizing problem behaviors in children (low behavior regulation including subscales for hyperactivity-impulsivity, aggression, and inattention) increased parenting stress a few years later. As a whole, the study indicated "a simultaneous and long-term negative spillover between employee and parent roles as well as child outcomes" (U. Kinnunen \& Rantanen, 2006, p. 241).

Conclusions. Unemployment periods become longer and accumulate with age. Unemployment affects distress if it lowers self-esteem and economic situation. Both individual drift for selection and social causation operate between long-term unemployment and psychological distress. Individual characteristics often function indirectly by explaining selection onto low education or problem behavior, which are risks for becoming and remaining employed. The distress consequences of unemployment may be amplified in individuals who are more vulnerable to distress. An unstable career has negative consequences for health and marital relationship. The effects of job insecurity on children are mediated by parents' well-being and childrearing practices.

\subsection{Work-family balance}

\subsubsection{A stress theory approach to work-family interface}

Family and work are important domains of life with various demands placed upon an individual. The study of the work-family interface started from the role stress theory, according to which, managing multiple roles creates strain and conflicts between the demands of the roles. A conflict in which the demands of the family 
role interfere with one's ability to perform the work role is called a family to work conflict (FWC) (Greenhaus \& Beutell, 1985). Another direction in the bidirectional work-family conflict are when demands at work spillover to the home. It is called a work-to-family conflict (WFC). Time spent at work may take away time spent at home, and demands of work may make an individual too fatigued to manage family life.

Frequency of conflict. Work-family conflict was assessed in the JYLS at ages 36, 42, and 50 using a four-item questionnaire (two items for WFC, e.g., "How often does your job or career keep you from spending the amount of time you would like to spend with your family?" and two items for FWC, e.g., "How often does your home life interfere with your responsibilities at work, such as getting to work on time, accomplishing daily tasks, or working overtime?”) (Rantanen, U. Kinnunen, Pulkkinen, \& Kokko, 2012). It was found that the mean level conflict in both directions remained around the same from age 36 to 42 to 50 . However, the frequencies of the two types of conflicts were different. It was more often seen that work takes time from the family than in reverse: about 20 percent of the participants at different ages had experienced work demands that often or very often negatively affected family roles, but only around 3 percent had experienced family demands that often or very often negatively affected work performances. The mean levels of WFC and FWC did not differ significantly between men and women for the ages measured. Thus, work-family conflict was relevant for women and men throughout midlife, not only for parents (or mothers) of small children. Neuroticism as a personality trait increased an individual's vulnerability to experience work-family conflict in both directions, and psychological distress (Rantanen, Pulkkinen, \& U. Kinnunen, 2005).

Developmental trajectories. The means hide heterogeneity among the respondents. Heterogeneity in the development trajectories of work-family conflict existed as shown by latent profile analyses (Rantanen et al., 2012). For 38 percent of the sample, the work-family conflict was stable low across ages; for 55 percent it decreased and for 5 percent it increased. The stable low trajectory was related to lower demands in work and family throughout the study period. Most participants in this trajectory were lower white-collar employees whose average weekly working hours were around 38 hours, and whose youngest child had passed toddler age when the study period started (at age 36).

The decrease of the conflict from work demands to family (WFC) was associated with diminishing work- and family-related demands with age, which was caused by the decrease in weekly working hours and in the number of children living at home. The small group of participants who experienced increasing work-family conflict, had, on average, two children living at home at age 50 (as at age 36), and they had an increasing number of hours worked weekly ranging from 45 hours a week at age 36 to more than 50 hours a week at age 50 . The increasing workfamily conflict trajectory was associated with reports of more job exhaustion and depressive symptoms in middle age (but not at age 36) compared with the other trajectories. It indicates the negative consequences of prolonged work-family conflict. 
The work-to-family conflict was experienced at least sometimes by the participants who were in higher white-collar occupations (62 percent), more frequently than those who were in lower white-collar occupations (33 percent). Participants in blue-collar occupations were between them (45 percent). Differences between the occupational groups did not exist in the family-to-work conflict: 5 percent to 12 percent had experienced it.

A question of whether job exhaustion precedes work-family conflict or whether work-family conflict precedes job exhaustion can only be analyzed with a longitudinal data. Rantanen, U. Kinnunen, Feldt, and Pulkkinen (2008) showed that job exhaustion did not have these associations. Neither WFC nor FWC had long-term reciprocal associations with job exhaustion, low marital adjustment, parental stress, or psychological distress over 6 years in the JYLS data or over 1 year in another longitudinal data.

Work-family conflict on the practical level is manageable in Finland where the state has an active role in reconciling the demands of work and family lives. Both mothers and fathers benefit from a well-functioning public daycare system, the legal right to reduce their working hours when children are young, the right to stay at home with a sick child for 4 days, and the right to stay at home until the child is 3 years old and to maintain the work place for that time.

\subsubsection{A role enhancement theory approach to work-family interface}

Another approach to the work-family interface, besides the stress theory approach, is the role enhancement theory, according to which, participation in multiple roles increases opportunities for growth and better functioning in other life domains (U. Kinnunen, Feldt, Geurts, \& Pulkkinen, 2006). Work and family are not only in conflict. In general, the family-work interface was more positive than negative. Working makes the person enjoy time at home, and relaxation at home gives energy and positive mood for work, as illustrated by the frequency ( percentage) of "often" or "very often" responses to the following questions at age 50.

- Work $\rightarrow$ Family: Because I work, I enjoy my time at home (66 percent)

- Work $\rightarrow$ Family: Because of my work, I am more able to put home-related matters into perspective (27 percent)

- Family $\rightarrow$ Work: Because I relax and regain my energy at home, I can better focus on performing my work (60 percent)

- $\quad$ Family $\rightarrow$ Work: Because of my home life, I am more able to put work-related matters into perspective (56 percent).

Well-being. In a study based on the JYLS data (U. Kinnunen et al., 2006), different types of work-family interface were compared. The results indicated for work-to-family interface, that positive work-to-family spillover was associated with lower job exhaustion and lower psychological stress. It means that it is good for a person's mental well-being if he/she can cheerfully come home after a successful 
day at work and if his/her positive mood affects positively the atmosphere at home. In contrast, negative work-to-family spillover was associated with job exhaustion and psychological distress. Thus, general well-being and well-being at work were reflected in work-to-family interface.

Family-to-work interface was not similarly connected to well-being. Positive family-to-work spillover (e.g., "after spending time with your spouse/family, you go to work in a good mood, and your mood affects the atmosphere at work") was not directly linked to lower job exhaustion and lower psychological distress. Neither was the negative family-to-work spillover linked to them; it was only associated with low marital satisfaction. The results indicated, however, that having children at home increased a risk of negative spillover from family to work, but at the same time, it increased positive work-to-family spillover. It means that for parents, work may offer a positive mood and possibly skills that are positively reflected in the family life despite the negative side.

Work-family balance. A $2 \times 2$ typology for work-family balance based on high and low WFC and high and low work-family enrichment (WFE) was created and studied by Rantanen, U. Kinnunen, and Pulkkinen (2013). WFC refers to perceived difficulty in fulfilling conflicting work and family roles. WFE, in turn, refers to perceived easy fulfilling of work and family roles due to beneficial skills, support, and resources received from the participation in both domains.

In this typology of work-family balance, there are two types of balance that involve low perceived conflict (see Figure 13.3). A beneficial type that results from low perceived conflict and high enrichment; and a passive type that results from low conflict and low enrichment. There are also two types of balance that involve high perceived conflict: an active type that results from high conflict and high enrichment; and a harmful type that results from high conflict and low enrichment. Each work-family balance type had typical personality traits at age 42 (Rantanen et al., 2013):

\begin{tabular}{c|c|c|}
\cline { 2 - 3 } Low & $\begin{array}{c}\text { Passive (26\%) } \\
\text { E- C- N+ A+ } \\
\text { Work-family } \\
\text { enrichment }\end{array}$ & $\begin{array}{c}\text { Harmful (9\%) } \\
\text { E- C- N+ A- }\end{array}$ \\
\cline { 2 - 3 } High & $\begin{array}{c}\text { Beneficial (48\%) } \\
\text { E+ C+ N- A+ }\end{array}$ & $\begin{array}{c}\text { Active (16\%) } \\
\text { E+ C- N+ A- }\end{array}$ \\
\cline { 2 - 2 } & Low Hork-family conflict High
\end{tabular}

FIGURE 13.3 The experience of work-family conflict and enrichment in relation to personality traits at age 42 .

Source: Rantanen, Kinnunen, \& Pulkkinen (2013). Results summarized by Pulkkinen.

Note: $\mathrm{E}=$ extraversion, $\mathrm{C}=$ conscientiousness, $\mathrm{N}=$ neuroticism, $\mathrm{A}=$ agreeableness; $+/$ - above/below the sample mean. 
- The beneficial type participants were extraverted and conscientious; they were more agreeable, but less neurotic than the harmful type participants.

- The harmful type participants were low in extraversion and conscientiousness but high in neuroticism.

- The active type participants were extraverted and neurotic.

- The passive type participants were low in extraversion and neuroticism but high in agreeableness.

In line with the previous findings by Rantanen et al. (2005), which showed that neuroticism makes people vulnerable to the experience of work-family conflict, the high-conflict (harmful and active) type participants were more neurotic than the beneficial type. Agreeableness, in turn, was characteristic of low conflict types. High conscientiousness was characteristic of the beneficial type (Rantanen et al., 2013).

This typology has some correspondence to the model for Personality and Psychological Functioning (cf. Section 8.2), particularly in regard to the beneficial and harmful type. The beneficial type participants, who could balance demands of work and family and considered these roles complementary and supporting for each other, shared characteristics of individuals with the Resilient personality profile. On the contrary, the harmful type participants, who perceived high conflict between the demands of work and family and did not see them as supporting for each other, shared characteristics of individuals with the Brittle personality profile.

The work balance types differed in their organization of daily life (Rantanen et al., 2013). The weekly working hours were higher in the active and harmful types than in the beneficial and passive type participants, and consequently, the active and harmful types felt that they had less personal time than the beneficial and passive types. Weekly domestic work hours and the feeling of giving up time for others were also higher in the active type than in the beneficial and passive type participants. These connections show that personality traits may affect the investment that an individual makes in various roles, organization of activities, and experiences of conflicts.

\subsubsection{Successful reconciliation}

The JYLS participants were asked at age 50 to assess on a general level how well they had been able to reconcile work and family/private life. Most respondents (around 70 percent) chose either well or very well; 3 percent from the men and 1 percent from the women chose the option poorly or very poorly. The rest assessed that the reconciliation had taken place moderately. Thus, a great majority assessed that the reconciliation between work and family was good.

At age 42, the participants were asked whether different arrangements would help reconcile work and family. (The scale was: no, somewhat, much.) Their wishes for flexible and shortened working hours, supervision of children in the afternoon, and part-time work reflected Finnish society in which a day care system is good for children, but when children go to school at age 7 , services are unsatisfactory and 
children often have to stay alone for hours when parents are working. The rates of "much" responses were as follows:

- $\quad$ Flexible working hours (65 percent).

- Arrangement of children's afternoon activity by school (61 percent).

- Shortened daily working hours for one parent (60 percent).

- $\quad$ Pay for homemaking parent when children are small (53 percent).

- Increased opportunity for part-time work (49 percent).

- Parents excluded from evening and shift work/given preference for daytime hours (49 percent).

- Nursing of sick children arranged by employer (48 percent).

- Possibility of employing a childminder with tax rebate (44 percent).

- Tax rebated for salary paid for domestic work (cleaning, caring) (39 percent).

- $\quad$ Paternal leave for one month (38 percent).

Another type of aspect in the reconciliation of work and family concerns the extent to which people want to keep work and family as separate as possible (Rantanen \& Räikkönen, 2010). Among the JYLS participants at age 50, 46 percent wanted to keep work and family separate, and only 14 percent wanted to do work-related matters during their free time. However, 44 percent wished to be available for private matters at work; 8 percent did not want it. Thus, the participants, on average, wished for flexibility from work, but did not want to afford flexibility to their private life. Men were more flexible than women in both respects. Also, the participants in higher white-collar occupations were more flexible than those in lower white-collar and blue-collar occupations.

Conclusions. The family-work interface was more positive than negative in middle age. Positive experiences with work affected positively the atmosphere at home and an individual's mental well-being. Balancing the demands of work and family and perceiving them as complementary was associated with the same personality traits as the Resilient personality profile, whereas perceiving conflicts between the demands and not perceiving them as supporting to each other was associated with the Brittle personality profile.

\subsection{Recovery from work and the content of free time}

Finland is a work-centered society. Work conditions dominate public discussion and political decision making; the impact of political decisions on families and children are not prominent criteria for decision making. Nevertheless, on the personal level at age 50, work did not give as much pleasure as the other important domains of life: family, leisure pursuits, and friends (cf. Section 6.3.1). The family was first place from age 27 to 50 (Pulkkinen \& Polet, 2010b). Work dropped gradually to fourth place: at age 42 in the men and at age 50 in the women. Leisure pursuits correspondingly improved its rank order. 
Recovery. Recovery from work was not a big problem among the participants at age 50. It was assessed by asking the participants, whether they could forget about work, kick back and relax, do relaxing things, and take time for leisure. Two-thirds of the participants (66 percent) agreed with these statements somewhat or fully. Responses to another question proved the same: "In general, how well do you recover from the stress caused by your work after the working day?" The vast majority of the participants responded that they recovered from work well (30 percent) or quite well (52 percent); 15 percent felt that they recovered moderately and 3 percent felt their recovery was quite poor. None of the participants indicated that he or she recovered poorly.

Relaxation and psychological detachment, which are two components of recovery experiences, were measured by using the Recovery Experience Questionnaire, for instance: "I use the time to relax" and "I distance myself from work." The more time pressure the participants felt at work, the poorer were psychological detachment, relaxation, and the subjective evaluation of recovery, as shown by Jalonen et al. (2015). This study also indicated that job skill discretion (cf. Section 13.2.2) was associated with better subjective recovery experience. Recovery from work was more difficult in higher white-collar occupations than in lower white-collar occupations (Rantanen \& Räikkönen, 2010).

Content of free time. To study the content of free time, the participants were presented a list of 57 leisure activities with a request to indicate on a five-point scale how often they were engaged in each of them. The most common activities were reading newspapers and watching TV, but the participants also took care of their physical fitness with some gender differences (Pulkkinen \& Polet, 2010c). More typical of women than of men were walking and gymnastics, gardening, reading novels, taking care of pets, crosswords, dancing, and studies. More typical of men than of women were lotteries, spectator sports, skiing, skating, ball games, fishing and hunting, repairing machines, sitting in bars and pubs, and playing with amusement machines. The long list did not cover all activities in which the participants engaged. They added, for instance, working in various associations, parish, and voluntary organizations, water sports, motor cycling, and home-related exercises such as snow and forest work, and walking the dog. Many participants were supervisors of children's hobbies.

In the area of arts, fewer participants were active. At some point of their life, 29 percent of women and 21 percent of men had played an instrument, but at age 50, these figures were 11 and 3 percent, respectively. Also, singing in a choir had previously been more common, but at age 50, it was found only in a few percent. Creative activity in writing, acting in a theater, and visual arts was found in 12 percent of the participants.

For some leisure activities, it was possible to compare their frequencies at ages 27, 36, and 50 (Pulkkinen \& Polet, 2010c). Reading books decreased with age. At all ages, women had read books more than men. At age 50, 84 percent of the women and 57 percent of the men had read at least one book during the last 6 months. Handicrafts and doing joinery decreased in the women but increased in 
the men with age; at age 50, one-third of the women and men spent their free time with them. Age differences in going to concerts, exhibitions, and theater did not exist, but women were more active than men. When all these arts were considered, two-thirds of the women and a half of the men had visited cultural events at least once during the last 6 months. Some participants were active in several types of leisure activities. The activities were not mutually exclusive but complementary. This was seen, for instance, in the interest both in physical exercise and arts. The number of activities was not associated with satisfaction with one's free time.

As a special aspect of activities, the participants were asked at age 50 about their use of modern information technology. Computers had become common in work places; only 5 percent had not used a computer. Nevertheless, the majority (60 percent) of those who used a computer at work did not use it at home. Those who used it at home did it, on average, for 1 to 2 hours a week; some used it for 20 to 30 hours a week. About 18 percent of the participants admitted some dependency on the use of a computer as indicated by staying on the internet longer than he or she had meant. The data were collected in 2009. Since a rapid development in smart phones and other electronic equipment took place, their use has largely penetrated the population, this will also be likely in this age cohort born in 1959 .

Satisfaction. Ten percent of the men and women were very satisfied with the current content of their free time, and most participants were quite satisfied. High satisfaction was associated with the content of physical exercises in a specific way. The more intensively men and women exercised, that is, they walked, ran, or exercised at least for half an hour at a time so that their pulse rose and they were sweating, the more satisfied they were with the content of their leisure. The intensive exercise at least four times a week was practiced by 22 percent of the men and the women. As a motivation for heavy exercise participants (particularly men), they exercised in order to improve their mood (emotion regulation). It was possibly associated with the secretion of endorphins due to heavy exercise.

Other associations between satisfaction with the current content of free-time activities and the frequency of one's participation in activities were not found except for doing joinery in men; the more men were doing something with their hands, the more satisfied they were with their free time. It was an interesting finding. Many men have no opportunities to do these activities at home in an urban environment. In Finland, it is common to have a summer (holiday) cottage and activities there fulfill the needs of working by hand. Living in a one-family house (46 percent of the participants) also gives more opportunities for free-time activities than living in an apartment building (32 percent). The participants who lived in a one-family house were more often (64 percent) very satisfied with their habitation than those who lived in an apartment (32 percent). Nevertheless, satisfaction with habitation and content of free time were not associated with each other. There are other reasons for the difference in satisfaction with habitation, such as the space available. Dissatisfaction with free time was associated with poorer health and poorer income, which limited participation in pleasure-giving activities. More men than women were quite or very dissatisfied ( 24 percent and 11 percent, respectively). 
Friends and relatives. Time with friends may also be relaxing and help recover from work and other stresses. The participants were asked how many close friends they had. The mean number of friends was higher in the men (4.5) than in the women (3.3) at age 50 , but more men (17 percent) than women ( 4 percent) were lacking a confidant with whom one can speak about his or her personal matters and problems (Pulkkinen \& Polet, 2010b). Satisfaction with the current state of friendships was generally good: one-fifth was very satisfied, two-thirds satisfied, and only a few were very unsatisfied. The similar distribution without gender differences was already found at ages 36 and 42 . Satisfaction was slightly associated with the number of friends, but the quantity of friends explained only a small proportion of the satisfaction with friendships. Possibly even one confidant may make a person satisfied with friendships.

People also had social contacts with relatives. The participants were asked at age 50 how many relatives (siblings, aunts, uncles, cousins) they had. The numbers varied greatly, from 2 to 200. One-tenth of the participants felt closeness to their relatives very much, and one-tenth did not feel closeness at all. The distributions did not differ between the men and women, although there was a tendency that there were more men than women who said that they did not feel closeness and togetherness with their relatives. One indicator of how close the relationships with friends and relatives are is whether the doors are open for them to visit. One-fifth of the participants mentioned that relatives and friends visit their homes weekly, but the monthly basis for visits was more common (about 45 percent). Only a few said that they did not visit at all. There was no gender difference in this respect.

Most participants (80 percent) said that they know many of their neighbors and greet them and chat with them (Pulkkinen, 2010b). Two-thirds also said that they had got help from them if needed. Only a few said that they did not know their neighbors and that they did not get assistance. Almost one-third said that they thought they would get help if needed. Nevertheless, the neighbors did not visit the participants as often as the relatives and friends did: one-fifth of the men and one-third of the women said that the neighbors did not visit them at all. The category of acquaintances that visited the participants' homes most rarely was their colleagues. Half of the women and one-third of the men said that their colleagues did not visit their homes at all. Women's full-time work outside the home has made them busy with the household and children in their free time. More women than men also wanted to keep work and family separate.

Conclusions. The JYLS participants had versatile interests during their free time at age 50. High satisfaction with the content of free time was associated with the intensity of physical exercise in men and women. Women were more active than men in cultural interests. Contacts with friends and relatives were satisfactory. Recovery from work was not a big problem in middle age, but time pressure at work, particularly in higher white-collar occupations, was associated with poorer recovery. 
$\Longrightarrow$ Taylor \& Francis Taylor \& Francis Group

http://taylorandfrancis.com 


\section{PART IV}

Epilogue 
$\Longrightarrow$ Taylor \& Francis Taylor \& Francis Group

http://taylorandfrancis.com 


\section{4}

\section{NAVIGATING THROUGH THE SPHERES OF LIFE}

\subsection{Middle age: a prime time}

The main domains of adult life were illustrated in Figure 9.1 by propellers for family, work, leisure, and health. These domains are related to each other in workfamily balance, recovery from work through leisure activities, health-promoting or health-compromising behavior, and care. In middle age, social functioning in these domains was mostly positive. The concept of the midlife crisis exaggerates individuals' re-evaluation of their life, which is generally experienced positively. Average descriptions of people's functioning conceal individual differences which exist on all layers of personality. Unique individuals have, however, common features in self-regulation with its components of emotion and behavior regulation which are reflected in their social and psychological functioning.

Adulthood is a long period of life, and may last even 80 years after late adolescence. There is no established way of categorizing it into periods, but middle adulthood is generally defined as covering the ages from 40 to 60 years (Lachman, Teshale, \& Agrigoroaei, 2015). In the JYLS, the first data collection in adulthood was at age 27 , which can be seen as the time of early young adulthood, and the second data collection took place at age 36 , which represents the time of later young adulthood. The third data collection was at age 42, which is the time of early middle adulthood, and the fourth data collection took place at age 50, in the midst of middle age. The terms middle adulthood, middle age, and midlife are often used interchangeably in the literature. In this book, the concept of middle age is used to cover from early middle age, at age 42 , to the middle of middle age at age 50.

Middle age is the prime time for women (Mitchell \& Helson, 1990) and other studies support this view as also being true for men (Lachman et al., 2015). Middleaged people have been found to fare well in many aspects of their lives. They generally feel well physically and psychologically, have good relationships, and are satisfied 
with the different aspects of their lives. In the minority of the participants, various problems accumulated. The JYLS findings on middle-aged people's average life in each domain of life, concurs with the view of middle age as being a prime time of life. References to original publications are not repeated in the following summary of each domain and the intersection of the domains presented in Figure 9.1.

Family. Ninety percent of middle-aged people lived with a partner, mostly in a marriage. Close to one-third had divorced by the time they were 50 , but their present relationship with their spouse or a common-law partner was found to be good. In addition, the participants' relationships with the older and younger generations in their families were good. The most fertile time was during the early 30s. The participants' parental identity developed through the adult years. Identity achievement was associated with high levels of nurturing of their children, which is part of child-centered parenting. In child-centered parenting, the parents sensitively organize their behavior from the perspective of the child and provide warmth, guidance, and sustained interest in the child's activities. It promotes the development of adaptive behavior in children characterized by high self-regulation.

Work-family balance. A role enhancement theory on the work-family interface received greater confirmation in middle age than a conflict model. Around half of the participants had experienced that performance in one role positively affected their performance in the other role. The positive spillover from work to family was associated with lower job exhaustion and less psychological stress at work. At different ages, one-fifth of the participants had experienced that work demands often negatively affected their roles in the family, and only a few participants had experienced that family demands negatively affected their performance at work.

Work. Most participants regarded work as a profession, which means that their personal development needs were connected to work. Close to 90 percent were satisfied with the choice of their occupation. Most participants were employees. Women more often than men worked in lower white-collar occupations, while men were more often in blue-collar occupations. The women's salary was, however, 20 percent lower than the men's salary in middle age. The unemployment rate was around 8 percent at age 50 , but it had varied during the participants' life depending on the economic situation in Finland. Career paths were mostly stable, in other words, the participants mainly continued to work in the same field. The unstable career line, which included unemployment, was found in 18 percent of men and 15 percent of women. Long-term unemployment, lasting 1 year or more, tended to be chaining in the same individuals. The stagnated career line, including at least around 4 years of unemployment during the last 8 years, was found in 5 percent of the participants at age 50 . Job insecurity was associated with allostatic load, which refers to bodily reactions to stress. Unemployment created distress if it lowered the person's self-esteem or worsened his/her economic situation. The effects of unemployment on the children were mediated by the parents' well-being. One-sixth of the participants (13 percent of men, 18 percent of women) lived below the poverty line at age 50 .

Recovery. Recovery from work was not a big problem at the age of 50 . The vast majority assessed said that they recovered well from the stress caused by work. 
Similar responses were received for the question of whether participants were able to forget about work, do relaxing things, and take time out for leisure activities.

Leisure. Middle-aged people were active in their leisure time and had a variety of different interests, although there were differences in the amount of leisure activities between the participants. High satisfaction with one's free time was associated with intensive physical exercise and not with the number of activities. Satisfaction with the state of friendship was generally good. Participants had, on average, four to five friends, but close to one-fifth of the men had no confidant. Leisure activities in adolescence rather often included behavior that was against social norms and the law, but criminal convictions concentrated in a small group of individuals and they were rare in middle age.

Health-compromising behavior. All the participants had used alcohol at some point in time. Starting to drink at an early age predicted heavy drinking and suffering from problems caused by drinking in middle age. Men drank more alcohol than women. Drinking to intoxication is relatively common in Finland, as seen in that one-third of middle-aged men and one-tenth of women reported that they got drunk on a weekly basis. Problem drinking was defined based on the participant having experienced problems caused by drinking and having elevated scores in alcoholism screening tests; it was found in 39 percent of men and 22 percent of women. At age 50, several problems caused by drinking and the high amount of consumption of alcohol were found in 18 percent of the men and 6 percent of the women. The use of alcohol increased in Finland during the time when the research participants were in adolescence. Possibly, the research sample represents a "wet generation," which differs from recent generations who drink less alcohol and who start drinking later in life. Awareness of the risks of the use of alcohol has increased, as has also happened in relation to cigarette smoking. Smoking decreased with age. The use of illicit drugs was rare.

Health. Most participants assessed their health as being good and only 7 percent assessed it as poor (2 percent very poor). Heavy drinking increases the risk of early death and of developing metabolic syndrome, which in itself is a condition that can lead to cardiovascular disease and diabetes. Indicators of the risk factors associated with metabolic syndrome risk include insulin resistance, abdominal obesity, hypertension, dyslipidemia, and microalbuminuria. The incidence of metabolic syndrome among the participants doubled from age 42 to 50 , when it was found in half of the men and in a quarter of the women. Obesity was found at the same rates at age 50. Those who were overweight in childhood tended to be overweight in adulthood. Also, having a systolic blood pressure in adolescence is a strong predictor for having systolic blood pressure in middle age.

Care. Middle-aged adults were focused on their nuclear families and taking care of their own and often also their spouse's children, who were all living in the same household (some children part-time due to joint custody). The participants' grandparents had passed away but they lived on in their grandchildren's good memories. The most positive memories were associated with the grandparents' support and taking care of them on a fairly regular basis. The participants' mothers were mostly alive, as were about half of the fathers, and both their mothers and fathers generally 
lived independently in their own homes. Only a few of the participants provided financial support, or gave practical help to their parents on a weekly basis, but most participants gave emotional support to their parents and kept in regular contact with them. Members of the sandwich generation, in which a middle-aged person has to take care of their own children, grandchildren, and parents, did not exist in great numbers among the JYLS participants. The Finnish welfare state provided public services for both child care and old age people's care. More lately, the state has aimed at the scenario where aging people should live at their homes with the help of visiting nurses. The burden of the care of an aging person falls on his or her aging spouse and adult children.

The participants were becoming grandparents. The relationship with their grandchildren was emotionally close, but the grandparents seldom took care of their grandchildren on a regular basis; each child had a right to a full-time public daycare independent of the parents' work situation. This right was limited to 20 hours a week in 2016 on a choice of local authorities, if one of the parents was at home. This change may increase the need for the grandparents' help in childcare. Changes in legislation and policy demonstrate the dependency of people's functioning in different spheres of life on societal matters.

\subsection{Midlife transition}

The research results do not indicate the existence of a normative midlife crisis, as reviewed by Kokko (2010a). Helson (1997, p. 34) states that "No major theorist argues for a normative midlife crisis in the sense of psychological disturbance." A large longitudinal study, the Midlife in the United States (MIDUS,) shows, accordingly, that a crisis is not a typical midlife phenomenon (Lachman et al., 2015). The MIDUS was started in 1995 with a sample of around 7,000 participants aged 25 to 75 years (Brim, Ryff, \& Kessler, 2004) and, until the present, new data have been collected at about 10 -year intervals.

The conception of a midlife crisis has a background in popular writings (e.g., Jacques, 1965; Sheehy, 1976), based largely on clinical samples as noted by Lachman et al. (2015). Levinson, Darrow, Klein, Levinson, and McKee (1978) described polarities in men's lives in their 40s and their reactions to them, which were named as crises. "The term crisis comes from Eriksonian theory and is not associated with breakdown or with any particular time of life. It means that there is heightened concern and a need to make a choice" (Helson, 1997, p. 31). Around half of the JYLS participants acknowledged that they had experienced periods when they had re-assessed their life goals and changed their ways of life (Kokko, 2010b). When the word crisis was used in a question posed to participants, affirmative responses about crises were received from 40 percent, but for many of them, this means the reflecting on their life goals. Some individuals undertake this kind of reflection at different stages of their lives.

In the JYLS, life satisfaction and psychological well-being remained very stable across the adult years and social well-being increased from age 42 to 50 . People in middle age also find their life meaningful: uncertainty about meaningfulness was 
only found in a few percent (Kokko, 2010b). In mid-life, people may become more aware of the passing of time and start to think of the meaning of life in a new way, and perhaps find a new life path for the rest of their lives, but new choices are generally experienced positively. A "crisis" is not an appropriate term to use to describe the re-evaluation of one's life and its goals and values. The experience of purpose in life is seen as one of the important goals of human development (Bengtson, Gans, Putney, \& Silverstein, 2009).

Researchers have argued for years that a generalized conception of a midlife crisis is erroneous and harmful to the respect and reputation of middle-aged people, for instance, in the labor market. As early as 1997, Helson stated that it was time to move on from debating the midlife crisis. She proposed that the expression midlife transition be used instead, which is less likely to cause misunderstandings than the expression a midlife crisis. Lachman et al. (2015) describe a transition in midlife as a pivotal period for balancing growth and decline and linking earlier and later periods of life. Middle-aged people play a central role in the family, workplace, and in society at large. The MIDUS shows that the well-being of middle-aged people is more positive than is generally believed, and the JYLS findings confirm this.

Individuals who experience a serious crisis at any age tend to also experience crises at other ages. This suggests that some individuals are more vulnerable to psychological problems than others and is possibly associated with their personality profile having a high level of neuroticism (the Brittle type). Serious crises may also result from the accumulation of problems in life due to a person's style of life including, for instance, heavy drinking or risk-taking behavior. The consequences of these activities often emerge in middle age (Elliott \& Vaitilingam, 2008).

\subsection{The person functioning in the different spheres of life}

The description of people's average functioning in various domains of life conceals differences between individuals that may cause an even larger variation within a group in social and psychological functioning than the variation between age groups, as reviewed by Pulkkinen (2000) for cognitive functioning. Possible reasons for differences between individuals are often sought from external factors, such as socioeconomic background, but less often from the individuals themselves. However, as noted by Helson (1997) the properties of an individuals' life depend on the environment and on the personality. The study of personality as a source of variation between individuals in psychological and social functioning was one of the main aims of the JYLS.

In the metaphorical picture of different spheres of life (Figure 9.1), a person in the middle is steering his or her life through the domains. The energy comes from the person's characteristics of acting, goals and values, and existential experiences of the past, present, and future; and, in general, from his or her psychological and social functioning capacities in the actual context. The participants of the JYLS were described in Part II on the three layers proposed by McAdams (2015): the person as social actor; the person as motivated agent, and the person as autobiographical author. On each layer, individual differences existed. 
The person as social actor was analyzed within the model of how individual differences in adult personality unfold from socioemotional behavior in childhood (Figure 4.1). Four developmental paths were conceptualized and identified, associated with psychological and social functioning up to middle age as summarized in Figure 5.1 and Box 5.1. The basic hypothesis was that individual differences in socioemotional behavior can be described - not only based on whether a person acts or not, but also on the basis of whether a person cognitively controls the emotional and behavioral aspects of an act. Data were analyzed with variableand person-oriented approaches by using several data sets and statistical procedures for finding ways and categories to describe differences between the participants. Irrespective of the nature of the data and methods of analysis, a similar type of variation was found in which individual differences were organized in terms of social activity (extraversion, approach tendency) and self-regulation. The same terminology was used for the styles of life, personal styles, and personality profiles to indicate the basic directions of individual differences. The names of the styles do not refer to categorical concepts (types), but rather to the corners of the two-dimensional map. The "neighbor" styles may be combined in individuals' behaviors to different degrees. Individuals may also be rather "ordinary," somewhere in the middle of the continua, without a distinctive style of life.

Individuals with the Resilient, Brittle, Undercontrolled, and Overcontrolled styles of life steer their lives in ways that differ from each other in many respects, such as in individuals' relations to themselves, other people, and society. Their associations with psychological functioning were summarized in Figure 8.1. Psychological functioning refers to internal criteria for overall adaptation to life. It covers mental well-being and various aspects of self-reflection such as identity formation, personal control over one's life, and self-esteem. The Resilient and Brittle individuals differed systematically in various indicators of psychological functioning so that mental well-being and other aspects of psychological functioning were more positive in the Resilient than in the Brittle individuals.

A general developmental trend in adult personality could also be described within the two-dimensional mode model: the development toward increasing selfregulation by middle age. However, it did not occur in the individuals who were the lowest in self-regulation, particularly in the Brittle, which means that individual differences in self-regulation increased. It also suggests increasing differences in psychological functioning. Problems in mental well-being in middle age and beyond would deserve more attention.

Differences between the participants also existed on the level of the person as motivated agent. These differences were described in terms of the patterns and timing of transition to adulthood, life structures that consist of important things in people's lives and values. Personality characteristics are associated with individual differences in goals and values. On the level of the person as autobiographical author, everybody is unique. Nevertheless, certain commonalities could be found between individuals' ways of processing life. Comparisons between them from a specified perspective, for instance, identity formation and a sense of control over one's life were also made with measures available. 
A holistic approach to a human being includes the idea that he/she is a whole. "The totality derives its characteristic features and properties from the interaction among the elements involved, not from the effect of each isolated part on the totality," as Magnusson (2000, p. 42) spells out. Personality characteristics, motivations, and personal interpretations of life form a totality that functions in a context and is dependent on underlying biological processes. It is important to be aware of it. However, scientists also have to focus their attention on details in order to understand the function of the parts in the totality. 


\section{5}

\section{THE INTEGRATED MODEL FOR INDIVIDUAL DIFFERENCES IN SOCIAL AND PSYCHOLOGICAL FUNCTIONING}

The metaphor of having to fit hundreds of pieces together to make a jigsaw puzzle is a good one for writing this synthesis of results. In the picture on the jigsaw puzzle, models for individual differences have come up as dominant figures and helped to fill in the details of the picture. As noted by Stikker (2012), a model is not the same as reality, but it is a simplified picture invented by a researcher, a schematic representation of a phenomenon. Although a model is never complete, it may satisfy a researcher's desire to discover order in the structure and dynamics of a phenomenon.

Individual differences in personality have been presented in this book within a two-dimensional framework, starting by outlining children's differences in socioemotional behavior (Figure 1.2). The next stage was to include developmental directions into the model (Figure 4.1). Empirical findings supporting the assumptions about different developmental paths were presented in the two-dimensional scheme (Figure 5.1), as well as the results with regards to the relationships between personality profiles and psychological functioning (Figure 8.1).

In this chapter, empirical findings on connections between individual differences in personality and social functioning are summarized and presented with psychological functioning. Connections of the participants' social functioning with their socioemotional behavior in childhood and adolescence, and with their lifestyles and personality traits in adulthood, were analyzed in many studies, although not systematically across domains and time. The comparisons that were available showed that successful functioning was associated with constructive and compliant behaviors in childhood and extraversion, conscientiousness, and agreeableness in adulthood. Problems in social functioning were, in turn, associated with aggressive behavior and noncompliance in childhood and neuroticism in adulthood. The results presented in Chapters 10 to 13 are summarized in Box 15.1 without repeating references to original studies. 


\section{BOX 15.1 SOCIAL FUNCTIONING IN DIFFERENT DOMAINS OF LIFE IN RELATION TO THE PARTICIPANTS' CHARACTERISTICS IN CHILDHOOD (C) AND ADULTHOOD (A): RESEARCH FINDINGS (PART III) SUMMARIZED}

Family domain and care

Secure attachment at age 36

Fearful attachment at age 36

Good marital quality, women

Good marital quality, men

Divorce by age 36

No divorce

Dissatisfaction with partner at age 42 Low parenting stress

High parenting stress

Parental identity, achieved

Parental identity, diffusion (men)

Work domain and work-family balance Success at school

A lack of post-comprehensive education High occupational attainment, women

men

Low occupational attainment, women

men

Higher income at age 43

Longer duration of unemployment:
Associated characteristics: examples

A: Extraversion, self-esteem, mental well-being

A: Neuroticism, depression, anxiety

C: Constructive and active behavior

C: Not aggressive, anxious, or labile behavior

C: Aggressive behavior

C: Compliant (men); older age at marriage (women)

A: Neuroticism

A: Extraversion, self-esteem; identity commitment (men)

A: Neuroticism, low self-esteem

A: Being a parent, generativity, child-centered parenting (nurturance, awareness of children's whereabouts); psychological and social well-being (men)

A: Low nurturance; parenting stress (men)

C: Constructive behavior

C: Social anxiety

C: Constructive and active

A: Extraversion

C: Compliant and constructive

A: Agreeableness

C: Anxious, passive

A: Neuroticism

C: Emotionally labile, poor school success

C: Constructive behavior

C: Aggressive behavior; age 14: school maladjustment, anxiety, low school success, low constructiveness

A: Low education, heavy drinking 
Occupational well-being: Engaged

Occupational well-being: Burnout

Occupational well-being: "Bored out"

Higher work-family conflict

Work-family balance: Beneficial

Work-family balance: Harmful

Health domain and health behavior

Being accident prone

Poor self-assessed health, age 36

Good self-assessed health

Heavy drinking

Refraining from substance use

Metabolic syndrome

Leisure domain and recovery from work

Large social network

Organized leisure activities

Home-oriented hobbies

No hobbies, watch TV

Interest in violence viewing, adults

Nonoffending:

Obligations to society

Nonoffending
A: Resilient personality profile

A: Brittle personality profile

A: Undercontrolled personality profile

A: Neuroticism

A: Extraversion, conscientiousness, agreeableness

A: Low extraversion, high neuroticism

C: Disobedient to the teacher, poor school success, low anxiety (males), high anxiety (females)

Age 14: Low behavior regulation

A: Heavy drinking, regular smoking, neuroticism, low extraversion

A: Thriver lifestyle, Resilient personality profile

Age 14: Aggressive behavior, emotional lability and low in constructive and compliant behavior

A: Undercontrolled and Brittle lifestyles

C: Compliant behavior

A: Overcontrolled lifestyle

C: Early onset of drinking

A: Heavy drinking

C: Constructive and active behavior

C: Constructive behavior; Thriver lifestyle

C: Compliant behavior

A: Overcontrolled lifestyle

C: Anxious behavior

A: Loser lifestyle

C/A: Aggressiveness

A: Previous violence viewing Compliance, low aggressive behavior

A: Agreeableness and conscientiousness

C: Constructive behavior

A: Thriver lifestyle

C: Compliance, low aggression

A: Agreeableness, conscientiousness 
Criminal offending

Temperance in the way of life

Temperance (above 75th percentile)

Temperance (below 25th percentile)
Age 14: Low self-regulation (lacking in concentration, aggressiveness, norm-breaking behavior), poor school success, low in constructive behavior

A: Sensation seeking, neuroticism, impulsivity and disinhibition

C: Constructive and compliant behavior

A: Conformity, conscientiousness, psychological well-being, life satisfaction, and self-esteem

C: High aggressive, low constructive, and compliant behavior

Age 14: Problem behaviors, poor school results, early onset of drinking

A: Short education, high disinhibition and impulsiveness, and low conformity, conscientiousness, psychological well-being, satisfaction, and self-esteem.

Successful social functioning. In the domains of family and care, successful social functioning was found in the participant's relationship with his/her partner and in child-rearing. The personality characteristics associated with good relationships with partners were already found in childhood: lower levels of aggressive behavior for women and men and in a higher level of constructive behavior for women and a higher level of compliance for men. In adulthood, positive emotionality through extraversion and mental well-being were associated with secure attachment. Parents' extraversion was associated with low parenting stress, and the extraverted parents reported that they were highly nurturing of their children; children, however, assessed that there was more nurturing in the home atmosphere when the parents were not high in extraversion. Also, life experiences mattered: the participants' parental identity developed when their children were born, and women were at lower risk of divorce if they were older when they got married.

In the domain of work, success at work, indicated by occupational status, career orientation, income, and low levels of dropping out of education was associated with constructive behavior in childhood. In men, compliance and agreeableness were also associated with success at work. The Engaged type (in terms of wellbeing at work) was characteristic of the Resilient personality profile in adulthood, 
defined by high extraversion and conscientiousness. Also, the Beneficial type (in terms of work-family balance) was associated with high extraversion, conscientiousness, and agreeableness.

In the domains of health good self-assessed health was associated with the Resilient personality profile in adulthood characterized by high conscientiousness. Hampson et al. (2016) have shown that high conscientiousness already in childhood was associated with good self-assessed health, whereas decreasing health in adulthood was associated with lower conscientiousness. Conscientiousness (i.e., self-discipline and goal-directness) may contribute to health through a healthy lifestyle, seen, for instance, in the controlled use of alcohol in the JYLS. Heavy drinking was preceded by the early start of the use of alcohol and problem behaviors in adolescence; it was associated with the Reveller and Loser styles of life. Refraining from the use of alcohol and smoking was characteristic of the Overcontrolled lifestyle.

A large social network in adulthood was associated with constructive and active behavior in childhood. Following the rules of society, as indicated by nonoffending, was associated with lower aggressiveness and higher compliance in childhood and with higher agreeableness and conscientiousness in adulthood (when participants were compared with persistent offenders). In general, temperate behavior, defined by controlled drinking, no smoking, normal body mass index, and no criminal record in adulthood was associated with high constructive and compliant behavior and low aggressiveness in childhood, and with conformity, conscientiousness, self-control, and emotional and psychological well-being in adulthood.

Problems in social functioning. In the domain of the family, problems in functioning were found in the participant's relationship to his/her partner and in his/her childrearing. Indicators of problems in functioning for the former were dissatisfaction with the partner, fearful attachment, divorce, and poor marital quality. The personality characteristics associated with these problems were aggressiveness in childhood and higher neuroticism in adulthood. Indicators of problems in functioning in relation to child-rearing were high parenting stress and low levels of nurturing. In adulthood, they were associated with high neuroticism. Identity diffusion for parenting in men was, in turn, associated with high parenting stress and low levels of nurturing.

In the domain of work, low career orientation, temporary jobs, long duration of unemployment, and dropping out were associated with high aggressiveness and other behavior problems in childhood and adolescence and with anxiety, together with poor school success and low constructive behavior. In adulthood, they were associated with low agreeableness and low extraversion. The Burnout type (with regards to well-being at work) was characterized by the Brittle personality profile in adulthood, defined by low extraversion, low conscientiousness, and high neuroticism, whereas the "Bored-out" type (in terms of well-being at work) was characterized by the Undercontrolled personality profile, defined by high extraversion and low conscientiousness. A low ability to cope with monotony is associated with the Undercontrolled style of life, which fits the finding on being bored at 
work. The harmful type in terms of work-family balance was associated with low extraversion and high neuroticism.

In the domain of health, being accident prone was associated with noncompliance (disobeying teacher), poor school success, and low anxiety in men (but with high anxiety in women). Poor self-assessed health was associated with heavy drinking, smoking, and low behavior regulation. Heavy drinking, in turn, was a risk factor for metabolic syndrome. Low temperance in middle age defined by excessive drinking, regular smoking, being overweight (or being obese in the case of men), and having a criminal record was preceded by high aggressive behavior and low constructive and compliant behavior in childhood and it was associated with high disinhibition and impulsivity in adulthood.

Problems in social functioning such as unstable career, heavy drinking, and violations against social norms tended to concentrate in the same individuals. Preceding multi-problem behavior was often seen in maladjustment to school indicating low self-regulation. Aggressive behavior predicts later problems in social functioning, but only if it is highly elevated (above 75th percentile); high aggression tends to be accompanied by other problems. Risk factors do not usually function as single predictors of problem behaviors. Criminal offending was predicted by high aggressiveness when it was combined with poor school success. Also lacking concentration and lacking constructive behavior predicted criminal offending. In adulthood, criminal offending was associated with sensation seeking, neuroticism, impulsivity, and disinhibition.

In middle age, some participants had many problems (unstable career line, not being employed, problem drinking, health problems, and poverty) accumulated. For the men (53 percent) and women (56 percent) who had none of these problems at age 50, the likelihood of belonging to the personality profile group, called Resilient, was significant. The male participants (15 percent), who had at least three problems out of five, had been over-represented in the group for very aggressive children. This was not found in women, but for the women (11 percent) who had at least three problems, the likelihood of belonging to the personality profile group, called Brittle, in adulthood was significant. The difference in not having social functioning problems or having many of them concurs with the participants' differences in psychological functioning such as mental well-being, which was higher in the Resilient profile than in the Brittle profile (cf. Figure 8.1).

The integrated model. Individual differences in social functioning are presented schematically in Figure 15.1 together with individual differences in psychological functioning (see Figure 8.1). The model finalizes my theoretical reasoning and summarizes empirical findings. Four developmental paths form the core of the model in the figure depicting individuals' differences in steering their lives. They are described within the framework of a person's approach or avoidance tendency that often manifests itself in active versus passive behavior and in the self-regulation of the expression of these tendencies. While high versus low psychological functioning was associated with the Resilient versus Brittle personality, social functioning was related to self-regulation more generally. Successful social functioning was 


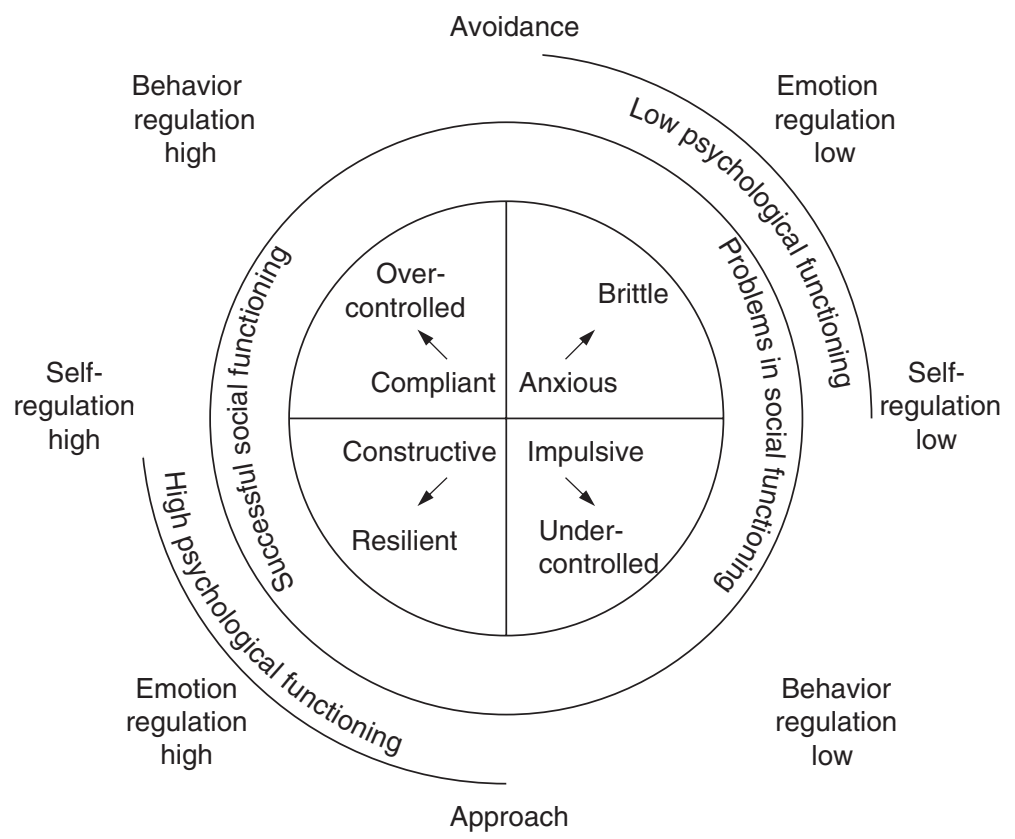

FIGURE 15.1 The integrated model for individual differences in social and psychological functioning.

connected to the Resilient and Overcontrolled personality, and problems in social functioning to the Brittle and Underconrolled personality. Thus, social functioning was connected to both aspects of self-regulation, behavior and emotion regulation, and psychological functioning to emotion regulation.

There was a rather systematic gender difference in that successful social functioning was connected to constructive behavior in men and women, but in the men, it was also associated with high compliance, and in women with extraversion. Compliance may involve too much passivity in women for social success, whereas extraversion may involve too much impulsivity in men to warrant successful social functioning (Pulkkinen, 2009).

Psychological functioning and social functioning correlate with each other. The accumulation of social functioning problems is associated with low psychological functioning, and a lack of social functioning problems is correspondingly associated with high psychological functioning. An individual's achievements in the education and labor markets, and the quantity and quality of social relationships, affect his or her psychological functioning, but in general, causal relationships between social and psychological functioning are limited. Common causes lie on a deeper level of individual differences. 


\section{6}

\section{A CREDO}

A person was metaphorically described as steering his or her life through various domains by means of an engine that receives energy from the person's characteristics, goals and values, and mental well-being. Furthermore, individual differences in social and psychological functioning were described in a series of schematic models. The use of the metaphor and models does not mean that the conception of the human being in this book is mechanistic. The goal was to illustrate the core role of an individual as an agent of his or her life.

In his phenomenological psychological analysis, Juha Perttula (1998) emphasized the need for a researcher to disclose the conception of the human being that he or she has adopted in his/her investigation. His own model included three principles: nestedness, holism, and pluralism. The principle of nestedness means that a researcher cannot avoid making ontological commitments; the principle of holism means that a human being operates as a whole; and the principle of pluralism means that there are various ways to be a human being. I concur with him in these principles.

Ontological commitments refer to conceptions about the nature of existence, for instance, the relationship between mind and body. In my own work, the longitudinal study grew from the idea that human beings are not only animals or bodies who react to external events in instinctual ways. Human beings are capable of reflecting on their own behavior and emotions and perceiving complex situations from many perspectives. I believed that these capacities exist in the human mind and affect an individual's behavior already in childhood. In this final chapter, it is with pleasure that I return to childhood after a 50 -year journey.

In the path from childhood to adulthood, there are different parallel and complementary processes in human development in the physical, mental, and spiritual realms. The first process is the individuation of a person so that he/she can unfold his/her inborn uniqueness in all these aspects (Pulkkinen, 1984). The second is socialization into society, which means the adaptation of an individual into society 
in order to enable him or her to function successfully with other individuals. The third process is an individual's integration into the belief systems of the culture. Belief systems may vary from the material to the spiritual. An individual's development in these processes may be promoted by encouraging the development of his or her individuality, by guiding his or her adaptation to the complex society, and by enriching his or her experiences through cultural masterpieces and spiritual views. Likewise, an individual's development may be restricted by shaping him or her to a predetermined model, by neglecting to provide guidance, and by limiting his/her cultural and spiritual experiences. Variations in individuals' life experiences are reflected in their actions.

In another context, I have described different views of the child and the implications of these views for child-rearing and education (Pulkkinen, 2014a). My image of the child is in line with the image of the child presented by Matthes (2013) and the Learning for Well-Being Community (Kickbush, 2012). It has been further formulated within the Alliance for Childhood European Network Group (2017) as follows:

- Children are endowed with inner potential to grow, learn and communicate and to participate in their own way and with their own rights and responsibilities within society.

- The child is an agent of his/her own life in relation with others. The child is not an object to be pushed and modeled into a shape by the adult.

- Adults who are sensitive to children's needs are there to help unfold their uniqueness.

- The quality of the children's relationships with adults and other children affects their growth as human beings in either a positive or negative way.

- Children are co-creators of our world and transform it.

- Human development is a process of unfolding an individual's uniqueness in which mutually interacting biological, psychological, social, cultural, spiritual and societal factors interplay.

For the comparison, I present the view of the child in the reformed national core curriculum for the Finnish comprehensive basic school, which was in force from the autumn term of 2016. Its formulation concurs with the formulation of the image of the child presented above in highlighting the fundamental value of every pupil as a unique individual. It crystallizes the basis of Finnish education with everybody's right to high-quality education with the result that inequality in child well-being in Finland is one of the smallest in the world (Unicef, 2016). This formulation can be applied to child-centered social services for children by replacing education by services.

Comprehensive basic education is founded on the inherent value of childhood. Every child is unique and valuable as he or she is. Every child has a right to evolve to his or her full measure as a human being and member of society. In this process the child needs encouragement and individualized support and an experience that he or she is listened to at school, that 
his or her opinions are valued, and that his or her learning and well-being are important. What is important is the experience that he or she can build together with other people the positive functioning and well-being of his or her community.

(OPH, 2015, p. 15; italics and translation by Pulkkinen, 2016)

The categorization of individuals based on their behaviors or lifestyles may seem contradictory when the focus is on each person's uniqueness. Therefore, a conceptual clarification is needed. The uniqueness of individuals refers to their innate potentials, which may be physical, social, cognitive, emotional, artistic, or other potentials, enabling an individual to find his or her place in the wholeness of the living system. All places in society are valuable because the wholeness does not function without its parts. In order to function in one's place in harmony with other people and to feel mentally well, an individual needs meta-level capacities such as self-regulation. The present book shows the significance of self-regulation for social and psychological functioning. The categorization was made in respect to individuals' self-regulation, not in respect to their unique potentials.

Knowledge of developmental processes and the factors associated with them may help parents, other educators, coaches, managers, and politicians to find ways of improving the quality of life to support the unfolding of individuals' uniqueness and reducing the risk of suppressing or misleading this developmental process. Respecting uniqueness demands sensitivity in order to observe the interests and innate potentials of individuals and to encourage them to become agents of their lives. Knowledge about, for instance, the importance of constructive behavior for the child's further development can be applied to child-rearing at home, child care in day care centers, education at school, and coaching for particular hobbies. In accordance with the findings of the JYLS, Sampson (2016) remarks that it is not only cognitive skills that are needed for adult success, but also noncognitive skills including personal character, especially self-control, are needed. He suggests that in childhood interventions both self-control and social control should be considered. In accordance with this, Blair and Raver (2015) argue that "fostering selfregulation is a primary way of fostering school readiness" (2015, p. 723).

A key question is how to guide the child to process emotions in an anticipatory way so that he/she can maintain an active and positive approach to the matters in question and retain empathy for people involved. Adapting to the rules of society is needed but with the addition of flexibility and rational thinking. Compliance and conformity to meaningless commands may momentarily enable an individual to function with other people but will be harmful in the long term. Moral and ethical aspects should be included in the process of promoting behavior regulation.

The improvement of particular skills and competences without considering the wider context is not sufficient in interventions, as Schoon (2006) remarks. One should understand the functional meaning of the competence holistically and how it develops in context. An example of a successful intervention for the improvement of social competencies was an experiment at schools that I conducted to apply 
research findings to practice (Pulkkinen, 2012b). By organizing club activities at school and by changing the structure of a school day, the learning environment became more child-friendly and richer. It affected children positively. Particularly cultural activities such as participating in music, and arts and crafts improved, besides children's unique artistic skills, their school achievement and self-regulation indicated by concentration, persistence, and constructive behavior. These results in Finland concur with findings in other countries as reviewed by Pulkkinen (2016): high quality extracurricular activities at school may enrich the learning environment and have longstanding positive impacts on the pupils' development.

Among adults, likewise, working in a way that allows for creativity and learning new things and skills is most satisfactory and motivating. The consideration of the uniqueness of an adult as a worker is a challenge for the management of work. The low levels of pleasure that middle-aged participants said that they received from their work, in comparison with family, leisure, and friends, suggests that something might be done to improve the organization and management of work to better fit the needs of individuals.

Although childhood behavior is associated with adult outcomes, it does not mean that human development only takes place in childhood. As expressed by Schoon (2006, p. 163) "The notion that there is one basic mechanism underlying adjustment in different contexts and at different life stages is untenable." Personality and its capacities develop through adulthood. With age, psychological development depends increasingly on self-education, which includes increasing self-reflection and awareness of oneself, other people, society, and global issues in the world. An example of changes in awareness is sustainability thinking, which has increased immensely during the past few decades (Caradonna, 2014). Changes are continuous throughout life in human beings and nature. Having a high level of personal choice in a change is associated with the positive evaluation of the change and its impact on a lasting reorientation in life. 


\section{REFERENCES}

Achenbach, T. M., \& Edelbrock, C. S. (1983). Manual of the child behavior checklist and revised child behavior profile. Burlington, VT: University of Vermont, Department of Psychiatry.

Achenbach, T. M., McGonaughy, S. H., \& Howell, C. T. (1987). Child/adolescent behavioral and emotional problems: Implications of cross-informant correlations of situational specificity. Psychological Bulletin, 101, 213-32.

af Klinteberg, B. (1996). Biology, norms, and personality: A developmental perspective. Neuropsychobiology, 34, 146-54.

Ainsworth, M. D. S., Blehar, M. C., Waters, E., \& Wall, S. (1978). Patterns of attachment: A psychological study of the strange situation. Hillsdale, NJ: Erlbaum.

Alatupa, S., Pulkki-Råback, L., Hintsanen, M., Elovainio, M., Mullola, S., \& KeltikangasJärvinen, L. (2013). Disruptive behavior in childhood and socioeconomic position in adulthood: A prospective study over 27 years. International Journal of Public Health, 58, 247-256.

Alliance for Childhood (2017). Available online at http://www.allianceforchildhood.eu/ image-of-the-child.

Allport, G. W. (1937). Personality: A psychological interpretation. New York, NY: Holt, Rinehart \& Winston.

Antonovsky, A. (1987). Unraveling the mystery of health: How people manage stress and stay well. San Francisco, CA: Jossey-Bass.

Arnett, J. J. (2000). Emerging adulthood: A theory of development from the late teens through the twenties. American Psychologist, 55, 469-80.

Arnett, J. J. (2004). Emerging adulthood: The winding road from the late teens through the twenties. New York, NY: Oxford University Press.

Aro, H. (1988). Stress, development and psychosomatic symptoms in adolescence. A study of 14- to 16-year-old school children. (ActaUnversitatis Tamperensis Series A, No. 242). Tampere, Finland: University of Tampere.

Atkinson, J. W. (1964). An introduction to motivation. Princeton, NJ: Van Nostrand.

Aunola, K., Nurmi, J.-E., Onatsu-Arvilommi, T., \& Pulkkinen, L. (1999). The role of parents' self-esteem, mastery-orientation and social background in their parenting styles. Scandinavian Journal of Psychology, 40, 307-17. 
Baldwin, W. (1993). The consequences of early childbearing: A perspective. Journal of Research on Adolescence, 3, 349-52.

Baltes, P. B. (1983). Life-span developmental psychology: Observation on history and theory revisited. In R. M. Lerner (Ed.), Developmental psychology: Historical and philosophical perspectives (pp. 79-111). Hillsdale, NJ: Erlbaum.

Baltes, P. B. (1997). On the incomplete architecture of human ontogeny: Selection, optimization, and compensation as foundation for developmental theory. American Psychologist, $52,366-80$.

Baltes, P. B., Lindenberger, U., \& Staudinger, U. M. (1998). Life-span theory in developmental psychology. In R. M. Lerner (Ed.) \& W. Damon (Series Ed.), Handbook of child psychology: Vol. I. Theoretical models of human development (5th ed., pp. 1029-143). New York, NY: Wiley.

Bandura, A. (1973). Aggression: A social learning analysis. Englewood Cliffs, NJ: Prentice-Hall.

Bandura, A. (1982). Self-efficacy in changing societies. New York, NY: Cambridge University Press.

Bandura, A. \& Walters, R. H. (1963). Social learning and personality development. New York, NY: Holt, Rinehart \& Winston.

Bartholomew, K. (1990). Avoidance of intimacy: An attachment perspective. Journal of Personality and Social Relationships, 7, 147-78.

Bartholomew, K., \& Horowitz, L. M. (1991). Attachment styles among young adults: A test of a four-category model. Journal of Personality and Social Psychology, 61, 226-44.

Baumrind, D. (1971). Current patterns of parental authority. Developmental Psychology Monographs (Part 2), 4, 1-103.

Beckeley, A. L., Caspi, A., Harrington, H., Houts, R.M., Mcgee, T.R., Morgan, N., Schroeder, F., Ramrakha, S., Poulton, R., \& Moffitt, T. E. (2016). Adult-onset offenders: Is a tailored theory warranted? Journal of Criminal Justice, 46, 64-81.

Bengtson, V. L. (1985). Diversity and symbolism in the grandparental role. In V. Bengtson \& J. F. Robertson (Eds.), Grandparenthood (pp. 11-25). Beverly Hills, CA: Sage.

Bengtson, V. L., Gans, D., Putney, N. M., \& Silverstein, M. (2009). Theories about age and aging. In V. L. Bengtson, M. Silverstein, M. Putney, \& D. Gans (Eds.), Handbook of theories of aging (2nd edition, pp. 3-24). New York, NY: Springer.

Benoit, D., \& Parker, K. C. H (1994). Stability and transmission of attachment across three generations. Child Development, 65, 1444-56.

Bergman, L. R., \& Magnusson, D. (1997). A person-oriented approach in research on developmental psychopathology. Development and Psychopathology, 9, 291-319.

Bergman, L. R., Magnusson, D., \& El-Khouri, B. M. (2003). Studying individual development in an interindividual context: A person-oriented approach. Mahwah, NJ: Erlbaum.

Birren, J. E., \& Hedlund, B. (1987). Contributions of autobiography to developmental psychology. In N. Eisenberg (Ed.), Contemporary topics in developmental psychology (pp. 394-415). New York, NY: Wiley.

Bjerstedt, A. (1963). Sociometriska metoder. Uppsala, Sweden: Almqvist \& Wicksells.

Blair, C., \& Raver, C. C. (2015), School readiness and self-regulation: A developmental psychobiological approach. Annual Review of Psychology, 66, 711-31.

Block, J. (1971). Lives through time. Berkeley, CA: Bankroft Books.

Block, J. (2002). Personality as an affect-processing system: Toward an integrative theory. Hilldsdale, NJ: Erlbaum.

Block, J. H., \& Block, J. (1980). The role of ego-control and ego-resiliency in the organization of behavior. In W. A. Collins (Ed.), Development of cognition, affect, and social relations (Vol. 13, pp. 39-101). Hilldsdale, NJ: Lawrence Erlbaum Associates. 
Brandtstädter, J. (1984). Personal and social control over development: Some implications of an action perspective in life-span developmental psychology. In P. B. Baltes \& O.G. Brim, Jr. (Eds.), Life-span development and behavior (Vol. 6, pp. 1-32). San Diego, CA: Academic Press.

Brim, O. G., Ryff, C. D., \& Kessler, R. C. (2004). How healthy are we? A national study of well-being at midlife. Chicago, IL: Chicago University Press.

Bronfenbrenner, U. (1979). The ecology of human development. Cambridge, MA: Harvard University Press.

Bühler, C. (1933). Der menschliche Lebenslauf als psychologische Problem. Leipzig: Hirzel.

Busby, D. M., Christensen, C., Crane, D. R., \& Larson, J. H. (1995). A revision of the Dyadic Adjustment Scale for use with distressed and nondistressed couples: Construct hierarchy and multidimensional scales. Journal of Marital and Family Therapy, 21, 289-308.

Buss, A. H. (1961). The psychology of aggression. New York, NY: Wiley.

Buss, A. H., \& Perry, M. (1992). The aggression questionnaire. Journal of Personality and Social Psychology, 63, 452-9.

Cairns, R. B., \& Cairns, B. D. (1994). Lifelines and risks: Pathways of youth in our time. New York, NY: Cambridge University Press.

Campos, J. J., Campos, R. G., \& Barrett, K. C. (1989). Emergent themes in the study of emotional development and emotion regulation. Developmental Psychology, 25, 394-402.

Caprara, G. V., Barbaranelli, C., Pastorelli, C., Bandura, A., \& Zimbardo, P. G. (2000). Prosocial foundations of children's academic achievement. Psychological Science, 11, 302-6.

Caprara, G.V., \& Zimbardo, P. G. (1996). Aggregation and amplification of marginal deviations in the social construction of personality and maladjustment. European Journal of Personality, 10, 79-110.

Caradonna, J. L. (2014). Sustainability: A history. Oxford, UK: Oxford University Press.

Carstensen, L.L. (1993). Motivation for social contact across the life span: A theory of socioemotional selectivity. In J. E. Edwards (Ed.), Nebraska Symposium on Motivation: Developmental perspectives on motivation (pp. 209-54). Lincoln, NE: University of Nebraska Press.

Carstensen, L.L., Pasupathi, M., Mayr, U., \& Nesselroade, J. R. (2000). Emotional experience in everyday life across the adult life span. Journal of Personality and Social Psychology, 79, 644-55.

Carver, C. S. (2005). Impulse and constraint: Perspectives from personality psychology, convergence with theory in other areas, and potential for integration. Personality and Social Psychology Review, 9, 312-33.

Caspi, A., Bem, D. J., \& Elder, G. H. Jr. (1989). Continuities and consequences of interactional styles across the life course. Journal of Personality, 52, 375-406.

Caspi, A., Moffitt, T. E., Thornton, A., Freedman, D., Amell, J. A., Harrington, H., Smeijers, J., \& Silva, P. A. (1996). The life history calendar: A research and clinical assessment method for collecting retrospective event-history data. International Journal of Methods in Psychiatric Research, 6, 101-14.

Caspi, A., Roberts, B. W., \& Shiner, R. L. (2005). Personality development: Stability and change. Annual Review of Psychology, 56, 433-84.

Caspi, A., \& Shiner, R. L. (2006). Personality development. In R. M. Lerner (Ed.) \& W. Damon (Series Ed.), Handbook of child psychology: Vol. 3. Social, emotional, and personality development (6th ed., pp. 300-65). New York, NY: Wiley.

Cattell, R. B., \& Coan, R. W. (1957). Child personality structure as revealed in teacher's ratings. Journal of Clinical Psychology, 13, 315-27.

Chao, R. K., \& Kohlberg, L. (1994). Beyond parental control and authoritarian parenting style: Understanding Chinese parenting through the cultural notion of training. Child Development, 65, 1111-19. 
Cicchetti, D., Ganiban, J., \& Barnett, D. (1991). Contributions from the study of high-risk populations to understanding the development of emotion regulation. In J. Garber \& K. A. Dodge (Eds.), The development of emotion regulation and dysregulation (pp. 15-48). New York, NY: Cambridge University Press.

Cicchetti, D., \& Toth, S. L. (1991). A developmental perspective on internalizing and externalizing disorders. In D. Cicchetti \& S. L. Toth (Eds.), Internalizing and externalizing expression of dysfunction. Rochester Symposium on Developmental Psychopathology (Vol. 2, pp. 1-19). Hillsdale, NJ: Erlbaum.

Clausen, J. A. (1995). Gender, contexts, and turning points in adults' lives. In P. Moen, G. H. Elder Jr. \& K. Luscher (Eds.), Examining lives in context: Perspective on the ecology of human development (pp. 365-89). Washington, DC: APA.

Cole, P. M., Michel, M. K., \& Teti, L. (1994). The development of emotion regulation and dysregulation: A clinical perspective. In N. A. Fox (Ed.), The development of emotion regulation: Biological and behavioral considerations (pp. 73-100). (Monographs of the Society for Research in Child Development, 59, 2-3, Serial No. 240). Chicago, IL: University of Chicago Press.

Collins, W. A. (2003). Historical perspectives on contemporary research in social development. In P. K. Smith \& G. H. Hart (Eds.), Blackwell Handbook of childhood social development (pp. 3-23). Malden, MA: Blackwell.

Collins Cobuild: English Dictionary for Advanced Learners (2001, 3rd ed.). Glascow, UK: HarperCollins Publishers.

Conger, R. D., Ge, X.-J., \& Lorenz, F. O. (1994). Economic stress and marital relations. In R. D. Conger \& G. H. Elder Jr. (Eds.), Families in troubled times (pp. 187-203). New York, NY: Walter de Gruyter.

Costa, P. T. Jr., \& McCrae, R. R. (1985). The NEO personality inventory. Odessa, FL: Psychological Assessment Resources.

Costa, P. T. Jr., \& McCrae, R. R. (1989). The NEO/NEO-FFI manual supplement. Odessa, FL: Psychological Assessment Resources.

Cronbach, L. J. (1960). Essentials of psychological testing. New York, NY: Harper \& Brothers.

Crowne, D. P., \& Marlowe, D. (1960). A new scale of social desirability independent of psychopathology. Journal of Consulting Psychology, 24, 349-54.

Crowne, D. P., \& Marlowe, D. (1964). The approval motive: Studies in evaluative dependence. New York, NY: Wiley.

Daly, M., Delaney, L., Egan, M., \& Baumeister, R. F. (2015). Childhood self-control and unemployment throughout the life span: Evidence from two British cohorts. Psychological Science, 26, 709-23.

Denissen, J. J. A., van Aken, M. A, G., Penke, L., \& Wood, D. (2013). Self-regulation underlies temperament and personality: An integrative developmental framework. Child Development Perspectives, 7, 225-60.

Depue, R. (1987). General Behavior Inventory. Ithaca, NY: Cornell University: Department of Psychology.

De Raad, B., \& Kokkonen, M. (2000). Traits and emotions: A review of their structure and management. European Journal of Personality, 14, 477-96.

DeYoung, C. G. (2014). Cybernetic Big Five theory. Journal of Research in Personality, 56, 33-58.

Dick, D. M., Bernard, M., Aliev, F., Viken, R. J., Pulkkinen, L., Kaprio, J., \& Rose, R. J. (2009). The role of socio-regional factors in moderating genetic influences on early adolescent behavior problems and alcohol use. Alcoholism: Clinical \& Experimental Research, $33,1739-48$.

Diener, E., Emmons, R. A., Larsen, R.J., \& Griffin, S. (1985). The satisfaction with life scale. Journal of Personality Assessment, 48, 71-5. 
Dodge, K. A. (1991). The structure and function of reactive and proactive aggression. In T. Pepler \& K. H. Rubin (Eds.), The development and treatment of childhood aggression (pp. 201-18). Hillsdale, NJ: Erlbaum.

Dodge, K. A., Bates, J. E., \& Pettit, G. S. (1990). Mechanisms in the cycle of violence. Science, 250, 1678-83.

Dollard, J., \& Miller, N. E. (1950). Personality and psychotherapy: An analysis in terms of learning, thinking and culture. New York, NY: McGraw.

Donellan, M. B., \& Robins, R. W. (2010). Resilient, overcontrolled, and undercontrolled personality types: Issues and controversies. Social and Personality Psychology Compass, 4, 1070-83.

Dubow, E. F., Huesmann, L. R., Boxer, P., Pulkkinen, L., \& Kokko, K. (2006). Middle childhood and adolescent contextual and personal predictors of adult educational and occupational outcomes: A mediational model in two countries. Developmental Psychology, 42, 937-49.

Duckworth, A. L., \& Steinberg, L. (2015). Unpacking self-control. Child Development Perspectives, 9, 32-7.

Duncan, G. J., Bergman, L. R., Duckworth, K., Kokko, K., Lyyra, A-L., Mettzger, M., Pulkkinen, L., \& Simonton, S. (2012). Child skills and behaviors in intergenerational inequality. In J. Ermisch, M. Jäntti \& T. Smeeding (Eds.), From parents to children: The intergenerational transmission of advantage (pp. 207-34). New York, NY: Russel Sage Foundation.

Edwards, A. L. (1957). The social desirability variable in personality assessment and research. New York, NY: Dryden.

Eisenberg, N. (2014). Following the data (and sometimes theory). The career of a socioemotional scientist. In R. M. Lerner, A. C. Petersen, R. K. Silbereisen \& J. Brooks-Gunn (Eds.), The developmental science of adolescence: History through autobiography (pp. 124-33). New York, NY: Psychology Press Taylor \& Francis Group.

Eisenberg, N., \& Fabes, R. A. (1992). Emotion, regulation, and the development of social competence. In M. S. Clark (Ed.), Emotion and social behavior (pp. 119-50). Newbury Park, CA: Sage.

Eisenberg, N., Duckworth, A., Spinrad, T., \& Valiente, C. (2014). Conscientiousness: Origins in childhood? Developmental Psychology, 50, 1331-49.

Ekman, G. (1952). Differentiell psykologi. Stockholm, Sweden: Geber.

Elder, G. H. Jr. (1974). Children of the Great Depression. Chicago, IL: University of Chicago Press.

Elder, G. H. Jr. (1998). The life course and human development. In R. M. Lerner (Ed.) \& W. Damon (Series Ed.), Handbook of child psychology: Vol. I. Theoretical models of human development (5th ed., pp. 939-91). New York, NY: Wiley.

Elliott, J., \& Vaitilingam, R. (Eds.) (2008). Now we are 50: Key finding from the National Child Development Study. London, UK: The Centre for Longitudinal Studies.

Elo, A.-L., Leppänen, A., Lindström, K., \& Ropponen, T. (1990). TSK. Miten käytät työstressikyselyä [OSQ. How to use Occupational Stress Questionnaire]. Helsinki, Finland: Finnish Institute of Occupational Health.

Emmons, R. A. (1986). Personal strivings: an approach to personality and subjective wellbeing. Journal of Personality and Social Psychology, 51, 1058-68.

Erikson, E. H. (1950). Childhood and society. New York, NY: Norton.

Ewing, J. A. (1984). Detecting alcoholism: The CAGE Questionnaire. JAMA, 252, 1905-7.

Eysenck, H. J., \& Eysenck, S. B. G. (1963). On the dual nature of extraversion. British Journal of Social and Clinical Psychology, 2, 46-55.

Eysenck, H. J., \& Eysenck, S. B. G. (1975). Manual of the Eysenck Personality Questionnaire. London, UK: Hodder and Stoughton. 
Eysenck, H. J., \& Rachman, S. (1965). The causes and cures of neurosis: An introduction to modern behavior therapy based on learning theory and the principles of conditioning. San Diego, CA: Knap.

Eysenck, S. B. G. (1965). A new scale for personality measurements in children. British Journal of Educational Psychology, 35, 362-7.

Fadjukoff, P. (2007). Identity formation in adulthood. (Jyväskylä Studies in Education, Psychology and Social Research No. 319). Jyväskylä, Finland: Jyväskylä University Printing House.

Fadjukoff, P., Kokko, K., \& Pulkkinen, L. (2007). Implications of timing of entering adulthood for identity achievement. Journal of Adolescent Research, 22, 504-30.

Fadjukoff, P., Kokko, K., \& Pulkkinen, L. (2010). Changing economic conditions and identity formation in adulthood. European Psychologist, 15, 293-303.

Fadjukoff, P., \& Pulkkinen, L. (2006). Identity formation, personal control over development, and well-being. In L. Pulkkinen, J. Kaprio \& R. J. Rose (Eds.), Socioemotional development and health from adolescence to adulthood (pp. 265-85). New York, NY: Cambridge University Press.

Fadjukoff, P., Pulkkinen, L., \& Feldt, T. (2007). Role of personality styles in identity formation. In Fadjukoff, P. Identity formation in adulthood. (Jyväskylä Studies in Education, Psychology and Social Research, No. 319). Jyväskylä, Finland: Jyväskylä University Printing House.

Fadjukoff, P., Pulkkinen, L., \& Kokko, K. (2005). Identity processes in adulthood: Diverging domains. Identity: An International Journal of Theory and Research, 5, 1-20.

Fadjukoff, P., Pulkkinen, L., \& Kokko, K. (2016). Identity Formation in adulthood: A longitudinal study from age 27 to 50. Identity: An International Journal of Theory and Research, $16,8-23$.

Fadjukoff, P., Pulkkinen, L., Lyyra, A.-L., \& Kokko, K. (2016). Parental identity and its relation to parenting and psychological functioning in middle age. Parenting: Science and Practice, 16, 87-107.

Farrington, D. P. (1986). Stepping stones to adult criminal careers. In D. Olweus, J. Block \& M. Radge-Yarrow (Eds.), Development of antisocial and prosocial behavior. Research theories and issues (pp. 359-84). Orlando, FL: Academic Press.

Farrington, D. P. (2010). Families and crime. In J. Q. Wilson \& J. Petersilia (Eds.), Crime and public policy (3rd ed., pp. 130-57). Oxford, UK: Oxford University Press.

Farrington, D. P., Ttofi, M. M., \& Coid, J. W. (2009). Development of adolescent-limited, late-onset, and persistent offenders from age 8 to 48. Aggressive behavior, 35, 150-63.

Feldt, T., Kokko, K., Kinnunen, U., \& Pulkkinen, L. (2005). The role of family background, school success, and career orientation in the development of sense of coherence. European Psychologist, 10, 298-308.

Feldt, T., Mäkikangas, A., \& Aunola, K. (2006). Sense of coherence and optimism. In L. Pulkkinen, J. Kaprio \& R. J. Rose (Eds.), Socioemotional development and health from adolescence to adulthood (pp. 286-305). New York, NY: Cambridge University Press.

Feldt, T., Metsäpelto, R.-L., Kinnunen, U., \& Pulkkinen, L. (2007). Sense of coherence and five-factor approach to personality: Conceptual relationships. European Psychologist, 12, $165-72$.

Feshbach, S. (1964). The function of aggression and the regulation of aggressive drive. Psychological Review, 71, 257-72.

Freitas, A. L., \& Downey, G. (1998). Resilience: A dynamic perspective. International Journal of Behavioral Development, 22, 263-85.

Frick, P. J., Lahey, B. B., Loeber, R., Tannenbaum, L., Van Horn, Y., \& Christ, M. A. G. (1993). Oppositional defiant disorder and conduct disorder: a meta-analytic review of 
factor analyses and cross-validation in a clinic sample. Clinical Psychology Review, 13, 319-40.

Friedman, H. S., \& Kern, M. L. (2014). Personality, well-being, and health. Annual Review of Psychology, 65, 719-42.

Frijda, N. H. (1986). The emotions. Cambridge, UK: Cambridge University Press.

Frone, M. R., Russell, M., \& Cooper, M. L. (1992). Antecedents and outcomes of workfamily conflict: Testing a model of the work-family interface. Journal of Applied Psychology, $77,65-78$.

Furstenberg, F. F., Brooks-Gunn, J., \& Morgan, S. P. (1987). Adolescent mothers in later life. New York, NY: Cambridge University Press.

Garmezy, N. (1985). Stress-resistant children: The search for protective factors. In J. E. Stevenson (Ed.), Recent research in developmental psychopathology (pp. 213-33). Oxford, UK: Pergamon.

Gerris, J. R. M., Vermulst, A. A., van Boxtel, D. A. A. M., Janssens, J. M. A. M., van Zutphen, R. A. H., \& Felling, A. J. A. (1993). Parenting in Dutch families. Nijmegen, Netherlands: University of Nijmegen, Institute of Family Studies.

Geurts, S. (2000). SWING: Survey Work-home Interaction. Nijmegen, Netherlands: University of Nijmegen.

Giorgi, A. (1988). Sketch of a psychological phenomenological method. In A. Giorgi (Ed.), Phenomenology and psychological research (pp. 8-22). Pittsburgh, PA: Duquesne University.

Glueck, S., \& Glueck, E. (1950). Unraveling juvenile delinquency. New York, NY: The Commonwealth Fund.

Gommans, R., \& Cillessen, A. H. N. (2015). Nominating under constraints: A systematic comparison of unlimited and limited peer nomination methodologies in elementary school. International Journal of Behavioral Development, 39, 77-86.

Goodman, R. (1997). The Strengths and Difficulties Questionnaire: A research note. Journal of Child Psychology and Psychiatry, 38, 581-6.

Gottfredson, M. R., \& Hirschi, T. (1990). A general theory of crime. Stanford, CA: Stanford University Press.

Granberg, M. (2003). Onko tunneälyllä yhteyttä työelämään [Is emotional intelligence associated with work]? Master's Thesis, Department of Psychology, University of Jyväskylä, Finland.

Greenhaus, J. H., \& Beutell, N. J. (1985). Sources and conflict between work and family roles. Academy of Management Review, 10, 76-88.

Haapasalo, J. (1990). Eysenckin persoonallisuuskyselyn ja Zuckermanin elämyyshakuisuusasteikon faktorirakenteet ja pisteitys Suomessa [Scoring and factor structure of the Eysenck Personality Questionnaire and Zuckerman Sensation Seeking Scales in Finland]. (Reports from the Department of Psychology, No. 311). University of Jyväskylä, Finland.

Hall, C. S., \& Lindzey, G. (1957). Theories of personality. New York, NY: Wiley.

Hämäläinen, M., \& Pulkkinen, L. (1995). Aggressive and non-prosocial behaviour as precursors of criminality. Studies on Crime and Crime Prevention, 4, 6-21.

Hämäläinen, M., \& Pulkkinen, L. (1996). Problem behavior as a precursor of male criminality. Development and Psychopathology, 8, 443-55.

Hämäläinen, M., Pulkkinen, L., Allik, J., \& Pulver, A. (1994). NESTA-persoonallisuustestin standardointi "viiden suuren" persoonallisuuden piirteen arvioimiseksi [Standardization of a Big Five personality test, NESTA]. (Reports from the Department of Psychology, No. 327). University of Jyväskylä, Finland.

Hampson, S. E., \& Goldberg, L. R. (2006). A first large cohort study of personality trait stability over the 40 years between elementary school and midlife. Journal of Personality and Social Psychology, 91, 763-79. 
Hampson, S. E., Edmonds, G. W., Barckley, M., Goldberg, L. R., Dubanoski, J. P., \& Hillier, T. A. (2016). A Big Five approach to self-regulation: personality traits and health trajectories in the Hawaii longitudinal study of personality and health. Psychology, Health E Medicine, 21, 152-62.

Hartshorne, H., May, M. A., \& Maller, J. B. (1929). Studies in the nature of character: II Studies in service and self-control. New York, NY: Macmillan.

Hautmann, C., Eichelberger, I., Hanisch, C., Plück, J., Wlater, D., \& Döpfner, M. (2015). Association between parental emotional symptoms and child antisocial behaviour: What is specific and is it mediated by parenting? International Journal of Behavioral Development, $39,43-52$.

Havighurst, R. (1953). Human development and education. New York, NY: Longmans.

Heikkinen, R.-L. (2013). Sosiaalinen toimintakyky ja sen arviointikeinot [The assessment of social capacity]. Gerontologia, 27, 386-96.

Helson, R. (1997). The self in middle age. In M. E. Lachman \& J. B. James (Eds.), Multiple paths of midlife development (pp. 21-43). Chicago, IL: The University of Chicago Press.

Hibell, B., Andersson, B., Bjarnason, T., Kokkevi, A., Morgan, M., \& Narusk, A. (1977). The 1995 ESPAD Report: Alcohol and other drug use among students in 26 European countries. (The Swedish Council for Information on Alcohol and Other Drugs and the Pompidou Group at the Council of Europe). Stockholm, Sweden: Modin.

Hietalahti, M., Rantanen, J., \& Kokko, K. (2016). Do life goals mediate the link between personality traits and mental well-being? The Journal of Happiness $\mathcal{E}$ Well-Being, 4, 72-89.

Hietalahti, M., Tolvanen, A., \& Kokko, K. (2015). Values and personal life investment in middle-age: Measures and relations. Journal of Adult Development, 22, 206-20.

Hietalahti, M., Tolvanen, A., \& Kokko, K. (2016). Relationships between personality traits and values in middle-age. Manuscript in revision in Journal of Individual Differences.

Hirsjärvi, S., \& Hurme, H. (1980). Teemahaastattelu [Thematic interview]. Helsinki, Finland: Gaudeamus.

Hogan, D. P., \& Astone, N. M. (1986). The transition to adulthood. Annual Review of Sociology, 12, 109-30.

Hu, L. T., \& Bentler, P. M. (1999). Cutoff criteria for fit indices in covariance structure analysis: Conventional criteria versus new alternatives. Structural Equation Modeling, 6, 1-55.

Hurme, H. (1976). Käyttäytymisen itsekontrollin taustaedellytysten metodinen tarkastelu [A methodological analysis of the backgrounds of self-control of behavior]. (Reports from the Department of Psychology, No. 175). University of Jyväskylä, Finland.

Hurme, H. (2006). Grandparents as resource factors in the family. In L. Pulkkinen, J. Kaprio $\&$ R. J. Rose (Eds.), Socioemotional development and health from adolescence to adulthood (pp. 245-62). New York, NY: Cambridge University Press.

Hyde, J. S. (2014). Gender similarities and differences. Annual Review of Psychology, 65, 373-98.

Immonen, M., \& Kokko, K. (2008). Epänormatiivisten elämäntapahtumien ajoittuminen aikuisiässä ja yhteydet psyykkiseen hyvinvointiin [Timing of non-normative life events and mental well-being in adulthood]. Psykologia, 43, 4-17.

Izard, C., Stark, K., Trentacosta, C., Schultz, D. (2008). Beyond emotion regulation: Emotion utilization and adaptive functioning. Child Development Perspectives, 2, 156-63.

Jacques, E. (1965). Death and mid-life crisis. International Journal of Psychoanalysis, 46, 502-14. Jaffe, S. (2002). Pathways to adversity in young adulthood among early child bearers. Journal of Family Psychology, 16, 38-49.

Jahoda, M., Lazarsfeld, P. F., \& Zeisl, H. (1972). Marienthal: The sociography of an unemployed community. London, UK: Tavistock. (Original work published 1933.)

Jalonen, N., Kinnunen, M-L., Pulkkinen, L., \& Kokko, K. (2015). Job skill discretion and emotion control strategies as antecedents of recovery from work. European Journal of Work and Organizational Psychology, 24, 389-401. 
Jessor, R., Donovan, J. E., \& Costa, F. M. (1991). Beyond adolescence. Problem behavior and young adult development. New York, NY: Cambridge University Press.

Jessor, R., \& Jessor, S. L. (1977). Problem behavior and psychosocial development: A longitudinal study of youth. New York, NY: Academic Press.

Jokisaari, M. (2007). Attainment and reflection: The role of social capital and regrets in developmental regulation. (Jyväskylä Studies in Education, Psychology and Social Research, No. 346). Jyväskylä, Finland: Jyväskylä University Printing House.

Jones, H. E. (1938). The California Adolescent Growth Study. Journal of Education Research, $31,561-7$.

Junger-Tas, J., Terlouw, J.-G., \& Klein, M.W. (Eds.) (1994). Delinquent behavior among young people in the western world. First results of the International Self-Report Delinquency study. Amsterdam, Netherlands: Kluger Publications.

Juujärvi, P. (2003). A three-level analysis of reactive aggression among children. (Jyväskylä Studies in Education, Psychology and Social Research, No. 229). Jyväskylä, Finland: Jyväskylä University Printing House.

Juujärvi, P., Kooistra, L., Kaartinen, J., \& Pulkkinen, L. (2001). An aggression machine. V. Determinants in reactive aggression revisited. Aggressive Behavior, 27, 430-45.

Kagan, J. (1965). Reflection-impulsivity and reading ability in primary grade children. Child Development, 36, 609-28.

Kagan, J. (1980). Perspectives on continuity. In O. G. Brim Jr. \& J. Kagan (Eds.), Constancy and change in human development (pp. 26-74). Cambridge, MA: Harvard University Press.

Kagan, J. (1983). Developmental categories and the promise of connectivity. In R. M. Lerner (Ed.), Developmental psychology: Historical and philosophical perspectives (pp. 29-54). Hillsdale, N.J.: Erlbaum.

Kagan, J., \& Moss, H. A. (1962). Birth to maturity. New York, NY: Wiley.

Kahn, H. \& Wiener, A. J. (1967). The year 2000: A framework for speculation on the next thirtytree years. New York, NY: Macmillan.

Kananoja, A. (1983). Mitä on sosiaalinen toimintakyky? [What is social functioning?] Kuntoutus, 3, 3-10.

Kanfer, F. H., \& Karoly, R. (1972). Self-control: A behavioristic excursion into the lion's den. Behavior Therapy, 3, 398-416.

Kaplan, B. (1983). A trio of trials. In R. M. Lerner (Ed.), Developmental psychology: Historical and philosophical perspectives (pp. 185-227). Hillsdale, N.J.: Lawrence Erlbaum.

Karasek, R.A. (1979). Job demands, job decision latitude, and mental strain: Implications for job redesign. Administrative Science Quarterly, 24, 285-308.

Karasek, R. A. (1985). Job Content Questionnaire and user's guide. Lowell, MA: University of Massachusetts Lowell, Department of Work Environment.

Karasek, R. A., \& Theorell, T. (1990). Stress, productivity and reconstruction of working life. New York, NY: Basic Books.

Kendall, P. C., \& Wilcox, L. E. (1979). Self-control in children: Development of a rating scale. Journal of Consulting and Clinical Psychology, 47, 1020-9.

Keyes, C. L. M. (1998). Social well-being. Social Psychology Quarterly, 61, 121-40.

Keyes, C. L. M. (2002). The mental health continuum: From languishing to flourishing in life. Journal of Health and Social Research, 43, 207-22.

Keyes, C. L. M., \& Waterman, M. B. (2003). Dimensions of well-being and mental health in adulthood. In M. H. Bornstein, L. Davidson, C. L. M. Keyes, K. A. Moore \& The Center for Child Well-Being (Eds.), Well-being: Positive development across the life course (pp. 477-497). Mahwah, NJ: Lawrence Erlbaum Associates. 
Kickbush, I. (2012). Learning for well-being: A policy priority for children and youth in Europe: $A$ process for change. With the collaboration of J. Gordon and L. O'Toole, drafted on behalf of the Learning for Well-being Consortium of Foundations in Europe. Brussels, Belgium: Universal Education Foundation.

King, L. A., \& Emmons, R. A. (1990). Conflict over emotional expression: Psychological and physical correlates. Journal of Personality and Social Psychology, 58, 864-77.

Kinnunen, M.-L. (2010). Terveys [Health]. In L. Pulkkinen \& K. Kokko (Eds.), Keskiikä elämänvaiheena [Middle age as a stage of life]. (Reports from the Department of Psychology, No. 352, pp. 66-74). University of Jyväskylä, Finland. Available online at http://urn.fi/URN:ISBN:978-951-39-3894-9 (accessed 2 February 2017).

Kinnunen, U., \& Feldt, T. (2004). Economic stress and marital adjustment among couples. Analyses at the dyadic level. European Journal of Social Psychology, 34, 519-32.

Kinnunen, U., \& Pulkkinen, L. (1998). Linking economic stress to marital quality among Finnish marital couples: Mediator effects. Journal of Family Issues, 19, 705-24.

Kinnunen, U., \& Pulkkinen, L. (2001). Linking job characteristics to parenting behavior via job-related affect. In J. R. M. Gerris (Ed.), Dynamics of Parenting (pp. 233-49). Leuven, Belgium: Garant-Uitgevers.

Kinnunen, U., \& Pulkkinen, L. (2003). Childhood socioemotional characteristics as antecedents of marital stability and quality. European Psychologist, 8, 223-37.

Kinnunen, U., \& Rantanen, J. (2006). Parental work and children's behavior: The mediator roles of partner relationship and parenthood. In L. Pulkkinen, J. Kaprio \& R. J. Rose (Eds.), Socioemotional development and health from adolescence to adulthood (pp. 228-44). New York, NY: Cambridge University Press.

Kinnunen, M.-L., Kaprio, J., \& Pulkkinen, L. (2005). Allostatic load of men and women in early middle age. Journal of Individual Differences, 26, 20-8.

Kinnunen, M.-L., Kokkonen, M., Kaprio, J., \& Pulkkinen, L. (2005). The associations of emotion regulation and dysregulation with the metabolic syndrome factor. Journal of Psychosomatic Research, 58, 513-21.

Kinnunen, U., Feldt, T., Geurts, S., \& Pulkkinen, L. (2006). Types of work-family interface: Well-being correlates of negative and positive spillover between work and family. Scandinavian Journal of Psychology, 47, 149-62.

Kinnunen, M.-L., Feldt, T., Kinnunen, U., Kaprio, J., \& Pulkkinen, L. (2006). Association between long-term job strain and metabolic syndrome factor across sex and occupation. Journal of Individual Differences, 27, 151-61.

Kinnunen, M.-L., Pietiläinen, K., \& Rissanen, A. (2006). Body size and overweight from birth to adulthood. In L. Pulkkinen, J. Kaprio \& R. J. Rose (Eds.), Socioemotional development and health from adolescence to adulthood (pp. 95-107). New York, NY: Cambridge University Press.

Kinnunen, M.-L., Feldt, T., Kinnunen, U., \& Pulkkinen, L. (2008). Self-esteem: An antecedent or a consequence of social support and psychosomatic symptoms? Cross-lagged associations in adulthood. Journal of Research in Personality, 42, 333-47.

Kinnunen, M.-L., Metsäpelto, R. L., Feldt, T., Kokko, K., Tolvanen, A., Kinnunen, U., Leppänen, E., \& Pulkkinen, L. (2012). Personality profiles and health: Longitudinal evidence among Finnish adults. Scandinavian Journal of Psychology, 53, 512-22.

Kivimäki, M., Elovainio, M., Kokko, K., Pulkkinen, L., Kortteinen, M., \& Tuomikoski, H. (2003). Hostility, unemployment and health status: Testing three theoretical models. Social Science \& Medicine, 56, 2139-52.

Kivimäki, M., Kinnunen, M.-L., Pitkänen, T., Vahtera, J., Elovainio, M., \& Pulkkinen, L. (2004). Contribution of early and adult factors to socioeconomic variation in blood pressure: Thirty-four-year follow-up study of school children. Psychosomatic Medicine, 66, 184-9. 
Kochanska, G. (1990). Maternal beliefs as long-term predictors of mother-child interaction and report. Child Development, 61, 1934-1943.

Kokko, K. (2001). Antecedents and consequences of long-term unemployment. (Jyväskylä Studies in Education, Psychology and Social Research, No. 183). Jyväskylä, Finland: Jyväskylä University Printing House.

Kokko, K. (2006). Unemployment and psychological distress, and education as a resource factor for employment. In L. Pulkkinen, J. Kaprio \& R. J. Rose (Eds.), Socioemotional development and health from adolescence to adulthood (pp. 306-27). New York, NY: Cambridge University Press.

Kokko, K. (2010a). Keski-iän määrittelyä ja kuvailua [Definition and description of middleage]. In L. Pulkkinen \& K. Kokko (Eds.), Keski-ikä elämänvaiheena [Middle age as a stage of life]. (Reports from the Department of Psychology, No. 352, pp. 1-4). University of Jyväskylä, Finland. Available online at http://urn.fi/URN:ISBN:978-951-39-3894-9 (accessed 2 February 2017).

Kokko, K. (2010b). Psyykkinen hyvinvointi [Psychic well-being.] In L. Pulkkinen \& K. Kokko (Eds.), Keski-ikä elämänvaiheena [Middle age as a stage of life]. (Reports from the Department of Psychology, No. 352, pp. 91-6). University of Jyväskylä, Finland. Available online at http://urn.fi/URN:ISBN:978-951-39-3894-9 (accessed 2 February 2017).

Kokko, K. (2010c). Työ [Work]. In L. Pulkkinen \& K. Kokko (Eds.), Keski-ikä elämänvaiheena [Middle age as a stage of life]. (Reports from the Department of Psychology, No. 352, pp. 32-9). University of Jyväskylä, Finland. Available online at http://urn.fi/ URN:ISBN:978-951-39-3894-9 (accessed 2 February 2017).

Kokko, K., Bergman, L. R., \& Pulkkinen, L. (2003). Child personality characteristics and selection into long-term unemployment in Finnish and Swedish longitudinal samples. International Journal of Behavioral Development, 27, 134-44.

Kokko, K., Korkalainen, A., Lyyra, A.-L., \& Feldt, T. (2013). Structure and continuity of well-being in mid-adulthood: A longitudinal study. Journal of Happiness Study, 14, 99-114.

Kokko, K., \& Pulkkinen, L. (1998). Unemployment and psychological distress: Mediator effects. Journal of Adult Development, 5, 205-17.

Kokko, K., \& Pulkkinen, L. (2000). Aggression in childhood and long-term unemployment in adulthood: A cycle of maladaptation and some protective factors. Developmental Psychology, 36, 463-72.

Kokko, K., \& Pulkkinen, L. (2005). Stability of aggressive behavior from childhood to middle age in women and men. Aggressive Behavior, 31, 485-97.

Kokko, K., Pulkkinen, L., Huesmann, L. R., Dubow, E. F., \& Boxer, P. (2009). Intensity of aggression in childhood as a predictor of different forms of adult aggression: A twocountry (Finland and United States) analysis. Journal of Research on Adolescence, 19, 9-34.

Kokko, K., Pulkkinen, L., \& Mesiäinen, P. (2009). Timing of parenthood in relation to other life transitions and adult social functioning. International Journal of Behavioral Development, 33, 356-65.

Kokko, K., Pulkkinen, L., Mesiäinen, P., \& Lyyra, A.-L. (2008). Trajectories based on postcomprehensive and higher education: Their correlates and antecedents. Journal of Social Issues, 64, 59-76.

Kokko, K., Pulkkinen, L., \& Puustinen, M. (2000). Selection into long-term unemployment and its psychological consequences. International Journal of Behavioral Development, 24, 310-20.

Kokko, K., Rantanen, J., \& Pulkkinen, L. (2015). Associations between mental well-being and personality from a life-span perspective. In M. Blatný (Ed.), Personality and well-being across the life-span (pp. 134-59). New York, NY: Palgrave Macmillan. 
Kokko, K., \& Shehadeh, H. (in press). Aikuisuuden persoonallisuuden kehitys: sopeutumista vai kasvua? [Personality development in adulthood: Adjustment or growth?] In E. Kallio (Ed.), Ajattelun kehitys aikuisuudessa - kohti moninäkökulmaisuutta [Development of adult thinking - towards multiple perspectives]. Jyväskylä, Finland: Suomen kasvatustieteellisen seuran tutkimuksia.

Kokko, K., Simonton, S., Dubow, E. F., Lansford, J. E., Olson, S. L., Huesmann, L. R., Boxer, P., Pulkkinen, L., Bates, J. E., Dodge, K. A., \& Pettit, G. S. (2014). Country, sex, and parent occupational status: Moderators of the continuity of aggression from childhood to adulthood. Aggressive Behavior, 40, 553-67.

Kokko, K., Tolvanen, A., \& Pulkkinen, L. (2013). Associations between personality traits and psychological well-being across time in middle adulthood. Journal of Research in Personality, 47, 748-56.

Kokkonen, M., \& Kinnunen, M.-L. (2006). Emotion regulation and well-being. In L. Pulkkinen, J. Kaprio \& R. J. Rose (Eds.), Socioemotional development and health from adolescence to adulthood (pp. 197-208). New York, NY: Cambridge University Press.

Kokkonen, M., Kinnunen, T., \& Pulkkinen, L. (2002). Direct and indirect effects of adolescent self-control of emotions and behavioral expression on adult health outcomes. Psychology and Health, 17, 657-70.

Kontula, O., \& Koskela, K. (1991). Mustasukkaisuus ja terveys [Jealousy and health]. Helsinki, Finland: Sosiaali-ja terveyshallitus.

Kooistra, L., Tolvanen, A., Mäkiaho, A., \& Pulkkinen, L. (2001). Criminal offence and mental health problems in adulthood: Modelling the predictive relationship with inattentiveness. Unpublished manuscript.

Kopp, C. B. (1982). Antecedents of self-regulation: A developmental perspective. Developmental Psychology, 18, 199-214.

Kristenson, H., \& Trell, E. (1982). Indicators of alcohol consumption: Comparisons between a questionnaire (Mm-MAST), interviews and gammaglutamyl transferase (GGT) in a health survey of middle-aged males. British Journal of Addiction, 77, 297-304.

Kroger, J. (2000). Identity development: Adolescence through adulthood. Thousand Oaks, CA: Sage Publications.

Kroger, J., Martinussen, M., \& Marcia, J. E. (2010). Identity status change during adolescence and young adulthood: A meta-analysis. Journal of Adolescence, 33, 683-98.

Lachman, M. E., Teshale, S., \& Agrigoroaei, S. (2015). Midlife as a pivotal period in the life course: Balancing growth and decline at the crossroads of youth and old age. International Journal of Behavioral Development, 39, 20-31.

Lahtela, L., Rajala, A-M., Kokko, K., Räikkönen, E., \& Feldt, T. (2014). Katumusten yhteydet eheyteen ja hyvinvointiin keski-iässä [Regrets and their associations with ego integrity and well-being in midlife]. Psykologia, 49, 4-22.

Larsen, R. J., \& Prizmic, Z. (2004). Affect regulation. In R. F. Baumeister \& K. D. Vohs (Eds.), Handbook of self-regulation: Research theory, and application (pp. 40-61). New York, NY: Guilford Press.

Laursen, B., Little, T.D., \& Card, N. A. (Eds.) (2012). Handbook of developmental research methods. New York, NY: Guilford Press.

Laursen, B., Pulkkinen, L., \& Adams, R. (2002). The antecedents and correlates of agreeableness in adulthood. Developmental Psychology, 38, 591-603.

Lazarus, R. S. (1966). Psychological stress and the coping process. New York, NY: McGraw-Hill. Lazarus, R. S., \& Folkman, S. (1984). Stress, appraisal and coping. New York, NY: Springer.

Lehto, J. E., Juujärvi, P., Kooistra, L., \& Pulkkinen, L. (2003). Dimensions of executive functioning: evidence from children. British Journal of Developmental Psychology, 21, 59-80. 
Lehto, J. E., Pulkkinen, L., \& Juujärvi, P. (2002). Executive functioning and personality: Evidence for a common origin. Unpublished manuscript.

Lerner, R. M. (1983). The history of philosophy and the philosophy of history in developmental psychology: A view of the issues. In R. M. Lerner (Ed.), Developmental psychology: Historical and philosophical perspectives (pp. 3-26). Hillsdale, NJ.: Erlbaum.

Levinson, D. J. (1986). A conception of adult development. American Psychologist, 41, 3-13.

Levinson, D. J., Darrow, C. N., Klein, E. B., Levinson, M. H., \& McKee, B. (1978). The seasons on a man's life. New York, NY: Knopf.

Little, B. R. (1983). Personal projects: A rationale and method for investigation. Environment and Behavior, 15, 273-309.

Lloyd, B. H., \& Abidin, R. R. (1985). Revision of the Parent Stress Index. Journal of Pediatric Psychology, 10, 169-77.

Loeber, R. \& Dishion, T. (1983). Early predictors of male delinquency: A review. Psychological Bulletin, 94, 68-99.

Loeber, R., Slot, N.W., \& Southamer-Loeber, M. (2006). A three-dimensional, cumulative developmental model of serious delinquency. In P.-O. H. Wikström \& R. J. Sampson (Eds.), The explanation of crime: Context, mechanisms, and development (pp. 153-94). Cambridge, UK: Cambridge University Press.

Luria, A. R. (1961). The role of speech in the regulation of normal and abnormal behavior. New York, NY: Leveright.

Maccoby, E., \& Martin, J. A. (1983). Socialization in the context of the family. In E. M. Hetherington (Ed.), P. H. Mussen (Series Ed.), Handbook of child psychology. Vol. 4. Socialization, personality, and social development (pp. 1-101). New York, NY: Wiley.

Macfarlane, J. W. (1938). Studies in child guidance: I. Methodology of data collection and organization. Monographs of the Society for Research in Child Development, 3 (Whole No. 19).

Magnusson, D. (1998). The logic and implications of a person-oriented approach. In R. B. Cairns, L. R. Bergman \& J. Kagan (Eds.), Methods and models of studying the individual. (pp. 33-63). Thousand Oaks, CA: Sage.

Magnusson, D. (2000). The individual as the organizing principle in psychological inquiry: A holistic approach. In L. R. Bergman, R. B. Cairns, L.-G. Nilsson \& L. Nystedt (Eds.), Developmental science and the holistic approach (pp. 33-47). Mahwah, NJ: Erlbaum.

Magnusson, D., \& Bergman, L. R. (1988). Individual and variable-based approaches to longitudinal research on early risk factors. In M. Rutter (Ed.), Studies of Psychosocial Risk: The power of Longitudinal Data (pp. 45-61). New York, NY: Cambridge University Press.

Mahoney, J. L., Larson, R. W., \& Eccles, J. S. (2005). Organized activities as contexts of development. Mahwah, NJ: Erlbaum.

Main, M., \& Weston, D. R. (1981). The quality of the toddler's relationships to mother and father: Related to conflict behavior and the readiness to establish new relationships. Child Development, 52, 932-40.

Mäkikangas, A., Rantanen, J., Bakker, A. B., Kinnunen, M.-L., Pulkkinen, L., \& Kokko, K. (2015). The circumplex model of occupational well-being: Its relation with personality. Journal for Person-Oriented Research, 1, 115-29.

Männikkö, K. (2001). Adult attachment styles: A person-oriented approach. (Jyväskylä Studies in Education, Psychology and Social Research, No. 185). Jyväskylä, Finland: Jyväskylä University Printing House.

Männikkö, K., \& Pulkkinen, L. (2001). Parenting and personality styles. A long-term longitudinal approach. In J.R.M. Gerris (Ed.), Dynamics of Parenting (pp. 179-95). Leuven, Belgium: Garant-Uitgevers.

Marcia, J. E. (1966). Development and validation of ego-identity status. Journal of Personality and Social Psychology, 3, 551-8. 
Marcia, J. E. (1980). Identity in adolescence. In J. Adelson (Ed.), Handbook of adolescent psychology (pp. 159-87). New York, NY: Wiley.

Marcia, J. E. (2002). Identity and psychosocial development in adulthood. Identity: An International Journal of Theory and Research, 2, 7-28.

Marini, M. (1984). Age and sequencing norms in the transition to adulthood. Social Forces, 63, 229-44.

Mascaro, N., Rosen, D. H., \& Morey, L. C. (2004). The development, construct validity, and clinical utility of the spiritual meaning scale. Personality and Individual Differences, 37 , 845-60.

Maslach, C, \& Jackson, S. (1986). MBI: Maslach Burnout Inventory Manual (2nd ed.). Palo Alto, CA: Consulting Psychologists Press.

Maslow, A. (1954). Motivation and personality. New York, NY: Harper.

Masten A. A., \& Coatsworth, J. D. (1995). Competence, resilience, and psychopathology. In D. Cicchetti \& D. J. Cohen (Ed.), Developmental psychopathology. Vol. 2: Risk, disorder, and adaptation (pp. 715-52). New York, NY: Wiley.

Masten, A. A., Roisman, G. I., Long, J. D., Burt, K. B., Obradović, J., Riley, J. R., \& Tellegen, A. (2005). Developmental cascades: Linking academic achievement and externalizing and internalizing symptoms over 20 years. Developmental Psychology, 41, 733-46.

Matthes, M. (2013). Improving the quality of childhood in Europe: The social organisation of a grass root initiative and its strategy. In B. Heys, M. Matthes \& P. Sullivan (Eds.), Improving the quality of childhood in Europe 2013 (pp. 13-27). Forest Row, UK: ECSWE. Available online at http://allianceforchildhood.eu/publications (accessed 2 February 2017).

Maughan, B., \& Champion, L. (1990). Risk and protective factors in the transition to young adulthood. In P. B. Baltes \& M. Baltes (Eds.), Successful aging: Research and theory (pp. 296-331). Cambridge, UK: Cambridge University Press.

Mayer, J. D., \& Gaschke, Y. N. (1988). The experience and meta-experience of mood. Journal of Personality and Social Psychology, 55, 102-11.

Mayer, J. D., \& Salovey, P. (1997). What is emotional intelligence? In P. Salovey \& D. J. Sluter (Eds.), Emotional development and emotional intelligence: Educational implications (pp. 3-31). New York, NY: Basic Books.

Mayer, J. D., \& Stevens, A. A. (1994). An emerging understanding of the reflective (meta-) experience of mood. Journal of Research in Personality, 28, 351-73.

McAdams, D. P. (2015). Three lines of personality development: A conceptual itinerary. European Psychologist, 20, 252-64.

McAdams, D. P., \& Adler, J. M. (2006). How does personality develop? In D. K. Mroczek \& T. D. Little (Eds.), Handbook of personality development (pp. 469-92). Mahwah, NJ: Erlbaum.

McAdams, D. P., \& Olson, B. D. (2010). Personality development: Continuity and change over the life course. Annual Review of Psychology, 61, 517-42.

McAdams, D. P., \& Pals, J. L. (2006). A new Big Five: fundamental principles for an integrative science of personality. American Psychologist, 61, 204-17.

McClelland, D. C., \& Apicella, F. S. (1945). A functional classification of verbal reactions to experimentally induced failure. Journal of Abnormal and Social Psychology, 40, 376-90.

McCrae, R. R., \& Costa, P. T., Jr. (2003). Personality in adulthood: A five-factor theory perspective (2nd ed.). New York, NY: Guilford.

McEwen, B., \& Stellar, E. (2003). Stress and the individual: Mechanism leading to disease. Archives of Internal Medicine, 153, 2093-101.

Merrick, E. N. (1995). Adolescent childbearing as career "choice": Perspective from an ecological context. Journal of Counseling and Development, 73, 288-95. 
Metsäpelto, R.-L., \& Juujärvi, P. (2006). Parental knowledge and family atmosphere in relation to children's socioemotional behavior. In L. Pulkkinen, J. Kaprio \& R. J. Rose (Eds.), Socioemotional development and health from adolescence to adulthood (pp. 209-27). New York, NY: Cambridge University Press.

Metsäpelto, R.-L., Polet, J., Kokko, K., Rantanen, J., Kinnunen, M.-L., Pitkänen, T., Lyyra, A.-L., \& Pulkkinen, L. (2010). Tutkimuksen toteutus [The execution of the study]. In L. Pulkkinen \& K. Kokko (Eds.), Keski-ikä elämänvaiheena [Middle age as a stage of life]. (Reports from the Department of Psychology, No. 352, pp. 5-19). University of Jyväskylä, Finland. Available online at http://urn.fi/URN:ISBN:978-951-39-3894-9 (accessed 3 February 2017).

Metsäpelto, R.-L., \& Pulkkinen, L. (2003). Personality traits and parenting: Neuroticism, extraversion and openness to experience as discriminative factors. European Journal of Personality, 17, 59-78.

Metsäpelto, R.-L., \& Pulkkinen, L. (2005). The moderating effect of extraversion on the relation between self-reported and observed parenting. Journal of Applied Developmental Psychology, 26, 371-84.

Metsäpelto, R.-L., Pulkkinen, L., \& Poikkeus, A.-M. (2001). A search for parenting style: A cross-situational analysis of parental behavior. Genetic, Social and General Psychology Monographs, 127, 169-92.

Miller, N. E. (1959). Liberalization of basic S-R concepts: extension to conflict behavior, motivation, and social learning. In S. Koch (Ed.), Psychology: a study of a science (Vol. 2, pp. 196-292). New York, NY: McGraw-Hill.

Ministry of Education (2016). Education system in Finland. Available online at http://www. oph.fi/download/146428_Finnish_Education_in_a_Nutshell.pdf (accessed 18 July 2016).

Mischel, W. (1968). Personality and assessment. New York, NY: Wiley.

Mischel, W. (1974). Processes in delay of gratification. New York, NY: Academic Press.

Mischel, W., \& Ayduk, O. (2004). Willpower in a cognitive-affective processing system: The dynamics of delay of gratification. In R. F. Baumeister \& K. D. Vohs (Eds.), Handbook of self-regulation: Research theory, and application (pp. 99-129). New York, NY: Guilford Press.

Mitchell, V., \& Helson, R. (1990). Women's prime of life. Psychology of Women Quarterly, 14, 451-70.

Miyake, A., Friedman, N. P., Emerson, M.J., Witzki, A. H., Howerter,A., \& Wager, T. D. (2000). The unity and diversity of executive functions and their contributions to complex 'Frontal Lobe' tasks: A latent variable analysis. Cognitive Psychology, 41, 49-100.

Moffitt, T. E. (1993). "Life-Course-Persistent" and "Adolescence-Limited" antisocial behavior: A developmental taxonomy. Psychological Review, 100, 674-701.

Moffitt, T. E., Caspi, A., Dickson, N., Silva, P. A., \& Stanton, W. (1996). Childhood-onset versus adolescent-onset antisocial conduct in males: Natural history from age 3 to 18 . Developmental Psychopathology, 8, 399-424.

Moffitt, T. E, Caspi, A., Harrington, H., \& Milne, B. J. (2002). Males on the life-coursepersistent and adolescence-limited antisocial pathways: Follow-up at age 26 years. Developmental Psychopathology, 14, 179-207.

Moffitt, T. E., Caspi, A., Rutter, M., \& Silva, P. A. (2001). Sex differences in antisocial behavior: Conduct disorder, delinquency, and violence in the Dunedin Longitudinal Study. Cambridge, UK: Cambridge University Press.

Morris, W., \& Reilly, N. (1987). Toward the self-regulation of mood: Theory and research. Motivation and Emotion, 11, 215-49. 
Mueller, M., \& Elder, G. H. Jr. (2003). Family contingencies across generations: Grandparent-grandchild relationships in holistic perspective. Journal of Marriage and Family, 65, 404-17.

Murray. H. A. (1938). Explorations in personality. New York, NY: Oxford University Press.

Mussen, P. H., \& Eisenberg-Berg, N. (1977). Roots of caring, sharing, and helping: The development of prosocial behavior in children. San Francisco. CA: Freeman.

Mustonen, A. (1997). Media violence and its audience. (Jyväskylä Studies in Education, Psychology and Social Research, No 135). Jyväskylä, Finland: Jyväskylä University Printing House.

Muthén, L. K., \& Muthén, B. O. (1998-2015). Mplus User's Guide (7th ed.). Los Angeles, CA: Muthén \& Muthén.

Nagin, D. S. (1999). Analyzing developmental trajectories: A semi-parametric, group-based approach. Psychological Methods, 4, 139-57.

Nagin, D. S. (2005). Group-based modeling of development. Cambridge, MA: Harvard University Press.

National Institute for Health and Welfare (2017). Available online at http://www.thl. fi/attachments/kouluterveyskysely/Tulokset/ktkysely_kokomaa_2004_2013_pk.pdf and http://www.thl.fi/attachments/kouluterveyskysely/Tulokset/ktkysely_kokomaa_2006_ 2015_pk.pdf (accessed 8 February 2017).

Neufeld, G., \& Maté, G. (2004). Hold on to your kids: Why parents need to matter more than peers. Toronto: Vintage Canada.

Neugarten, B. L., Moore, J. W., \& Lowe, J. C. (1965). Age norms, age constraints, and adult socialization. The American Journal of Sociology, 70, 710-17.

Nikander, T. (1998). Fertility and family surveys in countries of the ECE region: Standard country report, Finland. New York, NY: United Nations.

Nurmi, J.-E. (1989). Adolescents' orientation to the future: development of interests and plans, and related attributions and affects, in the life-span context. (Commentationes Scientiarum Socialium, No. 39). Helsinki, Finland: Societas Scientiarum Fennica.

Nurmi, J.-E., Salmela-Aro, K., \& Haavisto, T. (1995). The strategy and attribution questionnaire: Psychometric properties. European Journal of Psychological Assessment, 11, 108-21.

Olson, W. C. (1949). Child development. Boston: D. C. Heath and Company.

Olweus, D. (1969). Prediction of aggression. (On the basis of a projective test.) Stockholm, Sweden: Scandinavian Test Corporation.

Olweus, D. (1979). Stability of aggressive reaction patterns in males: a review. Psychological Bulletin, 86, 852-75.

OPH (2015). Perusopetuksen opetussuunnitelman perusteet 2014. [National core curriculum for basic education 2014.] Opetushallitus. Määräykset ja ohjeet [Finnish National Board of Education] 2014:96. Tampere, Finland: Juvenes Print.

Orth, U., Trzesniewski, K. H., \& Robin, R. W. (2010). Self-esteem development from young adulthood to old age: A cohort-sequential longitudinal study. Journal of Personality and Social Psychology, 9, 645-58.

Perho, H., \& Korhonen, M. (1993). Elämänvaiheiden onnellisuus ja sisältö keski-iän kynnyksellä. [Happiness of life stages in front of middle age.] Gerontologia, 7, 271-85.

Perttula, J. (1998). The experienced life-fabrics of young men. (Jyväskylä Studies in Education, Psychology and Social Research, No. 136). Jyväskylä, Finland: Jyväskylä University Printing House.

Peterson, C., \& Seligman, M. E. P. (2004). Character strengths and virtues: A handbook and classification. New York, NY: Oxford University Press.

Piquero, A. R., Moffitt, T. E., \& Wright, B. E. (2007). Self-control and criminal career dimensions. Journal of Contemporary Criminal Justice, 23, 72-89. 
Pitkänen, L. (1963a). Kyselylomake-, lauseentäydennys-ja Rosenzweig-tyyppisten testien käytöstä lasten persoonallisuuden piirteiden mittaamisessa. [Inventories, sentence completions and Rosenzweig-test as measures of children's personality characteristics.] (Reports from the Department of Psychology, No. 59). University of Jyväskylä, Finland.

Pitkänen, L. (1963b). The effect of spaced vs. massed presentation of aggression items on verbal aggressive responses of children. Scandinavian Journal of Psychology, 4, 55-64.

Pitkänen, L. (1966). Havaittavan aggression monidimensionaalisuudesta [Multidimensionality of observable aggression.] (Licenciate thesis, Department of Psychology.) University of Jyväskylä, Finland.

Pitkänen, L. (1968). Aggressiivisuuspiirre käyttäytymisen kuvauksen kaksiulotteisessa viitekehyksessä [Aggression within the two-dimensional framework of behavior.] (Reports from the Department of Psychology, No. 84). University of Jyväskylä, Finland.

Pitkänen, L. (1969). A descriptive model of aggression and nonaggression with applications to children's behaviour. (Jyväskylä Studies in Education, Psychology and Social Research, No. 19). Jyväskylä, Finland: University of Jyväskylä. Available online at http://users.jyu. fi/ leapulkk/dissertation.html (accessed 3 February 2017).

Pitkänen, L. (1973a). An aggression machine: I The intensity of aggressive defence aroused by aggressive offence. Scandinavian Journal of Psychology, 14, 56-64.

Pitkänen, L. (1973b). An aggression machine: II. Interindividual differences in the aggressive defence responses aroused by varying stimulus conditions. Scandinavian Journal of Psychology, 14, 65-74.

Pitkänen, L. (1973c). An aggression machine: III. The stability of aggressive and nonaggressive patterns of behaviour. Scandinavian Journal of Psychology, 14, 75-77.

Pitkänen, T. (2006). Alcohol drinking behavior and its developmental antecedents. (Jyväskylä Studies in Education, Psychology and Social Research, No. 293). Jyväskylä, Finland: Jyväskylä University Printing House.

Pitkänen, T. (2010). Päihteiden käyttö aikuisiässä [Substance use in adulthood]. In L. Pulkkinen \& K. Kokko (Eds.), Keski-ikä elämänvaiheena [Middle age as a stage of life]. (Reports from the Department of Psychology, No. 352, pp. 56-65). University of Jyväskylä, Finland. Available online at http://urn.fi/URN:ISBN:978-951-39-3894-9 (accessed 3 February 2017).

Pitkänen, T., Kokko, K., Lyyra, A.-L., \& Pulkkinen, L. (2008). A developmental approach to alcohol drinking behaviour in adulthood: A follow-up study from age 8 to age 42 . Addiction, 103, 48-68.

Pitkänen, T., Lyyra, A.-L., \& Pulkkinen, L. (2005). Age of onset of drinking and the use of alcohol in adulthood: a follow-up study from age 8-42 for females and males. Addiction, 100, 652-61.

Pitkänen-Pulkkinen, L. (1977). Effects of simulation programmes on the development of self-control. In C.F.M. van Lieshout \& D.J. Ingram (Eds.), Stimulation of social development in school (pp. 176-90). Amsterdam, Netherlands: Swets \& Zeitlinger.

Pitkänen-Pulkkinen, L. (1980a). The child in the family. Nordisk Psykologi, 32, 147-57.

Pitkänen-Pulkkinen, L. (1980b). An aggression machine. IV: Concurrent and predictive validity over ten years. Scandinavian Journal of Psychology, 21, 275-81.

Pitkänen-Pulkkinen, L. (1981a). Concurrent and predictive validity of self-reported aggressiveness. Aggressive Behavior, 7, 97-110.

Pitkänen-Pulkkinen, L. (1981b). Long-term studies on the characteristics of aggressive and nonaggressive juveniles. In P.F. Brain \& D. Benton (Eds.), Multidisciplinary approaches to aggression research (pp. 225-43). Amsterdam, Netherlands: Elsevier/North-Holland Biomedical Press.

Pulkkinen, L. (1977). Kotikasvatuksen psykologia [Psychology of upbringing.] Jyväskylä, Finland: Gummerus. 
Pulkkinen, L. (1982). Self-control and continuity from childhood to adolescence. In B. P. Baltes \& O.G. Brim, Jr. (Eds.), Life-span development and behavior (Vol. 4, pp. 63-105). New York, NY: Academic Press.

Pulkkinen, L. (1983a). The search for alternatives to aggression. In A. P. Goldstein \& M. Segall (Eds.), Aggression in global perspective (pp. 104-44). New York, NY: Pergamon Press.

Pulkkinen, L. (1983b). Youthful smoking and drinking in a longitudinal perspective. Journal of Youth and Adolescence, 12, 253-83.

Pulkkinen, L. (1984). Nuoret ja kotikasvatus [Youth and upbringing]. Helsinki, Finland: Otava.

Pulkkinen, L. (1985). Continuity and discontinuity in personality development and the problem of age stages. In C. J. Brainers \& V. F. Reyna (Eds.), Developmental psychology (pp. 427-37). Amsterdam, Netherlands: Elsevier/North Holland.

Pulkkinen, L. (1986). The role of impulse control in the development of antisocial and prosocial behavior. In D. Olweus, J. Block \& M. Radke-Yarrow (Eds.), Development of antisocial and prosocial behavior: Theories, research, and issues (pp. 149-75). Orlando, FL: Academic Press.

Pulkkinen, L. (1987). Offensive and defensive aggression in humans: A longitudinal perspective. Aggressive Behavior, 13, 197-212.

Pulkkinen, L. (1988). Delinquent development: theoretical and empirical considerations. In M. Rutter (Ed.), The power of longitudinal data: Studies of risk and protective factors for psychosocial disorders (pp. 184-99). Cambridge, UK: Cambridge University Press.

Pulkkinen, L. (1989a). Predictability of the length of educational routes and the development of career lines. Scandinavian Journal of Educational Research, 33, 203-14.

Pulkkinen, L. (1989b). Transition to adulthood. In M. A. Luszcz \& T. Nettlebeck (Eds.), Psychological development: Perspectives across the life-span (pp. 321-33). Amsterdam, Netherlands: Elsevier, North-Holland Science Publishers, and the presentation given in the XXIV International Congress of Psychology, Sydney, Australia, 1988.

Pulkkinen, L. (1990a). Adult life-styles and their precursors in social behaviour of children and adolescents. European Journal of Personality, 4, 237-51.

Pulkkinen, L. (1990b). Home atmosphere and adolescent future orientation. European Journal of Psychology of Education, 5, 33-43.

Pulkkinen, L. (1990c). Young adults' health and its antecedents in evolving life-styles. In K. Hurrelmann \& F. Lösel (Eds.), Health hazards in adolescence (pp. 67-90). Berlin, Germany: de Gruyter.

Pulkkinen, L. (1992a). Life-styles in personality development. European Journal of Personality, 6, 139-55.

Pulkkinen, L. (1992b). The path to adulthood for aggressively inclined girls. In K. Björkqvist \& P. Niemelä (Eds.), Of mice and women: Aspects of female aggression (pp. 113-21). San Diego, CA: Academic Press.

Pulkkinen, L. (1995). Behavioral precursors to accidents and resulting physical impairment. Child Development, 66, 1660-79.

Pulkkinen, L. (1996a). Female and male personality styles: A typological and developmental analysis. Journal of Personality and Social Psychology, 70, 1288-306.

Pulkkinen, L. (1996b). Proactive and reactive aggression in early adolescence as precursors to anti- and prosocial behavior in young adults. Aggressive Behavior, 22, 241-57.

Pulkkinen, L. (1998). Levels of longitudinal data differing in complexity and the study of continuity in personality characteristics. In R. B. Cairns, L. R. Bergman \& J. Kagan (Eds.), Methods and models for studying the individual (pp. 161-84). Beverly Hills, CA: Sage.

Pulkkinen, L. (2000). Developmental psychology II: Adulthood and aging. In K. Pawlik \& M. R. Rosenzweig (Eds.), The International Handbook of Psychology (pp. 261-82). London, UK: SAGE. 
Pulkkinen, L. (2001). Antisocial behavior in childhood and adolescence. In N. Smelser \& P. Baltes (Eds.), International Encyclopedia of the Social and Behavioral Sciences (Vol. 1, pp. 549-53). Oxford, UK: Pergamon.

Pulkkinen, L. (2002). Social development and its risk factors. In C. von Hofsten \& L. Bäckman (Eds.), Psychology at the Turn of the Millennium, Vol. 2: Social, Developmental, and Clinical Perspectives (pp. 53-76). East Sussex, UK: Psychology Press.

Pulkkinen, L. (2004). A longitudinal study on social development as an impetus for school reform towards an integrated school day. European Psychologist, 9, 125-41.

Pulkkinen, L. (2006). The Jyväskylä Longitudinal Study of Personality and Social Development (JYLS). In L. Pulkkinen, J. Kaprio \& R. J. Rose (Eds.), Socioemotional development and health from adolescence to adulthood (pp. 29-55). New York, NY: Cambridge University Press.

Pulkkinen, L. (2009). Personality - a resource or risk for successful development. Scandinavian Journal of Psychology, 50, 602-10.

Pulkkinen, L. (2010a). Miltä keski-ikä näyttää? [What does middle-age look like?] In L. Pulkkinen \& K. Kokko (Eds.), Keski-ikä elämänvaiheena [Middle age as a stage of life.] (Reports from the Department of Psychology, No. 352, pp. 97-106). University of Jyväskylä, Finland. Available online at http://urn.fi/URN:ISBN:978-951-39-3894-9 (accessed 3 February 2017).

Pulkkinen, L. (2010b). Perhe [Family]. In L. Pulkkinen \& K. Kokko (Eds.), Keski-ikä elämänvaiheena [Middle age as a stage of life]. (Reports from the Department of Psychology, No. 352, pp. 26-31). University of Jyväskylä, Finland. Available online at http://urn.fi/ URN:ISBN:978-951-39-3894-9 (accessed 3 February 2017).

Pulkkinen, L. (2012a). Family factors in the development of antisocial behavior. In T. Bliesener, A. Beelman \& M. Stemmler (Eds.), Antisocial behavior and crime: Contributions of developmental and evaluation research to prevention and intervention (pp. 89-108). Göttingen, Germany: Hogrefe.

Pulkkinen, L. (2012b). The integrated school day: Improving the educational offering of schools in Finland. In C. Clouder, B. Heys, M. Matthes, \& P. Sullivan (Eds.), Improving the quality of childhood in Europe 2012 (pp. 40-67). Forest Row, UK: ECSWE. Available online at http://allianceforchildhood.eu/publications (accessed 3 February 2017).

Pulkkinen, L. (2014a). Positive human development: A spontaneous or guided process? Policy lessons from 45 years of longitudinal research in Finland. In M. Matthes, L. Pulkkinen, L. M. Pinto \& C. Clouder (Eds.), Improving the quality of childhood in Europe 2014 (Vol. 5, pp. 58-73). Brussels, Belgium: Alliance for Childhood European Network Foundation. Available online at http://allianceforchildhood.eu/publications (accessed 3 February 2017).

Pulkkinen, L. (2014b). Self-control at the heart of successful development. In R. M. Lerner, A. C. Petersen, R. K. Silbereisen \& J. Brooks-Gunn (Eds.), The developmental science of adolescence: History through autobiography (pp. 373-85). New York, NY: Psychology Press Taylor \& Francis Group.

Pulkkinen, L. (2015). Individual differences that should be considered in the prevention of juvenile delinquency. In A. Sözüer (Ed.), Juvenile justice: Academic papers (Part 2, pp. 1443-61). Ankara, Turkey: Adalet.

Pulkkinen, L. (2016). An inspiring school day: An effort to transform research findings into policy. In M. Matthes, L. Pulkkinen, B. Heys, C. Clouder \& L. M. Pinto (Eds.), Improving the quality of childhood in Europe (Vol. 6, pp. 52-69). Brussels, Belgium: Alliance for Childhood European Network Foundation. Available online at http://alliancefor childhood.eu/publications (accessed 3 February 2017).

Pulkkinen, L. (2017, in press). Longitudinal Study of Personality and Social Development: Insights about aggression after 5 decades. In A. T. Vazsonyi, D. J. Flannery, \& M. DeLisi 
(Eds.), The Cambridge Handbook of Violent Behavior and Aggression (2nd ed.). Cambridge, UK: Cambridge University Press.

Pulkkinen, L., \& Caspi, A. (2002). Personality and paths to successful development: An overview. In L. Pulkkinen \& A. Caspi (Eds.), Paths to successful development (pp. 1-16). Cambridge, UK: Cambridge University Press.

Pulkkinen, L., Feldt, T., \& Kokko, K. (2005). Personality in young adulthood and functioning in middle age. In S. Willis \& M. Martin (Eds.), Middle Adulthood: A lifespan perspective (pp. 99-141). Thousand Oaks, CA: Sage.

Pulkkinen, L., Feldt, T., \& Kokko, K. (2006). Adaptive behavior in childhood as an antecedent of psychological functioning in early middle age: Linkage via career orientation. Social Indicators Research, 77, 171-95.

Pulkkinen, L., Fyrstén, S., Kinnunen, U., Kinnunen, M-L., Pitkänen, T., \& Kokko, K. (2003). 40+ Erään ikäluokan selviytymistarina [40+ A successful transition to middle adulthood in a cohort of Finns.] (Reports from the Department of Psychology, No. 349). University of Jyväskylä, Finland.

Pulkkinen, L., \& Hämäläinen, M. (1995). Low self-control as a precursor to crime and accidents in a Finnish longitudinal study. Criminal Behaviour and Mental Health, 5, 424-38.

Pulkkinen, L., Heikkinen, A., Markkanen, T., \& Ranta, M. (1977). Näin ohjaan lastani: Lasten itsehallinnan harjoitusohjelma [Guiding my child: A program for developing selfcontrol in children]. Jyväskylä, Finland: Gummerus.

Pulkkinen, L., \& Hurme, H. (1984). Aggression as a predictor of weak self-control. In L. Pulkkinen \& P. Lyytinen (Eds.), Human action and personality: Essays in honour of Martti Takala (pp. 172-89). (Jyväskylä Studies in Education, Psychology and Social Research, No. 54). University of Jyväskylä, Finland.

Pulkkinen, L., \& Kallio, E. (1988). Nuoren aikuisen suhde tupakointiin [Smoking in young adulthood.] (National Board of Health, Report No. 7). Helsinki, Finland: Valtion painatuskeskus.

Pulkkinen, L., Kaprio, J., \& Rose, R. J. (1999). Peers, teachers, and parents as assessors of the behavioural and emotional problems of twins and adjustment: The Multidimensional Peer Nomination Inventory. Twin Research, 2, 274-85.

Pulkkinen, L., \& Kokko, K. (2000). Identity development in adulthood: A longitudinal study. Journal of Research in Personality, 34, 445-70.

Pulkkinen, L., \& Kokko, K. (2012). Foundational issues in longitudinal data collection. In B. Laursen, T. D. Little, \& N. A. Card (Eds.), Handbook of developmental research methods (pp. 129-47). New York, NY: Guilford Press.

Pulkkinen, L., \& Kokko, K. (2015). Polarization of individual differences in well-being. A paper presented at the14th European Congress of Psychology, Milan, July, 2015.

Pulkkinen, L., \& Kokko, K. (2017). The Jyväskylä Longitudinal Study of Personality and Social Development: Measures used for the study of major topics in different data waves. Finnish Social Science Data Archive, Tampere, Finland.

Pulkkinen, L., Kokko, K., \& Rantanen, J. (2012). Paths from socioemotional behavior in middle childhood to personality in middle adulthood. Developmental Psychology, 48, 1283-91.

Pulkkinen, L., Lyyra, A.-L., \& Kokko, K. (2009). Life success of males on nonoffender, adolescence-limited, persistent and adult-onset antisocial pathways: Follow-up from age 8 to 42. Aggressive Behavior, 35, 117-35.

Pulkkinen, L., Lyyra, A.-L., \& Kokko, K. (2011). Is social capital a mediator between selfcontrol and psychological and social functioning across 34 years? International Journal of Behavioral Development, 35, 475-81. 
Pulkkinen, L., Männikkö, K., \& Nurmi, J.-E. (2000). Self-description and personality styles. In L. R. Bergman, R. B. Cairns, L.-G. Nilsson \& L. Nystedt (Eds.), Developmental science and the holistic approach (pp. 265-80). Mahwah, NJ: Erlbaum.

Pulkkinen, L., Nurmi, J.-E., \& Kokko, K. (2002). Individual differences in personal goals in mid-thirties. In L. Pulkkinen \& A. Caspi (Eds.), Paths to successful development: personality in the life course (pp. 331-52). Cambridge, UK: Cambridge University Press.

Pulkkinen, L., Nygren, H., \& Kokko, K. (2002). Successful development: Childhood antecedents of adaptive psychosocial functioning in adulthood. Journal of Adult Development, 9, 251-65.

Pulkkinen, L., Ohranen, M., \& Tolvanen, A. (1999). Personality antecedents of career orientation and stability among women compared to men. Journal of Vocational Behavior, 54, 37-58.

Pulkkinen, L., \& Pitkänen, T. (1993). Continuities in aggressive behavior from childhood to adulthood. Aggressive Behavior, 19, 249-63.

Pulkkinen, L., \& Pitkänen, T. (1994). A prospective study of the precursors to problem drinking in young adulthood. Journal of Studies on Alcohol, 55, 578-87.

Pulkkinen, L., \& Pitkänen, T. (2010). Temperance and the strengths of personality: Evidence from a 35-year longitudinal study. In R. Schwarzer \& P. A. Frensch (Eds.), Personality, human development, and culture: International perspectives on psychological science (Vol. 2, pp. 127-40). Hove, UK: Psychology Press.

Pulkkinen, L., \& Polet, J. (2010a). Koulutus, toimeentulo ja asuminen [Education, livelihood, and habitation]. In L. Pulkkinen \& K. Kokko (Eds.). Keski-ikä elämänvaiheena [Middle age as a stage of life]. (Reports from the Department of Psychology, No. 352, pp. 20-5). University of Jyväskylä, Finland. Available online at http://urn.fi/URN:ISBN:978-951-39-3894-9 (accessed 3 February 2017).

Pulkkinen, L., \& Polet, J. (2010b). Tyydytystä ja huolta aiheuttavat asiat elämässä [Sources of satisfactions and worries in life]. In L. Pulkkinen \& K. Kokko (Eds.), Keski-ikä elämänvaiheena [Middle age as a stage of life.] (Reports from the Department of Psychology No. 352, pp. 75-90). University of Jyväskylä, Finland. Available online at http://urn.fi/ URN:ISBN:978-951-39-3894-9 (accessed 3 February 2017).

Pulkkinen, L., \& Polet, J. (2010c). Vapaa-aika [Free time]. In L. Pulkkinen \& K. Kokko (Eds.), Keski-ikä elämänvaiheena [Middle age as a stage of life]. (Reports from the Department of Psychology, No. 352, pp. 49-55). University of Jyväskylä, Finland. Available online at http://urn.fi/URN:ISBN:978-951-39-3894-9 (accessed 3 February 2017).

Pulkkinen, L., Räikkönen, E., Kinnunen, M.-L., \& Kokko, K. (2013). A new model for the relations between longitudinal personality profiles and psychological functioning through middle age. A paper presented in the Conference of the International Society for the Study of Individual Differences. Barcelona, July 23-25, 2013.

Pulkkinen, L., \& Rönkä, A. (1994). Personal control over development, identity formation, and future orientation as components of life orientation: A developmental approach. Developmental Psychology, 30, 260-71.

Pulkkinen, L., Savioja, K., Juujärvi, P., Kokkonen, M., \& Metsäpelto, R.-L. (2000). Emootion ja käyttäytymisen sä̈telyn tutkimus [A study of emotion and behavior regulation]. (Reports from the Department of Psychology, No. 344). University of Jyväskylä, Finland.

Pulkkinen, L., \& Tremblay, R. E. (1992). Patterns of boys' social adjustment in two cultures and at different ages: A longitudinal perspective. International Journal of Behavioural Development, 15, 527-53.

Pulkkinen, L., Virtanen, T., af Klinteberg, B., \& Magnusson, D. (2000). Child behaviour and adult personality: Comparisons between criminality groups in Finland and Sweden. Criminal Behaviour and Mental Health, 10, 155-69. 
Pulver, A., Allik, J., Pulkkinen, L., \& Hämäläinen, M. (1995). A Big Five Personality Inventory in two non-Indo-European languages. European Journal of Personality, 9, 109-24.

Puohiniemi, M. (1995). Values, consumer attitudes and behaviour: An application of Schwartz's value theory to the analysis of consumer behaviour and attitudes in two national samples. (Department of Social Psychology, Research Report No. 5). University of Helsinki, Finland.

Puustinen, M., Kokkonen, M., Tolvanen, A., \& Pulkkinen, L. (2004). Children's help seeking and impulsivity. Learning and Individual Differences, 14, 231-46.

Quinton, D., \& Rutter, M. (1988). Parenting breakdown: The making and breaking of intergenerational links. (Studies in Deprivation and Disadvantage No. 14). Aldershot, UK: Avebury.

Räikkönen, E., Kokko, K., \& Rantanen, J. (2011). Timing of adult transitions: Antecedents and implications for psychological functioning. European Psychologist, 16, 314-24.

Räikkönen, E., Kokko, K., Chen, M., \& Pulkkinen, L. (2012). Patterns of adult roles, their antecedents and psychosocial wellbeing correlates among Finns born in 1959. Longitudinal and Life Course Studies, 3, 211-27.

Rantanen, J., \& Räikkönen, E. (2010). Työn laadulliset piirteet, hyvinvointi työssä sekä työn ja perheen yhteen sovittaminen [The quality of work, well-being at work, and work-family interface]. In L. Pulkkinen \& K. Kokko (Eds.), Keski-ikä elämänvaiheena [Middle age as a stage of life]. (Reports from the Department of Psychology, No. 352, pp. 40-8). University of Jyväskylä, Finland. Available online at http://urn.fi/URN:ISBN:978-951-39-3894-9 (accessed 3 February 2017).

Rantanen, J., Feldt, T., Hakanen, J. J., Kokko, K., Huhtala, M., Pulkkinen, L., \& Schaufeli, W. (2015). Cross-national and longitudinal investigation of a short measure of workaholism. Industrial Health, 53, 113-23.

Rantanen, J., Kinnunen, U., Feldt, T., \& Pulkkinen, L. (2008). The work-family conflict and psychological well-being: Stability and cross-lagged relations within one- and sixyear follow-ups. Journal of Vocational Behavior, 73, 37-51.

Rantanen, J., Kinnunen, U., \& Pulkkinen, L. (2013). The role of personality and role engagement in work-family balance. Horizons of Psychology, 22, 14-26.

Rantanen, J., Kinnunen, U., Pulkkinen, L., \& Kokko, K. (2012). Developmental trajectories of work-family conflict for Finnish workers in midlife. Journal of Occupational and Health Psychology, 17, 290-303.

Rantanen, J., Metsäpelto, R-L, Feldt, T., Pulkkinen, L., \& Kokko, K. (2007). Long-term stability in the Big Five personality traits in adulthood. Scandinavian Journal of Psychology, 48, 511-18.

Rantanen, J., Pulkkinen, L., \& Kinnunen, U. (2005). The Big Five personality dimensions, work-family conflict, and psychological distress: A longitudinal view. Journal of Individual Differences, 26, 155-66.

Rantanen, J., Tillemann, K., Metsäpelto, R-L., Kokko, K., \& Pulkkinen, L. (2015). Longitudinal study on reciprocity between personality traits and parenting stress. International Journal of Behavioral Development, 39, 65-76.

Rauhala, L. (1983). Ihmiskäsitys ihmistyössä. [Conception of man in the work with humans.] Helsinki, Finland: Gaudeamus.

Rauhala, U. (1966). Suomalaisen yhteiskunnan sosiaalinen kerrostuneisuus. [Social structure of Finnish society]. Porvoo, Finland: WSOY.

Read, S., \& Suutama, T. (2008). Elämän tarkoituksellisuuden muutokset iäkkäillä ihmisillä 16 vuoden pitkittäistutkimuksessa. [Changes in meaning in life among older persons in a 16-year longitudinal study]. Gerontologia, 22, 130-9.

Rimpelä, M. (1980). Tupakoinnin alkaminen [Onset of smoking among Finnish youth]. (Kansanterveystieteen julkaisuja, No. M56). University of Tampere, Finland. 
Roberts, B. W., Walton, K. E., \& Viechtbauer, W. (2006). Patterns of mean-level change in personality traits across the life course: A meta-analysis of longitudinal studies. Psychological Bulletin, 132, 1-25.

Roberts, G. C., Block, J. H., \& Block, J. (1984). Continuity and change in parents' childrearing practices. Child Development, 55, 586-97.

Roger, D., \& Nesshoeven, W. (1987). The construction and preliminary validation of a scale for measuring emotional control. Personality and Individual Differences, 8, 527-34.

Rokka, P. \& Sundelin, A. (1993). Nuorten naisten elämän orientaatio Straussin lähestymistavalla tarkasteltuna [Life orientation of young women as analyzed with the Grounded Theory by Strauss]. (Master's thesis, Department of Psychology.) University of Jyväskylä, Finland.

Romanov, K., Hatakka, M., Keskinen, E., Laaksonen, H., Kaprio, J., Rose, R. J. \& Koskenvuo, M. (1994). Self-reported hostility and suicidal acts, accidents, and accidental deaths: A prospective study of 21,443 adults aged 25 to 59. Psychosomatic Medicine, 56, 328-36.

Rönkä, A. (1999). The accumulation of problems of social functioning: Outer, inner, and behavioral strands. (Jyväskylä Studies in Education, Psychology and Social Research, No. 148). Jyväskylä, Finland: Jyväskylä University Printing House.

Rönkä, A., \& Pulkkinen, L. (1995). Accumulation of problems in social functioning in young adulthood: A developmental approach. Journal of Personality and Social Psychology, 69, 381-91.

Rönkä, A., \& Pulkkinen, L. (1998). Work involvement and timing of motherhood in the accumulation of problems in social functioning in young women. Journal of Research on Adolescence, 8, 221-39.

Rönkä, A., Kinnunen, U., \& Pulkkinen, L. (2000). The accumulation of problems of social functioning as a long-term process: women and men compared. International Journal of Behavioral Development, 24, 442-50.

Rönkä, A., Kinnunen, U., \& Pulkkinen, L. (2001). Continuity in problems of social functioning in adulthood: a cumulative perspective. Journal of Adult Development, 8, 161-71.

Rönkä, A., Oravala, S., \& Pulkkinen, L. (2002). I met this wife of mine and things got onto a better track. Journal of Adolescence, 25, 47-63.

Rönkä, A., Oravala, S., \& Pulkkinen, L. (2003). Turning points in adult's lives: The effects of gender and the amount of choice. Journal of Adult Development, 10, 203-15.

Rose, R. J. (2006a). Introduction. In L. Pulkkinen, J. Kaprio \& R. J. Rose (Eds.), Socioemotional development and health from adolescence to adulthood (pp. 1-25). New York, NY: Cambridge University Press.

Rose, R. J. (2006b). Genetic and environmental influences on social behavior and health. In L. Pulkkinen, J. Kaprio \& R. J. Rose (Eds.), Socioemotional development and health from adolescence to adulthood (pp. 56-75). New York, NY: Cambridge University Press.

Rose, R. J., Dick, D. M., Viken, R. J., Pulkkinen, L., \& Kaprio, J. (2001). Drinking or abstaining at age 14? A Genetic-epidemiological study. Alcoholism: Clinical E Experimental Research, 25, 1594-604.

Rosenbaum, M. (1980). A schedule for assessing self-control behaviors: Preliminary findings. Behavior Therapy, 11, 109-21.

Rosenberg, M. (1965). Society and the adolescent self-image. Princeton, NJ: Princeton University Press.

Rothbart, M. K. (2011). Becoming who we are: Temperament and personality in development. New York, NY: Guilford Press.

Rothbart, M., \& Bates, J. E. (1998). Temperament. In W. Damon \& N. Eisenberg (Eds.), Handbook of child psychology (5th ed.):. Social, emotional and personality development (Vol. 3, pp. 105-76). New York, NY: Wiley. 
Rothbart, M., \& Derryberry, D. (1981). Development of individual differences in temperament. In M. E. Lamb \& A. L. Brown (Eds.), Advances in developmental psychology (Vol. 1, pp. 37-86). Hillsdale, NJ: Erlbaum.

Rothbart, M. K., Ahadi, S. A., \& Evans, D. E. (2000). Temperament and personality: Origins and outcomes. Journal of Personality and Social Psychology, 78, 122-35.

Rubin, A. M. (1983). Television uses \& gratifications: The interactions of viewing patterns and motivations. Journal of Broadcasting, 27, 37-51.

Rusk, R. D., \& Waters, L. (2014). A psycho-social system approach to well-being: Empirically deriving the Five Domains of Positive Functioning. The Journal of Positive Psychology, 9, 1-12.

Rutter, M. (1967). A children's behaviour questionnaire for completion by teachers: preliminary findings, Child Psychology \& Psychiatry \& Allied Disciplines, 8, 1-11.

Rutter, M. (1994). Concepts of causation, tests of causal mechanisms, and implications for intervention. In A. C. Petersen \& J. T. Mortimer (Eds.), Youth unemployment and society (pp. 147-71). Cambridge, UK: Cambridge University Press.

Rutter, M. (1996). Transitions and turning points in developmental psychopathology: as applied to the age span between childhood and mid-adulthood. International Journal of Behavioral Development, 19, 603-26.

Rutter, M., Giller, H., \& Hagel, A. (1998). Antisocial behavior by young people. Cambridge, UK: Cambridge University Press.

Rutter, M., Quinton, D., \& Hill, J. (1990). Adult outcome of institution-reared children: Males and females compared. In L. N. Robins \& M. Rutter (Eds.), Straight and devious pathways from childhood to adulthood (pp. 135-57). New York, NY: Cambridge University Press.

Ryan, R. M., \& Deci, E. L. (2001). On happiness and human potentials: A review of research on hedonic and eudaimonic well-being. Annual Review of Psychology, 52, 141-66.

Ryan, R. M., \& Deci, E. L. (2006). Self-regulation and the problem of human autonomy: Does psychology need choice, self-determination, and will? Journal of Personality, 74, 1557-85.

Ryff, C. D. (1989). Happiness is everything or is it? Explorations on the meaning of psychological well-being. Journal of Personality and Social Psychology, 57, 1069-81.

Ryff, C. D., \& Heincke, S. G. (1983). Subjective organization of personality in adulthood and aging. Journal of Personality and Social Psychology, 44, 807-16.

Ryff, C. D., \& Keyes, C. L. M. (1995). The structure of psychological well-being revisited. Journal of Personality and Social Psychology, 69, 719-27.

Saarni, C. (1989). Children's understanding of strategic control of emotional expression in social transactions. In C. Saarni \& P. Harris (Eds.), Children's understanding of emotion (pp. 181-208). New York, NY: Cambridge University Press.

Sahlberg, P. (2011). Finnish lessons: What can the world learn from educational change in Finland. New York, NY: Teachers College Press.

Sainio, P. (2013). Työn merkitys 50-vuotiailla: ennakoivat tekijät ja seuraamukset [Significance of work at age 50: antecedents and consequences]. Master's thesis, Department of Psychology, University of Jyväskylä, Finland.

Salmela-Aro, K., Kiuru, N., Nurmi, J.E., \& Eerola, M. (2014). Antecedents and consequences of transitional pathways to adulthood among university students: 18-year longitudinal study. Journal of Adult Development, 21, 48-58.

Salovey, P., Mayer, J. D., \& Caruso, D. (2005). The positive psychology of emotional intelligence. In C. R. Snyder \& S. J. Lopez (Eds.), Handbook of positive psychology (pp. 159-71). New York, NY: Oxford University Press.

Sampson, R. J. (2016). The characterological imperative: On Heckman, Humphries, and Kautz's the myth of achievement tests: The GED and the role of character in American life. Journal of Economic Literature, 54, 493-513. 
Savolainen, J., Mason, W. A., Lyyra, A.-L., Pulkkinen, L., Kokko, K. (2017). Antisocial and human capital pathways to socioeconomic exclusion: A 42-year prospective study. Developmental Psychology (in press).

Schachter, S., \& Singer, J. E. (1962). Cognitive, social, and psychological determinants of emotional state. Psychological Review, 69, 379-99.

Schalling, D. (1986). The development of the KSP inventory. In B. af Klinteberg, D. Schalling, and D. Magnusson (Eds.), Self-report assessment of personality traits. (Reports from the Project Individual Development and Adjustment, No. 64, pp. 1-8). Department of Psychology, University of Stockholm, Sweden.

Schaufeli, W. B., Bakker, A. B., \& Salanova, M. (2006). The measurement of work engagement with a short questionnaire. A cross-national study. Educational and Psychological Measurement, 66, 701-16.

Schaufeli, W. B., Taris, T. W., \& Bakker, A. B. (2008). It takes two to tango: Workaholism is working excessively and working compulsively. In R.J. Burke \& C. L. Cooper (Eds.), The long work hours culture: Causes, consequences and choices (pp. 203-25). Bingley, UK: Emerald Publishing.

Scheier, M., \& Carver, C. S. (1985). Optimism, coping, and health: Assessment and implications of generalized outcome expectancies. Health Psychology, 4, 219-47.

Schleicher, A. (2011). The quality of childhood: Evidence from the Programme for International Student Assessment (PISA). In C. Clouder, B. Heys, M. Matthes, P. Sullivan (Eds.), Improving the quality of childhood in Europe 2011 (pp. 31-67). Forest Row, UK: ECSWE. Available online at www.allianceforchildhood.eu/publications (accessed 3 February 2017).

Schoon, I. (2006). Risk and resilience: Adaptations in changing times. New York, NY: Cambridge University Press.

Schütte, N. S., Malouff, J. M., Hall, L. E., Haggerty, D. J., Cooper, J. T., Golden, C. J., \& Dornheim, L. (1998). Development and validation of a measure of emotional intelligence. Personality and Individual Differences, 25, 167-77.

Schwartz, S. H. (1992). Universals in the content and structure of values: Theory and empirical tests in 20 countries. In M. Zanna (Ed.), Advances in experimental social psychology (Vol. 25, pp. 1-65). New York, NY: Academic Press.

Schwartz, S. H. (2012). Values and religion in adolescent development: Cross-national and comparative evidence. In G. Trommsdorff \& X. Chen (Eds.), Values, religion, and culture in adolescent development (pp. 97-122). New York, NY: Cambridge University Press.

Seligman, M. E. P., \& Csikszentmihalyi, M. (2000). Positive psychology: An introduction. American Psychologist, 55, 5-14.

Selzer, M. L. (1971). The Michigan Alcoholism Screening Test: The quest for a new diagnostic instrument. American Journal of Psychiatry, 127, 1653-58.

Serbin, L. A., Peters, P. L., McAffer, V. J., \& Schwartzman, A. E. (1991). Childhood aggression and withdrawal as predictors of adolescent pregnancy, early parenthood, and environmental risk for the next generation. Canadian Journal of Behavioural Science, 23, 318-31.

Sheehy, G. (1976). Passages. New York, NY: Dutton.

Shiner, R. L. (2006). Temperament and personality in childhood. In D. K. Mroczek and T. D. Little (Eds.), Handbook of personality development (pp. 213-230). Mahwah, NJ: Lawrence Erlbaum Associates.

Shulman, S., Barr, T., Livneh, Y., Nurmi, J.-E., Vasalampi., K., Pratt, M. (2015). Career pursuit pathways among emerging adult men and women: Psychosocial correlates and precursors. International Journal of Behavioral Development, 39, 9-19.

Silbereisen, R., \& Noack, P. (1988). On the constructive role of problem behavior on adolescence. In N. Bolger, A. Caspi, G. Downey \& M. Moorehouse (Eds.), Persons in context: Developmental processes (pp. 152-80). New York, NY: Cambridge University Press. 
Sillanaukee, P., Kiianmaa, K., Roine, R., \& Seppä, K. (1992). Alkoholin suurkulutuksen kriteerit [The criteria for heavy drinking.]. Suomen Lääkärilehti, 47, 2919-21.

Silventoinen, K., Lahelma. E., \& Kaprio, J. (2006). Self-rated health: Precursors and implications. In L. Pulkkinen, J. Kaprio \& R. J. Rose (Eds.), Socioemotional development and health from adolescence to adulthood (pp. 146-75). New York, NY: Cambridge University Press.

Silverstein, M., \& Bengtson, V. (1997). Intergenerational solidarity and the structure of adult-parent relationships in American families. American Journal of Sociology, 103, 429-60.

Sinisalo, P. (1986). Työvoimaura ja yksilönkehitys [Career line and personal development: A follow-up of North-Karelian youth]. (Manpower Research Series, No. 63). Helsinki: Ministry of Labour.

Sinkkonen, M., \& Pulkkinen, L. (1996). Elämänrakenne parhaassa aikuisiässä [Life structure in the prime time of adulthood.] (Reports from the Department of Psychology, No. 333). University of Jyväskylä, Finland.

Solantaus, T., Leinonen, J., \& Punamäki, R.-L. (2004). Children's mental health in times of economic recession. Replication and extension of the family stress model. Developmental Psychology, 40, 412-29.

Sonnentag, S., \& Fritz, C. (2007). The Recovery Experience Questionnaire: Development and validation of a measure for assessing recuperation and unwinding from work. Journal of Occupational Health Psychology, 12, 204-21.

Spanier, G. B. (1976). Measuring dyadic adjustment: New scales for assessing the quality of marriage and similar dyads. Journal of Marriage and Family, 38, 15-28.

Spence, J. T., \& Robbins, A. S. (1992). Workaholism: definition, measurement, and preliminary results. Journal of Personality Assessment, 58, 160-78.

Statista: The Statistical Portal (2017). Available online at https://www.statista.com/statistics/593447/consumption-of-alcoholic-beverages-in-finland/.

Stattin, H., \& Kerr, M. (2000). Parental monitoring: A reinterpretation. Child Development, $71,1072-85$.

Stattin, H., \& Magnusson, D. (1989). The role of early aggressive behaviour in the frequency, seriousness, and type of later crime. Journal of Consulting and Clinical Psychology, 29, 710-18.

Staub, E. (1979). Positive social behavior and morality. Vol. 1-2. New York, NY: Academic Press.

Staudinger, U. M. (1996). Psychologische Produktivität and Selbstentfalung im Alter. In M. Baltes \& L. Montada (Eds.), Productives Leben im Alter (pp. 344-73). Frankfurt: Campus.

Staudinger, U.M., \& Fleeson, W. (1996). Self and personality in old and very old age: A sample case of resilience? Development and Psychopathology, 8, 867-85.

Staudinger, U. M., \& Kunzmann, U. (2005). Positive adult personality development: Adjustment and/or growth? European Psychologist, 10, 320-9.

Steel, P., Schmidt, J., \& Shultz, J. (2008). Refining the relationship between personality and subjective well-being. Psychological Bulletin, 132, 138-61.

Steele, H., Steele, M., \& Fonagy, P. (1996). Associations among attachment classifications of mothers, fathers and their infants. Child Development, 67, 541-55.

Stephen, J., Fraser, E., \& Marcia, J. E. (1992). Moratorium-achievement (MAMA) cycles in lifespan identity development. Journal of Adolescence, 15, 283-300.

Stikker, A. (2012). Three windows on eternity: Exploring evolution and human destiny. London, UK: Watkins Publishing.

Strauss, A. (1990). Basics of qualitative research: Grounded Theory procedures and techniques. Newbury Park, CA: Sage.

Straus, M. A. (1992). Manual for the Conflict Tactics Scales (CTS). Durham, NH: University of New Hampshire. 
Straus, M. A., \& Hamby, S. L. (1996). The revised conflict tactics scale (CTS2). Journal of Family Issues, 17, 283-317.

Suomalainen, S., Kokkonen, M., \& Pulkkinen, L. (2004). Neuroottisuus ja mustasukkaisuus parisuhteen laadun heikentäjinä (Neuroticism and jealousy as factors weakening the quality of a relationship). Psykologia, 39, 370-81.

Takala, M. (1979). Perheen elämäntapa, vanhempien kasvatustietoisuus ja lasten sosiaalinen kehitys [The way of life of the family, parents' consciousness of child-rearing, and children's social development]. (Reports from the Department of Psychology, No. 219). University of Jyväskylä, Finland.

Takala, M., \& Pitkänen, L. (1963). Level of activation and goal-directed behaviour: The effect of chlorpromazine on aggressive, achievement-oriented and affiliation-oriented verbal responses. Scandinavian Journal of Psychology, 4, 115-22.

Terman, L. M. \& Oden, M. H. (1959). Genetic studies of genius: $V$. The gifted group at mid-life. Stanford, CA: Stanford University Press.

Thin, N. (2016). Positive social planning. In J. Stephen (Ed.), Positive psychology in practice. (2nd ed., pp. 751-72). Hoboken, NJ: Wiley.

Thomae, H. (1979). The concept of development and life-span developmental psychology. In P. B. Baltes \& O. G. Brim, Jr. (Eds.), Life-span development and behavior (Vol. 2. pp. 282-312). New York, NY: Academic Press.

Thompson, E. R. (2007). Development and validation of an internationally reliable shortform of the Positive and Negative Affect Schedule (PANAS). Journal of Cross-Cultural Psychology, 38, 227-42.

Tremblay, R. E. (2000). The development of aggressive behavior during childhood: What have we learned in the past century? International Journal of Behavioral Development, 24, 129-41.

Trommsdorff, G. (1986). Future time orientation and its relevance for development as action. In R. K. Silbereisen, K. Eyferth \& G. Rudinger (Eds.), Development as action in context: Problem behavior in normal youth development (pp. 121-36). Berlin: Springer-Verlag.

Ttofi, M. M., Farrington, D. P., \& Lösel, F. (2012). School bullying as a predictor of violence later in life. A systematic review and meta-analysis of prospective longitudinal studies. Aggression \& Violent Behavior, 17, 405-18.

Unicef (2016). Fairness for children: A league table of inequality in child well-being in rich countries. (Innocenti Report Card 13.) Florence, Italy: Unicef.

Universitas 21 (2013). U21 Ranking of national higher education systems 2013. Melbourne Institute of Applied Economic and Social Research, University of Melbourne, Australia. Available online at http://www.universitas21.com/news/details/96/u21-ranking-ofnational-higher-education-systems-2013 (5 March 2016).

Vaalamo, I., Pulkkinen, L., Kinnunen, T., Kaprio, J., \& Rose, R. J. (2002). Interactive effects of internalizing and externalizing problem behaviors on recurrent pain in children. Journal of Pediatric Psychology, 27, 245-57.

Van den Bulck, J. (1995). The selective viewer. Defining (Flemish) viewer types. European Journal of Communication, 10, 147-77.

Van IJzendoorn, M. H. (1995). Adult attachment representations, parental responsiveness, and infant attachment: A meta-analysis on the predictive value of the Adult Attachment Interview. Psychological Bulletin, 117, 387-403.

Van Steenbergen, E. F., Ellemers, N., \& Mooijaart, A. (2007). How work and family can facilitate each other: distinct types of work-family facilitation and outcomes for women and men. Journal of Occupational Health Psychology, 12, 279-300.

Viinikainen, J., \& Kokko, K. (2012). Personality traits and unemployment: Evidence from longitudinal data. Journal of Economic Psychology, 33, 1204-22. 
Viinikainen, J., Kokko, K., Pulkkinen, L., \& Pehkonen, J. (2010). Personality and labour market income: Evidence from longitudinal data. Labour, 24, 201-20.

Viinikainen, J., Kokko, K., Pulkkinen, L., \& Pehkonen, J. (2014). Labour market performance of dropouts: The role of personality. Journal of Economic Studies, 41, 453-68.

Virtanen, M., Kivimäki, M., Elovainio, M., Vahtera, J., Kokko, K., \& Pulkkinen, L. (2005). Mental health and hostility as predictors of temporary employment: Evidence from two prospective studies. Social Science \& Medicine, 61, 2084-95.

Vohs, K. D., \& Baumeister, R. F. (2004). Understanding self-regulation: An introduction. In R. F. Baumeister \& K. D. Vohs (Eds.), Handbook of self-regulation: Research theory, and application (pp. 1-9). New York: Guilford Press.

Voydanoff, P. (1990). Economic distress and family relations: A review of the eighties. Journal of Marriage and the Family, 52, 1099-115.

Vygotsky, L. S. (1962). Thought and Language. New York: Wiley.

Walder, L., Abelson, R., Eron, L., Banta, T., \& Laulicht, J. (1961). Development of a peerrating measure of aggression. Psychological Report, 9, 497-556.

Walker, R. N. (1967). Some temperament traits in children as viewed by their peers, their teachers, and themselves. Monographs of the Society for Research in Child Development, Serial No. 114.

Warr, P. (1994). A conceptual framework for the study of work and mental health. Work and Stress, 8, 84-97.

Watson, D., \& Clark, L. A. (1977). Extraversion and its positive emotional core. In R. Hogan, J. Johnson \& S. Briggs (Eds.), Handbook of Personality Psychology (pp. 767-93). San Diego, CA: Academic Press.

Watson, D., \& Tellegen, A. (1985). Towards a consensual structure of mood. Psychological Bulletin, 98, 219-35.

Werner, E. E., \& Smith, R. S. (1992). Overcoming the odds: High risk children from birth to adulthood. Ithaca, NY: Cornell University Press.

Werner, E. E., \& Smith, R. S. (2001). Journeys from childhood to midlife: Risk, resilience, and recovery. Ithaca, NY: Cornell University Press.

WHO (1980). International Classification of Impairments, Disabilities, and Handicaps: A manual of classification relating to the consequences of disease. Geneva: World Health Organization. Available online at http://whqlibdoc.who.int/publications/1980/924154 1261_eng.pdf (accessed 3 February 2017).

Wikipedia (2016). Updated 13 January 2016. Available online at https://en.wikipedia.org/ wiki/Finnish_war_reparations_to_the_Soviet_Union (5 March 2016).

Wilkinson, R. (2011). Inequality and the wellbeing of adults and childhood in rich countries. In C. Clouder, B. Heys, M. Matthes \& P. Sullivan (Eds.), Improving the quality of childhood in Europe 2011 (Vol. 2, pp. 62-79). Forest Row, UK: ECSWE. Available online at www.allianceforchildhood.eu/publications (accessed 3 February 2017).

Wilkinson, R., \& Pickett, K. (2010). The spirit level: Why equality is better for everyone. London, UK: Penguin Books.

Wittenborn, J. R. (1956). A study of adaptive children I - II. Psychological Monographs, 70, Nos. 1-3.

Ylälahti, E.-L. (1981). Naisten alkoholinkäytön muutoksista 1970-luvulla [Changes in female use of alcohol in the 1970s]. Alkoholipolitiikka, 46, 3-16.

Ylinentalo, O. (1965). Persoonallisuusinventaario kansakoulun ala-asteelle [A personality inventory for elementary school children]. (Centre for Educational Research, No. 13). University of Jyväskylä, Finland. 
York, K. L., \& John, O. P. (1992). The four faces of Eve: A typological analysis of women's personality at midlife. Journal of Personality and Social Psychology, 63, 494-508.

Zedeck, S., \& Mosier, K. L. (1990). Work in the family and employing organization. American Psychologist, 45, 240-51.

Zuckerman, M. (1979). Sensation seeking: Beyond the optimal level of arousal. Hillsdale, NJ: Erlbaum. 


\section{AUTHOR INDEX}

Abelson, R. 23

Abidin, R. R. 34, 213

Achenbach, T. M. 48, 51, 66

Adams, R. 97-8

Adler, J. M. 62-3

af Klinteberg, B. 15, 95, 194

Agrigoroaei, S. 255, 258-9

Ahadi, S. A. 32, 101

Ainsworth, M. D. S. 211

Alatupa, S. 228

Aliev, F. 194

Alliance for Childhood 270

Allik, J. 22, 31

Allport, G. W. 62, 90

Amell, J.A. 30

Andersson, B. 192

Antonovsky, A. 34, 134

Apicella, F. S. 7

Arnett, J. J. 106, 132

Aro, H. 35

Astone, N. M. 106

Atkinson, J.W. 4

Aunola, K. 34, 135-6, 214, 216-17

Ayduk, O. 51

Bakker, A. B. 35, 235-6

Baldwin, W. 225

Baltes, P. B. 43, 63, 156

Bandura, A. 4, 47, 115, 177

Banta, T. 23

Barbaranelli, C. 115

Barckley, M. 266

Barnett, D. 52
Barr, T. 116

Barrett, K. C. 52

Bartholomew, K. 34, 211-13

Bates, J. E. 17, 58, 99

Baumeister, R. F. 50, 175, 238

Baumrind, D. 81

Beckeley, A. L. 187

Bem, D. J. 176

Bengtson, V. L. 218-19, 259

Benoit, D. 213

Bentler, P. M. 56

Bergman, L. R. 15, 36, 40, 171, 176, 238

Bernard, M. 194

Beutell, N. J. 244

Birren, J. E. 29

Bjarnason, T. 192

Bjerstedt, $\AA 23$

Blair, C. 58,271

Blehar, M. C. 211

Block, J. 34, 40, 59-61, 85, 87, 125

Block, J. H. 34, 59

Boxer, P. 17, 23, 99-100, 228

Brandtstädter, J. 32, 134

Brim, O. G. 258

Bronfenbrenner, U. 84

Brooks-Gunn, J. 225

Bühler, C. 122

Burt, K. B. 176

Busby, D. M. 34

Buss, A. H. 5, 31

Cairns, B. D. 67

Cairns, R. B. 67 
Campos, J.J. 52

Campos, R. G. 52

Caprara, G.V. 115, 177

Caradonna, J. L. 272

Card, N. A. 40

Carlsson, Rae 125

Carstensen, L.L. 53

Caruso, D. 52, 54

Carver, C. S. 135

Caspi, A. 30, 51, 67, 91, 95, 101, 103, 156, 176, 181, 187

Cattell, R. B. 31

Champion, L. 177, 179

Chao, R. K. 34

Chen, M. 108-9, 111

Christ, M. A. G. 166

Christensen, C. 34

Cicchetti, D. 48, 52

Cillessen, A. H. N. 23

Clark, L. A. 150

Clausen, J. A. 137

Coan, R.W. 31

Coatsworth, J. D. 156, 159

Coid, J. W. 184

Cole, P. M. 52

Collins, W. A. 155

Conger, R. D. 241-2

Cooper, J.T. 32

Cooper, M. L. 34

Costa, F. M. 189

Costa, P.T., Jr. 22, 31, 88, 97

Crane, D. R., 34

Cronbach, L. J. 22

Crowne, D. P. 7, 42

Csikszentmihalyi, M. 6, 158

Daly, M. 175, 238

Darrow, C. N. 258

Deci, E. L. 33, 63, 142

Delaney, L. 175, 238

Denissen, J. J.A. 96

Depue, R. 34

De Raad, B. 149

Derryberry, D. 58

DeYoung, C. G. 149

Dick, D. M. 194-6

Dickson, N. 181

Diener, E. 33

Dishion, T. 167-8

Dodge, K. A. 17, 99, 170

Dollard, J. 4

Donellan, M. B. 61, 87

Donovan, J. E. 189

Döpfner, M. 74
Dornheim, L. 32

Downey, G. 163-4

Dubanoski, J. P. 266

Dubow, E. F. 15, 17, 23, 99-100, 226, 228

Duckworth, A. 48, 103

Duckworth, K. 113

Duncan, G. J. 113

Eccles, J. S. 188

Edelbrock, C. 48, 51

Edmonds, G. W. 266

Edwards, A. L. 42

Eerola, M. 110

Egan, M. 175, 238

Eichelberger, I. 74

Eisenberg, N. 47, 52, 103

Eisenberg-Berg, N. 6

Ekman, G. 4

Elder, G. H. Jr. 156, 176, 219, 241

El-Khouri, B. M. 36

Ellemers, N. 35

Elliott, J. 259

Elo, A.-L. 34

Elovainio, M. 203, 228, 238, 240

Emerson, M. J. 55

Emmons, R. A. 32-3, 122

Erikson, E. H. 63, 130, 156

Eron, L. 23

Evans, D. E. 32, 101

Ewing, J. A. 35

Eysenck, H. J. 31, 48, 71

Eysenck, S. B. G. 31, 71

Fabes, R.A. 47

Fadjukoff, P. 29, 131-4, 214-15

Farrington, D. P. 166, 170, 184

Feldt, T. 87-8, 90, 92, 95, 133, 135-7, 139, 142-4, 146, 151, 202, 228, 234, 242, 245

Felling, A. J. A. 34

Feshbach, S. 7

Fleeson, W. 33

Folkman, S. 241

Fonagy, P. 213

Fraser, E. 132

Freedman, D. 30

Freitas, A. L. 163-4

Frick, P. J. 166

Friedman, H. S. 150

Friedman, N. P. 55

Frijda, N. H. 51

Fritz, C. 35

Frone, M. R. 34

Furstenberg, F. F. 225

Fyrstén, S. 20, 200, 213, 223, 237 
Ganiban, J. 52

Gans, D. 259

Garmezy, N.

Gaschke,Y. N. 32

Ge, X.-J. 241-2

Gerris, J. R. M. 34

Geurts, S. 35, 245

Giller, H. 165

Giorgi, A. 126

Glueck, E. 187

Glueck, S. 187

Goldberg, L. R. 103, 266

Golden, C. J. 32

Gommans, R. 23

Goodman, R. 51

Gottfredson, M. R. 172, 187

Granberg, M. 231

Greenhaus, J. H. 244

Griffin, S. 33

Haapasalo, J. 32

Haavisto, T. 32, 214, 217

Hagel, A. 165

Haggerty, D. J. 32

Hall, C. S. 4

Hall, L. E. 32

Hämäläinen, M. 22, 31, 166-8, 175

Hamby, S. L. 34

Hampson, S. E. 103, 266

Hanisch, C. 74

Harrington, H. 30,181, 187

Hartshorne, H. 23

Hatakka, M. 200

Hautmann, C. 74

Havighurst, R. 106, 156

Hedlund, B. 29

Heikkinen, A. 54

Heikkinen, R.-L. 156

Heincke, S. G. 34, 139

Helson, R. 255, 258-9

Hibell, B. 192

Hietalahti, M. 118, 120-1, 150

Hill, J. 177, 225

Hillier,T.A. 266

Hintsanen, M. 228

Hirschi, T. 172, 187

Hirsjärvi, S. 28

Hogan, D. P. 106

Horowitz, L. M. 34, 212

Houts, R.M. 187

Howell, C. T. 66

Howerter, A. 55

Hu, L. T. 56

Huesmann, L. R. 17, 23, 99-100, 226, 228
Huesmann, R. 15

Hurme, H. 28, 84, 167, 220-2

Hyde, J. S. 71

Immonen, M. 139

Izard, C. 52

Jackson, S. 34

Jacques, E. 258

Jaffe, S. 226

Jahoda, M. 27, 237

Jalonen, N. 234, 249

Janssens, J. M.A. M. 34

Jessor, R. 176, 189

Jessor, S. L. 176, 189

John, O. P. 87

Jokisaari, M. 33

Jones, H. E. 3

Junger-Tas, J. 35

Juujärvi, P. 55-7, 70, 216-17

Kaartinen, J. 70

Kagan, J. 3, 9, 43, 49, 67, 102

Kahn, H. 34, 231

Kallio, E. 197-8

Kananoja, A. 155

Kanfer, F. H. 47

Kaplan, B. 43

Kaprio, J. 48, 53, 194-6, 200-3, 234, 240-1

Karasek, R.A. 34, 229, 234

Karoly, R. 47

Keltikangas-Järvinen, L. 228

Kendall, P. C. 51, 56

Kern, M. L. 150

Kerr, M. 217

Keskinen, E. 200

Kessler, R. C. 258

Keyes, C. L. M. 34, 142-3, 229

Kickbush, I. 270

Kiianmaa, K. 191

King, L. A. 32

Kinnunen, M.-L. 19-20, 51, 53, 87-8, 92, 96, 120, 136, 146-7, 151, 193, 200-3, 207, 213, 223, 234-7, 240-1, 249

Kinnunen, T. 48, 201

Kinnunen, U. 20, 74, 87-8, 110, 135-6, 146, $151,179,185,200,202,210-11,213$, 223-4, 227, 237, 241-7

Kiuru, N. 110

Kivimäki, M. 203, 238, 240

Klein, E. B. 258

Klein, M.W. 35

Kochanska, G. 34

Kohlberg, L. 34 
Kokkevi,A. 192

Kokko, K. 12, 15, 17, 19-20, 22-3, 29, $31,33,37,40,87-8,92,95-6,99-101$, 108-11, 113, 116-18, 120-2, 124, 132-3, 135-7, 139, 142-7, 150-1, 159-62, 180-1, 184-5, 190-1, 193-6, 200, 207, 213-16, 223-8, 230-40, 244, 249, 258-9

Kokkonen, M. 51, 53, 55, 149, 201, 209

Kontula, O. 34

Kooistra, L. 55-6, 70

Kopp, C. B. 49

Korhonen, M. 33

Korkalainen, A. 142-4

Kortteinen, M. 238

Koskela, K. 34

Koskenvuo, M. 200

Kristenson, H. 35

Kroger, J. 131-2

Kunzmann, U. 91, 95

Laaksonen, H. 200

Lachman, M. E. 255, 258-9

Lahelma. E. 203

Lahey, B. B. 166

Lahtela, L. 139

Landsford, Janet 15

Lansford, J. E. 17, 99

Larsen, R. J. 33, 52-3, 149

Larson, J. H. 34

Larson, R. W. 188

Laulicht, J. 23

Laursen, B. 40, 97-8

Lazarsfeld, P. F. 237

Lazarus, R. S. 7, 241

Lehto, J. E. 55-7

Leinonen, J. 242

Leppänen, A. 34

Leppänen, E. 87-8, 146, 151

Lerner, R. M. 43

Levinson, D. J. 106, 117, 126, 258

Levinson, M. H. 258

Lindenberger, U. 156

Lindström, K. 34

Lindzey, G. 4

Little, T. D. 40

Livneh,Y. 116

Lloyd, B. H. 34, 213

Loeber, R. 166-8, 187

Long, J. D. 176

Lorenz, F. O. 241-2

Lösel, F. 170

Lowe, J. C. 105

Luria, A. R. 47
Lyyra, A.-L. 19-20, 33, 113, 116, 120, 142-4, 161, 180-1, 184-5, 189-91, 193-6, 207, 214, 223, 226-7

Macfarlane, J.W. 3

Magnusson, D. 36, 40, 85, 125, 171-2, 176, 194, 261

Mahoney, J. L. 188

Main, M. 211

Mäkikangas, A. 135-6, 235-6

Maller, J. B. 23

Malouff, J. M. 32

Männikkö, K. 160, 212-13, 216

Marcia, J. E. 29, 130, 132-3, 214

Marini, M. 106

Markkanen, T. 54

Marlowe, D. 7, 42

Martinussen, M. 132

Mascaro, N. 33

Maslach, C. 34

Maslow, A. 156

Mason, W. A. 180

Masten, A.A. 156, 159, 176

Maté, G. 79

Matthes, M. 270

Maughan, B. 177, 179

May, M.A. 23

Mayer, J. D. 32, 52-4

Mayr, U. 53

McAdams, D. P. 62-4, 91, 96, 104-5, 125, $130,137,259$

McAffer,V. J. 225

McClelland, D. C. 7

McCrae, R. R. 22, 31, 88, 97

McEwen, B. 240

Mcgee, T. R. 187

McGonaughy, S. H. 66

McKee, B. 258

Merrick, E. N. 225

Mesiäinen, P. 116, 226-7

Metsäpelto, R.-L. 19-20, 87-8, 92, 95, 120, 135, 146, 151, 193, 207, 214, 216-17, 223

Mettzger, M. 113

Michel, M. K. 52

Miller, N. E. 4, 6

Milne, B. J. 181

Ministry of Education 113

Mischel, W. 51, 68, 70

Mitchell,V. 255

Miyake, A. 55

Moffitt, T. E. 30, 51, 181, 184, 187-8

Mooijaart, A. 35

Moore, J.W. 105 
Morey, L. C. 33

Morgan, M. 192

Morgan, N. 187

Morgan, S. P. 225

Morris, W. 49, 51

Mosier, K. L. 241

Moss, H. A. 3, 102

Mueller, M. 219

Mullola, S. 228

Murray, H.A. 9, 122

Mussen, P. H. 6

Mustonen, A. 169

Muthén, B. O. 36

Muthén, L. K. 36

Nagin, D. S. 37

Narusk, A. 192

National Institute for Health and Welfare 192

Nesselroade, J. R. 53

Nesshoeven, W. 32

Neufeld, G. 79

Neugarten, B. L. 105

Nikander, T. 107

Noack, P. 189

Nurmi, J.-E. 32, 34, 110, 116-18, 122, 124, 159, 214, 216-17

Obradović, J. 176

Oden, M. H. 3

Ohranen, M. 229

Olson, B. D. 63, 104

Olson, S. L. 17, 99

Olson, W. C. 4

Olweus, D. 42, 67, 98

Onatsu-Arvilommi, T. 34, 214, 216-17

Oravala, S. 137-41

Orth, U. 136

Pals, J. L. 62, 105, 130

Parker, K. C. H. 213

Pastorelli, C. 115

Pasupathi, M. 53

Pehkonen, J. 230-2

Penke, L. 96

Perho, H. 33

Perry, M. 31

Perttula, J. 126-7, 134, 269

Peters, P. L. 225

Peterson, C. 50, 203-4

Pettit, G. S. 17,99

Pickett, K. 100

Piquero, A. R. 187

Pitkänen, L. 4, 6-7, 10, 21, 26, 42, 54, 65, $67-9,71$
Pitkänen, T. 19-20, 33, 50, 66, 120, 168, 189-97, 200, 203-5, 207, 213, 223, 237

Pitkänen-Pulkkinen, L. 54, 66-7, 69-70, 84

Plück, J. 74

Poikkeus, A.-M. 217

Polet, J. 19-20, 117-20, 144, 193, 207, 223-4, 232, 248-9, 251

Poulton, R. 187

Pratt, M. 116

Prizmic, Z. 52-3, 149

Pulkkinen, L. 7, 9-10, 17-23, 27, 29-34, $37,40,47-51,53-8,66-7,69-72,74-6$, $79,81-5,87-8,90-2,95-101,104$, 106-11, 113-20, 122, 124, 132-41, 143-7, 150-1, 156-7, 159-63, 165-72, 174-5, 177, 179-81, 184-5, 187-91, 193-9, 200-5, 207-11, 213-17, 223-32, 234-6, 238, 240-2, 244-9, 251, 259, 268-72

Pulkki-Råback, L. 228

Pulver, A. 22, 31

Punamäki, R.-L. 242

Puohiniemi, M. 33

Putney, N. M. 259

Puustinen, M. 55, 238

Quinton, D. 141, 177, 225

Rachman, S. 48

Räikkönen, E. 92, 96, 108-11, 139, 147, 233-5, 248-9

Rajala, A-M. 139

Ramrakha, S. 187

Ranta, M. 54

Rantanen, J. 19-20, 92, 95, 100-1, 108, 110-11, 120-1, 143-5, 150, 193, 207. $214,223,233-6,242-9$

Rauhala, L. 126, 128

Rauhala, U. 17

Raver, C. C. 58,271

Read, S. 33

Reilly, N. 49, 51

Riley, J. R. 176

Rimpelä, M. 196

Robbins, A. S. 235

Roberts, B.W. 91, 95, 101, 103

Roberts, G. C. 34

Robin, R.W. 136

Robins, R.W. 61, 87

Roger, D. 32

Roine, R. 191

Roisman, G. I. 176

Rokka, P. 126, 128

Romanov, K. 200 
Rönkä, A. 32, 74, 134, 137-41, 176-7, 179, 185, 224-7

Ropponen, T. 34

Rose, R. J. 10, 12, 40, 48, 194-6, 200

Rosen, D. H. 33

Rosenbaum, M. 32

Rosenberg, M. 32, 136

Rothbart, M. K. 32, 58, 101, 149

Rubin, A. M. 33

Rusk, R. D. 158

Russell, M. 34

Rutter, M. 51, 137, 141, 164-5, 177, 187, 225, 238

Ryan, R. M. 33, 63, 142

Ryff, C. D. 33-4, 139, 142-3, 258

Saarni, C. 49

Sahlberg, P. 113

Sainio, P. 232, 235

Salanova, M. 35

Salmela-Aro, K. 32, 110, 214, 217

Salovey, P. 32, 52, 54

Savolainen, J. 180

Schachter, S. 7

Schalling, D. 31

Schaufeli, W. B. 35

Scheier, M. 135

Schleicher, A. 184

Schmidt, J. 145-6, 150

Schoon, I. 15, 61, 159, 271-2

Schroeder, F. 187

Schultz, D. 52

Schütte, N. S. 32

Schwartz, S. H. 33, 120-1

Schwartzman, A. E. 225

Seligman, M. E. P. 6, 50, 158, 203-4

Selzer, M. L. 35

Seppä, K. 191

Serbin, L.A. 225

Sheehy, G. 258

Shiner, R. L. 57, 67, 91, 95, 101, 103

Shulman, S. 116

Shultz, J. 145-6, 150

Silbereisen, R. 189

Sillanaukee, P. 191

Silva, P. A. 30, 51, 181, 187

Silventoinen, K. 203

Silverstein, M. 218, 259

Simonton, S. 17, 99, 113

Singer, J. E. 7

Sinisalo, P. 224

Sinkkonen, M. 200

Slot, N. W. 187

Smeijers, J. 30

Smith, R.S. 61, 159, 164, 219, 225
Solantaus, T. 242

Sonnentag, S. 35

Spanier, G. B. 34

Spence, J.T. 235

Spinrad, T. 103

Stanford, Nevitt 125

Stanton, W. 181

Stark, K. 52

Statista:The Statistic Portal 191

Stattin, H. 172, 217

Staub, E. 6

Staudinger, U. M. 33, 91, 95, 119, 156

Steel, P. 145-6, 150

Steele, H. 213

Steele, M. 213

Steinberg, L. 48

Stellar, E. 240

Stephen, J. 132

Stevens, A. A. 32, 53

Stikker, A. 262

Southamer-Loeber, M. 187

Straus, M. A. 34

Strauss, A. 128

Sundelin, A. 126, 128

Suomalainen, S. 209

Suutama, T. 33

Takala, M. 4, 111

Tannenbaum, L. 166

Taris, T.W. 35

Tellegen, A. 149, 176

Terlouw, J.-G. 35

Terman, L. M. 3

Teshale, S. 255, 258-9

Teti, L. 52

Theorell, T. 229, 234

Thin, N. 155

Thomae, H. 43

Thornton, A. 30

Tillemann, K. 92, 214

Tolvanen, A. 55, 87-8, 92, 118, 120, 143, 145-6, 151, 229

Toth, S. L. 48

Trell, E. 35

Tremblay, R. E. 6, 15, 171, 175

Trentacosta, C. 52

Trommsdorff, G. 107

Trzesniewski, K. H. 136

Ttofi, M. M. 170, 184

Tuomikoski, H. 238

Unicef 12, 270

Vaalamo, I. 48

Vahtera, J. 203, 240 
Vaitilingam, R. 259

Valiente, C. 103

van Aken, M. A, G. 96

van Boxtel, D. A. A. M. 34

Van den Bulck, J. 33

Van Horn,Y. 166

Van Ijzendoorn, M. H. 213

Van Steenbergen, E. F. 35

van Zutphen, R. A. H. 34

Vasalampi., K. 116

Vermulst, A. A. 34

Viechtbauer,W. 95

Viinikainen, J. 230-2, 239

Viken, R. J. 194, 195-6

Virtanen, M. 240

Virtanen, T. 194

Vohs, K. D. 50

Voydanoff, P. 241

Vygotsky, L. S. 47

Wager,T. D. 55

Walder, L. 23

Walker, R. N. 22

Wall, S. 211

Walters, R. H. 4

Walton, K. E. 95
Warr, P. 34

Waterman, M. B. 229

Waters, E. 211

Waters, L. 158

Watson, D. 149-50

Werner, E. E. 61, 159, 164, 219, 225

Weston, D. R. 211

WHO 156

Wiener, A. J. 34, 231

Wikipedia 13

Wilcox, L. E. 51, 56

Wilkinson, R. 12, 100, 184

Wittenborn, J. R. 7

Witzki, A. H. 55

Wlater, D. 74

Wood, D. 96

Wright, B. E. 187

Ylälahti, E.-L. 191

Ylinentalo, O. 31

York, K. L. 87

Zedeck, S. 241

Zeisl, H. 237

Zimbardo, P. G. 115, 177

Zuckerman, M. 31 


\section{SUBJECT INDEX}

accidents 11, 190, 198-9: accident prone 264, 267; predictors of 199-200; see also injuries activity/passivity: aspects assessed 25; behavioral/social 7, 9, 48, 56-9, 71-8, 97, 101-3, 116, 150-1, 161, 182-3, 229-30, 260

adaptation: characteristic $62,105,214$; conflicted 85-7, 127; positive 86-7, 97, 127 adaptive behavior 51,243

adulthood $3,19,30,40,43,62,76,79$, 87, 91-2,109-10, 126-7, 181-3, 185, 192; categories of 255 ; continuity from childhood to 96-104, 182, 190-2, 198, 205, 229-30, 262-8; development in 130-5, $144,159,166,168,176,179-80,189$, 192-3, 202, 257, 272; self-perceived 106, 132; see also adult transitions; middle age Adult Temperament Questionnaire (ATQ) 32, 101; see also temperament

adult transitions/adulthood, transition into: definition 28, 106-7: age-stratified 105,112 ; assessment 27,30 ; education, completing 107, 213; full-time work, entering 107, 114-15, 213; individualistic 105, 112, 132; intimate relationship, forming 107; parent, becoming 107, 138, 213; parental home, moving from 107, 213; patterns/groups of 108-11; timing, of entering 108, 112, 260; see also middle age; parenthood

affect/affectivity, positive/negative 32-3, 101,149-50; see also well-being age-graded tasks 156; see also developmental tasks

aggression: defensive aggression 5-6, 26; descriptive model of 5,169 ; direct/ indirect aggression 5-6, 24, 31, 69, 95-6, 169; facial aggression 5, 167; insights about 171 ; intensity of $5,69,169$; model of 5,169 ; motivational sequence of 5 ; offensive aggression 5-6; physical aggression 5-6, 24, 34, 69, 98-9, 167, $174,179,238$; proactive aggression 5-6, 21, 24, 26, 69, 169-71, 183, 187; reactive aggression 5-6, 21, 24, 26, 69, 169-71; verbal aggression 5, 24, 31, 69, 95, 174, 182

Aggression Machine (PAM) 21, 69-70; computerized 70

Aggression Questionnaire 31; see also question series

aggressive act 5-6

aggressive behavior $6,8,14,21-3,49$, 66-7, 73, 76, 98, 162, 167-76, 180, 185, 188, 194, 198, 228, 238-40; 262-3, 267; aspects assessed 24, 69-70; see also behavior regulation; developmental paths/lines

aggressiveness 26, 51, 69-70, 97, 101-2, 115, 139, 167-8, 172-5, 177-8, 183, 194, 205, 264-6

agreeableness 86, 88-97, 101, 103,121,

$123,135,145-6,183,210,229-30,239$, 246-7, 262-5

agreement between informants 65 
alcohol: consumption see alcoholism; drinking alcohol; use of alcohol; alcoholism 30, 186, 194, 257; alcoholism screening test CAGE 35, 189, 195-6; Malmö Modification of the MAST (mm-MAST) 35, 189

allostasis/allostatic load 240-1, 256

antecedent-focused 52-4, 149; see also emotion regulation

antisocial behavior 26, 74, 163-9, 181, 187; definition of 165; predictor of 167-9, 171-4, 185; risk of 175,188 ; see also criminal behavior

anxious behavior/anxiety $8,21,48$, 50-3, 56-7, 61, 66-7, 71-3, 75-6, 84-5, 88, 96-7, 101, 114-15, 133, 139, 143, 146-8, $159,169,171,175,178,182-3,194,199$, 211-12, 229, 236-40, 262-8; aspects assessed 25, 31,95; see also developmental paths/lines; emotion regulation

approach-avoidance tendency 6

assortative mating 74, 185

attachment 63, 79, 155; adult attachment

211, 213; clusters 212; Relationship

Questionnaire 34, 212; styles of 34, 211

authoritarian 34, 82, 216

authoritative 81,216

back translation method 22

behavior regulation 48, 50-1, 71-2, 74, 76, 82-3, 96, 98, 115, 133, 151, 158, $168,170,175,183,188,194,200-1$, 210-11, 228, 230, 238, 243, 266-8; definition of 50 ; norm-focused 51 ; self-focused 50-1

Big Five personality traits/Five Factor Model 31, 88, 90-1, 94, 145-6, 212, 236; see also agreeableness; conscientiousness; extraversion; neuroticism; openness to experience

blood pressure 35, 146, 201, 203, 240, 257

body mass index (BMI) 146, 202-5, 266; see also obesity

Brief Mood Introspection Scale (BMIS) 32 brittle 50, 52, 59-61, 75-7, 80, 86-92, 94, 96-7, 124, 128, 133, 136-7, 144, 146-8, 151, 194, 198, 199-201, 212-14, 236, 240, 247-8, 259-60, 264-8; see also clusters; developmental paths/lines; profiles; style of life

bullying, bully 155, 170-1, 188

care: functions/activities 73, 81, 119, 157-8, 188, 207, 218-22, 257-8, 262 career line/path: changeable 224-5, 237, 240; stability of 159-60, 179, 224, 226, 228, 240; stable 224-5, 236, 240, 242; unstable $98,110,123,174,177-80$, 224-7, 237, 240-3, 256, 267

cascading effects 176,179

case studies 84

causation hypothesis 238-9

character strength 203-4

children of participants 14, 22, 30, 55-6, 70-4, 109, 111, 129, 185, 196, 209, 213; number of 20, 207-8; see also offspring study

child-centered home/atmosphere 134, 160-1, 216; see also parenting

child-centered parents see parenting

child-rearing see parenting

Child-Rearing Practices Questionnaire 34, 215-16

circumplex 48, 149

clusters, labeling of 61,86 ; personal styles for 86-7, 90, 97, 122-4, 132-3, 144; see also attachment; data analysis; health; personal goals; problem behavior

cognitive control 7-10, 47, 53-4, 260 cognitive factors/capacities/skills 56, 116, $159,181,271$

cognitive mood management 53

cognitive tests 39,55

common-law partner/marriage/relationship 138, 207, 209-10, 256; co-morbidity 48, 175-6

comparative study 23, 98, 111, 171, 174, 228,238

compliant behavior/compliance 8-9, 21, $48,50-1,56-8,66-7,71-5,84,88,91$, 96-8, 101, 115, 160-1, 168, 170, 174, 182-3, 194, 197-9, 205, 210, 217, 228, 243, 262-8, 271; aspects assessed 24; see also behavior regulation; developmental paths/lines

concentration: lack of 25, 76-7, 98, 170, 172-5, 182-3, 188, 265, 267; problems of 176,180

conception of a man/human being 126, 128; see also image of the child conformity 31, 95-6, 101-3, 120-1, 205, 265-6

conscientiousness 88-91, 92-5, 101-3, 121, 145-6, 148-51, 183, 205, 212, 230, 236, 246-7, 263-6

constructive behavior/constructiveness 8, 21, 48, 50-2, 54, 56-8, 66-8, 71-7, $84,96,101,113-16,160-2,170,174-5$, 
182-3, 187, 194, 198, 205, 239, 263-8, $271-2$; as an antecedent of 228-30, 236; aspects assessed 9, 24, 163, 211, 228; see also developmental paths/lines; emotion regulation

continuity: contemporary 176 ; cumulative 176, 180; definition of 49, 67; differential 67; discontinuity 91, 104, 126-8, 129; heterotypic 49; homotypic 49; interactive 176,180

control over one's development/life 30, 111, 126-7, 130, 134-5, 140, 147-8, 260; Personal Control over one's Development Questionnaire 32, 134 coping with 7, 9, 24, 48, 54, 59, 139-40, $155,163,188,211,228,238$

core capacity 206

corporal/physical punishment 12, 72, 134, 160,184

crime, general theory of 172,187

criminal behavior/offending: arrests $86,98,166-9,172-5,177-8,181$, 189, 225; convictions 166, 175, 189 , 257; predictions, accuracy/linearity 167-8; records 35, 166; Self-Report Delinquency Scale 35, 181 see also antisocial behavior; offenders; register data

criminal offenses: alcohol-related 167, 189; associated with 79-80,112,171, 174, $177,180,187$; number of 166 ; thefts 166 , 172 ; types of $166-9,181-2$; violence 167 ; see also offenders

criminal responsibility age $36,165,181$ cross-sectional study $3,6,10,40,145$; see also longitudinal study

culture, cultural activities and life 33,83 , 111, 120, 123, 131, 204, 233, 249, 251, 270, 272; see also domains of life, value; values, Personal Value Task

data analysis: advanced statistical methods 37,42 ; analysis of variance (ANOVA) 36; cluster analysis/clustering 37, 71, $85,87-8,122,171,199,212$; configural frequency analysis 37,97 ; factor analysis $36,56,71,74,83,85,101,120,216$; latent class analysis 108 ; latent profile analysis 37,88 , 235, 244; latent growth curve analysis $36,145,175$; LISREL $36-7,74,160,163$; multivariate analysis of covariance 148; path analysis 36-7; regression analysis $36,186,195,210$; SEM (structural equation modeling)
36-7, 56, 92, 95, 99, 101; statistical software 36 ; trajectory analysis 37,116 , 145-6, 202, 226; see also adult transitions; clusters; factor score; patterns of behavior; profiles; sample

data collection: additional 21-2; methods 22-36; prospective 42,160, 168, 180, 216; retrospective 160,184, 190,216; stages/ waves of 13-14, 16-19, 40, 255; see also offspring study; sample

delay of gratification 60

delinquency 172, 187; Self-Report

Delinquency Scale 35, 181

demographic variables: sample representativeness

depression 31, 88, 124, 144, 150, 159, 209 , 225, 237, 241-2, 263; Depression Scale 34, 143; depressive symptoms 34,51 , 56-7, 98, 110, 146-8, 212, 236, 239

development: life-course 63, 141, 156, 165; life-span 43, 156; positive/successful 10, 49, 91, 97, 115, 156, 159, 163, 219, 226; study of 43; see also developmental stages, tasks; holism/holistic; life structure personality, approaches

developmental paths/lines 75, 77, 102, 260, 262, 267; childhood, age 8 75-6; early adolescence, age 14 75-9; late adolescence, age 2075,79 ; young adulthood, age 27 75, 79-80; upbringing and living conditions for 81-5; see also aggressive behavior; anxious behavior; compliant behavior; constructive behavior; orientation to, home; orientation to, indifference; orientation to, peers; orientation to, responsibility; style of life developmental stages 40, 63, 156, 258, 272 developmental tasks 106, 122-4, 156, 177 diseases 33, 35, 198, 200-1, 241; see also health disobedience, disobeying teacher 73-4, 163, $165,175,185,199$

divorce 107, 123-4, 128-9, 138, 207-10, 242, 255, 263, 266; parental 84, 178, 184 , 195 ; risk of 265

doctoral dissertation $3,5,70,128$

domains of life: adulthood 157-8, 243, 248, $259,262-5$, 269; follow-up study 22 ; investment 117-18; pleasure 117, 158, 248; satisfaction 33 , value $118-21,157,189$, 243, 259; see also identity; spheres of life drinking alcohol 77-8, 85, 86-7, 106, 112, 140, 189-96, 198, 225; abusive 189; age of onset/start 182-3, 189-90, 227; controlled 160-1, 266; critical age 190; drinking 
problems 74, 178-9; epidemiological risk limit 191-2; frequency of 190; heavy drinking 11, 20, 109, 129, 133, 177, 185-6, 188, 192, 199-201, 212, 225, 241, 257, 259, 267; intoxication 190, 192-3, 197, 204, 257; legal age 189; parental drinking 139-40, 160-1, 178, 184, 195-6, 225; problem drinking $98,123,162-3,171$, 192-4, 197, 238, 264-7; see also alcoholism; use of alcohol

dropout/dropping out 140, 188, 230-1, 236, 265-6

drugs, experimenting/using 53, 188, 197, 257

Dutch Work Addiction Scale (DUWAS) see workaholism

economic recession $11,29,118,122,132$, $225,237-8,242$

economic strain/stress 241-2; see also income

education: choice of 113-14; continuing education 116, 226; length of 77-80, 107-8, 111-15, 123, 132, 135, 180, 185, 196-7, 203, 205, 215-16, 225, 227-30, 238-40, 243, 263-5, 268; off-time education $116,226-7$; on-time education 116,226 ; of parents of the participants 17,114

educational career 173-4, 176, 225; see also adult transitions, patterns/groups of; career line

education system, Finnish 11-13, 100, 112, 220, 225, 270-1; comprehensive school 112; post-comprehensive education 112-13; tertiary education 113

ego/ego integrity/ego structures 9, 59, 61,139

emotional ambivalence 53; Ambivalence Over Expressiveness Questionnaire 32 emotional intelligence 54, 231; SelfReport Measure of Emotional Intelligence 32, 231

emotional lability/stability 23, 71, 96, 181 , 198, 238, 264; aspects assessed 25; see also self-regulation

Emotion Control Questionnaire 32, 205 emotion regulation $31,47,49-54,60$, $70,72,74,76,82-3,96,116,149-51$, $175,188,194,202,206,211,213,228$, 240, 268; antecedent-focused 52-3, 149; definition of 47,52 ; measures 32 ; program for improving 54 ; responsefocused 52-3; see also self-regulation
EPQ (Eysenck Personality Questionnaire) $31,76,80,182,205$

executive control/functions $7,48,54-8,60$; inhibition $55-8$; methods of study 55 ; shifting 55-6; updating 56-8; working memory 55-6

experienced life fabric, types of male experiences 126-7; see also phenomenological psychological analysis experimental research/study 4, 54, 69; extracurricular activities 188, 272 extraversion $4,9,31,48,76-8,80,86-96$, 101-4, 121, 123, 135, 145-6, 148-51, 183, 212, 214, 217, 229-30, 236, 239, 246-7, 262-8; dual nature 71

factor score $36,75,81,85$; see also data analysis

family: adversities 177-9; circumstances/ living conditions $81,83-5 ; 160-1$; continuity $126-9$; crime 184 ; orientation to $86-7,111,124$; significance of 119 , 138; values of 121; see also adult transition, patterns/groups of; child-centered home; domains of life; household, structure; life structure; parenting; relationships; socioeconomic status; spheres of life; work-to-family interface

family-work interface/conflict see workto-family interface

financial situation 33, 144, 185, 232-3, 241

Finland/Finnish 10-13

Finnish Social Science Data Archive 13, 22

FinnTwin12/twin sample 10, 48, 56, 194, 196, 203

free time activities $27-8,76,78-9,82,84$, $157,213,231$; content of $248-51$; see also leisure activities

friends $28,33,76-9,83,111,114,117,158$, $162,168,177,195,215,242-3,248$, $250-1,256,272$

future orientation 30, 107: Future Orientation Questionnaire 33, 118

gender difference $57,66,71,80,91,93,96$, 102-3, 113, 118, 131-3, 136, 138, 144, 162,179, 190-1, 197, 199, 202, 220-1, 223-4, 232, 249, 266

generations: comparisons across $71-2,114$; continuity across $73-4,185,195-6$; relationships between 218-22, 256

Generativity scale 34, 215

genetic analysis/factors 40, 74, 150 , 194-6, 203 
grade point average (GPA) 114, 160, 226; see also school success

grandparent 218-22, 257-8

Grounded Theory 128, 130

growth 33, 43, 64, 91, 95-6, 123, 142, 156, $189,206,245$

happiness $33,122,142-5,149,186,209$; see also well-being

harmonization of measures 42,98

health $30,35,53,117-19,121,124,140$, 150, 157-8, 234, 240; clusters for 199;

objective indicators of 146 ; risks of 198-200, 240; self-assessed/rated/ subjective $35,146-8,151,200-3,205$, 257, 264-6; see also medical examination; mental well-being, mental health; spheres of life

health behavior 35, 189-98: healthcompromising 157, 257; healthpromoting 157

hermeneutic tradition 126

holism/holistic 37, 40, 85, 125-6, 128, 260, 269; see also personality, approaches

hostility $34,88,97,200,210,239,242$

household: chores/tasks 111-12, 198, 209, 218, 220, 233, 251; structure 207-8, 257

hyperactivity $51,56-7,159,171,174,181$, $188,228,243$

identity: commitment 215-16, 263; development of 131, 133; domains 29, 130-3, 214; exploration 86-7; formation 130-3, 214-15, 218, 260; narrative identity 63, 125, 137; parental 214-15, 218; regression 132

identity status: diffusion $77,80,112$, 131-3, 262; foreclosure 131-3; identity achievement $77,80,131-3,262$; moratorium 131-3; overall 131

Identity Status Interview 29

image of the child 270

impulse/control 6-10, 23, 47-50, 53, 57, 59-60; see also model, impulse control impulsigenic 48

impulsivity, impulsiveness/impulsive 9, 25-6, 31, 48-51, 55, 71, 76, 86, 95, 98, $148,170,172,205,236,265,268$

income/salary 100, 107, 150, 180, 184, 186, 205, 230, 232-3, 236-7, 241, 250, $256,263,265$; see also economic strain; satisfaction with, income individuation 43, 156, 269; see also socialization inhibition see model for aggressive and nonaggressive behavior

injuries 35, 199

international comparison see comparative study

interview: semi-structured 27, 32; structured 28; thematic 27-8; see also Identity Status Interview

introversion 4, 9, 31, 48, 78, 80, 212, 217; see also extraversion

jealousy 34,209

job: demands 233-4; exhaustion 34, 233-5, 244-6, 256; insecurity $236,242-3,256$; Job Content Questionnaire 34, 234; Job Demand-Control model 234; Job Stress Survey 34; satisfaction 232-6; skill discretion 34, 234, 236, 249; strain 234 ; temporary 240, 266; see also career line; work

JYLS (Jyväskylä Longitudinal Study of Personality and Social Development) 6, 10; assessment methods 22-36; baseline data/study 14, 22; evaluation of choices 39-40; funding 12-15; goals of the study 9; major stages 14; see also data analysis; data collection; sample

Karolinska Scales of Personality (KSP): vulnerability trait $31,91,95-7$, 101, 159, 205

Kindergarten Study 5-6, 23, 68-9, 169

labor market 113, 136, 176, 178, 180, 224, 226-7, 230-1, 258, 268

latent profile analysis (LPA) see data analysis, profiles

legislation 12, 72, 74, 105, 158, 181, 191, 207, 258; Act of Child Custody 72

leisure activities $28,30,33,35,80,82-3,86$, 117, 144, 157-8, 168, 248-9, 257, 265; see also free time

life events 29-30, 105, 112, 137-40, 150; Life Event Inventory 29; nonnormative 112, 138; see also Life History Calendar

Life History Calendar 29-30, 181

life satisfaction 109-10, 139, 142-7, 149, 205, 258, 265; Life-Satisfaction Scale 33; see also satisfaction with; well-being

Life Situation Questionnaire (LSQ) 29-30, 32,189

life structure $32,106,117,119,121-4,126$, 260; Life Structure Inventory 32-3; see also domains of life 
lifestyle 28, 76; see also identity, domains; style of life

life success $91,181,185-7$

living conditions see family

Loner 75, 78-85, 175, 197; see also developmental paths/lines; style of life

longitudinal study 3-15; definition of 3; recommendations for 40-2; see also crosssectional study; data collection; JYLS

Loser 75, 77, 79-85, 107, 159, 175, 198, 265-6; see also developmental paths/lines; style of life

marital status $20,122,128,207-8$

marriage: intact 210; quality of 211; see also common-law partner; divorce

maturation 9, 43, 58, 91, 105-7, 182, 188, 214-15; hypothesis 187

medical examination 22, 29, 35; laboratory tests 35 ; saliva samples 40 ; Symptom

Check List 35; see also health

medicine, use of 199, 201

mental well-being 33-4, 36, 142-3, 148-9, 157, 248, 260, 263, 269; construct of 143; mental health/ill-health $34,123,143$, $185,226,239,240,242$; personality traits associated with $145,149-50$; tripartite model of 142-3; see also well-being meta-analysis $66-7,145,166$ metabolic syndrome 200-3, 234, 257 Meta-Evaluation Scale (MES) 32 metaphor, metaphorical xviii, 157, 259, 262, 269

Meta-Regulation Scale (MRS) 32

middle age/adulthood/midlife 19-20, 40, 63, 65, 95-6, 110, 117, 119-21, 158, 180, 197, 200, 213-14, 218, 220-1, 228, 230, 239, 244, 248, 251, 255-60, 267, 272; definition 255 ; "sandwich generation" 219, 222, 257; see also adulthood midlife transition/crisis 29, 258-9 missing information see sample model/framework of/for: aggression 5, 23, 69,161 ; aggressive and nonaggressive behavior, impulse control/twodimensional model 6-8, 10, 47, 49, 53, 67, 76; emotional and behavioral regulation 47-8; individual differences in social and psychological functioning, integrated 262-8; personality and psychological functioning 146-51, 201, 236; spheres of life 157; Unfolding of Socioemotional Behavior (USB) 49-52, 58-61, 65, 71, 74, 80, 82-3, 151, 159, 200, 228 monotony avoidance 31, 95-6, 148-9, 236

mood, positive/negative 53,143-4, 237; Brief Mood Introspection Scale (BMIS) 32

motherhood 128-9 early $174,179,225-7$; see also parenthood

Multidimensional Peer Nomination Inventory 51, 53

NEO-FFI (NEO-Five Factor Inventory) 31, 87-8, 91-3, 101, 182, 205

NEO-PI (NEO-Personality Inventory) 14, 22,31

neuropsychological basis/problems 22, 188 neuroticism 31, 48, 53, 77, 80, 86-9, 91-6, 101-3, 121-3, 134-5, 145-6, 149-51, 182-3, 209, 212, 214, 217-18, 229-30, $239,244,246-7,259,262-6$

neurotransmitters $149-50$

nonaggressive $6-9,14,21-3,42,69-70$, 169-70

noncognitive factors/capacities/skills 116, 271

noncompliance 199, 262

nonprosociality $174-5$; see also prosocial norm-breaking behavior $165,175,182-3$, 187,265

nurturance, nurturing 215-18, 221, 256, 263 ; see also parenting

obesity 201-2, 257; see also body mass index; overweight

occupational career 29, 130, 214; attainment 180, 228-9, 236, 263; see also career line/ path; identity, domains; occupational status, participants of

occupational status, participants of 20 , $78,80,95,116,132,185,203,223-32$, 241, 266: blue-collar 99, 184, 186, 223-4, 226-7, 233-5, 237, 245, 248, 256; white-collar 17, 113, 223-4, 226-7, 232, 234-5, 237, 244, 248-9, 251, 256; see also socioeconomic status of the family of origin

occupational well-being: types of 235; see also well-being

offenders: adolescence-limited 181-8; adultonset 181-8; nonoffenders 167-8, 181-8; persistent, life-course persistent $181-8$ offense categories see criminal offenses Offspring Study/sample 14, 22, 51, 54-5, $70-1,73,185,217,243$

openness to experience $88-9,92-5,101-2$, $123,145-6,151,217,230,236,239$ 
optimism 79, 116, 135-7, 146-8, 204, $226-7$

orientation to: home 78,81 ; indifference 76-78, 81; peers 77-9, 81, 194; responsibility $76-7,81$; see also developmental paths/lines; style of life overcontrolled 50-1, 61, 75-6, 78, 80, 86, 88-91, 94, 97, 124, 129, 133, 147-9, 236, 260, 264, 267-8; see also clusters; developmental paths/lines; profiles; style of life

overweight 202, 257, 267; see also body mass index; obesity

parenthood: early $107,179,225-7$; timing of 107-9, 179; see also adult transitions; motherhood

parenting: child-centered 72, 81-5, 107, 135, 160, 163, 184, 188, 196, 198, 206, 213, 215-18, 220-1, 226, 239, 242, 256; concerned 81,83; parent-centered 74, 81-5, 160, 196, 216; stress 34, 213-14, 216, 218, 242-3, 262-3, 266; Parenting Stress Scale 34; unconcerned 81, 83; see also identity, domains; identity, parental; nurturance participation rate see sample partner relationship see relationships, intimate/partner

patterns of behavior 4, 10, 21, 58, 71, 180; see also adult transitions; clusters; data analysis

peer nomination $16,18,21-7,42,51$, 55-6, 64-8, 71, 76, 97-8, 167, 170, 190-200, 228

peer relations/relationships $155,172-3$, $177-8$

person: as autobiographical author $62-3$, 125-41, 259; as motivated agent 62-3, $105-24,130,259$; as social actor 62-104, 130, 259

Personal Control over one's Development Questionnaire see control over one's development/life

personal goals 117 ; clusters for 122-3 personality: definition of 62 ; development of 43, 62, 125, 156; layers/levels of 62; see also developmental paths; growth; maturation; model, personality and psychological functioning

personality, approaches: idiographic 90 ; person-oriented $36-7,65,71,85,87$, 90, 145, 170-1, 260; variable-oriented 36-7, 65, 85, 87, 260; see also holism/ holistic personality inventory $14,22,30-1,42,76$; see also EPQ; NEO-FFI; NEO-PI

personality profiles see profiles personality trait see trait

Personal Life Investment Schedule 33, 117,122

personal styles see clusters

person-situation controversy 68 ; see also situation-specific behavior

phenomenological psychological analysis/ method 125-9, 269

PISA study 11, 184

Pleasure Ranking task 33, 117

political/politics $13,29,32,79-80,83$, 118-19, 123, 130-2, 233, 248, 271; see also identity, domains; values

Population Register Centre (PRC) 12, 18 positive development 10, 91, 97, 115, 159, $163,213,219,226$

positive functioning $33,143,158-9$, 162, 271

positive psychology $6,158,203$

poverty $177,180,256,267$

prevention, suggestions for $187-8$

prime time 255

problem behavior: clusters/patterns 171-4; externalizing/internalizing 48, 51, 56, 61, $159,165,194,217,243$; theory of 176

problem gravitation hypothesis 176

problems, accumulation of: chain of adversities 176; concentration of problems 176; co-occurrence 175-6; multi-problem predictors of 171 ; polarization 176, 180; strands of risk factors 176-7; see also risk factors profiles: analysis $37,88,235,244$; occupational well-being types 235-6; personality profiles $88-91$; profile groups 92-8, 146-9, 200-1, 212, 235-6, 240, 246; trajectories of work-family conflict 244; see also brittle; overcontrolled; resilient; undercontrolled

prosocial behavior/prosociality $6,162,171$, 174-5

protective factors, definition of 159 ; double function 163-4, 214, 239; study of 162-3; see also resource factors; risk factors

psychological distress 236, 239-40, 242-6 psychological functioning, definition of 156-7; indicators of 146-8; model of 148-51 problems 159 ; relation with social functioning 160, 267-8 psychological well-being see well-being 
psychosomatic symptoms 136, 185-6, 198-9, 201-2, 241

question series: reactive aggression 69; proactive aggression 69

reconciliation of work and family 35, 247-8 recovery from work: psychological detachment 249; Recovery Experience Questionnaire 249; relaxation 249 reflections 39-44; self-reflections 130, 157, 229 register data 22, 35-6, 166, 180-1 regrets 139; Regrets scale 33 relationships, intimate/partner 34,122 , 130, 158, 179, 185, 209-10, 213, 214, 242; Conflict Tactic Scales 34; Dyadic Adjustment Scale 34, 209; see also attachment; identity, domains; jealousy relatives 162,251

religion/religious 80, 119; activity 20; see also identity, domains

resilient 50, 52, 60-1, 75-80, 86-91, 94, 97, $114,122,129,133,136-7,144,146-8$, 151, 198, 200-1, 212-13, 228, 236, 240, 248, 260, 264-5, 267-8; in psychiatry 61, 139-40; see also clusters; developmental paths/lines; profiles; style of life resource factors, definition of 159-60; double function 164, 239; see also protective factors; risk factors

retention rate see sample

Reveller 75, 77, 79-84, 115, 159, 175, 194, 200, 266; see also developmental paths/ lines; style of life

risk factors 51, 61, 139-40, 158, 162, 170, 177-80, 187, 195, 196, 201, 216, 225-7, 241, 257, 266-7; definition of 159; inner strand of 177-8, 180; outer strand of 177-8, 180; see also protective factors; resource factors

risk-taking behavior 86, 182, 201, 225, 259

sample: attrition 17, 19-21, 41, 192; choice of 16; effective 17-18; eligible 17-18; initial 14, 18-19; intensively studied 21; missing information 37, 42; participation rate 17-19,92; representativeness of/ age cohort $12,17,20,41,113,119$, 207-8, 223-4, 250; retention rate 18-19; subsample/extreme groups 21, 69 satisfaction/dissatisfaction with: financial situation 33; free/leisure time 33, 112 , 136, 144, 250, 256; friendship 33, 251; housing 33 , 136, 144; income 144 ,
232-3; intimate relationship/partner 33-4, 144, 209-10, 246, 263, 266; job/ work/career/occupation 3, 33, 112, 117 , 136, 144, 224, 231, 234-6; life 30, 77-8, 80, 111-12, 127, 139, 142-4, 159-60; sources of $117,119,128,158,233$; Satisfaction with Life Scale 33; see also life satisfaction; well-being

school maladjustment 37, 70, 77-8, 162-3, 262

school starting age 16,112

school success $20,26,27,79,98,111$, 113-16, 123, 134-7, 160-3, 170-8, 182-3, 199, 205, 225-7, 229, 238, 263-7; see also grade point average

self-control 8, 10, 31, 47-51, 54, 56, 60, 70, 79, 103, 161, 172, 175, 187, 205, 238, 266, 271; Self-Control Check List 31; Self-Control Inventory 31; Self-Control Rating Scale (SCRS) 51, 56; Self-

Control Schedule (SCS) 32

self-determination theory 63

self-efficacy 60,177

self-esteem 32, 91, 110, 130, 133, 135-7, 140, 159-60, 186, 205, 212-13, 217, 228-9, 239, 243, 260, 263, 265; Self-

Esteem Scale 32, 136

self-rating 24, 26, 30, 69, 96, 98, 201

self-reflection 130, 157, 159, 260

self-regulation 10, 49, 54-60, 64, 71, 73-4, 76, 82-3, 85, 87, 91-2, 96-7, 101, 104, $116,151,158,160,162,168,170-2,178$, 180, 183, 185, 187, 196, 200-1, 203-6, 210-11, 213, 216, 220, 226, 239-40, 256, 260, 267-8, 271-2; aspects assessed 25, 161-3, 174-5, 182, 194, 228-30, 238-9, 243,265 ; definition of 50, 156; see also behavior regulation; emotion regulation

SEM (structural equation modeling) see data analysis

Sensation Seeking Scale (SSS) 31, 76, 182 sense of coherence 34, 134-6, 229:

Orientation to Life Questionnaire 34,134

sense of control over one's development/life see control over one's development/life situation-specific behavior 48-9, 68 smoking cigarettes 28, 30, 77-80, 85,

150, 170, 196-201, 205, 241, 257, 266;

mother's smoking 195

sociability $32,79,96,101-3$

social activity see activity/passivity

social capital 184

social development, definition of 156-7 
social functioning: definition of 155-7; in relation to $263-5$; integrated model of 267-8; positive 156; problems in 165-88; processes in positive functioning 158-64; successful 267-8

socialization experiences/process 51 , 58-9, 102, 155, 159-60, 269; see also individuation; Karolinska Scales of Personality social mobility $114,226-7$

social relationships: breadth of network 161-2; depth of relations 161-2 social well-being see well-being socioeconomic exclusion 180 socioeconomic status of the family of origin, background, parents' 20,81 , 83-5, 99-100, 110-11, 113-16, 132, 139, 160-1, 178, 184-5, 196, 203, 205, 220, 225, 230, 240; see also occupational status socioemotional behavior $24,41,63-8$, $114,151,170,183,259,262$; across generations 71-3; aspects assessed 24-6, 91, 96; definition of 49, 58-9; offender groups 182-3; partner relationship 210; predictors of 96-7, 100-4, 194, 197, 199200, 205, 228; theoretical frameworks 47-52; see also temperament socioemotional development 49-58, 74-6, 80, 158-9, 262-5; definition of 49, 64; see also developmental paths/lines spheres of life: comparison of satisfaction with 144; functioning in 157-8, 259-61; imbalance between 136; model 157; see also domains of life

spillover see work-to-family spiritual 33, 95, 120-1, 204, 269-70 Spiritual Meaning Scale 33 stability: absolute $37,43,92,95$; definition of 43,67 ; relative $36,43,66-8,92,95-6$, 136; structural 71; see also career line; continuity; emotional lability/stability statistical methods, software see data analysis Statistics Finland 12, 20, 113, 207

Strategic Attribution Questionnaire 32, 231 stress: feeling of 25, 34, 198-200, 240; see also economic strain; job, exhaustion; parenting, stress; work

style of life/lifestyle 28, 51-2, 58, 65; definition of 49; triangular model 49; unfolding of the styles of life 75-80; sustainability/sustainable 39, 41, 122, 206, 272; see also (for late adolescence) Loner; Loser; Reveller; Thriver; (for adulthood) brittle; overcontrolled; resilient; undercontrolled; (for anything else) clusters; developmental paths/lines; profiles synthesis $49,65,104$

teacher rating $18,22-7,64-8,71-3,76,97$, 101, 167, 199-200, 205, 228; choice of 42; Teacher Questionnaire 27; Teacher Rating Form of the Multidimensional Peer Nomination Inventory 55-6; teacher's predictions $115-16$

television viewing $35,53,79,111,169,249$, 265 ; see also violence viewing

temperament 9, 32, 58-9, 62, 100-4, 149-50, 159, 181; effortful control 32, 60,101; negative affectivity/emotionality 32, 101; surgency 32, 101; see also Adult Temperament Questionnaire temperance definition of 203-4; intemperate 205-6; temperate 204-6 theory of mind 62

Thriver 75, 77-85, 107, 113-14, 197, 200, 264; see also developmental paths/lines; style of life

trait: personality trait 5, 9, 30-1, 62, 85, 87-97, 101-4, 121, 144-51, 212, 217, 230, 235-6, 239, 244, 246-8, 262; see also agreeableness; conscientiousness; extraversion; Karolinska Scales of Personality, vulnerability trait; neuroticism; openness to experience trajectory see data analysis transition see adult transitions truancy $27,78,161-2,165-6,170,175,178$, $188,200,238$

turning point $29,126,137-41$

undercontrolled 50-1, 61, 75-8, 80, 86-92, 94, 96-7, 115, 124, 129, 133, 148-9, 151, 194, 198-200, 236, 260, 264-6, 268; see also clusters; developmental paths/lines; profiles; style of life

unemployment 78-80, 83, 85, 112, 115, 122, $124,139,175,179,185-6,224,230,237-43$, 256, 263, 265; long-term 37, 162-4, 237-9, 241, 243, 256; rate 20, 237, 256, unifying life theme 122,124 unique, uniqueness 10, 42, 44, 62, 90, 103, 106, 126-9, 156, 260, 269-72

University of Jyväskylä 4, 13, 29 upbringing see parenting USB (Unfolding of Socioemotional

Behavior) see model use of alcohol 20, 28, 35, 77-80, 110, 127 , 159-60, 182, 188-97, 201, 204-5, 209, 
226-7, 257, 266; assessment 28, 30, 35; quantity-frequency table 35,189 ; see also alcoholism; drinking alcohol

validity 41-2, 64-5; concurrent 65-6; predictive $69-70,172$; structural $71,74,90$

values: in middle age 120-1; Personal Value Task 32, 118; Schwartz Value Survey 33, 120; see also domains of life; life structure violence viewing 169, 264; see also television virtue 62, 203-4, 206

volition/volitional 48

way of life 111-12, 258, 265; definition of 111

well-being 22, 33, 95, 150, 199, 201, 233, 243 , $245,256,270$; concept of 142; emotional 33, 142-5, 151; occupational 235-6, 264, 266; personality and 142 ; psychological 33 , 110, 142-8, 159-62, 185-6, 205, 212, 215, 229, 240, 258, 265; Scales of Psychological Well-Being 33; Scales of Social Well-Being 34; social 34, 142-4, 216, 258; see also mental well-being women, types of: brittle 86 ; conflicted $85-7$; divorced women 128-9; individuated 87, 124, 136; married women 128-9; single women 128-9; traditional 87, 124; see also cluster; developmental paths/lines; profiles

work: demands at 34,244 ; hours 84,233 , 247; part-time 233,247 ; as a profession 231-2; shifts 85,233 ; significance of 34 ; as a sphere of life 223-51; stress 248, 256; see also job; well-being, occupational

workaholism 35, 233, 235; Dutch Work Addiction Scale 35

work engagement 35, 233; Utrecht Work Engagement Scale 35, 235

work-family balance 157, 243-6; role enhancement theory approach 245-7; stress theory approach 243-5; workfamily enrichment (WFE) 246; see also reconciliation of work and family work-to-family interface 245-6; conflict (FWC) 244-5, 248; conflict (WFC) 244-6; family-to-work interface 246; spillover 245-6, 256; see also profiles 